PACIFIC LINGUISTICS

Series D - 83

\title{
DICTIONARY OF PLANT NAMES IN THE LESSER SUNDA ISLANDS
}

Jilis A.J. Verheijen, SVD

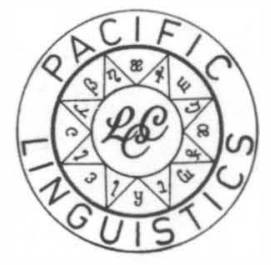

Department of Linguistics

Research School of Pacific Studies

THE AUSTRALIAN NATIONAL UNIVERSITY 
PACIFIC LINGUISTICS is issued through the Linguistic Circle of Canberra and consists of four series:

SERIES A: Occasional Papers

SERIES B: Monographs
SERIES C: Books

SERIES D: Special Publications

FOUNDING EDITOR: S.A. Wurm

EDITORIAL BOARD: K.A. Adelaar, T.E. Dutton, A.K. Pawley, M.D. Ross, D.T. Tryon

EDITORIAL ADVISERS:

B.W. Bender

University of Hawaii

David Bradley

La Trobe University

Michael G. Clyne

Monash University

S.H. Elbert

University of Hawaii

K.J. Fran lin

Summer Institute of Linguistics

W.W. Glover

Summer Institute of Linguistics

G.W. Grace

University of Hawaii

M.A.K. Halliday

University of Sydney

E. Haugen

Harvard University

A. Healey

Summer Institute of Linguistics

L.A. Hercus

Australian National University

John Lynch

University of Papua New Guinea
K.A. McElhanon

Summer Institute of Linguistics

H.P. McKaughan

University of Hawaii

P. Mühlhăusler

Bond University

G.N. O'Grady

University of Victoria, B.C.

K.L. Pi e

Summer Institute of Linguistics

E.C. Polomé University of Texas

Gillian San off University of Pennsylvania

W.A.L. Stokhof University of Leiden

B.K. T'sou

City Polytechnic of Hong Kong

E.M. Uhlenbeck University of Leiden

J.W.M. Verhaar

Divine Word Institute, Madang

C.L. Voorhoeve

University of Leiden

All correspondence concerning PACIFIC LINGUISTICS, including orders and subscriptions, should be addressed to:

\author{
PACIFIC LINGUISTICS \\ Department of Linguistics \\ Research School of Pacific Studies \\ The Australian National University \\ G.P.O. Box 4, Canberra, A.C.T. 2601 \\ Australia
}

Copyright (C) The Author

Typeset by Jeanette Coombes

Printed by A.N.U. Printing Service

The editors are indebted to the Australian National University for assistance in the production of this series

This publication was made possible by an initial grant from the Hunter Douglas Fund
First Published 1990

Maps drawn by Theo Baumann

Bound by F \& M Perfect Bookbinding

ISSN 0078-7566

ISBN 0858833999 


\section{TABLE OF CONTENTS}

FOREWORD iv

O. INTRODUCTION 1

1. VERNACULAR - TAXONOMIC LISTS 3

1.0 The structure of the lists 3

1.1 Rongga - taxonomic 7

$\begin{array}{lll}1.2 & \text { Ngadha - taxonomic } & 17\end{array}$

1.3 Endé - taxonomic 46

$\begin{array}{lll}1.4 & \text { Lio-taxonomic } & 59\end{array}$

$\begin{array}{lll}1.5 & \text { Bima - taxonomic } & 79\end{array}$

$\begin{array}{llr}1.6 & \text { Sumba-taxonomic } & 90\end{array}$

$\begin{array}{lll}1.7 & \text { Sawu - taxonomic } & 134\end{array}$

$\begin{array}{lll}1.8 & \text { Sika-taxonomic } & 142\end{array}$

1.9 'Solor' - taxonomic 157

$\begin{array}{ll}1.10 \text { Kédang - taxonomic } & 178\end{array}$

2. TAXONOMIC - VERNACULAR IN SOME 19 LANGUAGES 186

3. GROUPS AND FAMILIES WITH GENERA 251

APPENDIX I: INFORMANTS 259

APPENDIX II: SYNONYMOUS TAXA 260

MAPS 1-4 263

$\begin{array}{ll}\text { BIBLIOGRAPHY } & 267\end{array}$ 


\section{FOREWORD}

After writing the Dictionary of Manggarai Plant Names I felt challenged to prepare the present study.

In the fifties and early sixties I studied written sources and started collecting plants and their names in Ngadha. In the seventies and eighties I did occasional (but during holidays also well-planned) short research in the field outside Manggarai. The keen interest of several scientists inspired me to perform this task.

Though my time became very precious and other projects were pressing, I tried to finish this work as well as possible in the circumstances. A special consideration in doing so was the conviction that no-one else would take on this study in the foreseeable future, and that, because of an unstoppable dwindling in knowledge of wild plants, its execution would become almost impossible after a few decades. The interest shown by Pacific Linguistics in this work was a real moral support for me.

I am much obliged to Greshoff's Rumphius Fonds and Foundation Malesiana that partly enabled me to travel in Central Flores and Sumba in 1974. The latter Foundation again supported my fieldwork in eastem Flores in 1987. The Society of the Divine Word (SVD) procured the bulk of the expenses for the working out of my materials. In this the Society was faithful to its founder, Fr Arnold Janssen, a promoter of linguistic and anthropological studies by his missionaries, who himself was the author of a botanical prize essay (1858) at the University of Münster under the title 'Über die Monströsitäten bei den Gewächsen, zumal in den Blüthentheilen, ihre muthmaszlichen Ursachen und ihre Wirkung auf die Verrichtungen der Gewächse'.

I owe a great debt of gratitude to Mrs J. Ezard of Pacific Linguistics. She kindly corrected some serious and many smaller mistakes, made valuable suggestions, and ameliorated the inconsistent internal format of my manuscript.

Finally, I do hope that the given materials, which I did not obtain and work up easily, will be appreciated and used by agronomists, foresters and especially by etymologists.

Ruteng, 1 February 1988 


\section{INTRODUCTION}

\subsection{GENERAL REMARKS}

I would like first to direct the reader's attention to the Introduction in my Dictionary of Manggarai Plant Names (Verheijen 1982a:1-8). There many questions concerning the framework of a study similar to the present one are elucidated.

I do not need to repeat here my arguments in favour of the usefulness of a work like this for linguists, botanists, agriculturists and foresters.

My method of doing research both in the field and on the writing desk will become clear in the remarks with each of the vernacular-taxonomic lists (sections 1.1-1.10). In the Sumba list (1.6), especially, I discuss the problems and difficulties at great length. My remarks there can be regarded as a typical description of my method of working both by collecting in the country and by studying written records.

\subsection{LINGUISTIC PROBLEMS}

(a) I have succeeded in giving data from almost all languages of the so-called Bima-Sumba Group. Of course, there are great quantitative and qualitative differences. These languages are regarded by Blust (1980:11-12) as belonging to Central Malayo-Polynesian (CMP). Sika, East Flores (Solorese languages) and Kédang do not belong to the Bima-Sumba languages, from which they conspicuously differ by the inverted genitive and a kind of conjugation in many verbs. The present vocabularies of plant names may shed light on one minor aspect of the connection of the two groups by comparing their floral vocabulary.

(b) The orthography

My first principle is that the spelling should be recognisable by speakers of the languages in question. In the second place it should, within each language, be as consistent and simple as possible. These desiderata sometimes conflict with each other.

Since we have to consider many dialects and languages it is, of course, impossible to use consistent phonemic spelling. Every serious student of the lists in section 2 will observe that the pronunciation of the same symbols in the same line may be different. I give some examples.

As speakers of Bimanese and Sumbanese do not pronounce a pepet (schwa), the phoneme /e/ is the unrounded mid front vowel, whereas in other languages it is the pepet contrasting with /é/. And, because in Kédang the contrast between the unrounded higher and lower mid-front vowels is phonemic, both /é/ and /è/ are written in that language.

Similarly $r$, which is used in the Loura (Sumba), Endé and Rongga languages, probably represents different sounds.

On the other hand, the same sound is sometimes written with different symbols; for example, glottalised $d$ is represented in Bima and Sumba (Anakalangu dialect) by d, in Ngadha by dh, and in Sawu (Walker) by $\mathrm{d}^{\prime}$. 
The following are consistently used for the same sound throughout section 1 :

- (awa) for the smooth onset.

('awa, wa'a, wa') for the glottal stop. In Sika it precedes a glottalised r, 1 and w.

$/ \mathrm{gh} /$ is the voiced pharyngal spirant commonly used in western Flores languages.

\subsection{THE MAPS}

The maps are adapted to the special needs of this study and are not for general use.

The delimitation of the languages and dialects in Flores, except for those of the Solor Group, is only tentative, since there are no reliable maps available. 


\section{THE VERNACULAR - TAXONOMIC LISTS}

\subsection{THE STRUCTURE OF THE LISTS}

1.0.1 The ten lists below differ considerably from one another. That is a consequence of the variable availability of data, and also of the way in which each was obtained.

\subsubsection{THE POPULAR CLASSIFIERS}

In many languages people like to use classifiers which precede the proper plant names. This device marks the plant as a tree, a vine, a herb, a grass, a weed or a thomy plant. Where these are used, they are indicated by abbreviations of the terms, listed in the preliminary remarks for each language.

Their use is sometimes necessary, especially if the proper names are synonyms. In other cases an inseparable compound is formed, comparable with English 'silver-weed' or 'quaking-grass'. The attention given to classifiers is not equal in all these cultures, and I could not always pay sufficient attention to them. I have written comprehensively about the classifiers in Manggarai (Verheijen 1982b:3-4).

1.0.3 The vernacular names, sometimes preceded by a classifier, are alphabetically ordered in the second column. The common spelling of these names is dealt with under 0.2.(b). However, symbols that are specific to a certain language are listed in the preliminary remarks for each language.

1.0.4 In the next column, the localities where the name in question has been found may be given (in abbreviated form and listed in the preliminary remarks, also). Of course the range of use of the word may be much wider than indicated; each locality usually has its own dialect. The reader is referred to the maps.

1.0.5 In the fourth column the sources of identification, or of the first description, are sometimes entered. There are two groups of sources. In the first place are those authors, mostly linguists, who have specialised in a particular language. They have not usually used original scientific names. The other group is formed by the institute or persons who gave the identifications which are cited throughout the lists.

The abbreviations of the latter group are:

dft Hildebrand; see Bibliography

$\mathrm{H} \quad$ Heyne; see Bibliography

R Irinus Ros (my assistant)

RV Ros together with Verheijen

V Verheijen

3115 a number indicates the plant in my herbarium of which a duplicate is conserved in the Rijksherbarium at Leiden, Holland, where it was identified. 


\subsubsection{THE LAST COLUMN}

(a) The term 'taxonomic' is not quite adequate since descriptions are also given. In order to afford the users of this study the opportunity of easier further research, all the vernacular names I have encountered which are not yet identified are entered with a brief description or with a simple meaning.

In the texts of the descriptions the following abbreviations and signs are used:
a. and
cj. conjecture (the author's, concerning a botanical name)
cp. compare
cult. cultivated
fl. flower
fr. fruit
1. leaves
v. vide, see
w. with
? accuracy of following name or identification in doubt
$=$ the same as
$?=\quad$ probably the same as
smooth onset immediately before an initial vowel

glottal stop, used before the initial vowel of the syllable and finally after the last vowel.

Both ' and ' do not influence the alphabetical order. If two words only differ by 'or ' the form without them is placed first.

The abbreviations for specifically botanic terms are:

cf. confer, similar to

f. forma, a subdivision of a species

sp. $\quad$ species

spp. $\quad$ species (plural)

ssp. subspecies

var. varietas, a subdivision of a species

The remark 'many attributes' in this column refers to the vernacular name in the second column.

(b) The taxa, especially those of spermatophytes, are employed in accordance with the latest nomenclature found in Flora of Java. Therefore I use Bombax instead of Gossampinus and Luffa aegyptiaca instead of Luffa cylindrica. Incidently, a still later mutation was entered, such as Spondias malayana instead of Spondias pinnata. Incorrect spellings, like Areca catechu are rectified there too. The list of synonyms (Appendix III) will solve almost all doubts in this respect.

(c) The degree of accuracy of the given scientific identifications is far from consistent.

Complete accuracy is only possible if a plant with a correct vernacular name is identified by a qualified botanist. A reasonable degree of certainty is only attained if well-known plants are observed in nature by botanists or serious amateurs, or identified from herbarium samples or from decisive descriptions. 
It is well known that (vernacular) plant names often have many synonyms within the same dialect, and still more in cognate dialects - cp. Verheijen 1984:28. It is not always possible to adequately check the accuracy of these synonyms. For example, it appears that in the same article (p.29) a homonymous name was used for different plants.

Such mistakes (from a botanical point of view) in vernacular nomenclature are mostly explicable by the similar external appearance of plants, by their common properties and by the plants' use for the same purpose. Some of them, however, are for Schmutz (1977-80, and by personal communication) and me pure riddles. Our common experiences in the intensively researched Manggarai (Verheijen 1982b: 80ff.) prove this abundantly.

These factors of both polyonymy and polysemy can easily become sources of mistakes in identifying plants, and I too fell into this trap, discovering too late the existence of a homonym. In many cases in that work I put question marks, or otherwise indicated a conjecture, but also where no doubt was expressed mistakes did occur, besides slips of the pen. Therefore, if plants are to be used for medical purposes, these names should not be relied on.

People who like to be accurate and sure - among whom I modestly count myself - are only with great reluctance prepared to release data of this kind. I have, however, taken this risk as I know by experience that later findings often affirm serious assumptions and, even where doubts linger, the data can often prove to be very useful.

An example of difficulties with synonymous names is found in Onvlee's dictionary, in the following Kambera entries and their cognates in East Sumba dialects and in the West Sumba dialect of Wewewa:

$\begin{array}{lllllll} & \mathrm{Kb} & \mathrm{Mi} & \mathrm{Lw} & \mathrm{Ak} & \mathrm{Mb} & \mathrm{We} \\ \text { 1. } & \text { wengu } & \text { wengu } & \text { wengu } & \text { yengu } & \text { wengu } & \text { wengo } \\ \text { 2. } & \text { wíngu } & \text { wíngu } & \text { wengu } & \text { engu } & \text { yengu } & \text { (w)engo } \\ \text { 3. } & \text { yatangu } & \text { yatangu } & \text { yatangu } & \text { yengu } & \text { engu } & \text { (w)engo }\end{array}$

1,2 and 3 have in common that they are trees with edible fruit. 1 and 3 are trees that prefer a moist soil.

\subsubsection{THE ABSENT LISTS}

(a) Manggarai. In this language the Dictionary of Manggarai Plant Names (Verheijen 1982a) is available. Therefore no data are entered here.

(b) For different reasons no vernacular-taxonomic lists are given for the following languages, although they are entered in the taxonomic-vernacular section.

(i) The Manggarai dialects in Riung (FEM). They were studied, and plant names noted by me together with Mr Dadu, in 1976. They render interesting materials for the history of introduced plants.

(ii) Komodo. In Komodo: het eiland, het volk en de taal plant names are also entered in the wordlist, besides a taxonomic-vernacular list (Verheijen 1982b: 234-238). In Pulau Komodo: tanah, rakyat, dan bahasanya (Verheijen 1987: 260-266) this list is somewhat enlarged. 
(iii) Rembong. In Bahasa Rembong I (Verheijen 1977) the dictionary contains plant names; a taxonomic list is to be found in vol. III (Verheijen 1978: 89-99).

(iv) Lists are not given for the following 'languages' of the Manggarai Group: Waérana, Kepo' and Razong, nor for the Far-east Manggarai group of dialects. Mr Ros collected names in the Waérana language in 1973, in Kisol in 1970, and together with informants and myself in Mukun in 1975. Names from the Razong language were collected by myself in Kepo', with informants from the enclave of Razongkoé.

The 'languages' of Waérana and Razong both show primitive traits, as in most dialects of the Ngadha-Lio Group, for example a quinary counting system and the use of za'o 'I'.

We (Ros and Verheijen) studied the Kepo' in Watudopak in 1975, and later I was able to check them in Kepo' II, an enclave of early migrants near Waérana.

(v) From the Nagé language fewer names were collected from five of its dialects: Tédamudé, Bo'awaé and Raja. Our data were obtained without collecting plants and without identifying them in the field. These names belong mostly to very common plants.

(vi) From the Palu'é language, which is most cognate with Lio, only a hundred-odd names were collected during a two hours' stay. This happened at Nangahuré, a settlement of immigrants on the north coast near Ma'uméré. I had great difficulty with the pronunciation of the language, but as the names collected are of interest, I have very tentatively ventured to write them down. 


\subsection{RONGGA - TAXONOMIC}

\subsubsection{PRELIMINARY REMARKS}

(a) The language

(i) The Rongga district is situated in south-eastern Manggarai, but the \pm 4000 inhabitants speak a language that belongs to the Ngadha-Lio Group. As is the case with the cognate languages, all syllables are vocalic, viz. have no final consonants. The particle of negation mbiwa 'not' is characteristic.

(ii) Orthography

' $r$ ' corresponds with Manggarai / $/ \mathrm{s} /$ Rembong /j/ or $/ \mathrm{z} /$ and Kepo'/r/. It is the reflex of AN *d, *D and $*_{j}$ (an exception is rita). It seems to be a kind of alveolar flap and it approaches more ' $r$ ' than ' $z$ '. Some people spell it ' $z h$ '.

(b) Research and identification

In $1964 \mathrm{Mr}$ Ros made plant collections in the vicinity of Kisol. He did so again in 1970 and 1974, giving much attention to the vernacular names.

When finishing this manuscript I was able to note the names of many common plants that were not collected, and (therefore) not yet listed. At the same time the list that was already prepared could both linguistically and botanically be checked and enlarged.

The Manggarai influence often affected the names of plants that were introduced in the last few centuries.

(c) Abbreviations of classifiers

$\begin{array}{lll}\text { a } & \text { are } & \text { vine, liana } \\ \mathrm{k} & \text { kaju } & \text { tree, shrub, woody } \\ \mathrm{kr} & \mathrm{karo} & \text { thom, spike, thomy } \\ \mathrm{p} & \text { pu'u } & \text { tree, stool }\end{array}$

\subsubsection{THE LIST}

\begin{tabular}{|c|c|c|c|}
\hline \multirow{2}{*}{$\begin{array}{l}\mathrm{a} \\
\mathrm{k}\end{array}$} & anggu(r) & & Passiflora foetida \\
\hline & apupu & & Eucalyptus urophylla \\
\hline \multirow{2}{*}{ k } & ara & & Ficus \\
\hline & ara helo & & Ficus \\
\hline k & baso & & Ficus ?punctata \\
\hline a & bé & $983 / 5$ & Heterostemma acuminatum \\
\hline & bega & & Ficus depressa \\
\hline & beto & & Dendrocalamus asper \\
\hline & bila & 4295 & Crescentia cujete \\
\hline & bojé & & Erechtites valerianifolia \\
\hline & bolo & & ?Cordia \\
\hline $\mathrm{a}$ & boro & 4298 & Merremia vitifolia \\
\hline
\end{tabular}




\begin{tabular}{|c|c|c|c|}
\hline & dao (aré) & & Ipomoea batatas \\
\hline & dao mboa & & Manihot esculenta \\
\hline \multirow[t]{13}{*}{$\mathrm{kr}$} & dapé & & Opuntia ?elatior \\
\hline & dara & 1042 & Caesalpinia bonduc \\
\hline & déndé & & Pterocarpus indicus \\
\hline & denga & 4333 & Solanum erianthum \\
\hline & déngé & & Fungi \\
\hline & déngé ta'i jara & & Fungi \\
\hline & déngé toro & & Fungi, Agaricales \\
\hline & dero I & & Trema orientalis \\
\hline & ?dero II & & Erythrina orientalis \\
\hline & dingga & $973 / 5$ & Gmelina elliptica \\
\hline & éjé & & Citrullus lanatus \\
\hline & éko lako & 4313 & Uraria lagopodioides \\
\hline & ela & & Coix lacryma-jobi var. ma-yuen \\
\hline \multirow[t]{26}{*}{ k } & fai & & Albizia chinensis \\
\hline & fangé & & Paederia scandens \\
\hline & felu & & Aleurites moluccana \\
\hline & gegé & & Leucaena leucocephala \\
\hline & ghédo & & Panicum miliaceum \\
\hline & ghela & & Scleria terrestris \\
\hline & ghera élo & & Acacia verheijenii \\
\hline & ghumbu & & ?Gymnopetalum cochinchinensis \\
\hline & ha'é & & Carica papaya \\
\hline & hanggo & & Sterculia oblongata \\
\hline & hapé léwa & $1036 / 8$ & Dysoxylum gaudichaudianum \\
\hline & héa & & ?Luffa aegyptiaca \\
\hline & hembu & & Cassia fistula \\
\hline & héu & & Areca cathecu \\
\hline & hinga mota I & & Hemigraphis \\
\hline & hinga mota II & & Elephantopus scaber \\
\hline & hobo bara & & pulse \\
\hline & hobo mité & & pulse \\
\hline & hobo naké & & Vigna unguiculata \\
\hline & hobo piko & & pulse \\
\hline & hobo tana & & Arachis hypogaea \\
\hline & hoi & & Alstonia spectabilis \\
\hline & hosu & & Setaria italica \\
\hline & ila & & Schizostachyum blumii \\
\hline & indo & & Spondias malayana \\
\hline & indo waé & & ?Lannea coromandelica \\
\hline \multirow[t]{2}{*}{ k } & jaga (jangga) polo & 4289 & Cassia siamea \\
\hline & jambu & & Psidium guajava \\
\hline
\end{tabular}


jata

kabé

kabé ala

kaju laka

kalo menado

kambi ndari

karo mori

kasi kamba

kasi téu

kasi waé

kaso fai

kaso haki

kawa

kelu

kembo

kembo lako

kemuni

kénga

ké'o I

ké'o II

kerara

keri

keropo

keta

?kéwa

kinggo

kipi

kiso

kobé

koja

kolé

kopi

koro ito

koro kénga

koro méré

kota

kou

ko'u

k kowa

kowé nitu

kua

k kué

kuku
$960 / 2$

4318

$1089 / 91$

4290

$1104 / 6,4320$

$956 / 8$

4292

4311
Cassia occidentalis

Ficus fistulosa

Ficus

?Gendarussa vulgaris

Erythrina subumbrans

Phyllanthus virgatus

Leea (angulata)

Hyptis suaveolens

Cymbopogon citratus

Ocimum basilicum

Mallotus ricinoides

?Acalypha

Anthocephalus chinensis

Benincasa hispida

Morinda tomentosa

Morinda ?citrifolia

Murraya paniculata

Dioscorea sarasinii

= ela

Ardisia javanica

Artocarpus altilis (seedless)

Imperata cylindrica

Rauvolfia

Planchonella obovata

Dioscorea pentaphylla

Emilia sonchifolia

Pometia pinnata

?Globba marantina

Ficus balica

Canarium vulgare

Calotropis gigantea

Coffea

Capsicum frutescens

Smilax zeylanica

Capsicum annuum

Canna coccinea

Dioscorea hispida

Peltophorum pterocarpum

Oroxylum indicum

?Phaseolus

Flagellaria indica

Timonius timon

Schoutenia ovata 


\begin{tabular}{|c|c|c|c|}
\hline & kumba lebo & & $=$ wunu kupu \\
\hline & kumba sawa I & & cp. Ludwigia \\
\hline & kumba sawa II & & Pneumatopteris callosa \\
\hline & kuné I & & Curcuma viridiflora \\
\hline \multirow[t]{7}{*}{$\mathrm{k}$} & kuné II & & Alangium villosum var. parviflorum \\
\hline & kupé I & $963 / 6$ & Piliostigma malabaricum var. acidum \\
\hline & ?kupé JI & $998 / 1000$ & Dregea volubilis \\
\hline & kuru kodé & & Piper \\
\hline & ku'u & & Psophocarpus tetragonolobus \\
\hline & kuwu & & Pterygota horsfieldii \\
\hline & labu & & Sechium edule \\
\hline \multirow[t]{10}{*}{$\mathrm{k}$} & lala & & Pithecellobium \\
\hline & lando kata I & & Embelia \\
\hline & lando kata II & $1081 / 2$ & Hybanthus enneaspermus \\
\hline & lando kura & 4307 & Celosia argentea \\
\hline & lando réndo I & 4324 & Euphorbia hypericifolia \\
\hline & lando réndo II & & Fungi \\
\hline & lanu manu & & Orchidaceae \\
\hline & lanu wawi & & Pyrrosia \\
\hline & laru & & Myristica \\
\hline & lasi nggedo & & Chrysopogon aciculatus \\
\hline k & laté I & & Dendrocnide \\
\hline \multirow[t]{7}{*}{$\mathrm{a}$} & laté II & & vine, itching \\
\hline & lawi & & Acanthaceae \\
\hline & lawi lura & & tree, tall \\
\hline & léa & & Zingiber officinale \\
\hline & léa laja & & Languas galanga \\
\hline & léa naro & & Zingiber \\
\hline & leba & & Phaseolus lunatus \\
\hline \multirow[t]{11}{*}{ a } & léké & 4319 & Entada phaseoloides \\
\hline & lélu jawa & & Gossypium \\
\hline & lélu késo & 4332 & Gossypium hirsutum var. hirsutum \\
\hline & lélu nipo & & Gossypium \\
\hline & lembé raé I & & Drynaria \\
\hline & lembé raé II & & Lecanopteris carnosa \\
\hline & lenga & & Sesamum orientale \\
\hline & lenga wau I & 967/9 & Josephinia imperatricis \\
\hline & lenga wau II & & $?=$ ta'i wawi \\
\hline & lépa & & Sorghum saccharatum \\
\hline & lepi I & 4288 & Moghania lineata \\
\hline \multirow[t]{2}{*}{ a } & lepi II & 4315 & Phylacium bracteosum \\
\hline & lepi III & & Desmodium \\
\hline k & lepi IV & & Prunus wallaceana \\
\hline
\end{tabular}




\begin{tabular}{|c|c|c|}
\hline lésé & $995 / 7$ & Uvaria rufa \\
\hline lesi & & Pipturus argenteus \\
\hline li'é léwa & 4301 & Heterostemma \\
\hline linda & & ?Ageraturn conyzoides \\
\hline loko & $1098 / 1100$ & Ixora \\
\hline loko waé & & Syzygium \\
\hline lola & & Amorphophallus ?campanulatus \\
\hline longgo mori & & $?=$ karo mori \\
\hline ?longko kodé & $1125 / 7$ & Cerbera manghas \\
\hline lui & 4331 & Ziziphus \\
\hline $\mathrm{ma}$ & 4680 & Phaseolus lathyroides \\
\hline maghi & & Borassus sundaicus \\
\hline mali & & tree or shrub \\
\hline mata mela & 4306 & Desmodium triflorum \\
\hline mbako I & & Nicotiana tabacum \\
\hline mbako II & & tree w. good timber \\
\hline mbako roja & & Anaphalis longifolia \\
\hline mbapa & & Vitex pubescens \\
\hline mbara & & Solanum melongena \\
\hline mbara polo & & Solanum \\
\hline mbara woni & & Lycopersicon lycopersicum \\
\hline mbari & $1134 / 6$ & Harrisonia brownii \\
\hline mbaru jawa & & Jatropha curcas \\
\hline mbawa & $1107 / 9$ & Santalum album \\
\hline mbeba & & Doryxylum spinosum \\
\hline mbela & & Schizostachyum brachycladum \\
\hline mbera & & Melia azedarach \\
\hline mberu & & Casuarina junghuhniana (pantai) \\
\hline mbési & & Cucurbita moschata \\
\hline mbeté & & tree, tall w. straight branchless stem (bee-tree) \\
\hline mbeté jara & & Corchorus \\
\hline mbila & & Dillenia pentagyna \\
\hline mboa & & Ceiba pentandra \\
\hline mboa lada & & Bombax ceiba \\
\hline mboro & & Corypha utan \\
\hline mboro & & tree w. white fl. and violet racemose fr. \\
\hline mboti & & Amaranthus lividus \\
\hline mbungé & $970 / 2$ & Daturametel \\
\hline mé'a & & Spilanthes iabadicensis \\
\hline melu & & Rhaphidophora \\
\hline mépo I & & Stachytarpheta indica \\
\hline mépo II & & tree w. red fl. \\
\hline mesa & & Derris \\
\hline
\end{tabular}


k

k

k

a

a

$\begin{array}{ll}\text { k } & \text { néta } \\ & \text { néta mbatu } \\ & \text { neti } \\ & \text { neti lélu } \\ & \text { nganda liṛu } \\ & \text { nganga } \\ & \text { ngawu }\end{array}$

a ngwu

mesé

mindu

minggé

mo

moké

moké nitu

mondo

monggo

moro wio

muku

muku jawa I

muku jawa II

muku té'a I

muku té'a II

mundé

?mundu kura

muta

muti

namu

nanga

nanggé

nata

nata méngi

nata tanggo

nawu

ndaka

ndalu

ndanga

ndeka kodé

ndenu

nderé

ndéto

ndinga

ndinga lako

ndusu

nengi

néta
$1140 / 2$

4300

4314

4302

4310
Cordia monoica

Lygodium ?circinnatum

Trema orientalis

Artocarpus integer

Arenga pinnata

Caryota

Gardenia

Commelina

Acorus calamus

Musa paradisiaca

= ha'é

Gomphandra mappioides

Blumea balsamifera

Cayratia

Citrus spp.

Polygonum

Citrus

Lagerstroemia flos-reginae

Celtis tetrandra

Calamus

Tamarindus indica

Piper betle (race)

Piper betle (race)

Piper betle (race)

Passiflora foetida

Cucumis sativus (race)

Albizia procera

Kleinhovia hospita

Pittosporum moluccanum

Melochia cf. umbellata

Heteropogon contortus

Mucuna pruriens var. pruriens

Cinnamomum burmanni

Neolitsea

Melastoma

Canarium asperum

Anamirta cocculus

Lunasia amara

Breynia cernua

Micromelum pubescens

Amaranthus ?spinosus

?Randia / Ziziphus

Abelmoschus moschatus ssp. tuberosus 


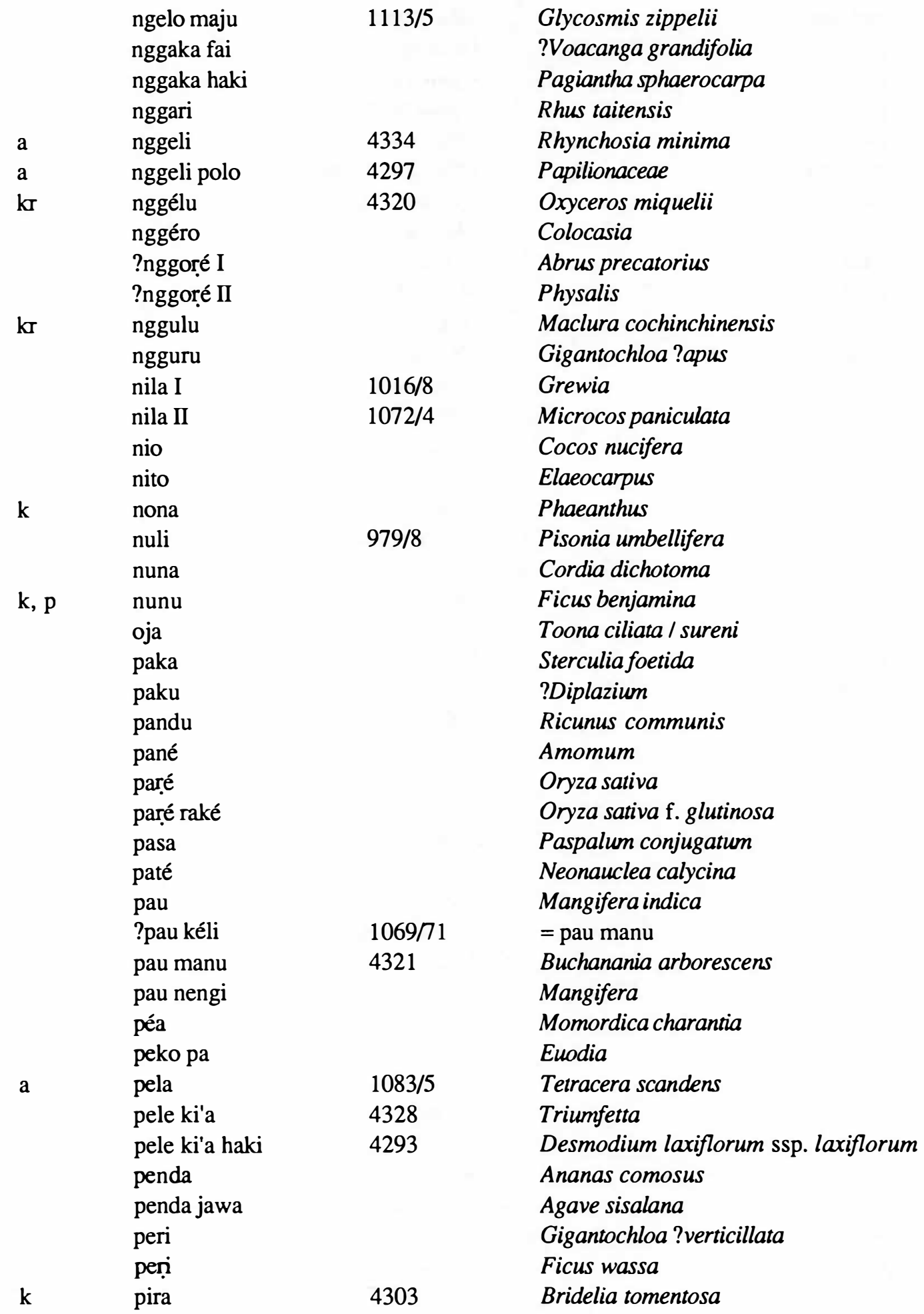




\begin{tabular}{|c|c|c|c|}
\hline & ponggo kura & & Elatostema \\
\hline & pui & & Decaspermum triflorum \\
\hline & pulu I & & $=$ pulu fai \\
\hline & pulu II & & $=$ pulu haki \\
\hline & pulu fai & & Urena lobata \\
\hline & pulu haki & & Triumfetta rhomboidea \\
\hline & puni & & Cyathea \\
\hline k & pura I & 4322 & Phaleria occandra \\
\hline & pura II & & Physalis \\
\hline & puréé & & Mallotus philippensis \\
\hline & ra'u & & Dracontomelon edule \\
\hline & remba & & Macaranga tanarius \\
\hline & résa & & Cyperaceae \\
\hline & résa haki & & Cyperaceae \\
\hline & rewa & 4330 & Melanolepis multiglandulosa \\
\hline & ringo & & Mimosa invisa \\
\hline & rita & & Alstonia scholaris \\
\hline & riti & & Amorphophallus (cult. w. smooth stem) \\
\hline & $\mathrm{ru}$ & 4299 & Salvia misella \\
\hline & rumbé & & Phaseolus aureus \\
\hline & runu & & Wedelia \\
\hline & runu waé & & Strobilanthes \\
\hline & sambi & $1054 / 6$ & Schleichera oleosa \\
\hline & sanggu sa & & Lichenes, Usnea \\
\hline & sapu & & Vernonia cinerea \\
\hline & sara walu & & Canavalia \\
\hline & saru walu & $1137 / 9$ & Leea angulata \\
\hline & sawa I & & Bidens \\
\hline & sawa II & & ?Pneumatopteris \\
\hline k & séko & & tree, tall w. very hard wood \\
\hline & séko kala & 4294 & Leguminosae \\
\hline & seko raké I & 4291 & Amaranthaceae \\
\hline & seko raké II & $1128 / 30$ & Fatoua pilosa \\
\hline & seku & & Kaempferia galanga \\
\hline $\mathrm{kr}$ & sepa & 4287 & Caesalpinia sappan \\
\hline & sésu (sénsus) & & Eupatorium (odoratum) \\
\hline & sewo & & ?Pteris \\
\hline & séwu & & Dioscorea \\
\hline & sia & & Alpinia \\
\hline & somu bara & & Allium sativum \\
\hline & somu toro & & Allium cepa var. ascalonicum \\
\hline & soro & & Grewia \\
\hline & sui & & Champereia manillana \\
\hline
\end{tabular}


a

suka

sulé

sura I

sura II

sura kumba

susu waé

ta'i ndala

ta'i tibo I

ta'i tibo II

ta'i wawi

tanggo

taru

taru jawa

taru kala

tawu

k

a

$\mathrm{kr}$

a

k

a

té'é

teko

tepo

tepu

teré

téwa ngamba

tewu

?timbu

timu

timu ka

tindu

toba

to'é

toka

tora

tuma

tuwa I

tuwa II

ua

uré

uté

uwi

uwi mboa

wajo

wako

wala

wana

waro

4285

Gnetum (gnemon)

Ficus septica

Dioscorea esculenta

$1039 / 41$

4323

4308

$989 / 91$

Ampelocissus

Dioscorea aculeata

Oxalis corniculata

Scurrula

Ehretia microphylla

Pupalia laplacea

Hyptis rhomboidea

= hobo naké

Indigofera

Cassia

4326

Tephrosia noctiflora

Lagenaria siceraria

Arytera

Colocasia esculenta

Costus speciosus

Claoxylon abbreviatum

Artocarpus elasticus

Drypetes neglecta

Saccharum officinarum

Phragmites karka

Cucumis sativus (race)

?Trichosanthes

Pisonia aculeata

Alocasia macrorrhiza

Bambusa blumeana

Centella asiatica

Lagenaria siceraria

Ipomoea obscura

Croton tiglium

$1095 / 7$

Derris polyphylla

Calamus / Daemonorops

Cajanus cajan

Aegle marmelos

Dioscorea alata

= dao mboa

Adenanthera pavonina

Themeda villosa

Planchonia valida

Smilax

Pterospermum diversifolium 


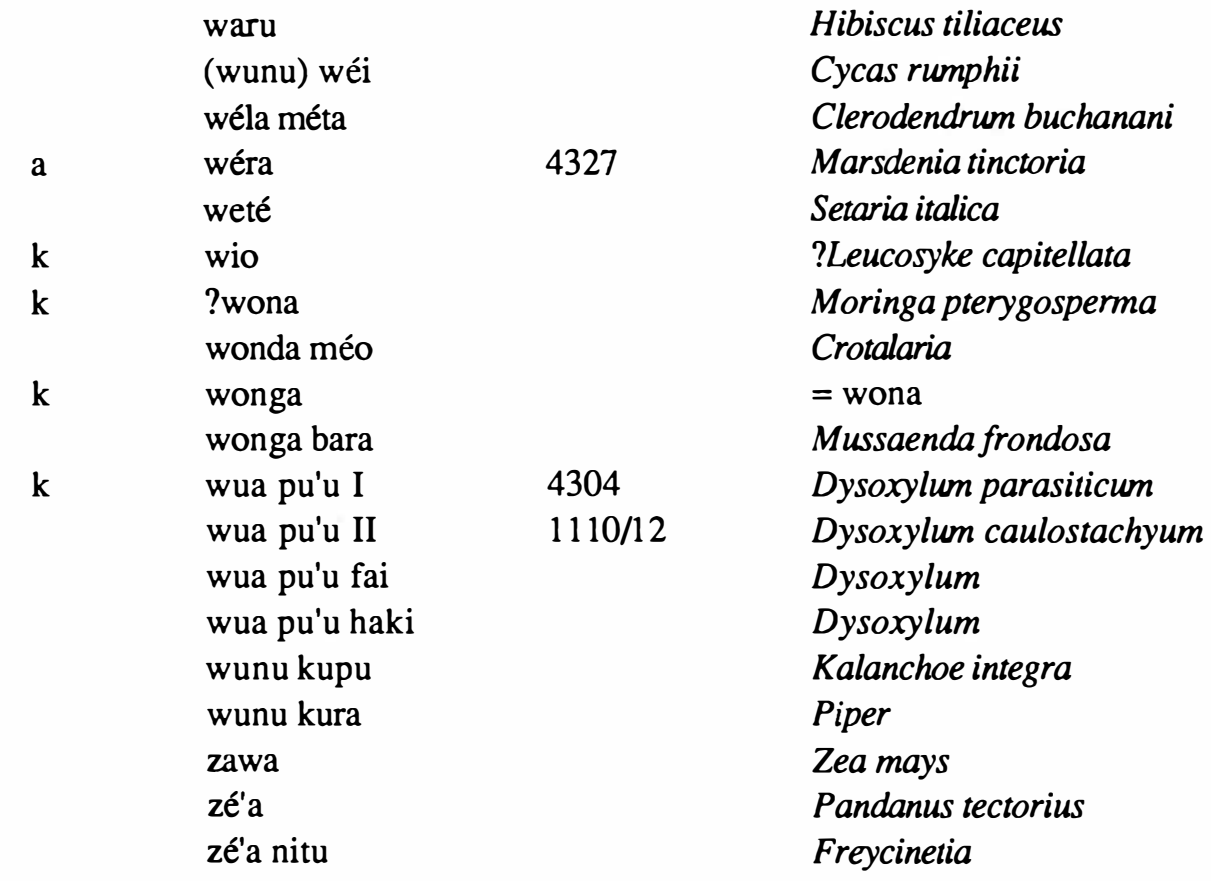




\subsection{NGADHA - TAXONOMIC}

\subsubsection{PRELIMINARY REMARKS}

(a) The language

(i) The Ngadha dialects show many sound shifts and considerable other mutual differences, resulting also in many synonymous names. I am not able to establish the grouping of these dialects.

(ii) Orthography and pronunciation

The glottal stop (') is very, very rare before an initial vowel, but common between vowels.

The smooth onset, which is normal before the initial vowel and often found before the vowel of a following syllable, is not spelled. This is the reverse of Amdt's orthography which uses ' $\mathrm{C}$ ' as a poor graphic substitute for " ", but does not spell the glottal stop.

'bh' and 'dh' are glottalised 'b' and 'd', a common regional spelling.

'gh' is regionally used for the voiced laryngal spirant.

h/ is voiceless and more or less fricative, depending on the dialect concerned; Djawanai (1977:10) spells it ' $x$ '.

' $j$ ' of ten corresponds with ' $z$ ' and ' $r$ ' in cognate words from other dialects.

' $w$ ' Amdt and also Djawanai (1977:10) spell it ' $v$ '.

(b) Research and identification

Only a few (tree) names were found in Hildebrand's list. In 1961-2 Mr I. Dahus collected plant names of Ngadha in the neighbourhood of Mataloko, providing their Manggarai equivalents.

Afterwards I took all the plant names in Arndt's (1961) dictionary. They formed a good starting base for further checking with informants.

In $1986 \mathrm{Mr}$ Ros spent two weeks identifying plants in the field. He collected at $1000 \mathrm{~m}$ in Mataloko, at about $600 \mathrm{~m}$ in Takatunga and Laja, near Ma'ubawa at the coast, and in So'a at a height of $\pm 400 \mathrm{~m}$. Frs H. Hermens and Ad Mommersteeg were of great help to him.

(c) Abbreviations

(i) Classifiers

$\begin{array}{lll}\text { a } & \text { ajé, aré, azé } & \text { vine, liana } \\ \mathrm{f} & \text { faré } & =\text { ajé } \\ \mathrm{k} & \text { kaju } & \text { tree, shrub, woody } \\ \mathrm{kb} & \text { koba } & =\text { ajé } \\ \mathrm{kr} & \text { karo } & \text { thorn(y), spike } \\ \mathrm{ku} & \text { kuru } & \text { grass, weed } \\ \mathrm{m} & \text { meré } & \text { weed, herb } \\ \mathrm{p} & \text { pu'u } & \text { palm, stool } \\ \mathrm{u} & \text { uta } & \text { vegetable } \\ \mathrm{w} & \text { wunu } & \text { leaf, herb }\end{array}$


(ii) Localities

$\mathrm{Bb}$ Boba

Bj Bajawa

Jr Jérébu'u

Lj Laja

Mb Ma'ubawa

MI Mataloko

Mn Manguléwa

So So'a

Tt Takatunga

Wj Watujaji

(iii) Additional sources

A Amdt

1.2.2 THE LIST

adé I

adé II

apu

ara

aré

MI

MI

aro pa

ata foka

azé watu

kr baba

k ba'i

m baka

baka azé

baka bési

$\mathrm{u}$

baka bhara

baka éu

baka koké

baka lélu

$\mathrm{u}$

baka mité

bako

?bako folo

bara I

bara II

$\mathrm{kb}$

MI

So

So

$\mathrm{Ml}$

So

$\mathrm{Tt}$

$\mathrm{Ml}$

M

A

So

$\mathrm{Tt}, \mathrm{Ml}$

$\mathrm{Ml}, \mathrm{Tt}$

$\mathrm{Ml}, \mathrm{Tt}$

$\mathrm{Ml}$, So

$\mathrm{Ml}, \mathrm{Tt}$

$\mathrm{Ml}, \mathrm{Tt}$

bara ba'i

bara karo

MI

bara kedhi

bara lai

A $\quad=$ laté $(\mathrm{kaju})$

= laté (meré)

A Fungi (mould)

Ficus (variegata)

$=\mathrm{kosu}$

Acronychia trifoliata

Persea americana

A liana type

A Rubus

A tree

5427 Blumea riparia

tree

$=$ muku té'a I

?Blumea

= baka lélu

?Vernonia

Erigeron sumatrensis

?Vernonia

Nicotiana tabacum

plant w. light violet fl.

Solanum melongena

= taga lai

?Cayratia

Solanum

Solanum spp.

Solanum melongena (small variety)

= taga lai 


\begin{tabular}{|c|c|c|c|c|}
\hline & bara mézé & & & = taga lai mézé \\
\hline & baru & $\mathrm{Ml}, \mathrm{Tt}$ & 5400 & Diplocyclos palmatus \\
\hline $\mathrm{k}$ & bazi & $\mathrm{Mb}$ & & Harrisonia brownii \\
\hline k & beké & So & & tree, large: wood for timber \\
\hline & béké ngata & & A & plant w. digitated 1. \\
\hline $\mathrm{k}$ & beko & M & & ?Cyrtandra \\
\hline & bela I & MI & & Schizostachyum brachycladum \\
\hline $\mathrm{ku}$ & bela II & M & 5413 & Panicum notatum \\
\hline w & belé biza & & & Drynaria \\
\hline & bena & So & & = lélu \\
\hline $\mathrm{p}$ & benga & So & 5432 & Solanum verbascifolium \\
\hline $\mathrm{k}$ & bé'o & Ml, So & & ?Euodia \\
\hline $\mathrm{k}, \mathrm{p}$ & beru & Ml, So & & Casuarina junghuhniana \\
\hline $\mathrm{kr}$ & bési I & M & & Rubus moluccanus \\
\hline & bési II & Ml, So & & Cucurbita moschata \\
\hline & bési jala & $\mathrm{Ml}$ & & = bési II \\
\hline & bési solo & So & & Trichosanthes villosa \\
\hline & beté kaba & $\mathrm{Ml}, \mathrm{Tt}$ & & Sida \\
\hline & beto & So & & Dendrocalamus asper \\
\hline & béwa & & A & ?= hopa béwa \\
\hline & béwo I & M & & Cassia sophera \\
\hline & béwo II & $\mathrm{Ml}, \mathrm{Mb}$ & & vine w. white latex \\
\hline & béwu & So & & = béwo II \\
\hline & béwu ré'é I & So & & Cassia tora \\
\hline & béwu ré'é II & So & & Orchidaceae \\
\hline & béwu ré'é III & So & & plant w. sharp odour \\
\hline $\mathrm{ku}$ & beza I & M & & Arthraxon hispidus \\
\hline $\mathrm{ku}$ & beza II & M & & Setaria palmifolia \\
\hline k & bhaka I & So & & Planchonia valida \\
\hline k & bhaka II & So & & Planchonella obovata \\
\hline $\mathrm{m}$ & bhara & So & 5426 & Vernonia \\
\hline k & bhaso & M & & Ficus sp. \\
\hline & bheé & $\mathrm{Tt}$ & & Fungi \\
\hline & bhena & $\mathrm{Tt}$, So & & (many attributes) = lélu \\
\hline & bheto I & So & & $=$ beto \\
\hline $\mathrm{ku}, \mathrm{m}$ & bheto II & $\mathrm{Ml}$, Tt, So & & Paspalum conjugatum \\
\hline ku & bheto III & M & & Ischaemum muticum \\
\hline $\mathrm{ku}$ & bheto IV & M & & Cyrtococcum accrescens \\
\hline & bheto nitu & So & & Dinochloa scandens \\
\hline $\mathrm{k}$ & bhéwo & & A & tree, in forest \\
\hline & bhéza bhaza & So & & Ipomoea batatos \\
\hline & bhogi bhéka & $\mathrm{Tt}$ & & Orchidaceae \\
\hline & bhoka & So & & $=$ tawu \\
\hline
\end{tabular}




\begin{tabular}{|c|c|c|c|c|}
\hline & bhoro & Mn & & Corypha utan \\
\hline & bhu tana & Tt, So & & Fungi \\
\hline & bhunga & So & & $=$ danga $I$ \\
\hline \multirow[t]{2}{*}{$\mathrm{k}, \mathrm{p}$} & bhuru bhara & So & & Ficus \\
\hline & bidi & Ml, So & & Averrhoa carambola \\
\hline \multirow[t]{2}{*}{$\mathrm{u}$} & ?bié & $\mathrm{Ml}, \mathrm{Tt}$ & & $=$ nilu $\mathrm{I}$ \\
\hline & biru & & A & plant \\
\hline \multirow[t]{3}{*}{ k } & biti & So & & tree, large; good timber \\
\hline & bo ba & MI & & Colocasia (wild species) \\
\hline & boa & Ml, So & & Ceiba pentandra \\
\hline \multirow[t]{10}{*}{$\mathrm{p}$} & boa jawa & So & & Bombax ceiba \\
\hline & boba & A & & shrub \\
\hline & boda & So & & = lu lima II \\
\hline & boé awa & M & & = goé awa \\
\hline & boé baé & & A & = wira waé \\
\hline & boka & M & & $=$ tawa \\
\hline & boko nawé & $\mathrm{Tt}$ & & $=$ li'é nawé \\
\hline & bolé kogo I & Ml & & $=$ boné ranga \\
\hline & bolé kogo II & $\mathrm{Ml}$ & 5398 & Lepidagathis \\
\hline & bolé kogo III & Ml & & Solanum nigrum \\
\hline \multirow[t]{6}{*}{$\mathrm{u}$} & bomo & So & & = kigo nipo \\
\hline & boné & M & & Galinsoga parviflora \\
\hline & boné ranga & $\mathrm{Ml}$ & & Physalis angulata \\
\hline & boné so'a & M & & $=$ boné \\
\hline & bopo & M & & shrub \\
\hline & boro & So & & $=$ bhoro \\
\hline \multirow[t]{17}{*}{$\mathbf{u}$} & boti & So & & Amaranthus (lividus) \\
\hline & boti karo & $\mathrm{Ml}$, So & & Amaranthus spinosus \\
\hline & boti modhé & So & & $=$ boti \\
\hline & bowo & M & & Orchidaceae \\
\hline & bu & & $\mathrm{dft}$ & $=$ beru \\
\hline & bu awa & M & & = goé awa \\
\hline & bu tana & M & & Fungi \\
\hline & bua bali & & A & plant, tuberous \\
\hline & bué awa & $\mathrm{Ml}$ & & = goé awa \\
\hline & buka & & A & plant, odorous \\
\hline & bunga ba'i & MI & & = wonga ba'i II \\
\hline & bungé I & $\mathrm{Ml}$, So & & Datura metel \\
\hline & bungé II & $\mathrm{Ml}, \mathrm{Tt}$ & & Cassia floribunda \\
\hline & bungé api I & $\mathrm{Ml}$ & & ?Vernonia cinerea \\
\hline & bungé api II & $\mathrm{Ml}$ & & herb w. white fl. \\
\hline & bungé api III & $\mathrm{Ml}$ & & Lepidagathis \\
\hline & bupu moku & So & 5444 & Eragrostis elongata \\
\hline
\end{tabular}


bura

u butu

a

k

k

$\mathrm{p}$

$\mathrm{kb}$

k

k

a butu hiku

daka

dalu

danga I

danga II

danga III

dara

daru

deki

deki tua

denu

déo

déra

dero I

dero II

déru

déto

dhao

dhao goa

dhao zé'é

dhara

dhari bhoka

dhédé

dhenu

dhéru

diga

dika

dodho bhara

dodho toro

(doloka

doza I

doza II

doza III

duré éjo

duri

duri kéri

duri kuru

duwa I

duwa II

eda

éjé
$\mathrm{Ml}, \mathrm{Tt}$

Ml, So

MI

So

So

M

Mauponggo

M

$\mathrm{Tt}$

MI

Ml, So

So

$\mathrm{Ml}$, So

So

MI

MI

MI

So

MI

MI

MI

MI

So

So

$\mathrm{Ml}, \mathrm{Tt}$

$\mathrm{Tt}$

MI

$\mathrm{Ml}, \mathrm{Tt}$

MI

MI

$\mathrm{Ml}$
A liana

$=$ jéko

Physalis

A

tree w. edible fr.

dft

Albizia procera

Acorus calamus

Kleinhovia hospita

Podocarpus

Caesalpinia major

$=$ dara

Fungi (on dead trunks, edible)

Fungi

Melochia cf. umbellata

A Trema orientalis

A tree

Erythrina subumbrans

$5457 \quad$ Erythrina ?orientalis

A Citrus (wild)

Mucuna pruriens var. pruriens

= ranga I (many attributes)

Ricinus communis

Ipomoea pes-caprae

$=$ dara

5440 Euphorbia (prostrata)

Pterocarpus indicus

$=$ denu

A $=$ déru

$=$ gulu I

Pandanus

Kaempferia

Kaempferia galanga

dft Pygeum)

Elattostachys

Chisocheton

Arytera litoralis

Melothria (perpusilla)

A Fungi

?Fungi

Fungi (edible)

Croton tiglium

liana, large

Bauhinia scandens

Benincasa hispida 


\begin{tabular}{|c|c|c|c|c|}
\hline & éjo & Ml & & $=$ suza \\
\hline & éko lako I & So & & Macaranga \\
\hline k & éko lako II & $\mathrm{Ml}, \mathrm{Lj}, \mathrm{Tt}$ & & Mallotus ricinoides \\
\hline \multirow[t]{3}{*}{ k } & ené & So & & Peltophorum pterocarpum \\
\hline & eré & & A & tree \\
\hline & fai & M & & Albizia chinensis \\
\hline \multirow[t]{6}{*}{ k } & fai bhara & So & & $=$ fai \\
\hline & fai toro & So & & Albizia falcataria \\
\hline & fai zé'é I & $\mathrm{Ml}$ & & $=$ rewu \\
\hline & fai zé'é II & M & & herb \\
\hline & faka tana & Ml & 5395 & Rorippa \\
\hline & fako & $\mathrm{Ml}$, Tt, So & & Fungi (many attributes) \\
\hline \multirow[t]{8}{*}{$\mathrm{a}, \mathrm{f}$} & fangé I & So & & Paederia scandens \\
\hline & fangé II & & A & ?Merremia \\
\hline & fangé bhara & $\mathrm{Bb}$ & 5414 & Hewittea sublobata \\
\hline & fao wako & So & & Themeda villosa \\
\hline & faré mengé & So & 5467 & ?Merremia \\
\hline & felu & & A & $=$ féo \\
\hline & féo & Ml, So & & Aleurites moluccana \\
\hline & fésa & $\mathrm{Ml}$ & & $=$ lebha fésa \\
\hline \multirow[t]{3}{*}{$\mathbf{a}$} & fi & $\mathrm{Tt}$ & & $=$ fili I/II \\
\hline & figo & Ml, So & & Strobilanthes \\
\hline & figo boté & So & 5466 & ?Lepidagathis \\
\hline $\mathbf{a}$ & fili I & M & & Phaseolus (wild) \\
\hline \multirow[t]{9}{*}{ a } & fili II & M & & Calopogonium mucunoides \\
\hline & fiso & & A & $=$ hiso \\
\hline & fitu I & Ml, So & & Fungi/Pycnoporus \\
\hline & fitu II & $\mathrm{Bj}$, So & & Fungi (many attributes) \\
\hline & foa & & A & Lichenes (on bark etc.) \\
\hline & fo'a & M & & Cucurbitaceae (edible) \\
\hline & fo'i fé'é & M & & Christella dentata \\
\hline & foja & So & & Mikania cordata \\
\hline & foro & M & & Ocimum basilicum \\
\hline \multirow[t]{2}{*}{ kb } & foza & So & & Ipomoea nil \\
\hline & fu lemu I & Ml & 5396 & Poaceae \\
\hline $\mathrm{m}$ & fu lemu II & $\mathrm{Ml}, \mathrm{Tt}$ & & Cyperus rotundus \\
\hline $\mathrm{m}$ & fu lemu III & Ml, So & & Fimbristylis ovata \\
\hline \multirow[t]{3}{*}{$k, p$} & futa & & & Cerbera \\
\hline & gadhi & & A & tree \\
\hline & gajo kéli & & $\mathrm{dft}$ & Myristicaceae \\
\hline $\mathrm{k}$ & gaka I & Ml, So & & Pagiantha sphaerocarpa \\
\hline k & gaka II & M & & Ficus fulva \\
\hline k & gari & Ml & & Rhus taitensis \\
\hline
\end{tabular}




\begin{tabular}{|c|c|c|c|c|}
\hline & gebu & & $\mathrm{dft}$ & Ardisia \\
\hline $\mathrm{k}$ & gegé & M & & Leucaena leucocephala \\
\hline \multirow[t]{8}{*}{$\mathrm{p}$} & ghaja & MI & 5412 & Inocarpus fagiferus \\
\hline & ghaka & Ml, So & & $=$ gaka I \\
\hline & ghédo & M & & Panicum miliaceum \\
\hline & ghé'é & Ml & & Mischocarpus sundaicus \\
\hline & ghésa & M & & Viburnum \\
\hline & ghogha & & A & plant \\
\hline & ghubu & M & 5399 & ?Trichosanthes (villosa) \\
\hline & ghuna & M & & tree \\
\hline $\mathrm{kr}$ & gi & $\mathrm{Ml}, \mathrm{Tt}$ & & Toddalia asiatica \\
\hline k & goa & So & & = waru jawa I \\
\hline $\mathrm{k}$ & goa toro & So & & Jatropha gossypifolia \\
\hline k & goé awa & M & & Psidium guajava \\
\hline \multirow[t]{13}{*}{$\mathrm{kb}$} & gogo & So & & Ichnocarpus moluccanus \\
\hline & gojé & & A & = gozé I \\
\hline & gola & MI & & Dioscorea (bulbifera?) \\
\hline & gozé I & Ml, So & & Abrus precatorius \\
\hline & gozé II & Ml & & Breynia cernua \\
\hline & gozé III & Ml & 5384 & Phaseolus ?pubescens \\
\hline & gozé IV & So & & Clematis \\
\hline & guawa & Ml & & = goé awa \\
\hline & gulé & Ml, So & & Fungi \\
\hline & gulé pau & So & & Fungi \\
\hline & $\begin{array}{l}\text { gulu I } \\
\text { gulu II }\end{array}$ & Ml, So & & $\begin{array}{l}\text { Maclura cochinchinensis } \\
=\text { méa }\end{array}$ \\
\hline & guru I & Ml, So & & Gigantochloa ?apus \\
\hline & guru II & M & & $=$ guru to'é \\
\hline \multirow[t]{3}{*}{$\mathrm{u}$} & guru III & So & & Commelina \\
\hline & guru butu & So & & Bambuseae \\
\hline & guru égé & $\mathrm{Ml}$ & & vine \\
\hline \multirow[t]{12}{*}{$f, w$} & guru hégé & So & 5423 & Asystasia \\
\hline & guru kéro & So & & bamboo, ornamental \\
\hline & guru to'é & Ml & & Bambusa cf. blumeana \\
\hline & gusi & & A & vine \\
\hline & haé & Ml, So & & = saé \\
\hline & ha'é & & A & $=$ padu \\
\hline & haé léwa & $\mathrm{Ml}$ & & = saé léwa \\
\hline & haé lé wa nipon & Ml, So & & Pennisetum spicatum \\
\hline & hago & M & & Sterculia oblongata \\
\hline & haha hoé & $\mathrm{Tt}$ & & Fungi \\
\hline & ha'o & & A & ?Daemonorops \\
\hline & hapa (jawa) & So & & Morus australis \\
\hline
\end{tabular}




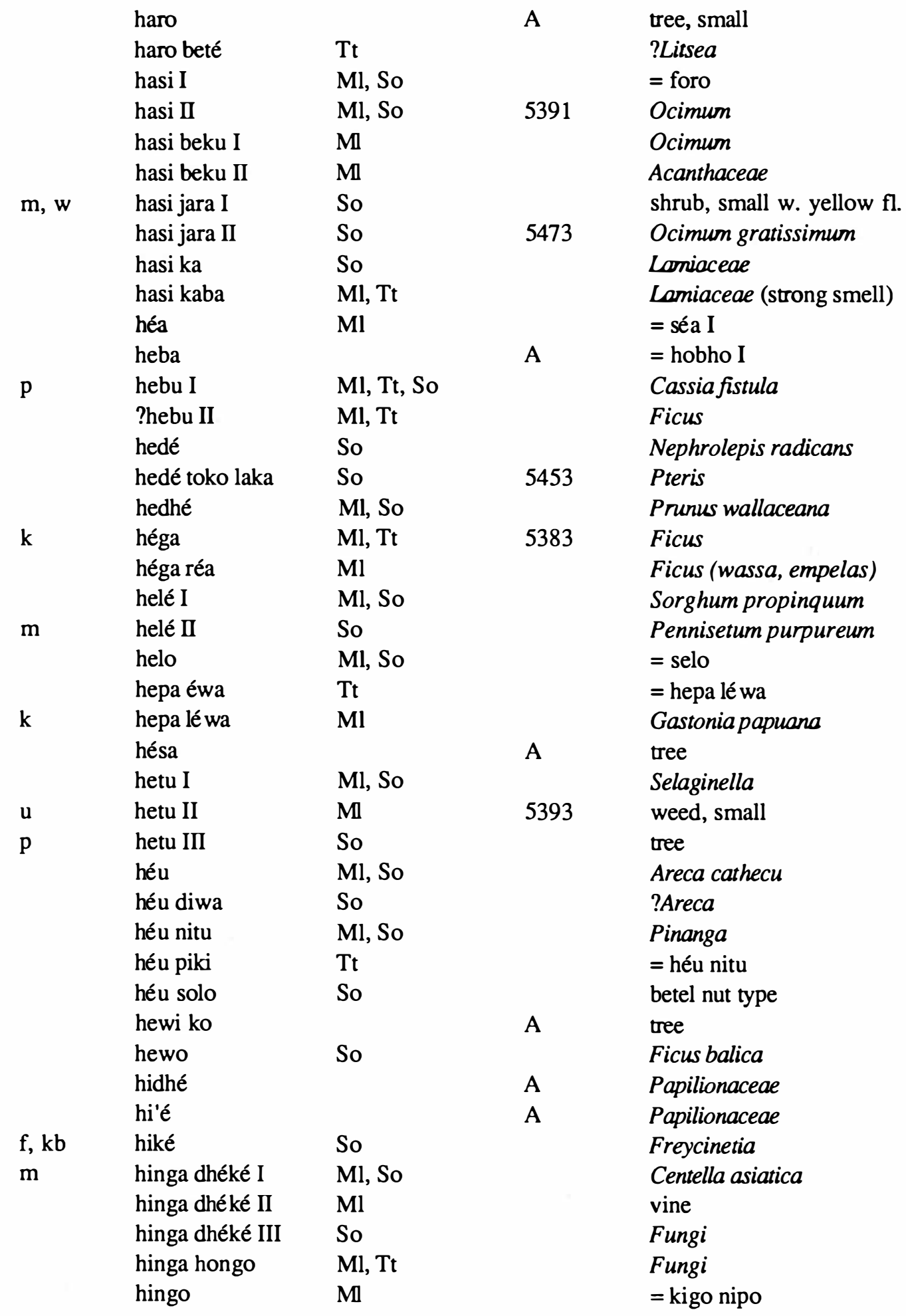




\begin{tabular}{|c|c|c|c|c|}
\hline & hingo nipo & Ml & & $=$ kigo nipo \\
\hline & hipa & $\mathrm{Ml}, \mathrm{Tt}$ & & $=$ zé'a nitu \\
\hline & hiso & So & & Dioscorea \\
\hline & ho & $\mathrm{Tt}$ & & = saé \\
\hline & hobho I & Ml, ?So & & Vigna unguiculata \\
\hline \multirow[t]{15}{*}{ k } & hobho II & $\mathrm{Ml}, \mathrm{Tt}$ & & Glochidion philippicum \\
\hline & hobho azé & $\mathrm{Ml}$ & & $=$ hobho I \\
\hline & hobho kaju I & Ml & & = rubhé \\
\hline & hobho kaju II & & A & Phaseolus vulgaris \\
\hline & hobho kaju III & & A & = uzé I \\
\hline & hobho nitu & Ml & & Papilionaceae \\
\hline & hobho tana & MI & & Arachis hypogaea \\
\hline & hobo & & A & $=$ hobho \\
\hline & hoi & & A & tree \\
\hline & ho'i & Ml & & $=$ talé \\
\hline & hongo & & A & Fungi \\
\hline & hopa béwa & So & 5483 & Dysoxylum ?gaudichaudianum \\
\hline & howé & & A & Papilionaceae \\
\hline & huku I & So & & $\begin{array}{l}\text { Platycerium bifurcatum ssp. } \\
\text { willinckii }\end{array}$ \\
\hline & huku II & $\mathrm{Tt}$ & & = lebé raé II \\
\hline \multirow[t]{4}{*}{$\mathrm{p}$} & huma & So & & $=$ rewu \\
\hline & $\mathrm{ibu}$ & $\mathrm{Ml}, \mathrm{Tt}$ & A & Asteraceae \\
\hline & ibu ngiu & M & & = koro kowé nitu \\
\hline & ila & Ml & & Schizostachyum blumii \\
\hline $\mathrm{m}$ & isi toko bhara & So & & Hyptis brevipes \\
\hline \multirow[t]{4}{*}{$\mathrm{m}$} & isi toko laka & So & & Hyptis rhomboidea \\
\hline & izé & So & & = éjé \\
\hline & jawa I & Ml & & = haé léwa \\
\hline & jawa II & & A & = haé \\
\hline \multirow[t]{8}{*}{$\mathrm{u}$} & jawa III & Ml & & ?Morus \\
\hline & jé'a & & A & = zé'a \\
\hline & jebu & Ml & & = goé awa \\
\hline & jéko & $\mathrm{Tt}$ & & Melastoma (?)polyantha \\
\hline & jeru & Ml & & Citrus aurantifolia \\
\hline & joi joi & Ml & & vine, wild \\
\hline & ka beka & $\mathrm{Tt}$ & & $=$ ra'a beka \\
\hline & ka rara & Ml & & $=$ kerara \\
\hline $\mathrm{kr}$ & kaba I & Ml & & Rubus fraxinifolius \\
\hline \multirow[t]{4}{*}{$\mathrm{kr}$} & kaba II & Ml & & = méa \\
\hline & kabé azé & $\mathrm{Bb}$ & & Cissus \\
\hline & kabi jata & $\mathrm{Tt}$ & & = lebé biza II \\
\hline & kabu ranga & So & & $=$ ranga lédha \\
\hline
\end{tabular}




\begin{tabular}{|c|c|c|c|c|}
\hline & kabu sengasu & Ml & & Elephantopus scaber \\
\hline & kadu & $\mathrm{Ml}, \mathrm{Tt}$ & & Bulbophyllum \\
\hline \multirow[t]{6}{*}{$k, p$} & ka'é & So & & $=$ ghaja \\
\hline & kaka bheto & M & & plant, edible w. sourish 1. \\
\hline & kaka kaju I & MI & & Ficus \\
\hline & kaka kaju II & $\mathrm{Ml}, \mathrm{Tt}$ & & vine \\
\hline & kaka kaju III & Ml & 2787 & Asplenium \\
\hline & kako & Ml & & Ipomoea aquatica \\
\hline \multirow[t]{4}{*}{$\mathrm{u}$} & kala & $\mathrm{Bb}, \mathrm{Ml}, \mathrm{Tt}$ & & $=$ kobho \\
\hline & kala beka & Ml & & $=$ ra'a beka \\
\hline & kalo & MI & & herb \\
\hline & kanga paké I & $\mathrm{Tt}$ & & = kungu paké \\
\hline \multirow[t]{5}{*}{$\mathrm{u}$} & $\begin{array}{l}\text { kanga paké II } \\
\text { kapu I }\end{array}$ & $\mathrm{Ml}, \mathrm{Lj}, \mathrm{Tt}$ & A & $\begin{array}{l}\text { Polypodiaceae } \\
\text { tree }\end{array}$ \\
\hline & kapu II & Ml & & Apiaceae \\
\hline & kara & & A & herb, creeping \\
\hline & kasi & & A & $=$ foro \\
\hline & kasi beku & & & = hasi beku II \\
\hline $\mathrm{kb}$ & katé & So & & Tetrastigma \\
\hline $\mathrm{kb}$ & katé bara & So & & Cayratia \\
\hline \multirow[t]{6}{*}{$\mathrm{kb}$} & katé toro & & & ?Rourea minor \\
\hline & kato jara & $\mathrm{Ml}$ & & Fungi \\
\hline & kato kadhé & & A & Fungi \\
\hline & kato kodhé & Ml, Tt, So & & Fungi \\
\hline & katu & & $\mathrm{dft}$ & $=$ dalu \\
\hline & kéa & Ml & & Bambuseae \\
\hline \multirow[t]{6}{*}{$\mathrm{kr}$} & kébé & Ml & & = bési I \\
\hline & kébé bési & & A & plant w. large 1. \\
\hline & kebhu & Ml & & = séwo \\
\hline & kebo & Ml, So & & Morinda \\
\hline & kebu & Ml, So & & = séwo \\
\hline & kebu kéwa & & A & plant, tuberous, wild \\
\hline k & keda I & $\mathrm{Ml}, \mathrm{Tt}$ & & = danga II \\
\hline $\mathrm{u}$ & keda II & $\mathrm{Tt}$ & & Cordia monoica \\
\hline k & $\begin{array}{l}\text { kedha I } \\
\text { kedha II }\end{array}$ & Ml, So & A & $\begin{array}{l}\text { Cordia dichotoma } \\
\text { vegetable, medicinal }\end{array}$ \\
\hline $\mathbf{w}$ & kedha III & So & 5456 & Deeringia (amaranthoides) \\
\hline \multirow[t]{6}{*}{$\mathrm{p}$} & kedondo & So & & Spondias dulcis \\
\hline & kelo & & A & $=$ selo \\
\hline & kelu & So, Jr & & = séa I \\
\hline & ?kéna & & A & $=$ tawu \\
\hline & ?kéngé ké'o & So & & plant, tuberous \\
\hline & kéngo & & A & Ficus \\
\hline
\end{tabular}




\begin{tabular}{|c|c|c|c|}
\hline ké'o & Ml, So & & Coix lacryma-jobi var. ma-yuen \\
\hline képa & So & A & Coix lacryma-jobi var. agrestis \\
\hline kéra & Ml & & shrub \\
\hline kéra zé'a & So & & $=$ peda I \\
\hline kerara & Ml & & Artocarpus altilis \\
\hline keri & So & & = kéri \\
\hline kéri & $\mathrm{Ml}$ & & Imperata cylindrica \\
\hline késa & $\mathrm{Tt}$ & & = ghésa \\
\hline kesi I & Ml & & Canarium asperum \\
\hline kesi II & So & & Lannea grandis \\
\hline kesi jawa & MI & & tree; good timber \\
\hline keta & Ml & & Planchonella obovata \\
\hline ketapa & So & & Terminalia catoppa \\
\hline kéu I & & A & = héu I \\
\hline kéu II & & $\mathrm{dft}$ & Ehretia acuminata \\
\hline kéu III & & $\mathrm{dft}$ & Vernonia arborea \\
\hline kéu IV & $\mathrm{Tt}$ & & $=$ selo \\
\hline kéu V & $\mathrm{Tt}$ & & = séa I \\
\hline kéwa & Ml, So & & $\begin{array}{l}\text { Dioscorea (pentaphylla) (many } \\
\text { attributes) }\end{array}$ \\
\hline ki & & A & = kéri \\
\hline kigo & $\mathrm{Ml}, \mathrm{Tt}$ & & Emilia sonchifolia \\
\hline kigo nipo & M & & Erechtites valerianifolia \\
\hline kigo wio I & Mn & & = kigo \\
\hline ?kigo wio II & & & Sonchus \\
\hline kipi & & A & = li'é kozé \\
\hline koba & So & & = nagé II \\
\hline koba karo & So & & $=$ sepa \\
\hline koba katé (toro) & So & & Rourea minor \\
\hline koba maza & So & & = fili I/II \\
\hline kobé & Ml & & shrub, l. and wood violettish \\
\hline kobho & Ml & 5415 & Melothria \\
\hline koi pada & $\mathrm{Tt}$ & & Acanthaceae \\
\hline koja & MI & & Canarium vulgare \\
\hline koké & So & & = kigo \\
\hline kolé I & So & & Calotropis gigantea \\
\hline kolé II & $\mathrm{Ml}$ & & Asclepiadaceae \\
\hline koli & So & & $=$ maghi \\
\hline kopa dhola & & A & Cucurbitaceae \\
\hline kopi I & Ml, So & & Coffea \\
\hline kopi II & & A & weed \\
\hline koré I & $\mathrm{Ml}$ & & tree \\
\hline koré II & $\mathrm{Ml}$ & & = lebé raé II \\
\hline koré III & $\mathrm{Ml}$ & & = belé biza \\
\hline
\end{tabular}




\begin{tabular}{|c|c|c|c|c|}
\hline & koré IV & $\mathrm{Ml}$ & & Vanda \\
\hline & koro & Ml, So & & Capsicum frutescens \\
\hline & koro kedhi & So & & $=$ koro \\
\hline & koro kowé & MI & & Phaseolus pubescens \\
\hline & koro kowé nitu & M & & Papilionaceae \\
\hline & koro mézé & Ml, So & & Capsicum annuum \\
\hline & koro wolo & $\mathrm{M}$ & & $=$ nata butu I \\
\hline & kosu & $\mathrm{M}$ & & Oryza sativa \\
\hline & kota & Ml, So & & Canna coccinea \\
\hline & koto I & $\mathrm{Ml}$ & & Liliaceae \\
\hline & koto II & & A & ?Dioscorea \\
\hline & kou & & A & Dioscorea hispida \\
\hline & ko'u & $\mathrm{Ml}$, So & & $=\mathrm{dhenu}$ \\
\hline $\mathrm{kb}$ & kowé I & So & 5465 & Phaseolus \\
\hline & kowé II & So & & $=$ koro kowé \\
\hline & koza & So & & $=$ rori $\mathrm{II}$ \\
\hline & kua I & $\mathrm{Ml}, \mathrm{Tt}$, So & & Flagellaria indica \\
\hline & kua II & $\mathrm{Tt}$ & & $=$ tawu \\
\hline & kubu & Ml & & $=$ tawu \\
\hline $\mathrm{u}$ & kuja & MI & & Canscora \\
\hline & kuju I & & & Poaceae \\
\hline & kuju II & & A & ?Litsea velutina \\
\hline & kuku & $\mathrm{Ml}$, So & & Schoutenia ovata \\
\hline & kula & So & & $=$ tawu \\
\hline & kumis kucing & So & & Orthosiphon aristatus \\
\hline & kuné & $\mathrm{Ml}, \mathrm{Tt}$, So & & Curcuma viridiflora \\
\hline & kungu méo & So & 5434 & Hibiscus (surattensis) \\
\hline & kungu paké & $\mathrm{Ml}$ & & Lycopodium cernuum \\
\hline & kungu ro'a & $\mathrm{Ml}, \mathrm{Tt}$ & & $=$ kungu paké \\
\hline & kungu sama roga & So & 3656 & = sama roga \\
\hline $\mathrm{u}$ & kupa & So & & Talinum \\
\hline $\mathrm{p}$ & kupé I & Ml, So & 5487 & Piliostigma malabaricum \\
\hline & kupé II & $\mathrm{Ml}, \mathrm{Tt}$ & & Fungi \\
\hline & kuru peti & So & 5448 & Eragrostis tenella \\
\hline$f, k b$ & kuwu & So & & Cucurbitaceae (wild) \\
\hline $\mathrm{u}$ & kuza I & $\mathrm{Ml}, \mathrm{Tt}$ & & Polygonum chinense \\
\hline $\mathrm{u}$ & kuza II & M & & Ludwigia \\
\hline & kuzu & & A & $=$ kuju II \\
\hline & labu jepa & $\mathrm{Ml}$, So & & Sechium edule \\
\hline & ladé I & $\mathrm{Ml}$ & & = laté $(\mathrm{kaju})$ \\
\hline & ladé II & & & = laté (méré) \\
\hline & lado kobo & So & & Fungi \\
\hline & lado rédo & So & & = rédo-rado \\
\hline
\end{tabular}




\begin{tabular}{|c|c|c|c|c|}
\hline & ?ladu & & A & ?Musa \\
\hline w & laé & & A & Cycas \\
\hline k & laiI & & A & tree w. long ensiform 1. \\
\hline w & lai II & So & 3703 & Pyrrosia longifolia \\
\hline \multirow[t]{5}{*}{ w } & lai III & So & & $=$ laé \\
\hline & lai koba & So & & vine \\
\hline & lai léwa & So & 5481 & Ophioglossurn (pendulum) \\
\hline & laja & $\mathrm{Ml}, \mathrm{Tt}$ & & Languas galanga \\
\hline & lala & & A & tree; causes itching \\
\hline \multirow[t]{3}{*}{ k } & lala logé & So & & tree; latex for caulking \\
\hline & lamu & & A & Algae \\
\hline & lamu toro & So & & $=$ gegé \\
\hline \multirow[t]{8}{*}{$\mathrm{p}$} & lanu I & So & & tree, small \\
\hline & lanu II & So & & $=$ ngusu \\
\hline & lasé hui I & & A & Bidens \\
\hline & lasé hui II & & A & vine \\
\hline & lasé kodhé & Ml & & Pittosporum (moluccanum) \\
\hline & lasu hui & So & & vine $w$. edible fr. \\
\hline & lasu lako & Mn & 4412 & Cyperacus pilosus \\
\hline & lasu mula & So & & Achyranthes aspera \\
\hline \multirow[t]{2}{*}{$k, p$} & laté (kaju) & So & & Dendrocnide \\
\hline & laté (méré) & So & & Laportea \\
\hline \multirow[t]{17}{*}{ w } & lawi & $\mathrm{Ml}$ & 4422 & Justicia gendarussa \\
\hline & léa & Ml, So & & Zingiber officinale \\
\hline & léa kaba & $\mathrm{Ml}, \mathrm{Tt}$ & & Zingiber \\
\hline & léa kogha & So & & Zingiber \\
\hline & léa laja & So & & $=$ laja \\
\hline & léa lako & Ml, So & & Globba marantina \\
\hline & léa manu & $\mathrm{Ml}, \mathrm{Tt}$ & & = léa kogha \\
\hline & léa naro & $\mathrm{Ml}$ & & Zingiber \\
\hline & léa rega I & So & & = léa kaba \\
\hline & léa rega II & So & & Amomum \\
\hline & léa rega III & So & & Zingiber \\
\hline & lebé denu & $\mathrm{Tt}$ & & Fungi \\
\hline & lebé biza & $\mathrm{Ml}$ & & Nephrolepis (hirsutula) \\
\hline & lebé raé I & & & = lebé biza \\
\hline & lebé raé II & $\mathrm{Ml}$ & & Asplenium nidus \\
\hline & lebha & Ml, So & & Phaseolus spp. (many attributes) \\
\hline & lebha fésa & So & & Phaseolus lunatus \\
\hline $\mathrm{u}$ & léda & $\mathrm{Ml}, \mathrm{Tt}$ & & Commelina (benghalensis) \\
\hline $\mathrm{u}$ & léda mézé & $\mathrm{Ml}$ & & Commelina (diffusa) \\
\hline $\mathrm{u}$ & léda waé I & So & 5435 & Borreria \\
\hline $\mathrm{u}$ & léda waé II & So & 5486 & Eclipta (prostrata) \\
\hline
\end{tabular}


k ledu

léké

léké nitu

léké salo

?léké solé

lélé I

lélé II

lelu I

lelu II

lélu

$\mathrm{m}$

w

$\mathrm{kb}$

p, k

$\mathrm{k}$

k

k

k

lema badha

lema kaba

lema mori I

lema mori II

lémo

lemu

lenga

lenga nitu

léo

lésé I

lésé II

lesi I

lesi II

lewi

lewi watu

li'é bara

li'é ha'é

li'é hiku

li'é kozé

li'é lésé

li'é lo

li'é logo I

li'é logo II

li'é nawé

li'é zepé

liko dué

p

lima léwa

lima ro'a

lipé

lité

lobha

lobo toro I

k
Ml, So

Ml

4691

So

MI

So

Ml

So

M

$\mathrm{Ml}$, So

So

So

So

So

So

$\mathrm{Bb}, \mathrm{Tt}$

$\mathrm{Ml}$

MI

M

So

So

So

MI

$\mathrm{Ml}, \mathrm{Tt}$

Ml

M

$\mathrm{Ml}, \mathrm{Tt}$

$\mathrm{Ml}, \mathrm{Bw}$

Ml

So

$\mathrm{Ml}, \mathrm{Tt}$

$\mathrm{Ml}$, So

Ml

MI

$\mathrm{Ml}$, So

MI

A
Homalium tomentosum

Entada phaseoloides

Mucuna

= léké nitu

Pholidota

Maoutia

tree, large

Phragmites karka

Arundina graminifolia

Gossypium

herb, small w. red 1 . and fr.

Saurauia

Agave sisalana

Aloe (cult.)

Citrus grandis

A $\quad$ = fu lemu I/IIIII

Sesomwn orientale

$5443 \quad$ Cleome (gynandra)

= sulé

Uvaria

Envatomia

$5390 \quad ?=$ zama

Boehmeria

$=$ lamu

$5442 \quad$ Pilea microphylla

Solanum

A padu

vine

Abrus precatorius

tree, coastal w.edible fr.

A tree w. hard wood

5392 Euphorbia (prostrata)

Phyllanthus

Ficus fistulosa (var. tengerensis)

A tree

Fungi

?Doryxylum spinosum

?Schefflera

Mucuna pruriens f. utilis

Homalanthus

Symplocos

= hepa léwa

Glochidion 


\begin{tabular}{|c|c|c|c|}
\hline lobo toro III & $\mathrm{Ml}, \mathrm{Tt}$ & & = déo \\
\hline lobo toro IV & So & & vine \\
\hline lodé & $\mathrm{Ml}$ & & Polygonum chinense \\
\hline lodé kedhi & Ml & & Polygonum \\
\hline lodé polo & $\mathrm{Bw}$ & & herb \\
\hline logo mité & $\mathrm{Ml}$ & & Polypodiaceae \\
\hline logo ngélu & Ml & & Fungi \\
\hline logo ngéu & $\mathrm{Tt}$ & & = logo ngélu \\
\hline logo teké & $\mathrm{Ml}$ & & Polypodiaceae \\
\hline loko & $\mathrm{Ml}, \mathrm{Wj}$, So & & Syzygium \\
\hline lola & MI & & Amorphophallus (campanulatus) \\
\hline lopa kaju & & & vine, epiphytic \\
\hline lowa I & $\mathrm{Ml}$, So & & Melia azedarach \\
\hline lowa II & So & & Dioscorea \\
\hline lowé & & A & plant, tuberous \\
\hline lowo & & A & plant, tuberous \\
\hline lu lima I & Ml, So & & Cucurbitaceae \\
\hline lu lima II & MI & 5387 & Luffa aegyptiaca \\
\hline lua méo & & & Fungi (mould) \\
\hline luba & MI & dft & Pterospermum javanicum \\
\hline lui & $\mathrm{Ml}, \mathrm{Lj}, \mathrm{Tt}$ & $\mathrm{dft}$ & Fraxinus griffithii \\
\hline luku & $\mathrm{Ml}, \mathrm{Tt}$ & 5403 & Syzygium aqueum \\
\hline lulu lima & & A & $=$ lu lima I \\
\hline lumu I & & & Magnolia glauca \\
\hline lumu II & Ml & & Musci \\
\hline lumu méo & $\mathrm{Ml}$ & & $=$ lumu II \\
\hline luni & Ml & & shrub, small, thorny \\
\hline maghi & M & & Borassus sundaicus \\
\hline maja & $\mathrm{Ml}, \mathrm{Tt}$ & & Anthocephalus chinensis \\
\hline mako & So & & Ipomoea alba \\
\hline mala & So & & shrub, small, thorny \\
\hline mama & So & & $=\mathrm{kosu}$ \\
\hline mama raké & So & & Oryza sativa f. glutinosa \\
\hline manu I & Ml, So & & Cinnamomum burmanni \\
\hline manu II & $\mathrm{Bw}, \mathrm{Wj}, \mathrm{Ml}$ & & Neolitsea \\
\hline manu III & So & & Mallotus philippensis \\
\hline manu lalu I & $\mathrm{Ml}, \mathrm{Tt}$ & & Enicostemma axillaris \\
\hline manu lalu II & $\mathrm{Tt}$ & & $=$ lasu lako \\
\hline mara & Ml & & Pometia pinnata \\
\hline $\operatorname{mar}$ & $\mathrm{Ml}, \mathrm{Tt}$ & & Alstonia spectabilis \\
\hline masi & M & & Boehmeria \\
\hline mata mela & $\mathrm{Ml}, \mathrm{Tt}$, So & 5439 & Oxalis corniculata \\
\hline matu & $\mathrm{Mn}$ & & Amorphophallus campanulatus \\
\hline
\end{tabular}




\begin{tabular}{|c|c|c|c|c|}
\hline k & maza I & Ml & & $=\operatorname{mara}$ \\
\hline \multirow[t]{5}{*}{$\mathrm{kb}$} & maza II & So & & $=$ fili $I / I I$ \\
\hline & maza bhara & MI & & tree \\
\hline & maza toro & Ml & & tree \\
\hline & mazo pubu & & A & Fungi \\
\hline & mbenga & So & & $=$ pesu benga \\
\hline $\mathrm{kr}$ & méa & $\mathrm{Ml}$, So & & Mimosa invisa \\
\hline $\mathrm{kr}$ & mé'a I & & & = méa \\
\hline \multirow[t]{5}{*}{$\mathbf{w}$} & mé'a II & Ml & & Spilanthes iabadicensis \\
\hline & mé'a ha'é & So & & Asteraceae \\
\hline & méa waé & So & & Asteraceae \\
\hline & mego & Ml & & tuber, wild \\
\hline & méké hé'é & So & 5488 & Canavalia \\
\hline $\mathrm{kb}$ & melu & Ml, So & & Rhaphidophora \\
\hline \multirow[t]{4}{*}{ f } & mengé & So & 5467 & Ipomoea \\
\hline & méngi & $\mathrm{Ml}$ & & $=$ nata méngi \\
\hline & meré & $\mathrm{Ml}$ & & = léda waé I \\
\hline & meru & $\mathrm{Ml}, \mathrm{Tt}$ & & Sericocalyx asper \\
\hline \multirow[t]{2}{*}{$\mathrm{k}$} & mési & $\mathrm{Ml}$, Tt, So & & Erythrina (subumbrans) \\
\hline & meso beki & & & $=$ lebé biza \\
\hline $\mathrm{kb}, \mathrm{f}$ & méta & So & & = néta I \\
\hline \multirow[t]{2}{*}{$\mathrm{m}, \mathrm{w}$} & meza & Ml, So & & = beza II \\
\hline & mezé & & A & vine \\
\hline \multirow[t]{4}{*}{$\mathrm{p}$} & mi goa & So & & ?Annona \\
\hline & midho & So & & $=$ nidho \\
\hline & midhu & So & 3622 & Pteris ensiformis \\
\hline & midu & $\mathrm{Wj}$, So & & zé'a nitu \\
\hline \multirow[t]{9}{*}{ w } & $\operatorname{mizu}$ & So & & Aneilema \\
\hline & mo & So & & $=$ naka \\
\hline & ?moga & & A & plant, poisonous \\
\hline & mogo & So & & = kigo nipo \\
\hline & moja & $\mathrm{Ml}$ & & Semecarpus forstenii \\
\hline & moké & MI & & $=$ tua \\
\hline & ?moko (?cemoko) & & $\mathrm{dft}$ & Pithecellobium \\
\hline & molo & So & & Crinum asiaticum \\
\hline & momi & & A & $=$ uwi momi \\
\hline \multirow[t]{7}{*}{$\mathrm{m}$} & moro api & So & & herb \\
\hline & moro bétu I & Ml, So & & Melastoma \\
\hline & moro bétu II & $\mathrm{Bb}$ & & plant \\
\hline & moro bowo & $\mathrm{Ml}, \mathrm{Tt}$ & & Portulaca oleracea \\
\hline & moro dala & & & Loranthaceae \\
\hline & moro etu & MI & & Clematis (pickeringii) \\
\hline & moro kutu & So & & shrub \\
\hline
\end{tabular}




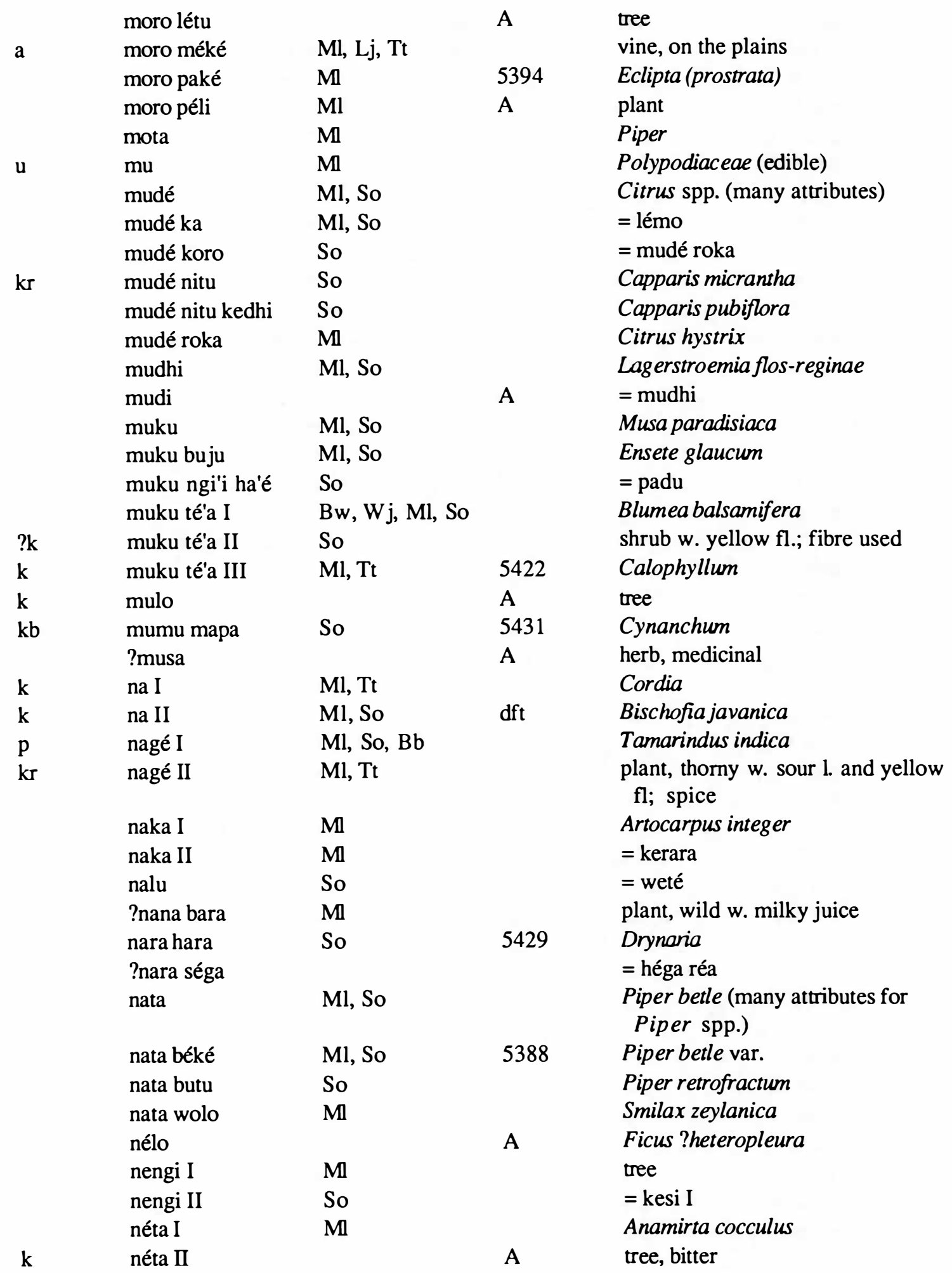




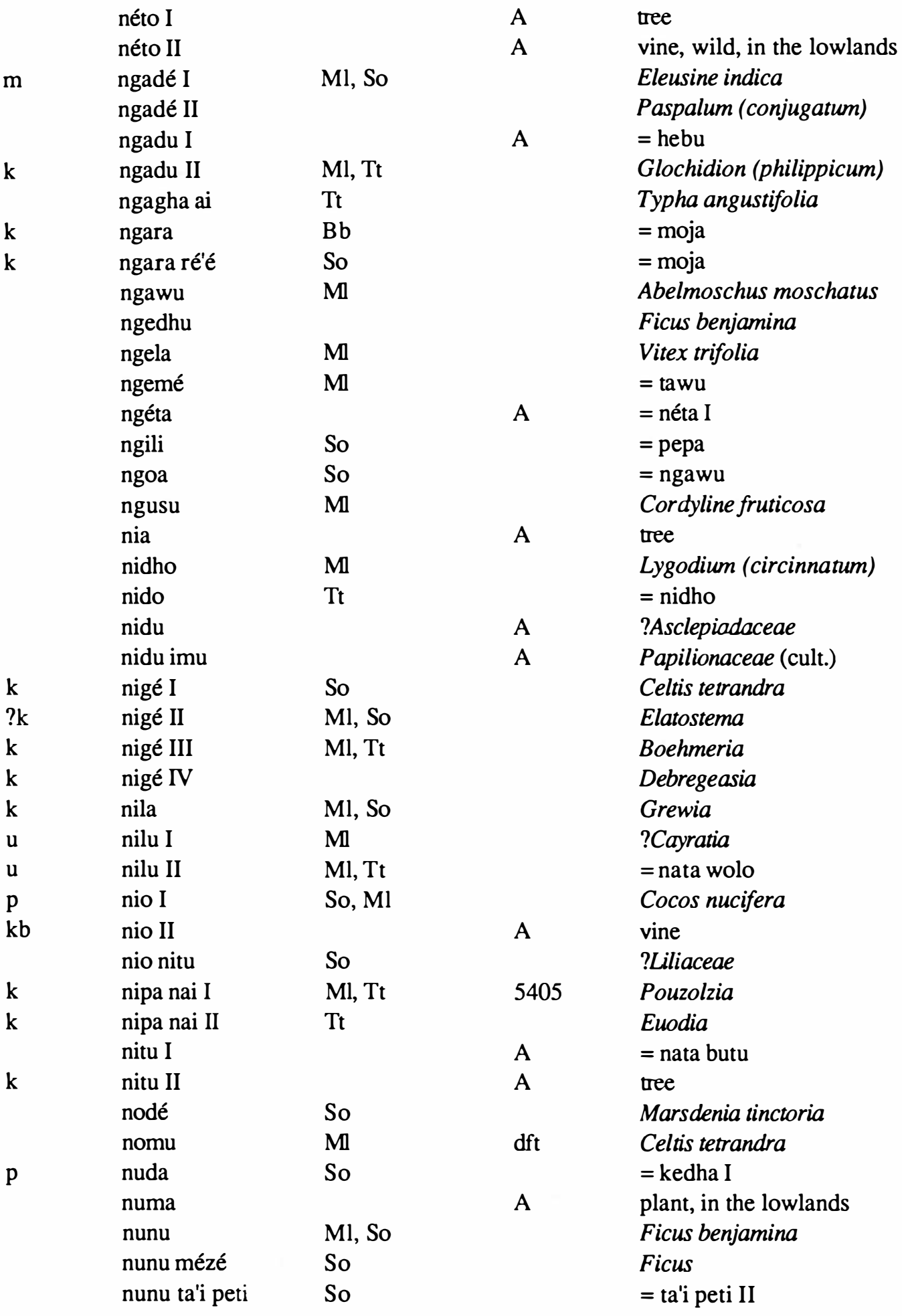




\begin{tabular}{|c|c|c|c|c|}
\hline & obho & M & & $=$ hobho I \\
\hline & odo & Ml, So & & $=\mathrm{kou}$ \\
\hline & ogo mité & $\mathrm{Tt}$ & & $=$ logo mité \\
\hline \multirow[t]{5}{*}{ k } & oja & Ml, So & $\mathrm{dft}$ & Toona sureni \\
\hline & ora goa & Ml, Tt, So & & Ageratum conyzoides \\
\hline & oza & So & & $=$ oja \\
\hline & oza laba & So & & tree, wood hard and stringy \\
\hline & oza weté & So & & tree, tall w. round fr. \\
\hline \multirow[t]{7}{*}{$\mathrm{p}$} & padu & Ml, So & & Canica papaja \\
\hline & padu goa & $\mathrm{Ml}$, So, $\mathrm{Tt}$ & 5419 & Ricinus communis \\
\hline & paga & & A & Momordica charantia \\
\hline & paga péa & Ml & & $=$ paga \\
\hline & paja I & Ml & & Dicranopteris pubigera \\
\hline & paja II & MI & & Pagiantha sphaerocarpa \\
\hline & paka & MI & & Sterculia foetida \\
\hline \multirow[t]{2}{*}{ w } & paka do I & So & 5433 & Laportea \\
\hline & paka do II & & & Fatoua pilosa \\
\hline w & paka do III & So & & Villebrunea \\
\hline $\mathrm{u}$ & paku & Ml, So & & Diplazium \\
\hline \multirow[t]{3}{*}{$\mathrm{u}$} & paku wolo & So & & Dryopteris \\
\hline & pangi & So & & = saé léwa \\
\hline & pangi jawa & So & & $=$ saé \\
\hline $\mathrm{kb}$ & papo I & $\mathrm{Ml}, \mathrm{Tt}$ & 5402 & Gymnopetalum cochinchinensis \\
\hline \multirow[t]{12}{*}{$\mathrm{kb}$} & papo II & & & Coccinia grandis \\
\hline & para & & A & tree, thorny \\
\hline & paré & Ml & & $=\mathrm{kosu}$ \\
\hline & paré nitu & M & & $=$ ngagha ai \\
\hline & patola & M & & Polypodiaceae \\
\hline & pau & Ml, So & & $\begin{array}{l}\text { Mangifera indica (several attributes } \\
\text { indicating races) }\end{array}$ \\
\hline & pau ro'a & $\mathrm{Ml}, \mathrm{Tt}$ & & Mangifera \\
\hline & pau séko & So & & Buchanania arborescens \\
\hline & pau téo I & So & & Anacardium occidentale \\
\hline & pau téo II & So & & $=$ luku \\
\hline & paza I & & & $=$ paja I \\
\hline & paza II & $\mathrm{Ml}, \mathrm{Tt}$ & & = paja II \\
\hline \multirow[t]{6}{*}{$\mathrm{kb}$} & péa & So & & $=$ paga \\
\hline & pebu & M & & tree, good timber \\
\hline & peda I & Ml & & Ananas comosus \\
\hline & peda II & Ml & & Pandanus (wild) \\
\hline & ?pedi & $\mathrm{Ml}, \mathrm{Tt}$ & & Abutilon \\
\hline & pégo & & A & Pandanus \\
\hline \multirow[t]{2}{*}{ k } & pela & $\mathrm{Ml}, \mathrm{Tt}$ & & Ficus ampelas \\
\hline & pepa & & A & Sorghum propinquum \\
\hline
\end{tabular}




\begin{tabular}{|c|c|c|c|c|}
\hline k & pépi I & So & & $=$ ngela \\
\hline k & pépi II & $\mathrm{Ml}, \mathrm{Tt}$, So & 5408 & Desmodium \\
\hline \multirow[t]{4}{*}{$\mathrm{kb}$} & pera & So & 5463 & ?Melothria \\
\hline & peri & Ml, So & & Gigantochloa ?verticillata \\
\hline & peria & Ml & & $=$ paga \\
\hline & $\begin{array}{l}\text { pesu benga } \\
\text { pidi }\end{array}$ & Ml & & $\begin{array}{l}\text { Lycianthes banahaensis } \\
\text { Ficus }\end{array}$ \\
\hline \multirow[t]{2}{*}{ k } & ?pini & Ml, So & & Podocarpus amanus \\
\hline & pira & & A & shrub w. edible fr. \\
\hline \multirow[t]{3}{*}{ k } & po & $\mathrm{Ml}, \mathrm{Tt}$ & & Mallotus ricinoides \\
\hline & pobo & $\mathrm{Tt}$ & & = baka lélu \\
\hline & poé & & A & tree \\
\hline k & polé & Ml & & Mallotus philippensis \\
\hline $\mathrm{m}$ & ponga & So & 5446 & Asclepias curassavica \\
\hline k & poni & $\mathrm{Ml}, \mathrm{Tt}$, So & & Cyathea \\
\hline \multirow[t]{3}{*}{ w } & poso reké & So & 5469 & Achyranthes \\
\hline & $\mathrm{pu}$ & & & $=$ pulu III \\
\hline & pubu & Ml, So & & Poaceae \\
\hline $\mathrm{m}$ & pubu neka (kedhi) & So & & Eulalia leschenaultiana \\
\hline $\mathrm{m}$ & pubu neka (mézé) & So & & Eragrostis japonica \\
\hline $\mathrm{m}$ & pubu waé & $\mathrm{Lj}, \mathrm{Tt}, \mathrm{Ml}$ & 3164 & Pogonatherum crinitum \\
\hline $\mathrm{u}$ & pudi & $\mathrm{Ml}, \mathrm{Tt}$ & & Costus speciosus \\
\hline $\mathrm{p}$ & pulu I & Ml, So & & Urena lobata \\
\hline \multirow[t]{7}{*}{$\mathrm{p}$} & pulu II & So & & Triumfetta \\
\hline & pulu III & $\mathrm{Tt}$ & & Cyperus \\
\hline & pulu fai & So & & $=$ pulu I \\
\hline & pulu haki & So & & $=$ pulu II \\
\hline & pura bheto & So & & Fungi \\
\hline & ?puré & Ml & & Mallotus philippensis \\
\hline & $\mathrm{ra}$ & & A & tree \\
\hline \multirow[t]{4}{*}{ k } & ra'a & So & & shrub, l. violettish \\
\hline & ra'a beka & Ml & & Clerodendrum buchanani \\
\hline & rabhu I & Ml & & Ficus \\
\hline & rabhu II & Ml, So & & Cyperus (kyllingia) \\
\hline \multirow[t]{6}{*}{$\mathrm{m}$} & rabhu III & Ml & & Cyperus brevifolius \\
\hline & rabu & & A & $=$ rabhu I \\
\hline & rabu wawi & So & 5460 & Cyperus \\
\hline & rada & & $\mathrm{dft}$ & Syzygium \\
\hline & raku & & A & plant, edible \\
\hline & rama & So, $\mathrm{Tt}$ & & Corchorus \\
\hline \multirow[t]{2}{*}{$\mathrm{m}$} & rama tuba & So & & Erigeron \\
\hline & ?ranga I & MI & & Ipomoea batatas \\
\hline k & ranga II & Ml & & tree \\
\hline
\end{tabular}




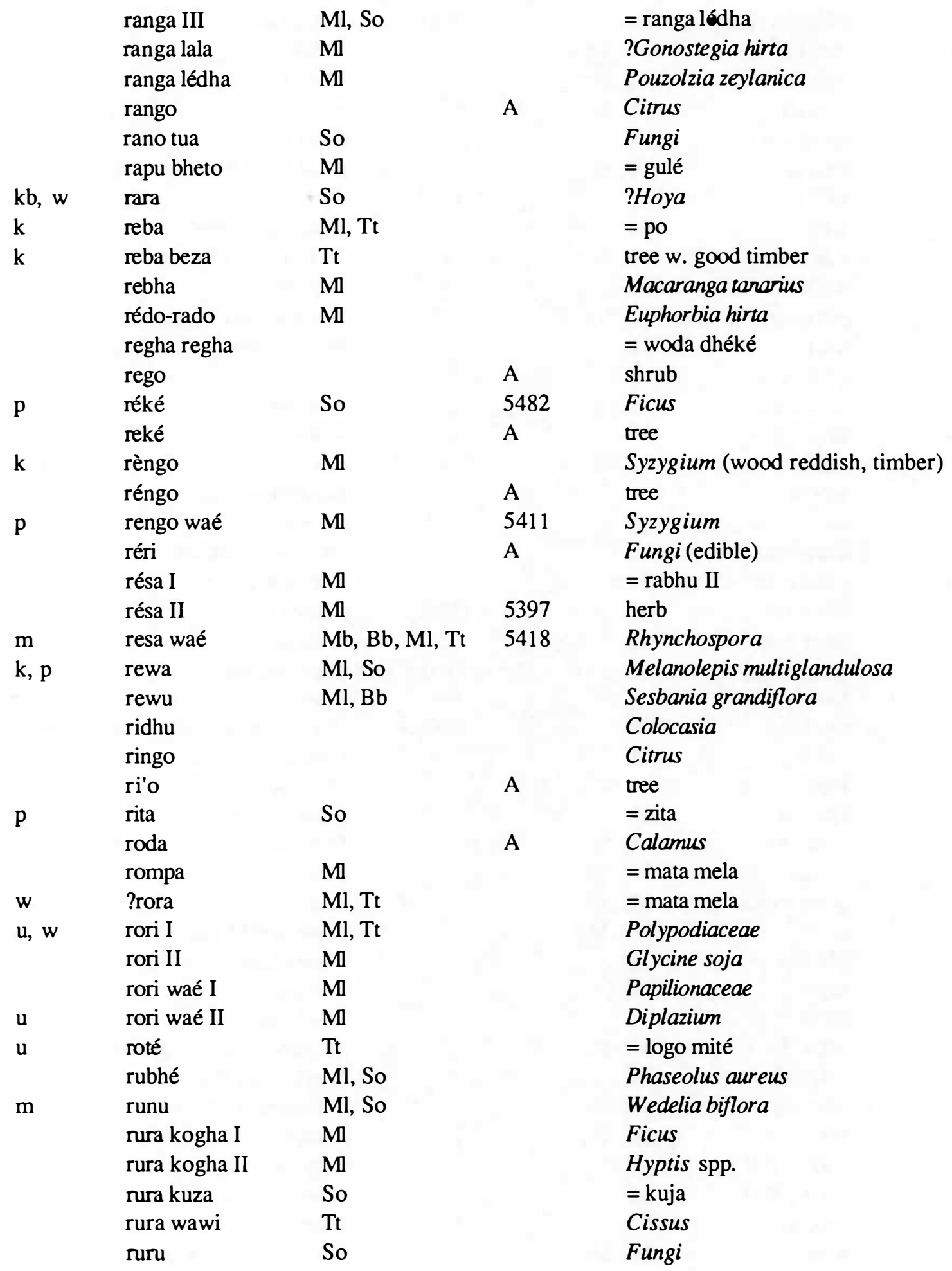




\begin{tabular}{|c|c|c|c|c|}
\hline & (wonga) ruru jawa & So & 5430 & Asystasia \\
\hline k & ruto I & Ml, So & & ?Blumea \\
\hline \multirow{5}{*}{ k } & ?ruto II & $\mathrm{Ml}, \mathrm{Tt}$ & & Ficus (w. large 1.) \\
\hline & ruto III & Ml, So & & $=$ ngedhu \\
\hline & ruto jawa & Ml & & Ficus \\
\hline & ruto nunu & Ml, So & & Ficus (w. hanging roots) \\
\hline & sa & & A & tree \\
\hline $\mathrm{k}, \mathrm{p}$ & sabi & M & & Schleichera oleosa \\
\hline \multirow[t]{3}{*}{ w } & sabi roto & $\mathrm{Ml}, \mathrm{Tt}$ & 5404 & Andrographis (paniculata) \\
\hline & saé & $\mathrm{Mn}$ & & Zea mays \\
\hline & saé léwa & $\mathrm{Ml}, \mathrm{Ng}$ & & Sorghum saccharatum \\
\hline \multirow[t]{3}{*}{ k } & saka & $\mathrm{Ml}, \mathrm{Tt}$ & & Melastoma \\
\hline & saka béka & M & & Cyperaceae \\
\hline & saka dula & M & & Acalypha \\
\hline $\mathbf{w}$ & saka kélé & Mn & & Malvaceae \\
\hline k & sala & $\mathrm{Ml}, \mathrm{Tt}$ & & tree \\
\hline \multirow[t]{3}{*}{$\mathrm{p}$} & sala luli & So & & Barleria prionitis \\
\hline & sama & M & & Pachyrrhizus erosus \\
\hline & sama jawa & M & & Dioscorea sarasinii \\
\hline \multirow[t]{6}{*}{ w } & sama roga & So & & Dryopteris \\
\hline & sama séko & So & $(5451)$ & $=$ sama \\
\hline & sama tora & So & & $=$ sama \\
\hline & sama wago & So & 5450 & Leguminosae \\
\hline & sama waru & & A & tuber \\
\hline & sao I & M & 5382 & Cucurbitaceae (wild, small) \\
\hline $\mathrm{u}$ & sao II & M & & Luffa acutangula \\
\hline \multirow[t]{3}{*}{$\mathrm{u}$} & sapa & & A & vegetable \\
\hline & sara kai & So & & Annona muricata \\
\hline & saru walu & Ml, So & & Leea indica \\
\hline \multirow[t]{14}{*}{$\mathrm{u}$} & sawa & Ml, So & & Bidens \\
\hline & sawa lebha & Ml, So & & Phaseolus \\
\hline & séa I & $\mathrm{Mn}, \mathrm{Ml}$ & & Benincasa hispida \\
\hline & séa II & $\mathrm{Mn}$ & & $=$ koro kowé \\
\hline & séga & $\mathrm{Mn}$ & & = héga réa \\
\hline & ?séki & & & tuber, edible \\
\hline & séko & Ml, So & & Psophocarpus tetragonolobus \\
\hline & séko déu I & So & & Eupatorium inulifolium \\
\hline & séko dé'u II & So & & Eupatorium odoratum \\
\hline & séko si'é I & $\mathrm{Ml}, \mathrm{Mn}$ & & = séko dé'u I \\
\hline & séko si'é II & M & & $=$ ngela \\
\hline & séko si'é III & M & & = wonga bhara II \\
\hline & séko si'é IV & $\mathrm{Tt}$ & & $=$ weka koba \\
\hline & seku & Ml, So & & Kaempferia galanga \\
\hline
\end{tabular}




\begin{tabular}{|c|c|c|c|c|}
\hline & seku dhéu & & & tree \\
\hline \multirow[t]{4}{*}{$\mathrm{kr}$} & sela & Ml & & Opuntia (elatior) \\
\hline & sela kedhi & & & $=$ rabhu II \\
\hline & selé & & A & = helé I \\
\hline & selo & $\mathrm{Mn}$ & A & Ficus (w. large fr.) \\
\hline \multirow{18}{*}{$\begin{array}{l}\mathrm{f}, \mathrm{kb} \\
\mathrm{kr}\end{array}$} & sengsara & So & & Passiflora edulis \\
\hline & sepa & Ml, So & & Caesalpinia sappan \\
\hline & séra & $\mathrm{Ml}$ & & Amomum \\
\hline & séra bhara I & $\mathrm{Ml}, \mathrm{Tt}$ & & Amomum \\
\hline & séra bahara II & $\mathrm{Tt}$ & & Alpinia \\
\hline & séra ro & $\mathrm{Tt}$ & & = séra bhara I \\
\hline & séra toro & Ml & & Zingiberaceae \\
\hline & séso & & A & plant, tuberous \\
\hline & sésu & $\mathrm{Ml}, \mathrm{Mn}$ & & = séko dé'u I \\
\hline & séu & $\mathrm{Mn}$ & & = héu \\
\hline & séu nitu & $\mathrm{Mn}$ & & = héu nitu \\
\hline & sewa rongo & MI & & Hyptis suaveolens \\
\hline & séwo & $\mathrm{Mn}$ & & Dioscorea aculeata \\
\hline & sewo & Ml & & $=$ hewo \\
\hline & séwu & & A & Dioscorea \\
\hline & sidhi & & A & Papilionaceae \\
\hline & sigu migu & Ml & & Polypodiaceae \\
\hline & siko si'é & $\mathrm{Ml}, \mathrm{Mn}$ & & = séko dé'u I/II \\
\hline \multirow[t]{12}{*}{$\mathrm{m}$} & siku deké I & So & & Paspalum conjugatum \\
\hline & siku deké II & & & $=\mathrm{waba}$ \\
\hline & sisa & & A & tree \\
\hline & (?sisoni & & dft & Elaeocarpus) \\
\hline & sobho & $\mathrm{Mn}$ & & $=$ hobho I \\
\hline & sobho tana & $\mathrm{Mn}$ & & $=$ hobho tana \\
\hline & soi & So & 5444 & Physalis \\
\hline & soi kedhi & Ml, So & & Solanum \\
\hline & so'i I & & A & Solanum nigrum \\
\hline & so'i II & & & $=$ talé \\
\hline & somu & $\mathrm{Ml}$, So & & Allium cepa var. ascalonicum \\
\hline & somu sunga & Ml, So & & Allium sativum \\
\hline k & su & So & A & tree, large \\
\hline $\mathrm{kr}$ & suda & & A & tree, thorny \\
\hline \multirow[t]{3}{*}{$\mathrm{f}, \mathrm{kb}$} & sui & So & & Ichnocarpus \\
\hline & sui koba I & Ml & 5410 & Pycnarrhena longifolia \\
\hline & sui koba II & So & & ?Ichnocarpus \\
\hline \multirow[t]{3}{*}{$\mathrm{k}, \mathrm{u}$} & su'i & $\mathrm{Ml}$ & & Champereia manillana \\
\hline & suja & So & & $=$ suza \\
\hline & suka & & A & Gnetum gnemon \\
\hline
\end{tabular}




\begin{tabular}{|c|c|c|c|}
\hline sulé & Ml & & Ficus septica \\
\hline sulu I & & A & = witu II \\
\hline sulu II & & A & tree \\
\hline suma & $\mathrm{Mn}$ & A & $=$ rewu \\
\hline suna toro & $\mathrm{Ml}$, So & & $=$ somu \\
\hline sunga & $\mathrm{Ml}$, So & & $=$ somu sunga \\
\hline sunga nitu & $\mathrm{Ml}$ & & $=\mathrm{kadu}$ \\
\hline sunga wolo & Mn & & ?Phyllanthus \\
\hline supu & & A & $=$ suza \\
\hline sura & Ma'ukéli & & $=$ suza \\
\hline susu benu & & A & $=$ susu penu I \\
\hline susu kaba & & A & Fungi \\
\hline susu penu I & Ml & & Euphorbia barnhartii \\
\hline susu penu II & & A & ?Sonchus \\
\hline susu témé & & A & $=$ susu penu I \\
\hline susu waw I & & & = kigo nipo \\
\hline susu wawi II & & A & Fungi \\
\hline suwo & $\mathrm{Jr}$ & & = wonga bhara I \\
\hline suwu & & A & $?=$ suwo \\
\hline suza & Ml, So & & Dioscorea esculenta \\
\hline suza zé'é & $\mathrm{Ml}$ & 5406 & Stephania \\
\hline tadu banga & So & $(5452)$ & plant w. bent thorns \\
\hline ta'é & & A & plant \\
\hline taga ko & $\mathrm{Tt}$ & & Vernonia cinerea \\
\hline taga kolo I & So & 5441 & Phyllanthus \\
\hline taga kolo II & So & 5445 & Euphorbia \\
\hline taga laé & & A & $=$ taga lai \\
\hline taga lai & MI & & Lycopersicon lycopersicum \\
\hline taga lai goa & So & & $=$ taga lai mézé \\
\hline taga lai kedhi & So & & $=$ taga lai (small fr.) \\
\hline taga lai mézé & So & & $=$ taga lai goa \\
\hline ta'i bara & So & & $=$ padu goa \\
\hline ta'i ha I & So & & tree \\
\hline ta'i ha II & & A & Fungi \\
\hline ta'i ha'é & So & & $=$ ta'i wawi I \\
\hline ta'i jara & $\mathrm{Tt}$ & & Fungi \\
\hline ta'i kaba & Ml & & Fungi \\
\hline ta'i kogha & So & 5455 & Cenchrus \\
\hline ta'i laja & & A & Fungi (mould) \\
\hline ta'i peti I & Ml & & Ficus heteropleura \\
\hline ta'i peti II & So & & Ficus \\
\hline ta'i su'a & & & Fungi \\
\hline ta'i wawi I & Ml & & Hyptis brevipes \\
\hline
\end{tabular}




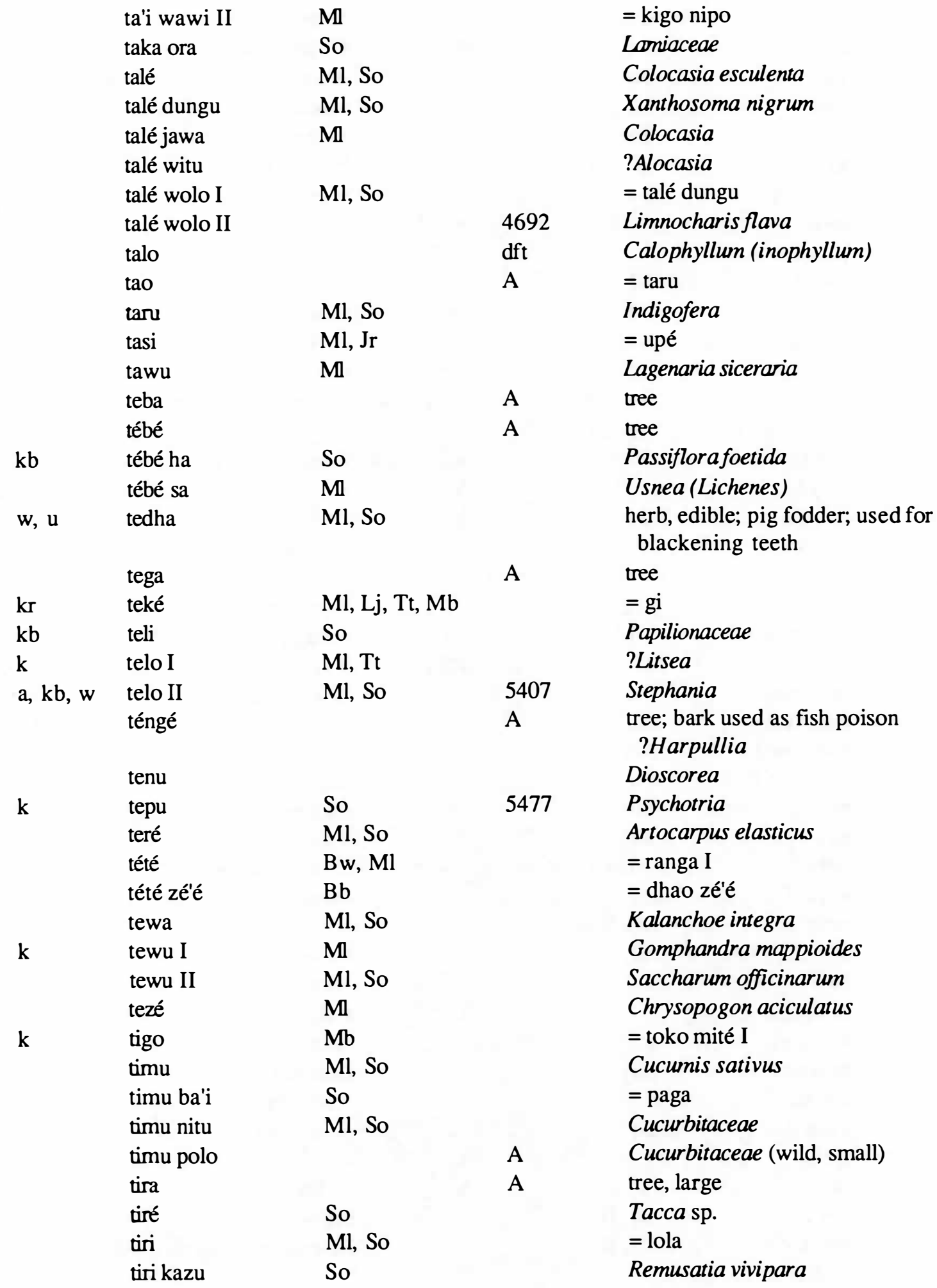




\begin{tabular}{|c|c|c|c|c|}
\hline & tiro taro & $\mathrm{Tt}$ & & Fungi \\
\hline \multirow[t]{7}{*}{$\mathrm{kr}$} & titu & $\mathrm{Bb}, \mathrm{Ml}$ & 5416 & Caesalpinia \\
\hline & tizo & So & & Amorphophallus (variabilis) \\
\hline & toba & Ml & & Homalomena \\
\hline & tobo tizo & So & & $=$ tizo \\
\hline & to'é & So & & $=$ guru to'é \\
\hline & togo édé & So & & $=$ sela \\
\hline & toko & & A & tree \\
\hline \multirow[t]{2}{*}{$\mathrm{m}$} & toko ika & & & Poaceae \\
\hline & toko kosu & Ml & & Fungi (large) \\
\hline \multirow[t]{3}{*}{ k } & toko mité I & $\mathrm{Mn}$ & 5409 & Bridelia \\
\hline & toko mité II & $\mathrm{Ml}, \mathrm{Tt}$ & & Polypodiaceae \\
\hline & toko tuki & & A & tree \\
\hline \multirow[t]{2}{*}{$\mathrm{k}$} & toko weté I & So & 5461,5462 & Breynia (racemosa) \\
\hline & toko weté II & Ml, So & & Breynia vitis-idaea \\
\hline \multirow[t]{7}{*}{$\mathrm{m}$} & toré faga neka & So & 5453,5454 & Digitaria setigera \\
\hline & toro I & & A & = lobo toro $\mathrm{I} / \mathrm{II}$ \\
\hline & toro II & So & & = bara I \\
\hline & toro karo & So & & Solanum (ferox) \\
\hline & toza & & A & $=\operatorname{tawu}$ \\
\hline & tua & $\mathrm{Ml}$, So & & Arenga pinnata \\
\hline & tua maghi & $\mathrm{Ml}$ & & $=$ maghi \\
\hline k & tui & $\mathrm{Tt}$ & 5421 & $=$ tuli \\
\hline \multirow[t]{2}{*}{$f, k b$} & tuka kutu & So & & ?Tinospora \\
\hline & tuka lako & MI & & Cyperaceae \\
\hline \multirow[t]{2}{*}{$\mathbf{a}$} & tuki toko I & $\mathrm{Ml}, \mathrm{Tt}$ & & Stemona \\
\hline & tuki toko II & $\mathrm{M}$ & & Cissus discolor \\
\hline \multirow[t]{3}{*}{ k } & tuli & MI & 5421 & Radermachera \\
\hline & turu lulu & Ml & & Fungi \\
\hline & tuwa I & Ml, So & & Croton tiglium \\
\hline \multirow[t]{11}{*}{$\mathrm{kb}$} & tuwa II & So & & Derris \\
\hline & tuwa ika & Ml, So & & $=$ tuwa II \\
\hline & ua & $\mathrm{Ml}$ & & Calamus \\
\hline & ?u'a & So & & $=\mathrm{ua}$ \\
\hline & ua maghi & & A & Calamus \\
\hline & ua manu & Ml & & ?Daemonorops \\
\hline & ua wawi & Ml & & Calamus \\
\hline & u'a manu & So & & $=$ ua manu \\
\hline & u'a wawi & So & & $=$ ua wawi \\
\hline & ujé & & A & = uzé I \\
\hline & uku & & A & $?=$ luku \\
\hline k & ular & $\mathrm{Ml}, \mathrm{Tt}$ & & Euphorbia \\
\hline $\mathrm{m}$ & uné ika & $\mathrm{Ml}, \mathrm{Tt}$ & & Desmodium ?heterocarpum \\
\hline
\end{tabular}




\begin{tabular}{|c|c|c|c|}
\hline uni & & A & tree \\
\hline upé & Ml, So & & Timonius cf. timon \\
\hline ura kogha I & So & & Sida acuta \\
\hline ura kogha II & So & & Sida rhombifolia \\
\hline ura kogha III & So & & herb \\
\hline uré & $\mathrm{Tt}$ & & = uzé I \\
\hline uté I & Ml & & Aegle marmelos \\
\hline uté II & MI & & Citrus \\
\hline uwi & Ml, So & & $\begin{array}{l}\text { Dioscorea alata (many attributes } \\
\text { indicating species and races) }\end{array}$ \\
\hline uwi jawa & Ml, So & & Manihot esculenta \\
\hline uwi momi & So & 5438 & Polycarpaea sumbana \\
\hline uza zé'é & $\mathrm{Ml}$ & & Vernonia cinerea \\
\hline uzé I & Ml & & Cajanus cajan \\
\hline uzé II & So & & tree, large \\
\hline wa & & A & tree \\
\hline waba & $\mathrm{Bb}, \mathrm{Ml}, \mathrm{Tt}$ & 5417 & Dactyloctenium aegyptium \\
\hline waé & $\mathrm{Tt}$ & & =waro I \\
\hline waé eda & $\mathrm{Ml}$ & & Bauhinia lingua \\
\hline wago I & $\mathrm{Ml}$ & & = déto \\
\hline wago II & $\mathrm{Ml}$ & & plant, wild, tuberous \\
\hline wajo I & & A & tree, large $w$. edible 1. \\
\hline wajo II & & A & Fungi \\
\hline wajo péli & MI & & = wajo II \\
\hline wako I & $\mathrm{Ml}$, So, $\mathrm{Tt}$ & & $=$ fao wako \\
\hline wako II & & A & tree w. yellowish wood \\
\hline wako laé & M & & Scleria terrestris \\
\hline wako mengé & Ml, So & & Cymbopogon citratus \\
\hline wako nawé & So & & $=$ wako mengé \\
\hline wako waé & Ml & & $=$ ngagha ai \\
\hline waku & So & & Pandanus \\
\hline wala & Ml & & Planchonia valida \\
\hline waro I & Ml & & Pterospermum diversifolium \\
\hline waro II & & A & Cucurbitaceae \\
\hline waru I & Ml, So & & Hibiscus tiliaceus \\
\hline waru II & & A & gourd \\
\hline waru jawa I & Ml, So & & Jatropha curcas \\
\hline waru jawa II & Ml & & = hapa (jawa) \\
\hline waru kéra & & A & tree \\
\hline waru waé & So & 5466 & Lepidagathis \\
\hline waru wégé & Ml & & $=$ waru jawa I \\
\hline waru wégé toro & Ml & 5420 & $=$ goa toro \\
\hline wata & So & & = weté or ghédo \\
\hline wau I & So & & Ageratum conyzoides \\
\hline
\end{tabular}




\begin{tabular}{|c|c|c|c|c|}
\hline $\mathrm{m}$ & wau II & So & & = sewa rongo \\
\hline $\mathrm{m}$ & wau III & So & 5428 & Salvia riparia \\
\hline $\mathrm{p}$ & weka I & Ml, So & & Desmodium \\
\hline \multirow[t]{2}{*}{ k } & weka II & $\mathrm{Ml}, \mathrm{Tt}$ & & plant, medicinal \\
\hline & weka koba & Ml & & Phylaceum bracteosum \\
\hline \multirow[t]{2}{*}{ w } & weka raké & So & 5472 & Desmodium strigosum \\
\hline & weté & MI & & Setaria italica \\
\hline \multirow{14}{*}{$\begin{array}{l}\mathrm{m} \\
\mathrm{m}\end{array}$} & weté nitu I & $\mathrm{Ml}, \mathrm{Tt}$ & 4654 & $=\mathrm{fu}$ lemu II \\
\hline & weté nitu II & M & & Echinochloa colonum \\
\hline & wié waé I & & A & Fungi \\
\hline & wié waé II & MI & & Elatostema \\
\hline & ?wiku & & 4729 & Polyscias scutellaria \\
\hline & wiku waé I & $\mathrm{Ml} \mathrm{Tt}$ & & Urticaceae \\
\hline & wiku waé II & Ml, So & A & Colocasia \\
\hline & wiku waé III & So & & Costus speciosus \\
\hline & wira & $\mathrm{Ml}$, So & & Alocasia macrorrhiza \\
\hline & wira waé & So & & Colocasia \\
\hline & witu I & MI & & Fungi \\
\hline & witu II & MI & & Saccharum spontaneum \\
\hline & wizu & So & & Thysanolaena maxima \\
\hline & woda dhéké & MI & & Crotalaria \\
\hline \multirow[t]{17}{*}{ k } & woi & $\mathrm{Ml}, \mathrm{Tt}$ & & Leucosyke capitellata \\
\hline & woli & MI & & tree \\
\hline & wona & Ml & & Moringa pterygosperma \\
\hline & wonga & So & & $=$ wona \\
\hline & wonga ba'i I & & & Cassia \\
\hline & wonga ba'i II & Ml & & Tithonia diversifolia \\
\hline & wonga bhara I & $\mathrm{Ml}, \mathrm{Tt}$ & & Mussaenda frondosa \\
\hline & wonga bhara II & Ml & & Pluchea indica \\
\hline & wonga bhara III & & & = seko dé'u I \\
\hline & wonga boa & $\mathrm{Bb}$, So & & $=$ boa \\
\hline & wonga bunga & So & & = wonga ba'i II \\
\hline & wonga dhonu & $\mathrm{Tt}$ & & = kigo nipo \\
\hline & wonga sésé & MI & & Cassia alata \\
\hline & wonga wau I & So & & Elsholtzia pubescens \\
\hline & wonga wau II & So & & Tournefortia \\
\hline & woni & & A & $=$ bara I \\
\hline & wowa I & & & shrub w. white fl. \\
\hline w & wowa II & So & & $=$ ra'a beka \\
\hline \multirow{4}{*}{$\mathrm{m}$} & woza paké & So & 5437 & Hedyotis \\
\hline & woza watu & & A & tree \\
\hline & wua dhara & So & & $=$ dara \\
\hline & wua nua & & A & Trichomanes \\
\hline
\end{tabular}




\begin{tabular}{|c|c|c|c|c|}
\hline \multirow[t]{2}{*}{ k } & wua pu'u & So & 5475 & Ficus \\
\hline & wua soi & So & 5444 & $=$ soi \\
\hline k & wulu I & So & 5474 & Streblus \\
\hline \multirow[t]{11}{*}{$\mathrm{p}$} & wulu II & Ml, So & & $=$ ila \\
\hline & wunu kéra & & A & plant, coastal \\
\hline & ?wunu léwa & Ml & A & Anaphalis longifolia \\
\hline & wunu mési & & A & plant, medicinal \\
\hline & wunu rori & & A & Polypodiaceae \\
\hline & wuti & & A & tree \\
\hline & wuwu & $\mathrm{Ml}$, So Bb & & $=$ paka \\
\hline & zabo & & A & tree \\
\hline & zabu & $\mathrm{Bb}$ & & = goé awa \\
\hline & zama & MI & 5389 & Pipturus argenteus \\
\hline & zama dhéké & Ml & & Urticaceae \\
\hline \multirow{4}{*}{ k } & zama nitu & So & 4416 & Boehmeria \\
\hline & zama witu & & & Leucosyke capitellata \\
\hline & zau & & A & tree \\
\hline & za'u I & Ml & A & Dracontomelon edule \\
\hline \multirow[t]{6}{*}{ w } & za'u II & Ml & & herb \\
\hline & zé'a & MI & & Pandanus tectorius \\
\hline & zé'a nitu & Ml & & Freycinetia \\
\hline & zéko & & A & Papilionaceae \\
\hline & zepé & MI & & ?Corchorus \\
\hline & zeru & & A & $=$ jeru \\
\hline \multirow[t]{3}{*}{$\mathrm{p}$} & zi'o & So & 5476 & Ficus \\
\hline & zita & Ml & & Alstonia scholaris \\
\hline & zita ré'é & $\mathrm{Bb}$ & & Rauvolfia \\
\hline
\end{tabular}




\subsection{ENDE - TAXONOMIC}

\subsubsection{PRELIMINARY REMARKS}

(a) The language

There is to my knowledge nothing published about the Endé language except Van Suchtelen's wordlist. Many insiders think that it is most cognate with Lio.

A conspicuous sound in the language (except in Nuabosi) is a kind of supradental trill or flap which of ten corresponds with $N /$ in Lio and Ngadha. It is here spelled ' $r$ '. ' $d h$ ' is the same sound (glottalised 'd') as in Ngadha.

(b) Research and identification

Many (tree) names were able to be taken from Hildebrand's list. As Ende was formerly the centre of the government in Flores, the forestry department worked there more intensively than elsewhere. This was very useful for the present work, because we (Mr Ros and myself) did not examine the forests. Our research, 1-5 July 1974, was in the region up to 500m. Many plants could be identified in the field, others were found by descriptions, and many were collected together with their names.

(c) Abbreviations

(i) Classifiers

$\begin{array}{lll}\mathrm{k} & \text { kaju } & \text { tree, shrub } \\ \mathrm{kb} & \text { komba } & \text { vine, liana } \\ \mathrm{kr} & \text { karo } & \text { thorn(y) } \\ \mathrm{ku} & \text { kuru } & \text { grass } \\ \mathrm{mb} & \text { mbené } & \text { weed, herb } \\ \mathrm{u} & \text { uta } & \text { vegetable } \\ \mathrm{w} & \text { wunu } & \text { leaf, herb }\end{array}$

(ii) Localities

$\begin{array}{lll}\text { Ln } & \text { Lianggéré } & ( \pm 500 \mathrm{~m} \text { above sea-level }) \\ \mathrm{Mb} & \text { Mbomba } & (0-100 \mathrm{~m} \text { above sea-level }) \\ \mathrm{Nb} & \text { Nuabosi } & ( \pm 250 \mathrm{~m} \text { above sea-level }) \\ \mathrm{Rr} & \text { Roworéké } & ( \pm 20 \mathrm{~m} \text { above sea-level }) \\ \mathrm{Ws} & \text { Watusipi } & ( \pm 100 \mathrm{~m} \text { above sea-level })\end{array}$

1.3.2 THE LIST

\begin{tabular}{|c|c|c|c|c|}
\hline & adhé & $\mathrm{Rr}, \mathrm{Nb}$ & RV & Amorphophallus \\
\hline k & aé I & & $\mathrm{dft}$ & Turpinia sphaerocarpa \\
\hline $\mathrm{u}$ & $\begin{array}{l}\text { aé II } \\
\text { aé isa }\end{array}$ & $\mathrm{Nb}, \mathrm{Mb}$ & RV & $\begin{array}{l}=\text { nasi } \\
\text { plant }\end{array}$ \\
\hline k & ago & $\mathrm{Nb}, \mathrm{Ln}$ & 3724 & Sterculia oblongata \\
\hline k & ai & & $\mathrm{dft}$ & Ficus oblongata \\
\hline & ako & $\mathrm{Rr}, \mathrm{Mb}$ & & Acorus calamus \\
\hline
\end{tabular}




\begin{tabular}{|c|c|c|c|c|}
\hline k & alu jawa & & $\mathrm{dft}$ & Dysoxylum \\
\hline k & ana bara & & $\mathrm{dft}$ & Drypetes longifolia \\
\hline k & ana bua & & $\mathrm{dft}$ & Rapanea \\
\hline \multirow[t]{2}{*}{ k } & ana kéa & & dft & Ehretia acuminata \\
\hline & ana na & $\mathrm{Rr}, \mathrm{Nb}$ & RV & Ananas comosus \\
\hline \multirow[t]{3}{*}{ k } & ana wonda & $\mathrm{Mb}$ & & $=$ wendi wonda \\
\hline & andé ponda & Ws & RV & Crotalaia \\
\hline & andé wonda & Ws & RV & Crotalaia \\
\hline k & angi & $\mathrm{Nb}$ & RV & = mara méké \\
\hline $\mathrm{kr}$ & ango & $\operatorname{Ln}$ & & $?=$ lodo \\
\hline k & anudi & & dft & Dysoxylum \\
\hline \multirow[t]{2}{*}{ k } & ara & $\mathrm{Rr}, \mathrm{Nb}, \mathrm{Ln}, \mathrm{Mb}$ & 3565 & Oryza sativa \\
\hline & aré & $\mathrm{Rr}$ & RV & Ficus \\
\hline k & aré mbaru & & & tree or shrub \\
\hline \multirow[t]{2}{*}{ k } & aré nana & & dft & Litsea \\
\hline & aré taki & & & Oryza sativa f. glutinosa \\
\hline k & ata & & & Annona squamosa \\
\hline \multirow[t]{3}{*}{ k } & até ro'a & & $\mathrm{dft}$ & Dysoxylum \\
\hline & $\mathrm{au}$ & $\mathrm{Rr}, \mathrm{Ln}$ & RV & Gigantochloa ?apus \\
\hline & au ré'é & $\mathrm{Rr}, \mathrm{Ln}$ & 3745 & Panicum notatum \\
\hline \multirow[t]{2}{*}{$\mathrm{kr}$} & banga & $\mathrm{Nb}$ & 3677 & Acanthospermum hispidum \\
\hline & bapa & $\mathrm{Rr}, \mathrm{Nb}$ & RV & Neonauclea calycina \\
\hline k & baru & $\mathrm{Rr}$ & $\mathrm{dft}$ & Syzygium \\
\hline \multirow[t]{9}{*}{ k } & baru aé & $\mathrm{Rr}$ & dft & Polyosma \\
\hline & basé & $\mathrm{Rr}, \mathrm{Nb}$ & RV & Pagiantha sphaerocarpa \\
\hline & béo ré'é & $\mathrm{Rr}, \mathrm{Mb}$ & 3581 & Guettarda speciosa \\
\hline & bési & $\mathrm{Rr}, \mathrm{Ln}$ & & Cucurbita moschata \\
\hline & beto & $\mathrm{Rr}, \mathrm{Ln}$ & & Dendrocalamus asper \\
\hline & bibi & $\operatorname{Ln}$ & & $=$ gegé \\
\hline & boa & Ln & & $=$ mboa II \\
\hline & bobo ré'é & $\operatorname{Ln}$ & & = ruba ré'é \\
\hline & boda & $\mathrm{Mb}$ & & Luffa aegyptiaca \\
\hline k & bonggi boja & & $\mathrm{dft}$ & Dysoxylum \\
\hline w & bongo & & & see: wunu mbongo \\
\hline \multirow[t]{3}{*}{ k } & bosé gaga & & dft & Fagraea \\
\hline & bu boréé & $\mathrm{Mb}$ & 3585 & Vernonia \\
\hline & bua nona & $\mathrm{Ln}, \mathrm{Mb}$ & & Annona squamosa \\
\hline \multirow[t]{6}{*}{$\mathrm{k}$} & ?buju & & $\mathrm{dft}$ & Elaeocarpus floribundus \\
\hline & bunga malu & $\mathrm{Rr}, \mathrm{Ln}$ & & $=\operatorname{mimosa}$ \\
\hline & bunga tonjo & $\mathrm{Mb}$ & 3594 & Catharanthus roseus \\
\hline & daga lai dhili & $\mathrm{Rr}, \mathrm{Mb}$ & & $\begin{array}{l}\text { Lycopersicon lycopersicum (wild } \\
\text { variety) }\end{array}$ \\
\hline & daga lai méré & $\mathrm{Rr}, \mathrm{Mb}$ & & Lycopersicon lycopersicum \\
\hline & daka & $\mathrm{Rr}$ & & Cucumis sativus \\
\hline
\end{tabular}




\begin{tabular}{|c|c|c|c|c|}
\hline & denu (dhenu) & $\mathrm{Rr}, \mathrm{Nb}, \mathrm{Ln}$ & RV & Melochia umbellata \\
\hline $\mathrm{k}$ & dhéo & $\mathrm{Nb}$ & $\mathrm{dft}$ & Trema orientalis \\
\hline $\mathrm{kb}$ & dhoi kamba & $\mathrm{Mb}$ & $?$ & Toxocarpus \\
\hline $\mathrm{kb}$ & dhoi tandu & & & plant \\
\hline \multirow{9}{*}{$\mathrm{k}$} & dhombo & $\mathrm{Rr}, \mathrm{Mb}$ & RV & Cassia fistula \\
\hline & égo légo & $\mathrm{Nb}$ & RV & Piper betle \\
\hline & éko méo I & $\mathrm{Nb}$ & 3658 & Neyraudia arundinacea \\
\hline & éko méo II & $\mathrm{Nb}$ & 3659 & Pennisetum macrostachyum \\
\hline & éko ndé'u & $\mathrm{Mb}$ & RV & Vitex trifolia \\
\hline & éko rako & $\mathrm{Ln}, \mathrm{Mb}$ & & ?Sorghum propinquum \\
\hline & éko té'u & $\mathrm{Rr}$ & & weed or grass, creeping \\
\hline & epo & $\mathrm{Rr}, \mathrm{Nb}$ & 3638 & Cordia \\
\hline & éra & $\mathrm{Rr}, \mathrm{Nb}, \mathrm{Ln}$ & 3681 & Scleria \\
\hline \multirow[t]{9}{*}{ k } & ero & $\mathrm{Nb}, \mathrm{Ln}$ & RV & Ficus \\
\hline & éu & $\mathrm{Rr}, \mathrm{Mb}$ & RV & Areca cathecu \\
\hline & fai & $\mathrm{Rr}, \mathrm{Ln}$ & $\mathrm{dft}$ & Albizia chinensis \\
\hline & fako & $\mathrm{Rr}, \mathrm{Ws}$ & & Fungi \\
\hline & fako tana & Ws & & $=$ hako tana \\
\hline & fangé & Ws & & ?Salvia cf. riparia \\
\hline & féo & $\mathrm{Rr}, \mathrm{Ln}$ & & Aleurites moluccana \\
\hline & fi'i ji & $\mathrm{Rr}, \mathrm{Nb}, \mathrm{Mb}$ & 3691 & Dischidia \\
\hline & fi'i juri & & & plant \\
\hline $\mathrm{k}$ & fo koki & & $\mathrm{dft}$ & Distylium stellare \\
\hline \multirow[t]{4}{*}{$\mathrm{k}$} & gagé kéli & & $\mathrm{dft}$ & Dysoxylum \\
\hline & gai & $\mathrm{Rr}, \mathrm{Ln}$ & RV & Themeda villosa \\
\hline & gai ara & $\mathrm{Mb}$ & 3689 & Coelorachis rottboellioides \\
\hline & gana wawi & $\mathrm{Rr}, \mathrm{Mb}$ & & Annonaceae \\
\hline \multirow[t]{13}{*}{ k } & gari & $\mathrm{Ln}$ & $\mathrm{dft}$ & see: nggari \\
\hline & gau & $\mathrm{Nb}$ & $\mathrm{RV}$ & Cordyline fruticosa \\
\hline & gegé & $\mathrm{Rr}, \mathrm{Mb}$ & $\mathrm{RV}$ & Leucaena leucocephala \\
\hline & gora gosa & $\mathrm{Rr}, \mathrm{Ln}$ & 3734 & Hibiscus surattensis \\
\hline & goru & $\operatorname{Ln}$ & & see: nggoru \\
\hline & gosa gora & $\mathrm{Ln}$ & & $=$ gora gosa \\
\hline & guawa & $\mathrm{Mb}$ & & Psidium guajava \\
\hline & hako & $\mathrm{Rr}$ & & $=$ fako \\
\hline & hako jara & $\mathrm{Rr}$ & $\mathrm{RV}$ & Fungi \\
\hline & hako tana & $\mathrm{Rr}, \mathrm{Mb}$ & RV & Fungi \\
\hline & hangé & $\mathrm{Rr}, \mathrm{Mb}$ & & $=$ fangé \\
\hline & hésa kungu jata & $\mathrm{Mb}$ & & Papilionaceae \\
\hline & hinga té'u & $\mathrm{Rr}, \mathrm{Ln}$ & 3740 & Leguminosae \\
\hline \multirow[t]{3}{*}{$\mathrm{kb}$} & ho'a & $\mathrm{Rr}, \mathrm{Mb}$ & RV & Ipomoea \\
\hline & huma & $\mathrm{Rr}, \mathrm{Nb}$ & $\mathrm{RV}$ & Sesbania grandiflora \\
\hline & huta & $\mathrm{Rr}, \mathrm{Nb}$ & 3637 & Cerbera manghas \\
\hline
\end{tabular}




\begin{tabular}{|c|c|c|c|c|}
\hline & ingga I & $\mathrm{Rr}, \mathrm{Nb}$ & 3647 & Moonia moluccana \\
\hline & ingga II & $\mathrm{Ln}$ & 3735 & Hypoestes \\
\hline & ira & $\mathrm{Mb}$ & & Jatropha curcas \\
\hline & iṛa meru I & $\mathrm{Mb}$ & & Jatropha gossypifolia \\
\hline & ira meru II & $\mathrm{Mb}$ & 3623 & Rhaphidophora \\
\hline & isa I & $\operatorname{Ln}$ & 3744 & Apluda mutica \\
\hline & isa II & $\mathrm{Mb}$ & RV & Terminalia catappa \\
\hline & jaga sa'i & $\mathrm{Rr}$ & RV & Ervatamia \\
\hline & jara ndora & $\mathrm{Rr}$ & & Colocasia \\
\hline & jawa & $\mathrm{Rr}, \mathrm{Nb}$ & RV & Zea mays \\
\hline & jawé I & & $\mathrm{dft}$ & Toona sureni/ciliaris \\
\hline & jawé II & & $\mathrm{dft}$ & Polyosma \\
\hline & jepa & $\mathrm{Rr}$ & RV & $=$ labu jepa \\
\hline k & jita & $\mathrm{Rr}, \mathrm{Ln}, \mathrm{Mb}$ & RV & Alstonia scholaris \\
\hline k & junu & $\mathrm{Rr}, \mathrm{Ln}$ & 3730 & Grewia \\
\hline $\mathrm{ku}$ & kabi & & $\mathrm{H}$ & Dactyloctenium aegyptium \\
\hline k & kabo & & $\mathrm{dft}$ & Myristica \\
\hline & kaju nio & $\mathrm{Nb}$ & & Harmsiopanax \\
\hline & kaju wawi & & $\mathrm{dft}$ & $=$ wela masa \\
\hline k & kaka rawa & & dft & Dysoxylum \\
\hline & kamba mbonggi & $\mathrm{Rr}, \mathrm{Mb}$ & 3708 & Malvaceae \\
\hline & kangga & $\mathrm{Rr}, \mathrm{Nb}$ & RV & Wendlandia \\
\hline & kangga au & $\mathrm{Mb}$ & 3586 & Boerhavia \\
\hline & kara & $\mathrm{Ln}$ & & ?Phaseolus \\
\hline & karara & & $\mathrm{H}$ & Artocarpus altilis (seedless form) \\
\hline & karo bési & $\mathrm{Rr}, \mathrm{Nb}$ & 3631 & Rubus \\
\hline k & kata roko & & $\mathrm{dft}$ & Tarenna incerta \\
\hline $\mathrm{kb}$ & katé & $\mathrm{Rr}, \mathrm{Ln}, \mathrm{Mb}$ & & ?Embelia \\
\hline k & kawu & & $\mathrm{dft}$ & $=$ buju \\
\hline $\mathrm{kb}$ & kedho & $\mathrm{Rr}, \mathrm{Nb}$ & RV & Byttneria flaccida \\
\hline & kedho ké & $\mathrm{Mb}$ & & plant \\
\hline & kembo & $\mathrm{Rr}, \mathrm{Mb}$ & RV & Morinda \\
\hline & ?kéra & $\mathrm{Rr}, \mathrm{Nb}, \mathrm{Mb}$ & 3649 & Moghania \\
\hline & kere kanda & $\mathrm{Nb}$ & & plant \\
\hline & kere kéba & $\mathrm{Nb}, \mathrm{Mb}$ & 3590,3669 & Elephantopus scaber \\
\hline & kere kéba haki & $\mathrm{Nb}$ & 3672 & Youngia japonica \\
\hline k & ?kesa & & & Dysoxylum densiflorum \\
\hline k & kesi & $\mathrm{Mb}$ & RV & Lannea coromandelica \\
\hline k & kesi éndé & $\mathrm{Rr}, \mathrm{Mb}$ & & ?Spathodea campanulatus \\
\hline k & kesi tana & $\operatorname{Ln}$ & RV & $=$ kangga \\
\hline k & keta & $\mathrm{Rr}, \mathrm{Nb}$ & dft, RV & Planchonella duclitan \\
\hline k & kéu & $\mathrm{Rr}$ & dft & Calophyllum soulattri \\
\hline k & kéu té'a & & $\mathrm{dft}$ & Litsea \\
\hline
\end{tabular}




\begin{tabular}{|c|c|c|c|c|}
\hline \multirow[t]{5}{*}{ k } & kewu & $\mathrm{Rr}, \mathrm{Nb}$ & RV & $=\mathrm{na}$ \\
\hline & ki & & $\mathrm{RV}, \mathrm{H}$ & Imperata cylindrica \\
\hline & kinggo & $\mathrm{Rr}, \mathrm{Nb}$ & 3678 & Emilia sonchifolia \\
\hline & kinggo nipo & $\mathrm{Mb}$ & & Erechtites valerianifolia \\
\hline & kinggo wawi & $\mathrm{Mb}$ & & Asteraceae \\
\hline k & kitu & $\mathrm{Rr}$ & $\mathrm{dft}$ & Euphorbiaceae \\
\hline k & kobé & & $\mathrm{dft}$ & = ana kéa \\
\hline k & koja & $\mathrm{Rr}, \mathrm{Mb}$ & $\mathrm{dft}$ & Canarium vulgare \\
\hline k & koja una & $\mathrm{Rr}$ & & tree or shrub \\
\hline \multirow[t]{13}{*}{ k } & koja wanda & & dft & $=$ nengi \\
\hline & koki & $\mathrm{Rr}, \mathrm{Mb}$ & 3690 & Ficus ?heteropleura \\
\hline & koko & $\mathrm{Rr}, \mathrm{Nb}$ & RV & $=$ wonga kota \\
\hline & koko woso ré'é & $\mathrm{Rr}, \mathrm{Ln}$ & RV & ?Canna \\
\hline & komba monda & $\mathrm{Rr}, \mathrm{Ln}$ & 3741 & Ipomoea obscura \\
\hline & kopi I & $\mathrm{Mb}$ & RV & Coffea \\
\hline & kopi II & & RV & Abelmoschus esculentus \\
\hline & koré & $\mathrm{Rr}, \mathrm{Ln}, \mathrm{Mb}$ & RV & Calotropis gigantea \\
\hline & korị & & RV & Borassus flabellifer \\
\hline & koro dhiki & $\mathrm{Rr}, \mathrm{Mb}$ & $\mathrm{RV}, \mathrm{H}$ & Capsicum frutescens \\
\hline & koro méré & $\mathrm{Rr}, \mathrm{Mb}$ & RV & Capsicum annuum \\
\hline & koro raru & $\mathrm{Mb}$ & 3608 & Barleria prionitis \\
\hline & koro wuro & & RV & Piper \\
\hline kb & kua I & $\mathrm{Nb}, \mathrm{Ln}$ & RV & Flagellaria indica \\
\hline k & kua II & & dft & Sarcospermum paniculatum \\
\hline \multirow[t]{4}{*}{ k } & kua III & & dft & $=$ aé II \\
\hline & kubi dhéké I & $\mathrm{Rr}, \mathrm{Nb}$ & 3664 & $\begin{array}{l}\text { Desmodium velutinum ssp. } \\
\text { velutinum }\end{array}$ \\
\hline & kubi dhéké II & $\mathrm{Mb}$ & 3716 & Moghania \\
\hline & kubi dhéké hai & $\mathrm{Nb}$ & 3663 & Desmodium \\
\hline \multirow[t]{6}{*}{ k } & kuku & & RV & Schoutenia ovata \\
\hline & kuku manu & $\mathrm{Ln}$ & 3727 & Phymatodes scolopendria \\
\hline & kulu & $\mathrm{Mb}$ & & ?Artocarpus altilis (seeded form) \\
\hline & kumi rongo I & $\mathrm{Mb}$ & 3569 & Pouzolzia \\
\hline & $\begin{array}{l}\text { kumi rongo II } \\
\text { kuné }\end{array}$ & $\mathrm{Nb}$ & 3676 & $\begin{array}{l}\text { Euphorbia } \\
\text { Curcuma viridiflora }\end{array}$ \\
\hline & kura & $\mathrm{Rr}, \mathrm{Mb}$ & 3627 & Fungi \\
\hline \multirow[t]{7}{*}{ k } & kuro & $\mathrm{Rr}$ & & Pygeum \\
\hline & kuru ba'i & $\mathrm{Nb}$ & & Paspalum conjugatum \\
\hline & kuru kamba & $\mathrm{Rr}, \mathrm{Ln}, \mathrm{Mb}$ & 3618 & Poaceae \\
\hline & kuru mengé & $\mathrm{Mb}$ & 3617 & Cyperus brevifolius \\
\hline & kuru oro & & $\mathrm{H}$ & Brachiaria subquadripara \\
\hline & $\begin{array}{l}\text { kuru weté } \\
\text { kutu }\end{array}$ & $\mathrm{Mb}$ & 4272 & $\begin{array}{l}\text { Setaria adhaerens } \\
\text { weed }\end{array}$ \\
\hline & kuwu & $\mathrm{Nb}$ & & Trichosanthes villosa \\
\hline
\end{tabular}




\begin{tabular}{|c|c|c|c|c|}
\hline \multirow{3}{*}{ k } & labu jepa & \multicolumn{2}{|l|}{$\mathrm{Rr}$} & \multirow{2}{*}{$\begin{array}{l}\text { Sechium edule } \\
\text { Fraxinus griffithii }\end{array}$} \\
\hline & ladu & & & \\
\hline & laka toba & $\mathrm{Nb}, \mathrm{Mb}$ & 3666 & Lawsonia inermis \\
\hline k & lalu & & dft & Drypetes \\
\hline k & lalu mité & & dft & Syzygium \\
\hline k & lama kamba & & dft & $=$ kua II \\
\hline k & lanu & Ln & $\mathrm{dft}$ & Albizia procera \\
\hline k & lawi éré & & dft & Syzygium \\
\hline k & lélé wula & & dft & Paratrophis philippinensis \\
\hline k & lodo & & $\mathrm{dft}$ & Randia \\
\hline k & lombo toro & & & $=$ rombo toro \\
\hline k & luku & & $\mathrm{dft}$ & Syzygium \\
\hline k & lula & & & plant \\
\hline & lula ré'é & & & plant \\
\hline & magé & $\mathrm{Ln}$ & & $=$ magi \\
\hline & magi & & RV & Tamarindus indica \\
\hline k & maja I & & $\mathrm{dft}$ & Crypteronia paniculata \\
\hline k & maja II & & dft & Elaeocarpus sphaericus \\
\hline $\mathrm{kb}$ & mako & $\mathrm{Rr}, \mathrm{Nb}, \mathrm{Ln}$ & 3742 & Ipomoea nil \\
\hline k & mara méké & $\mathrm{Ln}$ & 3720 & Pittosporum moluccanum \\
\hline & mata ika & $\mathrm{Rr}, \mathrm{Nb}$ & 3686 & Desmodium \\
\hline & mata mité & $\mathrm{Rr}, \mathrm{Mb}$ & & Papilionaceae \\
\hline & mata rera I & $\mathrm{Mb}$ & 3576 & Breynia vitis-idaea \\
\hline & mata rera II & $\mathrm{Nb}, \mathrm{Ln}$ & 3650 & Bridelia tomentosa \\
\hline k & mbaja & & $\mathrm{dft}$ & Pygeum \\
\hline w & mbaka & $\mathrm{Rr}, \mathrm{Nb}, \mathrm{Ln}$ & RV & $=$ kinggo \\
\hline & mbaka keri & $\mathrm{Nb}$ & RV & Erigeron sumatrensis \\
\hline k & mbapa & & $\mathrm{dft}$ & Premne \\
\hline & mbara roto & $\mathrm{Mb}$ & 3592 & Hibiscus vitifolius \\
\hline & mbaté kamba & $\mathrm{Nb}$ & & Sida \\
\hline & mbawu & $\mathrm{Nb}$ & & Maoutia \\
\hline k & mbera & $\mathrm{Rr}$ & & Melia azedarach \\
\hline & mbera héko I & $\mathrm{Mb}$ & 3611 & Abroma \\
\hline & mbera héko II & $\mathrm{Mb}$ & 3705 & Abroma \\
\hline & mbera nawu & $\mathrm{Rr}, \mathrm{Mb}$ & & Abelmoschus moschatus \\
\hline & mbera roto & $\mathrm{Mb}$ & & $?=$ mbara roto \\
\hline & mbera wawi & & & plant, prickly \\
\hline $\mathrm{kb}$ & mbéré wawi & $\mathrm{Nb}$ & & Embelia \\
\hline & mberi & $\mathrm{Rr}, \mathrm{Ln}$ & & Solanum melongena \\
\hline & mberi ré'é I & $\mathrm{Nb}, \mathrm{Mb}$ & 3579 & Solanum indicum \\
\hline & mberi ré'é II & $\mathrm{Mb}$ & 3580 & Solanum \\
\hline & mberi ré'é III & $\operatorname{Ln}$ & 3721 & Solanum erianthum \\
\hline & mbeté jara & $\operatorname{Ln}$ & & Sida \\
\hline
\end{tabular}




\begin{tabular}{|c|c|c|c|c|}
\hline \multirow[t]{3}{*}{ k } & mbéwa & & $\mathrm{dft}$ & Pygeum \\
\hline & mbéwo & $\mathrm{Rr}, \mathrm{Nb}, \mathrm{Mb}$ & 3707 & Cassia \\
\hline & mbindi I & $\mathrm{Rr}, \mathrm{Nb}, \mathrm{Mb}$ & 3671 & Averrhoa bilimbi \\
\hline \multirow[t]{2}{*}{$\mathrm{ku}$} & mbindi II & $\mathrm{Nb}$ & 3653 & Paspalum \\
\hline & mbisi kojo & $\mathrm{Rr}, \mathrm{Nb}$ & RV & Centella asiatica \\
\hline k & mboa I & $\mathrm{Rr}, \mathrm{Mb}$ & & Ceiba pentandra \\
\hline \multirow[t]{2}{*}{ k } & mboa II & $\operatorname{Ln}$ & & $=$ mboko ronggo I \\
\hline & mboa ré'é & $\mathrm{Rr}, \mathrm{Mb}$ & 3604 & Melastoma polyanthum \\
\hline \multirow[t]{4}{*}{ k } & mboja & & dft & $=$ tanggo \\
\hline & mboko ronggo I & & $\mathrm{dft}$ & Bombax ceiba \\
\hline & mboko ronggo II & $\mathrm{Rr}, \mathrm{Mb}, \mathrm{Ws}$ & 3599 & Phyllanthus virgatus \\
\hline & mboko sa & & $\mathrm{H}$ & Piper nignum \\
\hline \multirow[t]{10}{*}{$\mathrm{kb}$} & mbonggi & $\mathrm{Ln}$ & & Convolvulaceae \\
\hline & mbongo & $\mathrm{Mb}$ & & see: wunu mbongo \\
\hline & mboti & $\mathrm{Mb}$ & RV & Amaranthus \\
\hline & mboti karo & $\mathrm{Nb}$ & & Amaranthus (spinosus) \\
\hline & mboti ngadu rịu & $\mathrm{Mb}$ & 3566 & Amaranthus \\
\hline & mboti pesa & $\mathrm{Mb}$ & & Amaranthus lividus \\
\hline & mboti ré'é & $\mathrm{Mb}$ & & Amaranthus (?spinosus) \\
\hline & $\mathrm{mbu}$ & $\mathrm{Nb}$ & & Casuarina \\
\hline & mbu'a huja & $\mathrm{Nb}$ & 3665 & Cyperus \\
\hline & mbué tana & & $\mathrm{H}$ & Arachis hypogaea \\
\hline \multirow[t]{21}{*}{ k } & mbuju & & & $=? \mathrm{buju}$ \\
\hline & mburi mbedo & Ln & RV & Eupatorium odoratum \\
\hline & mbutu mité & $\mathrm{Mb}$ & 3713 & Abrus \\
\hline & meja & $\mathrm{Nb}$ & & Setaria palmifolia \\
\hline & meké & & & herb \\
\hline & meké jara & $\mathrm{Rr}, \mathrm{Nb}$ & & Corchorus \\
\hline & meké ré'é & & 4728 & $\begin{array}{l}\text { Desmodium velutinum ssp. } \\
\text { velutinum }\end{array}$ \\
\hline & meké reké I & $\mathrm{Mb}$ & 3575 & Sida elongata var. balica \\
\hline & meké reké II & $\mathrm{Mb}$ & 3577 & Cyathula prostrata \\
\hline & meké reké III & $\mathrm{Mb}$ & 3600 & Fatoua pilosa \\
\hline & memu & $\mathrm{Nb}$ & 3673 & Pteris biaurita \\
\hline & méngi & $\mathrm{Rr}, \mathrm{Mb}$ & & Piper betle \\
\hline & mera & $\mathrm{Mb}$ & & see: niru mera \\
\hline & mera gera & $\mathrm{Nb}$ & RV & Drymaria cordata \\
\hline & merpara & $\mathrm{Mb}$ & 3609 & Cissus \\
\hline & mersawa & $\mathrm{Nb}$ & RV & Bidens \\
\hline & meru I & $\mathrm{Rr}$ & RV & Ficus ?fistulosa \\
\hline & meru II & $\operatorname{Ln}$ & & Ficus wassa \\
\hline & mimosa & $\mathrm{Mb}$ & RV & Mimosa invisa \\
\hline & mimosa fai & $\mathrm{Nb}$ & 3683 & Cassia \\
\hline & moké & $\mathrm{Rr}, \mathrm{Ln}$ & H, R V & Arenga pinnata \\
\hline
\end{tabular}




\begin{tabular}{|c|c|c|c|c|}
\hline & monggo & $\mathrm{Rr}, \mathrm{Mb}$ & 3582 & Commelina \\
\hline & moru & $\mathrm{Mb}$ & 3701 & Ficus \\
\hline \multirow[t]{3}{*}{$\mathrm{kb}$} & mosa & $\mathrm{Nb}$ & 3633 & Mucuna acuminata \\
\hline & muku & $\mathrm{Rr}, \mathrm{Mb}$ & $\mathrm{RV}$ & Musa paradisiaca \\
\hline & muku ronggo & $\mathrm{Mb}$ & 3688 & Phyllanthus \\
\hline k & mundé & & $\mathrm{H}, \mathrm{RV}$ & Citrus grandis \\
\hline k & mundi & & $\mathrm{dft}$ & Lagerstroemia flos-reginae \\
\hline \multirow[t]{2}{*}{ k } & na & & $\mathrm{dft}$ & Bischofia javanica \\
\hline & nagha & $\mathrm{Nb}$ & & Cucurbitaceae \\
\hline k & naka & & & Artocarpus integer \\
\hline k & naka kéli & & $\mathrm{dft}$ & Planchonella \\
\hline k & naka ré'é & & $\mathrm{dft}$ & Planchonella obovata \\
\hline k & naka wara & & $\mathrm{H}$ & Artocarpus heterophyllus \\
\hline $\mathrm{kb}$ & namo & $\mathrm{Mb}$ & & Cassytha \\
\hline k & namu & & $\mathrm{dft}$ & Ulmus lanceaefolia \\
\hline $\mathrm{kb}$ & nana & $\operatorname{Ln}$ & & ?Ichnocarpus moluccanus \\
\hline k & nanggé & $\mathrm{Nb}, \mathrm{Mb}$ & & $=$ magi \\
\hline \multirow[t]{5}{*}{$\mathrm{u}$} & nasi & $\mathrm{Rr}$ & & Talinum triangulare \\
\hline & nata & $\mathrm{Mb}$ & & Piper betle \\
\hline & nawé & $\mathrm{Ln}, \mathrm{Mb}$ & & Cymbopogon citratus \\
\hline & nawu & $\mathrm{Rr}$ & & $=$ mbera nawu \\
\hline & ndaka & & & see: daka \\
\hline $\mathrm{kb}$ & ndaru & $\mathrm{Nb}$ & $\mathrm{RV}$ & ?Caesalpinia bonduc \\
\hline k & ndawa I & $\mathbf{M b}$ & 3564 & Brucea javanica \\
\hline \multirow[t]{5}{*}{$\mathrm{k}$} & ndawa II & $\operatorname{Ln}$ & 3733 & Micromelum hirsutum \\
\hline & ndepu & $\mathrm{Nb}$ & 3632 & Dioscorea \\
\hline & ndéto & $\operatorname{Ln}$ & $\mathrm{RV}$ & $=$ mosa \\
\hline & ndimu & & & Cucumis sativus var. \\
\hline & ndombo ndo'o & $\mathrm{Mb}$ & 3561 & Dendrophthoe \\
\hline \multirow[t]{5}{*}{ k } & ndopo & & $\mathrm{dft}$ & Planchonella linggensis \\
\hline & ndora bima & $\operatorname{Ln}$ & $\mathrm{RV}$ & Pachyrrhizus erosus \\
\hline & ndoro & & & Piper \\
\hline & nduru kaju & & & plant \\
\hline & nengi & $\mathrm{Nb}$ & 3635 & Canarium asperum \\
\hline \multirow[t]{8}{*}{ k } & neta batu & & $\mathrm{dft}$ & Engelhardia spicata \\
\hline & ngaga ié & $\mathrm{Nb}$ & 3636 & Dysoxylum parasiticum \\
\hline & nggara ngganda & $\mathrm{Mb}$ & 3574 & Passiflora foetida \\
\hline & nggari & $\mathrm{Nb}$ & 3642 & Rhus taitensis \\
\hline & nggendi mité & $\mathrm{Nb}$ & $\mathrm{RV}$ & Homalanthus peltatus \\
\hline & nggéo & $\mathrm{Mb}$ & RV & Dioscorea cp. pentaphylla \\
\hline & nggoé nggawa & $\operatorname{Ln}$ & $\mathrm{RV}$ & $=$ guawa \\
\hline & nggoru & $\mathrm{Nb}, \mathrm{Ln}$ & 3738,3668 & Pouzolzia \\
\hline $\mathrm{k}$ & nggura & & $\mathrm{dft}$ & Loplacea \\
\hline
\end{tabular}




\begin{tabular}{|c|c|c|c|c|}
\hline \multirow[t]{2}{*}{$\mathrm{kb}$} & ngidho & $\mathrm{Mb}$ & RV & Lygodium ?circinnatum \\
\hline & ngidho ré'é & $\mathrm{Mb}$ & $\mathrm{RV}, 3573$ & Lygodium japonicum \\
\hline \multirow[t]{4}{*}{$\mathrm{kr}$} & ngighi & $\mathrm{Mb}$ & & $\begin{array}{l}\text { tree w. small bent thorns, greer } \\
\text { fl. at stem }\end{array}$ \\
\hline & nigi sogi & $\operatorname{Ln}$ & 3731 & Toddalia asiatica \\
\hline & nindi & & & Coccinia grandis \\
\hline & nio & $\mathrm{Mb}$ & RV & Cocos nucifera \\
\hline k & nipa nai & $\mathrm{Mb}, \mathrm{Nb}$ & 3634 & Leea \\
\hline \multirow[t]{5}{*}{ k } & nira & $\operatorname{Ln}$ & RV & Grewia \\
\hline & niru mera & $\mathrm{Ln}$ & RV & Oxalis corniculata \\
\hline & noki & $\mathrm{Nb}$ & & ?Rosaceae \\
\hline & nunu & & & Ficus \\
\hline & nunu ra & $\operatorname{Ln}$ & & Ficus \\
\hline $\mathrm{k}$ & oja & & $\mathrm{dft}$ & Dysoxylum \\
\hline \multirow[t]{3}{*}{$\mathrm{k}$} & oja kapa & & $\mathrm{dft}$ & Siphonodon celastrineus \\
\hline & ondo & $\operatorname{Ln}$ & RV & Dioscorea hispida \\
\hline & ora & $\mathrm{Rr}, \mathrm{Mb}$ & RV & Acanthaceae \\
\hline \multirow[t]{11}{*}{$\mathrm{mb}$} & oṛa ia & $\mathrm{Mb}$ & RV & Ageratum conyzoides \\
\hline & oro & $\mathrm{Rr}, \mathrm{Nb}$ & 3657 & Sorghum saccharatum \\
\hline & oro jawa & & $\mathrm{H}$ & $=$ jawa \\
\hline & owo & & & tree \\
\hline & pa & & & ?Acronychia \\
\hline & pa wau & $\mathrm{Ln}$ & 3732 & Euodia \\
\hline & paku I & $\mathrm{Mb}$ & 3615 & Nephrolepis hirsutula \\
\hline & paku II & $\mathrm{Mb}$ & 3572 & Pteris vittata \\
\hline & paku manu I & $\mathrm{Mb}$ & 3706 & Tectaria siifolia \\
\hline & paku manu II & $\mathrm{Ln}$ & 3722 & Tectaria crenata \\
\hline & paku téki I & $\mathrm{Nb}$ & 3654 & Nephrolepis biserrata \\
\hline \multirow[t]{3}{*}{$\mathrm{kb}$} & paku téki II & & & vine, rather thick \\
\hline & paré ui & & & ?Oryza sativa \\
\hline & pau & $\mathrm{Rr}, \mathrm{Ln}$ & RV & Mangifera indica \\
\hline k & pau katé & & $\mathrm{dft}$ & Mangifera \\
\hline k & pau kéli & $\mathrm{Rr}, \mathrm{Nb}$ & 3630 & Buchanania arborescens \\
\hline $\mathrm{k}$ & pau rẹ̣u & $\mathrm{Mb}$ & 3593 & Manfigera timorensis \\
\hline $\mathrm{kb}$ & pendi & & & Tetracera scandens \\
\hline $\mathrm{kb}$ & penggi & & & Tetracera scandens \\
\hline \multirow[t]{2}{*}{$\mathrm{kr}$} & pera & $\mathrm{Rr}, \mathrm{Mb}$ & $\mathrm{RV}$ & Opuntia \\
\hline & peri & $\mathrm{Rr}, \mathrm{Nb}, \mathrm{Ln}$ & RV & Gigantochloa ?verticillata \\
\hline k & peto & & $\mathrm{dft}$ & Podocarpus imbricatus \\
\hline k & pira & $\mathrm{Rr}$ & $\mathrm{dft}$ & Pithecellobium umbellatum \\
\hline \multirow[t]{3}{*}{$\mathrm{k}$} & pira kéli & & $\mathrm{dft}$ & Bridelia \\
\hline & pombo & $\mathrm{Rr}, \mathrm{Ln}, \mathrm{Mb}$ & 3725 & Gynura grandifolia \\
\hline & pudi & $\mathrm{Nb}$ & & $=$ puri \\
\hline $\mathrm{ku}$ & pumbu & & RV & Poaceae \\
\hline
\end{tabular}




\begin{tabular}{|c|c|c|c|c|}
\hline & puri & $\mathrm{Rr}$ & RV & Costus speciosus \\
\hline & puru I & $\mathrm{Ln}, \mathrm{Mb}$ & 3563 & Triumfetta \\
\hline & puru II & $\mathrm{Mb}$ & 3710 & Triumfetta \\
\hline & ?puru fai & $\mathrm{Nb}$ & & Urena lobata \\
\hline $\mathrm{u}$ & $\mathrm{ra}$ & $\mathrm{Rr}$ & & Ficus \\
\hline & ragé & $\mathrm{Ln}$ & & Uvaria \\
\hline & rama & $\mathrm{Rr}, \mathrm{Nb}, \mathrm{Ln}$ & 3682 & Pipturus \\
\hline & ramu & $\mathrm{Rr}$ & RV & Musci \\
\hline k & ranu & & & $=$ lanu \\
\hline k & ra'u I & & $\mathrm{dft}$ & Dracontomelon edule \\
\hline k & ra'u II & & $\mathrm{dft}$ & $?=$ tapa seké \\
\hline k & ra'u mbopo & & $\mathrm{dft}$ & Aglaia ganggo \\
\hline & réba & $\mathrm{Nb}, \mathrm{Mb}$ & RV & Momordica charantia \\
\hline & rebe ré'é & $\mathrm{Rr}$ & & = ruba ré'é \\
\hline & rema manu & $\mathrm{Nb}$ & & plant \\
\hline & rema mori lo'o & $\mathrm{Mb}$ & 3597 & Indigofera linifolia \\
\hline k & rema réwa & & & tree w. yellow fl. \\
\hline & rẹmé & $\operatorname{Ln}$ & RV & ?Mallotus ricinoides \\
\hline & remi man & $\mathrm{Rr}$ & 3675 & Borreria levis \\
\hline k & rena & $\mathrm{Rr}$ & $\mathrm{dft}$ & Salix tetrasperma \\
\hline & répa reko & $\mathrm{Rr}, \mathrm{Nb}, \mathrm{Ln}$ & 3661,3717 & Selaginella plana \\
\hline & repo & & 3613 & Selaginella willdenowii \\
\hline k & réréé & $\mathrm{Rr}, \mathrm{Nb}$ & 3640 & Ficus \\
\hline & rẹéé mberé & $\mathrm{Rr}, \mathrm{Nb}$ & 3643 & Ficus \\
\hline & rẹéé méré & & RV & Ficus \\
\hline & reri I & $\mathrm{Rr}, \mathrm{Nb}$ & 3670 & cf. Melothria \\
\hline $\mathrm{kb}$ & $\begin{array}{l}\text { reri II } \\
\text { reri III }\end{array}$ & $\mathrm{Nb}$ & 3652 & $\begin{array}{l}\text { Lycianthes leve } \\
\text { epiphyte, climbing w. coarse } \\
\text { l. and red fr. }\end{array}$ \\
\hline $\mathrm{u}$ & rerị IV & $\mathrm{Mb}$ & RV & Pormulaca oleracea \\
\hline & rẹeri aré & $\mathrm{Rr}, \mathrm{Mb}, \mathrm{Ws}$ & 3570 & Polycarpaea zollingeri \\
\hline & reru & $\mathrm{Rr}, \mathrm{Ln}$ & & Polypodiaceae \\
\hline & reru ré'é & $\mathrm{Nb}$ & RV & ?Cyperus tenuiculmis \\
\hline & rewa & $\mathrm{Nb}$ & & Melanolepis multiglandulosa \\
\hline & rewo (rewo) & $\operatorname{Ln}$ & & $=$ mbera nawu \\
\hline & rịma réwa & $\mathrm{Rr}, \mathrm{Mb}$ & 3610 & Dysoxylum \\
\hline & rinu & $\mathrm{Rr}, \mathrm{Nb}, \mathrm{Mb}$ & & = wonga bara II \\
\hline & robho & $\mathrm{Rr}, \mathrm{Mb}, \mathrm{Ws}$ & RV & ?Amorphophallus \\
\hline & rojo & $\mathrm{Rr}, \mathrm{Nb}$ & RV & Ensete glaucum \\
\hline & rombo toro & $\mathrm{Rr}, \mathrm{Mb}$ & & Glochidion \\
\hline & rosé & $\mathrm{Rr}, \mathrm{Nb}, \mathrm{Ln}$ & RV & Colocasia esculenta \\
\hline & ruba ré'é & $\mathrm{Rr}, \mathrm{Ln}$ & 3702 & Hyptis suaveolens \\
\hline k & ruku & $\mathrm{Rr}$ & dft & Syzygium \\
\hline & runu & $\mathrm{Nb}, \mathrm{Ln}$ & 3674 & Wedelia urticaefolia \\
\hline
\end{tabular}




\begin{tabular}{|c|c|c|c|c|}
\hline \multirow{8}{*}{$\mathrm{k}$} & runu jawa & $\mathrm{Mb}$ & 3601 & Wedelia biflora \\
\hline & saka & & $\mathrm{dft}$ & Stemonurus \\
\hline & sambé roto & $\mathrm{Ln}$ & & herb, medicinal \\
\hline & sambé ruto & $\mathrm{Mb}$ & 3591 & Andrographis paniculata \\
\hline & sambi & $\mathrm{Rr}, \mathrm{Mb}$ & $\mathrm{RV}$ & Schleichera oleosa \\
\hline & sara & $\mathrm{Rr}, \mathrm{Ln}$ & & ?Lecanopteris carnosa \\
\hline & sara koré & $\mathrm{Rr}$ & & Polypodiaceae \\
\hline & sara waru & $\mathrm{Rr}, \mathrm{Mb}$ & RV & Leea angulata \\
\hline \multirow{3}{*}{$\begin{array}{l}\mathrm{u} \\
\mathrm{k}\end{array}$} & sasi & $\mathrm{Nb}$ & $\mathrm{RV}$ & $=$ nasi \\
\hline & sati & & $\mathrm{dft}$ & Celtis tetrandra \\
\hline & sawa ra & $\operatorname{Ln}$ & 3743 & Cissus \\
\hline k & sawo kaka? I & $\mathrm{Rr}$ & $\mathrm{dft}$ & Terminalia 'mollis' \\
\hline \multirow[t]{8}{*}{$\hat{k}$} & sawo kaka II & & $\mathrm{dft}$ & Sterculia \\
\hline & sebé wéa & $\mathrm{Nb}$ & RV & Crinum asiaticum \\
\hline & sega sa'i & $\mathrm{Nb}, \mathrm{Ln}$ & $\mathrm{RV}$ & $=$ jaga sa'i \\
\hline & segé & $\mathrm{Ln}$ & RV & Pithecellobium tomentellum \\
\hline & segé sa'i & $\mathrm{Ln}, \mathrm{Mb}$ & & $=$ jaga sa'i \\
\hline & ségo régo & $\mathrm{Mb}$ & 3587 & Leguminosae \\
\hline & selé & & & herb w. rose fl. \\
\hline & sendo & $\mathrm{Rr}, \mathrm{Ln}$ & 3736 & Cardiospermum halicacabuin \\
\hline $\mathrm{kb}$ & sepi muku & $\operatorname{Ln}$ & 3739 & Merremia unbellata \\
\hline \multirow[t]{2}{*}{$\mathrm{kb}$} & seri & & $\mathrm{RV}$ & Ficus ?punctata \\
\hline & sewo toto & $\mathrm{Ln}$ & & herb, tall, many branches \\
\hline \multirow[t]{2}{*}{ k } & ?sié (?sé) & & $\mathrm{dft}$ & $=$ tapa seké \\
\hline & sigo & $\mathrm{Ln}, \mathrm{Mb}$ & 3729 & Trema tomentosa \\
\hline k & singgi bara & & $\mathrm{dft}$ & Dysoxylum \\
\hline k & singgi mité & & $\mathrm{dft}$ & Syzygium \\
\hline $\mathrm{k}$ & soké & & $\mathrm{dft}$ & Gyrinops cumingiana \\
\hline \multirow[t]{2}{*}{$\mathrm{k}$} & soki & $\mathrm{Rr}$ & RV & Clerodendrum buchanani \\
\hline & soro mbika & $\mathrm{Nb}$ & & $=$ soki \\
\hline w & soso & $\mathrm{Rr}, \mathrm{Nb}$ & 3656 & Dryopteris purpurascens \\
\hline w & sui & $\mathrm{Rr}, \mathrm{Mb}$ & & ?Champereia manillana \\
\hline \multirow[t]{9}{*}{ k } & suka & $\mathrm{Mb}$ & & Gnetum \\
\hline & suka ré'é & $\mathrm{Mb}$ & $\mathrm{RV}$ & tree w. inedible 1. \\
\hline & $\begin{array}{l}\text { suka ria } \\
\text { supé }\end{array}$ & $\mathrm{Mb}$ & 3606 & $\begin{array}{l}\text { Phaleria octandra } \\
\text { vegetable, bitter }\end{array}$ \\
\hline & suré & $\mathrm{Rr}, \mathrm{Nb}, \mathrm{Mb}$ & RV & Ficus septica \\
\hline & ?suru mika & $\mathrm{Nb}$ & & $?=$ soki \\
\hline & susu meti & $\mathrm{Rr}, \mathrm{Mb}$ & 3619,4275 & Heteropogon contortus \\
\hline & taga & $\mathrm{Rr}, \mathrm{Nb}$ & RV & Calamus \\
\hline & ta'i manu & $\mathrm{Rr}, \mathrm{Ln}$ & $\mathrm{RV}$ & Vernonia cinerea \\
\hline & ta'i ra & $\operatorname{Ln}$ & 3723 & Kalanchoe cf. integra \\
\hline k & talo & & $\mathrm{dft}$ & Litsea \\
\hline
\end{tabular}




$$
\begin{aligned}
& \text { k } \\
& k \\
& k \\
& k
\end{aligned}
$$

k

k

k

k

k

k

$\mathrm{kb}$

$\mathrm{kb}$

$\mathrm{k}$
$\mathrm{k}$
$\mathrm{k}$

k

$\begin{array}{ll}\text { tanggo } & \\ \text { tapa seké } & \\ \text { taro } & \\ \text { taro kéli } & \\ \text { taru } & \\ \text { taru ndu'a } & \mathrm{Rr}, \mathrm{Mb} \\ \text { tawu I } & \mathrm{Rr}, \mathrm{Mb} \\ \text { tawu II } & \\ \text { tenggo loho } & \\ \text { tepu } & \\ \text { teré } & \mathrm{Rr}, \mathrm{Nb}, \mathrm{Mb} \\ \text { tewu } & \mathrm{Rr}, \mathrm{Nb}, \mathrm{Ln} \\ \text { tiri } & \mathrm{Rr}, \mathrm{Mb} \\ \text { tiri ré'é } & \mathrm{Rr} \\ \text { toko mbako } & \mathrm{Mb} \\ \text { toko mbo } & \\ \text { toko mbonga } & \mathrm{Rr}, \mathrm{Ln} \\ \text { toko monda } & \mathrm{Nb} \\ \text { toko retu } & \mathrm{Rr}, \mathrm{Nb} \\ \text { toko tenggo } & \mathrm{Mb} \\ \text { toko toro } & \mathrm{Rr}, \mathrm{Nb} \\ \text { tuku tobhé } & \mathrm{Nb} \\ \text { tungo } & \mathrm{Rr}, \mathrm{Ln} \\ \text { tutu po } & \mathrm{Nb} \\ \text { tuwa I } & \mathrm{Nb} \\ \text { tuwa II } & \mathrm{Rr} \\ \text { ua } & \mathrm{Ln} \\ \text { ua ré'é } & \mathrm{Rr}, \mathrm{Ln} \\ \text { ua taga } & \\ \text { uju bima ré'é I } & \mathrm{Rr}, \mathrm{Nb} \\ \text { una ika } & \mathrm{Mb}, \mathrm{Nb} \\ \text { upé } & \\ \text { ura } & \\ \text { uru aja } \\ \text { uru talo } \\ \text { uru waté } \\ \text { uta }\end{array}$

$\mathrm{dft}$

dft

dft

$\mathrm{dft}$

3598

3651

3639

3589

RV

3684

RV

RV

3719

RV

3603

$\mathrm{H}$

3687

3700

dft

dft

dft

3648

3567

RV

3726

$\mathrm{dft}$
Meliosma ferruginea

Mastixia rostrata

Syzygium

Platea

Indigofera

Euphorbia schuhmannii

Engelhardia spicata

= tanggo

plant

Acanthaceae

Artocarpus elasticus

Saccharum officinarum

Amorphophallus

Amorphophallus variabilis

Tarenna incerta

plant, new, adventitious

Hyptis longipes

Stephania

Tridax procumbens

Gendarussa vulgaris

plant

Asparagus racemosus

Citrus

Mallotus ricinoides

Derris

Ryssopterys timoriensis

Calomus

Ischaemum

Calamus

Colocasia esculenta

Desmodium

Timonius cf. timon

Cajanus cajan

Litsea

Litsea

Litsea velutina

= uta ba'i

Carica papaya

Dioscorea alata

Ipomoea pes-tigridis

Manihot esculenta

Clematis

Helicia 
k

k

k

k

$\mathrm{ku}$

k

k

k

k

\begin{tabular}{|c|c|c|c|}
\hline wae & $\mathrm{Rr}, \mathrm{Ln}$ & dft, RV & Pterospermum diversifolium \\
\hline wa'i ré'é & $\mathrm{Nb}$ & 3662 & Leguminosae \\
\hline wajo & $\mathrm{Rr}$ & $\mathrm{dft}$ & Adenanthera pavonina \\
\hline wana & $\mathrm{Mb}$ & & ?Smilax zeylanica \\
\hline wara I & $\mathrm{Rr}, \mathrm{Nb}, \mathrm{Ln}$ & RV & Planchonia valida \\
\hline wara II & & $\mathrm{dft}$ & Terminalia \\
\hline waru & $\mathrm{Rr}, \mathrm{Ln}, \mathrm{Mb}$ & RV & Hibiscus tiliaceus \\
\hline waru jawa & & $\mathrm{dft}$ & Litsea \\
\hline wasa mburi & $\mathrm{Rr}, \mathrm{Ln}$ & & plant \\
\hline wasa ṛima & $\mathrm{Rr}, \mathrm{Mb}$ & 3588 & Acalypha indica \\
\hline wela masa & & $\mathrm{dft}$ & Acer laurinum \\
\hline wena na & & $\mathrm{dft}$ & Palaquium \\
\hline wendi wonda & $\mathrm{Mb}$ & 3571 & Physalis micrantha \\
\hline wendo ro'a & $\mathrm{Nb}$ & 3641 & Cassia \\
\hline wira & & & Colocasia \\
\hline wira toro & $\mathrm{Nb}$ & 3644 & Alocasia \\
\hline witu & $\mathrm{Rr}, \mathrm{Nb}$ & $\mathrm{RV}$ & Saccharum spontaneum \\
\hline wolo angi & & $\mathrm{dft}$ & Planchonella nitida \\
\hline wona & $\mathrm{Rr}, \mathrm{Mb}$ & RV & Moringa pterygosperma \\
\hline ?wonda ro'a & $\mathrm{Rr}, \mathrm{Nb}$ & & Crotalaria \\
\hline wonga bara I & $\mathrm{Mb}$ & & Mussaenda frondosa \\
\hline wonga bara II & & & Eupatorium inulifolium \\
\hline wonga kota & $\mathrm{Mb}$ & & Canna coccinea \\
\hline wonga mata & $\mathrm{Mb}$ & 3583 & cf. Lepidagathis \\
\hline wonga wonda & $\mathrm{Mb}$ & & $=$ wendi wonda \\
\hline wora mbata & $\mathrm{Mb}$ & 3607 & Tournefortia \\
\hline wowo & & $\mathrm{dft}$ & Sarcosperma paniculatum \\
\hline wunu butu & & $\mathrm{dft}$ & Mimusops elengi \\
\hline wunu gaga & & $\mathrm{dft}$ & Tarenna incerta \\
\hline wunu mbongo & $\mathrm{Mb}$ & 4267 & Ehretia microphylla \\
\hline wunu mera & $\mathrm{Ln}, \mathrm{Mb}$ & 3562 & $=$ niru mera \\
\hline wunu meré & $\mathrm{Mb}$ & 4274 & Bridelia \\
\hline wura & $\mathrm{Rr}, \mathrm{Ln}, \mathrm{Mb}$ & 3578 & Macaranga \\
\hline wuru & $\mathrm{Rr}, \mathrm{Nb}, \mathrm{Ln}$ & $\mathrm{RV}$ & Schizostachyum blumii \\
\hline wuwu & $\mathrm{Rr}, \mathrm{Mb}$ & RV & Sterculia foetida \\
\hline
\end{tabular}




\subsection{LIO - TAXONOMIC}

\subsubsection{PRELIMINARY REMARKS}

(a) The language

The Lio language, which is spoken in the western part of the civil district of Sika, as well as in the former kingdom of Lio, resembles closely that of Endé. It has several rather different dialects; compare the terms for 'vine' below, and the sound-shift of $k>h$ in the south-east.

(b) Research and identification

I used Hildebrand's list and Heyne's book with their identifications. Amdt provided many plant names in his dictionary (Amdt 1934), many of which I was able to identify with the help of informants.

Mr Ros and I did research in the highlands of Moni, also climbing the Kelimuti, in 1974. We could identify many plants in the field and collected others. In 1987 we spent three days in the lowlands of Watunéso. There we checked our list with excellent informants, Mrs Veronika Aés and Ms W. Nggana, who gave data about the district of Detu Kéli in northern Lio. Besides that we did some identif ying in the field.

Fr Dirk Visser's hospitality and knowledge of the people and environment were very usef ul to us.

In Ndona my list was checked with Mr A.W. Soré.

(c) Abbreviations

(i) Classifiers

$\begin{array}{lll}\mathrm{a} & \text { ajé } & \text { vine, liana } \\ \mathrm{b} & \text { bené } & \text { weed (also for mushrooms) } \\ \mathrm{g} & \text { gulu } & \text { thorn(y), spike } \\ \mathrm{hb} & \text { hoba } & =\text { ajé } \\ \mathrm{k} & \mathrm{kaju} & \text { tree, shrub } \\ \mathrm{kb} & \mathrm{koba} & =\text { ajé } \\ \mathrm{kr} & \text { karo } & \text { thom } \\ \mathrm{ku} & \text { kuru } & \text { grass } \\ \mathrm{p} & \text { pu'u } & \text { palm, stool } \\ \mathrm{t} & \text { toko } & \text { stem, stalk (of high grasses) } \\ \mathrm{u} & \text { uta } & \text { vegetable, herb } \\ \mathrm{ub} & \text { uba } & =\text { ajé } \\ \mathrm{w} & \text { wunu } & \text { leaf, herb }\end{array}$

(ii) Localities

$\begin{array}{ll}\text { Dk } & \text { Detukeli } \\ \mathrm{Ds} & \text { Detusoko } \\ \mathrm{Mn} & \text { Moni } \\ \mathrm{Nd} & \text { Ndona } \\ \mathrm{Pg} & \text { Paga (Amdt 'P') } \\ \mathrm{Wj} & \text { Wolojita (A 'V') } \\ \mathrm{Wn} & \text { Watunéso (A 'N') }\end{array}$


If no place is named by Arndt, the plant name concerned is from Mbuli or Lisé Detu, which are situated in south-east Lio.

(iii) Additional sources

A Amdt

\subsubsection{THE LIST}

ub aé I

k aé II

aé na

k aé nona

hb ajé moda

k amo

ana baga

ana gebu

?ana na

$k$ ana ndawa

k

k

$\mathrm{kb}$ anggo I

$\mathrm{hb}$ anggo II

$\mathrm{kb} \quad$ ara I

k ara II

aré na

k aré nana

k aré ngoni

aré nona

asa uwi

ata niti

p au

$\mathrm{k}, \mathrm{p} \quad$ baké

bako

k balu

$\mathrm{k}$ balu aé

k balu mité

k balu weté

k bama

k bapa bao

bara béwa

bara kowé

k basé

k baso

bela
$\mathrm{Mn}, \mathrm{Nd}$

Nd

Dk

Nd

$\mathrm{Mn}, \mathrm{Nd}$

$\mathrm{Mn}$

$\mathrm{Mn}$

$\mathrm{Nd}, \mathrm{Dk}$

$\mathrm{Nd}$

Nd, Dk

$\mathrm{Dk}$

$\mathrm{Mn}, \mathrm{Nd}$

$\mathrm{Nd}$

$\mathrm{Mn}, \mathrm{Nd}$

$\mathrm{Mn}, \mathrm{Nd}$

$\mathrm{Mn}, \mathrm{Nd}$

$\mathrm{Nd}$

$\mathrm{Nd}$

Dk

$\mathrm{Mn}, \mathrm{Nd}$

$\mathrm{Nd}, \mathrm{Dk}$

$\mathrm{Mn}, \mathrm{Nd}$

$\mathrm{Mn}, \mathrm{Nd}$ vegetable

Podocarpus ?neriifolius

= aré na

$=$ aré nona

A

vine or liana

tree

$3777 \quad$ Cyperus cf. brevifolius

A

plant

= aré na

shrub

$=$ ata niti

dft Weinmannia

Passiflorafoetida

Vitis vinifera

A plant, tuberous, thomy

Ficus variegata

Ananas comosus

Litsea

= kaba ana ko'o

Annona squamosa

?Dioscorea

plant

Gigantochloa ?apus

?Ficus

Nicotiana tabacum

Syzygium

Polyosma

tree or shrub

tree or shrub

?Arytera litoralis

tree

plant, tuberous

Cyperus ?rotundus

Pagiantha sphaerocarpa

?Ficus

Schizostachyum brachycladum 


\begin{tabular}{|c|c|c|c|c|}
\hline $\mathrm{kb}$ & bela ba'i & $\mathrm{Mn}, \mathrm{Nd}$ & & Cucurbitaceae \\
\hline & bela wula & $\mathrm{Mn}, \mathrm{Nd}$ & & ?Schizostachyum \\
\hline & bengu & $\mathrm{Mn}$ & & $=$ gau \\
\hline k & bera & $\mathrm{Mn}, \mathrm{Nd}$ & $\mathrm{dft}$ & Melia azedarach \\
\hline b & bera bela & & $\mathrm{H}$ & Brachiaria subquadripara \\
\hline & beréné (bon) & $\mathrm{Nd}$ & & Phaseolus vulgaris \\
\hline & berkowé & $\mathrm{Mn}$ & & = bara kowé \\
\hline $\mathrm{kb}$ & bési & $\mathrm{Mn}, \mathrm{Nd}$ & & Cucurbita moschata \\
\hline $\mathrm{b}$ & beté jara & $\mathrm{Nd}$ & & Sida \\
\hline & beté kamba & Dk & & Sida acuta \\
\hline & bewu & Mn, Nggéla & & Lindernia \\
\hline & bheto & $\mathrm{Mn}, \mathrm{Nd}$ & & Dendrocalamus asper \\
\hline & bheto laka & $\mathrm{Nd}$ & & Bambuseae \\
\hline & bheto nitu & $\mathrm{Nd}$ & & = gai kiu \\
\hline $\mathrm{p}$ & biji & $\mathrm{Mn}, \mathrm{Nd}$ & & Pinanga \\
\hline $\mathrm{h}$ & bila & Dk & & Crescentia cujete \\
\hline $\mathrm{kb}$ & $\begin{array}{l}\text { bima } \\
\text { bima beku }\end{array}$ & Wn & & $\begin{array}{l}\text { = ndora bima } \\
\text { pulse }\end{array}$ \\
\hline k & bira & & & tree \\
\hline k & boa & $\mathrm{Mn}, \mathrm{Nd}$ & & Ceiba pentandra \\
\hline k & boa jawa & Dk & & $=$ boa \\
\hline k & boa ré'é & Dk & & Bombax ceiba \\
\hline k & boi & & A & tree \\
\hline k & bongo & $\mathrm{Nd}, \mathrm{Dk}$ & & Ficus ?fistulosa \\
\hline & bora & $\mathrm{Mn}$ & 3774 & Moonia moluccana \\
\hline $\mathrm{p}$ & boro & $\mathrm{Mn}$ & & Corypha utan \\
\hline $\mathrm{u}$ & boti & $\mathrm{Nd}$ & 3749 & Amaranthus (lividus) \\
\hline b & boti gulu & $\mathrm{Nd}$ & & Amaranthus ?spinosus \\
\hline & boti puti & $\mathrm{Mn}, \mathrm{Nd}$ & & $=$ boti \\
\hline k & bu I & $\mathrm{Nd}, \mathrm{Dk}$ & 3826 & Casuarina junghuhniana \\
\hline k & bu II & Dk & & Casuarina equisetifolia \\
\hline b & bu béwa & & & $=$ lesé \\
\hline b & bu boré & & & Hyptis suaveolens \\
\hline k & bu fo & & A & tree \\
\hline & bu mbéwa & & & $=$ sora \\
\hline $\mathrm{k}$ & bu rua & & A & tree \\
\hline $\mathrm{kb}$ & bué & $\mathrm{Nd}$ & & $\begin{array}{l}\text { (many attributes indicating several } \\
\text { species and varieties of pulses) }\end{array}$ \\
\hline & bué bewu & & & pulse \\
\hline & bué bosi & & & = beréné (bon) \\
\hline & bué duké & $\mathrm{Nd}$ & 3761 & Canavalia \\
\hline & bué jo & $\mathrm{Nd}$ & & pulse \\
\hline & bué kaju & $\mathrm{Nd}$ & & Phaseolus aureus \\
\hline & bué kaju roja & & & Phaseolus \\
\hline
\end{tabular}




\begin{tabular}{|c|c|c|c|c|}
\hline & \multicolumn{2}{|l|}{ bué mengé } & \multirow[t]{2}{*}{3775} & pulse \\
\hline & bué nggodho & $\mathrm{Nd}$ & & $=$ nggodho \\
\hline & bué nggoli & $\mathrm{Nd}$ & & Phaseolus pubescens \\
\hline & bué rako & $\mathrm{Nd}$ & & = bué uta \\
\hline & bué ria & $\mathrm{Nd}$ & & $=$ lipé \\
\hline & bué rumbi & Dk & & $=$ lipé \\
\hline & bué sé'a & $\mathrm{Nd}$ & & Phaseolus \\
\hline & bué tana & $\mathrm{Nd}$ & $\mathrm{H}$ & Arachis hypogaea \\
\hline & bué tola & $\mathrm{Nd}$ & & pulse \\
\hline & bué uta & & & Vigna unguiculata \\
\hline k & bunga & & & Sambucus canadensis \\
\hline k & bunga ba'i & & & Tithonia diversifolia \\
\hline $\mathrm{kb}$ & daka & & $\mathrm{H}$ & Cucumis sativus \\
\hline $\mathrm{u}$ & dala & & & Polypodiaceae \\
\hline k & dama I & & & Jatropha curcas \\
\hline k & dama II & $\mathrm{Nd}$ & & $=$ pandu \\
\hline g & degu lengu & $\mathrm{Mn}, \mathrm{Nd}$ & 3748 & Mimosa invisa \\
\hline k & denu & $\mathrm{Nd}$ & & Melochia cf. umbellata \\
\hline $\mathrm{k}$ & déo & $\mathrm{Nd}$ & & Trema orientalis \\
\hline $\mathrm{kr}$ & déré mera & $\mathrm{Mn}$ & & Cuphea hookeriana (cj.) \\
\hline k & dero & $\mathrm{Mn}$ & & Erythrina orientalis \\
\hline k & dero belanda & & & Erythrina subumbrans \\
\hline $\mathrm{p}$ & doba & $\mathrm{Mn}$ & & $=$ doba lenga \\
\hline $\mathrm{p}$ & doba lenga & $\mathrm{Mn}, \mathrm{Nd}$ & & Sesamum orientale \\
\hline & domba & Dk & & = fésa \\
\hline $\mathrm{kb}$ & dowé & $\mathrm{Nd}$ & & = séko \\
\hline k & duli I & $\mathrm{Pg}$ & & tree \\
\hline & duli II & & & ?Colocasia \\
\hline $\mathrm{u}$ & edi & $\mathrm{Nd}$ & & = ruba ré'é I/II \\
\hline $\mathrm{t}$ & éko lako & $\mathrm{Nd}, \mathrm{Dk}$ & & $=$ gai \\
\hline k & éko ndé'u & & dft & Eurya \\
\hline $\mathrm{kb}$ & ?endu & $\mathrm{Mn}$ & & Calopogonium mucronoides \\
\hline hb & éndu I & & A & Papilionaceae \\
\hline $\mathrm{ku}$ & éndu II & & & Poaceae \\
\hline $\mathrm{ku}$ & éra laé & $\mathrm{Nd}, \mathrm{Dk}$ & & = éré lai \\
\hline $\mathrm{ku}$ & éré lai & $\mathrm{Mn}$ & 3813 & Carex vesiculosa \\
\hline $\mathrm{u}$ & eri & $\mathrm{Nd}$ & & Fungi \\
\hline $\mathrm{u}$ & éru & & 3763 & Pothomorphe \\
\hline $\mathrm{p}$ & éwu & Dk & & ?Musa \\
\hline k & fai & $\mathrm{Nd}$ & dft & Albizia chinensis \\
\hline $\mathrm{u}$ & fako & & & Fungi (many attributes) \\
\hline a & fangé I & & & Paederia scandens \\
\hline & fangé II & $\mathrm{Mn}, \mathrm{Nd}$ & 3789 & Salvia riparia \\
\hline
\end{tabular}




\begin{tabular}{|c|c|c|c|c|}
\hline $\mathrm{p}$ & faté & $\mathrm{Nd}$ & & ?Ocimum \\
\hline $\mathrm{p}$ & faté mela & & & Ocimum basilicum \\
\hline $\mathrm{p}$ & féndo & $\mathrm{Nd}$ & & Amomum \\
\hline $\mathrm{k}$ & féo & & & Aleurites moluccana \\
\hline \multirow[t]{2}{*}{$\mathrm{u}$} & fésa & & 3765 & ?Phaseolus lunatus \\
\hline & fu'a ré'é & $\mathrm{Pg}$ & A & plant, thorny, coastal \\
\hline $\mathrm{k}$ & funa & & A & tree \\
\hline $\mathrm{k}$ & funga & $\mathrm{Mn}, \mathrm{Nd}, \mathrm{Dk}$ & & Ficus \\
\hline $\mathrm{k}$ & futa & $\mathrm{Nd}$ & & $\begin{array}{l}\text { tree w. sticky latex; cuttings for } \\
\text { fences }\end{array}$ \\
\hline $\mathrm{k}$ & ga awa & $\mathrm{Wn}$ & & $=$ nggo awa \\
\hline $\mathrm{t}$ & gai & $\mathrm{Nd}$ & & Saccharum spontaneum \\
\hline $\mathrm{t}$ & gai ara & & & grass, tall, or small bamboo \\
\hline $\mathrm{t}$ & gai géla & $\mathrm{Dk}$ & & ?Poaceae \\
\hline $\mathrm{t}$ & gai kiu & & & ?Thysanolaena maxima \\
\hline \multirow[t]{2}{*}{$\mathrm{k}$} & gari & $\mathrm{Nd}$ & $\mathrm{dft}$ & Rhus taitensis \\
\hline & gau & $\mathrm{Nd}$ & & Cordyline fruticosa \\
\hline $\mathrm{k}$ & gegé I & $\mathrm{Nd}$ & & tree, small w. hard wood \\
\hline \multirow[t]{3}{*}{$\mathrm{k}$} & gegé II & $\mathrm{Mn}$ & & Leucaena leucocephala \\
\hline & gera & & A & $=$ kengga I \\
\hline & gero & Dk & & Amorphophallus variabilis \\
\hline k & gila & & A & tree \\
\hline $\mathrm{u}$ & ?go & $\mathrm{Nd}$ & & Colocasia \\
\hline $\mathrm{u}$ & go salu & $\mathrm{Dk}$ & & Cucurbitaceae \\
\hline $\mathrm{u}$ & gola & & A & ?Cucumis sativus \\
\hline $\mathrm{k}$ & golkar & Dk & & Eupatorium odoratum \\
\hline \multirow[t]{2}{*}{$\mathrm{k}$} & gulu béwa & $\mathrm{Nd}, \mathrm{Dk}$ & A & Maclura cochinchinensis \\
\hline & gulu gua & $\mathrm{Nd}, \mathrm{Dk}$ & A & Leea ?angulata \\
\hline \multirow[t]{2}{*}{$\mathrm{k}$} & gulu karo & $\mathrm{Nd}$ & A & plant, thomy, coastal \\
\hline & gulu meta & $\mathrm{Nd}$ & A & shrub, evergreen w. bent thoms \\
\hline $\mathrm{k}$ & gunu & $\mathrm{Mn}$ & $\mathrm{dft}$ & Grewia \\
\hline \multirow[t]{4}{*}{ k } & hago & Wn & $\mathrm{dft}$ & Sterculia oblongata \\
\hline & hai latu & $\mathrm{Mn}$ & & Triumfetta \\
\hline & hapa loi & & & $=$ loi \\
\hline & héa & Wn & & Benincasa hispida \\
\hline $\mathrm{k}$ & hebu & $\mathrm{Nd}$ & & Homalanthus peltatus \\
\hline a & héko & & A & vine; used as cord \\
\hline $\mathrm{k}$ & helo & $\mathrm{Mn}$ & 3822 & Ficus \\
\hline $\mathrm{kb}$ & héna & & & = kéna \\
\hline $\mathrm{u}$ & hengga & & & vegetable \\
\hline $\mathrm{k}$ & hepo & & & $=\mathrm{kepo}$ \\
\hline $\mathrm{k}$ & hera & $\mathrm{Wn}$ & & ?Erythrina \\
\hline u & hésa & & & vegetable \\
\hline b & hewi & & A & herb; used against boils \\
\hline
\end{tabular}




$\begin{array}{lll}\mathrm{k} & \text { hewo } & \\ & \text { hi } & \text { Wn } \\ \mathrm{u} & \text { higo } & \\ \mathrm{kb} & \text { hipi } & \\ \mathrm{k} & \text { hoki } & \text { Mn } \\ \mathrm{u} & \text { hongo } & \\ \mathrm{p} & \text { horo } & \\ & \text { horo ria } & \end{array}$

$\mathrm{hb}, \mathrm{kb} \quad \mathrm{iju} r \mathrm{ra}$

u iré

hb jala pébé I

u jala pébé II

$\mathrm{k}, \mathrm{p} \quad$ janga

$\mathrm{ku} \quad$ jara

u jata

kb jawa $\quad$ jawa ka

u jepa

k jita

k ju

k junu

k

p ka('é) jawa

k kago

$\mathrm{u}$

k

$\mathrm{k}$

$\mathrm{k}$

$\mathrm{k}$ ila melu I

ila melu II

ina koba

ingga

jo wawo

kaba ana ko'o

kaju mani

kaju té'a

kaju wawi

Dk

$\mathrm{Nd}$

Nd, Dk

Dk

$\mathrm{Nd}$

Dk

Dk

Dk

$\mathrm{Nd}$

Nggéla, Wn, Nd

Dk

$\mathrm{Nd}$

$\mathrm{Nd}$

Nd

Dk

$\mathrm{Mn}$

Nd, Dk

Ds

$\mathrm{Mn}, \mathrm{Nd}$

$\mathrm{Nd}$

$\mathrm{Nd}$

Nd

Dk

Mn, Nd

Dk

kali raga

kalo wajo

kapok kéli

kara I

kara II

karo niki

karo pela
Nd

Dk

Nd

$\mathrm{Mn}, \mathrm{Nd}$
?= kinga kewo

$=\mathrm{ki}$

$=$ kigo

$\mathrm{A}=\mathrm{kipi}$

$3755=$ koki

A $=$ kongo

$=$ koro I

Capsicum annuum

A Ipomoea ?nil

Rhaphidophora

Jatropha gossypifolia

Buddleja asiatica

Sericocalyx asper

A vegetable

A vine

Fungi

Kleinhovia hospita

= soko belanda

Fungi

Zea mays

= nggéjé

Sechium edule

Alstonia scholaris

A = weté I

tree, very bitter

dft Grewia

3802 Vaccinium timorense

Homalanthus peltatus

$=$ muku jawa

= hago

Cinnamomum burmanni

tree, small w. yellow wood and thorny l.; firewood

tree

Coccinia grandis

Acorus calamus

= kalé raga

Adenanthera pavonina

dft Meliosma ferruginea

$=$ kerara

Uvaria rufa

shrub, thorny; for catching flying foxes

Opuntia elatior 


\begin{tabular}{|c|c|c|c|c|}
\hline ku & kasa kamba & $\mathrm{Mn}, \mathrm{Nd}$ & 3778 & Cyperus \\
\hline $\mathrm{kb}$ & katé & & & liana, long \\
\hline $\mathrm{kb}, \mathrm{u}$ & kéa & $\mathrm{Mn}, \mathrm{Nd}, \mathrm{Dk}$ & & = héa \\
\hline k & keba & $\mathrm{Nd}$ & $\mathrm{dft}$ & Pometia pinnata \\
\hline k & kebu I & Dk & & $=$ hebu \\
\hline \multirow[t]{2}{*}{$\mathrm{u}$} & kebu II & $\mathrm{Dk}$ & A & marine plant \\
\hline & kejawa & Ds & & $=$ muku jawa \\
\hline k & kéju & & A & tree w. edible fr. \\
\hline $\mathrm{kb}$ & keju repé & $\mathrm{Mn}$ & A & vine \\
\hline \multirow[t]{2}{*}{$\mathrm{t}$} & kela & $\mathrm{Mn}, \mathrm{Nd}$ & & Phragmites karka \\
\hline & kela meta & $\mathrm{Ds}, \mathrm{Dk}$ & A & grass, tall \\
\hline k & kelo & $\mathrm{Nd}$ & & $=$ helo \\
\hline k & kembo & $\mathrm{Mn}, \mathrm{Nd}$ & & Morinda \\
\hline $\mathrm{kb}$ & kéna & $\mathrm{Mn}, \mathrm{Nd}$ & & Lagenaria siceraria \\
\hline \multirow[t]{2}{*}{$? \mathrm{u}$} & kéngé & & & Fungi \\
\hline & kengga I & $\mathrm{Mn}, \mathrm{Nd}$ & A & Bambusa blumeana \\
\hline \multirow[t]{8}{*}{$\mathrm{u}$} & kengga II & & & $=$ hengga \\
\hline & kenggé & $\mathrm{Nd}$ & & Fungi \\
\hline & kéo I & $\mathrm{Mn}, \mathrm{Nd}$ & & Coix lacryma-jobi var. ma-yuen \\
\hline & ké'o II & $\mathrm{Pg}$ & & $=$ jawa \\
\hline & ké'o mbapé & & & = ké'o I \\
\hline & ké'o mboka & Dk & & = ké'o I \\
\hline & ké'o mui & $\mathrm{Nd}$ & & Coix lacryma-jobi var. agrestis \\
\hline & ké'o oto & $\mathrm{Dk}$ & & $=$ ké'o mui \\
\hline $\mathrm{u}$ & kepa & $\mathrm{Mn}$ & 3791 & Amomum \\
\hline k & kepo & $\mathrm{Mn}, \mathrm{Nd}$ & & Cordia dichotoma \\
\hline k & ?kepo kéli & & & Cordia \\
\hline k & kera & $\mathrm{Nd}, \mathrm{Dk}$ & & tree \\
\hline k & kéra & $\mathrm{Nd}$ & A & tree, firewood, cuttings planted \\
\hline k & kerara & $\mathrm{Dk}, \mathrm{Wn}$ & & Artocarpus altilis (seedless) \\
\hline k & kerinu & $\mathrm{Mn}, \mathrm{Nd}$ & & Eupatorium inulifolium \\
\hline k & kesi & $\mathrm{Mn}, \mathrm{Nd}$ & & Lannea coromandelica \\
\hline $\mathrm{p}$ & kéu & $\mathrm{Mn}, \mathrm{Nd}$ & $\mathrm{H}, \mathrm{RV}$ & Areca cathecu \\
\hline $\mathrm{k}$ & ké'u té'a & $\mathrm{Dk}$ & & Alangium villosum \\
\hline $\mathrm{kb}$ & kéwa & & & Dioscorea \\
\hline k & kewi & $?$ & & ?Rauvolfia \\
\hline \multirow[t]{3}{*}{ k } & kewo & $\mathrm{Nd}$ & & $=$ kinga kewo \\
\hline & ki & $\mathrm{Nd}$ & $\mathrm{H}$ & Imperata cylindrica \\
\hline & kigo & & & Asteraceae \\
\hline \multirow[t]{2}{*}{$\mathrm{u}$} & kigo paré & $\mathrm{Mn}, \mathrm{Nd}$ & & Emilia sonchifolia \\
\hline & kigo ria & & & Gynura \\
\hline \multirow[t]{2}{*}{$\mathrm{u}$} & ki'i & $\mathrm{Mn}, \mathrm{Nd}$ & & Fungi \\
\hline & kinga kewo & $\mathrm{Dk}$ & & Ficus balica \\
\hline
\end{tabular}




\begin{tabular}{|c|c|c|c|c|}
\hline & kipa I & $\mathrm{Nd}$ & & Dicranopteris pubigera \\
\hline $\mathrm{ku}$ & kipa II & Dk & & $=$ soko belanda \\
\hline \multirow[t]{2}{*}{$\mathrm{a}, \mathrm{kb}$} & kipi & $\mathrm{Mn}$ & & $\begin{array}{l}\text { liana, very long and strong w. red } \\
\text { edible fr. }\end{array}$ \\
\hline & kisa I & & & $=$ ndora \\
\hline k & kisa II & Dk & & tree; wood used for building boats \\
\hline k & kitu koka & Dk & & $?=$ koja \\
\hline k & koja & $\mathrm{Nd}$ & $\mathrm{H}$ & Canarium vulgare \\
\hline k & koja nengi & $\mathrm{Nd}$ & $\mathrm{dft}$ & $=\mathrm{kesi}$ \\
\hline k & koki & $\mathrm{Nd}$ & & Ficus \\
\hline $\mathrm{p}$ & koko woso & $\mathrm{Mn}, \mathrm{Nd}$ & & Canna coccinea \\
\hline $\mathrm{k}$ & kolé & $\mathrm{Nd}$ & & Calotropis gigantea \\
\hline $\mathrm{p}$ & koli & $\mathrm{Nd}$ & & Borassus sundaicus \\
\hline $\mathrm{u}$ & konga & $\mathrm{Nd}$ & A & vegetable \\
\hline $\mathrm{u}$ & kongo & $\mathrm{Mn}, \mathrm{Nd}$ & & Fungi \\
\hline \multirow[t]{3}{*}{$\mathrm{k}$} & kora & $\mathrm{Nd}$ & & tree, on mountains \\
\hline & kora wolo & & A & plant, medicinal \\
\hline & koré I & & 3752 & ?Asplenium nidus \\
\hline k & koré II & Dk & & $?$ \\
\hline $\mathrm{p}$ & koro I & $\mathrm{Nd}$ & & Capsicum frutescens \\
\hline $\mathrm{u}$ & koro II & $\mathrm{Mn}, \mathrm{Nd}$ & & Acanthaceae \\
\hline \multirow[t]{5}{*}{$\mathrm{k}$} & $\begin{array}{l}\text { koro ndé } \\
\text { koro ria }\end{array}$ & $\mathrm{Dk}$ & & $\begin{array}{l}\text { Artocarpus heterophyllus } \\
=\text { horo ria }\end{array}$ \\
\hline & koro wolo & $\mathrm{Mn}, \mathrm{Dk}$ & & Piper \\
\hline & kosi & Dk & & Amorphophallus \\
\hline & koto I & $\mathrm{Pg}$ & & plant, tuberous \\
\hline & koto II & & $\mathrm{H}$ & $=$ daka \\
\hline \multirow[t]{3}{*}{ k } & koto koto & & & ?Mangifera \\
\hline & kowé & $\mathrm{Nd}$ & & Papilionaceae \\
\hline & kowo & $\mathrm{Nd}$ & & $?=$ kembo \\
\hline $\mathrm{kb}$ & kua & $\mathrm{Nd}$ & & Flagellaria indica \\
\hline k & kuku & & & Schoutenia obovata \\
\hline k & kuku mbeku I & $\mathrm{Nd}$ & & Leea indica \\
\hline b & kuku mbeku II & $\mathrm{Nd}$ & & Lycopodium cernuum \\
\hline $\mathrm{u}$ & kula & $\mathrm{Nd}$ & & Fungi \\
\hline k & kuma & $\mathrm{Nd}$ & & Sesbania grandiflora \\
\hline \multirow[t]{7}{*}{ k } & kuma wundu & & & $\begin{array}{l}\text { tree; juice for preserving fishing- } \\
\text { lines, etc. }\end{array}$ \\
\hline & kumi laka & $\mathrm{Mn}$ & 3794 & Acanthaceae \\
\hline & kumi lamu I & & & $? M u s c i$ \\
\hline & kumi lamu II & Dk & & Lichenes, Usnea \\
\hline & kumi lélé & $\mathrm{Nd}$ & & = kumi lamu II \\
\hline & kumi nitu & & 3827 & = kumi lamu II \\
\hline & kumi rongo & & & Poaceae \\
\hline
\end{tabular}




\begin{tabular}{|c|c|c|c|c|}
\hline \multirow[t]{2}{*}{ b } & kumi rusa I & $\mathrm{Mn}$ & & Euphorbia hirta \\
\hline & kumi rusa II & $\mathrm{Dk}$ & & $=$ kuku mbeku II \\
\hline $\mathrm{p}$ & kuné & Nd & & Curcuma viridiflora \\
\hline \multirow{6}{*}{$\mathrm{k}$} & kupé & Dk & & Piliostigma malabaricum \\
\hline & kuru ba'i & & & ?Paspalum conjugatum \\
\hline & kuru kamba & & & Eleusine indica \\
\hline & kuru paré & $\mathrm{Mn}$ & 3746 & Echinochloa crus-galli \\
\hline & kuru weté & $\mathrm{Nd}$ & 3780 & Eragrostis warburgii \\
\hline & kuwu & $\mathrm{Nd}$ & & Cucurbitaceae \\
\hline $\mathrm{p}, \mathrm{k}$ & la & $\mathrm{Mn}, \mathrm{Nd}$ & & Mallotus philippensis \\
\hline u & lada & Dk & & Nasturtium officinale \\
\hline k & ladu & $\mathrm{Nd}$ & & Areca \\
\hline hb & laga tana & & A & vine \\
\hline $\mathrm{p}$ & laja I & Nd & & Languas galanga \\
\hline \multirow[t]{2}{*}{$\mathrm{p}$} & laja II & Dk & & Costus speciosus \\
\hline & laka jawa & $\mathrm{Nd}$ & & Areca \\
\hline \multirow[t]{4}{*}{$\mathrm{k}$} & laka toba & $\mathrm{Nd}, \mathrm{Dk}$ & & tree; 1. for nail-polish \\
\hline & lako raki & $\mathrm{Mn}$ & & Salvia riparia \\
\hline & lamu & $\mathrm{Mn}, \mathrm{Nd}$ & 3825 & Algae \\
\hline & lamu ka'u & $\mathrm{Mn}, \mathrm{Nd}$ & & Musci \\
\hline $\mathrm{k}$ & landé I & $\mathrm{Nd}$ & & Dendrocnide \\
\hline \multirow[t]{2}{*}{$\mathrm{kb}$} & landé II & Dk & & Laportea \\
\hline & landé mesi & $\mathrm{Dk}$ & & $=$ laté mesi \\
\hline $\mathrm{k}$ & landé wuja & $\mathrm{Nd}, \mathrm{Dk}$ & & Laportea ?aestuans \\
\hline $\mathrm{k}$ & langga & $\mathrm{Mn}, \mathrm{Nd}, \mathrm{Dk}$ & & Vitex trifolia \\
\hline $\mathrm{k}$ & lanu & $\mathrm{Nd}$ & $\mathrm{dft}$ & Albizia procera \\
\hline \multirow[t]{2}{*}{$\mathrm{k}$} & laté & Lisé & & = landé $\mathrm{I} / \mathrm{II}$ \\
\hline & laté mesi & Wn & & $\begin{array}{l}\text { plant } w \text {. large woolly l.; used } \\
\text { against itch }\end{array}$ \\
\hline $\mathrm{p}$ & léa & $\mathrm{Mn}, \mathrm{Nd}$ & & Zingiber officinale \\
\hline \multirow[t]{2}{*}{$\mathrm{u}$} & léba & $\mathrm{Mn}$ & & Momordica charantia \\
\hline & lege féa & & & ?Desmodium \\
\hline $\mathrm{p}$ & léi & & & Cycas rumphii \\
\hline $\mathrm{kb}$ & léké & $\mathrm{Mn}, \mathrm{Nd}$ & & Entada phaseoloides \\
\hline $\mathrm{kb}$ & léké mboja & $\mathrm{Mn}, \mathrm{Nd}$ & & vine, tripartite l. w. round leaflets \\
\hline \multirow[t]{2}{*}{$\mathrm{p}, \mathrm{k}$} & lélé I & $\mathrm{Mn}$ & & Ficus ?benjamina \\
\hline & lélé II & $\mathrm{Mn}$ & & Ficus heteropleura \\
\hline $\mathrm{k}$ & lélé dhéndé & $\mathrm{Dk}$ & & Ficus \\
\hline $\mathrm{k}$ & lélé fai & $\mathrm{Dk}$ & & $=$ lélé $\mathrm{I}$ \\
\hline $\mathrm{k}$ & lélé halo & $\mathrm{Mn}$ & & Ficus \\
\hline $\mathrm{k}$ & lélé kaki & $\mathrm{Dk}$ & & Ficus \\
\hline $\mathrm{kb}$ & lélé kalo & $\mathrm{Nd}$ & & Ficus \\
\hline $\mathrm{k}$ & lélé panda & & & Ficus \\
\hline u & lélu & $\mathrm{Nd}$ & & Polypodiaceae (edible) \\
\hline
\end{tabular}




\begin{tabular}{|c|c|c|c|}
\hline $\mathrm{p}$ & lélu & $\mathrm{Mn}$ & \\
\hline \multirow[t]{2}{*}{$\mathrm{u}$} & lelu kela I & $\mathrm{Nd}$ & \\
\hline & $\begin{array}{l}\text { lelu kela II } \\
\text { lélu ré'é }\end{array}$ & Dk & \\
\hline \multirow[t]{3}{*}{ k } & lema kamba & $\mathrm{Mn}, \mathrm{Nd}$ & 3816 \\
\hline & lema manu & $\mathrm{Mn}, \mathrm{Nd}$ & 3781 \\
\hline & lema mori & $\mathrm{Mn}, \mathrm{Nd}$ & \\
\hline $\mathrm{kb}$ & lema nda'i & $\mathrm{Mn}$ & 3795 \\
\hline k & lemé & $\mathrm{Mn}$ & 3753 \\
\hline $\mathrm{ku}$ & lénda au & $\mathrm{Nd}$ & \\
\hline \multirow[t]{2}{*}{$\mathrm{p}$} & lenga & $\mathrm{Nd}$ & \\
\hline & lepé kata & Dk & \\
\hline $\mathrm{u}$ & leri & $\mathrm{Nd}$ & \\
\hline $\mathrm{p}$ & lesa & Dk & \\
\hline $\mathrm{b}$ & lesé & $\mathrm{Mn}, \mathrm{Nd}$ & \\
\hline k & linggé & $\mathrm{Mn}$ & A \\
\hline $\mathrm{u}$ & lipé & $\mathrm{Mn}, \mathrm{Nd}$ & 3823 \\
\hline \multirow[t]{2}{*}{$\mathrm{ku}$} & lité I & $\mathrm{Mn}, \mathrm{Nd}$ & \\
\hline & lité II & Dk & \\
\hline \multirow[t]{3}{*}{$\mathrm{p}$} & lo lai & $\mathrm{Mn}, \mathrm{Nd}$ & \\
\hline & loba I & $\mathrm{Nd}$ & \\
\hline & lob(h)a II & Nd, Dk & \\
\hline k & loi & Dk & \\
\hline $\mathrm{p}$ & lojo & & \\
\hline k & loko féa & & 3764 \\
\hline$n$ & $\begin{array}{l}\text { loko fudhé } \\
\text { lolo }\end{array}$ & $\mathrm{Mn}$ Nd & \\
\hline
\end{tabular}

Gossypium

vegetable, at riverside

Polypodiaceae (not edible)

Malvaceae

Saurauia verheijenii

Hedyotis corymbosa

Agave sisalana

Alternanthera

Melanolepis multiglandulosa

Commelina

= doba lenga

$=$ besu

Portulaca oleracea

Spondias malayana

Equisetum debile

tree

Mucuna pruriens f. utilis

Setaria palmifolia

Sorghum propinquum

$=$ léi

Raphanus sativus

plant, reddish, medicinal and for dyeing

Alstonia spectabilis

Ensete glaucum

Moghania

herb, medicinal

Sorghum saccharatum (many attributes pointing to races)

Pennisetum spicatum

Pycnarrhena

= lolo kowé

Poaceae

A = lolo kowé

see: wonga londa I, II

dft Glochidion

marine vegetable

Dioscorea sarasinii

Ipomoea

plant, smelling

dft

Syzygium aqueum

Syzygium

marine plant

$\mathrm{dft}$ 


\begin{tabular}{|c|c|c|c|c|}
\hline k & $\begin{array}{l}\text { magé } \\
\text { magé koba }\end{array}$ & $\begin{array}{l}\mathrm{Mn}, \mathrm{Nd} \\
\mathrm{Dk}\end{array}$ & $\mathrm{H}, \mathrm{dft}$ & $\begin{array}{l}\text { Tamarindus indica } \\
\text { plant, 1. resemble those of the } \\
\text { tamarind }\end{array}$ \\
\hline \multirow[t]{2}{*}{ hb } & mako & & A & vine \\
\hline & mako mala & $\mathrm{Mn}$ & & plant, at riverside \\
\hline k & mara pau & $\mathrm{Nd}$ & $\mathrm{dft}$ & Litsea tomentosa \\
\hline k & $\operatorname{mar}$ & $\mathrm{Nd}$ & & tree w. good timber \\
\hline k & mata mbusu & & A & tree w. good timber \\
\hline \multirow[t]{2}{*}{ k } & mata muri & & A & tree w. white latex \\
\hline & mata ndoro & & & Urticaceae \\
\hline \multirow[t]{2}{*}{ k } & mati & & A & tree \\
\hline & mbaka & & & Asteraceae \\
\hline \multirow[t]{2}{*}{$\mathrm{b}, \mathrm{u}$} & mbaka jégha & $\mathrm{Mn}, \mathrm{Nd}$ & 3818 & $=$ mbaka nipo \\
\hline & mbaka lodhu & & A & plant \\
\hline \multirow[t]{2}{*}{$\mathbf{u}$} & mbaka nipo & $\mathrm{Nd}$ & & Erechtites valerianifolia \\
\hline & mbako & $\mathrm{Mn}, \mathrm{Nd}$ & & $=$ bako \\
\hline k & mbangga & $\mathrm{Mn}, \mathrm{Nd}$ & & Citrus \\
\hline \multirow[t]{3}{*}{$\mathrm{p}$} & mbangga rai & $\mathrm{Nd}$ & & ?Asteraceae \\
\hline & mbanggo & $\mathrm{Mn}$ & & Dioscorea aculeata \\
\hline & mbapé & $\mathrm{Mn}$ & & = ké'o I \\
\hline k & mbawu & $\mathrm{Nd}$ & & Leucosyke capitellata \\
\hline k & mbawu noko & $\mathrm{Nd}$ & & Maoutia \\
\hline $\mathrm{t}$ & mbenga & Dk & & Hyptis rhomboidea \\
\hline $\mathrm{u}$ & mbéré wawi & $\mathrm{Nd}$ & & tree w. sourish 1. \\
\hline \multirow[t]{2}{*}{$\mathrm{u}$} & mberi & & & Solanum melongena \\
\hline & mberi mbombo & $\mathrm{Nd}$ & & $=$ mbira mbombo \\
\hline $\mathbf{u}$ & $\begin{array}{l}\text { mberi wula } \\
\text { mbeta }\end{array}$ & $\mathrm{Nd}$ & & $\begin{array}{l}\text { Solanum melongena var. } \\
\text { marine plant }\end{array}$ \\
\hline k & mbéwa & Nd, Dk & A & tree \\
\hline k & mbéwo & $\mathrm{Mn}, \mathrm{Nd}$ & 3797 & Cassia \\
\hline k & mbindi & $\mathrm{Mn}, \mathrm{Nd}$ & & Averrhoa carambola \\
\hline \multirow[t]{3}{*}{$\mathrm{k}$} & mbindi wala & Dk & & Averrhoa bilimbi \\
\hline & mbira mbombo & $\mathrm{Mn}$ & 3747 & Rubus \\
\hline & mbiri mbombo & Dk & & $=$ mbira mbombo \\
\hline \multirow[t]{2}{*}{ k } & mbiru & $\mathrm{Nd}$ & & $?$ \\
\hline & mboa & $\mathrm{Mn}, \mathrm{Nd}$ & 3814 & Melastoma \\
\hline k & mboi & Dk & & Ficus ampelas \\
\hline $\mathrm{kb}$ & mboja I & $\mathrm{Mn}$ & & = léké mboja \\
\hline k & mboja II & & dft & Pygeum \\
\hline \multirow[t]{5}{*}{$\mathrm{u}$} & mboko féo & $\mathrm{Nd}$ & & Fungi \\
\hline & mboko kipi & & & = kipi \\
\hline & mboko kusi & Dk & & Pyrrosia \\
\hline & mboko longgo & & 3779 & Phyllanthus amarus \\
\hline & mboko meké & $\mathrm{Nd}$ & & Corchorus \\
\hline
\end{tabular}




\begin{tabular}{|c|c|c|c|c|}
\hline & mboko néka I & Dk & & Caesalpinia ?major \\
\hline & mboko néka II & $\mathrm{Nd}$ & A & water plant \\
\hline & mboko ona & & & $?=$ mboko néka I \\
\hline & mboko para & $\mathrm{Nd}$ & & $=$ para \\
\hline & mboko pulu & & & $?=$ pulu I/II \\
\hline & mboko repé & & A & $?=$ repé $\mathrm{I} / \mathrm{II}$ \\
\hline & mboko sa & & A & Piper nigrum \\
\hline & mboko uwi & & A & Dioscorea pentaphylla \\
\hline $\mathrm{ub}, \mathrm{hb}, \mathrm{a}$ & mbonggi & $\mathrm{Mn}, \mathrm{Nd}$ & A & ?Ipomoea \\
\hline $\mathrm{k}$ & mbungé & $\mathrm{Mn}, \mathrm{Nd}$ & & Datura metel \\
\hline & mburi mbombo I & $\mathrm{Mn}$ & 3799 & Rubus alceaefolius \\
\hline & mburi mbombo II & Nd, Dk & & Solanum \\
\hline & mé'a I & $\mathrm{Mn}$ & 3750 & Synedrella nodiflora \\
\hline & mé'a II & Dk & & Spilanthes iabadicensis \\
\hline & meja & & & Setaria palmifolia \\
\hline & meka reké & Dk & & ?Fatoua pilosa \\
\hline $\mathrm{ku}$ & meké & $\mathrm{Nd}$ & & Chrysopogon aciculatus \\
\hline b & mela & $\mathrm{Nd}$ & & Oxalis corniculata \\
\hline $\mathrm{b}$ & mela ji'é & & & $=$ mela \\
\hline $\mathrm{b}$ & mela ré'é & $\mathrm{Mn}, \mathrm{Nd}$ & & Drymaria cordata \\
\hline & melu & $\mathrm{Mn}, \mathrm{Nd}$ & & $=$ dama I/II \\
\hline k & melu funga & & & tree \\
\hline & méngi & & & $=$ nata \\
\hline $\mathrm{u}$ & méra & & & marine plant \\
\hline k & méra pélé & $\mathrm{Nd}$ & & Semecarpus forstenii \\
\hline & mer(e) pélé & $\mathrm{Nd}$ & & = méra pélé \\
\hline b & mersawa & & & $=$ mora sawa \\
\hline k & meru & $\mathrm{Mn}, \mathrm{Nd}$ & 3751 & Ficus ?wassa \\
\hline k & meta leja & $\mathrm{Mn}, \mathrm{Nd}$ & 3768 & Bridelia tomentosa \\
\hline & mimosa & Dk & & Mimosa invisa \\
\hline ?kb & modi & & & Dioscorea ?bulbifera \\
\hline ku & modu & $\mathrm{Mn}, \mathrm{Nd}, \mathrm{Dk}$ & 3829 & Cyperus cf. brevifolius \\
\hline $\mathrm{kb}$ & mogo & $\mathrm{Nd}$ & & Commelina \\
\hline $\mathrm{b}, \mathrm{k}$ & moka & $\mathrm{Mn}, \mathrm{Nd}$ & 3756 & Dysoxylum \\
\hline $\mathrm{p}$ & moké & $\mathrm{Mn}, \mathrm{Nd}$ & & Arenga pinnata \\
\hline $\mathrm{a}, \mathrm{hb}$ & monda & $\mathrm{Nd}$ & & ?Dioscorea \\
\hline kb & monda ré'é & Dk & & ?Stemona \\
\hline ku & monggo & Dk & & $=$ mogo \\
\hline k & mora a & & & Morus australis \\
\hline & mora ro'a & $\mathrm{Mn}, \mathrm{Nd}$ & 3769 & Dendrophthoe \\
\hline b & mora sawa & & & ?Bidens \\
\hline $\mathrm{kb}$ & moro & $\mathrm{Nd}$ & & Derris \\
\hline $\mathrm{kb}$ & moro nipa & Dk & & ?Menispermaceae \\
\hline
\end{tabular}




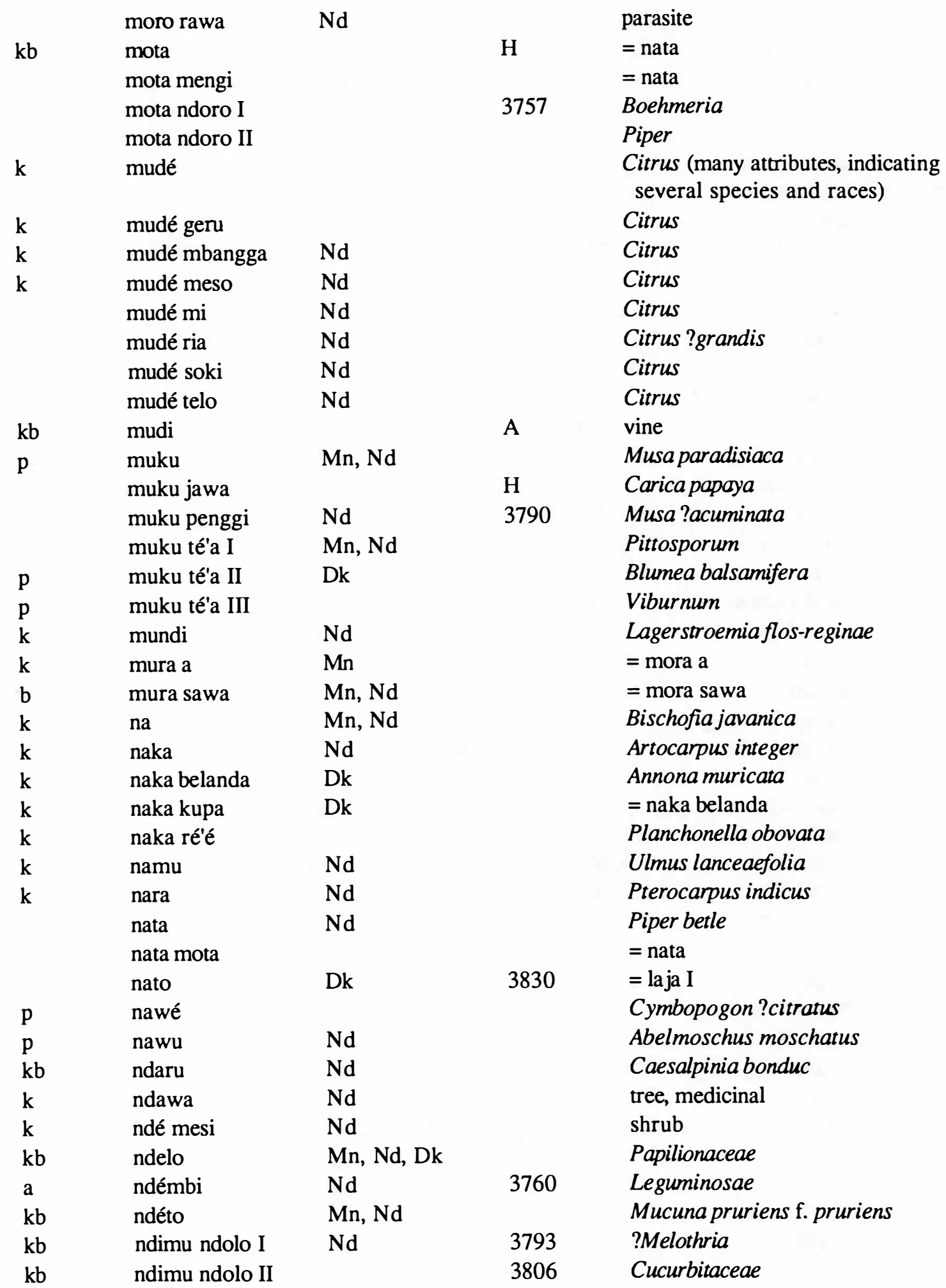




\begin{tabular}{|c|c|c|c|c|}
\hline $\mathrm{p}, \mathrm{w}$ & ndoko & $\mathrm{Pg}, \mathrm{Nd}$ & A & $=$ waku \\
\hline k & ndoko ré'é & $\mathrm{Pg}$ & A & tree \\
\hline $\mathrm{p}, \mathrm{k}$ & ndopo & & A & Cassia fistula \\
\hline \multirow[t]{2}{*}{$\mathrm{kb}$} & ndora & $\mathrm{Nd}$ & & $\begin{array}{l}\text { Ipomoea batatas (many attributes } \\
\text { indicating races) }\end{array}$ \\
\hline & ndora bima & & & Pachyrrhizus erosus \\
\hline $\mathrm{kb}$ & ndora ndu'a & $\mathrm{Nd}$ & & Ipomoea alba \\
\hline $\mathbf{w}$ & ndoro & & & $=$ mota ndoro $\mathrm{I} / \mathrm{II}$ \\
\hline \multirow[t]{2}{*}{ k } & nduru I & $\mathrm{Nd}, \mathrm{Dk}$ & & tree, tall, erect, deciduous \\
\hline & nduru II & & & Fungi \\
\hline $\mathrm{u}$ & ndutu & $\mathrm{Nd}$ & & Fungi \\
\hline k & néka & $\mathrm{Nd}$ & & $?=$ mboko néka $\mathrm{I} / \mathrm{II}$ \\
\hline \multirow[t]{2}{*}{ k } & nengi & Dk & & $=$ kesi \\
\hline & néro & & & Polypodiaceae \\
\hline$h b, k b, u b$ & néta & $\mathrm{Mn}, \mathrm{Nd}, \mathrm{Dk}$ & & Anamirta cocculus \\
\hline $\mathrm{kb}$ & ngadu liru & Lisé, Nd & & Sida acuta \\
\hline k & ngala kutu & Nd & dft & Sarcosperma paniculatum \\
\hline \multirow[t]{3}{*}{$\mathrm{u}$} & ngara liru & $\mathrm{Mn}, \mathrm{Nd}, \mathrm{Dk}$ & & Sida rhombifolia \\
\hline & ngeku ro'a I & Nd & A & parasite, on trees \\
\hline & ngeku ro'a II & Dk & & Fungi (edible) \\
\hline $\mathrm{p}$ & ngeti kamba & & & ?Sida \\
\hline k & nggada denu & & A & tree \\
\hline k & nggaja & $\mathrm{Nd}$ & A & ?Inocarpus fagiferus \\
\hline $\mathrm{t}$ & nggaju & Dk & & $=$ gai \\
\hline \multirow[t]{2}{*}{$\mathrm{t}$} & nggaju kiu & & & Thysanolaena maxima \\
\hline & ?nggaju soko & & A & Calanus \\
\hline k & nggaké I & & & Bauhinia \\
\hline $\mathbf{a}$ & nggaké II & & & Bauhinia ?hirsuta \\
\hline $\mathrm{u}$ & nggako & $\mathrm{Nd}$ & & Ipomoea aquatica \\
\hline $\mathrm{kb}$ & nggéjé & $\mathrm{Mn}, \mathrm{Nd}$ & & Citrullus lanatus \\
\hline k & nggéndi & $\mathrm{Mn}, \mathrm{Nd}, \mathrm{Dk}$ & & Leguminosae \\
\hline k & nggo awa & $\mathrm{Mn}$ & & Psidium guajava \\
\hline k & nggoa awa & $\mathrm{Mn}, \mathrm{Nd}$ & & $=$ nggo awa \\
\hline k & nggoé awa & Dk & & $=$ nggo awa \\
\hline k & nggoé nggawa & & & $=$ nggo awa \\
\hline $\mathrm{u}$ & nggodho & $\mathrm{Mn}, \mathrm{Nd}$ & & Glycine soja \\
\hline k & nggoji & $\mathrm{Mn}, \mathrm{Nd}$ & A & tree \\
\hline \multirow[t]{3}{*}{$\mathrm{kb}$} & nggoli & $\mathrm{Mn}, \mathrm{Nd}$ & 3807 & ?Phaseolus \\
\hline & nggoli bali & $\mathrm{Nd}$ & 3770 & = bué nggoli \\
\hline & nggolu & $\mathrm{Mn}, \mathrm{Nd}$ & 3758 & Leguminosae \\
\hline g & nggowi & $\mathrm{Nd}$ & A & shrub, thorny \\
\hline \multirow[t]{2}{*}{$\mathrm{g}, \mathrm{kb}$} & nggowi niki & Nd, Dk & & $?=$ karo niki \\
\hline & ngguru wai/waé & Wn & & $=$ gulu gua \\
\hline
\end{tabular}




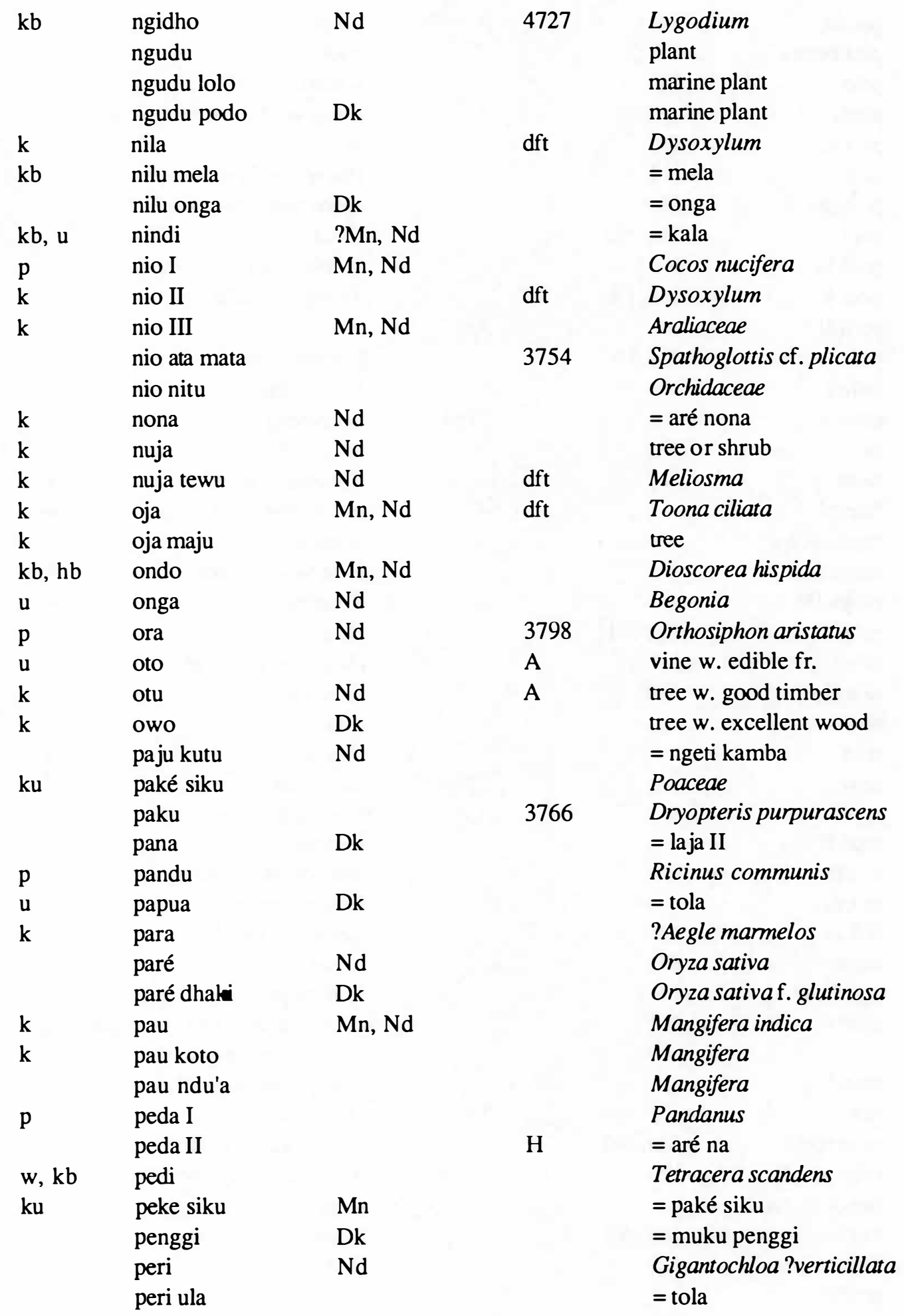




\begin{tabular}{|c|c|c|c|c|}
\hline \multirow{4}{*}{ k } & perula & \multirow{4}{*}{$\mathrm{Dk}$} & \multirow[t]{3}{*}{ A } & \multirow{2}{*}{$\begin{array}{l}=\text { tola } \\
=\text { tola }\end{array}$} \\
\hline & peta pepua & & & \\
\hline & péto & & & $=$ keba \\
\hline & pinda & & A & plant, wild, tuberous \\
\hline k & pipi té'a & Dk & & tree, small \\
\hline k & pira & $\mathrm{Nd}$ & & tree w. hard timber \\
\hline $\mathrm{p}$ & po lina & $\mathrm{Dk}$ & & Alocasia macrorrhiza \\
\hline $\mathrm{p}$ & poni & $\mathrm{Mn}, \mathrm{Nd}$ & & Cyathea \\
\hline \multirow[t]{2}{*}{$\mathrm{p}$} & poni lo & $\mathrm{Nd}$ & & Cyatheaceae \\
\hline & pudi I & $\mathrm{Mn}, \mathrm{Dk}$ & & Amorphophallus \\
\hline $\mathrm{k}$ & pudi II & & A & tree \\
\hline $\mathrm{u}$ & puli & Nd, Dk & & Cucurbitaceae \\
\hline $\mathrm{p}$ & pulu I & & & Urena lobata \\
\hline $\mathrm{p}$ & pulu II & $\mathrm{Nd}$ & 3786 & Triumfetta \\
\hline $\mathrm{k}$ & ra & $\mathrm{Nd}$ & & tree, large \\
\hline \multirow[t]{3}{*}{$\mathrm{k}$} & rama & $\mathrm{Nd}$ & & Pipturus argenteus \\
\hline & ?randé & & A & plant, medicinal \\
\hline & randé bewu & & A & $=$ randé \\
\hline $\mathrm{u}$ & ranga I & $\mathrm{Nd}$ & & Pouzolzia zeylanica \\
\hline $\mathrm{k}$ & ranga II & & & Pouzolzia \\
\hline $\mathrm{k}$ & ra'u & $\mathrm{Pg}, \mathrm{Nd}$ & & Dracontomelon edule \\
\hline \multirow[t]{3}{*}{$\mathrm{p}$} & ré'a & $\mathrm{Nd}$ & & Pandanus tectorius \\
\hline & ré'a jawa & Wn & & $=$ aré na \\
\hline & redo & & A & $=$ bué tana \\
\hline $\mathrm{ku}$ & rega & & & Poaceae \\
\hline $\mathrm{ku}$ & repa & & 3796 & Cenchrus brownii \\
\hline \multirow[t]{2}{*}{ k } & repé I & $\mathrm{Nd}$ & A & tree; bark for binding \\
\hline & repé II & & & Desmodium \\
\hline $\mathrm{k}, \mathrm{p}$ & ro kéi & Dk & & ?Artocarpus glaucus \\
\hline \multirow[t]{4}{*}{$\mathrm{u}$} & ro tela & & & Coleus amboinicus \\
\hline & roé aé & Dk & & Colocasia (wild) \\
\hline & rogo & Dk & & Algae \\
\hline & rolé fose & Dk & & $=$ mora sawa \\
\hline $\mathrm{p}$ & rosé & $\mathrm{Nd}$ & & $\begin{array}{l}\text { Colocasia esculenta (many attributes } \\
\text { indicating races) }\end{array}$ \\
\hline k & roso & & A & tree, poisonous \\
\hline $\mathrm{u}$ & rua & & $?$ & vegetable \\
\hline b & ruba ré'é I & $\mathrm{Mn}, \mathrm{Nd}$ & & Galinsoga parviflora \\
\hline \multirow[t]{2}{*}{$\mathrm{b}$} & ruba ré'é II & Wn & & Ageratum conyzoides \\
\hline & rungu ranga & & A & plant \\
\hline b & runu & $\mathrm{Mn}, \mathrm{Nd}$ & & Wedelia urticaefolia \\
\hline $\mathrm{u}$ & sala & $\mathrm{Nd}$ & & Polygonum \\
\hline k & sambi & $\mathrm{Nd}$ & & Schleichera oleosa \\
\hline $\mathrm{u}$ & sambi roto & Dk & & vegetable, bitter, wild \\
\hline
\end{tabular}




\begin{tabular}{|c|c|c|c|c|}
\hline \multirow[t]{2}{*}{ k } & sambi tombo & $\mathrm{Pg}$ & A & tree \\
\hline & sara mbira & $\mathrm{Mn}, \mathrm{Nd}, \mathrm{Dk}$ & & Remusatia vivipara \\
\hline k & sawa koka & & & tree w. useful timber \\
\hline $\mathrm{ku}$ & sawu I & & 3782 & Oplismenus compositus \\
\hline ku & sawu II & & 3812 & Themeda \\
\hline \multirow[t]{4}{*}{ k } & sé & Nd, Dk & A & $?$ \\
\hline & séba (aé) & Nd, Dk & A & Monochoria vaginalis \\
\hline & séba nggako & & A & = nggako \\
\hline & sé'é I & & 3788 & Hewittia sublobata \\
\hline b & sééé II & & & ?Euphorbia \\
\hline $\mathrm{kb}$ & séko & $\mathrm{Nd}$ & 3759 & Psophocarpus tetragonolobus \\
\hline $\mathrm{p}$ & seku & $\mathrm{Nd}$ & & Kaempferia galanga \\
\hline \multirow[t]{3}{*}{$\mathrm{kb}$} & seli & $\mathrm{Nd}, \mathrm{Dk}$ & & ?Freycinetia \\
\hline & semu & & A & plant, strong-smelling \\
\hline & sendo & & A & plant \\
\hline \multirow[t]{2}{*}{ k } & séra & & A & tree \\
\hline & sermélé & Dk & & $=$ mbindi \\
\hline k & seti I & $\mathrm{Mn}$ & $\mathrm{dft}$ & Celtis tetrandra \\
\hline k & seti II & Dk & & Ficus \\
\hline k & sigo & $\mathrm{Wj}$ & A & tree \\
\hline k & singgi & $\mathrm{Nd}$ & $\mathrm{dft}$ & Syzygium \\
\hline \multirow[t]{3}{*}{ k } & singgi mité & & $A, d f t$ & Linociera \\
\hline & singgi wela & $\mathrm{Nd}$ & & tree \\
\hline & soki & $\mathrm{Nd}$ & & $=$ tuga soki \\
\hline \multirow[t]{5}{*}{$\mathrm{t}$} & soko & $\mathrm{Nd}$ & & $=$ gai \\
\hline & soko ajé & & A & grass, tall \\
\hline & soko belanda & $\mathrm{Nd}$ & & Pennisetum purpureum \\
\hline & soko roto & & A & grass, tall \\
\hline & somu & $\mathrm{Nd}$ & & Allium cepa var. ascalonicum \\
\hline $\mathrm{t}$ & sora & $\mathrm{Mn}$ & & Erigeron sumatrensis \\
\hline \multirow[t]{2}{*}{$\mathrm{u}$} & soso & Nd & & Polypodiaceae \\
\hline & suga sala & Dk & & Achyranthes aspera \\
\hline $\mathrm{u}, \mathrm{k}$ & sui & & A & Champereia manillana \\
\hline k & sui suka & $\mathrm{Nd}$ & & Phaleria octandra \\
\hline $\mathrm{kb}$ & su'i I & Dk & A & vine, medicinal \\
\hline k & su'i II & Dk & & shrub \\
\hline $\mathrm{kb}$ & suja & $\mathrm{Nd}$ & & Dioscorea esculenta \\
\hline \multirow[t]{2}{*}{$\mathrm{k}$} & sulé & $\mathrm{Mn}$ & & Ficus septica \\
\hline & sunga I & & A & Allium sativum \\
\hline k & sunga II & $\mathrm{Nd}$ & A & tree, smells as garlic when felled \\
\hline k & susu lako & $\mathrm{Mn}, \mathrm{Nd}$ & A & tree w. long 1. \\
\hline \multirow[t]{2}{*}{$\mathbf{a}$} & taga & $\mathrm{Nd}$ & & Calamus \\
\hline & taga kata & Dk & & $=$ taga manu \\
\hline
\end{tabular}




\begin{tabular}{|c|c|c|c|c|}
\hline \multirow{4}{*}{$\begin{array}{l}\mathrm{u} \\
\mathrm{u}\end{array}$} & \multicolumn{2}{|l|}{$\begin{array}{l}\text { taga manu } \\
\text { ta'i jara I }\end{array}$} & \multirow[t]{4}{*}{ A } & \multirow{5}{*}{$\begin{array}{l}\text { Myrsinaceae } \\
\text { Fungi } \\
\text { herb, medicinal } \\
\text { Anaphalis longifolia } \\
\text { Vernonia }\end{array}$} \\
\hline & ta'i jara I & & & \\
\hline & ta'i jara II & & & \\
\hline & ta'i nitu & & & \\
\hline w & ta'i wawi & $\mathrm{Mn}, \mathrm{Nd}$ & & \\
\hline $\mathrm{kb}, \mathrm{k}$ & tali té'u I & $\mathrm{Mn}, \mathrm{Nd}$ & 3808 & Dumasia villosa \\
\hline $\mathrm{kb}$ & tali té'u II & Dk & & Abrus precatorius \\
\hline k & tandu wani & & A & shrub \\
\hline k & tanggo & $\mathrm{Nd}$ & A & Ficus fulva \\
\hline k & tangi & Dk & & $\begin{array}{l}\text { shrub; bark is substitute for betel } \\
\text { nu t }\end{array}$ \\
\hline k & tangi mura ro'a & & 3792 & Ficus ?punctata \\
\hline $\mathrm{p}$ & taro & $\mathrm{Nd}$ & & Indigofera \\
\hline $\mathrm{p}$ & $\tan$ & Dk & A & $=$ taro \\
\hline k & ta'u & & A & tree w. edible fr. \\
\hline k & ta'u jara & & A & shrub \\
\hline $\mathrm{kb}$ & tawé & $\mathrm{Nd}, \mathrm{Dk}$ & A & vine; substitute for betel nut \\
\hline $\mathrm{p}, \mathrm{k}$ & té'a & & & tree or shrub \\
\hline $\mathrm{p}$ & teka jawa & Ds & & $=$ muku jawa \\
\hline $\mathrm{u}$ & tembu & $\mathrm{Nd}$ & & Fungi \\
\hline & tembu moké & $\mathrm{Nd}$ & & Fungi \\
\hline & teni & & A & ?Dioscorea \\
\hline & tenu & Dk & & $=$ teni \\
\hline k & tepe déké & $\mathrm{Mn}$ & & Turpinia \\
\hline $\mathrm{u}$ & tepé tana & & & Fungi \\
\hline $\mathrm{u}$ & tepu & $\mathrm{Nd}$ & A & plant, wild, medicinal \\
\hline k & teré & Nd, Dk & & Artocarpus elasticus \\
\hline & teru & & & $=$ wuja \\
\hline $\mathrm{b}$ & tété kadho & Dk & A & Centella asiatica \\
\hline & tété nggebhé & $\mathrm{Nd}$ & & = tété kadho \\
\hline b & tété wati & & A & = tété kadho \\
\hline $\mathrm{p}$ & tewu & $\mathrm{Nd}$ & & Saccharum officinarum \\
\hline $\mathrm{kb}$ & timu & $\mathrm{Nd}$ & & $=$ daka \\
\hline & tiri & $\mathrm{Nd}$ & & Amorphophallus \\
\hline & tola & $\mathrm{Nd}$ & & Luffa aegyptiaca \\
\hline $\mathrm{u}$ & tonga & $\mathrm{Nd}$ & & herb \\
\hline $\mathrm{u}$ & toro & $\mathrm{Nd}$ & & $=$ mberi \\
\hline $\mathrm{p}$ & toro pisi & & & Solanum torvum \\
\hline k & tuga & $\mathrm{Nd}$ & & Citrus \\
\hline k & tuga lo'o & $\mathrm{Mn}$ & & Citrus \\
\hline k & tuga ria & $\mathrm{Mn}$ & & Citrus grandis \\
\hline k & tuga soki & & & Citrus \\
\hline k & tuga sowa & Dk & & $=$ tuga ria \\
\hline hb & tuwa & Nd, Dk & A & liana, water-containing \\
\hline
\end{tabular}




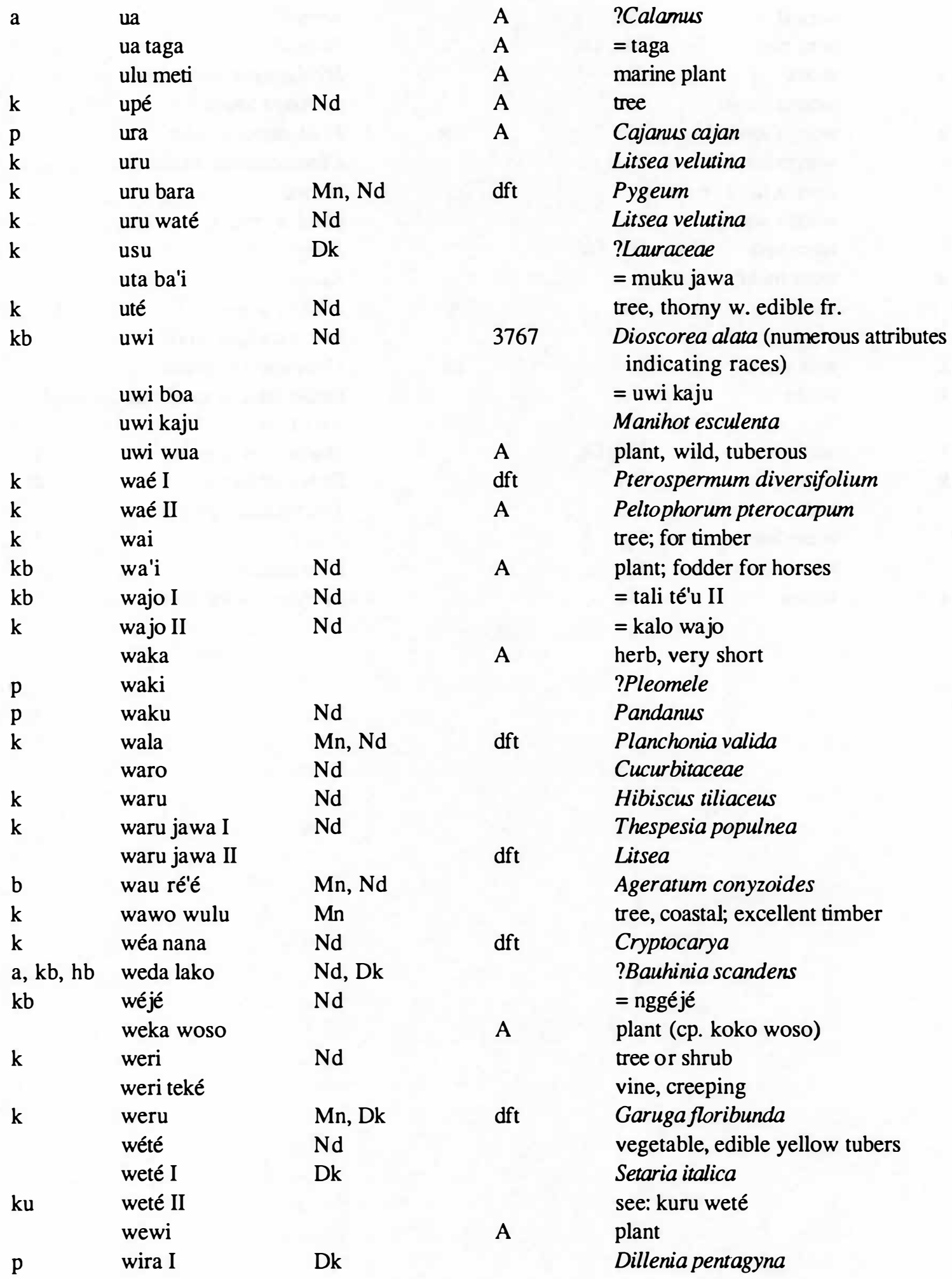


$\mathrm{u}$

k

u

k

k

k

k

k

k wira II

wira rua

wona

wonga bingu

wonga londa I

wonga londa II

wonga runu

wonga wai

wora bata

wora moké

woso

wowo

wua wulu

wudu

wuja

wuka

wulu

wunu kapa

wuso weka

wuwu
Wn

$\mathrm{Mn}, \mathrm{Dk}$

$\mathrm{Nd}$

Wn

Nd

$\mathrm{Nd}$

Pg, Dk

Nd

Dk

Nd, Dk

Wn

$\mathrm{Nd}$

$\mathrm{Pg}$

Dk

Dk
$=$ rosé

?= rosé

Moringa pterygosperma

= wonga londa II

$\mathrm{dft}$

Mallotus ricinoides

Clerodendrum buchanani

= runu

plant w. red fl.

Fungi

Fungi

A $\quad=$ koko woso

tree; excellent wood

dft Gironniera cuspidata

shrub; bark is substitute for betel nut

Macaranga tanarius

Sterculia foetida

Schizostachyum blumii

A $\quad ?=$ leri

Desmodium

Pterygota horsfieldii 
1.5 BIMA - TAXONOMIC

\subsubsection{PRELIMINARY REMARKS}

(a) The language

(i) Bima is the westernmost language of the 'Bima-Sumba Group' and, in wider context, of CMP (Central Malayo-Polynesian, after Blust 1980:10-11). Dialectal words cited are from Kolo and Réo/Pota.

(ii) Orthography and pronunciation

/e/ is the unrounded mid front vowel. I do not think that Jonker's/e/ and /è/, or his /o/ and /ò/, form different phonemes.

'ḍ' and 'ḅ' are glottalised 'b' and 'd'.

(b) Research and identification

A good many names of identified plants were able to be taken from Hildebrand's 'daftar' and some from Heyne's work.

Jonker's dictionary provided me with several other plant names. I tried to identify them with the aid of Bimanese settlers in Pota in 1980. We identified a number of plants in the field - Ros in Réo, myself in Pota.

I also consulted the Bimanese-Indonesian dictionary by Sahidu. It contains no taxonomic names, but enabled me to add some names and to identify others with the help of descriptions or Indonesian translations.

(c) Abbreviations

(i) Classifiers

$\begin{array}{lll}\text { a } & \text { ai } & \text { liana, vine } \\ \text { f } & \text { fu'u } & \text { tree, stool } \\ \text { h } & \text { haju } & \text { tree } \\ \text { hu } & \text { humpa } & \text { vine } \\ \text { mp } & \text { mpori } & \text { grass, rushes } \\ \text { r } & \text { ro'o } & \text { leaf, herb }\end{array}$

(ii) Localities

$\begin{array}{ll}\text { Kl } & \text { Kolo } \\ \mathrm{Pt} & \text { Pota } \\ \text { Ré } & \text { Réo }\end{array}$

(iii) Additional sources

J Jonker

S Sahidu 


\subsubsection{THE LIST}

aduria

anggu

anggu

aruna I

aruna II

aruna ai

arunana

arundae I

mp ati

ati (ka)ndolo

banggulae

?bara I

?bara II

barungku

bawa

bawe

belere

bembe

bila

binawa

bintango

bintango na'e

biru

boa

bohobangi

bue

buka

?bulu

buri

cacingi

cacoro

cangke

cira

daem se

?dalima

damse

dau

dimu

dobu

due

?dumu

?dumu kunci
$\mathrm{H}$

V

$\mathrm{J}, \mathrm{H}$

V

$\mathrm{dft}$

V

$\mathrm{H}$

J

$\mathrm{H}$

$\mathrm{dft}$

$\mathrm{dft}$

J

J

$\mathrm{Pt}$

$\mathrm{Pt}$

J, V

$\mathrm{V}$

$\mathrm{dft}$

$\mathrm{dft}$

$\mathrm{dft}$

V

R

$\mathrm{dft}$

V

V

$\mathrm{H}$

$\mathrm{H}$

V

J

J

J, V

$\mathrm{R}$

J

V

$\mathrm{H}$

$\mathrm{H}$

J

V

V

$\mathrm{H}$
= duria

Vitis vinifera

Passiflora foetida

Ananas comosus

= aruna ai

Agave sisalana

Mimusops elengi

Acanthaceae

Imperata cylindrica

Saccharum spontaneum

Zingiber purpureum

Gyrocarpus americanus

Tetrameles nudiflora

Papilionaceae

Allium cepa var. ascalonicum

Pongamia pinnata

?Euphorbiaceae

Datura metel

Aegle marmelos

Cordia subcordata

Calophyllum soulattri

Calophyllum ?inophyllum

Leucosyke capitellata

Canavalia

Syzygium

Vigna unguiculata

Monochoria vaginalis

Piper betle var. siriboa

Pachyrrhizus erosus

Capparis

Fungi

Syzygium aromaticum

Maclura cochinchinensis

= uwi haju

Punica granatum

= uwi haju

Indigofera sp.

Cucumis sativus

Saccharum officinarum

Ficus benjamina

Curcuma

Boesenbergia pandurata 
dungga

dungga buti

dungga jurutuli

dungga ncia

dungga niu

duria

duwa

duwa tutu

duwa wua

duwe

eja

?eja wilu

?enggo

fanda I

fanda II

fanda dipi

fanda mengi

fanda ngaha

fare

fare keta

feli

fimpi

fo'o

fo'o doro

fo'o wuḅa

gadu

gandarisa I

gandarisa II

garanji

garoso I

garoso II

garoso kaya

garoso ?mbolo

garoso rui

garu

guni

haju me'e I

haju me'e II

hala

hala henggo

handa

?heci

hei
$\mathrm{Pt}$

J

V

V

J

V

J

V

V

V

$\mathrm{H}$

Ré

J, R

$\mathrm{dft}$

V

H

V

H

$\mathrm{H}$

dft

dft

$\mathrm{H}$

df $t$

V

$\mathrm{H}$

H

V

Pt

$\mathrm{Pt}$

Ré

Ré

H

V

R

V

R

dft

V

V

dft

V

V

J, V

dft

Citrus

Citrus

Citrus ?aurantium

Citrus aurantifolia

Citrus grandis

Durio zibethinus

Derris

Derris ?elliptica

?Croton tiglium

Syzygium cumini

= uwi eja

?Dioscorea

Rhus taitensis

$=$ aruna I

= fanda dipi

Pandanus tectorius var.

Pandanus tectorius var. laevis

$=$ aruna I

Oryza sativa

Oryza sativa f. glutinosa

Streblus asper

Intsia bijuga

Mangifera indica

Mangifera

?Mangifera timorensis

$=$ lede

Gendarussa vulgaris

Barleria prionitis

Spinifex littoreus

Annona muricata

= garoso kaya

Annona squamosa

Annona reticulata

= garoso I

Lansium

?Corchorus capsularis

Diospyros littorea

Diospyros 'toposia'

Benincasa hispida

Cucurbitaceae

Mucuna pruriens var. pruriens

Canarium asperum

Urticaceae 


\begin{tabular}{|c|c|c|c|c|}
\hline & humpa leu & Ré & $\mathrm{R}$ & Entada phaseoloides \\
\hline & huni & & $\mathrm{H}$ & Curcuma viridiflora \\
\hline & inci & & $\mathrm{H}$ & Spondias malayana \\
\hline $\mathrm{h}$ & isu & & $\mathbf{J}$ & Albizia saponaria \\
\hline & jago & & J & Zea mays \\
\hline & jambu & & J & Psidiurn guajava \\
\hline & jati & & $\mathbf{J}$ & Tectona grandis \\
\hline & jene mawa (ra) & & J & Rosa sp. \\
\hline & jinta & & $\mathrm{J}$ & Cuminum cyminum \\
\hline & kabatula & Ré & V & Euphorbia tirucalli \\
\hline $\mathrm{mp}$ & kaḅisa & $\mathrm{Pt}$ & V & Cyperaceae \\
\hline $\mathrm{mp}$ & kaḅisa na'e & $\mathrm{Pt}$ & V & Typha angustifolia (cj.) \\
\hline $\mathrm{mp}$ & kaḅisa to'i & $\mathrm{Pt}$ & V & Cyperaceae \\
\hline & kaḅoe & & $\mathrm{H}$ & Phaseolus radiatus \\
\hline & kaboe jao & & V & Phaseolus aureus \\
\hline & kacunda & & $\mathrm{H}$ & ?Tacca leontopetaloides \\
\hline & kaḍara & & $\mathrm{H}$ & Caesalpinia bonduc \\
\hline & kadele & & V & Glycine soja \\
\hline & ?kaḍui dolu janga & & $\mathbf{J}$ & Solanum sp. \\
\hline & kadui lako & & & Solanum sp. \\
\hline & kaḍui (na'e) & & $\mathbf{J}$ & Solanum melongena \\
\hline & kadui paranggi & & V & Lycopersicon lycopersicum \\
\hline & kaḍui rui & & $\mathrm{J}$ & Solanum sp. \\
\hline & ?kadui tarende & & & ?Solanum sp. \\
\hline & kahawa & & $\mathrm{J}$ & Coffea arabica \\
\hline . & ?kaju mani(sa) & & $\mathbf{J}$ & Cinnamomum ?burmannii \\
\hline & kakapi & $\mathrm{Pt}$ & V & Crotalaria sp. \\
\hline & kaki'i & & V & ?Ceropegia sp. \\
\hline & kalaki & & & vegetable, bitter, creeping \\
\hline & kalaku & & $\mathrm{dft}$ & Diospyros malabarica \\
\hline$? \mathrm{mp}$ & kalalu & $\mathrm{Pt}$ & V & Cyperaceae \\
\hline & kaleli & & $\mathrm{H}$ & Aleurites moluccana \\
\hline & kalende & & $\mathrm{H}$ & Citrullus lanatus \\
\hline & kalimone & $\mathrm{Pt}$ & V & Chonemorpha / Ichnocarpus \\
\hline & kalo & & $\mathbf{J}$ & Musa paradisiaca \\
\hline & kalo goa & & $\mathrm{S}$ & ?Musa sp. \\
\hline & kalo linci & $\mathrm{Pt}$ & V & ?Ensete glaucum \\
\hline & kamboi & Ré & $\mathrm{R}$ & $=$ taride \\
\hline & kalompe mesa & & & $?$ \\
\hline & kamboja I & & V & Plumeria acuminata \\
\hline & kamboja II & & V & Ipomoea \\
\hline & kamonca & $\mathrm{Pt}$ & V & Alangium villosum \\
\hline & kampaja & & $\mathrm{H}$ & $=$ panja \\
\hline
\end{tabular}




\begin{tabular}{|c|c|c|c|}
\hline kampuja & & $\mathrm{J}, \mathrm{S}$ & Zingiber aromaticum \\
\hline kamuni & & $\mathrm{H}$ & Murraya paniculata \\
\hline kanahi & & $\mathrm{dft}$ & Cassia fistula \\
\hline kananga & & $\mathbf{J}$ & ?Cananga odorata \\
\hline kananga wuḅa & & $\mathrm{H}$ & Gmelina asiatica \\
\hline kanari & & $\mathrm{H}$ & Canarium vulgare \\
\hline kanto & & $\mathrm{J}$ & plant, tuberous \\
\hline kanturu & & $\mathrm{S}$ & ?Musa sp. \\
\hline kantusu & & dft & $\begin{array}{l}\text { Palaquium ?amboinense I } \\
\text { ?obovatum (cp. lahodo) }\end{array}$ \\
\hline ka'o & & $\mathrm{J}, \mathrm{S}$ & plant, tuberous, wild \\
\hline kapala & & $\mathrm{H}$ & Myristica frangrans \\
\hline kapanca & & $\mathrm{H}$ & Lawsonia inermis \\
\hline kapanca cina & & V & ?Aglaia odorata \\
\hline kapu'u & & dft & Dysoxylum caulostachyum \\
\hline kara & $\mathrm{Pt}$ & V & Acacia farnesiana / tomentosa \\
\hline karake & $\mathrm{Pt}$ & V & Cayratia ?trifolia \\
\hline karaki & & & $?$ \\
\hline karana isu & & $\mathrm{dft}$ & Ganophyllum falcatum \\
\hline karara & & $\mathrm{H}$ & Artocarpus altilis (seedless) \\
\hline karebe & $\mathrm{Pt}$ & V & Eleocharis dulcis \\
\hline katanga & Ré & & Jatropha curcas \\
\hline katanga jawa & & $\mathrm{R}$ & $=$ tatanga $\mathrm{I}$ \\
\hline katangga & Ré & V & Millingtonia hortensis \\
\hline katawi & & $\mathrm{dft}$ & Buchanania arborescens \\
\hline katipu & & dft & Planchonia valida \\
\hline ?katipu doro & & $\mathrm{dft}$ & Garcinia sp. \\
\hline katumba & & $\mathrm{H}$ & Coriandrum sativum \\
\hline katungga & $\mathrm{Kl}$ & $\mathbf{J}$ & $=$ dungga \\
\hline kaua I & & $\mathrm{J}$ & Calamus sp. \\
\hline kaua II & & V & Flagellaria indica \\
\hline $\operatorname{kemba}(n g)$ & $\mathrm{Pt}$ & V & Eupatorium odoratum \\
\hline kenu I & $\mathrm{Pt}$ & V & Phragmites karka \\
\hline kenu II & & $\mathrm{J}$ & $\begin{array}{l}\text { ?Saccharum spontaneum (cp. ati } \\
\text { (ka)ndolo) }\end{array}$ \\
\hline keu (dial) & & $\mathrm{H}$ & Bambuseae \\
\hline koja & $\mathrm{Pt}$ & V & $=$ rapa mila \\
\hline kolo & & $\mathrm{H}$ & Artocarpus altilis (seeded) \\
\hline kombakasi & & $\mathrm{J}, \mathrm{S}$ & vine w. medicinal 1 . \\
\hline konca I & & $\mathrm{dft}$ & Nauclea coadunata \\
\hline konca II & $\mathrm{Pt}$ & V & Anthocephalus cadamba \\
\hline konca doro & & dft & Litsea accedentoides \\
\hline kopa & & & Parkia roxburghii \\
\hline kope & $\mathrm{Pt}$ & V & Canavalia sp. \\
\hline
\end{tabular}




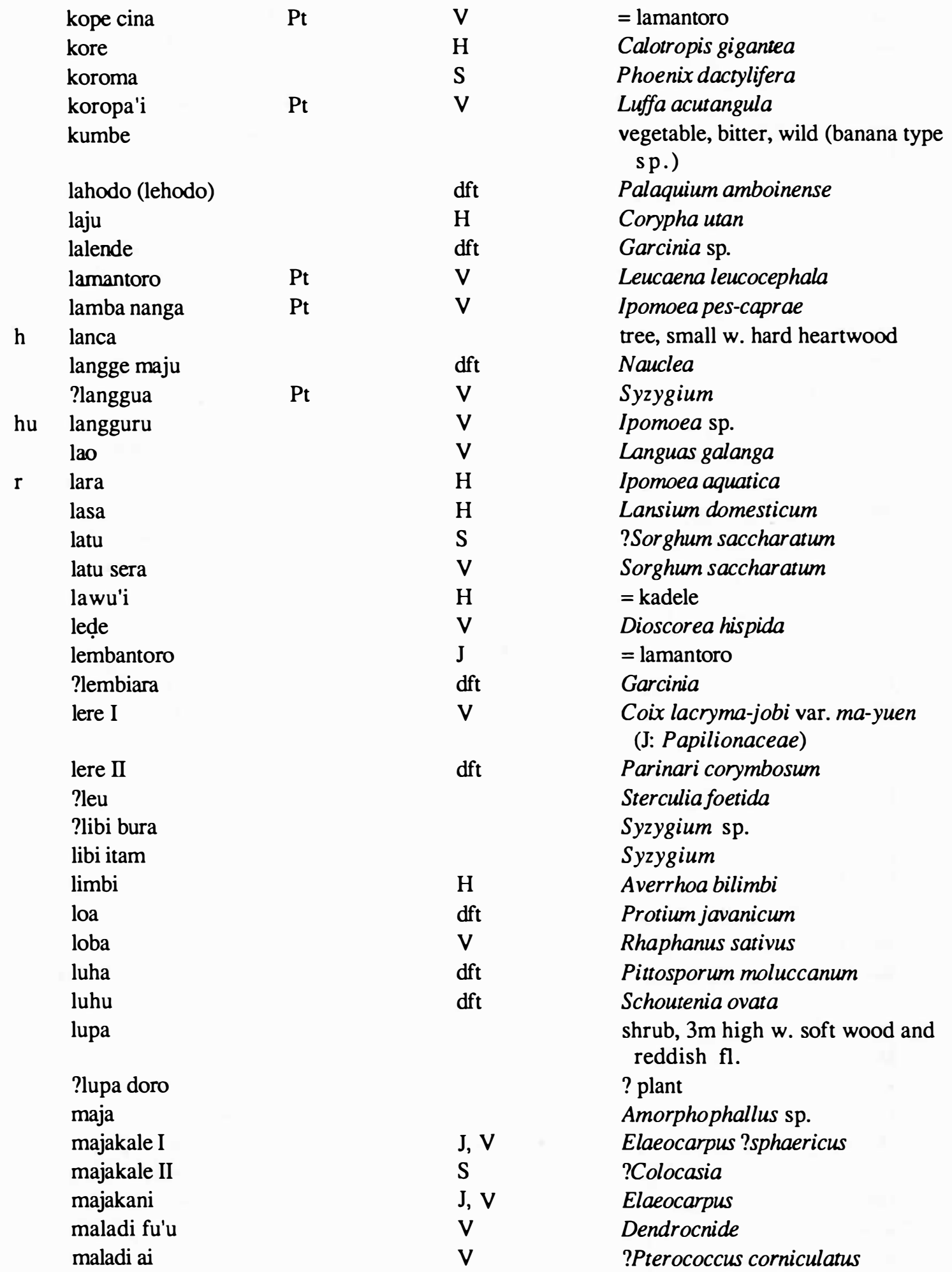




\begin{tabular}{|c|c|c|c|}
\hline maladi ara & & $\mathrm{J}, \mathrm{V}$ & ?Laportea \\
\hline mangge & & $\mathrm{H}$ & Tamarindus indica \\
\hline manggusta & & $\mathbf{J}$ & Garcinia mangostana \\
\hline mantau & & $\mathrm{H}, \mathrm{dft}$ & Calophyllum inophyllum \\
\hline marica & & V & Piper nigrum \\
\hline mbora & & dft & Peltophorum pterocarpum \\
\hline mboro & & dft & Ehretia acuminata \\
\hline menga & & $\mathrm{dft}$ & Melia azedarach \\
\hline mengi & $\mathrm{Pt}$ & V & Toona sureni \\
\hline mila & & & Schizostachyum blumii \\
\hline miro & & $\mathrm{J}, \mathrm{H}$ & Calamus sp. \\
\hline ?miro jopu & & $\mathrm{J}$ & Calamus sp. \\
\hline monggo & & $\mathrm{dft}$ & Syzygium sp. \\
\hline mpafa & & $\mathrm{dft}$ & Premna tomentosa \\
\hline mpaja & & $S$ & $=$ panja \\
\hline mpelo & & $\mathrm{dft}$ & Buchanania arborescens \\
\hline mpiu I & & $\mathrm{dft}$ & Diospyros malabarica \\
\hline ?mpiu II & & dft & Litsea accedentoides \\
\hline mpori pa'i & & $\mathrm{S}$ & bitter grass \\
\hline mundu & & $\mathrm{H}$ & Jasminum sambac \\
\hline nadu & & $\mathrm{H}$ & Amaranthus \\
\hline nadu rui & & V & Amaranthus spinosus \\
\hline nahi & & $\mathrm{H}$ & Piper betle var. siriboa I \\
\hline ?nanga & $\mathrm{Kl}$ & $\mathbf{J}$ & $=$ nangga \\
\hline nangga & & $\mathrm{H}$ & Artocarpus integer \\
\hline nangga doro & & $\mathrm{dft}$ & Planchonella obovata \\
\hline nangga karao & & V & Artocarpus ?heterophyllus \\
\hline nao & & $\mathrm{H}$ & Arenga pinnata \\
\hline nara & & $\mathrm{H}$ & Pterocarpus indicus \\
\hline nara wadu & & V & Pterocarpus indicus f. echinocarpus \\
\hline ncambu wera & $\mathrm{Pt}$ & dft & Exocarpus latifolius \\
\hline ncuna & & $\mathrm{H}$ & Allium sativum \\
\hline ndango & & V & Acorus calamus \\
\hline ndangu/a mada & & V & Homalium tomentosum \\
\hline ndaru I & & dft & Albizia procera \\
\hline ?ndaru II & & $\mathrm{J}, \mathrm{S}$ & Lagerstroemia \\
\hline ?ndola & & $\mathrm{J}, \mathrm{S}$ & plant, creeping, reddish \\
\hline ndolo & & $\mathrm{S}$ & $=$ ati $(\mathrm{ka})$ ndolo \\
\hline nenggi & & $\mathrm{J}$ & Artocarpus ?integer II \\
\hline ngame & & V & Cyperaceae \\
\hline nggala & & dft & Planchonella duclitan \\
\hline ?nggo & & & $=$ ?enggo \\
\hline nipa & $\mathrm{Pt}$ & V & Nypa fruiticans \\
\hline
\end{tabular}




\begin{tabular}{|c|c|c|c|}
\hline \multirow{4}{*}{$\begin{array}{l}\text { ni'u } \\
\text { njala-njala } \\
\text { ?nonu I } \\
\text { nonu II }\end{array}$} & & $\mathbf{J}$ & Cocos nucifera \\
\hline & & V & Cassytha filiformis \\
\hline & & & Morinda citrifolia I \\
\hline & & & $\begin{array}{l}\text { shrub; white latex of edible fr. is } \\
\text { medicinal }\end{array}$ \\
\hline ntanga & & & Kleinhovia hospita \\
\hline ?ntimu I & & & ?Flacourtia (rukam) \\
\hline ntimu II & & & ?Neonauclea excelsa \\
\hline ntonu & $\mathrm{Pt}$ & V & Melochia ?umbellata \\
\hline ?ntonu bura & & $\mathrm{dft}$ & Litsea \\
\hline ntowa & & $\mathbf{J}$ & plant \\
\hline ?nuna & $\mathrm{Pt}$ & V & Cordia ?dichotoma \\
\hline oka de & $\mathrm{Pt}$ & V & $=\operatorname{kemba}(\mathrm{ng})$ \\
\hline o'o I & & $\mathrm{H}$ & Bambuseae \\
\hline o'o II & & V & ?Gigantochloa apus \\
\hline o'o ai & & V & ?Dinochloa scandens \\
\hline o'o hamia & & & $=$ mila \\
\hline o'o kunci & & & Gigantochloa sp. \\
\hline o'o nteri & & & Schizostachyum brachycladum \\
\hline o'o pa'i & & V & Gigantochloa ?verticillata \\
\hline ?o'o potu & & V & Dendrocalomus asper \\
\hline o'o ragi & & & $?$ \\
\hline o'o rui & & V & Bambusa blumeana \\
\hline o'o todo & & & bamboo type \\
\hline pacingi & & $\mathrm{R}$ & tree, large $w$. edible fr. \\
\hline padu peo & $\mathrm{Pt}$ & V & ?Acanthaceae \\
\hline palawu & & $\mathrm{H}$ & Sesbania grandiflora \\
\hline pampa & & $\mathrm{dft}$ & Vitex pubescens \\
\hline panja & & $\mathrm{H}$ & Carica papaja \\
\hline paramau & & $\mathrm{J}, \mathrm{V}$ & Sida rhombifolia \\
\hline paranggi & & V & = kadui paranggi \\
\hline paria & & $\mathrm{J}, \mathrm{V}$ & Momordica charantia \\
\hline parongge & & $\mathrm{H}$ & Moringa pterygosperma \\
\hline paropa & & V & Sonneratia alba \\
\hline pataha & & $\mathrm{J}, \mathrm{V}$ & Ocimum \\
\hline pataha doro & & & ?Ocimum sp. \\
\hline pataha kolo & & & ?Ocimum sp. \\
\hline pataha londe & Ré & $\mathrm{R}$ & Ocimum basilicum \\
\hline pataha mengi & & & $?=$ pataha londe \\
\hline pataha mpori I & & $\mathrm{J}, \mathrm{V}$ & Cymbopogon citratus \\
\hline pataha mpori II & $\mathrm{Pt}$ & V & ?Hyptis suaveolens \\
\hline pato I & & $\mathrm{dft}$ & Buchanania arborescens \\
\hline pato II & & $\mathrm{dft}$ & Neonauclea calycina \\
\hline patola & & V & Luffa ?acutangula \\
\hline
\end{tabular}




\begin{tabular}{|c|c|c|c|}
\hline & V & Oplismenus compositus \\
\hline perdeke & & V & Achyranthes hispidus \\
\hline permau & & & $=$ paramau \\
\hline peto & & J & Erythrina sp. \\
\hline peto radarasa & & $\mathbf{J}$ & Erythrina sp. \\
\hline pingga & & $\mathrm{dft}$ & Celtis wightii \\
\hline pode & & $\mathrm{H}$ & Parkia speciosa \\
\hline ponda & & $\mathrm{J}, \mathrm{V}$ & Cucurbita moschata \\
\hline ponda mbolo & & $\mathrm{J}, \mathrm{V}$ & Crescentia cujete \\
\hline ponda naru & & & Lagenaria siceraria var. \\
\hline ponda ndali & & $\mathrm{J}, \mathrm{V}$ & Luffa aegyptiaca \\
\hline ponda nono & $\mathrm{Pt}$ & V & Lagenaria siceraria var. \\
\hline puci anggi & & $\mathrm{H}$ & Cynometra cauliflora \\
\hline puteri malu & $\mathrm{Pt}$ & V & Mimosa invisa \\
\hline ra mila & & $S$ & $?=$ rapa mila \\
\hline raca & & & tree, large, densely branched \\
\hline rambuta(n) & & $\mathrm{J}$ & Nephelium lappaceum \\
\hline rangga & & $\mathrm{H}$ & Ziziphus nummularia \\
\hline rapa & & $\mathrm{H}$ & $=$ rapa mila \\
\hline rapa mila & & S & Arachis hypogaea \\
\hline rau & & $\mathrm{J}, \mathrm{V}$ & Dracontomelon edule \\
\hline rea & & $\mathrm{H}$ & Zingiber officinale \\
\hline rida & & $\mathrm{dft}$ & Alstonia scholaris \\
\hline ringa & & $\mathrm{H}$ & Sesamum orientale \\
\hline ringi & & $\mathrm{H}$ & Ceiba pentandra \\
\hline ringi wuba & & V & Bombax ceiba \\
\hline rino ai & & $\mathrm{dft}$ & Grewia eriocarpa \\
\hline rino wadu & & $\mathrm{dft}$ & Grewia sp. \\
\hline risa & & & plant \\
\hline rondu & & V & Lagerstroemia flos-reginae \\
\hline rondu keha & & dft & Premna sp. \\
\hline rontu & & $S$ & tree \\
\hline ronu & & $S$ & tree \\
\hline ro'o koja & & V & Papilionaceae \\
\hline ropa & & & $=$ paropa \\
\hline rufe & & $\mathrm{dft}$ & Piliostigma malabaricum \\
\hline rui ntangu & $\mathrm{Pt}$ & V & Opuntia ?elatior \\
\hline rui peo & & V & Caesalpinia crista \\
\hline sabia I & & $(\mathrm{H})$ & Piper retrofractum \\
\hline sabia II & & $S$ & = saha na'é \\
\hline sada bura & & $\mathrm{dft}$ & Madhuca sp. \\
\hline sada kala & & $\mathrm{dft}$ & Palaquium sp. \\
\hline safare & Pt, Ré & $\mathrm{R}$ & tree, tall w. good timber \\
\hline
\end{tabular}




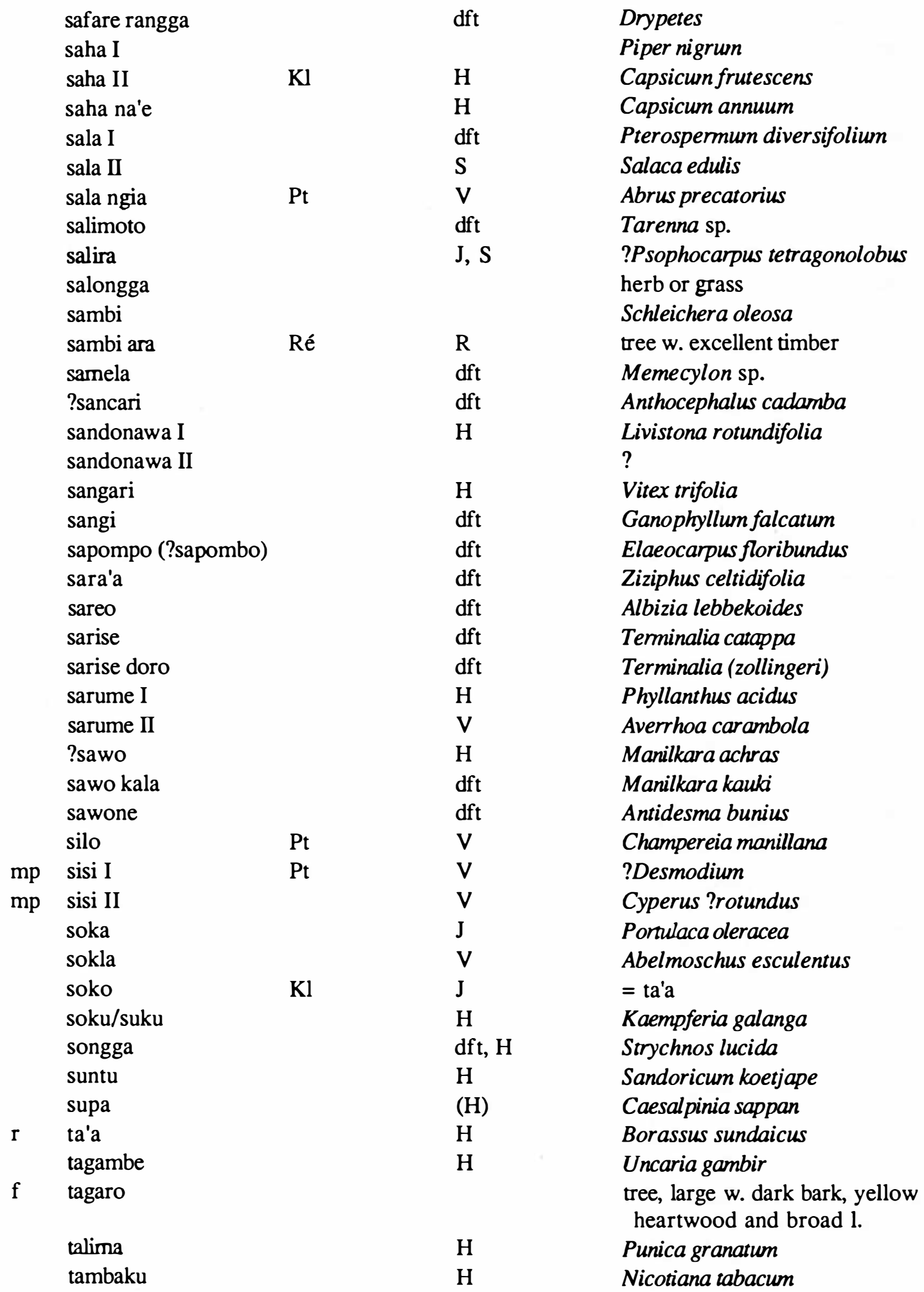




\begin{tabular}{|c|c|c|c|}
\hline tampoli & $\mathrm{Pt}$ & V & Hibiscus \\
\hline $\operatorname{tanju}$ & & $\mathrm{H}$ & Mimusops elengi \\
\hline taride & $\mathrm{Pt}$ & V & Lantana camara \\
\hline tatanga I & & $\mathrm{J}, \mathrm{V}$ & Ricinus communis \\
\hline tatanga II & Ré & V & $=$ katanga \\
\hline temba & & $\mathrm{dft}$ & Albizia lebbeck \\
\hline ?timu I & & $\mathrm{dft}$ & cp. ntimu II \\
\hline timu II & $\mathrm{Kl}$ & $\mathrm{H}$ & $=\operatorname{dimu}$ \\
\hline tombe langgiri & Ré & $\mathbf{R}$ & Physalis \\
\hline tomberowa & & J & plant \\
\hline tonggu ala & $\mathrm{Pt}$ & V & Diospyros javanica \\
\hline tonjo(ng) & $\mathrm{Pt}$ & V & Nelumbo nucifera \\
\hline (n)tui & $\mathrm{Pt}$ & V & Dolichandrone spathacea \\
\hline tula & & V & Alstonia spectabilis \\
\hline ?tupa & & $\mathrm{H}$ & $=$ ni'u \\
\hline u'a & & J & Areca cathecu \\
\hline uwi eja & & $\mathrm{H}$ & Colocasia esculenta \\
\hline uwi (gunu) & & $\mathrm{H}$ & Ipomoea batatas \\
\hline uwi haju & Pt, Ré & V & Manihot esculenta \\
\hline uwi jara & $\mathrm{Pt}$ & V & ?Merremia umbellata \\
\hline uwi rama & & & $=$ uwi (gunu) \\
\hline (uwi) sura I & & V & Dioscorea esculenta \\
\hline uwi sura II & & V & Dioscorea aculeata \\
\hline uwi tandi'i & & $\mathrm{J}, \mathrm{V}$ & $=($ uwi) sura I \\
\hline wako & & $\mathrm{dft}$ & Rhizophora apiculata \\
\hline wau & & $\mathrm{H}$ & Hibiscus tiliaceus \\
\hline wau moti & & V & Thespesia populnea \\
\hline wia & & $\mathrm{H}$ & Alocasia macrorrhiza \\
\hline witi & & V & Setaria italica (J: pulse) \\
\hline wiu & & dft & Garuga floribunda \\
\hline woḍi & & S, V & Ficus rumphii \\
\hline ?wolo & & J & Gossypium sp. \\
\hline wu(w)a podu & & V & ?Anamirta cocculus \\
\hline ?wudiwonja / wonca (s) & & $\mathrm{J}, \mathrm{V}$ & ?Ficus sp. \\
\hline wudu jara & & $\mathbf{R}$ & Voacanga \\
\hline wunta & & $\mathbf{J}$ & $=$ wolo \\
\hline ?wuré & & $\mathrm{J}, \mathrm{V}$ & Dolichos lablab \\
\hline wure ḅoa & & S & $=$ wure \\
\hline wuwu & $\mathrm{Pt}$ & V & $=\mathrm{leu}$ \\
\hline
\end{tabular}




\subsection{SUMBA - TAXONOMIC}

\subsubsection{PRELIMINARY REMARKS}

I dwell a bit longer on the introduction to this vernacular list, as research in the field in Sumba was longer and the study more intensive.

(a) The language

(i) For practical purposes (especially in section 2) I divide the Sumba dialects into an eastern group and a western group. This division is based on notes which Onvlee (1973:165) made in 1936, though in his dictionary (Onvlee 1984:ix, xvi) he apparently evades a decision.

The vocabularies of the many dialects are greatly different, for example the word for 'tree' is ai in $\mathrm{Ak}$ and $\mathrm{Kb}$, ia in $\mathrm{Mi}, \epsilon$ in $\mathrm{Lw}$, yai in $\mathrm{Mb}$, wasu in $\mathrm{Ww}$, ghazu in Lr, wasu in $\mathrm{Ll}$, ghasu in $\mathrm{Tr}$ and ghayo in Ko. The dialects commonly coincide with (old) civil boundaries, and are named after those of ten small districts. Only the names of the dialects from which plant names are noted are shown on the map (Map 4, p.266). This is based on the map I found in Kapita (1976). The (modern) civil districts (kabupaten and kecamatan) are not indicated.

(ii) Orthography and pronunciation

I use Onvlee's latest orthography.

The Sumba languages differ greatly from the vast majority of Indonesian languages by the fact that vowel-length and stress have phonemic values.

Short syllables are marked by ' ', and long ones by ' ', on the respective vowels. These marked syllables are always stressed. Otherwise, syllables with vowels of medium length are commonly stressed on the penultimate syllable of the base word.

As Sumbanese dialects are (officially) known as having only vocalic syllables, an unstressed vowel called by Onvlee 'hulpvokaal', namely '- $u$ ' in eastern Sumba, and '-a' or '-o' in western dialects, is added to an originally final consonant. However, I heard in the pronunciation of John Mata Rara many names without the additional '- $u$ ', although Kapita proposed to spell them anyway. Also Dowa several times omitted the additional '-o' sound. Possibly they have been influenced by school-teaching, where they have to pronounce consonantal endings, or by maps and the use of them by non-Sumbanese officials.

Because outsiders can hardly distingush the original from the extended form, we have the stressed syllables in words of three or more syllables (without " " or ' ') indicated by a preceding ', e.g. ka'lobungu, la'mbunguru, 'litapu.

Only once, in the last stage of checking, I found a final glottal stop, in the word tuwa' in Loura.

Glottalised $b$ and $d$ are found in Anakalangu, and are spelled 'ḅ' and ' $d$ '.

In one case I deviated from Onvlee's and Kapita's orthography, namely by accepting iwi at the side of $i w i$, writing them consistently iwi (I) and iwi (II). I did this because of finding (noting) in dialects ùwi and àwi (cp. Dioscorea hispida in section 2), and in view of the queer homonymy of such common plants as the rattan and a very well known poisonous tuber.

Sumbanese dialects don't have the pepet; the phoneme /e/ is the higher or lower mid-front vowel. 
In Kodi, Tanarighu and Loura we find a kind of laryngal spirant or trill, here spelt 'gh'.

A final long and stressed syllable, formerly written with doubled vowel as in, halii and yuu, is now spelt (after Onvlee) halĭ, yú.

A diacritic '."' is put on the second of two consecutive vowels to indicate that no diphthong is meant, e.g. pau contrasts with paï.

(b) Research and identification

My first acquaintance with Sumbanese plant names was in 1962, when collecting data for word comparison in my Manggarai dictionary.

I collected materials from Dammerman's article with 43 identified species and some 80 vemacular names from 'East Sumba and Loura'. Hildebrand's list yielded 63 names for some 50 tree species, mostly from eastem Sumba. Also Heyne's work gave identified plant names which, however, of ten had to be emendated. Finally Wielenga named many plants, mostly without scientific names, but luckily with several dialectal synonyms.

When I started my botanic-linguistic research in Sumba 12 July 1974 with the above data, I had the good luck of coming upon a wonderful new source. Through the kindness of $\mathrm{Mr} \mathrm{K}$. Windi I was able to note some 300 plant names, of which many were identified, from Kapita's provisional Kambera-Indonesian wordlist. Mr Windi kindly taught me about the orthography and pronunciation of the Kambera dialect, which very much helped my three weeks' research in the field. This made up somewhat for the absence of Oemboe H. Kapita.

I collected with Mr Irinus Ros some 350 dried plants together with their names. They were kindly identified in the Rijksherbarium of Leiden (numbers in the list). Besides this, we could with more or less certainty identify plants in the field.

As several informants who spoke other dialects wandered about with us, it became possible to note also many names of the Kodi, Wewewa and Karera dialects.

We began collecting intensively in the neighbourhood of Waingapu on the way to westem Sumba and when returning from there. Our research in the wide vicinity of We'etebula in Loura was very fruitful. Then we climbed the mountain Yawila and visited for some days Waikabubak, especially Pogobina. Back in Waingapu we were able to check our collection and verify many names with the help of informants from western Sumba. Then we collected for five days in Melolo and Rindi.

This research only progressed so smoothly thanks to the great hospitality and every kind of help given by the Frs Redemptorists in Waingapu, We'etebula, Waikabubak and Melolo.

Afterwards I was able to add 33 names of grasses that were collected by the veterinary surgeon Piet Hoekstra and identified in the herbarium of the Kebun Raya Indonesia in Bogor. The scientific names were updated by Dr J. F. Veldkamp in Leiden.

In 1977 Oemboe H. Kapita, the excellent connoisseur of the Sumbanese culture and dialects, kindly corrected my provisional list.

In 1987 I was able to check with the published dictionaries of Kapita and of Onvlee. Synonymous names from the latter source were added, especially from the dialects of Anakalangu, Lewa, Mamboru and Wewewa. 
In the final stage I added a few names from the village of Totoka in Loura as given by Mr Yosef Dowa.

(c) Abbreviations

(i) Classifiers

The classifiers were not able to be established with any certainty.

(ii) Localities

Onvlee's and Kapita's usage is followed in writing place names. Where a form occurs in both East and West Sumba dialects, the eastern locations are given first, separated from the western locations by a semicolon.

$\begin{array}{ll}\text { Ak } & \text { Anakalangu } \\ \text { ES } & \text { East Sumba (somewhere) } \\ \text { ESb } & \text { East Sumba (in most dialects) } \\ \text { Kb } & \text { Kambera } \\ \text { Kn } & \text { Kanatangu } \\ \text { Ko } & \text { Kodi } \\ \text { Kr } & \text { Karera } \\ \text { L } & \text { Lauli (Lolina) } \\ \text { Lr } & \text { Loura (Laora, Laura) } \\ \text { Lw } & \text { Lewa } \\ \text { Mb } & \text { Mamboru (Mamboro) } \\ \text { Mi } & \text { Mangili } \\ \text { Mn } & \text { Melolo } \\ \text { Np } & \text { Napu } \\ \text { Rn } & \text { Rindi } \\ \text { Tb } & \text { Tabundungu } \\ \text { Tm } & \text { Tarimbangu } \\ \text { Tr } & \text { Tanarighu } \\ \text { Tt } & \text { Totoka } \\ \text { Ww } & \text { Wewewa (Waijewa) } \\ \text { Wk } & \text { Wanukaka } \\ \text { WS } & \text { West Sumba (somewhere) } \\ \text { WSb } & \text { West Sumba (in most dialects) } \\ & \end{array}$

(iii) Additional sources
D Dammerman
O Onvlee
OK Oemboe H. Kapita
PH Piet Hoekstra
W Wielenga
YD Yosef Dowa 


\subsubsection{THE LIST}

\begin{tabular}{|c|c|c|c|}
\hline abalawi & Ak & $\mathrm{O}$ & $=$ ambalaï \\
\hline ago kùra & $\mathrm{u}$ & RV & = anggo kùra \\
\hline ai bàhi & $\mathrm{Kb}$ & 3856 & Cassia \\
\hline ai iju & $\mathrm{Kb}$ & OK & Maclura cochinchinensis \\
\hline ai mítengu & ES & $\mathrm{D}$ & = ai mítingu \\
\hline ai mítingu & $\mathrm{Kb}$ & $\mathrm{D}, \mathrm{O}$ & Diospyros \\
\hline ai rara & $\mathrm{Kr}$ & dft & Parinari corymbosum \\
\hline áke & Ko & RV & = ka'nanggaru \\
\hline áku & Ko & RV & $=$ híkilu \\
\hline alaka & $\mathrm{Mb}$ & RV & = 'alaku \\
\hline 'alaku & $\mathrm{ESb}$ & $\mathrm{O}$ & ?Uvaria \\
\hline àlango & Ww & $\mathrm{O}$ & = yàlangu \\
\hline àle & $\mathrm{Lr}$ & RV & vine, finger-thick \\
\hline àlongu & $\mathrm{Ak}, \mathrm{Mb}$ & $\mathrm{O}$ & = yàlangu \\
\hline àmba & $\mathrm{Kb}, \mathrm{Tm}, \mathrm{Tb}$ & $\mathbf{W}, \mathrm{O}$ & = kamàru \\
\hline ambalaï & $\mathrm{ESb}$ & RV & Lycopersicon lycopersicum \\
\hline analalu & $\mathrm{Kb}$ & $\mathrm{OK}$ & creeping vine \\
\hline àndala & $\mathrm{Lr}$ & RV & tree w. medicinal $l$. \\
\hline anggalai & $\mathrm{Rn}, \mathrm{Mb}$ & RV & $=$ ambalaï \\
\hline anggo kùra & Ww, Lr, Ko & RV & ?Cleistanthus \\
\hline anjangi & ES & $\mathrm{dft}$ & $=$ kanjangi \\
\hline ?apet & WS & $\mathrm{H}$ & Ischaemwm timorense \\
\hline au & $\mathrm{ESb}$ & RV & Gigantochloa ?apus \\
\hline au bàta & Lw & OK & $=$ tàringu \\
\hline au 'bokulu & $\mathrm{Kb}$ & RV & = tàringu \\
\hline au búku & & & Bambuseae (w. narrow hole) \\
\hline au jawa & $\mathrm{Rn}, \mathrm{Kr}, \mathrm{Kb}$ & & ?Gigantochloa \\
\hline auhu & Lw & W & $=$ uhu \\
\hline ausu & $\mathrm{Ak}, \mathrm{Mb}$ & $\mathrm{O}$ & $=\mathrm{uhu}$ \\
\hline auwi & Ak & $\mathbf{O}$ & $=$ iwi I \\
\hline àwi & $\mathrm{Kr}$ & RV & $=$ ìwi II \\
\hline ?bakara wai & ES & dft & Litsea velutina \\
\hline bakara wato & $\mathrm{Rn}$ & RV & $=$ ?bakara wai \\
\hline baku & $\mathrm{Ak} ; \mathrm{Tt}$ & W & $=$ hambaku \\
\hline baku sawu & Ak & $\mathrm{O}$ & = mbaku haü I \\
\hline bàla & $\mathrm{Tt}$ & V, YD & $=$ uhu kanu \\
\hline ?bala ria & $\mathrm{Kb}$ & 3893 & Eranthemum \\
\hline barapola & ES & $\mathrm{D}$ & Anaphalis longifolia \\
\hline bawa & $\mathrm{ESb}$ & $\mathrm{OK}, \mathrm{O}$ & $=$ labawa \\
\hline bawa kawaii & $\mathrm{Kb}$ & OK, RV & Allium sativum \\
\hline 'bawangu & $\mathrm{Kb} ; \mathrm{Ww}$ & W & $=$ labawa \\
\hline ?bengga kada yìbi & Ww & RV & $=$ ghasu ghula \\
\hline
\end{tabular}




\begin{tabular}{|c|c|c|c|}
\hline bengge & Ww & $\mathrm{O}$ & $=$ benggi \\
\hline benggi & $\mathrm{Mi}, \mathrm{Kb}$ & OK & Coriandrum sativum \\
\hline bila & $\mathrm{Ll}, \mathrm{Lr}$ & $\mathrm{D}, \mathrm{OK}$ & = wa'bila \\
\hline bila?wae jeje & Ww & 4063 & Acanthaceae \\
\hline bìlya & Ko & RV & = wa'bila \\
\hline ?bok & ES & $\mathrm{dft}$ & Podocarpus amanus \\
\hline bỏla & Ww & OK & $=$ uhu kanu \\
\hline 'borungu & Ak & $\mathrm{O}$ & = mbúrungu \\
\hline bou 'taiko & $\operatorname{Tr}$ & RV & $=$ rumba wau \\
\hline bou kàto & $\mathrm{Ll}, \mathrm{Lr}$ & RV & $=$ rumba wau \\
\hline 'bubulo & Ko & RV & = kapuwa hambaku \\
\hline ?buka bilara & $\mathrm{Lr}, \mathrm{Tt}$ & RV & $=$ wulu nggàduku I \\
\hline bunga & $\mathrm{Kb}, \mathrm{Ak}$ & OK & Plumeria acuminata \\
\hline 'bungaru & Ak & $\mathrm{O}$ & = wàla 'mbunguru \\
\hline bùngga & $\mathrm{Lr}$ & $\mathrm{D}$ & = kajú I \\
\hline 'buoki & $\mathrm{u}$ & RV & = kondu 'kurangu II \\
\hline cekir & ?Lw & $\mathrm{H}$ & $=$ hakuru \\
\hline cuwa & Lw & $\mathrm{O}$ & $=$ tuwa \\
\hline dagha lára & Ko & RV & $=$ kahindu \\
\hline daisa & Ak & $\mathrm{O}$ & $=$ ndeha \\
\hline dána & $\mathrm{Lr}$ & $\mathrm{D}$ & = nítu \\
\hline danggalaza & $\mathrm{Lr}$ & RV & $=$ ambalä̈ \\
\hline dàru & Ak & $\mathrm{O}$ & = ndàru \\
\hline dàwara & $\mathrm{Mb} ; \mathrm{Ww}$ & RV & = dàwaru \\
\hline dàwaru & $\mathrm{ESb}$ & 4013 & Pinanga coronata \\
\hline 'deisa & $\mathrm{U}$ & 4125 & $?=$ ndeha \\
\hline dìkira & $\mathrm{Ll}, \mathrm{Ww}, \mathrm{Lr}, \mathrm{Tr}$ & RV, D & $=$ kahindu \\
\hline dìngira & $\mathrm{Ww}, \mathrm{Lr}, \mathrm{Tt}$ & RV & = 'wingiru \\
\hline dinjo & Ko & RV & $=$ ede II \\
\hline dinjo dawa & Ko & RV & $=$ injungu \\
\hline dòyo & Ko & RV & = kajú II \\
\hline dungga & Ww, Lr & RV & $=$ 'mulungu \\
\hline ede I & $\mathrm{Kr}$ & RV & $=$ injungu \\
\hline ede II & $\mathrm{Ll}, \mathrm{Lr}$ & RV & Spondias pinnata \\
\hline ede dawa & $\mathrm{Lr}$ & RV & $=$ injungu \\
\hline èlo & $\mathrm{Ll}, \mathrm{Ww}, \mathrm{Lr}$ & OK & $=$ halí \\
\hline ende & Ww & OK & = ede II \\
\hline ende dáwa & Ww & RV & $=$ injungu \\
\hline 'engala & $\mathrm{Ww}, \mathrm{Lr}$ & RV & = 'engalu \\
\hline 'engalu & ?Rn, Kb & RV & $\begin{array}{l}\text { Dioscorea ?sarasinii } \\
\text { (OK: D. pentaphylla) }\end{array}$ \\
\hline engo & Ww & OK & = wíngu \\
\hline 'engolo & Ko & RV & $=$ 'engalu \\
\hline
\end{tabular}




\begin{tabular}{|c|c|c|c|}
\hline engu & Ak & OK & = wíngu \\
\hline gabiru & Ak & $\mathrm{O}$ & $=$ nggamuru \\
\hline gaï & Ak & $\mathrm{O}$ & = nggaï \\
\hline gajung & $\mathrm{Rn}$ & RV & $=$ ngganju \\
\hline ganyo & $\mathrm{u}$ & RV & $=$ ngganju \\
\hline ghàgho & $\mathrm{Lr}$ & RV & $=$ nggai \\
\hline ghasu ghula & $\operatorname{Tr}$ & RV & Melastoma \\
\hline ghazu kamba & $\mathrm{Lr}, \mathrm{Tt}$ & $\mathrm{D}, \mathrm{YD}$ & $=$ karipi \\
\hline ghazu (m)boro & $\mathrm{Lr}$ & 3933 & = kàhi kataru \\
\hline ghasu we'e & $\mathrm{Lr}$ & RV & $=$ wasu we'e \\
\hline ghòlo & $\mathrm{Lr}$ & 4249 & Pisonia \\
\hline gho'o & $\mathrm{Lr}$ & W & $=\mathrm{au}$ \\
\hline ghudi & $\mathrm{Lr}, \mathrm{Tt}$ & RV & $=$ wudi \\
\hline ghulu manu & $\mathrm{Lr}$ & RV & Mallotus philippensis \\
\hline ghùnga & $\mathrm{Lr}$ & RV & = wunga III \\
\hline ghùngo & $\mathrm{Lr}$ & $\mathrm{D}$ & $=$ lawùngu \\
\hline ghùni rara & $\mathrm{Lr}$ & RV & $=$ wulu manu II \\
\hline 'ghunuta & $\mathrm{Lr}$ & RV & $=$ 'wundutu \\
\hline goka & Ak & $\mathrm{O}$ & $=$ nggoka \\
\hline hajewi & $\mathrm{Kb}$ & ?W & tree; yields dye \\
\hline haki & $\mathrm{Kb}$ & OK & tree, on the plains \\
\hline hakúro & Ko & RV & $=$ hakuru \\
\hline hakuru & $\mathrm{Mi}, ? \mathrm{~Kb}, \mathrm{Lw}$ & $\mathrm{O}$ & Kaempferia galanga \\
\hline halai & Lw & $\mathrm{dft}$ & $=$ halí \\
\hline halà(i)yo & Ko & RV & = halí \\
\hline halaya dín & $\mathrm{Kb}$ & OK & vine; roasted seeds are eaten \\
\hline halí & $\mathrm{Mi}, \mathrm{Rn}, \mathrm{Kr}, \mathrm{Kb}$ & RV & Alstonia spectabilis \\
\hline ha'lumuto & Ko & $\mathrm{O}$ & = 'lumutu \\
\hline ha'lurutu I & $\mathrm{Kr}, \mathrm{Kb}$ & 3848 & Achyranthes aspera \\
\hline ha'lurutu II & $\mathrm{Kr}, \mathrm{Kb}$ & RV & = kawúru wàla \\
\hline hamaili & $\mathrm{Kr}$ & 3937 & Grewia \\
\hline ha'mbaingu & $\mathrm{Kb}$ & OK & tree \\
\hline hambaku & ESb & W & Nicotiana tabacum \\
\hline ?hambeu & ES & $\mathrm{H}$ & = hambaku \\
\hline hambuli & $\mathrm{Mi}, \mathrm{Kb}$ & OK & $\begin{array}{l}\text { tree w. spiny bark; wood used } \\
\text { for guitar-case }\end{array}$ \\
\hline ha'milungo & Ko & 4242 & Ardisia \\
\hline hamú & $\mathrm{Kb}$ & $\mathrm{O}$ & water plant, tuberous \\
\hline hamui & $\mathrm{ESb}$ & OK & Neolitsea \\
\hline handa (OK: hànda) & $\mathrm{Kb}$ & $\mathrm{O}, \mathrm{OK}$ & ?Ficus \\
\hline handalo & Ko & RV & $?=$ 'hidiku \\
\hline $\begin{array}{l}\text { handána } \\
\text { hànggi }\end{array}$ & Lr, Ko & $\mathrm{O}$ & $\begin{array}{l}\text { = nítu } \\
\text { see: wàla hànggi }\end{array}$ \\
\hline
\end{tabular}




\begin{tabular}{|c|c|c|c|}
\hline hanjata & $\mathrm{Mi}, \mathrm{Kb}$ & $\mathrm{O}, \mathrm{OK}$ & tree w. poisonous fr. \\
\hline ha'njokaru tàki & $\mathbf{K r}$ & 3947,3977 & Leea angulata \\
\hline hàpangu & $\mathrm{Kb}$ & RV & Caesalpinia sappan \\
\hline hapoku & $\mathrm{Kb}$ & RV & = kapóka \\
\hline harakaya & $\mathrm{Kr}, \mathrm{Kb}$ & RV & Annona muricata \\
\hline harakayi & $\mathrm{Rn}$ & RV & $=$ harakaya \\
\hline harama njara & $\mathrm{Kb}$ & $\mathrm{OK}, \mathrm{D}, \mathrm{H}$ & Ficus pubinervis \\
\hline hàru & $\mathrm{Kb}$ & OK & = ndàru \\
\hline hawi & Lw & $\mathrm{O}$ & $=$ hewi \\
\hline hawúni & $\mathrm{Rn}, \mathrm{Kb}$ & OK & tree w. stinging hairs \\
\hline hayindu & $\mathrm{Rn}$ & $\mathrm{D}$ & $=$ kahindu \\
\hline heduku & Lw & $\mathrm{O}$ & = 'hidiku \\
\hline hei & $\mathbf{K r}$ & 4226 & Pisonia sylvestris \\
\hline hekulu & Lw & $\mathrm{O}$ & = hîkilu \\
\hline henggi & ?Kb & OK & ?= wàla hànggi \\
\hline hewi & $\mathrm{Kb}$ & OK & $\begin{array}{l}\text { tree; } 1 .(10 \times 20 \mathrm{~cm}) \text { eaten; } \\
\text { cuttings for living fences }\end{array}$ \\
\hline híbu manginu & $\mathrm{Kb}$ & $\mathrm{PH}$ & Eragrostis amabilis \\
\hline 'hidiku & $\mathrm{Mi}, \mathrm{Kb}$ & OK & tree w. medicinal $l$. \\
\hline hîkilu & $\mathrm{Kb}$ & OK & Acorus calamus \\
\hline hili & $\mathrm{Mi}, \mathrm{Rn}, \mathrm{Lw}$ & RV & = hili bara \\
\hline hili bara & $\mathrm{Kb}$ & RV & Colocasia esculenta \\
\hline hili jawa & $\mathbf{K r}$ & RV & $=$ hili bara \\
\hline hili làtangu & $\mathrm{Kb}$ & $\mathrm{O}$ & = piuru II \\
\hline hili manggaü & $\mathbf{K r}$ & RV & $=$ manggaii I \\
\hline hili 'mbukutu & $\mathrm{Kb}$ & $\mathrm{O}$ & $\begin{array}{l}\text { Colocasia (variety w. many } \\
\text { small tubers) }\end{array}$ \\
\hline hingiro & WS & $\mathrm{H}$ & = 'wingiru \\
\hline homa & $\mathrm{Mi}, \mathrm{Kb}, \mathrm{Lw}$ & OK & = 'huama \\
\hline horani & Lw & $\mathrm{O}$ & $=$ hureni \\
\hline 'huama & $\mathbf{K r}$ & $\mathrm{dft}$ & Gyrinops 'cumingiana' \\
\hline 'hubuku & $\mathbf{K r}$ & 4332 & Wrightia \\
\hline hugha & Ko & RV & $=$ huwa \\
\hline huli & Ko & $\mathrm{O}$ & $=$ hili bara \\
\hline huli dawa & ?Ko & RV & Colocasia \\
\hline huli káka & Ko & RV & Colocasia \\
\hline hulu katáka & $\mathbf{K r}$ & $\mathrm{dft}$ & Aglaia II \\
\hline huluku & ES & dft & = ai rara \\
\hline hunani & $\mathrm{Mi}, \mathrm{Kb}$ & OK & $=$ kahindu \\
\hline hurani & $\mathrm{Kr}$ & RV & $=$ hureni \\
\hline huranu & $\mathrm{Mb} ; \mathrm{Ko}$ & $\mathrm{O}$ & $=$ hureni \\
\hline hureni & $\mathrm{Rn}, \mathrm{Kb}$ & $\mathrm{dft}, \mathrm{D}, \mathrm{H}$ & Toona sureni \\
\hline huwa & $\mathrm{Kr}, \mathrm{Kb}$ & $\mathrm{D}$ & Gnetum gnemon \\
\hline
\end{tabular}




\begin{tabular}{|c|c|c|c|}
\hline idu & $\mathrm{Lr}$ & D & $=\mathrm{ai} \mathrm{iju}$ \\
\hline iji & $\mathrm{Kb}, \mathrm{Ak}$ & $\mathrm{RV}$ & $=$ ede II \\
\hline 'ilahu & $\mathrm{Kr}, \mathrm{Kb}$ & OK, RV & Saccharum spontanewm \\
\hline 1lyaho & Ko & RV & $=$ tibu \\
\hline inju jawa & $\mathrm{Rn}$ & $\mathrm{RV}$ & $=$ injungu \\
\hline injungu & $\mathrm{Kb}$ & $\mathrm{OK}, \mathrm{RV}$ & Spondias dulcis \\
\hline iri & $\mathrm{Kb}$ & $\mathrm{O}$ & ?Pandanus (without thoms) \\
\hline ?iwes & ?ES & $\mathrm{H}$ & $=$ mbúrungu \\
\hline iwi (I) & $\mathrm{Kb}$ & OK & Calamus \\
\hline ìwi (II) & $\mathrm{Rn}, \mathrm{Kb}$ & RV & Dioscorea hispida \\
\hline iwi manu & $\mathrm{Rn}$ & RV & $?=$ nggai \\
\hline ìwi maüku & $\mathrm{Kb}$ & W & = ìwi II \\
\hline ?iwi wé & $\mathrm{Rn}$ & RV & Calomus \\
\hline jàla alaku & $\mathrm{Mi}, \mathrm{Kb}, \mathrm{Lw}$ & $\mathrm{O}$ & = 'alaku \\
\hline jàla (búti) & $\mathrm{Kb}$ & OK & vine w. edible black fr. \\
\hline jami & ES & & shrub \\
\hline jamu & Lw & $\mathrm{O}$ & $=$ jami \\
\hline jangi & ES & $\mathrm{H}$ & $=$ kanjangi \\
\hline jàriku & $\mathrm{Mi}, \mathrm{Rn}, \mathrm{Kb}$ & RV & $=$ jàriku mayilu \\
\hline jariku 'bokulu & $\mathrm{Kb}$ & $\mathrm{O}$ & $=$ jàriku 'tobungu \\
\hline jàriku langga & $\mathrm{Rn}$ & RV & Citrus \\
\hline jàriku mayilu & $\mathrm{Kr}, \mathrm{Kb}$ & $\mathrm{OK}, \mathrm{RV}$ & Citnus aurantifolia \\
\hline jàriku rútu & $\mathrm{Kb}$ & $\mathrm{O}$ & tree, tall \\
\hline jàriku 'tobungu & $\mathrm{Rn}, \mathrm{Kb}$ & RV & Citrus grandis \\
\hline jàriku wihìku & $\mathrm{Kb}$ & OK & Citrus \\
\hline jaü & $\mathrm{Kb}$ & W & Fungi \\
\hline jimbu & $\mathrm{Kb}$ & ?RV & Ficus ?balica \\
\hline jiriku & Lw & $\mathrm{O}$ & $=$ jàriku \\
\hline jolu & Lw & $\mathrm{O}$ & $=$ wua júli \\
\hline ju'latingu & $\mathrm{Rn}, \mathrm{Kb}$ & $\mathrm{RV}, \mathrm{OK}$ & Dendrocnide \\
\hline júli & $\mathrm{Kb}$ & $\mathrm{O}$ & = wua júli \\
\hline kàba & Ko & $\mathrm{O}$ & $=$ nahu I \\
\hline ?kába I & $\mathrm{L}$ & RV & $=$ kopa lua \\
\hline kaba II & Ak & $\mathrm{O}$ & $=$ kamba I \\
\hline kaba lua (?luwa) & ?Kb & OK, O & $=$ kopa lua \\
\hline kabai & Ak & $\mathrm{O}$ & $=$ kambí \\
\hline kabai tana & Ak & $\mathrm{O}$ & $=$ manila I \\
\hline kabàketa & $\mathrm{L}$ & 4256 & Clematis \\
\hline kabala & $\mathrm{Tt}$ & v & Ficus $(?=$ ka'balaka $)$ \\
\hline ka'balaka & $\mathrm{u}$ & RV & Ficus (?= wàngga) \\
\hline kabáli biedu & Ww & 4138 & Maesa \\
\hline kabaru & $\mathrm{Mi}, \mathrm{Rn}, \mathrm{Kb}, \mathrm{Lw}$ & $3857, \mathrm{D}, \mathrm{H}$ & Hibiscus tiliaceus \\
\hline kabaunu & Lw & $\mathrm{O}$ & $=$ katambini \\
\hline
\end{tabular}




\begin{tabular}{|c|c|c|c|}
\hline kabé tana & $\mathbf{L}$ & $\mathbf{H}$ & $=$ manila I \\
\hline ka'bebaku & $\mathrm{Rn}, \mathrm{Kr}, \mathrm{Kb}$ & 4240 & Homalanthus populneus \\
\hline kabia karabo & $\mathrm{L}$ & RV & $=$ mapu 'mbelaru pingi I \\
\hline ?kabiya karambo & Ko & RV & $=$ mapu 'mbelaru pingi I \\
\hline kabóna I & Ww, Lr, Ko & RV & $=$ karua kapu \\
\hline kabóna II & Ko & RV & $=$ katambini \\
\hline kabota I & $\mathrm{Kb} ; \mathrm{Tr}$ & $\mathrm{O}$ & = ndúma kawini \\
\hline kabota II & Ko & $\mathrm{O}$ & $=$ nahu I \\
\hline kabota koba & Ww & ?OK & Amorphophallus ?campanulatus \\
\hline ka'bounyu & $\mathbf{L}$ & RV & $=$ katambini \\
\hline kabuaka & Ww & 4012 & Areca \\
\hline kabùkela & $\mathbf{L}$ & RV & = kalambàki \\
\hline kabùkela manu & $\mathbf{L}$ & RV & $=$ kandinu bara \\
\hline kabúni & Ko & RV & $=$ 'wingiru \\
\hline kabúnu & $\mathrm{Lr}$ & RV & $=$ katambini \\
\hline ka'buruko & Ko & RV & $=$ makiku \\
\hline kabusu & $\mathrm{L}$ & RV & = lita rùmba \\
\hline kabuzu & $\mathrm{Lr}$ & RV & = lita rùmba \\
\hline kaci katáro & Ww & ?RV & = kawìta kaba \\
\hline kadábu & $\mathrm{L}$ & RV & Pagiantha sphaerocarpa \\
\hline kadàmo ndima & $\mathrm{Ll}, \mathrm{Ww}, \mathrm{Lr}$ & RV & Ricinus communis \\
\hline kadàmo (pi'a) & $\mathrm{Ll}, \mathrm{Ww}, \mathrm{Lr}$ & RV & = padàmu humba \\
\hline kadángi & $\mathrm{Ww}, \mathrm{Lr}$ & $\mathrm{D}$ & $=$ kahala wau \\
\hline kadapu & Ak & $\mathrm{O}$ & $=$ tarúbu \\
\hline kadara & Ak & $\mathrm{O}$ & = kandara II \\
\hline kadauki & $\mathrm{Mi}, \mathrm{Kb}, \mathrm{Ak}$ & OK & Ficus \\
\hline kadauku & Lw & $\mathrm{O}$ & $=$ kadauki \\
\hline kaḍebu & Ak & $\mathrm{O}$ & $=$ kadembu \\
\hline kadembi & $\mathrm{Rn}$ & RV & $?=$ kadembu \\
\hline kadembu & $\mathrm{Kb}$ & $3869, \mathrm{OK}$ & Citrullus lanatus \\
\hline kadimbu & Mi & $\mathrm{O}$ & $=\mathrm{kadembu}$ \\
\hline ka'dinggiru & $\mathrm{Mi}, \mathrm{Kb}, \mathrm{Lw}$ & OK & Cucumis sativus \\
\hline ka'dinggiru kalaüi & $\mathrm{Kb}$ & 4006 & Cucurbitaceae \\
\hline kadíra & $\mathbf{L}$ & RV & $=$ 'paita \\
\hline kadito wàla & $\mathrm{Lr}$ & RV & $=$ kadito wòla \\
\hline kadito wòla & Ww & 3991 & Fatoua pilosa \\
\hline kadiu & Lw & $\mathrm{O}$ & $?=$ kajú II \\
\hline kadóke & $\mathrm{Ww}, \mathrm{Tt}$ & V, YD & $?=$ kadauki \\
\hline kadóke tana & $\mathrm{Lr}$ & 4246 & Mammea timorensis \\
\hline kadoki & Ko & $\mathrm{O}$ & $=$ kadauki \\
\hline kaduki & $\mathrm{Mb}$ & $\mathrm{O}$ & $=$ kadauki \\
\hline kadúru & $\mathrm{Kb}$ & $\mathrm{dft}, \mathrm{Ok}$ & Palaquium obovatum \\
\hline kadúru landu kaka & ES & dft & Cryptocarya \\
\hline
\end{tabular}




\begin{tabular}{|c|c|c|c|}
\hline kaghailo tana & $\mathrm{Lr}$ & RV & $=$ mangàta \\
\hline kaghóna & $\mathrm{Lr}$ & RV & = kawona \\
\hline kaha & Ko & RV & = kamàru \\
\hline kahàbaru & $\mathrm{Kb}$ & $\mathrm{D}, \mathrm{H}$ & Murraya paniculata \\
\hline kahaiku & $\mathrm{Mi}, \mathrm{Kb}$ & OK & $=$ ka'hauki \\
\hline kahala managhe & Ko & 3918 & Dendrophthoe \\
\hline kahala wau & $\mathrm{Kb}$ & OK & Hyptis suaveolens \\
\hline kahambi & $\mathrm{Mi}, \mathrm{Rn}, \mathrm{Kr}$ & $\mathrm{D}, \mathrm{H}$ & $=\mathrm{kahembi}$ \\
\hline kahambu & Lw & $\mathrm{O}$ & $=$ kahembi \\
\hline kahanga kiri & $\mathrm{Kb}$ & PH & Dichanthiwm caricoswm \\
\hline ka'hauki & $\mathrm{Rn}$ & RV & Fungi \\
\hline kahembi & $\mathrm{Kb}, \mathrm{Tm}$ & OK & Schleichera oleosa \\
\hline kàhi I & Mi, ?Kb; Ko & RV & Canariwm asperwm \\
\hline kàhi II & $\mathrm{Rn}, \mathrm{Ml}, \mathrm{Kr}, \mathrm{Kb}$ & RV & $=$ kàhi jawa \\
\hline ?kàhi jawa & $\mathrm{Kb}$ & RV & Lannea coromandelica \\
\hline kàhi kataru & $\mathrm{Kb}$ & 3933,4230 & Sapindus saponaria \\
\hline kahilu kamambi & $\mathrm{Kb}$ & RV & = kahilu kamemi \\
\hline kahilu kamemi & $\mathrm{Kr}$ & 3903,4147 & Pterocaulon sphacelatum \\
\hline kahilu wei & $\mathrm{Kb}$ & OK & Plantago major \\
\hline kahilu wúya & Rn & 4160 & Monochoria vaginalis \\
\hline kahindu & $\mathrm{Kb}$ & D & Sida rhombifolia \\
\hline kahomba & $\mathrm{Kb}$ & RV & Cyperaceae \\
\hline kahomba ('bokulu) & $\mathrm{Kb}$ & OK & Cyperus monocephalus \\
\hline kahomba kudu & $\mathrm{Kb}$ & OK & Cyperus rotundus \\
\hline kajailu & Ak & $\mathrm{O}$ & $=$ kanjilu \\
\hline kajawa & $\mathrm{Kb}, \mathrm{Lw}, \mathrm{Ak}$ & OK & Carica papaya \\
\hline kajiu & $\mathrm{Kb}$ & OK & = kajú I \\
\hline kajú I & ESb & $3861, \mathrm{D}$ & Casuarina cf. equisetifolia \\
\hline kajú II & Lw & dft, H, D & Podocarpus imbricatus \\
\hline kajú 'omangu & ES & $\mathrm{dft}$ & = kajú II \\
\hline kàka & $\mathrm{Kb}$ & OK & marine plant \\
\hline kakaha I & $\mathrm{Mi}, \mathrm{Kb}, \mathrm{Lw}$ & OK & tree, in forest; good timber \\
\hline kakaha II & $\mathrm{Kr}$ & 3851 & $\begin{array}{l}\text { Dicliptera (OK, O: } \\
\text { Graptophyllum hortense) }\end{array}$ \\
\hline kakaha III & $\mathrm{Rn}$ & 4148 & Peristrophe \\
\hline kakaha baina & $\mathrm{Kb}$ & $?$ & $?$ \\
\hline kakaha jawa & $\mathrm{Kb}$ & 3897 & Acanthaceae \\
\hline kakaha míni & $\mathrm{Kb}$ & RV & Barleria prionitis \\
\hline kakembalu & $\mathrm{Mi}, \mathrm{Kb}, \mathrm{Lw}$ & $\mathrm{O}$ & Fungi \\
\hline kakomba & $\mathrm{Kr}$ & dft & Apodytes \\
\hline kakuta & $\mathrm{Kb}$ & OK & marine vegetable \\
\hline kalada nggangga & $\mathrm{Rn}, \mathrm{Kr}, \mathrm{Kb}$ & 2853 & Abrus (precatorius) \\
\hline kalàhangu & $\mathrm{Kb}$ & $\mathrm{O}$ & vine \\
\hline
\end{tabular}




\begin{tabular}{|c|c|c|c|}
\hline kalaho & Ko & RV & = kapu ndala II \\
\hline kalaho ?uti & Ko & ?RV & = karòbo pùti \\
\hline kàlahu & $\mathrm{Kb}, \mathrm{Tm}, \mathrm{Lw}$ & OK & = karobu hàmu \\
\hline kalai & $\mathrm{Ak}, \mathrm{Mb} ; \mathrm{Ww}$ & OK & = kawaka \\
\hline kalailu I & $\mathrm{Tm}$ & $\mathbf{W}$ & = kalú \\
\hline kalailu II & $\mathrm{Kr}$ & RV & = kajawa \\
\hline kalaki kambe & $\mathrm{Lr}$ & 3973 & Glochidion angulaturn \\
\hline kalàla & ES & $\mathrm{RV}, \mathrm{D}$ & Opuntia elatior \\
\hline kalàla humba & $\mathrm{Kb}$ & 3914 & Pereskia \\
\hline kalàla jawa & $\mathrm{Kb}$ & RV & Euphorbia cf. antiquorum \\
\hline ka'lalapa & $\mathrm{Lr}$ & RV, D & $=$ kalàla \\
\hline kalalapo & Ko & RV & $=$ kalàla \\
\hline kalalingangu & $\mathrm{Kb}$ & $\mathrm{O}$ & Vitex trifolia \\
\hline kalamba & $\mathrm{Rn}, \mathrm{Kr}, \mathrm{Kb}$ & RV & Ficus?depressa \\
\hline kalambàki & $\mathbf{R}$ & RV & Ficus ?septica \\
\hline kalangga I & $\mathrm{Kr}, \mathrm{Kb}$ & D, 3895 & Ziziphus nwmmularia \\
\hline kalangga II & Ww, Lr & RV & $=$ tariwangu \\
\hline kalangga dawa & $\mathrm{Lr}$ & RV & $=$ harakaya \\
\hline kalangga jawa & $\mathrm{Rn}, \mathrm{Kr}$ & RV & $=$ tariwangu \\
\hline ?kalangga lúlu & Ko & RV & $=$ tariwangu \\
\hline kalangga ndima & $\mathrm{Lr}$ & RV & $=$ tariwangu \\
\hline kalara (kuta) & $\mathrm{Rn}, \mathrm{Kr}, \mathrm{Kb}$ & 4001 & Piper \\
\hline ?kalawau & ES & D & $=$ kahala wau \\
\hline kalayo & Ko & $\mathrm{O}$ & = kawaka \\
\hline ka'lelapa & $\mathrm{Ll}, \mathrm{Ww}$ & RV & = kalàla \\
\hline kalelu & Mi & OK & = kajawa \\
\hline kali & $\mathrm{Kn}$ & W & $=$ kalú \\
\hline kàli kúwa & $\mathrm{L}$ & 4126 & Ipomoea sepiaria \\
\hline kalia & Ww & 3966 & Fungi, Polyporus \\
\hline kalia rara & $\mathrm{Lr}$ & 3968 & Fungi, Pycnoporus \\
\hline kalia taiko karambo & $\mathrm{Ww}, \mathrm{Lr}$ & RV & = kapuda \\
\hline kalibu wä & $\mathrm{Kn}, \mathrm{Mi}$ & W & = wàla 'mbunguru \\
\hline kalighu & $\mathrm{Lr}$ & 4213 & Blwmea lacera \\
\hline kalihi & $\mathrm{Rn}, \mathrm{Kb}$ & $\mathrm{dft}, 4225$ & Ficus?pilosa \\
\hline kalijawa & $\mathrm{Kn}, \mathrm{Rn}$ & OK & $=$ kajawa \\
\hline kaliku & $\mathbf{R n}$ & RV & Commelina \\
\hline kalimbàki & $\mathrm{Kb}$ & $\mathrm{O}$ & $=$ ? kalambàki \\
\hline kalindu & Ko & RV & $?$ \\
\hline kalìpi & $\mathrm{Kr}$ & RV & $?$ \\
\hline kalisi & $\mathrm{Ak}, \mathrm{Mb}$ & $\mathrm{O}$ & $=$ kalihi \\
\hline kaliso & Ww & $\mathrm{RV}$ & = kalighu \\
\hline kalita búti & $\mathrm{Lr}$ & 3930 & Alpinia \\
\hline kalìti & Mi & $\mathrm{dft}$ & $=$ kalihi \\
\hline
\end{tabular}




\begin{tabular}{|c|c|c|c|}
\hline kalitu ahu & $\mathrm{Kb}$ & 3836 & Gyrocarpus \\
\hline ka'lobungu & $\mathrm{Kb}$ & OK & $=$ 'lobungu I \\
\hline kalogho & Lr, Ko & W & $=$ kalú \\
\hline kalogho dawa & Lr, Ko & RV & $=$ kajawa \\
\hline kalogho koki & Ko & $\mathrm{O}$ & = kalú yúmbulu \\
\hline kalotu & ES & $\mathrm{D}$ & $=$ kanoru \\
\hline kalowi kuki & $\mathrm{Mb}$ & $\mathrm{O}$ & = kalú yúmbulu \\
\hline kalowo & Ww & OK & = kalú \\
\hline kalowo dawa & Ww & OK & = kajawa \\
\hline kalowo kedu & Ww & $\mathrm{O}$ & = kalú yúmbulu \\
\hline kalowu & $\mathrm{Lw}, \mathrm{Ak}, \mathrm{Mb}$ & OK & = kalú \\
\hline kalowu jawa & $\mathrm{Mb}$ & $\mathrm{O}$ & $=$ kajawa \\
\hline kalowu sobulu & Ak & $\mathrm{O}$ & = kalú yúmbulu \\
\hline kalú & $\mathrm{Mi}, \mathrm{Rn}, \mathrm{Kr}, \mathrm{Kb}$ & OK & Musa paradisiaca \\
\hline kalú ai & $\mathrm{Rn}$ & RV & Sterculia ?oblongata \\
\hline kalú ata & Ww & RV & Arytera \\
\hline kalú húmbulu & $\mathbf{K b}$ & RV & kalú yúmbulu \\
\hline kalú jawa & $\mathrm{Kb}$ & $\mathrm{W}, \mathrm{O}$ & $=$ kajawa \\
\hline kalú yúmbulu & $\mathrm{Mi}, \mathrm{Kb}, \mathrm{Lw}$ & $\mathrm{O}$ & Ensete glaucum \\
\hline ka'lumbangu & $\mathrm{Rn}, \mathrm{Kb}$ & 3912 & Sterculia foetida \\
\hline kama & $\mathrm{Rn}$ & RV & = kamànggihu \\
\hline ka'madala & Ww & RV & Spilanthes iabadicensis \\
\hline ka'madala ndara & Ww & 4056 & Spilanthes grandiflora \\
\hline kamàge & $\mathrm{L}$ & RV & = kamànggihu \\
\hline kamàgisu & Ak & $\mathrm{O}$ & = kamànggihu \\
\hline ka'maili & $\mathrm{ESb}$ & OK & ?Setaria italica var. \\
\hline ka'maimbungu & $\mathrm{Rn}, ? \mathrm{~Kb}, \mathrm{Lw}$ & OK, RV & Colocasia \\
\hline kamàla kamba & $\mathrm{Rn}$ & RV & $=$ ghulu manu \\
\hline kamàla kóndu & $\mathrm{Rn}$ & RV & Boraginaceae \\
\hline kamàla lànga & $\mathrm{Rn}$ & RV & ?Corchorus \\
\hline kamàla lànga maràda & $\mathrm{Kr}, \mathrm{Kb}$ & 3876 & Corchorus \\
\hline kamàla litapu & $\mathrm{Kb}$ & $\mathrm{PH}$ & Paspalum scrobiculatum \\
\hline kamàla manila & $\mathrm{Kb}$ & RV & herb, resembles the peanut \\
\hline kamàla paú & $\mathrm{Kr}$ & $\mathrm{dft}$ & Buchanania arborescens \\
\hline kamàla tuwa & $\mathrm{Kb}$ & OK & tree \\
\hline kamàla uhu & $\mathrm{Kb}$ & $\mathrm{O}$ & Poaceae \\
\hline kamàla uhu kanu & $\mathrm{Kb}$ & PH & Setaria verticillata \\
\hline kamàla 'wataru & $\mathrm{Rn}, \mathrm{Kb}$ & RV & Sorghum ?propinquum \\
\hline kamàla wora & $\mathrm{Kr}$ & RV & shrub; cp. wora \\
\hline kamàngge & Ww, Lr & $\mathrm{D}, \mathrm{RV}$ & = kamànggihu \\
\hline kamàngge rewa I & Ww, Lr & RV & = wua júli \\
\hline kamàngge rewa II & Ww, Lr & RV & $=$ mapu \\
\hline kamànggihu & Mi, Kb, Lw & $\mathrm{RV}, \mathrm{O}$ & Coix lacryma-jobi var. ma-yuen \\
\hline
\end{tabular}




\begin{tabular}{|c|c|c|c|}
\hline kamanggisa & Ww & $\mathrm{O}$ & = kamànggihu \\
\hline kamàra I & $\mathrm{Tt}$ & V, YD & tree, large, in forest \\
\hline kamàra II & $\mathrm{L}$ & RV & Strobilanthes \\
\hline kamàra wèzi I & $\mathrm{Lr}$ & 3935 & Chompereia manillana \\
\hline kamàra wèzi II & $\mathrm{Lr}$ & 3997 & Cansjera leptostachya \\
\hline kamàru & ES & $\mathrm{D}$ & Tamarindus indica \\
\hline kamàru kawaü & $\mathrm{Kb}$ & $\mathrm{O}$ & vine w. edible sour 1. \\
\hline kamàru làngga & $\mathrm{Kr}$ & RV & $=$ kahala wau \\
\hline kamàru tehiku & $\mathrm{Kb}$ & OK & marine plant, edible \\
\hline (pingi) kamba I & Sumba & OK & Gossypium \\
\hline kamba II & ES & $\mathrm{dft}$ & Alangium chinense \\
\hline kamba hika & $\mathrm{Rn}, \mathrm{Kr}, \mathrm{Kb}$ & OK & = kopa lua \\
\hline kamba kadu & $\mathrm{Kb}$ & 3885 & Marsdenia villosa \\
\hline kamba watu & $\mathrm{Mb}$ & ? & $=$ kopa lua \\
\hline kambala & $\mathrm{Mi}, \mathrm{Kb}, \mathrm{Lw}$; Ww, Ko & $\mathrm{D}$ & Broussonetia papyrifera \\
\hline kambálaka I & $\mathrm{Ww}, \mathrm{Lr}$ & OK & = ka'balaka \\
\hline kambálaka II & $\mathrm{Mb}$ & $\mathrm{O}$ & $=$ kambala \\
\hline kambauni & $\mathrm{Kb}, \mathrm{Lw}$ & OK & $=$ katambini \\
\hline kàmbe & Ww & $\mathrm{O}$ & = kambí \\
\hline kàmbe lólo & WSb & RV & ?Vigna unguiculata \\
\hline kàmbe móro wiasa & $\mathrm{L}$ & RV & = kambí múru \\
\hline kàmbe móro wìaza & $\mathrm{Lr}$ & RV & = kambí múru \\
\hline kàmbe nónggo & $\mathrm{Lr}$ & 3955 & Phaseolus \\
\hline kàmbe ra'a & Ww & 4253 & Cissus \\
\hline kàmbe rara & $\mathrm{Tt}$ & YD & $=$ kàmbe ra'a \\
\hline kàmbe tana I & WS & RV & $=$ manila I \\
\hline kàmbe tana II & $\mathrm{Lr}$ & 3916 & Alysicarpus \\
\hline kambí & $\mathrm{Kb}$ & OK & Phaseolus \\
\hline kambí hàmu & $\mathrm{Kb}$ & OK & = kambí múru \\
\hline kambí haü & $\mathrm{Kb}$ & $\mathrm{RV}, \mathrm{OK}$ & = kambí múru \\
\hline kambí maràda & $\mathrm{Kb}$ & 3872 & Papilionaceae \\
\hline kambí múru & $\mathrm{Rn}$ & RV & Phaseolus aureus \\
\hline kambí tana & Ko & W & $=$ manila I \\
\hline kambila & $\operatorname{Tr}$ & RV & $=$ kapumbu tai manu \\
\hline ka'mborungu & $\mathbf{N p}$ & $\mathrm{H}, \mathrm{W}$ & = 'wataru \\
\hline kàmbu & $\mathrm{Mi}, \mathrm{Kr}, \mathrm{Lw}, \mathrm{Mb}$ & RV & $=\mathrm{kombu}$ \\
\hline kambu & ES & $\mathrm{dft}$ & = kamba II \\
\hline kámbu lawora I & $\mathrm{Kr}$ & 3967 & Tetrastigma \\
\hline kámbu lawora II & $\mathrm{Kr}$ & RV & $?=$ púpu \\
\hline kámbu la'wuara & $\mathrm{Kr}$ & $3866, \mathrm{H}$ & = wora \\
\hline kambua & $\mathrm{Mb}$ & $\mathrm{O}$ & = kambí \\
\hline kambùkela & Ww & 4037 & Glochidion glabrum \\
\hline kambùkela mene & Ww & 4036 & Ficus \\
\hline
\end{tabular}




\begin{tabular}{|c|c|c|c|}
\hline kambùkelo & Ko & RV & $?=$ kalambaki \\
\hline ka'mburungu & $\mathrm{Kb}$ & OK & = 'wataru \\
\hline kambúta & WS & RV & $=$ wanggakúli \\
\hline kambúta tagho & $\mathrm{Lr}$ & 3994 & Lepidagathis \\
\hline ka'megalu & Ak & $\mathrm{O}$ & $=$ ka'menggalu \\
\hline kameli & Ww, Lr, Ko & OK & = ka'maili \\
\hline ka'menggala & $\mathrm{Mb}$ & $\mathrm{O}$ & $=$ ka'menggalu \\
\hline ka'menggalu & $\mathrm{Mi}, \mathrm{Kb}, \mathrm{Lw}$ & $\mathrm{RV}, \mathrm{OK}$ & Dioscorea (OK: bulbifera) \\
\hline ka'menggeho & Ko & RV & = kamànggihu \\
\hline kamenggo & Ko & $\mathrm{O}$ & $=$ ka'menggalu \\
\hline kameru & $\mathrm{Kb}$ & OK & = kamàru \\
\hline kamia marèda & Ww & RV & Osbeckia ?dolichophylla \\
\hline kamímbingu & Mi & OK & = ka'maimbungu \\
\hline kamímbungu & $\mathrm{Mi}, \mathrm{Kb}$ & OK & = ka'maimbungu \\
\hline kamíti I & $\mathrm{Kb}$ & 3890 & Deeringia \\
\hline kamíti II & $\mathrm{Kr}$ & 3925 & Syzygium \\
\hline kanàbu & Ko & $\mathrm{O}$ & $=$ wora \\
\hline kanaka & $\mathrm{Kb}$ & $\mathrm{O}$ & tree; wood used for torches \\
\hline ka'nanggaru & $\mathrm{Mi}, \mathrm{Kr}, \mathrm{Kb}$ & RV & Dillenia ?pentagyna \\
\hline kanawa & $? \mathrm{~Kb}$ & dft & Pterocarpus indicus \\
\hline kandali & $\mathrm{Kb}$ & PH & Cymbopogon nardus \\
\hline kandalu & Lw & $\mathrm{O}$ & $=$ kandali \\
\hline kandàlu & Ww & RV & Polygonum chinense \\
\hline ka'ndangara & $\mathrm{Ww}, \mathrm{Lr}$ & RV & $=$ kanjangi \\
\hline kandangaro & Ko & $\mathrm{O}$ & $=$ kanjangi \\
\hline kandángi & $\mathrm{Ww}, \mathrm{Lr}$ & RV & $=$ kahala wau \\
\hline kandángi podu & $\mathrm{Ww}, \mathrm{Lr}$ & 3954 & Anisomeles indica \\
\hline kandangu I & $\mathrm{Kb}$ & RV & $=$ kandangu buku \\
\hline kandangu II & $\mathrm{Mi}, \mathrm{Rn}, \mathrm{Kr}, \mathrm{Kb}$ & RV & = kandangu hàmu \\
\hline kandangu buku & $\mathrm{Kb}$ & RV & Cymbopogon citratus \\
\hline kandangu hàmu & $\mathrm{Kb}$ & RV & Ocimum basilicum \\
\hline kandangu haü & $\mathrm{Rn}, \mathrm{Kb}$ & RV & herb; used for seasoning \\
\hline kandànu I & $\mathrm{Mi}, \mathrm{Kr}$ & OK & = kandinu mítingu \\
\hline kandànu II & Mi & RV & = kandinu II \\
\hline kandara I & $\mathrm{Kb}$ & $\mathrm{O}$ & $\begin{array}{l}\text { tree, thomy; heartwood used for } \\
\text { red dye }\end{array}$ \\
\hline kandara II & $\mathrm{Kb}$ & D & Acacia tomentosa \\
\hline kandaü lúku & $\mathrm{Lr}$ & 3919 & = kambùkela \\
\hline ka'ndaüngu & $\mathrm{Kb}$ & 3904 & Chloris barbata \\
\hline ka'ndaüngu bàkulu & Ak & $\mathrm{PH}$ & Digitaria adscendens \\
\hline ka'ndaüngu lúlu jungga I & $\mathrm{Kb}$ & PH & Cynodon dactylon \\
\hline ?ka'ndaüngu lúlu jungga II & $\mathrm{Kb}$ & $\mathrm{H}$ & ?Brachiaria reptans \\
\hline ka'ndaüngu mbata kambàku & $\mathrm{Kb}$ & PH & Dactyloctenium aegyptium \\
\hline
\end{tabular}




\begin{tabular}{|c|c|c|c|}
\hline kandaüngu witu & $?$ & $\mathrm{H}$ & $=$ kandali \\
\hline kandelu & $\mathrm{Mb} ; \mathrm{Ll}, \mathrm{Ww}, \mathrm{Lr}$ & $\mathrm{D}, \mathrm{OK}$ & $=$ kanjilu \\
\hline kandinu I & $\mathrm{Kb}, \mathrm{Lw}$ & OK & $=$ kandinu mítingu \\
\hline kandinu II & $\mathrm{Kb}$ & 4229 & Macaranga \\
\hline kandinu bara & Rn & RV & Macaranga ?tanarius \\
\hline kandinu kiku ahu & Rn & RV & Acalypha caturus \\
\hline kandinu mítingu & Rn & RV & Melochia cf. umbellata \\
\hline kandinu rara & $\mathrm{Kb}$ & $\mathrm{O}$ & tree, coastal \\
\hline kandora & $\mathrm{ESb} ; \mathrm{Ww}$ & OK & Calophyllum inophyllum \\
\hline kandoro & Ko & $\mathrm{O}$ & = 'yawilu \\
\hline ka'ndoruku & $\mathrm{Kr}$ & $\mathrm{dft}$ & = kadúru \\
\hline ka'ndulangu & $\mathrm{Kb}$ & OK & Cassia alata (OK) \\
\hline kandúru & $\mathrm{Mi}, \mathrm{Kr}, \mathrm{Kb}$ & RV & Solanum melongena \\
\hline kandúru ahu & $\mathrm{Rn}$ & RV & Solanum \\
\hline kandúru anggalai & $\mathrm{Kb}$ & $\mathrm{O}$ & = ambalaï \\
\hline kandúru 'paita & $\mathrm{Kb}$ & OK & Solanum \\
\hline kandúruko & Ko & 3953 & Desmodium \\
\hline ka'ngarika & $\mathrm{Kr}, \mathrm{Lr}$ & 3996 & Aneilema \\
\hline kangàta & $\mathrm{Mi}, \mathrm{Kb}, \mathrm{Lw}$ & $\mathrm{O}$ & $=$ mangàta \\
\hline ka'ngereko & Ko & RV & $=$ ka'ngarika \\
\hline kàngga riti & Ko & RV & = ríti kàngga \\
\hline kanggaruka & Ww & 4064 & Blwmea riparia f. angustifolia \\
\hline kangoka & $\mathrm{Kb}$ & OK & Algae (OK: ?Euchaema sp.) \\
\hline ka'ngorita & $\mathrm{Mb} ; \mathrm{Lr}$ & RV & $=$ ha'lurutu I \\
\hline kangórutu & Lw & $\mathrm{O}$ & = kangúrutu \\
\hline kangúrita & Ww & RV & $=$ ha'lurutu I \\
\hline kangúruku & $\mathrm{Ll}, \mathrm{Ww}$ & RV & = ka'ngarika \\
\hline kangúrutu I & $\mathrm{Kb}$ & PH & = mapu kangúrutu $\mathrm{I} / \mathrm{II}$ \\
\hline kangúrutu II & $\mathrm{Mi}, \mathrm{Kb}$ & $\mathrm{O}$ & ?Algae, ?Musci \\
\hline kani & Mi & OK & = uhu kanu \\
\hline kanigu & Ak & RV & $=$ kaninggu \\
\hline kanìki & $\mathrm{Ll}, \mathrm{Ww}, \mathrm{Lr}$ & RV & $=$ kawilu \\
\hline ka'niluka I & $\mathrm{L}$ & RV & Embelia \\
\hline ka'niluka II & Ww & RV & Garcinia dulcis \\
\hline kaninggo & Ww, Lr & $\mathrm{D}$ & $=$ kaninggu \\
\hline kaninggu & $\mathrm{ESb}$ & $\mathrm{D}$ & Cinnamomum bumanni \\
\hline kaninggyo & Ko & RV & $=$ kaninggu \\
\hline ka'njailu & Lw & $\mathrm{O}$ & $=$ kanjilu \\
\hline kanjangi & $\mathrm{Mi}, \mathrm{Kb}, \mathrm{Mb}$ & RV & Kleinhovia hospita \\
\hline kanjilu & $\mathrm{Mi}, \mathrm{Rn}, \mathrm{Kb}$ & D & Ficus variegata \\
\hline kanoru & ES & OK & Arenga pinnata \\
\hline kanu & $\mathrm{Kb}$ & RV & $=$ uhu kanu \\
\hline kanùbu & Ww, Lr & RV & $=$ wora \\
\hline
\end{tabular}




\begin{tabular}{|c|c|c|c|}
\hline kanùnu & $\mathrm{Kr}, \mathrm{Kb}$ & dft & Drypetes \\
\hline kapa & $\mathrm{Mi}, \mathrm{Kb}$ & $\mathrm{O}$ & plant, coastal (OK: sea animal) \\
\hline kapàdi & Ko & RV & $=$ ngganju \\
\hline kapàdu & Ko & RV & $=$ 'paita \\
\hline ka'paita & Ww, Ko & 4015 & Amomum \\
\hline kapaka & Mi, Lw; Ww, Lr, Ko & $\mathrm{D}$ & $=\mathrm{ka}$ 'lumbangu \\
\hline 'kapala kura & $\mathrm{L}$ & 4133 & Solanum \\
\hline 'kapala lera & $\mathrm{Lr}$ & RV & = kondu 'kurangu II \\
\hline kapalia & $\mathrm{Rn}$ & 4164 & Ixora \\
\hline 'kapalo & Ko & RV & = kondu 'kurangu II \\
\hline ka'panggalu & $\mathrm{Kb}$ & OK & shrub \\
\hline kapanggaru & $\mathrm{Kb}$ & $\mathrm{O}$ & plant w. curling 1. \\
\hline kapapa & $\mathrm{Mb} ; \mathrm{Ll}, \mathrm{Ww}, \mathrm{Ko}$ & RV & = ka'papangu II \\
\hline kapapa omangu & $\mathrm{Kr}$ & 3952 & Phaseolus \\
\hline kapara rí I & $\mathrm{Kb}$ & $(3838)$ & Phaseolus lunatus var. \\
\hline kapapa rí II & $\mathrm{Kb}$ & OK & ?Phaseolus vulgaris var. \\
\hline ka'papangu I & $\mathrm{Kb}$ & RV & Canavalia \\
\hline ka'papangu II & $\mathrm{ESb}$ & RV & Phaseolus lunatus var. \\
\hline ka'papangu uhu & $\mathrm{Kb}$ & $(3844)$ & Phaseolus lunatus var. \\
\hline kapàra & $\mathrm{Rn}$ & RV & = kapàraku \\
\hline kapàra luku & $\mathrm{Kr}$ & 4113,4090 & Debregeasia \\
\hline kapàraka & $\mathrm{Lr}$ & RV & = kapàraku \\
\hline kapàraku & $\mathrm{ESb}$ & ;,3924,3999 & Trema ?orientalis \\
\hline kapári 'teiyo & Ko & RV & = kapìra 'taiko \\
\hline kapáta I & $\mathrm{Ll}, \mathrm{Ww}, \mathrm{Lr}$ & RV & = karùngu \\
\hline kapáta II & $\mathrm{L}$ & 4123 & Cosmos caudatus \\
\hline kapehu & $\mathrm{Mi}, \mathrm{Kb}, \mathrm{Lw}$ & OK & tree w. hard heartwood \\
\hline kapeka & Ww & 4040 & Begonia isoptera \\
\hline ka'pelata & $\mathrm{Lr}$ & RV & = kapi'elata \\
\hline ka'pelata ndende & $\mathrm{Lr}$ & 3917 & Desmodium \\
\hline ka'pelato & Ko & 3926 & Moghania \\
\hline kaperi 'taiko mane & $\mathrm{L}$ & RV & Blumea \\
\hline kapia I & $\mathrm{Lr}$ & 3920 & Bridelia ovata \\
\hline kapia II & $\mathrm{L}$ & 4257 & ?Glochidion \\
\hline ka'pidihu & $\mathrm{Kb}$ & RV & Spinifex littoreus \\
\hline kapi'elata & $\mathrm{L}$ & RV & ?Desmodium \\
\hline ka'pilitu & $\mathrm{Kb}$ & $\mathrm{OK}$ & = ka'pulutu \\
\hline ka'pilutu & Lw & $\mathrm{O}$ & = ka'pulutu \\
\hline kapioho & Ko & $\mathrm{O}$ & = kapehu \\
\hline kapìra 'taiko & $\mathrm{Ll}, \mathrm{Tt}$ & RV & Blumea balsamifera \\
\hline kapìri 'taiko & Ww & RV & = kapìra 'taiko \\
\hline ka'pirihu & $\mathrm{Kb}$ & OK & ?= ka'pohaku mini II \\
\hline ka'pohaku I & $\mathrm{Kb}$ & $\mathrm{D}, \mathrm{RV}$ & Urena lobata \\
\hline
\end{tabular}




\begin{tabular}{|c|c|c|c|}
\hline ka'pohaku II & $\mathrm{Kb}$ & PH, 3862 & Cenchrus brownii \\
\hline ka'pohaku bai & $\mathrm{Kr}$ & RV & ?= ka'pohaku I \\
\hline ka'pohaku míni I & $\mathrm{Kr}$ & 3858 & Dicliptera \\
\hline ka'pohaku míni II & $\mathrm{Rn}, \mathrm{Kr}, \mathrm{Kb}$ & RV & Triumfetta ?rhomboidea \\
\hline kapóka & Ww, Lr & 3896 & Physalis miciantha \\
\hline kapoku & $\mathrm{Kb}$ & RV & = kapóka \\
\hline kapoleho & Ww, Lr, Ko & RV & = kapúli or púlihu \\
\hline kapolisa & $\mathrm{Mb}$ & $\mathrm{O}$ & = púlihu \\
\hline kapolusu & Ak & $\mathrm{O}$ & = púlihu \\
\hline kapongge & Ww & $\mathrm{O}$ & $=$ tambuki \\
\hline ka'popoka & $\mathrm{Ll}, \mathrm{Ww}, \mathrm{Tt}$ & $4122, \mathrm{PH}$ & = kapúmbu tai manu \\
\hline kapopoko & Ko & RV & $=$ 'mulungu \\
\hline kapora & $\mathrm{Kb}$ & $\mathrm{O}, \mathrm{OK}$ & vine; fl. and fr. are eaten \\
\hline ka'poraka & Ww & $\mathrm{O}$ & = kapàraku \\
\hline kaporatu & Ak & $\mathrm{O}$ & = ka'pohaku I \\
\hline ka'poreta & Ko & RV & = ka'pohaku I \\
\hline kaporitu & $\mathrm{Mb}$ & $\mathrm{O}$ & = ka'pohaku I \\
\hline ka'porota I & Ww, Lr & RV & = ka'pohaku I \\
\hline ka'porota II & $\mathrm{Lr}$ & $\mathrm{D}$ & = ka'pohaku míni II \\
\hline kaporoto & Ww & $\mathrm{O}$ & = ka'pohaku I \\
\hline kapu ndala I & Ko & 4264 & Gesneriaceae \\
\hline kapu ndala II & $\mathrm{ESb}$ & $\mathrm{RV}$ & Trichosanthes ?villosa \\
\hline kapúbungu & Ak & $\mathrm{O}$ & = ka'pumbungu \\
\hline kapuda & $\mathrm{Rn}, \mathrm{Kb}$ & OK & Fungi \\
\hline kapúli & $\mathrm{Kr}$ & 3993,3842 & Helicteres \\
\hline ka'pulihu & $\mathrm{Kb}$ & $\mathrm{RV}$ & $=$ 'pulihu \\
\hline ka'pulita & $\mathrm{Mb}$ & $\mathrm{O}$ & $=$ ka'pulutu \\
\hline ka'pulitu & Mi & OK & $=$ ka'pulutu \\
\hline ka'pulota & Ww & $\mathrm{RV}$ & $=$ ka'pulutu \\
\hline ka'puloto & Кo & $\mathrm{O}$ & $=$ ka'pulutu \\
\hline ka'pulutu & $\mathrm{Rn}, \mathrm{Kb}, \mathrm{Ak}$ & OK, 4176 & Ficus \\
\hline kapumbu & $\mathrm{Kb}$ & & = mapu kangúrutu ?II \\
\hline kapumbu tai manu & $\mathrm{Kb}$ & $4122, \mathrm{PH}$ & Apluda mutica \\
\hline kapúmbu & Ww, Lr, Ko & 3962 & Dichanthiwm?annularwn \\
\hline ka'pumbungu & $\mathrm{Kb}$ & OK & Poaceae \\
\hline kapùru 'taiko & $\mathrm{Lr}$ & RV & = kapìra 'taiko \\
\hline kapuwa hambaku & $\mathrm{Kr}$ & $\mathrm{RV}$ & Hyptis rhomboidea \\
\hline kapùza & Ww, Lr & $\mathrm{D}$ & = katíti \\
\hline karàbo & Ko & $\mathrm{O}$ & $=$ karobu \\
\hline karàbu & $\mathrm{Mi}, \mathrm{Kr}, \mathrm{Mb}$ & W & = karobu hàmu \\
\hline karàbu tunu & $\mathrm{Kr}$ & $\mathrm{RV}$ & Benincasa hispida \\
\hline karakápu & $\mathrm{Rn}$ & 4173 & Merremia vitifolia \\
\hline karaki & ESb & OK & Ficus (ampelas) (cj.) \\
\hline
\end{tabular}




\begin{tabular}{|c|c|c|c|}
\hline karaku & Lw & $\mathrm{O}$ & = karaki \\
\hline karàngu ahu & $\mathrm{Rn}, \mathrm{Kb}$ & 4174 & Crotalaria \\
\hline karánu & Ko & RV & $=$ hureni \\
\hline karara & Sumba & RV & Artocarpus altilis \\
\hline karara marapu & $\mathrm{Lr}$ & RV & $?=$ 'uowa \\
\hline karáwa & $\mathrm{Ll}, \mathrm{Ww}, \mathrm{Lr}$ & RV & $=$ tánda 'malangu I \\
\hline kara'wunangu & $\mathrm{Mi}, \mathrm{Kb}, \mathrm{Lw}$ & OK & vine, yellow, creeps on trees \\
\hline karawùrangu & $\mathrm{Kr}$ & $4044,\left(4045^{a}\right)$ & Selaginella willdenowii \\
\hline kàre & $\mathrm{Ll}, \mathrm{Ww}, \operatorname{Tr}$ & RV & $=$ kàri \\
\hline kareha & $\mathrm{Mi}, \mathrm{Kb}, \mathrm{Lw}$ & $\mathrm{H}$ & Cyperus monocephalus \\
\hline kareha lúku & $\mathrm{Rn}$ & 4161 & $\begin{array}{l}\text { Mitrasacme pygmaea var. } \\
\text { malaccensis }\end{array}$ \\
\hline kareha wai & $\mathrm{Kb}$ & $\mathrm{H}$ & Cyperus rotundus \\
\hline karenggu & $\mathrm{Lr}$ & RV & $=$ kiku manu II \\
\hline karenggu ala & Ww & 4254 & Cordyline fruticosa \\
\hline karere & Ww, Lr & RV & = ka'dinggiru \\
\hline karere koro & $\mathrm{L}$ & RV & Luffa acutangula \\
\hline kareri & $\mathrm{Ak}, \mathrm{Mb} ; \mathrm{Ww}$ & OK & $=\mathrm{ka}^{\prime}$ dinggiru \\
\hline kareru & Lw & $\mathrm{O}$ & $=$ ka'dinggiru \\
\hline karesa & $\mathrm{Mb}$ & $\mathrm{O}$ & $=$ kareha \\
\hline kareweto & Кo & $\mathrm{O}$ & = rútu I \\
\hline kàri & ESb & RV & Cycas ?rumphii \\
\hline kariho & Кo & RV & $=$ kahàbaru \\
\hline kariki & $\mathrm{Kb}$ & $\mathrm{O}$ & Fungi \\
\hline karikú(hu) & ES & $\mathrm{D}$ & = ngàndu kàriku \\
\hline ?karimboyo & Ko & RV & $=$ wudi \\
\hline karipatu & Ak & $\mathrm{O}$ & = ka'ripitu I \\
\hline karipi & $\mathrm{ESb}$ & $\mathrm{D}, \mathrm{O}$ & Piliostigma malabaricum \\
\hline ka'ripico & Ko & $\mathrm{RV}$ & = ka'ripitu I \\
\hline ka'ripico marapu & Ko & RV & = kalia rara \\
\hline karipita & $\mathrm{Mb} ; \mathrm{Ww}$ & $\mathrm{O}$ & = ka'ripitu II \\
\hline ka'ripito tai ndara & $\mathrm{Lr}$ & RV & Fungi \\
\hline ka'ripitu I & $\mathrm{Rn}, \mathrm{Kr}, \mathrm{Kb}$ & OK & Fungi \\
\hline ka'ripitu II & $\mathrm{Rn}, \mathrm{Kr}, \mathrm{Kb}$ & RV & Fungi, Hirneola \\
\hline karipu & Lw & $\mathrm{O}$ & $=$ karipi \\
\hline kaníri I & $\mathrm{Ll}, \mathrm{Tt}$ & $\mathrm{RV}$ & = kahàbaru \\
\hline karíri II & $\mathrm{ESb}$ & $\mathrm{H}, \mathrm{O}$ & $=$ ka'dinggiru \\
\hline kaníri kalaü & $\mathrm{Kr}$ & $\mathrm{RV}$ & = ka'dinggiru kalaü \\
\hline karìsi & $\mathrm{Mb}$ & OK & = kahàbaru \\
\hline karita & $\mathrm{Mi}, \mathrm{Kb}, \mathrm{Lw}$ & $\mathrm{O}$ & $=$ rita I \\
\hline karitaku I & $\mathrm{Kr}$ & 3958 & Oplismenus compositus \\
\hline karìtaku II & $\mathrm{Kb}$ & 3865 & Salvia \\
\hline karitaku kàhi & $\mathrm{L}$ & D & Canariwm vulgare \\
\hline
\end{tabular}




\begin{tabular}{|c|c|c|c|}
\hline ka'ritaku kasi & ES & D & = karitaku kàhi \\
\hline karìzi & $\mathrm{Lr}$ & $\mathrm{D}, \mathrm{H}$ & = kahàbaru \\
\hline karòbo & Ww, Lr & $\mathrm{OK}, \mathrm{RV}$ & $=\mathrm{karobu}$ \\
\hline karòbo dawa & $\mathrm{Tt}$ & v & Sechiwm edule \\
\hline karòbo jawa & $\mathrm{L}$ & $\mathrm{v}$ & = karòbo dawa \\
\hline karòbo pùti & $\mathrm{Ww}, \mathrm{Lr}$ & RV & Luffa aegyptiaca \\
\hline karobu & $\mathrm{Mi}, \mathrm{Kb}, \mathrm{Ak}$ & $\mathrm{D}, \mathrm{H}$ & $\begin{array}{l}\text { Lagenaria siceraria var. } \\
\text { (vegetable) }\end{array}$ \\
\hline karobu hàmu & $\mathrm{Kb}$ & RV & Cucurbita moschata \\
\hline karobu rara & $\mathrm{Kb}$ & $\mathrm{O}$ & = karobu hàmu \\
\hline karobu tunu & $\mathrm{Kb}$ & OK & $=$ karàbu tunu \\
\hline karoka & $\mathrm{Kb}, \mathrm{Mb}$ & $\mathrm{O}$ & tree w. strong wood \\
\hline karoku & $\mathrm{Ak}$ & $\mathrm{O}$ & $=$ karúku \\
\hline karóndungu & Lw & $\mathrm{O}$ & = kaníndingu \\
\hline 'karota & $\mathrm{Ll}, \mathrm{Ww}$ & RV & $=\operatorname{rami} I$ \\
\hline kàru & Lw & $\mathrm{O}$ & = kàri \\
\hline karua kapu & $\mathrm{Kb}$ & 3860 & Ipomoea pes-tigridis \\
\hline karùdi & Ww, Lr, Ko & $\mathrm{D}$ & $=$ kanoru \\
\hline karúku & $\mathrm{Mi}, \mathrm{Kb}, \mathrm{Lw}$ & OK & tree w. broad 1.; ?Ficus \\
\hline karúndingu & $\mathrm{Mi}, \mathrm{Kb}$ & OK & $\begin{array}{l}\text { tree; known for edible larvae in } \\
\text { rotted trunk }\end{array}$ \\
\hline karùngu & $\mathrm{Rn}$ & RV & Bidens \\
\hline karùpe I & Ww & $\mathrm{O}$ & $=$ karipi \\
\hline karùpe II & $\mathrm{Lr}$ & 4263 & Ampelocissus arachnoides \\
\hline karupi & Ak & $\mathrm{O}$ & $=$ karipi \\
\hline kása I & $\mathrm{L}$ & RV & = kamàru \\
\hline kása II & $\mathrm{Mb}$ & $\mathrm{O}$ & $=$ kakaha I \\
\hline kasambi & $? \mathrm{Mb}$ & $?$ & $=$ kahembi \\
\hline kàsi & $\mathrm{Ak}, \mathrm{Mb} ; \mathrm{Ll}$ & RV & $=$ kàhi jawa \\
\hline kasósa & $\mathrm{L}$ & RV & $=$ hureni \\
\hline ka'suoba & $\mathrm{L}$ & 4127 & Scirpus \\
\hline kata I & $\mathrm{Kr}, \mathrm{Kb} ; \mathrm{Ww}$ & OK & Lycopodium cernuum \\
\hline kata II & $\mathrm{Kr}, \mathrm{Kb}$; Ww, Lr, Ko & 3961 & Lygodiwm circinnatwm \\
\hline kata III & $\mathrm{Kb}$ & OK & Orchidaceae \\
\hline kata IV & $\mathrm{Kb}$; Lr, Ko & $\mathrm{O}$ & Polypodiaceae \\
\hline kata lólo & $\mathrm{Ww}$ & 4075 & $\begin{array}{l}\text { Lygodium scandens/ } \\
\text { microphyllum }\end{array}$ \\
\hline katàbi & $\mathrm{Kb}$ & OK & Ipomoea batatas \\
\hline katàbi jawa & $\mathrm{Kb}$ & OK & Solanum tuberosum \\
\hline katàbi lúku & $\mathrm{Rn}$ & 4157 & Convolvulaceae \\
\hline katàbi mondu & $\mathrm{Kb}$ & 3839 & Operculina turpethwm \\
\hline katàbi 'omangu & $\mathrm{Kb}$ & 3852 & Ipomoea obscura \\
\hline katàbu & $\mathrm{Np}$ & W & $=$ katàbi \\
\hline katambini & $\mathrm{Mi}, \mathrm{Kb}$ & $\mathrm{RV}, 4238$ & Phragmites karka \\
\hline
\end{tabular}




\begin{tabular}{|c|c|c|c|}
\hline katambuni & $\mathrm{Mi}, \mathrm{Rn}, \mathrm{Kr}$ & RV & $=$ katambini \\
\hline katanda tana & $\mathrm{Lr}$ & 3986 & Boerhavia \\
\hline ka'tanggara & $\mathrm{L}$ & RV & = ka'nanggaru \\
\hline ka'tanggaru & $\mathrm{Kb}$ & dft, 3886 & Millingtonia hortensis \\
\hline kàtango & Ko & $\mathrm{O}$ & = kàtangu \\
\hline kàtangu & $\mathrm{ESb}$ & $\mathrm{OK}, \mathrm{dft}$ & Planchonella duclitan \\
\hline katara kàde & $\operatorname{Tr}$ & 4255 & Eclipta prostrata \\
\hline katàri jala & $\mathrm{Kb}$ & RV & $=$ lamata \\
\hline katéte & $\mathrm{L}$ & RV & = katàbi \\
\hline katéti & Ak & OK & = katàbi \\
\hline kàti káka & $\mathrm{L}$ & $4121 \mathrm{~A}$ & Smithia \\
\hline kàti katàru I & ?Kr & dft & Sapindaceae \\
\hline kàti katàru II & $\mathrm{Kr}$ & 3934 & Dysoxylum parasiticum \\
\hline kàti tihu & $\mathrm{Kr}, \mathrm{Kb}$ & 3847 & Alternanthera \\
\hline katilara & $\mathrm{Ll}, \mathrm{Lr}$ & RV & Dioscorea \\
\hline katilu kawimbi & Ko & RV & ?Vitex pubescens \\
\hline katilu simbi & Ww, Tr & 4032 & Wendlandia \\
\hline ?katilu yìbi & $\mathrm{L}$ & RV & $=$ ghasu ghula \\
\hline katilyóro & Ko & RV & = katìlara \\
\hline katina & Ww & $\mathrm{O}$ & $=$ tibu hala \\
\hline ka'tinahu I & $\mathrm{Kb}$ & OK & $=$ tibu hala \\
\hline ka'tinahu II & $\mathrm{Mi}, \mathrm{Kb}, \mathrm{Lw}$ & $\mathrm{O}$ & tree w. hard wood \\
\hline ka'tinasa & $\mathrm{Mb}$ & $\mathrm{O}$ & $=$ ka'tinahu II \\
\hline ka'tinasu & Ak & $\mathrm{O}$ & = ka'tinahu II \\
\hline katinyaho & Ko & $\mathrm{O}$ & = ka'tinahu II \\
\hline katìri dála & $\mathrm{L}$ & RV & $=$ lamata \\
\hline katíti & $\mathrm{Kb}$ & $\mathrm{D}, \mathrm{H}$ & Lagenaria siceraria (for gourds) \\
\hline katówa kedu I & Ww & 4065 & Dendrobium \\
\hline katówa kedu II & Ww & 4091 & Myrmecodia \\
\hline katúa duóda (?ruoda) & $\mathrm{L}$ & RV & = kapuwa hambaku \\
\hline katúku malungo & Ko & RV & $=$ mòlu \\
\hline katungga & $\mathrm{Mb}$ & $\mathrm{O}$ & = walawina \\
\hline katunggu lawa & Ww, Lr & RV & $=$ tandala pua \\
\hline katunggu lawa ndende & Ww & 4068 & Scutellaria discolor \\
\hline katunggu lengi & Ww & 4030 & Claoxylon abbreviatum \\
\hline ?katùra lòdo & Ww & RV & = tangára lòdo \\
\hline kaungi & ES & $\mathrm{H}$ & $=$ 'wingiru \\
\hline kaura & Ak & $\mathrm{O}$ & = ?kandángi pòdu \\
\hline ka'wadaru & $\mathrm{Kb}$ & RV & ?Colocasia \\
\hline kawaka & Mi, Kb, Lw & $\mathrm{OK}$ & plant, creeping \\
\hline kawàlu & Mi, Rn, Kr, Tm; Ko & OK, W & $=$ kawilu \\
\hline kawàngo & $\mathrm{Ko}, \mathrm{Lr}$ & RV & $=$ kabaru \\
\hline kawangu & Ww & $\mathrm{O}$ & = kabaru \\
\hline
\end{tabular}




\begin{tabular}{|c|c|c|c|}
\hline kawaü & $\mathrm{Kb}$ & OK & = 'uowa \\
\hline kawawu & $\mathrm{Mb}, \mathrm{Ak}$ & $\mathrm{O}$ & = 'uowa \\
\hline kawia I & $\mathrm{Mi}, \mathrm{Kb}$ & OK & Acacia leucophloea \\
\hline kawia II & ?Kr & RV & $=$ marawìta \\
\hline ka'wiaka & Lw & $\mathrm{O}$ & $=$ marawìta \\
\hline kawilu & $\mathrm{Kb}, \mathrm{Lw}, \mathrm{Ak}, \mathrm{Mb}$ & RV & Aleurites moluccana \\
\hline kawindu & $\mathrm{Kb}$ & 3879 & Melochia corchorifolia \\
\hline kawìnga & $\mathrm{ESb}$ & $\mathrm{O}$ & vine, edible \\
\hline kawingyo & Ko & $\mathrm{O}$ & = kawìnga \\
\hline kawita kaba & $\mathrm{Kr}$ & $\mathrm{dft}$ & Linociera \\
\hline ka'woana & $\mathrm{Kn}$ & W & = kawona \\
\hline kawona & $\mathrm{Kn}, \mathrm{Kb}, \mathrm{Lw}, \mathrm{Mb}$; Ll,Ww & RV & Moringa pterygosperma \\
\hline kawongu au & $\mathrm{Kb}$ & $\mathrm{PH}, \mathrm{H}$ & Brachiaria ramosa \\
\hline kawowo & Lw & $\mathrm{O}$ & = kawú \\
\hline kawowu & $\mathrm{Ak}, \mathrm{Mb}$ & $\mathrm{O}$ & = kawú \\
\hline kawú & $\mathrm{Mi}, \mathrm{Kb}, \mathrm{Lw}$ & OK & Amorphophallus \\
\hline kawua & $\mathrm{Kr}, \mathrm{Kb}$ & V & = kabaru \\
\hline ka'wuana & $\mathrm{Rn}$ & RV & $=$ kawona \\
\hline kawuasa & Ak & $\mathrm{O}$ & = 'uwahu \\
\hline kawùlu uru I & $\mathrm{Kb}$ & PH & = ka'ndaüngu \\
\hline kawùlu uru II & $?$ & $?$ & $=$ wulu ùru $\mathrm{I}$ \\
\hline kawúru wàla & $\mathrm{Rn}$ & RV & Stachytarpheta indica \\
\hline kayàmbi & Rn & 4166 & Acrostichwm aureum \\
\hline kayàmbi oamangu & $\mathrm{Rn}$ & 4144 & Eranthemum \\
\hline kayàmbi uamangu & ES & $\mathrm{dft}$ & Engelhardia spicata \\
\hline kayu puti & $\mathrm{Kb}$ & $\mathrm{O}$ & = yàlangu \\
\hline ka'yumbangu & ES & $\mathrm{D}$ & $=\mathrm{ka}$ 'lumbangu \\
\hline káza & $\mathrm{Lr}$ & $\mathrm{D}$ & = kamàru \\
\hline káza lólo & Ww & RV & = kamàru kawaü \\
\hline kazi & Ww, Lr & OK & = kàhi jawa \\
\hline kazóza & Ww, Lr, Tt & RV & $=$ hureni \\
\hline kedare (kadara) & $\mathrm{Kr}$ & $\mathrm{D}, \mathrm{H}$ & = kandara II \\
\hline kei & Ww, Lr, Tt & $\mathrm{D}$ & = kàhi I \\
\hline kelor & $\mathrm{Kb}$ & OK & = kawona \\
\hline kèmbe & Ko & RV & = kambí \\
\hline kèmbe móro wiha & Ko & RV & = kambí múru \\
\hline kèmbe tana & Ko & RV & $=$ manila I \\
\hline kencalu & ?Lw & $\mathrm{D}$ & $=$ kanjilu \\
\hline kenda kabela & Lr & RV & = kámbu lawora I \\
\hline kère & Lr, Ko, Tt & RV & = kàri \\
\hline ?kèsi & $\mathrm{L}$ & RV & $=$ kàhi jawa \\
\hline 'ketoka & Ww, Lr, Tt & $\mathrm{D}$ & = kúnjuru \\
\hline ?keura (should be kawia?) & ES & $\mathrm{D}$ & $=$ marawìta \\
\hline
\end{tabular}




\begin{tabular}{|c|c|c|c|}
\hline kèzi & $\mathrm{Ww}, \mathrm{Tt}$ & RV & = kàhi jawa \\
\hline ki'i I & $\mathrm{Lr}$ & 3995 & cf. Andrographis \\
\hline ki'i II & $\mathrm{Lr}$ & 3987 & Hypoestes \\
\hline kiketaru & ES & $\mathrm{D}$ & = katilu kawimbi \\
\hline kiku ahu & $\mathrm{Kr}$ & RV & = karùngu \\
\hline kiku bòngga & Ko & RV & = kiku bòngga marapu \\
\hline kiku bòngga marapu & Ww & 4022 & Mallotus ricinoides \\
\hline kiku manu I & $\mathrm{Lr}$ or $\mathrm{Kr}$ & RV & Freycinetia \\
\hline kiku manu II & $\mathrm{Kr}$ & 4209 & Pleomele \\
\hline kiku meu & $\mathrm{Kb}$ & OK & herb \\
\hline kìru & $\mathrm{Ll}, \mathrm{Kr}$ & 4134 & Dysoxylum parasiticum \\
\hline kìru bára & Rn & 4215 & = kìru \\
\hline koba wai & Ak & RV & Centella asiatica \\
\hline koba we'e & $\mathrm{L}$ & RV & = koba wai \\
\hline kobu & Ak & $\mathrm{O}$ & $=\mathrm{kombu}$ \\
\hline 'kokuru & $\mathrm{ESb}$ & OK & Cocos nucifera \\
\hline 'kokuru búti & $\mathrm{Kb}$ & OK & tree, coastal \\
\hline 'kokuru ka'wulanga & $\mathrm{Kb}$ & OK & Cocos nucifera var. \\
\hline kombo & Ww, Lr, Ko & $\mathrm{D}, \mathrm{RV}$ & $=\mathrm{kombu}$ \\
\hline kombu & $\mathrm{Rn}, \mathrm{Kb}$ & OK & Morinda tomentosa \\
\hline kómi & Ww, Lr, Ko & OK & $=\mathrm{kahembi}$ \\
\hline komisa & $\mathrm{Mb}$ & $\mathrm{O}$ & $=$ kahembi \\
\hline kóndu & $\mathrm{Kr}$ & RV & Bischofia javanica \\
\hline kondu 'kurangu I & $\mathrm{Kb}$ & PH & Hackelochloa granularis \\
\hline kondu 'kurangu II & $\mathrm{Rn}$ & RV & Erechtites valerianifolia \\
\hline kopa & $\mathrm{ESb}$ & OK & $=$ rongu \\
\hline kopa lua & $\mathrm{Kb}$ & RV & Ceiba pentandra \\
\hline kopi & Sumba & RV & Coffea \\
\hline kopi nggangga & $\mathrm{Kr}, \mathrm{Kb}$ & RV & Leucaena leucocephala \\
\hline kopi 'riutu & $\mathrm{Rn}$ & RV & = kopi nggangga \\
\hline kopi rútu & $\mathrm{Kb}$ & OK & = kopi nggangga \\
\hline korondahi & Ko & RV & $=$ harakaya,$?=$ tariwangu \\
\hline koru & Ko & RV & = karere koro \\
\hline koru pòdu & $\mathrm{Tt}$ & V & $=$ karere koro \\
\hline 'kotera & Ww, Lr & RV & Ryssopterys \\
\hline (n) kowa & Mi & $\mathrm{O}$ & Mimosa pigra \\
\hline 'kowaku & $? \mathrm{Ll}$ & $\mathrm{H}$ & $=$ kuta I \\
\hline kubùkela manu & $\mathrm{L}$ & RV & $=$ kandinu bara \\
\hline kulá & $\mathbf{K b}$ & $\mathrm{O}, \mathrm{OK}$ & Vitex parviflora \\
\hline kulu & $\mathrm{Kb}$ & OK, D & Artocarpus altilis (seeded) \\
\hline kulu nderi & $\mathrm{Kb}$ & OK & Poaceae \\
\hline kúlu tana & $\mathrm{Kb}$ & OK & Fungi \\
\hline kúni & $\mathrm{Ll}, \mathrm{Ww}$ & RV & = 'wingiru \\
\hline
\end{tabular}




\begin{tabular}{|c|c|c|c|}
\hline kunita & WS & $\mathrm{H}$ & $=$ 'wingiru \\
\hline kúnjuru & $\mathrm{ESb}$ & RV, D & Cassia fistula \\
\hline kunu & $\mathrm{Mi}, \mathrm{Kb}, \mathrm{Lw}$ & OK & = kandángi pòdu \\
\hline kunu búti & $\mathrm{Mi}, \mathrm{Kb}$ & OK & $=$ kahala wau \\
\hline kunyi & Ak & $\mathrm{O}$ & = 'wingiru \\
\hline 'kuomi & $\mathrm{L}$ & RV & $=$ kahembi \\
\hline kùru kára I & $\mathrm{Lr}$ & 4247 & Menispermaceae \\
\hline kùru kára II & Ww & 4076 & Dipteris conjugata \\
\hline kùru wawi & $\mathrm{L}$ & 4082,4137 & Elephantopus scaber \\
\hline kuta I & $\mathrm{Rn}, \mathrm{Kr}$ & $\mathrm{H}$ & Piper betle (infertile variety) \\
\hline kuta II & $\mathrm{Kb}$ & $\mathrm{OK}$ & marine plant, edible \\
\hline kuta búti & $\mathrm{Kb}$ & OK & Piper sp. \\
\hline kùta ghazu & $\mathrm{Lr}$ & 4245 & Callicøpa \\
\hline kuta haü & $\mathrm{Kb}$ & RV & Piper \\
\hline kuta kalara & $\mathbf{K b}$ & OK & Piper \\
\hline kuta kawaï & $\mathrm{Kb}$ & $\mathrm{O}$ & Piper betle var. \\
\hline ku'u ghodo & $\mathrm{Lr}$ & RV & ?= ngàndu kàriku \\
\hline ku'u wodo & $\mathrm{Ww}, \mathrm{Tt}$ & RV, YD & ?= ngàndu kàriku \\
\hline làba & Ko & RV & $=$ winu \\
\hline làba malandi & Ko & RV & $=$ winu malanja \\
\hline labawa & $\mathrm{Mi}, \mathrm{Kb}, \mathrm{Lw}$ & OK, RV & $\begin{array}{l}\text { Allium cepa var. cepa } \\
\quad(\text { sativum) }\end{array}$ \\
\hline laboya & Ak & $\mathrm{O}$ & $=$ lamboya \\
\hline làbu & $\mathrm{Mb}$ & $?$ & = 'lobungu I \\
\hline làbungo & $\mathrm{Lr}, \mathrm{Tt}$ & RV, YD & = 'lobungu II \\
\hline làbungu & Mi, Lw, Ak & RV & = 'lobungu II \\
\hline la'bunguru & Ak & $\mathrm{O}$ & $=$ la'mbunguru \\
\hline ?ladi & ES & $\mathrm{H}$ & $=$ layia \\
\hline lagapa & ?L & 3998 & Lunasia cf. amara \\
\hline lagat & Ll, Ww, Lr & RV & $=$ ju'latingu \\
\hline laghughu & Ko & RV & = lawungu \\
\hline laghui & $\mathrm{Lr}$ & RV & = walawina \\
\hline laghùngo & $\mathrm{Lr}$ & RV & = lawungu \\
\hline laghùta & Ko & RV & $=$ lita rùmba \\
\hline lagódo & $\mathrm{L}$ & RV & = langgódo II \\
\hline lahona & $\mathrm{Kb}$ & $\mathrm{RV}, \mathrm{OK}$ & Alliwm cepa var. escalonicum \\
\hline la'huana & $\mathrm{Kr}$ & RV & $=$ lahona \\
\hline lailu & Ak & $\mathrm{O}$ & $=$ lelu \\
\hline laka & $\mathrm{Kb}$ & W & ?Lawsonia inermis \\
\hline lamata & $\mathrm{Kr}, \mathrm{Kb}$ & RV & Portulaca oleracea \\
\hline lamboya & $\mathrm{ESb}$ & OK & Curcuma zedoaria \\
\hline la'mbunguru & $\mathrm{Mi}, \mathrm{Kb}, \mathrm{Lw}$ & OK & $=$ wala 'mbunguru \\
\hline làme & Ww, Lr, Tt & RV & $=$ lamua \\
\hline
\end{tabular}




\begin{tabular}{|c|c|c|c|}
\hline lamtoro & $\mathbf{K b}$ & & = kopi nggangga \\
\hline lamtoro jawa & $\mathbf{W w}$ & 4117 & Acacia villosa f. glabra \\
\hline lamu & $\mathrm{Mi}, \mathrm{Kb}, \mathrm{Lw}$ & OK & marine plant; fr. edible \\
\hline lamua & $\mathrm{ESb}$ & $R V$, dft & Melia azedarach \\
\hline landa & $\mathrm{Mi}, \mathrm{Kb}, \mathrm{Lw}$ & $\mathrm{O}$ & Pandanus tectorius var. ?laevis \\
\hline lándu káka & $\mathrm{Rn}$ & 4145 & Dysoxylum gaudichaudianum \\
\hline lándu káka 'omangu & $\mathrm{Kb}$ & 4231 & ?Dysoxylum \\
\hline landusa & $\mathrm{Mb}$ & $\mathrm{O}$ & = ka'pidihu \\
\hline lànga & Sumba & RV & Sesamum orientale \\
\hline langae & Ww & $\mathrm{O}$ & $=$ langaha \\
\hline langaha & $\mathrm{Mi}, \mathrm{Rn}, \mathrm{Kb}$ & RV & Planchonia valida \\
\hline langai & $\mathrm{Mi}, \mathrm{Kr}, \mathrm{Tm}$ & $\mathrm{H}$ & = 'kokuru \\
\hline langasa & $\mathrm{Ak}, \mathrm{Mb}$ & $\mathrm{O}$ & $=$ langaha \\
\hline langgapa & $\mathrm{Kb}$ & $\mathrm{O}$ & tree \\
\hline langgapa lolo & $\mathrm{Lr}$ & 4052 & Lepidagathis \\
\hline langgate & Ww & $\mathrm{O}$ & = ju'latingu \\
\hline langgetu I & Ww, Lr & 4235 & ?Sterculiaceae \\
\hline langgetu II & Ww & RV & $=$ hamaili \\
\hline langgi wei & $\mathrm{Kb}$ & OK & plant, creeping \\
\hline langgìri & Ww, Lr, Tt & RV & ?Amomum \\
\hline langgiri meu & $\mathrm{Kb}$ & OK & ?Crotalaria \\
\hline ?langgítu & $\mathrm{Lr}$ & RV & $?=$ hamaili \\
\hline langgódo I & WS & RV & = langgúdu \\
\hline langgódo II & WSb & RV & ?Dioscorea ?pentaphylla \\
\hline langgoro maràda & $\mathrm{Kr} ; \mathrm{Ko}$ & 3898 & Crotalavia \\
\hline langgoti & Ak & $\mathbf{W}$ & $?=$ hamaili \\
\hline langgúdu & $\mathrm{Kr}, \mathrm{Kb}$ & 3939 & Tacca pabmate \\
\hline langgùtu & Ko & RV & $?=$ hamaili \\
\hline langira I & Mb; Kw, Ko & $\mathrm{O}$ & = ka'ndulangu \\
\hline langira II & $\mathrm{Rn}, \mathrm{Kr}, \mathrm{Kb} ; \mathrm{Ll}, \mathrm{Lr}$ & RV & Alangium ?villosum \\
\hline langirya & Ko & RV & = langira II \\
\hline languasu & $\mathrm{Mb}$ & $\mathrm{O}$ & = liza rónda \\
\hline lantoro & Ww, Lr & RV & $=$ kopi nggangga \\
\hline lapále & $\mathrm{Kr}$ & dft & Dysoxylum \\
\hline lapali & ES & dft & Chisocheton \\
\hline lari & $\mathrm{Mi}, \mathrm{Kb}, \mathrm{Lw}$ & $\mathrm{O}$ & $=\operatorname{laru} \mathrm{I}$ \\
\hline laru I & Mb; Ww, Ko & 4083 & Knema cinerea \\
\hline laru II & ES & $\mathrm{dft}$ & ?Garcinia \\
\hline lasona & $\mathrm{Tm}, \mathrm{Tb}, \mathrm{Mb}$ & W & = lahona \\
\hline lataho & Ko & RV & = kata lólo \\
\hline laungu & $\mathrm{Mi}, \mathrm{Kb}, \mathrm{Lw}$ & OK, O & $=$ lawungu \\
\hline lawanda & $\mathrm{Mi}, \mathrm{Kb}$ & $\mathrm{O}$ & Lavandula officinalis \\
\hline lawina & $\mathrm{Kb}$ & OK & $=$ walawina \\
\hline
\end{tabular}




\begin{tabular}{|c|c|c|c|}
\hline lawuhu & $\mathrm{Kb}$ & $\mathrm{RV}$ & ?Dioscorea \\
\hline lawui & Ww & OK & = walawina \\
\hline lawuji & $\mathrm{u}$ & $\mathrm{RV}$ & $=$ walawina \\
\hline lawungu & $\mathrm{Kb}, \mathrm{Ak}, \mathrm{Mb}$ & $\mathrm{RV}, \mathrm{OK}$ & Schoutenia ovata \\
\hline layia & $\mathrm{Kb}$ & W & Zingiber officinale \\
\hline leke & WSb & $\mathrm{RV}$ & $=$ 'alaku \\
\hline lelu & $\mathrm{ESb}$ & OK & marine plant, edible \\
\hline lème & Ko & $\mathrm{O}$ & $=$ lamua \\
\hline ?libra & $\mathrm{Lr}$ & $\mathrm{H}$ & Nypa fruticans \\
\hline licopo & Ko & $\mathrm{O}$ & = 'litapu I \\
\hline ligha rónda & Ko & RV & $=$ liza rónda \\
\hline limaru & Lw & $\mathrm{O}$ & $=$ limora \\
\hline limora & $\mathrm{Mi}, \mathrm{Kb}$ & OK & ?shrub, on the plains \\
\hline lindu watu I & ES & $\mathrm{dft}$ & Avicennia marina var. resinifera \\
\hline lindu watu II & $\mathrm{Kr}$ & $\mathrm{dft}$ & Anacardiaceae \\
\hline lino & $\mathrm{Ww}, \mathrm{Tt}$ & V & $?=$ linu I \\
\hline linu I & ES & 3938 & Glycosmis \\
\hline linu II & ESb & $\mathrm{dft}$ & Grewia ?eriocarpa \\
\hline linu III & $\mathrm{Lr}$ & $\mathrm{D}$ & $=$ hureni \\
\hline linu bara & $\mathrm{Kb}$ & $\mathrm{O}$ & tree w. white wood; cp. linu I+II \\
\hline linu kadu & $\mathrm{Kb}$ & $\mathrm{O}$ & $\begin{array}{l}\text { tree w. blackish wood; cp. linu } \\
\text { I+II }\end{array}$ \\
\hline lìnyo & Ko & $\mathrm{O}$ & $=$ hureni \\
\hline lipe & $\mathrm{Ww}, \mathrm{Lr}, \mathrm{Tt}$ & RV & $=$ lipi \\
\hline lipi & $\mathrm{Mi}, \mathrm{Kb} ; \mathrm{Ww}, \mathrm{Lr}$ & 4279 & Mucuna pruriens var. utilis \\
\hline lìpi pana & $\mathrm{Rn}$ & W & = úndi \\
\hline lìpu & Lw & $\mathrm{O}$ & $=$ lipi \\
\hline lipya & Ko & $\mathrm{O}$ & $=$ lipi \\
\hline lisa & $\mathbf{L}$ & RV & $?=$ layia \\
\hline lisa ráta & $\mathbf{L}$ & RV & $=$ liza rónda \\
\hline lisa 'ruoda & $\mathrm{L}$ & RV & $?=$ liza rónda \\
\hline lita rumba & $\mathrm{Rn}$ & RV & Dioscorea aculeata \\
\hline 'litangu & ESb & OK & $\begin{array}{l}\text { Dioscorea esculenta (O: Coleus } \\
\text { tuberosus) }\end{array}$ \\
\hline litapa & $\mathrm{Mb}$ & $\mathrm{O}$ & = 'litapu I \\
\hline 'litapu I & $\mathrm{Rn}, \mathrm{Kr}, \mathrm{Kb}$ & PH, (3927) & Rottboellia exaltata \\
\hline 'litapu II & $\mathrm{Mi}, \mathrm{Kb}$ & $\mathrm{O}$ & $\begin{array}{l}\text { shrub w. resinous wood; used } \\
\text { for torches }\end{array}$ \\
\hline 'litopa & $\mathrm{Ww}, \mathrm{Tt}$ & $\mathrm{OK}, \mathrm{YD}$ & = 'litapu I \\
\hline 'litopo & $\mathrm{Lr}$ & RV & = 'litapu I \\
\hline liza & Ww, Lr & RV & $=$ layia \\
\hline liza rónda & ?Ww, Lr & RV & Languas galanga \\
\hline loba & Sumba & $\mathrm{OK}, \mathrm{RV}$ & Peltophorum pterocarpwm \\
\hline lobo & ES & $\mathrm{D}$ & = 'lobungu II \\
\hline
\end{tabular}




\begin{tabular}{|c|c|c|c|}
\hline 'lobungu I & $\mathrm{Rn}, \mathrm{Kb}$ & RV & Decaspermwn \\
\hline 'lobungu II & $\mathrm{Kr}, \mathrm{Kb} ; \mathrm{Ll}$ & dft & Syzygium (lineatum) \\
\hline lòmo & Ko & RV & $=$ lamua \\
\hline lona & Ww, Lr & RV & $=$ lahona \\
\hline lona ghayo & Ko & RV & = bawa kawaü \\
\hline lona ghazu & $\mathrm{Lr}$ & RV & = bawa kawaü \\
\hline lona kaka & $\mathrm{Ww}, \mathrm{Ko}, \mathrm{Tt}$ & $\mathrm{V}, \mathrm{YD}$ & = bawa kawaü \\
\hline lona wasu & $\mathrm{L}$ & RV & = bawa kawaü \\
\hline longge kabàketa & Lr, Ko, Tt & 4252 & Clematis (lih móro) \\
\hline lua & $\mathrm{Kb}$ & OK & $=$ luwa \\
\hline lua ai & $\mathrm{Kb}$ & $\mathrm{H}$ & $=$ luwa ai \\
\hline lua pi'a & Ww & OK & $=$ luwa \\
\hline lugha dawa & Ko & RV & $=$ luwa ai \\
\hline lugha (piyo) & Ko & RV & $=$ luwa \\
\hline lukuta & Ww, Lr & RV & Drynaria \\
\hline lukutu I & $\mathrm{Kb}$ & OK & $=$ lukuta \\
\hline (rí) lukutu II & ESb & OK & marine plant, edible \\
\hline lúlu karara & $\mathrm{Kr}$ & 3988 & Hibiscus surattensis \\
\hline lúlu mbàlu & $\mathrm{Kb}$ & OK & vine, water-yielding, wild \\
\hline 'lumuta & $\mathrm{Mb} ; \mathrm{Ww}$ & $\mathrm{O}$ & $=$ 'lumutu \\
\hline 'lumutu & $\mathrm{ESb}$ & $\mathrm{O}$ & Algae \\
\hline lùnda & Ww, Lr & RV & $=$ tawai \\
\hline lundángo & Ko & RV & $=$ tawai \\
\hline lúnjuku & $\mathrm{Kb}$ & OK & marine plant, edible, reddish \\
\hline lùpe I & $\mathrm{L}$ & RV & Canavalia \\
\hline lùpe II & Ko & RV & $=$ lipi \\
\hline lupi & $\mathrm{Ak}$ & $\mathrm{O}$ & $=$ lipi \\
\hline lùri I & $\mathrm{L}$ & 3944 & Freycinetia \\
\hline lùri II & $\mathrm{Ll}, \mathrm{Ww}$ & $3944^{\mathrm{a}}$ & $?=$ uri, 'wakihu \\
\hline 'lutangu & Ak & OK & $=$ 'litangu \\
\hline luwa & $\mathrm{ESb} ; \mathrm{Ww}$ & RV & Dioscorea alata \\
\hline luwa ai & $\mathrm{Rn}, \mathrm{Kb}$ & RV & Manihot esculenta \\
\hline luwa ghazu & $\mathrm{Lr}$ & RV & $=$ luwa ai \\
\hline luwa ia & $\mathrm{Kr}$ & RV & $=$ luwa ai \\
\hline luwa pi'a & $\mathrm{Ll}, \mathrm{Ww}, \mathrm{Lr}$ & RV & $=$ luwa \\
\hline luwa rato & $\mathrm{Ww}$ & 4234 & Aristolochia tagala \\
\hline luwa tana & $\mathrm{Kb}$ & 3833 & Dioscorea \\
\hline luwa wasu & Ww & RV & $=$ luwa ai \\
\hline mada nu'u I & $\mathrm{L}$ & RV & $?=$ manda nu'u \\
\hline mada nu'u II & $\mathrm{L}$ & RV & $=$ susu nana \\
\hline madamu & Ak & $\mathrm{O}$ & = padàmu \\
\hline madáwa & $\mathrm{L}$ & $4111,(3970)$ & Orthosiphon aristatus \\
\hline madinu & Ak & $\mathrm{O}$ & = kandinu II \\
\hline
\end{tabular}




\begin{tabular}{|c|c|c|c|}
\hline madòmo & Ko & RV & $=$ padàmu humba \\
\hline madòmo dawa & Ko & RV & = kadàmo ndima \\
\hline madùbu & $\mathrm{Ll}, \mathrm{Tt}$ & 4109 & Loranthaceae \\
\hline magalaga & Ak & $\mathrm{O}$ & = manggalawa III \\
\hline ma'ghailo & $\mathrm{Lr}, \mathrm{Tt}$ & RV & $=$ ma'wailo \\
\hline maghela & Ko & $\mathrm{O}$ & = mayela II \\
\hline mahi & Ko & $\mathrm{O}$ & $=\mathrm{mahu}$ \\
\hline mahu & $\mathrm{ESb}$ & $\mathrm{O}$ & tree, in forest \\
\hline màja & $\mathrm{Rn}, \mathrm{Kb}$ & 3887 & Amaranthus (lividus) \\
\hline makèbela & $\mathrm{Lr}$ & 4248 & Hoya \\
\hline makiku & Ll, Ww, Lr & RV & Emilia sonchifolia \\
\hline maláho karimboyo & Ko & RV & = wota kamàmbi II \\
\hline malai rau & $\mathrm{Kr}$ & $\mathrm{dft}$ & Polyosma \\
\hline malangi & Ko & $\mathrm{O}$ & = kawìnga \\
\hline malere & $\mathrm{Kr} ; \mathrm{Ll}, \mathrm{Lr}, \mathrm{Ko}$ & RV & $=$ tandùla pua II \\
\hline malingata & W & 4048 & Scleria \\
\hline malíti I & Ll, Lr, Ko & RV & $=$ kalamba \\
\hline malíti II & $\mathrm{Ww}, \mathrm{Tt}$ & RV & $?=$ kalihi \\
\hline maloki & $\mathrm{Mi}, \mathrm{Kb}$ & OK & Ficus \\
\hline malóko & Lr & 3936,4004 & Ixora \\
\hline maloku & Lw & $\mathrm{O}$ & $=$ maloki \\
\hline malubu & $\mathrm{Mi}, \mathrm{Kb}, \mathrm{Lw}$ & OK, RV & Pterygota horsfieldii (cj.) \\
\hline malumbi & $\mathrm{Mb}$ & $\mathrm{O}$ & $=$ malubu \\
\hline mama & $\mathrm{Lr}$ & RV & $=$ winu \\
\hline mama lakawa & $\mathrm{Lr}$ & RV & $=$ pahawura I \\
\hline manda ngiyo & Ko & RV & $=$ manda nu'u \\
\hline manda nu'u & Ww & 4074 & Aralia montana \\
\hline mandagha & Lr, Ko & RV & ?= kandangu hàmu \\
\hline mandagha bùka & $\mathrm{Lr}$ & RV & = kandangu buku \\
\hline mandáwa & Ww & RV & $=$ kandangu hàmu \\
\hline mandáwa bùka & $\mathrm{Ll}, \mathrm{Ww}, \mathrm{Tr}$ & RV & $=$ kandangu buku \\
\hline mandònu I & $\mathrm{Ll}, \mathrm{Lr}$ & RV & $=$ kandinu mítingu \\
\hline mandònu II & Ww & OK & = kandinu II \\
\hline mandora & $\mathrm{Mi}, \mathrm{Kb}, \mathrm{Lw}$ & $\mathrm{O}$ & $=$ kandora \\
\hline mandubu & $\mathrm{Lr}$ & RV & $=$ madùbu \\
\hline manduyo & Ko & RV & = kandinu mítingu \\
\hline maneni & $\mathrm{Kb}$ & OK & ?Kalanchoe pinnata \\
\hline manera & Sumba & $\mathrm{dft}$ & Aglaia eusideroxylon \\
\hline mangàndingu & $\mathrm{Kb}$ & 3892 & Clerodendrum \\
\hline mangata & Ak, Mb; Ww & $\mathrm{O}$ & $=$ mangàta \\
\hline mangàta & $\mathrm{Mi}, \mathrm{Kb}, \mathrm{Lw}$ & RV, OK & Crinum asiaticum \\
\hline mangawa & ES & $\mathrm{dft}$ & Anthocephalus cadamba \\
\hline manggalawa I & Ko & $\mathrm{O}$ & = ambalaï \\
\hline
\end{tabular}




\begin{tabular}{|c|c|c|c|}
\hline manggalawa II & $\mathrm{Lr}$ & $\mathrm{D}, \mathrm{H}$ & = kandara II \\
\hline manggalawa III & $\mathrm{Mi}, \mathrm{Kb}, \mathrm{Mb} ; \mathrm{Ww}$ & OK & Myristica (fragrans) \\
\hline manggalewa & $\mathrm{Mb} ; \mathrm{Ww}$ & $\mathrm{O}$ & = kandara II \\
\hline manggaü I & $\mathrm{Rn}, \mathrm{Kb}$ & 3932 & Homalomena latifrons \\
\hline manggaü II & $\mathrm{Kr}$ & RV & ?Scindapsis/Rhaphidophora \\
\hline manggaü làmbata & $\mathrm{Kb}$ & $\mathrm{O}$ & vine \\
\hline 'manggita & $\mathrm{Mb}$ & W & $=$ 'menggitu \\
\hline 'manggitu & $\mathrm{Mi}, \mathrm{Kr}, \mathrm{Np}, \mathrm{Ak}, \mathrm{Wk}$ & W & $=$ 'menggitu \\
\hline 'mangico & Ko & RV & $=$ 'menggitu \\
\hline 'mangita & WSb & $\mathrm{D}, \mathrm{RV}$ & $=$ 'menggitu \\
\hline mangola & $\mathrm{Mi}, \mathrm{Kb}, \mathrm{Lw}$ & OK & tree \\
\hline maniéra & $\mathrm{Kr}$ & RV & $=$ manera \\
\hline manila I & $\mathrm{ESb}$ & $\mathrm{RV}, \mathrm{H}$ & Arachis hypogaea \\
\hline manila II & $\mathrm{Kb}$; Ww, Lr & OK & $=\operatorname{rami} I$ \\
\hline maninggatu & $\mathrm{Mi}, \mathrm{Kb}, \mathrm{Lw}$ & $\mathrm{O}$ & Scleria \\
\hline manjailu & $\mathrm{Mi}, \mathrm{Kb}$ & $\mathrm{O}$ & Ficus \\
\hline mantau & $\mathrm{ES}$ & $\mathrm{H}$ & $=$ kandora \\
\hline mapu & $\mathrm{Kr}, \mathrm{Kb}$ & 3864 & Themeda \\
\hline mapu bara I & $\mathrm{Kb}$ & $\mathrm{PH}$ & Sehima nervosum \\
\hline mapu bara II & $\mathrm{Kb}$ & PH & Chrysopogon gryllus \\
\hline mapu kahìriku & $\mathrm{Kb}$ & PH & Bothriochloa pertusa \\
\hline mapu kangúrutu I & $\mathrm{Kb}$ & PH & Dichanthium erectum \\
\hline mapu kangúrutu II & $\mathrm{Kb}$ & PH & Schizachyrium brevifolium \\
\hline mapu 'mbelaru pingi I & $\mathrm{Kb}$ & $?$ & Eleusine indica \\
\hline mapu 'mbelaru pingi II & $\mathrm{Kb}$ & PH & Heteropogon triticeus \\
\hline mapu múru kapuka & $\mathrm{Kb}$ & $\mathrm{O}$ & Poaceae \\
\hline mapu penji & $\mathrm{Kb}$ & PH & Themeda imberbis \\
\hline mapu rara & $\mathrm{Kb}$ & PH & $=$ rumba rara $I$ \\
\hline mapu wulu ihi kamambi & $\mathrm{Kb}$ & $\mathrm{PH}$ & Chrysopogon subtilis \\
\hline màra & $\mathrm{ESb}$ & OK & tree, straight \\
\hline màrahu rau & $\mathrm{Kr}$ & RV & $=$ màrahu rú \\
\hline màrahu rú & $\mathrm{Kb}$ & 3910 & Phyllanthus amarus \\
\hline maráka & Ww & 4069 & Acanthaceae \\
\hline ma'ranaka & Ww, Lr & 3989 & Mollugo pentaphylla \\
\hline marawàhi & $\mathrm{Kb}$ & $\mathrm{O}$ & shrub w. supple branches \\
\hline marawìta & $\mathrm{Lr}$ & $\mathrm{D}, \mathrm{H}$ & Albizia chinensis \\
\hline ma'regheta I & $\mathrm{Lr}$ & 3929 & Cyperus brevifolius \\
\hline ma'regheta II & $\mathrm{L}$ & $\mathrm{O}$ & = rútu I \\
\hline mareha & Ko & $\mathrm{O}$ & $=$ kareha \\
\hline ma'reweta & Ww & $\mathrm{O}$ & = rútu I \\
\hline mareza & Ww & $\mathrm{O}$ & $=$ kareha \\
\hline 'maripu & $\mathrm{Kr}$ & RV & = kamàla 'wataru \\
\hline marita & $\mathrm{Mi}, \mathrm{Kb}$ & $\mathrm{O}$ & $=$ rita I \\
\hline
\end{tabular}




\begin{tabular}{|c|c|c|c|}
\hline màritu & $\mathrm{Kb}$ & $\mathrm{O}$ & ?Poaceae \\
\hline maroto & Ww, Ko & $\mathrm{O}$ & = rútu II \\
\hline maroto kaghìki & $\mathrm{Lr}$ & RV & = jàriku mayilu \\
\hline maroto kaláda & $\mathrm{Ll}, \mathrm{Lr}$ & RV & $=$ jàriku 'tobungu \\
\hline maróto kawìki & $\mathrm{Ll}, \mathrm{Tt}$ & RV & = jàriku wihìku \\
\hline marotu & Ak & $\mathrm{O}$ & = rútu I \\
\hline marunggi & $\mathrm{Mi}, \mathrm{Kb}$ & $\mathrm{O}$ & $=$ kawona \\
\hline marunggu & Ko & $\mathrm{O}$ & $=$ kawona \\
\hline marùpe & Ko & RV & = karùpe II \\
\hline masu & $\mathrm{Mb}$ & $\mathrm{O}$ & $=$ mahu \\
\hline mata api & $\mathrm{L}$ & RV & = ka'lumbangu \\
\hline mata manu & $\mathrm{Kr}$ & RV & Mussaenda frondosa \\
\hline matu nuhi I & Ko & RV & $=\mathrm{mapu}$ \\
\hline matu nuhi II & Ko & RV & $=$ wua júli \\
\hline mawailo & Ww & 4265 & Phymatodes scolopendria \\
\hline mawona & Ak & $\mathrm{O}$ & $=$ kawona \\
\hline ma'yailo & Ko & RV & $=$ mawailo \\
\hline mayela I & $\mathrm{Kr}$ & 4212 & Orophea polycarpa \\
\hline mayela II & $\mathrm{ESb}$ & dft & Artocarpus glaucus \\
\hline mayela kalú & $\mathrm{Kr}$ & dft & Paratrophis philippinensis \\
\hline mazela & Ww & $\mathrm{O}$ & $=$ mayela I \\
\hline mazu & Ww & $\mathrm{O}$ & $=\mathrm{mahu}$ \\
\hline mbáha & Ko & RV & $=$ langaha \\
\hline mbaku & Ww, Lr, Ko & OK & = hambáku \\
\hline mbaku haü I & $\mathrm{ESb}$ & $\mathrm{RV}, \mathrm{O}$ & Capsicum frutescens \\
\hline mbaku haü II & $\mathrm{Kb}$ & $\mathrm{D}, \mathrm{OK}$ & = kajú II \\
\hline mbaku haii 'bokulu & $\mathrm{Kb}$ & RV & Capsicum annuum \\
\hline $\begin{array}{l}\text { mbaku zaü } \\
\text { mbàlu }\end{array}$ & Ww & $\mathrm{O}$ & $\begin{array}{l}\text { = mbaku haü I } \\
\text { see: lúlu mbàlu }\end{array}$ \\
\hline mbora & $\mathrm{Lr}$ & 3940 & $=$ pahaura \\
\hline mboro & WSb & RV & = mbúrungu \\
\hline mboru & $\mathrm{Mb}$ & $?$ & = mbúrungu \\
\hline mbórungu & Lw & $\mathrm{O}$ & = mbúrungu \\
\hline 'mbowila & $\mathrm{Ww}, \mathrm{Tt}$ & RV & tree \\
\hline mbówila & Ww & RV & $?=$ kondu kurangu II \\
\hline mbua lúlu & $\mathrm{Kb}$ & OK & marine vegetable \\
\hline mbula & ESb; Ko & $\mathrm{RV}, \mathrm{O}$ & Alocasia ?indica \\
\hline mbúli & $\mathrm{Kb}$ & OK & ?Calomus \\
\hline mbúlungu kawuku & $\mathrm{Rn}$ & 3909 & Heliotropium indicum \\
\hline mbúlungu pingi & $\mathrm{Mi}, \mathrm{Kb}$ & OK & $=$ kùru wawi \\
\hline mbunga & $\mathrm{ESb}$ & $\mathrm{O}$ & $?=$ bunga \\
\hline 'mbungura & $\mathrm{Mb}$ & $\mathrm{O}$ & $=$ wàla 'mbunguru \\
\hline 'mbunguru & $\mathrm{Mi}, \mathrm{Rn}, \mathrm{Kb}$ & 3894 & $=$ wàla 'mbunguru \\
\hline
\end{tabular}




\begin{tabular}{|c|c|c|c|}
\hline mbúrungu & $\mathrm{Mi}, \mathrm{Rn}, \mathrm{Kb}$ & RV & Conypha utan \\
\hline meli káka & Ww & RV & ?Medinilla \\
\hline memala & Ll, Ww & RV & $=$ kóndu \\
\hline mènde & Ww, Lr & RV & $?=$ ngùdu wawi \\
\hline 'menggitu & $\mathrm{Rn}, \mathrm{Kb}$ & $\mathrm{D}, \mathrm{H}$ & Borassus sundaicus \\
\hline meráka & Ww & 4069 & Strobilanthes \\
\hline mi'ejaka & $\mathrm{L}$ & 4141 & Acanthaceae \\
\hline minu we'e & $? \mathrm{Lr}$ & $4035 A$ & Litosanthes biflora \\
\hline moha & $\mathrm{Mi}, \mathrm{Kb}, \mathrm{Lw} ; \mathrm{KO}$ & $\mathrm{O}$ & tree, in forest \\
\hline mojoru & Ak & $\mathrm{O}$ & = kúnjuru \\
\hline 'mojura & $\mathrm{L}$ & RV & =kúnjuru \\
\hline mòlu & $\mathrm{Ll}, \mathrm{Lr}$ & RV & ?Rhaphidophora \\
\hline mòlu mane & $\mathrm{L}$ & RV & Costus speciosus \\
\hline mòlu manu & $\mathrm{Ll}, \mathrm{Tt}$ & 4132 & Pothos rumphii \\
\hline monga & $\mathrm{Kb}$ & $\mathrm{O}$ & vine, clings to trees \\
\hline monjura & $\mathrm{Mb} ; \mathrm{Ww}$ & $\mathrm{O}$ & = kúnjuru \\
\hline moras & $\mathrm{Tt}$ & V & Morus australis \\
\hline ?móro I & $\mathrm{Rn}$ & 3880 & Euphorbia prostrata \\
\hline móro II & ES & 4278 & cf. Omphalea \\
\hline móro III & Ww & 4025 & Flacourtia nukan \\
\hline móro kódi & $\mathrm{Lr}$ & 4003 & Cladogynos orientalis \\
\hline móro lóngge I & $\mathrm{Lr}, \mathrm{Tt}$ & 4250 & Exocarpus latifolius \\
\hline móro lóngge II & Ww & 4028 & Weinmannia blumei \\
\hline móro parìnggi & $\mathrm{Lr}$ & 4071 & Psilotum complanatum \\
\hline móro réwa & $\mathrm{Ll}, \mathrm{Tt}$ & 4128 & Phyllanthus amarus \\
\hline móro wuni & Ww & OK & = ka'ndulangu \\
\hline mosa & Ak & $\mathrm{O}$ & $=$ mahu \\
\hline moza & Ww & $\mathrm{O}$ & $=$ mahu \\
\hline 'muara & $\mathrm{Rn}$ & 4145 & Psoralea camborensis \\
\hline mudi & Ak & $\mathrm{O}$ & $=$ mundi \\
\hline muku & $\mathrm{Kb}$ & OK & = kalú \\
\hline 'mulungu & $\mathrm{Kr}$ & RV & Tetrastigma \\
\hline munde & Ww, Ko & RV & $=$ mundi \\
\hline munde bokólo & Ko & RV & = jàriku 'tobungu \\
\hline mundi & $\mathrm{Mi}, \mathrm{Kb}, \mathrm{Mb}$ & OK & Citrus \\
\hline mùne & Ko & RV & $=$ karipi \\
\hline múru kapuka & $\mathrm{Kb}$ & $\mathrm{PH}$ & Sorghum plumosum \\
\hline múru ma'ngondingu & $\mathrm{Kb}$ & OK & vine; medicinal \\
\hline múru nipa & $\mathrm{Kb}$ & OK & tree; used medicinally \\
\hline múru njara & $\mathrm{Rn}$ & 4165 & Enicostemma axillaris \\
\hline múru tamihiku & $\mathrm{Kb}$ & OK & shrub, small, on the plains \\
\hline múru wé & $\mathrm{Rn}$ & 4162 & Christia \\
\hline nába & Lr, Ko & 3931 & Canyotamitis \\
\hline
\end{tabular}




\begin{tabular}{|c|c|c|c|}
\hline nága & $\mathrm{L}$ & RV & $=$ nangga \\
\hline nága 'lukuta & $\mathrm{L}$ & RV & $=$ harakaya \\
\hline naho & Ko & $\mathrm{O}$ & $=$ nahu II \\
\hline nahu I & $\mathrm{Kb}$ & OK & Amorphophallus campanulatus \\
\hline nahu II & $\mathrm{Mi}, \mathrm{Kb}, \mathrm{Lw}$ & $\mathrm{O}$ & cf. Palaquium \\
\hline nalo & WS & RV & $=$ nara \\
\hline nangga & Sumba & RV & Artocarpus integer \\
\hline nara & $\mathrm{Rn}, \mathrm{Kr}, \mathrm{Kb}$ & RV & Themeda ?villosa \\
\hline naripa & Ww & $\mathrm{O}$ & $=$ 'neripu \\
\hline naripu & $\mathrm{Mi}$ & $\mathrm{O}$ & = 'neripu \\
\hline naripyo & Ko & $\mathrm{O}$ & = 'neripu \\
\hline narupu & Lw & $\mathrm{O}$ & = 'neripu \\
\hline nasu & $\mathrm{Ak}, \mathrm{Mb}$ & $\mathrm{O}$ & $=$ nahu I \\
\hline natu & Ak; Ww & $\mathrm{O}$ & $=$ nahu II \\
\hline nazo & Ww & $\mathrm{O}$ & $=$ nahu I \\
\hline ndàbo & Ww, Tt & 4024 & Pothomorphe subpeltata \\
\hline ndagha & Ko & RV & = kandangu hàmu \\
\hline ndaica & Lw & $\mathrm{O}$ & $=$ ndeha \\
\hline ndana & Ww & $\mathrm{O}$ & = nítu \\
\hline ndángi & $\mathrm{L}$ & RV & $=$ kahala wau \\
\hline ndàru & $\mathrm{ESb}$ & OK & Caesalpinia ?bonduc \\
\hline 'ndatara & Ww, Lr, Ko & $3974,(4241)$ & Schefflera elliptica \\
\hline ndaii & $\mathrm{Kb}$ & $\mathrm{O}$ & ?Fungi; cp. wulu ndaü I \\
\hline ndauluku & $\mathrm{Mi}, \mathrm{Kb}$ & $\mathrm{O}$ & Piper \\
\hline ndeha & Mi, Kb; Ko & $\mathrm{PH}$ & Panicum repens \\
\hline ndei & Ww & 4045 & Hypolytrum \\
\hline ndeiza & Ww & RV & $=$ ndeha \\
\hline ndelo & Ll, Lr, Ko & $\mathrm{RV}$ & = kadúru \\
\hline ndelo káka & Ww & 4043 & Ardisia \\
\hline nderi & $\mathrm{Kb}$ & OK & $=\mathrm{kulu}$ \\
\hline ndesa & $\mathrm{Mb}$ & $\mathrm{O}$ & $=$ ndeha \\
\hline ndia & $\mathrm{Kb}$ & OK & Ipomoea pes-caprae \\
\hline ndimu & Ko & $\mathrm{O}$ & $=$ nggaï \\
\hline ndongu & $\mathrm{Kb}$ & W, OK & Ceriops \\
\hline ndore & Ko & $\mathrm{O}$ & $?=$ ndúma kawini \\
\hline ndúma & Mi, Kb; Ko & $\mathrm{O}$ & = ndúma kawini \\
\hline ndúma kawini & $\mathrm{Rn}$ & 3948 & Amorphophallus \\
\hline ndúma míni & $\mathrm{Kr}$ & $\mathrm{RV}$ & Amorphophallus \\
\hline nepi & $\mathrm{Kb}$ & OK & epiphyte on trees \\
\hline 'neripu & $\mathrm{Kb}$ & $\mathrm{O}$ & Poaceae \\
\hline ngae & $\mathrm{Ll}, \mathrm{Lr}$ & RV & $=$ langaha \\
\hline 'ngaingo & $\mathrm{Ll}, \mathrm{Ww}, \mathrm{Tt}$ & OK & $=$ witu \\
\hline ngàndu kàriku & $\mathrm{Kb}$ & 3884 & Martynia annua \\
\hline
\end{tabular}




\begin{tabular}{|c|c|c|c|}
\hline nggai & $\mathrm{Kr}, \mathrm{Kb}$ & RV & Flagellaria indica \\
\hline nggai rídu & $\mathrm{Kb}$ & $\mathrm{O}$ & rattan, very thin \\
\hline nggaï & $\mathrm{ESb}$ & $\mathrm{D}, \mathrm{H}$ & Timonius timon \\
\hline nggambira & $\mathrm{Mb}$ & $\mathrm{O}$ & $=$ nggamuru \\
\hline nggambiryo & Ko & $\mathrm{O}$ & $=$ nggamuru \\
\hline nggamuru & $\mathrm{Mi}, \mathrm{Kb}, \mathrm{Lw}$ & $\mathrm{O}$ & Uncaria gambir \\
\hline nggánda & Ko & RV & $=$ mbaku haü I \\
\hline nggángga & $\mathrm{Ll}, \mathrm{Lr}$ & RV & $=$ mbaku haü I \\
\hline ngganja & $\mathrm{Kr}$ & RV & $=$ ngganju \\
\hline ngganju & $\mathrm{Kb}$ & RV & Canna coccinea \\
\hline nggano & Ww, Lr & RV & $=$ ngganju \\
\hline nggaü & Ko & RV & $=$ manggaü II \\
\hline 'nggoaka & $\mathrm{Kr}$ & $\mathrm{dft}$ & $=$ nggoka \\
\hline nggoka & ESb & OK & Aglaia I \\
\hline nggòko & Ko & W & ?Morinda citrifolia \\
\hline nggole & $\mathrm{Ww}, \mathrm{Tt}$ & 4021 & $=$ remi kawini \\
\hline nggule & Ww & $\mathrm{O}$ & $=$ wala 'mbunguru \\
\hline ngingnyo & Ko & $\mathrm{RV}$ & $=$ witu \\
\hline ngiu (ngio) & Ko & W & $=$ 'kokuru \\
\hline ngoka & ?WS & $\mathrm{dft}$ & Chisocheton (cp. nggoka) \\
\hline ngùdu wawi & $\mathrm{L}$ & 4016 & Pittosporum moluccanum \\
\hline ngura wawi & $\mathrm{L}$ & $\mathrm{RV}$ & = kùru wawi \\
\hline ní & $\mathrm{Mb}$ & W & = 'kokuru \\
\hline nipa & $\mathrm{Kb}$ & $\mathrm{O}$ & $?=$ Nypa fruticans \\
\hline nítu & $\mathrm{Mi}, \mathrm{Kb}, \mathrm{Lw}$ & $\mathrm{D}, \mathrm{OK}$ & Santalum album \\
\hline njángi & ES & $\mathrm{H}$ & $=$ kanjangi \\
\hline njati & Sumba & OK & Tectona grandis \\
\hline 'nundangu & $\mathrm{Mi}, \mathrm{Kb}$ & $\mathrm{D}$ & $=$ kanjangi \\
\hline nundi & $\mathrm{Mb}$ & $\mathrm{O}$ & = rútu II \\
\hline núnu & Ak & $?$ & $=$ tariwangu \\
\hline nu'u & Ww, Lr & W & $=$ 'kokuru \\
\hline nyariku & Ak & $\mathrm{O}$ & = 'neripu \\
\hline ?nyou & WS & $\mathrm{H}$ & $=$ 'kokuru \\
\hline ode & $\mathrm{Ll}, \mathrm{Lr}, \mathrm{Tt}$ & RV & = úndi \\
\hline odi & Ak & $\mathrm{O}$ & = úndi \\
\hline òkura & $\mathrm{Ll}, \mathrm{Ww}, \mathrm{Lr}$ & $\mathrm{H}$ & $=$ hakuru \\
\hline onde & Ww, Ko & RV & $=$ úndi \\
\hline ondi & $\mathrm{Mb}$ & $\mathrm{O}$ & $=$ úndi \\
\hline onggalu & Ko & W & $=\mathrm{au}$ \\
\hline oru & $\mathrm{Mb}$ & $\mathrm{O}$ & ?Bambuseae \\
\hline ou & $\mathrm{Mb}$ & $\mathrm{O}$ & $=\mathrm{au}$ \\
\hline owa & $\mathrm{Kb}$ & OK & = 'uowa \\
\hline pàda & $\mathrm{Ak}, \mathrm{Wk}$ & $\mathrm{O}$ & $=$ uri \\
\hline
\end{tabular}




$\begin{array}{llll}\text { padàmu } & \mathrm{ESb} & \mathrm{RV} & \text { Ricinus communis } \\ \text { padàmu humba } & \mathrm{Kr}, \mathrm{Kb} & \mathrm{RV} & \text { Jatropha curcas } \\ \text { pádu bola } & \mathrm{L} & \mathrm{RV} & \text { = lamata } \\ \text { paghu } & \mathrm{Lr}, \mathrm{Ko} & \mathrm{RV} & =\text { paü } \\ \text { pahaura } & \mathrm{Kr} & 3940,4002 & \text { Phyllanthus reticulatus } \\ \text { pahawura I } & \mathrm{Kb} & 3906 & \text { Phyllanthus hypericifolius } \\ \text { pahawura II } & \mathrm{Kb} & 3891 & \text { Breynia racemosa } \\ \text { pahìndu } & \mathrm{Kr} & \mathrm{RV} & =\text { kahindu } \\ \text { pahìndu ka'rambua } & \mathrm{Kr} & 3878 & \text { Sida parvifolia } \\ \text { paita } & \mathrm{Rn}, \mathrm{Kb} & \mathrm{RV} & \text { Momordica charantia } \\ \text { paku } & \mathrm{Ak}, \mathrm{Mb} & \mathrm{O} & =\text { paï } \\ \text { pàlo } & \mathrm{Ko} & \mathrm{O} & =\text { polu } \\ \text { pàlu } & \mathrm{Ak} & \mathrm{O} & =\text { polu } \\ \text { palu bola } & \mathrm{Tt} & \mathrm{V}, \mathrm{YD} & =\text { lamata } \\ \text { pamuhu } & \mathrm{Kb} & \mathrm{OK} & \text { tree, coastal; fine timber } \\ \text { pànda I } & \mathrm{Kb} & \mathrm{OK} & =\text { pànda ndima } \\ \text { pànda II } & \mathrm{Mi}, \mathrm{Lw}, \mathrm{Mb} ; \mathrm{Lr}, \mathrm{Ko} & \mathrm{RV} & =\text { uri } \\ \text { pànda dawa } & \mathrm{Ko} & \mathrm{RV} & =\text { pànda ndima } \\ \text { pànda jawa } & \mathrm{Kb} & \mathrm{H} & =\text { pànda ndima } \\ \text { pànda làma woya } & \mathrm{Ko} & \mathrm{RV} & =\text { rami I } \\ \text { pànda ndima } & \mathrm{Lr} & \mathrm{RV} & \text { Ananas comosus } \\ \text { pàndangu } & \mathrm{Mi}, \mathrm{Kb}, \mathrm{Lw} & \mathrm{OK} & =\text { uri } \\ \text { pandéra } & \mathrm{Ww}, \mathrm{Lr} & \mathrm{RV} & =\text { 'paita } \\ \text { paneta } & \mathrm{Ww}, \mathrm{Lr}, \mathrm{Ko} & \mathrm{RV} & =\text { pa'netangu } \\ \text { pa'netangu } & \mathrm{Kr}, \mathrm{Kb} & 4233 & \text { Anamirta cocculus } \\ \text { pa'niatangu } & \mathrm{Rn} & \mathrm{RV} & =\text { pa'netangu } \\ \text { pàpa } & \mathrm{Kb} & \mathrm{OK} & \text { Acrostichum } \\ \text { parau 'ramingu } & \mathrm{Kr} & \mathrm{RV} & \text { ?= kanjangi } \\ \text { pare } & \mathrm{WSb} & \mathrm{O} & =\text { uhu } \\ \text { pare kadito } & \mathrm{Tt} & \mathrm{V}, \mathrm{YD} & =\text { uhu kadita } \\ \text { pari } & \mathrm{ESb} & \mathrm{W} & =\text { uhu } \\ \text { pàri } & \mathrm{Kb} & \mathrm{OK} & =\text { kandara I } \\ \text { paróna } & \mathrm{Ww}, \mathrm{Lr} & \mathrm{RV} & =\text { màja } \\ \text { paruona } & \mathrm{L} & \mathrm{RV} & =\text { màja } \\ \text { paruona patara } & \mathrm{L} & \mathrm{RV} & \text { Amaranthus spinosus } \\ \text { patola } & \mathrm{Kb} & \mathrm{OK} & =\text { karòbo pùti } \\ \text { patopia } & \mathrm{Ww} & \mathrm{O} & =\text { polu } \\ \text { pàtungu } & \mathrm{Mb} & \mathrm{OK} & =\text { polu } \\ \text { pau } & \mathrm{ESb} & \mathrm{H}, \mathrm{RV} & \text { Mangifera indica } \\ \text { pau maràda } & \mathrm{Rn} & 4159 & \text { Asteraceae } \\ \text { pau 'omangu } & \mathrm{Kr}, \mathrm{Kb} & \mathrm{OK} & \text { Mangifera } \\ \text { paü } & \mathrm{WSb} & \mathrm{H}, \mathrm{RV} & \text { Diplazium } \text { ?esculentum } \\ \text { paü karambo } & 4018 & \text { Cyathea glabra } \\ & & & \\ & & & \end{array}$




\begin{tabular}{|c|c|c|c|}
\hline pawu & $-w^{2}=$ & RV & $=$ paü \\
\hline pa'yejangu & $\mathrm{Kb}$ & 3907 & Cleome gynandra \\
\hline payeti & $\mathrm{Kb}$ & OK & Gnemon \\
\hline pèdi & $\mathrm{Rn} ; \mathrm{Ll}, \mathrm{Ww}, \mathrm{Lr}$ & RV & Ficus wassa var. obversifolia \\
\hline pèdi káka & $\mathrm{Ll}, \mathrm{Lr}$ & 4106 & Ficus ?heteropleura \\
\hline pèdi mète & $\mathrm{Lr}$ & RV & Ficus \\
\hline pèdi nggángga & $\mathrm{Lr}$ & 3922 & Streblus asper \\
\hline pelu & $\mathrm{Kr}$ & RV & Vitaceae \\
\hline pelu mánu & $\mathrm{Mi}, \mathrm{Kb}$ & $\mathrm{OK}$ & = karùpe II \\
\hline pìdi & $\mathrm{Kr}, \mathrm{Kb}$ & OK & $=$ pèdi \\
\hline ?pìdi kalauki & $\mathrm{Kr}$ & OK & Ficus \\
\hline pípi & $\mathrm{Kb}$ & OK & tree, coastal \\
\hline pirahu & Lw & $\mathrm{O}$ & = ka'pirihu \\
\hline 'pirihu & $\mathrm{Kb}$ & OK, O & = ka'pirihu \\
\hline piripyo & Ko & $\mathrm{O}$ & = ka'pirihu \\
\hline 'pitingu & $?$ & $?$ & plant w. burrs \\
\hline pitungu & $\mathrm{Kb}$ & OK & $=$ tàringu \\
\hline piuru I & Ak & $\mathrm{O}$ & = tàringu \\
\hline piuru II & $\mathrm{Kb}$ & $\mathrm{O}$ & Colocasia \\
\hline po & $\mathrm{Mb}$ & $\mathrm{O}$ & $=\mathrm{pau}$ \\
\hline poda I & Ak & $\mathrm{O}$ & $=$ púnda \\
\hline poda II & $\mathrm{L}$ & RV & $=$ uri \\
\hline póka & $\operatorname{Tr}$ & 4110 & Cardiospermum halicacabum \\
\hline poka poka & $\operatorname{Tr}$ & RV & = kapóka \\
\hline polahari & $\mathrm{Kb}$ & OK & ?Alyxia reinwardtii \\
\hline polìhu & Lw & $\mathrm{O}$ & $=$ 'pulihu \\
\hline polo & Ko & $\mathrm{O}$ & $=$ polu \\
\hline polu & Mi, Kb, Lw & OK & Bambuseae \\
\hline ponda & Lw; Ko & $\mathrm{O}$ & = púnda \\
\hline pònda & Ww & RV & $=$ uri \\
\hline pònda (n)dima & $\mathrm{L}$ & $\mathrm{H}$ & = pànda ndima \\
\hline pòto & Ww, Ko & OK & $=$ tàringu \\
\hline pòto katilu & $\mathrm{Lr}, \mathrm{Tt}$ & RV & $?=$ tàringu \\
\hline pou & $? \mathrm{Lr}$ & RV & $=\mathrm{pau}$ \\
\hline pou ùta & $? \mathrm{Lr}$ & RV & = kamàla pau \\
\hline poyo & Ko & OK & $=\mathrm{pau}$ \\
\hline pula kalémbi & $\mathrm{Rn}$ & RV & ?Pyrrosia \\
\hline 'pulihu & $\mathrm{Mi}, \mathrm{Kb}$ & OK & tree, young l. gold coloured \\
\hline púnda & $\mathrm{Mi}, \mathrm{Kb}$ & OK & Syzygium \\
\hline 'puoku & $\mathrm{L}$ & RV & = wàla 'mbunguru \\
\hline púpu & $\mathrm{L}$ & 4142 & $\begin{array}{l}\text { Stephania japonica var. } \\
\text { timorensis }\end{array}$ \\
\hline puti & $?$ & $\mathrm{H}$ & Parkia speciosa \\
\hline
\end{tabular}




\begin{tabular}{|c|c|c|c|}
\hline pùtika & Ww, Lr, Tt & 4029 & ?Peristylis \\
\hline rábuka & Ww & 4139 & = mbúlungu kawuku \\
\hline ràdapa & $\mathrm{Lr}, \mathrm{Tt}$ & RV & $=$ walakeri \\
\hline rágata & $\mathrm{L}$ & 4119 & Nasturtium \\
\hline rambútan & $\mathrm{Rn}$ & RV & Passiflora ?foetida \\
\hline rame & Ll, Lr, Ko & RV & $=$ rami II \\
\hline rami I & $\mathrm{Rn}, \mathrm{Kr}, \mathrm{Kb}$ & RV & Agave sisalana \\
\hline rami II & $\mathrm{ESb}$ & 3946 & Pipturus \\
\hline rànga & $\mathrm{Mi}, \mathrm{Rn}$ & RV & $=$ rongu \\
\hline rangga & $\mathrm{Kb}$ & 3881 & Acacia farnesiana \\
\hline ràngu & $\mathrm{Mb}$ & $\mathrm{O}$ & $=$ rongu \\
\hline rànu I & Ko & RV & = lùri I \\
\hline rànu II & Ko & RV & = 'wakihu \\
\hline ràpu & Mb; Ko & RV & $=$ katàbi \\
\hline raü & $\mathrm{Kb}$ & OK & marine plant; edible \\
\hline rèdapa & Ww & RV & $=$ walakeri \\
\hline reha & $\mathrm{Kb}$ & OK & $=$ kareha \\
\hline remi & $\mathrm{Rn}$ & 4150 & $=$ rami II \\
\hline remi kawini & $\mathrm{Rn}$ & 4156 & Pogostemon cablin \\
\hline renu & $\mathrm{Tt}$ & V, YD & ?Pandanaceae \\
\hline ridi wówo & $\mathrm{L}$ & RV & = kapàra luku \\
\hline 'rienu & $\mathrm{L}$ & RV & $=$ renu \\
\hline rindi ghogho I & Ww, Lr & RV & = kapàra luku \\
\hline rindi ghogho II & $\mathrm{Lr}$ & RV & $=$ ghasu ghula \\
\hline rindi pare & Ko & RV & = kapàra luku \\
\hline rita I & Sumba & RV & Alstonia scholaris \\
\hline rita II & $\mathrm{Mb}$ & $\mathrm{O}$ & = rútu I \\
\hline ríti kàngga & Ww, Lr & 4088,4243 & Tabernaemontana orientalis \\
\hline ritya & Ko & RV & $=$ rita I \\
\hline ?riut otan & ES & $\mathrm{dft}$ & Albizia lebbeck \\
\hline robasa kabála & $\mathrm{L}$ & 4112 & Bridelia tomentosa \\
\hline ròbo & Ko & RV & = kamàla 'wataru \\
\hline ròngo I & Ww, Lr, Ko & RV & $=$ rongu \\
\hline ròngo II & Ww & $\mathrm{O}$ & $=$ kopa lua \\
\hline ròngo matomba & Ww & RV & $=$ rongu \\
\hline ròngo pi'a & $\mathrm{Lr}$ & RV & $=$ rongu \\
\hline rongu & $\mathrm{Kb}$ & OK & Bombax ceiba \\
\hline ròpu & Ww, Lr & RV & = katàbi \\
\hline rota & ESb; Ww, Ko & RV & Semecarpus \\
\hline rówa I & $\mathrm{Ll}, \mathrm{Lr}$ & RV & = 'uowa \\
\hline rówa II & $\mathrm{Lr}$ & D & $=$ kambala \\
\hline 'ruata & $\mathrm{Rn}$ & RV & $=$ rota \\
\hline rumba jawa & $\mathrm{Kb}$ & OK & Panicum maximum \\
\hline
\end{tabular}




\begin{tabular}{|c|c|c|c|}
\hline ?rumba mapu & & $\mathrm{H}$ & = wulu ndaü II \\
\hline rumba míni & $\mathrm{Kb}$ & $\mathrm{H}, \mathrm{PH}$ & Paspalidium flavidum \\
\hline rumba penji & ES & $\mathrm{H}$ & $=$ ka'ndaüngu \\
\hline rumba randi & $\mathrm{Rn}$ & $\mathrm{PH}$ & Echinochloa colonum \\
\hline rumba rara I & $\mathrm{Kb}$ & $\mathrm{PH}$ & Eulalia (fulva) \\
\hline rumba rara II & $\mathrm{Kb}$ & RV & = móro I \\
\hline rumba rendi & $\mathrm{Kb}$ & 3870 & $=$ rumba randi \\
\hline rumba wau & $\mathrm{Kr}$ & RV & Ageratum conyzoides \\
\hline rùmba wú 'teyo & Ko & RV & $=$ rumba wau \\
\hline rùngu rutuhu & $\mathrm{Rn}$ & 4151 & Euphorbia hypericifolia \\
\hline 'routa & $\mathrm{L}$ & RV & $=$ rota \\
\hline ruruka & $\mathrm{Mb}$ & $\mathrm{O}$ & $=$ 'rurungu \\
\hline 'rurungu & $\mathrm{Mi}, \mathrm{Kb}$ & OK & Heritiera littoralis (cj.) \\
\hline ruta bai & ?MI & 4211 & Acalypha indica \\
\hline ruta mète & $\operatorname{Tr}$ & RV & Borreria \\
\hline ruta wùni I & $\mathrm{Ll}, \mathrm{Tr}$ & 4130 & $=$ rùngu rutuhu \\
\hline ruta wùni II & $\operatorname{Tr}$ & RV & $=$ ruta mète \\
\hline rútu I & $\mathrm{Mi}, \mathrm{Kb}, \mathrm{Lw}$ & 4228 & Albizia lebbekoides \\
\hline rútu II & $\mathrm{Kb}$ & OK & Citrus \\
\hline rútu III & Ko & W & $=$ kuta I \\
\hline ruwa kapu & $\mathrm{Kr}$ & RV & = karua kapu \\
\hline saboli & Ak & $\mathrm{O}$ & $=$ hambuli \\
\hline sadana & Ak & $\mathrm{O}$ & = nítu \\
\hline sàgi & Ak & $\mathrm{O}$ & = wàla hànggi \\
\hline sakura & $\mathrm{Mb}$ & $\mathrm{O}$ & $=$ hakuru \\
\hline salai & $\mathrm{Mb}$ & $\mathrm{O}$ & = halí \\
\hline salau & Ak & $\mathrm{O}$ & = halí \\
\hline saluri & Ak & $\mathrm{O}$ & $?=$ uri \\
\hline samboli & $\mathrm{Mb}$ & $\mathrm{O}$ & $=$ hambuli \\
\hline samoi & $\mathrm{Mb}$ & $\mathrm{O}$ & $=$ hamui \\
\hline sandana & $\mathrm{Mb}$ & $\mathrm{O}$ & = nítu \\
\hline sànggi & $\mathrm{Mb}$ & $\mathrm{O}$ & = wàla hànggi \\
\hline sawi & $\mathrm{Ak}, \mathrm{Mb}$ & $\mathrm{O}$ & $=$ hewi \\
\hline sedí & $\mathrm{L}$ & RV & = hîkilu \\
\hline 'seduka & $\mathrm{Mb}$ & $\mathrm{O}$ & = 'hidiku \\
\hline 'seduku & Ak & $\mathrm{O}$ & = 'hidiku \\
\hline 'sekura & $\mathrm{L}$ & RV & = hîkilu \\
\hline 'sekuru & Ak & $\mathrm{O}$ & = hîkilu \\
\hline selu & Ak & $\mathrm{O}$ & $=$ hili bara \\
\hline 'sikura & $\mathrm{Tt}$ & V, YD & = hîkilu \\
\hline 'sikuru & Ak & $\mathrm{O}$ & $=$ hakuru \\
\hline sili & $\mathrm{Mb}$ & $\mathrm{O}$ & $=$ hili bara \\
\hline sirikaya & $\mathrm{Mb}$ & $\mathrm{O}$ & = harakaya \\
\hline
\end{tabular}




\begin{tabular}{|c|c|c|c|}
\hline suarani & Ak & OK & $=$ hureni \\
\hline suli wé & $\mathrm{Mb}$ & $\mathrm{O}$ & $=$ tarúbu \\
\hline surani & Ak & $\mathrm{O}$ & $=$ hureni \\
\hline susu nana & $\operatorname{Tr}$ & 4116 & Sida javensis ssp. javensis \\
\hline suwa & $\mathrm{Ak}, \mathrm{Mb}$ & $\mathrm{D}$ & $=$ huwa \\
\hline ta kalara & Ko & $\mathrm{RV}$ & $=$ kalara (kuta) \\
\hline tabàka & $\mathrm{Mi}, \mathrm{Kb}, \mathrm{Lw}$ & OK & tree $w$. curled 1. \\
\hline tabàla & $\mathrm{Ak}$ & $\mathrm{O}$ & = katíti \\
\hline taboku & Ak & $\mathrm{O}$ & $=$ tambuki \\
\hline tàbu & $\mathrm{Ml}, \mathrm{Kr}, \mathrm{Np}$ & $\mathrm{RV}$ & $=$ tíbu \\
\hline tàbu hala & $\mathrm{Kr}$ & $\mathrm{RV}$ & $=$ tibu hala \\
\hline tabùka & $\mathrm{Rn}, \mathrm{Kb}$ & 4179 & Excoecaria agallocha \\
\hline tàda ka'pihaku & $\mathrm{Kr}$ & 3959 & Macaranga \\
\hline tàda katàbi & $\mathrm{Kr}$ & $\mathrm{dft}$ & Pygeum/Prunus \\
\hline tàda múru & $\mathbf{K r}$ & $\mathrm{dft}$ & Syzygium \\
\hline tada wero & Ak & 0 & = yú karambua \\
\hline taghu reda & $\mathrm{Lr}$ & RV & $=$ kapu ndala II \\
\hline tai babu I & $\mathrm{R}, \mathrm{Kb}$ & 3900 & Abutilon auritum \\
\hline tai babu II & $\mathrm{Rn}$ & 3901 & $\begin{array}{l}\text { Abutilon indicum ssp. albeseens } \\
\text { var. australiense }\end{array}$ \\
\hline tai bahi & $\mathrm{Kb}$ & $\mathrm{O}$ & shrub \\
\hline tai monga & $\mathrm{Kb}$ & $\mathrm{O}$ & $=$ tai 'muanga \\
\hline tai 'muanga & $\mathrm{Rn}$ & 4149 & Cucurbitaceae \\
\hline 'taiko malawo & $\mathrm{Ll}, \mathrm{Ww}$ & 4107,4031 & Geniostoma rupestre \\
\hline 'taiko ndara & $\mathrm{Mb} ; \mathrm{Ww}$ & $\mathrm{RV}$ & = kapuwa hambaku \\
\hline 'taiko sapi I & $\mathrm{L}$ & 4124 & Synedrella nodiflora \\
\hline 'taiko sapi II & $\mathrm{L} \mathbf{l}$ & RV & = ruta mète \\
\hline 'taiko zapi & $\mathrm{Lr}$ & RV & $=$ ruta mète \\
\hline talàhu & Mi & $\mathrm{O}$ & $=$ talíhu \\
\hline tali rara & $\mathrm{Lr}, \mathrm{Tt}$ & 3942 & Combretum latifolium \\
\hline talihu & $\mathrm{Kb}$ & OK & Terminalia catappa \\
\hline tambaku & $\mathrm{Mb}$ & $\mathrm{O}$ & $=$ hambaku \\
\hline tambàla & $\mathrm{ESb}$ & OK & $=$ katíti \\
\hline tambèdala I & Ww & 4034 & Tetrastigma papillosum \\
\hline tambèdala II & Ww & 4038 & Cissus adnata \\
\hline tambele & Ko & $\mathrm{O}$ & = katíti \\
\hline tamboka & $\mathrm{Mb}$ & $\mathrm{O}$ & $=$ tambuki \\
\hline tamboku & Lw & $\mathrm{O}$ & $=$ tambuki \\
\hline tambuki & $\mathrm{Mi}, \mathrm{Rn}, \mathrm{Kb}$ & OK & Ipomoea aquatica \\
\hline tambúlu & $\mathrm{Kr}$ & $\mathrm{dft}$ & Glochidion \\
\hline tambùra I & $\mathrm{Kr}$ & $\mathrm{dft}$ & ?= bakara wai \\
\hline tambùra II & ES & $\mathrm{dft}$ & Cleidion javanicum \\
\hline tàme & $\mathrm{Ww}, \mathrm{Lr}$ & OK & $=$ ta'miangu \\
\hline
\end{tabular}




\begin{tabular}{|c|c|c|c|}
\hline ta'meangu & ES & dft & ?Pometia tomentosa \\
\hline tamia & Ko & $\mathrm{O}$ & $=$ ta'miangu \\
\hline $\begin{array}{l}\text { ta'miangu } \\
\text { taminu }\end{array}$ & $\mathrm{ESb}$ & $\begin{array}{l}\mathrm{RV}, \mathrm{OK} \\
\mathrm{H}\end{array}$ & $\begin{array}{l}\text { Schizostachyum blumii } \\
=\text { wunga III }\end{array}$ \\
\hline tàmu & Ko & RV & $=$ tòmu \\
\hline tánda 'malangu I & $\mathrm{Rn}, \mathrm{Kr}, \mathrm{Kb}$ & RV & Cassia \\
\hline tánda 'malangu II & $\mathrm{Kr}, \mathrm{Kb}$ & RV & Phyllanthus \\
\hline tánda 'malangu III & $\mathrm{Kr}$ & 3908 & Cassia tora \\
\hline tandala pua & $\mathrm{Kr}$ & 3949 & Vernonia wetarensis \\
\hline tande'i & $\mathrm{Ll}, \mathrm{Ww}, \mathrm{Lr}$ & 3963 & = 'litangu \\
\hline tandùla pua I & $\mathrm{Rn}$ & 4171 & Leucas flaccida \\
\hline tandùla pua II & $\mathrm{Rn}$ & RV & Wedelia ?urticaefolia \\
\hline ta'ngananga & $\mathrm{Mb}$ & $\mathrm{O}$ & $=$ ta'nganangu \\
\hline ta'nganangu & $\mathrm{ESb}$ & OK & Ficus \\
\hline tangára lòdo & $\mathrm{L}$ & RV & Clerodendrum \\
\hline tàpi & $\mathrm{Mb}$ & $\mathrm{O}$ & $=$ uri \\
\hline tàpu & $\mathrm{Mi}, \mathrm{Kb}, \mathrm{Lw}$ & $\mathrm{O}$ & Pandanaceae \\
\hline tapuha & $\mathrm{Mi}, \mathrm{Kb}, \mathrm{Lw}$ & OK & = katíti \\
\hline tapusa & $\mathrm{Ml}, \mathrm{Ak}$ & $\mathrm{O}$ & = katíti \\
\hline tara kàha & Ko & RV & $=$ tara maròmba \\
\hline tara 'kaito I & $\mathrm{Lr}$ & 3990 & Streblus \\
\hline tara 'kaito II & $\mathrm{Lr}$ & $\mathrm{D}, \mathrm{H}$ & $=$ kalangga I \\
\hline tara kuku mète & Ww & 4072 & Toddalia asiatica \\
\hline tara manu & $\mathrm{Kr}, \mathrm{Kb}$ & 3978 & Capparis pubiflora \\
\hline tara maràmba & $\mathrm{Lr}$ & RV & $=$ tara maròmba \\
\hline tara maròmba & Ww & RV & Mimosa invisa \\
\hline tara we'e & $\mathrm{Kr} ; \mathrm{Ll}, \mathrm{Ww}$ & RV & = kakaha míni \\
\hline tara wèri & Ww & 4062 & Rubus rosaefolius \\
\hline tara wìri lolo & $\mathrm{Ll}$ & 4066 & Rubus moluccanus var. discolor \\
\hline tarebu & Lw & $\mathrm{O}$ & $=$ tarúbu \\
\hline tàringu & $\mathrm{Mi}, \mathrm{Rn}, \mathrm{Kb}, \mathrm{Tb}$ & $\mathrm{RV}, \mathrm{O}$ & Dendrocalamus asper \\
\hline tariwangu & $\mathrm{Mi}, \mathrm{Kb}$ & RV & Annona squamosa \\
\hline tarúbu & $\mathrm{Mi}, \mathrm{Kb}$ & OK, RV & ?Alocasia \\
\hline tawai & $\mathrm{Kr}$ & RV & Cordia ?dichotoma \\
\hline tawu & Ww & RV & = katíti \\
\hline tebu & ES & $\mathrm{dft}$ & Helicia \\
\hline tebu bara & ES & $\mathrm{dft}$ & Ilex \\
\hline tei rara & Ko & RV & $=$ tali rara \\
\hline tera & $\mathrm{Lw}, \mathrm{Ak}, \mathrm{Mb}$ & $\mathrm{D}, \mathrm{OK}$ & Artocarpus elasticus \\
\hline 'terepa & $\mathrm{Ll}, \mathrm{Ww}, \mathrm{Lr}$ & RV & $\begin{array}{l}=\text { tera (D. Artocarpus } \\
\text { communis) }\end{array}$ \\
\hline 'terepo & Ko & $\mathrm{O}$ & $=$ tera \\
\hline tèringu & $\mathrm{Tm}$ & W & $=$ tàringu \\
\hline
\end{tabular}




\begin{tabular}{|c|c|c|c|}
\hline tíbu & $\mathrm{Kb}, \mathrm{Ak}$ & $\mathrm{O}$ & Saccharum officinarum \\
\hline tìbu & $\mathrm{Rn}$ & RV & $=$ tibu \\
\hline tìbu dàlu & $\mathrm{L}$ & RV & $=$ kandàlu \\
\hline tíbu hala & $\mathrm{Rn}, \mathrm{Kb}$ & RV & Saccharum arundinaceum \\
\hline 'tiemu & $\mathrm{L}$ & 4114 & Rhynchospora corymbosa \\
\hline túmbi tàda & $\mathrm{Kb}$ & $\mathrm{O}$ & water plant, tuberous \\
\hline timbu & $\mathrm{Lr}$ & $\mathrm{D}$ & =nggaï \\
\hline timbu tèke & $\mathrm{Lr}, \mathrm{Tt}$ & RV & = ha'njokaru tàki \\
\hline timo & Ww & $\mathrm{O}$ & = nggaï \\
\hline timu & $\mathrm{Mb}, \mathrm{Ak} ; \mathrm{Ll}, \mathrm{Ko}, \mathrm{Tt}$ & $\mathrm{O}$ & = nggaï \\
\hline timu tèke I & $\mathrm{Ll}$ & RV & $=$ ha'njokaru tàki \\
\hline timu tèke II & Ww & 4019 & Leea indica \\
\hline tíra & $\mathrm{Mi}, \mathrm{Kb}$ & OK & $=$ tera \\
\hline 'tiringu & $\mathrm{Kb}, \mathrm{Lw}$ & OK & $=$ tàringu \\
\hline tòbu & $\mathrm{Lw}, \mathrm{Ww}, \mathrm{Lr}, \mathrm{Tt}$ & OK & $=\mathrm{tibu}$ \\
\hline tòdola & $\mathrm{Ll}$ & 4140 & Eranthemum \\
\hline tòmu & $\mathrm{Ll}, \mathrm{Ww}$ & RV & Globba marantina \\
\hline toro & $\mathrm{Ww}, \mathrm{Tt}$ & OK & = kandúru \\
\hline toro bòngga & Ww & RV & = kandúru ahu \\
\hline toro danggalasa & $\mathrm{Ll}$ & RV & = ambalaï \\
\hline toro danggalaza & $\mathrm{Lr}$ & RV & = ambalaï \\
\hline toro ka'mauta & Ll, Ww, Ko & RV & = kandúru ahu \\
\hline toro lae & Tt & YD, V & = ambalaï \\
\hline toro manggaláwa & Ko & RV & = ambalaï \\
\hline toro pàdu & Ko & RV & $=$ toro wùli \\
\hline toro pòdu & $\mathrm{Lr}$ & RV & = kandúru ahu \\
\hline toro wùli & $\mathrm{Lr}$ & 4085 & Solanum torvum \\
\hline toru & $\mathrm{Tb}$ & W & = kandúru \\
\hline towi & $\mathrm{Mb}$ & $\mathrm{O}$ & $=$ tui \\
\hline toyu & Lw & $\mathrm{O}$ & $=$ tui \\
\hline tudu lawa & $\operatorname{Tr}$ & 4131 & $=$ tandala pua \\
\hline tugha & Ko & $\mathrm{O}$ & $=$ tuwa \\
\hline tugha pare & $\mathrm{Lr}$ & 3979 & Phylacium bracteosum \\
\hline tui & $\mathrm{Mi}, \mathrm{Kb}$ & OK & ?Radermachera \\
\hline tùmbu dàba & $\mathrm{Kr}$ & dft & Neonauclea excelsa \\
\hline tún & Kn, Lw & $\mathrm{W}, \mathrm{O}$ & = kandúru \\
\hline túru 'paita & ?Kb & $\mathrm{O}$ & Solanum \\
\hline 'turuku tana & $\mathrm{Kb}$ & 3850 & Boerhavia \\
\hline tuwa & ESb; Ww & RV & Derris ?elliptica \\
\hline tuwa' & $\mathrm{Tt}$ & V, YD & $=$ tuwa \\
\hline tuwa lati & $\mathrm{Kb}$ & $\mathrm{O}$ & plant; used for poisoning fish \\
\hline tuwa pare & $\mathrm{Tt}$ & YD & tree, large, in damp soil \\
\hline tuwa wewarungu & $\mathrm{Kb}$ & $\mathrm{O}$ & plant; used for poisoning fish \\
\hline
\end{tabular}




\begin{tabular}{|c|c|c|c|}
\hline ú & $\mathrm{Mb}$ & OK & $=\mathrm{au}$ \\
\hline ua réwa & $\mathrm{L} \mathbf{l}$ & RV & = pula kalémbi \\
\hline uasa & $\mathrm{Mb}$ & $\mathrm{O}$ & $=$ 'uwahu \\
\hline ughe & Ko & RV & $=$ iwi I \\
\hline ùghi & Ko & RV & = ìwi II \\
\hline uhu & $\mathrm{Rn}, \mathrm{Kr}, \mathrm{Kb}$ & $\mathrm{W}, \mathrm{H}$ & Oryza sativa \\
\hline uhu kadita & $\mathrm{Kb}$ & W & Oryza sativa f. glutinosa \\
\hline uhu kadito & $?$ & $\mathrm{H}$ & = usu kadita \\
\hline uhu kani & $\mathrm{Rn}$ & RV & $=$ uhu kanu \\
\hline uhu kanu & $\mathrm{Kb}$ & W & Setaria italica \\
\hline uhu manginu I & $\mathrm{Kr}, \mathrm{Kb}$ & $\mathrm{PH}$ & Setaria laxa \\
\hline uhu manginu II & $\mathrm{Kb}$ & PH & = ka'ritaku I \\
\hline uhu nggangga & $\mathrm{Kb}$ & $\mathrm{O}$ & vine \\
\hline ùhu rendi & $\mathrm{Kl}$ & $\mathrm{PH}$ & $?=$ rumba randi \\
\hline ui I & $\mathrm{Mi}, \mathrm{Kr}, \mathrm{Lw}, \mathrm{Np}$ & OK, W & $=$ iwi I \\
\hline ui II & $\mathrm{Mi}, \mathrm{Lw}$ & $\mathrm{O}$ & $=$ ìwi II \\
\hline ùka & $\mathrm{Ll}, \mathrm{Lr}, \mathrm{Tt}$ & RV & $=$ huwa \\
\hline uli & $\mathrm{Ww}, \mathrm{Lr}$ & OK & $=$ hili bara \\
\hline uli bùla & $\mathrm{Ll}$ & RV & ?Xanthosoma nigrum \\
\hline uli máne & $\mathrm{L}$ & 4266 & Colocasia \\
\hline uli mète & $\mathrm{Ll}, \mathrm{Tt}$ & RV & $=$ uli bùla \\
\hline ulu katáka & Ww, Lr & $\mathrm{dft}$ & = hulu katáka \\
\hline úndi & $\mathrm{Mi}, \mathrm{Kr}, \mathrm{Kb}$ & RV & Mucuna pruriens var. pruriens \\
\hline úndi pana & $\mathrm{Kb}$ & W & $?=$ úndi \\
\hline úndu & Lw & $\mathrm{O}$ & $=$ úndi \\
\hline 'uowa & $\mathrm{Rn}$ & 4251 & Melanolepis multiglandulosa \\
\hline upo & $\mathrm{Ll}, \mathrm{Lr}$ & RV & $=$ pau \\
\hline upo rúta & Ww, Lr & RV & $=$ pau 'omangu \\
\hline uri & $\mathrm{Mi}, \mathrm{Rn}, \mathrm{Kr}, \mathrm{Kb}$ & RV & Pandanus tectorius \\
\hline usu kadita & $\mathrm{Mb}$ & $\mathrm{H}$ & $=$ uhu kadita \\
\hline usu kanu & $\mathrm{Mb}$ & $\mathrm{H}$ & $=$ uhu kanu \\
\hline uta & Ww, Lr, Ko & RV & $=$ kuta I \\
\hline uta kalara & Ww, Ko & RV & $=$ kalara (kuta) \\
\hline uta 'waiyo & Ko & RV & $=$ uta we'e \\
\hline uta we'e & Ww, Lr & & $?$ \\
\hline 'uwahu & $\mathrm{Mi}, \mathrm{Kb}, \mathrm{Lw}$ & $\mathrm{O}$ & Pachyrrhizus erosus \\
\hline uwe & $\mathrm{Ll}, \mathrm{Ww}, \mathrm{Lr}$ & RV & $=$ iwi (I) \\
\hline ùwi (I) & $\mathrm{Mb} ; \mathrm{Ll}, \mathrm{Ww}, \mathrm{Lr}$ & RV & = ìwi (II) \\
\hline uwi (II) & $\mathrm{Ak}, \mathrm{Mb}$ & RV & $=$ iwi $(I)$ \\
\hline uwi (III) & $\mathrm{Lr}$ & D & $=$ wi'a \\
\hline uwi wé & Ww & RV & = iwi wé \\
\hline wa'bila & $\mathrm{Kr}, \mathrm{Kb}$ & RV, D & Aegle marmelos \\
\hline wàga & Ak & $\mathrm{O}$ & = wàngga II \\
\hline
\end{tabular}




\begin{tabular}{|c|c|c|c|}
\hline wagha & $\mathrm{Lr}$ & 3975 & Woodfordia fruticosa \\
\hline 'wakihu & $\mathrm{Rn}, \mathrm{Kr}, \mathrm{Kb}$ & $\mathrm{RV}$ & Pandanus \\
\hline wàla benggi & $\mathrm{Mi}, \mathrm{Kb}$ & OK & $=$ benggi \\
\hline wàla hànggi & ESb; Ko & $\mathrm{RV}$ & Apium graveolens \\
\hline wàla katàbi & $\mathrm{Kb}$ & 3859 & Ipomoea triloba \\
\hline wàla lia & $\mathrm{Kr}$ & $\mathrm{RV}$ & $=$ layia \\
\hline wàla 'mbunguru & $\mathrm{Kb}$ & OK & Datura metel \\
\hline wàla ngaha & $\mathrm{Kr}$ & RV & $=$ langaha \\
\hline wàla nggiri meu & $\mathrm{Kb}$ & OK & $=$ langgoro maràda \\
\hline wàla nggiri miau & ?Kr & $\mathrm{RV}$ & $=$ langgoro maràda \\
\hline wàla óna I & $\mathrm{Kr}$ & 4214 & Clematis \\
\hline wàla óna II & $\mathrm{Rn}, \mathrm{Kb}$ & 3849 & Vernonia moluccensis \\
\hline wàla ?penggi & $\mathrm{Kr}, ? \mathrm{~Kb}$ & (3834) & ?Eragrostis \\
\hline wàla wawi & $\mathrm{Lr}$ & RV & $=$ wòla wawi \\
\hline wàla wunga I & $\mathrm{Kb}$ & W & = wunga III \\
\hline ?wàla wunga II & ES & $\mathrm{H}$ & Foeniculum vulgare \\
\hline wàlakare & Ko & RV & $=$ walakeri \\
\hline wàlakari & $\mathrm{ESb}$ & RV & $=$ walakeri \\
\hline wàlakaru & Lw & $\mathrm{O}$ & $=$ walakeri \\
\hline walakeri & $\mathrm{Rn}, \mathrm{Kb}$ & RV & Erythrina orientalis/subumbrans \\
\hline walawí & Lw & $\mathrm{O}$ & = walawína \\
\hline walawina & $\mathrm{Mi}, \mathrm{Rn}, \mathrm{Kr}, \mathrm{Kb}$ & 3835 & Cajanus cajan \\
\hline walawinu & Ak & $\mathrm{O}$ & $=$ walawina \\
\hline walawiyo & Ko & RV & $=$ walawina \\
\hline wàngga I & Ww & 4017 & Cyreandra \\
\hline wàngga II & ESb; Ko & 4180 & Ficus benjamina \\
\hline wàngga III & $\mathrm{Kb}$ & 3867 & Ficus \\
\hline wanggakelu & Lw & $\mathrm{O}$ & = wanggakúli \\
\hline wanggakúli & $\mathrm{Mi}, \mathrm{Kb}$ & RV & Calotropis gigantea \\
\hline wanggatipi & Mi & OK & $=$ wanggatupi \\
\hline wanggatupi & $\mathrm{Kb}$ & OK & tree, on the plains \\
\hline wangkura & Ww & 4050 & Euonymus \\
\hline wangukeli & $\mathrm{Mb}$ & $\mathrm{O}$ & = wanggakúli \\
\hline wanu & $\mathrm{Mi}, \mathrm{Kb}, \mathrm{Lw}$ & OK & tree, coastal \\
\hline waru (loan?) & $\mathrm{Mi}, \mathrm{Kb}, \mathrm{Lw}$ & $\mathrm{O}$ & Hibiscus; cp. kabaru \\
\hline waru waka & $\mathrm{Kb}$ & $\mathrm{O}$ & Hibiscus (white hibiscus) \\
\hline wasu kamba & $\mathrm{L}$ & RV & $=$ karipi \\
\hline wasu lòma karàbo & $\mathrm{L}$ & RV & Ficus \\
\hline wasu rèketa & $\mathrm{L}$ & 4042 & Ryparosa \\
\hline wasu we'e & $\mathrm{L}$ & RV & Syzygium \\
\hline 'watara & Mb; WSb & W & $=$ 'wataru \\
\hline 'watara kajíji & $\mathrm{L}$ & RV & $=$ uhu kanu \\
\hline 'watara pi'a & $\mathrm{Ll}, \mathrm{Lr}$ & $\mathrm{D}$ & = 'wataru hàmu \\
\hline
\end{tabular}




\begin{tabular}{|c|c|c|c|}
\hline 'wataro pìyo & Ko & RV & = 'wataru hàmu \\
\hline 'wataru & $\mathrm{ESb}$ & & Zea mays \\
\hline 'wataru hàmu & $\mathrm{Rn}, \mathrm{Kb}$ & $\mathrm{D}, \mathrm{RV}$ & Sorghum saccharatum var. \\
\hline 'wataru hàmu 'unjuku & $\mathrm{Rn}$ & RV & $?=$ 'wataru hàmu \\
\hline 'wataru wuli & $\mathrm{Kr}$ & RV & = 'wataru hàmu \\
\hline 'waüru & $\mathrm{Kb}$ & OK & tree, in forest \\
\hline wàwo & Ww & RV & $=$ nggai \\
\hline wazu lòma karàmbo & Ww & RV & = wasu lòma karàbo \\
\hline wème ndara & Ww & 4055,4059 & Desmodium triflorum \\
\hline wengo & Ww & $\mathrm{O}, \mathrm{OK}$ & = wíngu \\
\hline wengu & $\mathrm{Kb}$ & OK & = wíngu \\
\hline wera & $\mathrm{Mi}, \mathrm{Kb}, \mathrm{Lw}, \mathrm{Mb}$ & OK & $\begin{array}{l}\text { tree, in forest; bark is a } \\
\text { substitutefor betel nut (cp } \\
\text { wero) }\end{array}$ \\
\hline wero & $\mathrm{Ww}, \mathrm{KO}$ & RV & = yú karambua \\
\hline weru & Ak & $\mathrm{O}$ & $=$ wera \\
\hline 'wewera & $\mathrm{Ll}$ & RV & Ficus ?fulva \\
\hline wí & $\mathrm{Mi}, \mathrm{Kb}, \mathrm{Lw}$ & OK & $=$ wi'a \\
\hline wia & $\mathrm{Ak}, \mathrm{Mb}$ & $\mathrm{O}$ & $=$ wi'a $/ \mathrm{mbula}$ \\
\hline wi'a & $\mathrm{Ll}, \mathrm{Ww}, \mathrm{Tt}$ & $\mathrm{D}, \mathrm{OK}, \mathrm{RV}$ & Alocasia macrorrhiza \\
\hline wi'a 'langita & $\mathrm{Lr}$ & RV & $=$ kùru wawi \\
\hline wi'a mareda & Ww & RV & $=$ kùru wawi \\
\hline wiaha & Ko & $\mathrm{O}$ & $=$ uhu \\
\hline wiaza & Ww & $\mathrm{O}$ & $=\mathrm{uhu}$ \\
\hline wìcu & Lw & $\mathrm{H}$ & $=$ witu \\
\hline widi ka'lauki & ES & dft & Calophyllum soulattri \\
\hline wila I & ES & $\mathrm{dft}$ & Gyrinops \\
\hline wila II & ES & dft & Gyrocarpus americanus \\
\hline 'wingira & $\mathrm{Mb}$ & $\mathrm{O}$ & = 'wingiru \\
\hline 'wingiru & $\mathrm{Mi}, \mathrm{Kb}$ & $\mathrm{H}$ & Curcuma viridiflora \\
\hline wíngu & $\mathrm{Kb}$ & OK & tree w. edible fr. \\
\hline 'winguru & Lw, Ak & $\mathrm{RV}, \mathrm{H}$ & = 'wingiru \\
\hline winja & $\mathrm{Rn}$ & 4155 & Alpinia \\
\hline wino & $\mathrm{Ll}, \mathrm{Ww}$ & $\mathrm{RV}$ & = winu \\
\hline wíno 'bioli & $\mathrm{L}$ & 4014 & ?Calanthe \\
\hline wino malandi & Ww & $\mathrm{RV}$ & $=$ winu malanja \\
\hline winu & ES & RV & Areca cathecu \\
\hline winu malanja & $\mathrm{Rn}, \mathrm{Kr}, \mathrm{Kb}$ & RV & Areca cathecu var. \\
\hline wíra & $\mathrm{Ll}$ & RV & $=$ rambútan \\
\hline wìti 'wundungu & $\mathrm{Kr}$ & $\mathrm{dft}$ & Neonauclea lanceolata \\
\hline witu & $\mathrm{ESb}$ & RV & Imperata cylindrica \\
\hline wíyo & Ko & RV & $=$ wi'a \\
\hline wóhu & $\mathrm{Rn}$ & RV & = 'uwahu \\
\hline
\end{tabular}




\begin{tabular}{|c|c|c|c|}
\hline wola & $\mathrm{Kb}$ & OK & = nába \\
\hline wòla kari & $\mathrm{L}$ & RV & $=$ walakeri \\
\hline wòla wawi & Ww & 3956 & Pouzolzia \\
\hline 'wolora & Ww & 4067 & Homalanthus populneus \\
\hline wona & $\mathrm{Kb}$ & $\mathrm{H}$ & = kawona \\
\hline wonjangi & Lw & $\mathrm{O}$ & $=$ kanjangi \\
\hline wo'o jáwa & $\mathrm{L}$ & RV & $=\mathrm{au}$ jawa \\
\hline wo'o pi'a & $\mathrm{Ll}, \mathrm{Ww}$ & RV & $=\mathrm{au}$ \\
\hline wo'o pòto & $\mathrm{Ll}$ & RV & = tàringu \\
\hline wora & $\mathrm{ESb} ; \mathrm{Ww}$ & $\mathrm{O}, \mathrm{W}$ & Indigofera \\
\hline wota kamàmbi I & $\mathrm{Rn}, \mathrm{Kr}$ & RV & Ervatania \\
\hline wota kamàmbi II & $\mathrm{Rn}$ & $3837, \mathrm{dft}$ & Voacanga \\
\hline wota kamembi & $\mathrm{Np}$ & RV & = wota kamàmbi II \\
\hline wota kamemi & Ak & RV & = wota kamàmbi II \\
\hline wowòhu & $\mathrm{Kb}$ & $\mathrm{O}$ & tree w. black wood \\
\hline wu kahìhi & Ko & RV & Loranthaceae \\
\hline wu maleka & Ko & RV & = 'alaku \\
\hline wu 'teyo & Ko & RV & $=$ rumba wau \\
\hline wua bila & $\mathrm{Kb}$ & RV & $=$ wa'bila \\
\hline wua júli & $\mathrm{Rn}, \mathrm{Kb}$ & $\mathrm{RV}, \mathrm{O}$ & Coix lacryma-jobi var. agrestis \\
\hline 'wuala & $\mathrm{Rn}$ & 3931 & = nába \\
\hline wuara & $\mathrm{Kr}$ & 3836 & $=$ wora \\
\hline wucu & Lw & $\mathrm{H}, \mathrm{O}$ & $=$ witu \\
\hline wudi & $\mathrm{ES}$ & 4224 & Ficus (OK: religiosa) \\
\hline wudu & $\mathrm{Lw}, \mathrm{Mb} ; \mathrm{Ww}, \mathrm{Ko}$ & $\mathrm{O}$ & $=$ wudi \\
\hline wùgha & Ko & RV & = nggai \\
\hline wulu jawu & $\mathrm{Ll}$ & RV & = wulu ndaü I \\
\hline wulu kalàla & $\mathrm{Kb}$ & RV & $=$ wulu nggàduku I \\
\hline wulu kalaü & $\mathrm{Kb}$ & PH & Aristida polyclados \\
\hline wulu kamàmbi & $\mathrm{Kb}$ & PH & Chloris virgata \\
\hline wulu manu I & Ll, Ko & RV & $=$ ghulu manu \\
\hline wulu manu II & $\mathrm{Kb}$ & 3882 & Celosia argentea \\
\hline wulu manu mane & $\mathrm{Ll}$ & 4136 & Pteris venulosa \\
\hline wulu ndaü I & $\mathrm{Mi}, \mathrm{Kb}, \mathrm{Lw}$ & OK & Fungi \\
\hline wulu ndaü II & Ww & RV & Chrysopogon aciculatus \\
\hline wulu nggàduku I & $\mathrm{Kb}$ & 3863 & Heteropogon contortus \\
\hline wulu nggàduku II & $\mathrm{Kr}$ & 3960 & $=$ mapu 'mbelaru pingi II \\
\hline wulu ùru I & $\mathrm{Kr}$ & 3871 & Eragrostis warburgii \\
\hline wulu ùru II & $\mathrm{Kb}$ & PH, H & = ka'ndaüngu \\
\hline wulu wae mète & Ww & 4070 & Scirpus \\
\hline wulu wawi & $\operatorname{Tr}$ & 4118 & Cyperus \\
\hline 'wundutu & $\mathrm{Kb}, \mathrm{Lw}$ & OK & Ficus glabella \\
\hline wunga I & $\mathrm{Kr}$ & OK & $=$ lawungu \\
\hline
\end{tabular}




\begin{tabular}{|c|c|c|c|}
\hline wunga II & ES & dft & Ziziphus \\
\hline wunga III & Sumba & $\mathrm{D}, \mathrm{H}$ & Sesbania grandiflora \\
\hline wunggitu & Mi & $\mathrm{O}$ & $=$ 'wundutu \\
\hline 'wunuta & Mb; Ll, Ww & RV & $=$ 'wundutu \\
\hline wunuto & Ko & $\mathrm{O}$ & $=$ 'wundutu \\
\hline wunutu & Ak & $\mathrm{O}$ & $=$ 'wundutu \\
\hline 'wuola & Lل] & RV & = 'nába \\
\hline wura kapáki & $\mathrm{Rn}$ & 4163 & Polygala malesiana \\
\hline wura wolu & $\mathrm{Kb}$ & OK & Phyllanthus pulcher \\
\hline wùru wàla & $\mathrm{Kr}$ & $\mathrm{dft}$ & Rhus taitensis \\
\hline wusu & $\mathrm{Mb}$ & W & $=$ witu \\
\hline wu'u katòpa & Ww & 4047 & Centotheca latifolia \\
\hline 'yaghila & Lr & RV & $?=$ hamúi \\
\hline yàlangu & $\mathrm{Mi}, \mathrm{Kb}, \mathrm{Lw}$ & OK & Melaleuca leucadendra \\
\hline yatangu & $\mathrm{Kb}$ & $\mathrm{OK}$ & $?=$ wíngu \\
\hline 'yawila & $\mathrm{Mb} ; \mathrm{Ww}$ & OK & $=$ 'yawilu \\
\hline 'yawilu & $\mathrm{ESb}$ & $\mathrm{OK}$ & ?Neolitsea \\
\hline yengu & $\mathrm{Mb}$ & OK & = wíngu \\
\hline yú karambua & $\mathrm{Kr}$ & RV & Pterospermum diversifolium \\
\hline yúmbulu & $\mathrm{ESb}$ & RV & = kalú yúmbulu \\
\hline zake & $\mathrm{Lr}$ & RV & = ka'nanggaru \\
\hline zànggi & Ww & $\mathrm{O}$ & = wàla hànggi \\
\hline 'zekera & Ww & $\mathrm{O}$ & = hîkilu \\
\hline 'zekura & $\mathrm{Lr}$ & RV & = hîkilu \\
\hline zuru ndena I & $\mathrm{Lr}$ & RV & $?=$ rambútan \\
\hline zuru ndena II & $\mathrm{Lr}$ & 3957 & $\begin{array}{l}\text { Abelmoschus manihot ssp. } \\
\text { tetraphyllus }\end{array}$ \\
\hline
\end{tabular}




\subsection{SAWU - TAXONOMIC}

\subsubsection{PRELIMINARY REMARKS}

(a) The language

(i) The language on the island of Ndao - the inhabitants say 'Dao' - is very cognate to Sawunese. Walker (1982:56) is inclined to regard it as a separate language. Fox considers it as a Sawunese dialect (Walker 1982:56), which was also my rather superficial impression during my visit to the islet (Verheijen 1976:3f.). A special dialect is spoken by the Sawunese colonies of Waingapu and Melolo on Sumba.

(ii) Orthography and pronunciation

The glottalised /b/, /d/,/g/ and /j/, respectively spelled ' $b$ ", ' $d$ "', ' $g$ '" and ' $\mathrm{j}$ ", following Walker, are characteristic sounds of Sawunese.

(b) Research and identification

(i) Wijngaarden's dictionary (1896) provides some unidentified plant names, Heyne gives several identified names, and Kapita and Onvlee mention some Sawunese equivalents in their Sumbanese works.

(ii) I myself collected a few plants and some names in Ndao in 1969. Ros and I visited Waingapu and Melolo on Eastern Sumba in 1974, made collections and noted names. Our collections were, as usual, identified in Leiden. We ourselves could further identify plants in the field and sometimes from descriptions. In 1982 I had the opportunity of checking my provisional list with help the of Mr Loren Wadu, a Sawunese in Ruteng.

(c) Abbreviations

(i) Classifiers

$\begin{array}{lll}\text { a } & \text { aj'u }^{\prime} u & \text { tree, shrub } \\ \mathrm{j}^{\prime} & \mathrm{j}^{\prime} \mathrm{u}^{\prime} \mathrm{u} & \text { grass, weed } \\ \mathrm{l} & \text { loro } & \text { vine } \\ \text { ro } & \text { rou } & \text { herb } \\ \mathrm{r} & \text { ru } & =\text { rou } \\ \text { wi } & \text { wila } & \text { flower } \\ \text { w } & \text { wo } & \text { fruit }\end{array}$

(ii) Localities

$\mathrm{Da}$ Ndao Is.

Ml Melolo/Waingapu

Sw Sawu Is.

(iii) Additional sources

OK Oemboe H. Kapita

Wg Wijngaarden 


\subsubsection{THE LIST}

\begin{tabular}{|c|c|c|c|c|}
\hline a & ai & $\mathrm{Da}$ & 2444 & Diospyros maritima \\
\hline $\mathbf{r}$ & aj'u dahi & Sw & Wg & marine plant \\
\hline \multirow[t]{2}{*}{$\mathbf{w}$} & aj’u jawa & M & & Annona squamosa \\
\hline & aj’u kéwawo & Sw & Wg & Opuntia elatior \\
\hline ?w & aj’u nona & Ml & RV & Annona muricata \\
\hline a & alo & Sw, Ml & 4184 & Wrightia \\
\hline \multirow[t]{2}{*}{ w } & ané & Sw & $\mathrm{RV}$ & Colocasia esculenta \\
\hline & aré I & Sw, Ml & RV & Oryza sativa \\
\hline \multirow[t]{2}{*}{$\mathbf{a}$} & aré II & Sw & & Pterocarpus indicus \\
\hline & aré laka & Sw & RV & Oryza sativa var. glutinosa \\
\hline $\mathbf{r}$ & bago & M & 4192 & Ceriops decandra \\
\hline \multirow[t]{2}{*}{ a } & bahi (behi) & Sw, Ml & 4190 & Cassia \\
\hline & beku & $\mathrm{Da}$ & 2420 & Suriana maritima \\
\hline \multirow[t]{3}{*}{$\mathrm{j}^{\prime}$} & bela képué & M & $\mathrm{RV}$ & Poaceae \\
\hline & bidara & $\mathrm{Da}$ & 2448 & Ziziphus nummularia \\
\hline & biko I & M & $4204 \mathrm{~A}$ & Asclepiadaceae \\
\hline ro & biko II & M & 4262 & Lumnitzera (littorea) \\
\hline \multirow[t]{2}{*}{ r } & bilu & Sw & Wg & herb \\
\hline & bóa & Sw, Ml & 4186 & Sonneratia alba \\
\hline \multirow[t]{5}{*}{$r$} & boro & Sw, Ml & RV & Corypha utan \\
\hline & bunga loro & Sw & $\mathrm{H}, \mathrm{Wg}$ & Jasminum sambac \\
\hline & d’ad'alu & $\mathrm{Da}$ & 2437 & Sesuvium portulacastrum \\
\hline & d’adi lai & Sw & $\mathrm{RV}$ & ?Loranthaceae \\
\hline & dao & Sw, Ml & & Indigofera \\
\hline w & d'eba nga'a & Sw, Ml & RV & Lagenaria siceraria (edible race) \\
\hline \multirow[t]{3}{*}{$\mathbf{w}$} & d'eba pedu & Sw & RV & Lagenaria siceraria (inedible) \\
\hline & $d^{\prime} e b u$ & Sw & $W g$ & Saccharum officinarum \\
\hline & deb'u loko & Sw, Ml & RV & Saccharum arundinaceum \\
\hline $\mathbf{w}$ & dimu dana & M & RV & Citrullus vulgaris \\
\hline \multirow[t]{2}{*}{$\mathbf{w}$} & dimu poro & Sw, Ml & RV & Cucumis sativus \\
\hline & doro & Sw & RV & Cucurbitaceae \\
\hline \multirow[t]{4}{*}{$\mathbf{r}$} & doro wa & Sw, Ml & RV & Cucurbitaceae \\
\hline & dudu kaha & Sw & $\mathrm{Wg}$ & = aj’́u kéwawo \\
\hline & dué & Sw, Ml & $\mathrm{H}, \mathrm{Wg}$ & Borassus sundaicus \\
\hline & dupé & $\mathrm{Da}$ & 2445 & Piliostigma malabaricum \\
\hline $\mathbf{w}$ & ehi & M & $\mathrm{RV}$ & ?Spondias dulcis \\
\hline$? w$ & éi képaka & & & Manihot esculenta \\
\hline \multirow[t]{2}{*}{$\mathrm{j}^{\prime}$} & gagé & M & 4198 & Dactyloctenium aegyptium \\
\hline & ganyo & Sw, Ml & RV & Canna indica \\
\hline \multirow[t]{3}{*}{ a } & g'edi & Sw, Ml & $\mathrm{RV}$ & Alstonia scholaris \\
\hline & gui & Ml & $\mathrm{H}$ & Calamus \\
\hline & guwi & M & RV & Dioscorea hispida \\
\hline
\end{tabular}




\begin{tabular}{|c|c|c|c|c|}
\hline \multirow[t]{3}{*}{$\mathbf{r}$} & hajo kako & Ml & RV & Ipomoea aquatica \\
\hline & hajo lada & Sw & RV & shrub, small w. sourish 1 \\
\hline & hajo paku & Ml & RV & Polypodiaceae (edible) \\
\hline \multirow[t]{2}{*}{$\mathbf{w}$} & hango & Sw, Ml & Wg & Carica papaya \\
\hline & hapé & Sw & $\mathrm{H}$ & Caesalpinia sappan \\
\hline \multirow[t]{2}{*}{$\mathbf{w}$} & haramélé & Sw & RV & ?Phyllanthus acidus \\
\hline & hau / (?hou) & $\mathrm{Da}$ & V & Hibiscus tiliaceus \\
\hline \multirow[t]{3}{*}{$j^{\prime}$} & hau mengi & $\mathrm{Da}$ & 2427 & Euphorbia hypericifolia \\
\hline & ?héboré ajūu & Sw & Wg & vine \\
\hline & hega & $\mathrm{Da}$ & & $=$ wega \\
\hline \multirow[t]{2}{*}{ wi } & hégé & Ml & & Apium graveolens \\
\hline & hég'udu jara & M & RV & Fungi \\
\hline \multirow[t]{7}{*}{$\mathbf{a}$} & heki & Ml & RV & tree, large \\
\hline & hélag’i & Ml & $\mathrm{H}$ & Tamarindus indica \\
\hline & hélag i ara & & RV & ?Leucaena leucocephala \\
\hline & hélag'i beni & Ml & 4202 & Cassia alasa \\
\hline & héliru & M & RV & Alstonia spectabilis \\
\hline & hémoé & Sw & & tree, large \\
\hline & hépaka & Sw & $\mathrm{H}$ & Michelia champaca \\
\hline $\mathbf{r}$ & hepé & & 4187 & Caesalpinia pulcherrima \\
\hline $\mathbf{r}$ & heré / (?héré) & Ml & & Cymbopogon citratus \\
\hline$j^{\prime}$ & hérégo & & 4199 & Echinochloa colonum \\
\hline $\mathrm{j}^{\prime}$ & hiha manu & Sw & $\mathrm{H}$ & Fimbristylis miliacea \\
\hline \multirow[t]{3}{*}{ w } & hili & Sw, Ml & & Capsicum annuum \\
\hline & hili j’ami & Sw & & Capsicum frutescens \\
\hline & hiri & Sw & Wg & Piper betle (race) \\
\hline w & hiwu & Sw & Wg & Dioscorea alasa \\
\hline w & hiwu inga & Mn & RV & Dioscorea ?sarasinii \\
\hline \multirow[t]{6}{*}{ w } & hiwu jawa & Ml & RV & Ipomoea batatas \\
\hline & hodo & $\mathrm{Da}$ & 2441 & Ammannia \\
\hline & hoka & Sw & $\mathrm{H}$ & $=$ naga \\
\hline & huki wou & Mn & RV & Acacia ?farnesiana \\
\hline & huna & Sw & $\mathrm{Wg}$ & $=$ lahuna \\
\hline & hunga & Sw, Ml & Wg & Allium sativum \\
\hline $\mathbf{r}$ & ?ila & MI & RV & $=(r u) j^{\prime} u^{\prime} u$ la \\
\hline \multirow[t]{2}{*}{ w } & jelima & Ml & RV & Punica granatwn \\
\hline & jeru & Sw & Wg & Citrus \\
\hline $\mathbf{w}$ & jeru ki'i & Mn & RV & Citrus nobilis \\
\hline \multirow[t]{3}{*}{ w } & jeru kébau & Ml & RV & Citrus grandis \\
\hline & jeru lému & MI & RV & Citrus \\
\hline & jeru menanga & Ml & 4258 & Finlaysonia obovata \\
\hline \multirow[t]{2}{*}{ w } & jeru neta' & Sw, Ml & & Citrus \\
\hline & jeru wo réna & & & ?= jeru kébau \\
\hline
\end{tabular}




\begin{tabular}{|c|c|c|c|c|}
\hline $\mathbf{r}$ & j'u'u kedub’u & M & 4200 & Chloris barbata \\
\hline $\mathbf{r}$ & j’u'u la & & RV & Saccharum spontanewm \\
\hline \multirow[t]{2}{*}{$\mathbf{r}$} & j’u'u méa & M & 4261 & Euphorbia \\
\hline & j'u'u méngi & $\mathbf{M}$ & $\mathrm{RV}$ & $=\left(j^{\prime} u^{\prime} u\right)$ wou méngi \\
\hline $\mathrm{r}$ & kaba loro I & M & Wg & Centella asiatica \\
\hline $\mathbf{r}$ & kaba loro II & M & RV & ?Ipomoea \\
\hline \multirow[t]{2}{*}{$\mathrm{j}^{\prime}$} & kaé & & RV & Poaceae \\
\hline & kaé huni & M & RV & ?Achyranthes aspera \\
\hline w & kaé ku'u & Sw, Ml & RV & Martynia annue \\
\hline$j^{\prime}$ & kai & Sw & $\mathrm{H}$ & Panicum repens \\
\hline a & kai wu & Sw & RV & Casuarina equisetifolia \\
\hline \multirow[t]{4}{*}{$j^{\prime}$} & kajepé & $\mathrm{Sw}, \mathrm{Da}$ & 2433,2435 & Merremia tridentata \\
\hline & kaka lobo & Sw, Ml & 4191 & Lindernia \\
\hline & kala kaha & M & RV & = aj’u kéwawo \\
\hline & kalai ngela bangi & $\mathrm{Da}$ & 2442 & Vitex trifolia ssp. littoralis \\
\hline a & karé & $\mathrm{Da}$ & Wg & Erythrina orientalis \\
\hline a & karé melanu & M & RV & Erythrina \\
\hline \multirow[t]{9}{*}{$\mathrm{a}$} & karé nga'a & M & & Erythrina ?subumbrans \\
\hline & karo' & $\mathrm{Da}$ & 2426 & Salsola kali \\
\hline & katapa nga'i & $\mathrm{Da}$ & 2439 & Terminalia catappa \\
\hline & keb'edu & M & RV & ?Derris \\
\hline & keb’o & M & RV & Morinda tomentosa \\
\hline & kebo' & $\mathrm{Da}$ & 2443 & Ficus obliqua \\
\hline & kebui aé & M & RV & Vigna unguiculata \\
\hline & kebui iki & Ml & $\mathrm{RV}$ & Phaseolus aureus \\
\hline & kéb’unu & Sw & $\mathrm{Wg}$ & Ficus \\
\hline w & kébuta nga'a & Ml & RV & Amorphophallus campanulatus \\
\hline \multirow[t]{4}{*}{ w } & kébuta ped'i & M & RV & Amorphophallus variabilis \\
\hline & ?kedudu & M & & ?Caryota \\
\hline & kéela & Sw & & $=$ wenyi \\
\hline & ?kéhabé & M & & $=$ kohabé \\
\hline w & kéhuré & Sw, Ml & RV & Dioscorea esculenta \\
\hline \multirow[t]{8}{*}{ w } & kéhuré edu & Sw & RV & Dioscorea ?aculeata \\
\hline & ?kela & Sw, Ml & $\mathrm{Wg}$ & $=$ wenyi \\
\hline & kélai léré & Sw & $\mathrm{Wg}$ & Centella asiatica \\
\hline & kélai linga jawa & Sw & $\mathrm{Wg}$ & Piper nigrum \\
\hline & kélaiwu & M & RV & Dendrocalamus asper \\
\hline & kélaiwu jawa & $\mathbf{M}$ & RV & ?Gigantochloa \\
\hline & kélaiwu wa & M & RV & Gigantochloa \\
\hline & kélara j’ami & Sw & & Piper \\
\hline \multirow[t]{3}{*}{1} & kelawa rai & Ml & 4195 & Merremia emarginata \\
\hline & kelela & Sw & Wg & $=$ wenyi \\
\hline & kelia & Ml & RV & Zingiber officinale \\
\hline
\end{tabular}




\begin{tabular}{|c|c|c|c|c|}
\hline & kelia beri & Sw & OK & Crinum asiaticum \\
\hline & kénana & Sw, Ml & $\mathrm{H}$ & Piper betle \\
\hline w & kéo & Ml & RV & Coix lacryma-jobi var. ma-yuen \\
\hline \multirow[t]{3}{*}{ a } & képaka & Sw, Ml & Wg, RV & Sterculia foetida \\
\hline & képapa edu & Sw & $\mathrm{Wg}$ & Papilionaceae (wild) \\
\hline & képapa nga'a & Sw & RV & Phaseolus ?lunatus \\
\hline $\mathrm{j}^{\prime}$ & képoké & Sw & $\mathrm{H}$ & Heteropogon contortus \\
\hline a & kérara & M & RV & Artocarpus altilis \\
\hline w & kérebo hawu & Ml & RV & Benincasa hispida \\
\hline \multirow[t]{4}{*}{$\mathbf{w}$} & kérebo jawa & M & RV & Cucurbita moschata \\
\hline & kéweru j'ami & Sw & Wg & Bombax ceiba \\
\hline & kéweru liha & Ml & RV & Ceiba pentandra \\
\hline & kéwunyi & $\mathrm{Ml}$ & $\mathrm{H}$ & Curcuma viridiflora \\
\hline \multirow[t]{2}{*}{$\mathbf{w}$} & ko & Sw, Ml & $\mathrm{H}$ & $=$ bidara \\
\hline & kobo deré I & Sw & & herb w. small 1. \\
\hline \multirow[t]{6}{*}{ ro } & kobo deré II & M & RV & Mimosa ?invisa \\
\hline & kohabé & Sw & RV & Schleichera oleosa \\
\hline & kolé (lari) & Sw, Ml & Wg & Calotropis gigantea \\
\hline & kolé wawi I & Sw & Wg & herb, wild w. small 1. \\
\hline & kolé wawi II & Mn & RV & Datura metel \\
\hline & kowi & Sw & Wg & Coffea robusta \\
\hline $\mathbf{r}$ & kunu roté & Ml & RV & Hyptis suaveolens \\
\hline $\mathbf{r}$ & kunu wa & Ml & RV & Lamiaceae \\
\hline \multirow[t]{2}{*}{$\mathbf{r}$} & kunu wango & Ml & 4197 & Salvia riparia \\
\hline & lahuna & Sw, Ml & RV & Allium cepa var. escalonicum \\
\hline \multirow[t]{2}{*}{ (r) } & laka & Sw & $\mathrm{Wg}$ & Lawsonia inermis \\
\hline & lakua & Mn & RV & Languas galanga \\
\hline \multirow[t]{3}{*}{ ro } & lama & Ml & RV & Portulaca oleracea \\
\hline & lasa & & $\mathrm{H}$ & Lansium domesticum \\
\hline & lata & Sw & Wg & Pandanus tectorius \\
\hline \multirow[t]{2}{*}{$\mathbf{r}$} & lata banga & Sw, Ml & Wg & Pandanus \\
\hline & $\begin{array}{l}\text { lata mengi } \\
\text { lawada }\end{array}$ & Sw & Wg, RV & $\begin{array}{l}\text { Pandanus amaryllifolius } \\
\text { tree }\end{array}$ \\
\hline w & lé & Ml & & Mucuna pruriens var. utilis \\
\hline \multirow[t]{2}{*}{$\mathbf{w}$} & lé edu & Ml & & = winga lé edu \\
\hline & ?lébi dué & & $\mathrm{Wg}$ & plant, climbing \\
\hline $\mathbf{w}$ & libi & & $\mathrm{H}$ & Averrhoa bilimbi \\
\hline \multirow[t]{6}{*}{$\mathbf{w}$} & libi haramélé & & $\mathrm{H}$ & = libi mélai \\
\hline & libi mélai & Sw & $\mathrm{H}$ & Averrhoa carambola \\
\hline & lilo géro moné & $\mathrm{Da}$ & 2432B & Euphorbia levis \\
\hline & lolo bangi & Ml & RV & Ricinus communis \\
\hline & lolo raé dou & Ml & RV & Jatropha curcas \\
\hline & lolo wa & Ml & RV & Jatropha ?gossypifolia \\
\hline
\end{tabular}


loro

lub'a

lub’a loko

lub’a méa

lub’a raé dou

a luki

a mangi

a maronggé

w matabalé

r méda'a bela

r méda'a duku

r méda'a kemu

médiri

méhia

r méluki

r mengahi

$j^{\prime} \quad$ mengu

ménila

menyaro

a

mera

merényo

moké (?moka)

w mu'u

(w) mu'u jawa

(w) naga

r na'i

na'i goi

na'i kéb’edu

na'i kébu

nana

r

nara

nena

nena edu

ngaka lobo

$j^{\prime} \quad$ nyebu délo

nyiu

nyiu hango

wi ona

w pau

pau rutu

péna'i holo

péna'i ki'i
Sw

Sw

Sw

Sw

$\mathrm{Da}$

MI

Sw

M

MI

M

MI

Sw

M

Sw, Ml

$\mathrm{MI}$

Sw

Sw

$\mathrm{Sw}$

Sw

Sw

M

Sw, Ml

Sw

Sw, Ml

Sw, Ml

MI

Sw

Sw, Ml

Sw

MI

M

MI

Sw

Sw

Sw

M

Sw, Ml

M

M

M
$=$ bunga loro

= woko jawa

tree

tree

Syzygium jambos

$=$ méluki

?Thespesia populnea

RV

RV

RV

RV

RV

RV

Wg

4185

$4260 \mathrm{~B}$

$4260 \mathrm{~A}$

$\mathrm{H}$

Wg

Wg

Wg

Wg

RV

Wg

Wg

RV

Wg

RV

RV

RV

RV

Wg

$\mathrm{H}$

Wg

Wg

RV

Wg

RV

RV

4188
= wona

Lycopersicon lycopersicum

?Ocimum

Ocimum basilicum

?Ocimum

Ficus

Ficus

Ficus

Avicennia

Eragrostis anabilis

Arachis hypogaea

Ficus

tree, large w. round 1.

tree, large

Arenga pinnata

Musa paradisiaca

$=$ hango

Artocarpus integer

Nicotiana tabacum

?Blumea

plant; bark or root mixed w.

palm wine

Acorus calamus

= nena

Themeda ?villosa

Ananas comosus

Agave sisalana

herb, at riverside

Eriochloa ramosa

Cocos nucifera

Cocos nucifera (race)

?Vernonia cinerea

Mangifera indica

?Buchanania arborescens

Anacardium occidentale

Tabernaemontana orientalis 


\begin{tabular}{|c|c|c|c|c|}
\hline \multirow[t]{3}{*}{ w } & péringi éi & M & RV & Momordica charantia \\
\hline & pesesa & $\mathrm{Da}$ & 2446 & Mallotus cf. moritzianus \\
\hline & peté & Sw & $\mathrm{H}$ & Parkia speciosa \\
\hline w & pétola & $\mathbf{M}$ & RV & $=$ tola \\
\hline w & poro loko & M & RV & ?Abrus \\
\hline \multirow[t]{5}{*}{$j^{\prime}$} & pudi wila & M & 4193 & Tridax procumbens \\
\hline & pulu téi & Sw, Ml & RV & ?Triumfetta \\
\hline & puri raho & M & 4206 & ?Pedilanthus / Pereskia \\
\hline & puta & Sw & $\mathrm{Wg}$ & tuber \\
\hline & puta nga'a & Sw & Wg & tuber (edible) \\
\hline $\mathbf{r}$ & raé & Mn & RV & Amaranthus \\
\hline \multirow[t]{2}{*}{$\mathrm{a}$} & rui & M & 3913 & ?Ceropegia \\
\hline & serbéi & Ml & RV & Morus ?australis \\
\hline \multirow[t]{4}{*}{$\mathbf{r}$} & taba & Sw, Ml & 4211 & Acalypha indica \\
\hline & taba rui I & M & 4259 & Ceropegia \\
\hline & taba rui II & M & RV & Euphorbia tirucalli \\
\hline & té & $\mathrm{Da}$ & 2436 & Vernonia \\
\hline $\mathbf{r}$ & tébai & Sw & $\mathrm{Wg}$ & herb, small \\
\hline \multirow[t]{6}{*}{$r$} & tédengi & $\mathbf{M}$ & 4204 & Rubiaceae \\
\hline & téi bahi & $\mathrm{Da}$ & 2440 & Capparis spinosa var. mariana \\
\hline & témié & Sw, Ml & $\mathrm{H}$ & Schizostachyum ?blumii \\
\hline & ténaga & Sw & $\mathrm{H}$ & Cananga odorata \\
\hline & téraé hawu & Sw, Ml & $W g$ & Sorghum saccharatum \\
\hline & téraé (jawa) & Sw & Wg & Zea mays \\
\hline \multirow[t]{5}{*}{ w } & teré & Sw, Ml & Wg & Solanum melongena \\
\hline & teré edu & Sw, Ml & RV & Solanum ?spinosum \\
\hline & téwo & Sw, Ml & RV & Phragmites karka \\
\hline & timu taka & $\mathrm{Da}$ & 2438 & Mukia maderaspatana \\
\hline & tola & Sw & Wg & Luffa aegyptiaca \\
\hline \multirow[t]{4}{*}{ w } & tori & Sw & Wg, RV & Cajanus cajan \\
\hline & uhu & Sw, Ml & RV & Setaria italica \\
\hline & waka & Sw & Wg, RV & ?Eleocharis dulcis \\
\hline & waka moné & M & 4201 & Boerhavia \\
\hline ro & walu goa & M & 4205 & $\begin{array}{l}\text { Abutilon indicum ssp. albescens } \\
\text { var. australierse }\end{array}$ \\
\hline a & wao & Sw & & $\begin{array}{l}\text { tree, tall w. small } 1 . \text { and round } \\
\text { inedible fr. }\end{array}$ \\
\hline \multirow[t]{2}{*}{$\mathbf{a}$} & wau & Sw, Ml & $\mathrm{Wg}, \mathrm{RV}$ & $=$ hau \\
\hline & wega & Sw & $\mathrm{Wg}$ & Ficus \\
\hline \multirow[t]{3}{*}{ w } & wéi képaka & Sw & $\mathrm{H}$ & ?= éi képaka \\
\hline & welu & Sw, Ml & RV & Aleurites moluccana \\
\hline & wengu I & M & $\mathrm{Wg}$ & Gossypium \\
\hline wi & wengu II & Sw, Ml & RV & Sesbania grandiflora \\
\hline
\end{tabular}




\begin{tabular}{|c|c|c|c|c|}
\hline & wengu kod'é & M & 4183 & Hibiscus vitifolius \\
\hline & wenyi & Sw, Ml & Wg & Areca cathecu \\
\hline & wenyi edu & Sw & RV & ?Pinanga \\
\hline $\mathrm{j}^{\prime}$ & wéro & Sw & Wg & $?=$ deb$^{\circ}$ u loko \\
\hline ro & widu & Ml & Wg & Imperata cylindrica \\
\hline \multirow[t]{6}{*}{$j^{\prime}$} & wila & Sw & $?$ & Poaceae \\
\hline & wila ona & M & & Vernonia cinerea var. parviflora \\
\hline & winga lé edu & Ml & RV & Mucuna pruriens var. pruriens \\
\hline & wo & Sw & RV & Bambuseae \\
\hline & wodo & Sw & RV & $=$ hodo \\
\hline & woko jawa & Sw & Wg & Psidium guajava \\
\hline a & wona & Sw, Ml & Wg & Moringa pterygosperma \\
\hline a & wou & Sw & Wg & tree, small \\
\hline \multirow[t]{2}{*}{$j^{\prime}$} & wou méngi & Ml & RV & ?Cyperus kyllingia \\
\hline & ?wowai & Sw & Wg & ?shrub, wild w. small red fr. \\
\hline \multirow[t]{2}{*}{ w } & wué kota & Sw & RV & Pachyrrhizus erosus \\
\hline & wué tédengi & M & & Asclepiadaceae \\
\hline $\mathbf{w}$ & wué wawi & M & 4189 & Leguminosae \\
\hline
\end{tabular}




\subsection{SIKA - TAXONOMIC}

\subsubsection{PRELIMINARY REMARKS}

(a) The language

(i) Sikanese is important as it is the westernmost language of the so-called 'Ambonese Group' of languages. A comparison with the plant names of the adjoining languages of the Bima-Sumba Group may yield interesting linguistic results. The differences between the dialects of Sika, Nita and Kangaé are small, but Tana $\mathrm{Ai}$ is considerably different. A conspicuous feature is that in several dialects final $/ \mathrm{n} /$ becomes $/ \mathrm{ng} /$.

(ii) Orthography and pronunciation

/é/ and /e/ are different phonemes.

The glottalised consonants are spelled ' 1 ', ' $r$ ' and ' $w$ '.

Special attention should be given to the phonemically different onsets of vowels: the 'smooth' onset is realised by the murmured or voiced ' $h$ ', and the 'rough' onset by the glottal stop. This applies to both initial and medial vowels. In order to simplify the orthography, only the glottal stop "' is spelled, contrary to Meyer's $(1937,1946)$ orthography. The vowels of the syllables not preceded by "' have a smooth onset.

The clusters $/ \mathrm{bl} /$ and $/ \mathrm{kl} /$ become in some dialects $/ \mathrm{br} /$ and $/ \mathrm{kr} /$.

(b) Research and identification

I owe several names of identified plants to the work of Hildebrand $(1940,1952)$ and one to the work of Heyne (1950).

I found very many names in Meyer's dictionary $(1937,1946)$, and a few in 'Tati lalang sara Sika II' by H. Bolscher (1985), all without identification. We tried to identify them with the help of informants; some could be identified in the field. Ros and myself also noted many new plants, chiefly around Watublapi and Ledalero.

Afterwards my provisional list was kindly checked by Fr Alo Mitan. Of course many names are used beyond the places where they were noted.

(c) Abbreviations

(i) Classifiers

$\begin{array}{lll}\text { 'a } & \text { 'ai } & \text { tree, shrub } \\ 1 & \text { lanang } & \text { creeping plant } \\ \text { t } & \text { tali } & \text { liana, vine } \\ \text { w } & \text { wa'an(g) } & \text { leaf, herb }\end{array}$

(ii) Dialects

$\begin{array}{ll}\text { Kn } & \text { Kangaé } \\ \mathrm{Nt} & \text { Nita } \\ \text { Sk } & \text { Sika } \\ \text { TA } & \text { Tana Ai }\end{array}$


(iii) Additional sources

B Bolscher

M Meyer

\subsubsection{THE LIST}

ahu i'ur

'ai aning

'ai ba'i

'ai bunga

'ai jawan

'ai mitang

'ai 'ohu

'ai ta'i

'ai telo

'aing bedi

aja

ajawa

ale

'anggor

'antas I

'antas II

ara

ara nanan

'arnana

'arnana kaki

$\operatorname{arun}(\mathrm{g})$

arung atong

arung bura

arung méran

arung wélang

'atar

atong

'atpokat

aur

ba basar

bahir

bain tuhung

baka

bakér

baki

bako
B

TA

dft

Sk

Sk

TA

TA

TA

B

M

M

M

M

M

M

M

(dft)

dft

M

M

TA

TA

weed, in gardens

Sida rhombifolia

= patar hading

?Millingtonia hortensis

= padu I

Diospyros ebenum

Manihot esculenta

Zingiberaceae

Nauclea (cj.)

tree, small

$=$ lelé I

$=$ lelé I

weed

Passiflora foetida

Annona muricata

= kaé nona

Ficus ?variegata

$=$ én $(\mathrm{g})$

?Planchonella obovata

Planchonella firme

Amaranthus sp.

Amaranthus spinosus

Amaranthus lividus

Amaranthus

M

?Portulaca oleracea / Sesuvium

portulacastrum

Vitis vinifera

$\mathbf{M}$

Amaranthus (spinosus)

Persea onericana

M

$\mathbf{M}$

Gigantochloa apus

Poaceae

Barleria prionitis

Cyathula prostrata

= kala ara

?= baki

Ensete glaucum

M Nicotiana tabacum 


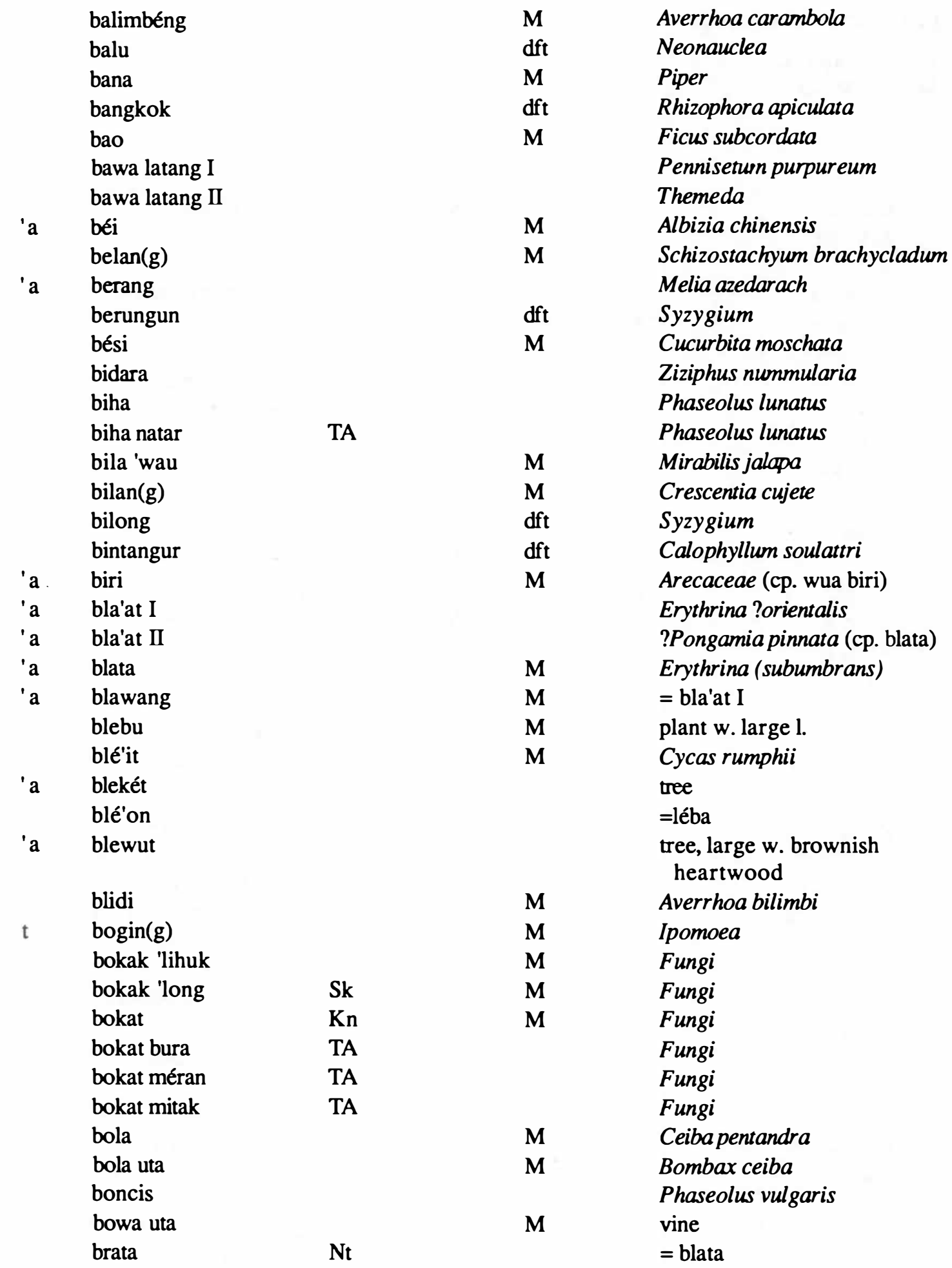




\begin{tabular}{|c|c|c|c|}
\hline bré'it & $\mathrm{Nt}$ & $\mathbf{M}$ & = blé'it \\
\hline bridi & $\mathrm{Nt}$ & & $=$ blidi \\
\hline budi & & & ?Lagerstroemiaflos-reginae \\
\hline bué & & & $\begin{array}{l}\text { Phaseolus aureus (w. green } \\
\text { seeds) }\end{array}$ \\
\hline bué lengi & & $\mathbf{M}$ & $\begin{array}{l}\text { Phaseolus aureus (w. brown } \\
\text { seeds) }\end{array}$ \\
\hline buké & & $\mathbf{M}$ & Morinda tomentosa \\
\hline buli bowa & & & ?Dioscorea \\
\hline buli bowa nata & & $\mathbf{M}$ & ?Dioscorea (edible tuber) \\
\hline buli bowa uta & & $\mathbf{M}$ & ?Dioscorea (inedible tuber) \\
\hline bunga baku & & $\mathbf{M}$ & Crinum asiaticum \\
\hline bunga mawar & & $\mathbf{M}$ & Rosa \\
\hline bunga méang & & & ?Cassia \\
\hline bunga méang waing & & & Mimosa invisa \\
\hline bunga raja & & & Duranta erecta \\
\hline bunga rebu & & & ?weed w. stinking fl. \\
\hline bunga tou & & $\mathbf{M}$ & Plumeria (acuminata) \\
\hline bur & & & ??Peltophorum pterocarpum \\
\hline buri wawi & & $\mathrm{dft}$ & Garcinia \\
\hline campaga & & & $=$ sampaga \\
\hline cendana laki & & & $=$ sampaga \\
\hline cermélé & TA & & = sermélé \\
\hline daé & & $\mathbf{M}$ & ?Elaeocarpus floribundus \\
\hline daga lais & & $\mathbf{M}$ & Lycopersicon lycopersicum \\
\hline dagar & & & Kleinhovia hospita \\
\hline dalima & & $\mathbf{M}$ & Punica granation \\
\hline dalo & & & Phragmites karka \\
\hline dama & & & tree \\
\hline damar jawa I & & & Jatropha curcas \\
\hline damar jawa II & & & Ricinus communis \\
\hline damar laka & & & Jatropha gossypifolia \\
\hline damar nilon & & & = damar jawa II \\
\hline ?dela & & & $=$ 'watar I \\
\hline denu & & $\mathbf{M}$ & Melochia cf. umbellata \\
\hline déru dédé & $\mathrm{Kn}$ & & $?=$ nderu dédé \\
\hline dihi & & $\mathbf{M}$ & tree, medium height \\
\hline diké & & $\mathbf{M}$ & tree, large; wood for boats \\
\hline dila I & TA & & Aegle marmelos \\
\hline dila II & $\mathrm{Kn}$ & & $=$ bilan $(\mathrm{g})$ \\
\hline ?dimun I & TA & & $=$ timun \\
\hline $\operatorname{dimun}(\mathrm{g}) \mathrm{II}$ & & $\mathbf{M}$ & Citrullus lanatus \\
\hline doko & & $\mathbf{M}$ & Pandanus (w. broad 1.) \\
\hline
\end{tabular}




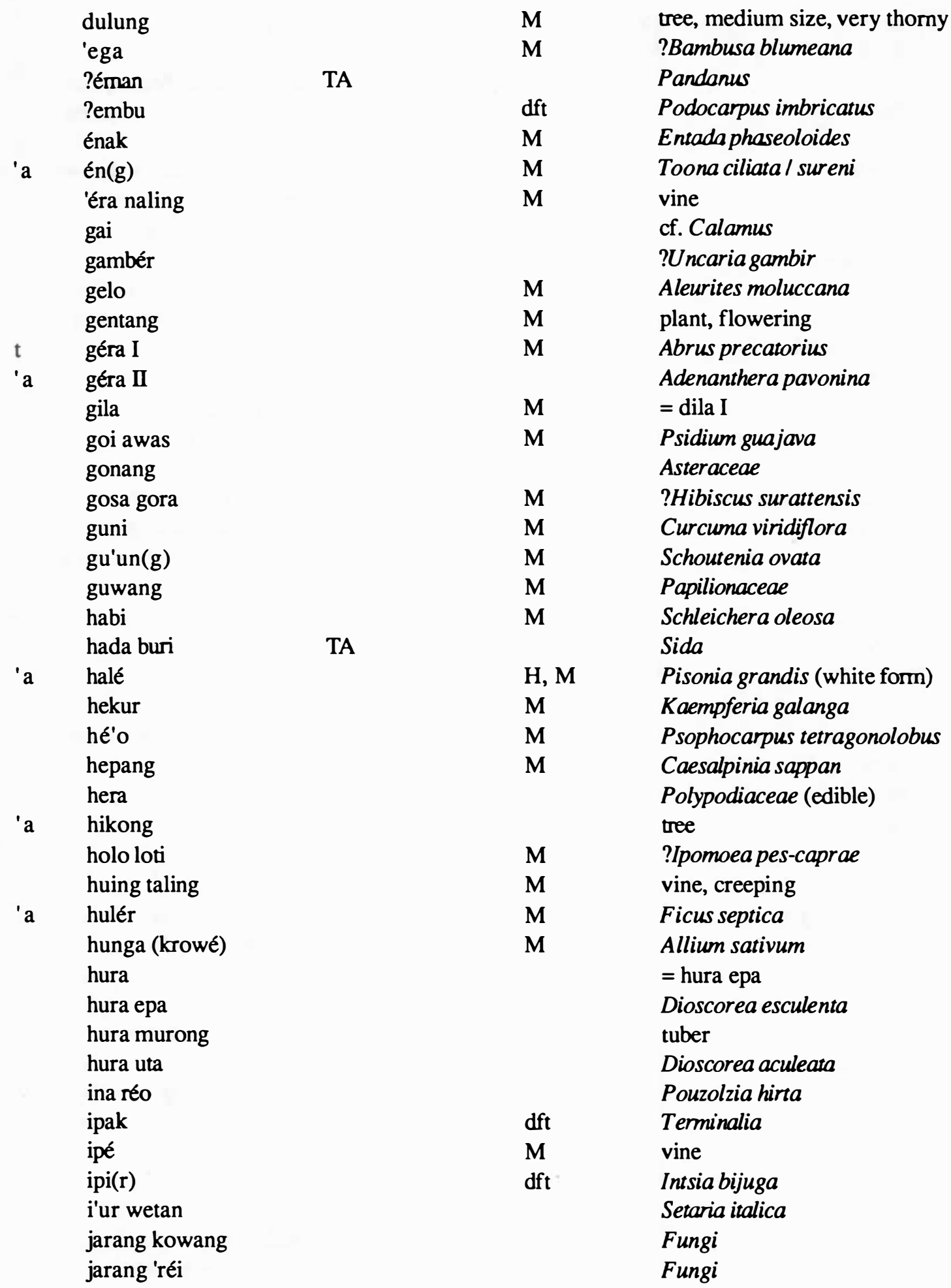




\begin{tabular}{|c|c|c|c|}
\hline jati & & & Tectona grandis \\
\hline jawa & TA & & $=\operatorname{dimun}(\mathrm{g}) \mathrm{II}$ \\
\hline johar & & & Cassia siamea \\
\hline ka nona & & & = kaé nona \\
\hline kabor & & & Cocos nucifera \\
\hline kadodo & & & Lannea coromandelica \\
\hline kaé nona & & & Annona squamosa (?reticulata) \\
\hline ?kaha & & $\mathbf{M}$ & ?Euphorbia \\
\hline kajo wair & & & Clerodendrum buchanani \\
\hline kala & & $\mathbf{M}$ & vine w. edible 1 . \\
\hline kala ara & & $\mathbf{M}$ & Artocarpus altilis (seedless) \\
\hline kala méon & & & = léba \\
\hline kala midi & $\mathrm{Kn}$ & $\mathbf{M}$ & vine w. edible 1. \\
\hline kali raga & & $\mathbf{M}$ & Acorus calamus \\
\hline kamét & & $\mathbf{M}$ & $\begin{array}{l}\text { shrub, coastal, thorny, } 3-4 \mathrm{~m} \\
\text { high (cp. klamét) }\end{array}$ \\
\hline kamodo & & $\mathbf{M}$ & Luffa acutangula \\
\hline kangkong & & $\mathbf{M}$ & Ipomoea aquatica \\
\hline kanonas & Sk & $\mathbf{M}$ & Annona \\
\hline kapa & & & Gossypium \\
\hline kara munti & & & $=$ kurmunting \\
\hline ?karang uru & & $\mathbf{M}$ & $?$ \\
\hline ?kata lari I & & RV & Cassia \\
\hline kata lari II & & & Crotalaria \\
\hline kéam & & dft & Cynometra ramiflora \\
\hline kébar & & & Cordyline fruticosa \\
\hline kebo & & & Morinda citrifolia \\
\hline kebojan & TA & & $=$ goi awas \\
\hline kédang & & $\mathbf{M}$ & Papilionaceae \\
\hline kégang & $\mathrm{Kn}$ & $\mathbf{M}$ & tree, large w. bitter fr. \\
\hline kélik gahu & & $\mathbf{M}$ & $\begin{array}{l}\text { vegetable w. palatable sour l., } \\
\text { white fl. }\end{array}$ \\
\hline keloti & & $\mathbf{M}$ & $?=$ kolo loti \\
\hline kepi & & & ?= réo \\
\hline kéru & $\mathrm{Kn}$ & & = kléru \\
\hline ?kéwa & & $\mathbf{M}$ & ?Dioscorea \\
\hline kibang & & & tree, large w. edible sour fr. \\
\hline klahé & TA & & $=$ klahi \\
\hline klahi & & $\mathbf{M}$ & Ocimum basilicum \\
\hline klahi $\operatorname{rotan}(g)$ & & $\mathbf{M}$ & Cymbopogon citratus \\
\hline klaka & & $\mathbf{M}$ & tree \\
\hline klakat & & $\mathbf{M}$ & $=$ krakat \\
\hline klamét & & $\mathbf{M}$ & vine, thorny (cp. kamét) \\
\hline
\end{tabular}




\begin{tabular}{|c|c|c|c|}
\hline klaté & $\mathrm{Kn}$ & $\mathbf{M}$ & Hyptis suaveolens \\
\hline klau & & $\mathbf{M}$ & tree, large \\
\hline klega & & $\mathbf{M}$ & vine w. edible fr. \\
\hline klégang & & & = kégang \\
\hline klekar & & & tree, large w. small leaflets \\
\hline klépok & & & $\begin{array}{l}\text { Papilionaceae (climbing; } 1 . \\
\text { eaten) }\end{array}$ \\
\hline kléra & & $\mathbf{M}$ & Benincasa hispida \\
\hline kléréng & & & ?= wéwé kléréng \\
\hline kléru & & & Pollia \\
\hline kléto & $\mathrm{Kn}$ & $\mathbf{M}$ & weed \\
\hline kletong & & $\mathbf{M}$ & marine vegetable \\
\hline klibang & & $\mathbf{M}$ & ?= kibang \\
\hline klogo & & $\mathbf{M}$ & marine Algae, reddish, edible \\
\hline kloko & & $\mathbf{M}$ & $=$ kroko \\
\hline klorot & & $\mathbf{M}$ & Triumfetta / Urena \\
\hline klorot apun & & & ?Achyranthes aspera \\
\hline klorot 'regét & $\mathrm{Kn}$ & RV & Capparis \\
\hline klukut & $\mathrm{TA}, \mathrm{Kn}$ & & Drynaria \\
\hline koang & & & Ficus balica \\
\hline kobu mang & & $\mathbf{M}$ & Agave sisalana \\
\hline koja & & $\mathbf{M}$ & Canarium vulgare \\
\hline koja koat & & $\mathbf{M}$ & $?$ \\
\hline koja lonang & & $\mathbf{M}$ & Sesamum orientale \\
\hline koja pepin & TA & & $=$ koja \\
\hline koja temuk & $\mathrm{Kn}$ & & Euphorbia hirta \\
\hline koja wela & & $\mathbf{M}$ & ? \\
\hline koko & & & $=$ kroko \\
\hline kolé & & $\mathbf{M}$ & $=$ 'ohu kolé \\
\hline koli & & $\mathbf{M}$ & Borassus sundaicus \\
\hline kolo loti & & $\mathbf{M}$ & Ipomoea pes-caprae \\
\hline komak & & & $\begin{array}{l}\text { Papilionaceae (creeping, } 1 \text { or } 2 \\
\text { beans in a pulse) }\end{array}$ \\
\hline konjawa & & & $=$ goi awas \\
\hline kopéng & & $\mathbf{M}$ & tree, small w. edible fr. \\
\hline kopi & & $\mathbf{M}$ & Coffea spp. \\
\hline koro & & $\mathbf{M}$ & $=$ koro geté \\
\hline koro anak & & & Capsicum frutescens \\
\hline koro blutuk & & & $=$ koro anak \\
\hline koro geté & & & Capsicum annuum \\
\hline ?koro 'iling & & $\mathbf{M}$ & Piper \\
\hline kota lari I & & & $=$ kurmunting \\
\hline kota lari II & & & = kata lari II \\
\hline
\end{tabular}




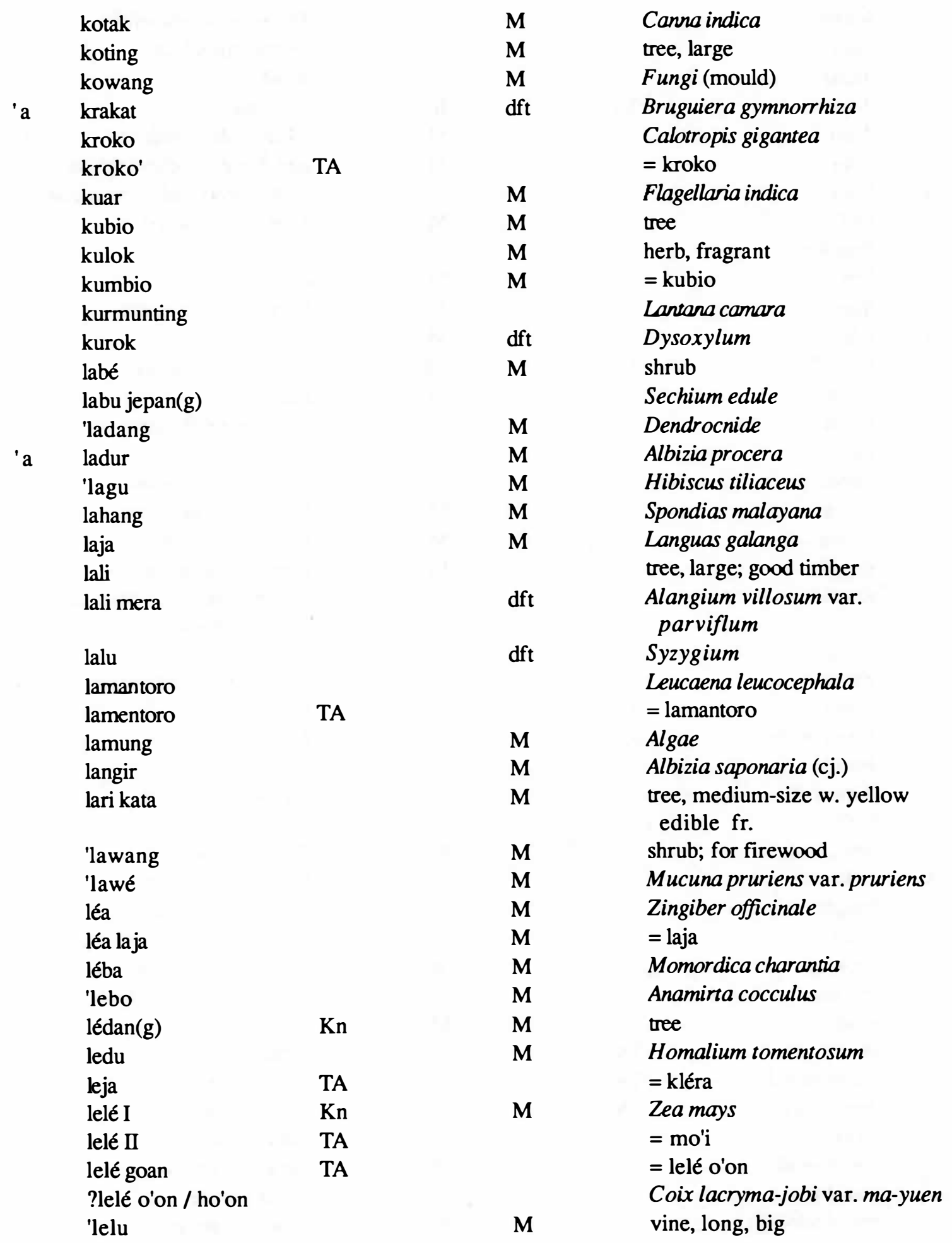




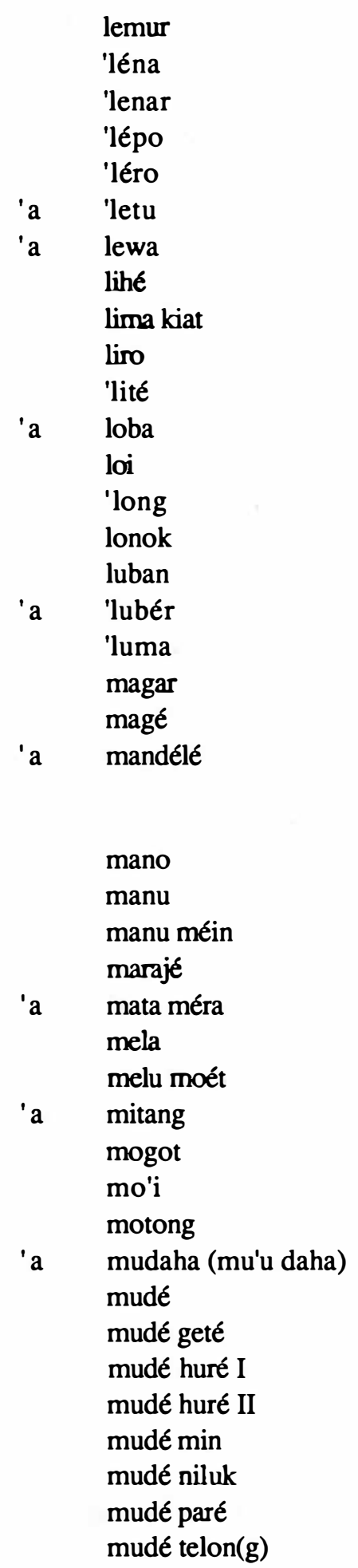

TA

TA

TA

$\mathrm{Kn}$

$\mathrm{Kn}$

\section{TA}

TA

TA
B

M

$\mathbf{M}$

M, dft

$\mathbf{M}$

$\mathbf{M}$

M

dft

M

M

$\mathbf{M}$

$\mathbf{M}$

M

$\mathbf{M}$

dft

Erechtites valerianifolia

Setaria palmifolia

shrub

Alocasia

?Blumea balsamifera

tree, large; excellent timber

Melanolepis multiglandulosa

Terminalis catappa

Canavalia

Ficus

Sorghum propinquum

?Glochidion

Alstonia spectabilis

Fungi

Elsholtzia pubescens

Amomum

Syzygium ?aqueum

Sesbania grandiflora

Dioscorea ?hispida

Tomarindus indica

tree, medium-size; black armbangles are cut out from wood

Cinnamomum burmanni

Uvaria

?

B shrub

Breynia vitis-idaea

Oxalis corniculata

M

$\mathrm{dft}$

$\mathbf{M}$

$\mathbf{M}$

M

Rhaphidophora (cj.)

Diospyros ebenum

Commelina diffusa

Coix lacryma-jobi var. agrestis

Moringa pterygosperna

herb or shrub, $2 \mathrm{~m}$ high

Citrus

= mudé paré

Citrus sinensis

$=$ mudé min

$\mathbf{M}$

Citrus nobilis

M Citrus aurantifolia

Citrus grandis

$\mathbf{M}$

Citrus aurantium 


$\begin{array}{lll}\text { 'a } & \text { muné } & \\ & \text { muti } & \\ & \text { mu'u } & \\ & \text { mu'u jawa } & \\ & \text { nakat } & \\ & \text { nakat jawa } & \\ \text { nakat wérén(g) } & \text { TA, Kn } \\ & \text { nalu } & \\ & \text { nalu épu } & \text { TA } \\ & \text { nalu lépu } & \text { TA } \\ & \text { ?natu } & \text { Kn } \\ & \text { nau } & \\ & \text { nawu } & \\ & \text { ndéru dédé } & \\ & \text { neni } & \\ \text { w } & \text { népar } & \\ & \text { newo } & \\ & \text { ?nila (nilo) } & \\ & \text { nita } & \\ & \text { nitu 'ohung } & \\ & \text { nitu petun } & \\ & \text { niur } & \\ \text { 'a } \quad \text { nu'é } & \text { TA }\end{array}$

nakat jawa$$
\text { nalu }
$$

nalu épu

nalu lépu

nawu

ndéru dédé

neni

newo

?nila (nilo)

nita

'ohung

nu'é

nunang

'a nupér

o'a

'oho ('ai)

'ohu

'ohu 'ai

'ohu do'én

'ohu jawar

'ohu kolé

'ohu krowé

'a ojan(g)

?orté lang

oto

'a padé

padu I

padu II

'a padun

paku
M

M

TA

M

$\mathbf{M}$

dft

RV

M

dft

M

$\mathbf{M}$

M

$\mathbf{M}$

TA

TA

TA

M

$\mathbf{M}$

$\mathbf{M}$

$\mathbf{M}$

$\mathbf{M}$
TA

TA
Artocarpus elastica

shrub, small w. edible fr. and

(large) 1.

Musa paradisiaca

= padu I

Artocarpus integer

Annona muricata

Artocarpus heterophyllus

Oryza sativa

Oryza sativa var. glutinosa

= nalu épu

= nakat wéréng

tree, small w. yellow 1.

Abelmoschus moschatus

Cyperus kyllingia

Canarium asperum

Eleusine indica

shrub w. black inedible 'beans'

Grewia eriocarpa

Sterculia oblongata

Myrmecodia (cj.)

Dinochloa scandens

Cocos nucifera

tree, small w. small speckled fr. attached to the twigs; often infested by edible larvae

Cordia ?subcordata

Timonius timon

Cassia fistula

Mimosa invisa

Dioscorea alata

= 'ai 'ohu

Platycerium bifurcatum

Ipomoea batatas

Dioscorea ?sarasinii

$=$ 'ohu

tree, ?tall w. thick stem

vine

Cucumis sativus (race)

tree, small; stem used for bows

Carica papaya

Ricinus

Euphorbia

Diplazium 


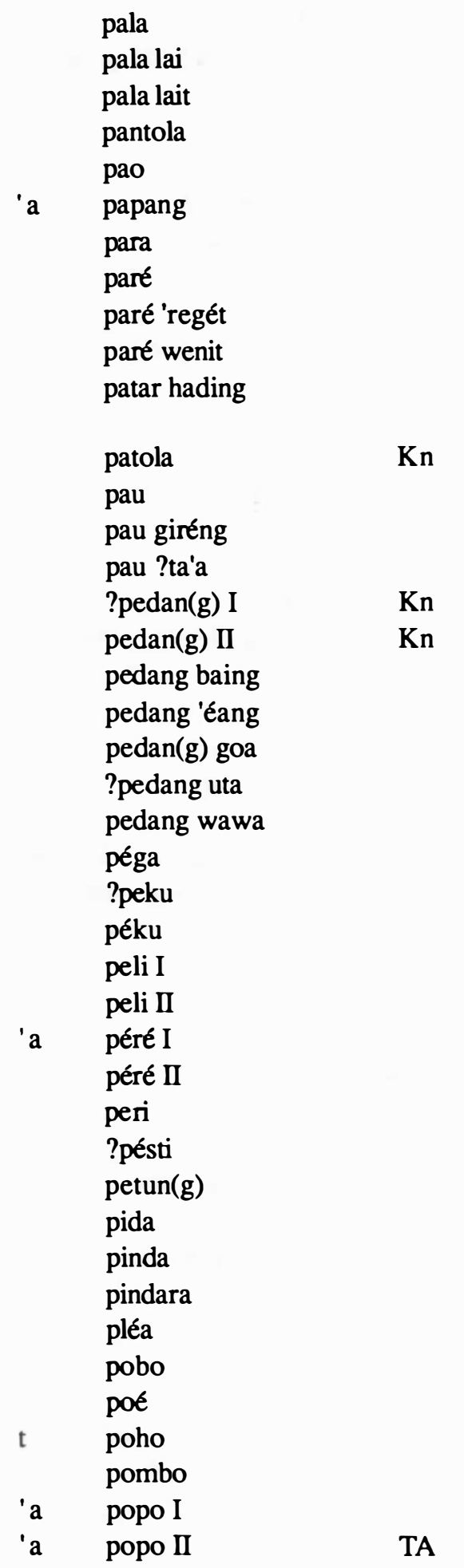

M

paré 'regét

paré wenit

patola

pau

pau gireng

?ta'a

?pedan(g) I

$\mathrm{Kn}$

pedang baing

pedang 'éang

pedan(g) goa

?pedang uta

pedang wawa

péga

péku

peli I

péré I

péré II

peri

?pésti

petun(g)

pindara

pléa

pobo

pombo

popo II
M

m

$\mathbf{M}$

M

Myristica fragrans

vine, creeping

= pala lai

= patola

Mangifera

Cordia

Pagiantha sphoerocarpa

Oryza sativa

Oryza sativa var. glutinosa

Sida

shrub or tree w. hard wood; bitter bark used against fever

$\mathbf{M}$

Luffa aegyptiaca

Mangifera indica

Anacardium occidentale

$\mathrm{dft}$

M

Mangifera

Pandanus

= pedang 'éang

M

$\mathbf{M}$

Opuntia ?elatior

Ananas comosus

= pedang baing

M

Pandanus amaryllifolius

= pedang baing

M

$\mathbf{M}$

$\mathbf{M}$

M

$\mathbf{M}$

M

$\mathbf{M}$

M

M

$\mathbf{M}$

$\mathbf{M}$

M

M

$\mathbf{M}$

shrub (Papilionaceae)

vine w. edible fr.

Mucuna pruriens var. utilis

Bambuseae

Gigantochloa ?verticillata

tree, large

vine, climbing

Ficus wassa / ampelas

tree

Dendrocalamus asper

Dioscorea hispida

Dioscorea (cp. pida)

Ziziphus nummularia

Dernis

$=$ lemur

tree, large

Paederia scandens

shrub

Eucalyptus alba

Musa 
'a popo bura

popo mérak

?popot

poté

puhé beta

puhu bura

ramu

rao

'rata oték

regon

'réhi bokat

?'réi bokat

'réi wawi

?'réi wi'ing

réo

reta

ri'i

ripit I

'a ripit II

rita

rité

roang dang

roé

'a roni

rosét

rotan

ru

ruha buén(g)

runu

sabu rongo

salur

sampaga

sang

saru 'wowong

sayur kupang

semara

sendana laki

sépé sénak

sermélé

sikun(g) jaga

singké
Kn

TA

TA, Kn

Sk

$\mathbf{M}$

M

M

M

M

M

dft

M

M

$\mathbf{M}$

M

M

M

$\mathbf{M}$

$M$

$M$

M

M

$M$

M

dft

$\begin{array}{ll} & \text { Talinum } \\ \text { Casuarina junghuhniana } \\ \text { dft sampaga } \\ \\ \text { ?Centrosema } \\ \text { Averrhoa carambola } \\ \text { Plumeria } \\ \text { Syzygium aromaticum }\end{array}$

Eucalyptus alba

Eucalyptus ?urophylla

Musa acuminata

Lagenaria siceraria

Centella asiatica

Mussaenda frondosa

$=$ blé'it

Syzygium

Bidens

= goi awas

Fungi

Fungi

Fungi

Fungi

Lannea coromandelica

Pandanus tectorius

Imperata cylindrica

epiphyte, on trees

tree, large

Alstonia scholaris

?= 'lité

vine; bark used as substitute for betel nut

tree

Polypodiaceae

Colocasia esculenta

Saccharum spontaneum

Casuarina equisetifolia I

Podocarpus?

Papilionaceae

Wedelia urticaefolia

Fungi

Trichosanthes anguina

Pterocarpus indicus

Piper nigrum

Crotalaria

Talinum

Casuarina junghuhniana

?Centrosema

Averrhoa carambola

Syzygium aromaticum 
soklat

sola

solo

somu

soro wowo

sunga

ta'a

ta'a koro

ta'a uta

tahi wakang

tali anggur

tali 'lawat (?blawat)

tana ra'i

tana ra'ing I

tana ra'ing II

tana unén

taor

$\operatorname{tarun}(\mathrm{g})$

tawu

tawu 'lewén

tebuk

teké liman

'a tenér

tepu

'a téru

té'u helon

té'u tilung

tewu

tiko

w tilu gelir

timun

tio mata

tiri I

tiri II

?toké

tolén(g)

ton bio

toro I

toro II

'a tour I

tour II

tu niluk
TA

$\mathrm{dft}$

TA

M

M

$\mathrm{Nt}$

dft

M

M

M

M

M

$\mathrm{Kn}$

M

M

M

M

$\mathbf{M}$

$M, d f t$
Abelmoschus esculenta

= patola

Myristica

Allium cepa var. escalonicum

Spilanthes iabadicensis

Allium sativum

Piper betle

Piper ?retrofractum

Piper betle

Algae

$=$ 'anggor

?= 'anggor

Selaginella

Loniaceae

Musci

Arachis hypogaea

Calophyllum soulattri I

inophyllum (cp. bintangur)

Indigofera

Lagenaria siceraria

= kamodo

Corypha pinnata

Polypodiaceae

tree, coastal, w. hard wood

Acanthaceae

Alstonia spectabilis

Poaceae

Fungi, Auricularia

Saccharum officinarum

?

Papilionaceae

Cucumis sativus

shrub

Amorphophallus ?campanulatus

Amorphophallus variabilis

Pandanus (broad-leafed)

Ficus

tree

Solanum melongena

Solanum aculeatum

Planchonia valida

Marnnea

Piliostigma malabaricum var. acidum 


\begin{tabular}{|c|c|c|c|}
\hline tua utan & & & Arenga pinnata \\
\hline tuka & & & Ipomoea batatas \\
\hline tuka boi & & & vine, tuberous \\
\hline tuka wawa & & & $=$ tuka wolanda \\
\hline tuka wolanda & & & Solanum tuberosum \\
\hline tulén & & dft & $\begin{array}{l}\text { Ricinocarpodendron } \\
\text { polystachyum }\end{array}$ \\
\hline tutur & & dft & Madhuca \\
\hline ?tuwun(g) & Sk, TA & & Caesalpinia ?crista \\
\hline unu kuta & & & $?$ \\
\hline unuk & & $\mathbf{M}$ & shrub; 1 . used as perfume \\
\hline 'urun aur & & & ?Capillipedium assimile \\
\hline 'urun paré & $\mathrm{Kn}$ & & Eleusine indica \\
\hline 'urun tali lanan & $\mathrm{Kn}$ & & Paspalum \\
\hline ('urun) tebé & & $\mathbf{M}$ & Heteropogon contortus \\
\hline utang & Sk & $\mathbf{M}$ & $=$ padu I \\
\hline utan $(\mathrm{g})$ halé & $\mathrm{Kn}$ & & Amaranthus \\
\hline utang hupér & & $\mathbf{M}$ & plant, creeping, bitter vegetable \\
\hline utang mérak & & $\mathbf{M}$ & Algae \\
\hline utang wetang & & $\mathbf{M}$ & green Algae \\
\hline wa'an bain & TA & & Mimosa invisa \\
\hline wado wa'i & & $\mathbf{M}$ & shrub; yields perfume \\
\hline wa'i & & $\mathbf{M}$ & vine, tuber \\
\hline wai talo & & & Zingiberaceae \\
\hline wangar & & $\mathbf{M}$ & ?Dioscorea \\
\hline waroting & & $\mathrm{dft}$ & Madhuca \\
\hline waru & TA & & Hibiscus tiliaceus \\
\hline 'watar I & & & Sorghum saccharatum \\
\hline 'watar II & TA & & Zea mays \\
\hline 'watar gahar & & & Sorghum (nitidum) \\
\hline wau mu'an & & & Selaginella \\
\hline wawi ta'in & & & Datura metel \\
\hline wawi toron & TA & & Solanum aculeatum \\
\hline wékak nalu & & $\mathrm{dft}$ & Neonauclea \\
\hline wélang & & & Porndaca oleracea \\
\hline wero pléan & & & Loranthaceae \\
\hline wéru & & & Semecarpus \\
\hline weru wégé & & & $=$ johar \\
\hline weru (werul-weru) & & $\mathbf{M}$ & ?Garugafloribunda \\
\hline wetan & $\mathrm{Kn}$ & & $=$ i'ur wetan \\
\hline ?wetan áhu & TA & & Setaria \\
\hline wetan sigar & TA & & $=$ i'ur wetan \\
\hline ?wetang wéting & & $\mathbf{M}$ & $=$ i'ur wetan \\
\hline
\end{tabular}




wéwé
wéwé glonak
wéwé kléréng
wéwé tana
'wiro
wua
wua biri
wua ladu
wuang golung
wuék
wuék tana
wuék wué
wukak
wulu
wuré
'wuta
wutik

TA

$\mathbf{M}$

TA

M

M

M

$\mathbf{M}$

TA

TA

M
M
M

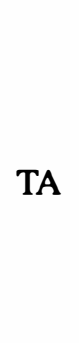

M

M

M

M

$\mathbf{M}$

$\mathbf{M}$
Vigna unguiculata

= wéwé

Phaseolus vulgaris

= wuék tana

Schizostachyum blumii

Areca cathecu

?Pinanga (cp. biri)

?Roystonea regia

Anacardium

Cajanus cajan

Arachis hypogaea
Papilionaceae (w. flat pulses)

Sterculia foetida

= 'wiro

Macaranga tanarius

Cerbera

Colocasia esculenta 


\section{9 'SOLOR' - TAXONOMIC}

\subsubsection{PRELIMINARY REMARKS}

(a) The language

(i) Keraf (1978) established, on the basis of lexicostatistical results, some 16 languages belonging to the Solorese Group of languages. He assumed, however, an $81 \%$ retention rate as the language limit. With a lower limit, as others maintain, this number would be drastically reduced.

Both Keraf's map with dialect/language boundaries and his lexicostatistical lists were of much help to me.

(ii) Orthography and pronunciation

It is clear that even between adjacent dialects, e.g. between Lewuka and Kalikasa, conspicuous sound-shifts are found. When checking our provisional list with informants from the same district later on, we heard final nasals instead of nasalised vowels, and smooth onsets instead of glottal stops. The smooth onset is not written here.

As our research lasted only ten days, with many hours on the way, and because we did not have informants with an overall knowledge of the dialects, we had to make dubious decisions concerning the orthography. Students should therefore use our materials with a critical attitude.

We spell assumedly nasalised vowels with ' ' '

(b) Research and identification

Many identified names of trees were taken from Hildebrand, other names were found in Heyne. These authors of ten use 'Solor' without specifying the precise place. Most names were identified by us in the field. Many plants were collected, and we got their identification from Leiden.

(c) Abbreviations

(i) Classifiers

$\begin{array}{lll}\text { e } & \text { ebong, ebô } & \text { tree } \\ \text { k } & \text { kajo, kajô, kayô, kayo', karo } & \text { tree, woody } \\ \text { kl, kr } & \text { klemet, lremet, keremê } & \text { grass, weed } \\ \text { t } & \text { talé, tali, talé, talé' } & \text { vine, 'liana } \\ \text { w } & \text { wulun } & \text { flower }\end{array}$

(ii) Localities

$\begin{array}{ll}\text { Bg } & \text { Bélogili } \\ \text { Kl } & \text { Kalikasa } \\ \text { Ll } & \text { Lewoléin } \\ \text { Lr } & \text { Larantuka } \\ \text { Lm } & \text { Lamelera } \\ \text { L } & \text { Lewotobi } \\ \text { Lw } & \text { Lewuka } \\ \text { Re } & \text { Rita-ebang } \\ \text { Sl } & \text { Solorese dialect (unspecified) } \\ \text { Wh } & \text { Witihama }\end{array}$


1.9.2 THE LIST

\begin{tabular}{|c|c|c|c|c|}
\hline & aho melan & $\mathrm{Lt}$ & RV & Oxalis corniculata \\
\hline & aho wéré & $\mathrm{Bg}$ & RV & = aho wéwén \\
\hline & aho wéwén & $\mathrm{Bg}$ & 5508 & Allmannia nodiflora \\
\hline & aho wéwér & $\mathrm{Bg}$ & RV & = aho wéwén \\
\hline & akasia & $\mathrm{Bg}, \mathrm{Ll}$ & RV & $=$ ta'é \\
\hline kl & aler I & $\mathrm{L}$ & RV & Spilanthes iabadicensis \\
\hline \multirow[t]{4}{*}{ kl } & aler II & $\mathrm{L}$ & RV & $=$ unu \\
\hline & amakéan & $\mathrm{Sl}$ & $\mathrm{dft}$ & Dysoxylum \\
\hline & ana-noa & Wh & V & = amona \\
\hline & anggor I & $\mathrm{Lt}$ & RV & Vitis vinifera \\
\hline $\mathrm{t}$ & anggor II & $\mathrm{L}$ & RV & $=$ bibis \\
\hline \multirow[t]{5}{*}{$\mathbf{k}$} & angin & $\mathrm{L}$ & RV & Casuarina junghuhniana \\
\hline & angin taing & $\mathrm{Lt}$ & RV & Lichenes, Usnea \\
\hline & anona I & $\mathrm{Sl}$ & $\mathrm{H}$ & $=$ ata belanda \\
\hline & anona II & $\mathrm{Sl}$ & $\mathrm{H}$ & $=$ amona \\
\hline & ansana (Ind.) & $\mathrm{Sl}$ & $\mathrm{dft}$ & $=$ kena'a \\
\hline \multirow[t]{14}{*}{ k } & apu & $\mathrm{Bg}$ & RV & ?Hibiscus \\
\hline & amona & $\mathrm{L}$ & RV & Annona reticulata \\
\hline & aru kramat & Sl & $\mathrm{H}$ & Bambusa ?blumeana \\
\hline & ata I & $\mathrm{L}$ & RV & $=$ ata belanda \\
\hline & ata II & $\operatorname{Re}$ & & Annona squamosa \\
\hline & ata belanda & $\mathrm{Bg}, \mathrm{Re}$ & RV & Annona muricata \\
\hline & ata blada & $\mathrm{Lw}$ & V & $=$ ata belanda \\
\hline & atfokat & Wh & V & $=$ atpokat \\
\hline & atpokat & $\mathrm{Lt}, \mathrm{Bg}$ & RV & Persea americana \\
\hline & $\mathrm{au}$ & $\mathrm{Bg}, \mathrm{Wh}$ & RV & $=a u^{\prime}$ \\
\hline & $\mathrm{au}^{\prime}$ & $\mathrm{L}$ & RV & Gigantochloa ?apus \\
\hline & $a^{\prime} u$ & $\mathrm{Bg}$ & RV & Amaranthus \\
\hline & au ora & $\mathrm{Bg}$ & RV & Dinochloa scandens \\
\hline & au petû & Sl & $\mathrm{H}$ & $=$ petû \\
\hline w & a'ung & $\mathrm{L}$ & RV & Amaranthus \\
\hline \multirow[t]{6}{*}{ w } & a'ung klamang & $\mathrm{L}$ & RV & Amaranthus ?spinosus \\
\hline & aur & Lw & V & ?Avicennia \\
\hline & aurarâ & Wh & V & $=$ jajang \\
\hline & babesi & $\mathrm{Lr}$ & dft & Ziziphus celtidifolia \\
\hline & babesi putih & $\mathrm{Sl}$ & $\mathrm{dft}$ & Ziziphus 'talanai' \\
\hline & baé & $\mathrm{L}$ & RV & Albizia chinensis \\
\hline \multirow[t]{2}{*}{$\mathbf{k}$} & bahi & Lt, Wh & V & $=$ kasambi \\
\hline & bahik & $\mathrm{L}$ & RV & Spathoglottis cf.plicata \\
\hline \multirow[t]{3}{*}{ ai } & baî & $\mathbf{L t}$ & RV & Mimosa invisa \\
\hline & bajâ & $\mathrm{L}$ & RV & Albizia falcataria \\
\hline & baki & $\mathrm{Lt}, \mathrm{Bg}$ & RV & $=$ muko baki \\
\hline
\end{tabular}




\begin{tabular}{|c|c|c|c|c|}
\hline & bako & $\mathrm{Lt}$ & RV & $=$ kebako \\
\hline & balu & Sl & $\mathrm{dft}$ & Syzygium \\
\hline & baner & Sl & $\mathrm{dft}$ & $=$ worungû \\
\hline & bao & $\mathrm{Lt}, \mathrm{Bg}, \mathrm{Wh}$ & V & Ficus benjamina \\
\hline & baor & Lw & V & $=$ bao \\
\hline \multirow[t]{14}{*}{ kl } & bawa batang & $\mathrm{Lt}$ & RV & Saccharum anundinaceum \\
\hline & bawâ burâ & $\mathrm{Bg}, \mathrm{Wh}$ & RV & Allium sativum \\
\hline & bawâ mé'â & $\mathrm{Lt}, \mathrm{Bg}$ & $\mathrm{RV}, \mathrm{H}$ & Allium cepa var. escalonicum \\
\hline & bayâ & Wh & V & Amaranthus \\
\hline & be oré & $\mathrm{Ll}$ & RV & = géi \\
\hline & belâ & Lw, Lt, Bg, Wh & RV & Schizostachyum brachycladum \\
\hline & belékâ & $\mathrm{Bg}$ & RV & $\begin{array}{l}\text { Lagenaria siceraria b) (water } \\
\text { gourd) }\end{array}$ \\
\hline & belibê & Wh & $\mathrm{V}$ & $=$ klibang \\
\hline & belibê & $? \mathrm{Lt}$ & $\mathrm{H}$ & Averrhoa carambola \\
\hline & belilé & Sl & $\mathrm{dft}$ & = klaét I \\
\hline & belimbî & $\mathrm{Bg}$ & RV & $=$ klibang \\
\hline & belo? & Sl & $\mathrm{dft}$ & Syzygium \\
\hline & belung & $\mathrm{Lt}$ & RV & Rhaphidophora \\
\hline & beluwak & Sl & $\mathrm{dft}$ & $=$ sengké \\
\hline \multirow[t]{4}{*}{ k } & bemia & $\mathrm{Bg}$ & RV & = baî \\
\hline & bengo' & $\mathrm{L}$ & RV & $=\mathrm{ipa}$ \\
\hline & bera papaung & Sl & $\mathrm{dft}$ & Buchanania arborescens \\
\hline & berenit & $\mathrm{Bg}$ & RV & shrub, thomy \\
\hline \multirow[t]{9}{*}{ k } & bérut & $\mathrm{u}$ & RV & Semecarpus forstenii \\
\hline & bési & $\mathrm{Lw}, \mathrm{Lt}, \mathrm{Bg}, \mathrm{Wh}$ & V & Cucurbita moschata \\
\hline & bési kélé & $\mathrm{L}$ & RV & $=$ kelotô \\
\hline & bési kélén & $\mathrm{Bg}$ & 5498 & Eclipta alba \\
\hline & bési kélét & $\mathrm{Bg}$ & 5494 & Hedyotis (Oldenlandia) \\
\hline & bibis & Wh & V & Passiflora foetida \\
\hline & bidara & Wh & V & Ziziphus nummularia \\
\hline & bilong & Sl & dft & Syzygium cumini \\
\hline & blata' I & $\mathrm{Lt}$ & RV & Erythrina (thomy variety) \\
\hline \multirow[t]{2}{*}{ k } & blata' II & $\mathrm{Lt}$ & RV & Erythrina \\
\hline & blé'it & $\mathrm{Lt}$ & RV & Cycas rumphii \\
\hline k & bluntas & $\mathrm{Lt}$ & 5512 & Pluchea (indica) \\
\hline \multirow[t]{4}{*}{ k } & blurat & $\mathrm{Lt}$ & 5516 & Trema cannabina \\
\hline & boncis & $\mathrm{Lt}, \mathrm{Wh}$ & V & Phaseolus vulgaris \\
\hline & bora & $\mathrm{Bg}$ & RV & Caryota mitis \\
\hline & boro & Sl & $\mathrm{dft}$ & Bruguiera parviflora \\
\hline \multirow[t]{3}{*}{ k } & ?boro & $\mathrm{L}$ & RV & Adenanthera ?pavonina \\
\hline & borot & Wh & $\mathrm{v}$ & $=$ té'ét \\
\hline & ?boru & $\mathrm{Bg}$ & RV & Stachytarpheta indica \\
\hline
\end{tabular}




\begin{tabular}{|c|c|c|c|c|}
\hline & bua sengsara & $\mathrm{Lt}$ & RV & Passiflora quadrangularis \\
\hline & buit & Wh & 4843 & Gyrocarpus americanus \\
\hline & bunga kerama' & Wh & V & $=$ baî \\
\hline \multirow[t]{10}{*}{$\mathbf{k}$} & cendana & $\mathrm{Lt}$ & RV & Santalum album \\
\hline & dagalaing & $\mathrm{Lt}$ & RV & = sagalai \\
\hline & dagalaing bélê & $\mathrm{Lt}$ & RV & $\begin{array}{l}\text { Lycopersicon lycopersicum } \\
\text { (w. large fr.) }\end{array}$ \\
\hline & dalima & $\mathrm{Bg}, \mathrm{Wh}$ & V & Punica granatwn \\
\hline & dami & Sl & dft & Pterospermum javanicum \\
\hline & danga' & $\mathrm{Lt}$ & RV & $=$ kedanga \\
\hline & darat & $\mathrm{Lt}$ & RV & $=$ bidara \\
\hline & dela & $\mathrm{Lw}, \mathrm{Bg}, \mathrm{Wh}$ & RV & Coix lacryma-jobi var. ma-yuen \\
\hline & delat & $\mathrm{L}$ & RV & $=$ lekâ tenga \\
\hline & delat goa & $\mathrm{Lt}$ & RV & $=$ dela \\
\hline k & dila & $\mathrm{Lt}$ & RV & $=$ kedila I \\
\hline k & dila' bélê I & $\mathrm{Lt}$ & $\mathrm{RV}$ & $=$ kedila II \\
\hline \multirow[t]{7}{*}{ k } & dila bélê II & $\mathrm{Lt}$ & RV & ?Euphorbia ?antiquorum \\
\hline & $\operatorname{dimu}$ & $\mathrm{Bg}$ & H, RV & Cucumis sativus \\
\hline & dimû & $\mathrm{Lt}, \mathrm{Wh}$ & V & Citrullus lanatus \\
\hline & dimû wata' & Wh & $\mathrm{V}$ & $=\operatorname{dimu}$ \\
\hline & dokal & $\mathrm{Lt}$ & RV & Fungi, Auricularia \\
\hline & doko & $\mathrm{Lt}, \mathrm{Bg}$ & RV & Pandanus (broad-leafed) \\
\hline & doto' & $\mathrm{Lt}$ & RV & = belékâ \\
\hline \multirow[t]{2}{*}{ k } & duling & $\mathrm{Lt}$ & 5527 & Pisonia umbelliflora \\
\hline & dulo kumang & $\mathrm{Lt}$ & RV & Maclura cochinchinensis \\
\hline \multirow[t]{2}{*}{ e } & dungeng & $\mathrm{L}$ & RV & $\begin{array}{l}\text { tree, large w. triparite } 1 . \text { and } \\
\text { violet petioles }\end{array}$ \\
\hline & éga & Wh & V & Crotalaria \\
\hline k & éhể & $\mathrm{Lt}, \mathrm{Bg}$ & RV & Spondias malayana \\
\hline \multirow[t]{3}{*}{$\mathbf{k}$} & ehéng & $\mathrm{L}$ & RV & Elaeocarpus floribundus \\
\hline & ékot & Wh & V & Lagenaria siceraria (small fr.) \\
\hline & fata & Sl & $\mathrm{H}$ & $=$ wata \\
\hline k & feno & $\mathrm{Lt}$ & RV & Melochia cf. umbellata \\
\hline \multirow[t]{3}{*}{$\mathbf{k}$} & ferak & $\mathrm{Lt}$ & RV & $=$ kerewak \\
\hline & foâ & $\mathrm{L}$ & RV & = kewoâ \\
\hline & fulor & Lw & V & $=$ wulo' \\
\hline \multirow[t]{5}{*}{ k } & furak & $\mathrm{u}$ & RV & shrub w. edible 1 and white fl. \\
\hline & futa & Sl & dft & $=$ futa \\
\hline & futa' & $\mathrm{Lt}$ & RV & Cerbera \\
\hline & gabe & $\mathrm{Lm}$ & $\mathrm{H}$. & Uncaria gambir \\
\hline & gaé & Sl & $\mathrm{H}$ & Inocarpus fagiferus \\
\hline \multirow[t]{2}{*}{$\mathbf{k}$} & gari' & $\mathrm{Lt}$ & RV & Rhus taitensis \\
\hline & géi & $\mathrm{Lt}$ & RV & Abrus precatorius \\
\hline
\end{tabular}




\begin{tabular}{|c|c|c|c|c|}
\hline & gélâ & $\mathrm{Lt}$ & RV & = gélâ burâ \\
\hline & gélâ burâ & $\mathrm{Bg}$ & RV & Portulaca oleracea \\
\hline & gélâ jawa & $\mathrm{Bg}$ & RV & = gélâ mé'â \\
\hline & gélâ mé'â & $\mathrm{Bg}$ & RV & Sesuvium portulacastrum \\
\hline & gemalo & Sl & $\mathrm{H}$ & $=\operatorname{ta}^{\prime} \hat{\mathbf{u}}$ \\
\hline & gému & Sl & $\mathbf{H}$ & $=$ gaé \\
\hline & gerung & $\mathrm{Kl}$ & V & $=\mathrm{lu}$ \\
\hline & geta & $\mathrm{L}$ & RV & Manihot glazovii \\
\hline & gewawa & $\mathrm{Bg}$ & RV & $=$ koa jawa \\
\hline k & hali I & Sl & $\mathrm{dft}$ & Aglaia ganggo \\
\hline \multirow[t]{2}{*}{$\hat{k}$} & hali II & Sl & $\mathrm{dft}$ & Dysoxylum \\
\hline & hekur & $\mathrm{Lt}$ & RV & $=$ seku \\
\hline k & hepang & $\mathrm{u}$ & RV & Caesalpinia sappan \\
\hline \multirow[t]{12}{*}{$\mathrm{t}$} & hepé & $\mathrm{Bg}$ & RV & Calopogonium mucunoides \\
\hline & herô lolô & Wh & V & Sida \\
\hline & hugaleng & $\mathrm{L}$ & RV & Achyranthes aspera \\
\hline & hura I & $\mathrm{Bg}$ & RV & $=$ uwé \\
\hline & hura II & $\mathrm{Bg}$ & RV & $=$ hura' \\
\hline & hura' & $\mathrm{Lt}, \mathrm{Wh}$ & $\mathrm{V}$ & Dioscorea esculenta \\
\hline & hura' lado & $\mathrm{Lt}$ & RV & Dioscorea aculeata \\
\hline & ?hura utan & $\mathrm{Bg}$ & RV & $=$ hura' lado \\
\hline & ikâ matan & $\mathrm{Lt}$ & RV & Breynia \\
\hline & ipa & Wh & $\mathbf{V}$ & Mucuna pruriens f. utilis \\
\hline & ipaj & $\mathrm{Lw}, \mathrm{Bg}$ & V & $=\mathrm{ipa}$ \\
\hline & ipê gingé & $\mathrm{Bg}$ & RV & Pennisetum spicatum \\
\hline \multirow[t]{9}{*}{ k } & ipek & & RV & ?Terminalia \\
\hline & ipi & $\mathrm{Sl}$ & $\mathrm{dft}$ & Intsia bijuga \\
\hline & ipij & $\mathrm{L}$ & RV & $=\mathrm{ipi}$ \\
\hline & ipis & $\mathrm{Bg}$ & RV & $=\mathrm{ipi}$ \\
\hline & ira & $\mathrm{Bg}$ & RV & ?Acacia farnesiana \\
\hline & jahom & Sl & $\mathrm{H}$ & $=$ waha' \\
\hline & jajang & Sl & $\mathrm{dft}$ & Toona ciliata / sureni \\
\hline & jambu ménté & $\mathrm{Bg}$ & RV & = wuâ golu \\
\hline & jambulan & $\mathrm{Lr}$ & $\mathrm{H}$ & $=$ bilong \\
\hline \multirow[t]{6}{*}{ k } & jati & $\mathrm{Lt}, \mathrm{Wh}$ & $\mathrm{V}, \mathrm{RV}$ & Tectona grandis \\
\hline & johar & $\mathrm{Lt}, \mathrm{Wh}$ & RV & Cassia siamea \\
\hline & kabahi & $\mathrm{Bg}, \mathrm{Sl}$ & $\mathrm{dft}, \mathrm{RV}$ & $=$ kasambi \\
\hline & kabako & Sl & $\mathrm{H}$ & $=$ kebako \\
\hline & kada waning & $\mathbf{L}$ & RV & Eupatorium inulifolium \\
\hline & kadanga & Sl & $\mathrm{H}$ & $=$ kedanga \\
\hline \multirow[t]{3}{*}{ k } & kadu ria & $\mathrm{u}$ & RV & Dillenia pentagyna \\
\hline & kaférak & ?Lm & $\mathrm{H}$ & ?= kawéra \\
\hline & kaha' & $\mathrm{Lt}, \mathrm{Wh}$ & RV & Opuntia \\
\hline
\end{tabular}




\begin{tabular}{|c|c|c|c|c|}
\hline & kakung baru & $\mathbf{L t}$ & RV & Hyptis suaveolens \\
\hline & kalado & $\mathrm{Lr}$ & $\mathrm{dft}$ & $=$ kledo $^{\prime}$ \\
\hline & kaladu & Sl & $\mathrm{dft}$ & $=$ kladu \\
\hline & kalikét & $\mathrm{Bg}$ & RV & Pennisetum purpureum \\
\hline & ?kalukê & $\mathrm{Bg}, \mathrm{Sl}$ & dft, RV & $=$ kepi' \\
\hline & kamboja & Lt, Wh & V & Plumeria acuminata \\
\hline & kami'é & Sl & $\mathrm{dft}, \mathrm{H}$ & $=$ kemi'é \\
\hline & kanda pistu & $\mathrm{Lr}$ & $\mathrm{dft}$ & = kluang \\
\hline & kang tupateng & $\mathrm{Lt}$ & RV & Dioscorea \\
\hline \multirow[t]{7}{*}{ w } & kangkung & $\mathrm{Lt}$ & RV & Ipomoea aquatica \\
\hline & kanila & Sl & $\mathrm{dft}$ & Grewia salutaris \\
\hline & kanona & $\mathrm{Bg}$ & RV & Cordia ?subcordata \\
\hline & kapape & Sl & $\mathrm{H}$ & $=$ kapuka \\
\hline & kape bola & $\mathrm{Lt}$ & RV & $=\mathbf{k a p o}^{\prime}$ \\
\hline & kape lolon & $\mathrm{Lt}$ & RV & = kapek I \\
\hline & kapek I & Bg, Wh & RV & Gossypium \\
\hline $\mathbf{k}$ & kapek II & $\mathrm{Bg}$ & RV & ?Corchorus \\
\hline \multirow[t]{25}{*}{$\mathbf{k}$} & kapek III & $\mathrm{Bg}$ & RV & Bombax ceiba \\
\hline & kapes & Lw & V & $=$ kapek I \\
\hline & kapo' & $\mathrm{Bg}$ & RV & Ceiba pentandra \\
\hline & kapuka & Sl & $\mathrm{H}$ & Eucalyptus alba \\
\hline & karcinta & $\mathrm{Lr}$ & $\mathrm{H}$ & Phyllanthus emblica \\
\hline & ?karo & Sl & $\mathrm{dft}$ & Dysoxylum \\
\hline & karo gula & Wh & 4839 & Samanea saman \\
\hline & karorot & $\mathrm{Bg}$ & RV & Triumfetta \\
\hline & kasambi & $\mathrm{Lr}$ & $\mathrm{dft}$ & Schleichera oleosa \\
\hline & kasi & $\mathrm{Lr}$ & $\mathrm{H}$ & $=$ paja \\
\hline & katawu pokang & Sl & $\mathrm{H}$ & $=$ ketebuk \\
\hline & katué & Sl & dft & Syzygium \\
\hline & kawéra & Sl & $\mathrm{H}$ & Artocarpus heterophyllus \\
\hline & kawuta' & $\mathrm{Bg}$ & RV & ?Excoecaria agallocha \\
\hline & kborot & Lw & V & $=$ téét \\
\hline & ké'a méin & $\mathrm{Lt}$ & RV & Euphorbia hirta \\
\hline & ke'ale I & Wh & 4837 & $=$ aler I \\
\hline & ke'ale II & Hinga & V & Galinsoga parviflora \\
\hline & kéam batu & Sl & $\mathrm{dft}$ & Myristica frangrans / ?fatua \\
\hline & kebako & $\mathrm{Bg}, \mathrm{Wh}$ & V & Nicotiana tabacum \\
\hline & kebawang & Lw & V & = bawâ mé'â \\
\hline & kebawang bujak & Lw & V & = bawâ burâ \\
\hline & kebelû & Wh & & $=$ padu bima \\
\hline & kebelung & Lw & & $=$ padu bima \\
\hline & kébo & $\mathrm{Bg}$ & RV & = kébol \\
\hline $\mathrm{t}$ & keboja I & $\mathrm{Bg}$ & 5489 & Operculina turpethum \\
\hline
\end{tabular}




\begin{tabular}{|c|c|c|c|c|}
\hline \multirow[t]{16}{*}{$\mathrm{t}$} & keboja II & $\mathrm{Bg}$ & 5511 & Ipomoea quamoclit \\
\hline & kébol & $\mathrm{Lw}, \mathrm{Lt}$ & H, RV & Arenga pinnata \\
\hline & kebû & $\mathrm{Lt}, \mathrm{Bg}$ & 5491 & Bidens \\
\hline & kebuké & $\mathrm{Lt}, \mathrm{Bg}$ & RV & $=$ kebuke' \\
\hline & kebuke' & Wh & V & Morinda tomentosa \\
\hline & kebuko & $\mathrm{Bg}, \mathrm{Lr}$ & RV & $=$ kebuke' $^{\prime}$ \\
\hline & keda & $\mathbf{L}$ & RV & $=$ keda $^{\prime}$ \\
\hline & keda' & $\mathrm{Bg}, \mathrm{Wh}$ & V & Mucuna pruriens f. pruriens \\
\hline & kedanga & $\mathrm{Bg}$ & RV & Kleinhovia hospita \\
\hline & kedar & Lw & V & $=$ keda $^{\prime}$ \\
\hline & kedelé & $\mathrm{Lt}$ & RV & Glycine soja \\
\hline & kédibu & $\mathrm{Lr}$ & $\mathrm{H}$ & Cordyline terminalis \\
\hline & kedila I & $\mathrm{Bg}$ & RV & Aegle marmelos \\
\hline & kedila II & $\mathrm{Bg}$ & RV & Crescentia cujete \\
\hline & kedola I & $\mathrm{Bg}$ & RV & Luffa acutangula \\
\hline & kedola II & $\mathrm{Bg}$ & RV & Luffa ?aegyptiaca \\
\hline \multirow[t]{16}{*}{$\mathbf{k}$} & kedondô I & $\mathrm{Lt}$ & RV & Spondias dulcis \\
\hline & ?kedondô II & Wh & V & $=$ éhề \\
\hline & kedo'ok & $\mathrm{Bg}$ & RV & Mallotus philippensis \\
\hline & kehawu & Wh & V & $=$ rokak I \\
\hline & kehulé & $\mathbf{L}$ & RV & $=$ kehulo' \\
\hline & kehulo' & $\mathrm{Bg}, \mathrm{Lr}$ & RV & Ficus septica \\
\hline & keka' & $\mathrm{Lt}, \mathrm{Bg}$ & H, RV & Bambusa ?blumeana \\
\hline & kekaj & Lw & V & $=$ keka' \\
\hline & kela & $\mathrm{Bg}$ & RV & $=$ klewak \\
\hline & kela' & $\mathbf{L}$ & RV & $=$ klewak \\
\hline & kela' utâ & $\mathrm{L}$ & RV & Trichosanthes villosa \\
\hline & kela'a & $\mathrm{Bg}$ & $\mathrm{H}, \mathrm{RV}$ & Benincasa hispida \\
\hline & keladi & $\mathrm{Lw}, \mathrm{Bg}, \mathrm{Wh}$ & RV & Colocasia esculenta \\
\hline & keladu & $\mathrm{Bg}$ & RV & $=$ kladu \\
\hline & kelapu & $\mathrm{Bg}$ & RV & Fatoua pilosa \\
\hline & kelebo & $\mathrm{Bg}$ & RV & = klebo I \\
\hline \multirow[t]{9}{*}{ k } & keledo & $\mathrm{Bg}$ & RV & $=$ kledo' $^{\prime}$ \\
\hline & keleka' para I & $\mathrm{Lr}$ & dft & $=$ kepara \\
\hline & keleka' para II & $\mathrm{Bg}$ & RV & Voacanga grandiflora \\
\hline & keléla & Wh & V & Lycopodium \\
\hline & kélféor & Lw & V & = wé'o \\
\hline & keliha & $\mathrm{Sl}$ & dft & Terminalia catappa \\
\hline & kelomo I & Wh & V & = bérut \\
\hline & kelomo II & Sl & dft & Ziziphus timoriensis \\
\hline & ?keloré & $\mathrm{Lr}$ & dft & Neonauclea calycina \\
\hline \multirow[t]{2}{*}{ k } & kelotô & $\mathrm{Bg}$ & RV & Vernonia cinerea \\
\hline & kélu & $\mathrm{Bg}$ & RV & Fungi \\
\hline
\end{tabular}




\begin{tabular}{|c|c|c|c|}
\hline keludu & Wh & V & $=$ kedila II \\
\hline keluk & $\mathrm{L}$ & RV & Bauhinia hirsuta \\
\hline kelupu & $\mathrm{Bg}$ & RV & $=$ lebok \\
\hline kélur dang & $\mathbf{L t}$ & RV & Fungi \\
\hline kemati & $\mathrm{Bg}$ & RV & = dagalaing bélê \\
\hline kematir & $\mathrm{Lr}$ & dft & $=$ sagalai \\
\hline kembang sepatu & $\mathbf{L}$ & RV & Hibiscus rosa-sinensis \\
\hline kemede' & Wh & V & $=$ hura' lado \\
\hline kemérî & $\mathrm{Bg}$ & 5495 & Vitex paniculata \\
\hline kemi'é & $\mathrm{Bg}$ & RV & Aleurites moluccana \\
\hline kemu'u au & $\mathrm{Bg}$ & RV & Poaceae \\
\hline kemu'un & Wh & V & Eulalia leschenaultiana \\
\hline kena & Sl & $\mathrm{dft}$ & $=$ kanona \\
\hline kena'a & $\mathrm{Bg}, \mathrm{Sl}$ & dft, RV & Pterocarpus indicus \\
\hline kenaha & Sl & $\mathrm{H}$ & $=$ kena'a \\
\hline kenapa & Wh & V & Ficus subcordata \\
\hline kenar & Lw & V & $=$ nenas \\
\hline kenarê & $\mathrm{L}$ & RV & $=$ kedila I \\
\hline kenari & Wh & V & $=\mathrm{koja}^{\prime}$ \\
\hline kenila & $\mathrm{Bg}$ & RV & $=$ kanila \\
\hline kenobak & $\mathrm{Bg}$ & RV & cf. Tylophora \\
\hline kenoé & Wh & V & Lamiaceae \\
\hline kentang & $\mathrm{Lt}$ & RV & Solanum tuberosum \\
\hline kenuna & $\mathrm{Bg}$ & RV & $=$ nunang \\
\hline kenutî & $\mathrm{Bg}$ & RV & $=$ nuting \\
\hline ke'olok & Wh & V & Caesalpinia \\
\hline képa I & $\mathrm{Lt}, \mathrm{Bg}$ & RV & Coleus amboinicus \\
\hline képa II & $\mathrm{Bg}$ & RV & = képa' \\
\hline képa' & $\mathrm{L}$ & RV & Pacchyrrhizus erosus \\
\hline képa' glého' & $\mathrm{L}$ & RV & = kipé \\
\hline kepara & $\mathrm{Bg}$ & RV & Pagiantha sphaerocarpa \\
\hline kepi' & $\mathrm{Lt}$ & RV & $\begin{array}{l}\text { Piliostigma malabaricum var. } \\
\text { acidum }\end{array}$ \\
\hline kepipi & $\mathrm{Bg}$ & RV & Cissus \\
\hline kepo & $\mathrm{Sl}$ & $\mathrm{dft}$ & = wukak \\
\hline képoh & $\mathrm{Lr}$ & $\mathrm{H}$ & = wukak \\
\hline kepok & Lw & V & $=\mathbf{k a p o}^{\prime}$ \\
\hline kepulut & Wh & V & ?Urena lobata \\
\hline kepunga' & Wh & V & = wawé' toron I \\
\hline kerang & Lw & V & $=$ kela'a \\
\hline kerbau tilun & $\mathrm{Lt}$ & RV & Kalanchoe pinnata \\
\hline keremét & $\mathrm{Bg}$ & RV & Poaceae \\
\hline keréra & $\mathrm{Bg}$ & RV & Ipomoea pes-caprae \\
\hline
\end{tabular}




\begin{tabular}{|c|c|c|c|}
\hline keretuk & $\mathrm{Bg}$ & RV & $=$ ketuk \\
\hline kerewak & $\mathrm{Bg}$ & RV & Macaranga tanarius \\
\hline keri & $\mathbf{L t}$ & RV & $=\operatorname{dimu}$ \\
\hline keroko & Wh & V & $=$ kroko' $^{\prime}$ \\
\hline keromé tilun & Wh & V & Fungi, Auricularia \\
\hline keronga & $\mathrm{Bg}$ & RV & = kapuka \\
\hline keroré & Wh & V & = keroré' \\
\hline keroré' & $\mathrm{Bg}$ & RV & Morinda citrifolia \\
\hline kéru & $\mathrm{Lt}, \mathrm{Bg}$ & 5520 & Pollia \\
\hline keruku I & $\mathrm{Bg}$ & 5493 & Heliotropium \\
\hline keruku II & $\mathrm{Bg}$ & RV & ?Ocimum \\
\hline kesâ & $\mathrm{Bg}$ & RV & Euphorbia prunifolia \\
\hline kesambi & Sl & $\mathrm{dft}$ & $=$ kasambi \\
\hline keso & Wh & V & = wé'o \\
\hline ketaka I & $\mathrm{Bg}$ & RV & Ocimum basilicum \\
\hline ketaka II & Wh & V & Ocimum sanctum \\
\hline ketaka bélâ & Wh & V & $=$ ketaka I \\
\hline ketapâ & Wh & V & $=$ keliha \\
\hline ketebuk & $\mathrm{Bg}, \mathrm{Wh}$ & RV & Corypha pinnata \\
\hline ketelak & $\mathrm{Bg}$ & RV & Physalis \\
\hline keteli & $\mathrm{Bg}$ & RV & Crotalaria \\
\hline keti'é & $\mathrm{Bg}, \mathrm{Wh}$ & H, RV & Amorphophallus variabilis \\
\hline ketoro & $\mathrm{Bg}, \mathrm{Wh}$ & V & Solanum melongena \\
\hline ketuk & $\mathrm{Lt}$ & RV & Canarium asperum \\
\hline keturu & $\mathrm{Bg}$ & RV & Caesalpinia \\
\hline ke'ulek & Wh & 4834 & Ficus \\
\hline kewaki & Wh & V & = blé'it \\
\hline kewanga I & $\mathrm{Bg}$ & RV & Capparis \\
\hline kewanga II & Wh & V & $=$ nunang \\
\hline kewéra & $\mathrm{Bg}$ & 5506 & Desmodium \\
\hline kewérak I & Wh & V & Artocar pus altilis (soeded) \\
\hline ?kewérak II & Wh & V & Artocarpus integer \\
\hline kewérak lala' & $\mathrm{Bg}$ & RV & Artocarpus integer \\
\hline kewikir & $\mathrm{Lr}$ & $\mathrm{dft}$ & $=$ kewikit \\
\hline kewikit & $\mathrm{L}$ & RV & Leea ?angulata \\
\hline kewoâ & $\mathrm{Bg}, \mathrm{Wh}$ & RV, V & Ficus balica \\
\hline kewoit & Wh & V & = uwé' jawâ \\
\hline kéwuk & $\mathbf{L t}$ & RV & Zingiberaceae \\
\hline kikê & $\mathrm{L}$ & RV & $=$ unu ale \\
\hline kinok & $\mathrm{Lt}$ & RV & tree, large, fr. blue when fallen \\
\hline kipé & $\mathrm{Bg}$ & RV & Phaseolus ?lunatus \\
\hline kla'a & $\mathrm{Lt}$ & RV & $=$ kela'a \\
\hline kladar I & $\mathrm{Lt}$ & RV & $=$ lete \\
\hline
\end{tabular}




\begin{tabular}{|c|c|c|c|c|}
\hline & kladar II & $\mathrm{Lt}$ & RV & $=$ keladi \\
\hline & kladar mé'â & $\mathrm{Lt}$ & RV & Colocasia \\
\hline & kladu & $\mathrm{Lt}$ & RV & Albizia procera \\
\hline & klaét I & $\mathrm{Lt}$ & RV & Melia azedarach \\
\hline \multirow[t]{3}{*}{ k } & klaét II & $\mathrm{Lt}$ & RV & $\begin{array}{l}\text { tree, large w. small round } \\
\text { yellow fr. }\end{array}$ \\
\hline & klapu tébang & $\mathrm{L}$ & RV & $=$ terata wulun loko \\
\hline & klaru méra & $\mathrm{Lt}$ & RV & Leea indica \\
\hline \multirow[t]{3}{*}{ kl } & klatê & $\mathrm{Lt}$ & 5528 & Laportea interrupta \\
\hline & klatê laking & $\mathrm{Lt}$ & RV & ?Dendrocnide \\
\hline & klatê waing & $\mathrm{Lt}$ & RV & Laportea?aestuans \\
\hline e & klawê I & $\mathrm{Lt}$ & RV & Ficus (small 1.) \\
\hline \multirow[t]{3}{*}{ k } & klawê II & $\mathrm{Lt}$ & 5530 & Ficus ?variegata \\
\hline & klebo I & $\mathrm{Lt}$ & RV & Anamirta cocculus \\
\hline & klebo II & $\mathrm{Lt}$ & RV & Ichnocarpus \\
\hline k & kledo' & $\mathrm{Lt}$ & RV & Homalium tomentosum \\
\hline \multirow[t]{2}{*}{ k } & kléhing & $\mathrm{Lt}$ & RV & $\begin{array}{l}\text { tree, large w. cordate } 1 \text {. and } \\
\text { slimy bark }\end{array}$ \\
\hline & klekar & $\mathrm{L}$ & RV & ?Rauvolfia \\
\hline kr & klélang & $\mathrm{Lt}$ & RV & Chrysopogon aciculatus \\
\hline \multirow[t]{5}{*}{ k } & kleming & $\mathrm{Lt}$ & RV & Cinnamomum burmanni \\
\hline & kléruk & Lw & V & $=$ wua' \\
\hline & klewak & $\mathrm{Lt}$ & RV & Lagenaria siceraria (for dishes) \\
\hline & klibang & $\mathbf{L}$ & RV & Averrhoa bilimbi \\
\hline & kligong & $\mathrm{Lt}$ & RV & Emilia sonchifolia \\
\hline k & klikar & $\mathrm{L}$ & RV & Wikstroemia androsaemifolia \\
\hline \multirow[t]{8}{*}{ kl } & klikét & $\mathrm{Lt}$ & RV & $=$ kalikét \\
\hline & klokô & $\mathrm{Lt}$ & RV & $=$ kroko' \\
\hline & klomé tilun I & $\mathrm{Lt}$ & RV & Fungi \\
\hline & klomé tilun II & $\mathrm{Lt}$ & RV & Ipomoea obscura \\
\hline & klonuk & Lt & RV & Fungi \\
\hline & kloré & Ili Apé & V & $=$ keroré' \\
\hline & klorér & Lw & V & $=$ keroré' \\
\hline & kloro & $\mathrm{L}$ & RV & Triumfetta \\
\hline \multirow[t]{2}{*}{$\mathrm{kl}$} & klorot & $\mathrm{Lt}$ & 5515 & Cenchrus \\
\hline & klowang & $\mathrm{Sl}$ & $\mathrm{H}$ & = kluang \\
\hline \multirow[t]{7}{*}{ k } & kluang & $\mathrm{Lt}$ & RV & Cassia fistula \\
\hline & ko & $\mathrm{L}$ & RV & = ladoméra \\
\hline & koa jawa & $\mathrm{Lt}$ & RV & Psidium guajava \\
\hline & kobu wéwél & $\mathrm{Lt}$ & RV & $=$ sisal \\
\hline & kobu wéwélê & $\mathrm{Lt}$ & RV & $=$ sisal \\
\hline & ko'it I & $\mathrm{Lt}$ & RV & Drynaria \\
\hline & ko'it II & $\mathrm{Bg}$ & RV & Hydnophytum formicarum \\
\hline
\end{tabular}




\begin{tabular}{|c|c|c|c|c|}
\hline & kojâ & $\mathrm{Lt}$ & RV & Ipomoea \\
\hline & koja' & $\mathbf{L}$ & RV & Canarium vulgare \\
\hline \multirow[t]{2}{*}{$t$} & kokô bolo' & $\mathrm{Lt}$ & RV & Mucuna \\
\hline & kolế & $\mathbf{L}$ & RV & Stemona \\
\hline \multirow[t]{21}{*}{$t$} & kolé' & $\mathrm{L}$ & RV & Dioscorea \\
\hline & kolér & Lw & V & $=$ koli \\
\hline & koli & $\mathrm{Lt}$ & RV & Borassus sundaicus \\
\hline & kolo énga I & $\mathrm{Bg}$ & RV & Dioscorea \\
\hline & kolo énga II & $\mathrm{Bg}$ & RV & Ipomoea (small 1.) \\
\hline & kolo énga III & $\mathrm{Bg}$ & $\mathbf{R V}$ & Ipomoea (large l.) \\
\hline & kolô poho' & $\mathrm{Bg}$ & RV & Paederia scandens \\
\hline & kopi & $\mathrm{Lt}, \mathrm{Bg}, \mathrm{Wh}$ & RV & Coffea \\
\hline & kopi soklat & $\mathrm{u}$ & $\mathbf{R V}$ & Abelmoschus esculentus \\
\hline & koro boang & $\mathrm{Lt}$ & RV & Lareara camara \\
\hline & koromé tilun & $\mathrm{Bg}$ & RV & Ipomoea cf. obscura \\
\hline & kotak & $\mathrm{L}$ & RV & Conna indica \\
\hline & kotat & $\mathrm{Lt}$ & RV & $=$ kotak \\
\hline & ?kpukut & Lw & V & Fungi, Auricularia \\
\hline & kroko' & $\mathrm{Bg}$ & RV & Calotropis gigantea \\
\hline & krokor & Lw & $\mathbf{V}$ & $=$ kroko \\
\hline & ktebuk & Lw & $\mathbf{V}$ & $=$ ketebuk \\
\hline & kû & $\mathrm{Lt}$ & RV & Ficus?variegata \\
\hline & kubak & $\mathrm{L}$ & RV & Dioscorea pentaphylla \\
\hline & kuda klorot & $\mathrm{L}$ & RV & $=$ kepulut \\
\hline & kuda ta'in & $\mathrm{Bg}$ & 5492 & Tridax procumbens \\
\hline $\mathrm{k}$ & kué' & $\mathrm{L}$ & RV & Champereia manillana \\
\hline \multirow[t]{2}{*}{$\mathrm{kl}$} & kujo' kumi & $\mathrm{Lt}$ & 5521 & Cyperus (rotundus) \\
\hline & kuka & Sl & $\mathrm{H}$ & $=\operatorname{dim} \hat{\mathrm{u}}$ \\
\hline $\mathrm{t}$ & kukak (kuka') & $\mathrm{L}$ & RV & Cucurbitaceae \\
\hline \multirow[t]{14}{*}{ k } & kukû & $\mathrm{Lt}, \mathrm{Bg}$ & RV & Schoutenia ovata \\
\hline & kukung & $\mathrm{Lr}$ & $\mathrm{dft}$ & $=$ kukû \\
\hline & kulat I & $\mathrm{L}$ & RV & Algae \\
\hline & kulat II (Ind.) & $\mathrm{Lt}, \mathrm{Wh}$ & V & Musci \\
\hline & kule' & $\mathrm{L}$ & RV & $=$ kehulo' \\
\hline & ?kulitaba & $\mathrm{Lr}$ & $\mathrm{dft}$ & $=$ lo'o I \\
\hline & kuma & $\mathrm{Lt}, \mathrm{Bg}, \mathrm{Wh}$ & RV & Curcuma viridiflora \\
\hline & kuma burâ & $\mathrm{u}$ & RV & ?Globba marantina \\
\hline & kuma léu & $\mathrm{Bg}$ & RV & Madhuca cp. malaccensis \\
\hline & kumas & Lw & $\mathbf{V}$ & $=$ kuma \\
\hline & kumur & $\mathrm{Kl}$ & $\mathbf{V}$ & = té'ét \\
\hline & ku'u I & $\mathrm{Bg}$ & RV & ?Areca \\
\hline & ?ku'u II & $\mathrm{Bg}$ & RV & Piper retrofractum \\
\hline & ku'u blurê & $\mathrm{Lt}$ & RV & $=$ sili \\
\hline
\end{tabular}




\begin{tabular}{|c|c|c|c|c|}
\hline & ku'u krowéng & $\mathbf{L t}$ & RV & $?=$ ku'u taling \\
\hline & ku'u léléng & $\mathrm{Lt}$ & RV & $=$ sili bélâ \\
\hline & ku'u taling & $\mathbf{L}$ & 5522 & Piper ?aduncum \\
\hline & ?kuwék I & Wh & V & $=a^{\prime}$ ung klamang \\
\hline & ?kuwék II & Wh & V & $=$ sola \\
\hline & kwaror & Lw & $\mathbf{V}$ & $=$ wata \\
\hline & kwaru olot & Lw & $\mathbf{V}$ & $=$ wata belolo \\
\hline & kwérak & Lw & $\mathbf{V}$ & Artocarpus altilis (seeded) \\
\hline & kwérak wato & $\mathbf{L}$ & RV & Artocarpus heterophyllus \\
\hline \multirow[t]{6}{*}{ k } & kwiki & $\mathrm{Bg}$ & RV & = kewikit \\
\hline & la'ak & $\mathrm{Bg}$ & RV & $=$ lékẻ \\
\hline & labu jepâ & Lt, Wh & V & Sechium edule \\
\hline & ladi & $\mathrm{Sl}$ & $\mathrm{dft}$ & Rhizophora stylosa \\
\hline & ladoméra & $\mathrm{Bg}$ & RV & Heteropogon contortus \\
\hline & laku mukung & Sl & $\mathrm{dft}$ & Dysoxylum \\
\hline \multirow[t]{3}{*}{ kl } & lalat & $\mathbf{L t}$ & RV & Themeda ?villosa \\
\hline & ?lalu meran & $\mathrm{Bg}$ & RV & Cyperaceae \\
\hline & lamatoro & $\mathrm{Lt}, \mathrm{Bg}$ & RV & Leucaena leucocephala \\
\hline \multirow[t]{10}{*}{$\mathbf{k}$} & lamtoro & Lw & V & $=$ lamatoro \\
\hline & lamu & $\mathbf{L}$ & RV & Algae (marine) \\
\hline & lamû & $\mathrm{Bg}$ & RV & Algae \\
\hline & laot & $\mathrm{Lw}, \mathrm{Bg}, \mathrm{Wh}$ & V & Languas galanga \\
\hline & léba' & $\mathrm{Lt}$ & RV & $=$ peria \\
\hline & lebok & $\mathbf{L}$ & RV & Solanum ?mawitianum \\
\hline & léi sédê & $\mathrm{Bg}$ & RV & Blumea \\
\hline & léjap & Lw & V & $=$ rerap \\
\hline & lekâ tenga & $\mathrm{Bg}$ & RV & Coix lacryma-jobi var. agrestis \\
\hline & lekê & $? \mathrm{Ll}$ & RV & Bambuseae \\
\hline \multirow[t]{10}{*}{$\mathrm{t}, \mathrm{k}$} & lékê & $\mathrm{Lt}$ & RV & Uvaria ?rufa \\
\hline & lengê & $\mathrm{Lt}$ & RV & Fungi \\
\hline & léra & & & $=$ rerap \\
\hline & leséng & $\mathrm{Lm}$ & RV & Spondias malayana \\
\hline & lete & $\mathrm{Bg}$ & RV & Alocasia macrorrhiza \\
\hline & lewa' & $\mathrm{Lt}$ & RV & Melanolepis multiglandulosa \\
\hline & lia' & Lt, Wh & RV & Zingiber officinale \\
\hline & lia' goa & $\mathbf{L}$ & RV & ?Amomum \\
\hline & lima bolak & $\mathrm{Bg}$ & RV & = ké'a méin \\
\hline & lima dada & Wh & V & $=$ ata belanda \\
\hline w & lima pait & $\mathbf{L}$ & 5526 & ?Trichosanthes \\
\hline \multirow[t]{4}{*}{ k } & lité I & $\mathbf{L t}$ & RV & Mallotus ricinifolius \\
\hline & lité II & $\mathrm{Bg}$ & RV & shrub \\
\hline & lolô & $\mathrm{Lt}, \mathrm{Bg}$ & RV & Piper betle \\
\hline & lolô buri & $\mathbf{L}$ & RV & Piper \\
\hline
\end{tabular}




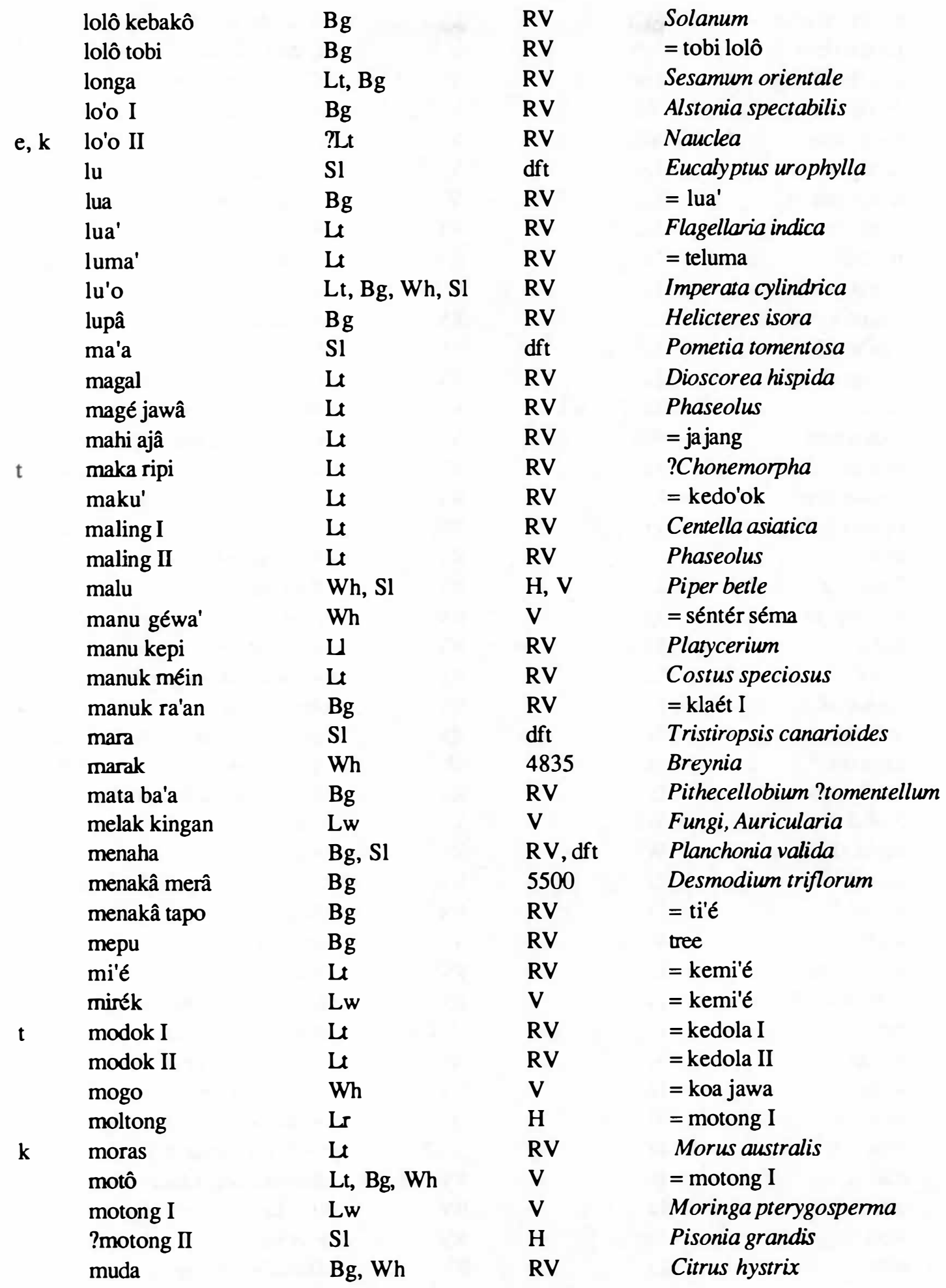




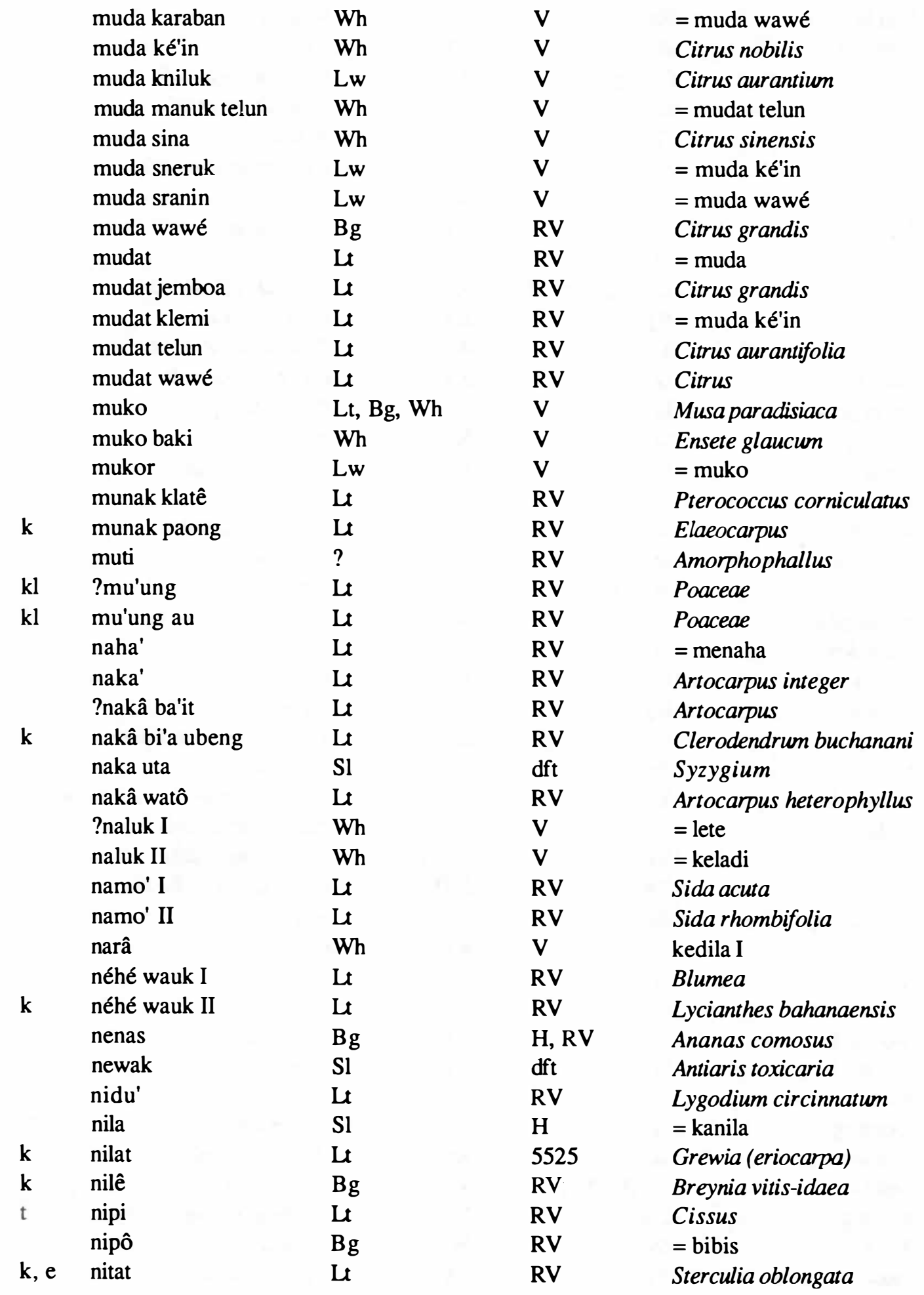




\begin{tabular}{|c|c|c|c|c|}
\hline & nodé & $\mathrm{Bg}$ & RV & Sida rhombifolia \\
\hline & nora & $\mathrm{Bg}$ & RV & $?$ \\
\hline & notâ & Wh & $\mathbf{V}$ & $=$ seré \\
\hline & nou & $\mathbf{L}$ & & = ué' nou \\
\hline & numo I & Sl & dft & Ficus pubinervis \\
\hline & numo II & Sl & $\mathrm{dft}$ & Syzygium \\
\hline & nunang & $\mathrm{Lt}$ & RV & Cordia dichotoma \\
\hline & nuting & $\mathrm{L}$ & $\mathbf{R V}$ & Lagerstroemia flos-reginae \\
\hline & nuwa & Wh & V & = pléa \\
\hline & nyanyilu & $\mathrm{Lr}, \mathrm{Sl}$ & dft & loan word; = kepi' \\
\hline & obo & $\mathrm{Bg}$ & RV & Amorphophallus \\
\hline & odo & $\mathrm{Bg}$ & V & Dioscorea ?pentophylla \\
\hline & okak & $\mathrm{Bg}$ & RV & Pittosporum \\
\hline $\mathrm{t}$ & olak & $\mathbf{L}$ & RV & ?Chonemorpha \\
\hline & olok & $\mathrm{Bg}$ & RV & Caesalpinia \\
\hline & ondo & $\mathbf{L}$ & RV & $=$ magal \\
\hline & orâ & $\mathrm{L}$ & RV & $=\mathrm{au}$ ora \\
\hline & oté kolang & $\mathrm{Lt}$ & RV & ?Cayratia trifolia \\
\hline & owa & Wh & V & = wukak \\
\hline & pador & Lw & V & $=$ padu' \\
\hline & padu' & Wh & V & Ricinus communis \\
\hline & padu aho tain & $\mathrm{Bg}$ & RV & ?Ricinus \\
\hline & padu bima & $\mathrm{Bg}$ & RV & Jatropha curcas \\
\hline & padu jawâ & $\mathrm{Bg}$ & RV & = padu bima \\
\hline & padu jawâ mé'â & $\mathrm{Bg}$ & RV & Jatropha gossypifolia \\
\hline & padu klou & $\mathrm{Bg}$ & RV & $=$ padu' \\
\hline & padu wuré & $\mathrm{Lt}$ & RV & $=$ padu bima \\
\hline & pahadé & $\mathrm{Bg}$ & RV & Harrisonia brownii \\
\hline $\mathrm{t}$ & paho & $\mathrm{Lt}$ & RV & = kolô poho' \\
\hline & paja & $\mathrm{Bg}$ & H, RV & Carica pepaya \\
\hline & pajam & Lw & V & $=$ paja \\
\hline & paku & Wh & V & Diplazium edule \\
\hline & pao & $\mathrm{Lt}, \mathrm{Bg}, \mathrm{Wh}$ & V & Mangifera indica \\
\hline & pao moko & $\mathbf{L t}$ & RV & = bera papaung \\
\hline & pao rengi & Wh & V & = wuâ golu \\
\hline & paor & Lw & V & $=$ pao \\
\hline $\mathbf{k}$ & parâ & L & RV & = kepara \\
\hline & pedâ & Bg, Wh & $V$ & $?=$ reta $\Pi$ \\
\hline & pedang & $\mathrm{Lt}$ & H, RV & $=$ nenas \\
\hline & pekok & $\mathrm{Lt}$ & RV & Euphorbia barnhariï \\
\hline & pelawan & $\mathrm{Lr}$ & $\mathrm{dft}$ & = kapuka \\
\hline & pelé & Wh & 4836 & Pterocaulon sphacelatum \\
\hline & pepu & $\mathbf{L t}$ & RV & Abelmoschus moschatus \\
\hline
\end{tabular}




\begin{tabular}{|c|c|c|c|c|}
\hline \multicolumn{5}{|r|}{ Andrographis } \\
\hline & perék & $\mathrm{Lt}$ & RV & Ficus ?heteropleura \\
\hline & peri & $\mathbf{L t}$ & RV & Gigantochloa ?verticillata \\
\hline & per̂ & $\mathrm{Bg}$ & RV & $=$ peri \\
\hline & peria & $\mathrm{Bg}, \mathrm{Wh}$ & V & Momordica charantia \\
\hline \multirow[t]{4}{*}{$\mathrm{kl}$} & permé & $\mathrm{Lt}$ & 5519 & Salvia \\
\hline & peté sina & Wh & V & $=$ lamatoro \\
\hline & petû & Lw, Lt, Bg & $\mathbf{V}$ & Dendrocalanus asper \\
\hline & petung & Lw & V & $=$ petû \\
\hline $\mathrm{t}$ & pi'a & $\mathrm{Lt}$ & RV & Tetracera scandens \\
\hline $\mathrm{t}$ & pléa & $\mathrm{Lt}, \mathrm{Bg}$ & RV & Derris \\
\hline \multirow[t]{4}{*}{ e } & poho' & $\mathrm{Lt}$ & RV & tree, tall w. large fr. \\
\hline & pola' & Wh & V & = kébol \\
\hline & popo I & Sl & $\mathrm{dft}$ & $=$ kapuka \\
\hline & popo II & $\mathrm{Lt}$ & RV & Musa cf. acuminata \\
\hline k & puho & $\mathrm{Bg}$ & RV & $=$ kapek I \\
\hline \multirow[t]{12}{*}{ e } & puho' & $\mathrm{Lt}$ & RV & $=$ kapek III \\
\hline & puho kapo & Wh & V & $=\mathbf{k a p o}^{\prime}$ \\
\hline & puho méan béle & Wh & V & $=$ kapek III \\
\hline & puhû ajû & $\mathrm{Bg}$ & 5497 & Clitorea (ternatea) \\
\hline & puhû burâ & $\mathrm{Bg}$ & RV & Eupatorium \\
\hline & puhû kerama & $\mathrm{Bg}$ & 5499 & Asteraceae \\
\hline & puhû terekat & $\mathrm{Bg}$ & 5510 & Cenchrus \\
\hline & puka & Sl & $\mathrm{dft}$ & $=$ kapuka \\
\hline & puka' & Wh & $\mathrm{dft}$ & $=$ kapuka \\
\hline & puka lelaki & Sl & $\mathrm{dft}$ & Eucalyptus urophylla \\
\hline & pukaj & Lw & $\mathbf{V}$ & $=$ kapuka \\
\hline & pukat & $\mathbf{L}$ & RV & = kapuka \\
\hline $\mathrm{t}$ & ra'a & $\mathrm{Lt}$ & 5513 & Cissus (discolor) \\
\hline k & raong & $\mathrm{Lt}$ & RV & Pometia pinnata \\
\hline \multirow[t]{3}{*}{$\mathrm{kr}$} & rata burâ & $\mathrm{Bg}$ & RV & Poaceae \\
\hline & réar & Lw & V & $=$ lu'o \\
\hline & regu & Sl & $\mathrm{dft}$ & = worungû \\
\hline \multirow[t]{9}{*}{ k } & réo & $\mathrm{Bg}, \mathrm{Lt}$ & RV & Lannea coromandelica \\
\hline & réo belanda & $\mathrm{Lt}$ & RV & Spathodea campanulata \\
\hline & rerap & Wh & V & Erythrina \\
\hline & reta I & $\mathrm{Lt}$ & RV & $\begin{array}{l}\text { Pandanus tectorius var. } \\
\text { littoralis }\end{array}$ \\
\hline & reta II & $\mathrm{L}$ & RV & Pandanus tectorius var. samak \\
\hline & rétor & Lw & V & $=$ rita \\
\hline & ribu wulung & $\mathrm{Lt}$ & RV & Erigeron sumatrensis \\
\hline & ripis & $\mathrm{Bg}$ & RV & tree, large, near the sea \\
\hline & rita & $\mathrm{Lt}, \mathrm{Bg}$ & H, RV & Alstonia scholaris \\
\hline
\end{tabular}




\begin{tabular}{|c|c|c|c|c|}
\hline & rokak I & $\mathbf{L t}$ & RV & Barleria prionitis \\
\hline & rokak II & $\mathbf{L}$ & RV & ?Capparis \\
\hline & romé tilu & $\mathrm{L}$ & RV & Ipomoea \\
\hline & rora & $\mathrm{Bg}$ & 5505 & Corchorus aestuans \\
\hline \multirow[t]{3}{*}{ k } & rowa & $\mathbf{L}$ & RV & $\begin{array}{l}\text { tree, large } w \text {. white latex and } \\
\text { cordate } 1 .\end{array}$ \\
\hline & ruha keboti & $\mathrm{Bg}$ & $\mathrm{RV}$ & $?$ \\
\hline & ruha ta'û & $\mathrm{Bg}$ & RV & ?Crotalavia \\
\hline \multirow[t]{12}{*}{ k } & ruho' & $\mathrm{L}$ & RV & Croton tiglium \\
\hline & sa'ang & Sl & $\mathrm{H}$ & Piper nigrum \\
\hline & sagalai & Lw, Wh & V & $\begin{array}{l}\text { Lycopersicon lycopersicum } \\
\text { (wild-growing variety) }\end{array}$ \\
\hline & sasi & $\mathrm{Bg}$ & RV & $=\operatorname{dimû}$ \\
\hline & sawan & Lw & V & = uwé' jawâ \\
\hline & sebo'ut & Wh & V & Euphorbia \\
\hline & segéi & Wh & V & = géi \\
\hline & segélâ & Wh & $\mathbf{V}$ & Spinifex littoreus \\
\hline & segelai & $\mathrm{u}$ & RV & = kakung baru \\
\hline & seku & $\mathrm{Bg}, \mathrm{Wh}$ & V & Kaempferia galanga \\
\hline & semara & $\mathrm{Lt}$ & RV & Casuarina junghuhniana \\
\hline & sengké & $\mathbf{L}$ & RV & Syzygium aromaticum \\
\hline $\mathrm{t}$ & séntér séma & $\mathrm{L}$ & 5518 & Centrosema \\
\hline \multirow[t]{8}{*}{$\mathrm{kl}$} & seré & $\mathrm{Lt}, \mathrm{Bg}$ & RV & Cymbopogon citratus \\
\hline & seré wangi & $\mathbf{L}$ & RV & Cymbopogon citratus (race) \\
\hline & silér & Lw & V & $=$ sili \\
\hline & sili & $\mathrm{Bg}$ & RV & Capsicum frutescens \\
\hline & sili bala & $\mathrm{Bg}$ & RV & = sili bélâ \\
\hline & sili bélâ & Wh & $\mathrm{V}$ & Capsicum annuwm \\
\hline & sili busê & Wh & $\mathbf{V}$ & $=$ sili \\
\hline & sili samo & $\mathrm{Lr}$ & $\mathrm{dft}$ & Antidesma ghaesembilla \\
\hline \multirow[t]{13}{*}{$\mathrm{kl}$} & sina séping & $\mathbf{L}$ & RV & Cyperaceae \\
\hline & sisal & Lw & V & Agave sisalana \\
\hline & soklat & $\mathrm{L}$ & RV & Theobroma cacao \\
\hline & sola & $\mathbf{L}$ & RV & Erechtites valerianifolia \\
\hline & sop & $\mathbf{L}$ & RV & Apium graveolens \\
\hline & soro kowa' & $\mathbf{L}$ & RV & $\begin{array}{l}\text { Cucurbitaceae (w. inedible } \\
\text { small, round, red fr.) }\end{array}$ \\
\hline & suka' & Wh & V & $=$ sisal \\
\hline & sukun & $\mathbf{L}$ & RV & Artocarpus altilis (seedless) \\
\hline & sura amen & Lw & V & = uwé' \\
\hline & sura kajor & Lw & V & = ué kajo \\
\hline & sura mojek & Lw & V & $=$ hura' \\
\hline & sura rotan & Lw & V & = hura' lado \\
\hline & ?surar & Lw & V & $=$ uwé' \\
\hline
\end{tabular}




\begin{tabular}{|c|c|c|c|c|}
\hline & suréng & $\mathrm{Lr}$ & dft & Toona sureni (sureni / ciliata) \\
\hline & ta'é & Sl & dft & Acacia oraria \\
\hline & tahâ & $\mathrm{Bg}$ & RV & $=$ waha' \\
\hline & tahâ tenaro & $\mathrm{Bg}$ & RV & $=$ waha' naro \\
\hline & Cahi lolo & $\mathrm{L}$ & RV & Sida \\
\hline & taka' & $\mathbf{L}$ & RV & $=$ ketaka I \\
\hline & tana one & Wh & V & = utâ tana \\
\hline kl & tana raking & $\mathbf{L}$ & 5524 & Lindernia (crustacea) \\
\hline & ta'o & $\mathrm{Lt}$ & RV & $=\mathrm{ta}^{\prime} \mathrm{u}$ \\
\hline & tapo & $\mathrm{Bg}, \mathrm{Wh}$ & H, RV & Cocos nucifera \\
\hline & tapo' & $\mathrm{Lt}$ & RV & $=$ tapo \\
\hline & tapor & Lw & V & $=$ tapo \\
\hline & tata & $\mathrm{Lr}$ & $\mathrm{H}$ & Ficus ?myriocarpa \\
\hline & ta'û & $\mathrm{Lt}, \mathrm{Bg}, \mathrm{Wh}$ & RV & Indigofera \\
\hline & taum & Lw & V & $=\operatorname{ta}^{\prime} \hat{\mathrm{u}}$ \\
\hline & tawau & Sl & $\mathrm{dft}$ & Calophyllum inophyllum \\
\hline & tebé & $\mathrm{Bg}$ & RV & $=$ tebo \\
\hline & tebe' & $\mathrm{Lt}, \mathrm{Bg}$ & RV & Saccharum spontaneum \\
\hline & tebo & $\mathrm{Bg}$ & RV & Phragmites karka \\
\hline & tebuk & $\mathrm{Lt}$ & RV & $=$ ketebuk \\
\hline k & teburô I & $\mathrm{Bg}$ & 5496 & Envatania \\
\hline $\mathrm{t}$ & teburô II & $\mathrm{Bg}$ & RV & ?Ipomoea \\
\hline & té'ét & $\mathrm{Lt}, \mathrm{Bg}$ & RV & Artocarpus elasticus \\
\hline & tefo & Sl & $\mathrm{H}$ & $=$ tewo \\
\hline k & telir I & $\mathrm{Lt}$ & RV & Murraya paniculata \\
\hline $\mathbf{k}$ & telir II & $\mathbf{L t}$ & RV & ?Dysoxylum arborescens \\
\hline & teluang & $\mathrm{Bg}$ & RV & $=$ kluang \\
\hline & teluma & $\mathrm{Bg}, \mathrm{Wh}$ & V & Sesbania grandiflora \\
\hline & tenai & Sl & $\mathrm{dft}$ & Tristiropsis canarioides \\
\hline kl & tengal & $\mathbf{L t}$ & RV & Thysanolaena (cj.) \\
\hline k & tenunu & $\mathrm{Bg}$ & 5504 & Pithecellobium umbellatum \\
\hline kl & tepo & $\mathrm{Lt}$ & 5529 & ?Pseuderanthemum \\
\hline & terata wulun loko & $\mathrm{Bg}$ & RV & Sericocalyx asper \\
\hline & teria & Lw & V & $=$ peria \\
\hline & tewa'u & Wh & V & $=$ tebe $^{\prime}$ \\
\hline & tewo & $\mathrm{Lt}, \mathrm{Bg}, \mathrm{Wh}$ & V & Saccharum officinarum \\
\hline & ti'é & $\mathrm{Lt}$ & RV & Amorphophallus campanulatus \\
\hline & ima & Sl & dft & Syzygium \\
\hline & timu & SI & dft & Timonius timon \\
\hline & tobi & $\mathrm{Lt}, \mathrm{Wh}, \mathrm{Sl}$ & $\mathrm{RV}, \mathrm{H}, \mathrm{dft}$ & Tamarindus indica \\
\hline & tobi lolô & $\mathrm{Bg}, \mathrm{Lr}$ & RV & Phyllanthus \\
\hline & tobi makî & Wh & V & $?=$ tobi lolô \\
\hline & tobi ronê & $\mathrm{Lt}$ & RV & $?=$ tobi lolô \\
\hline
\end{tabular}




\begin{tabular}{|c|c|c|c|c|}
\hline \multirow{3}{*}{$\begin{array}{l}w \\
k\end{array}$} & tolang & $\mathrm{u}$ & $\mathrm{RV}$ & $?=$ kedola $\mathrm{I} / \mathrm{II}$ \\
\hline & toléng & $\mathrm{Lt}$ & RV & tree, large \\
\hline & tomu & $\mathrm{L}$ & RV & $=$ laot \\
\hline k & tonê I & $\mathrm{u}$ & RV & Vitex trifolia \\
\hline \multirow[t]{2}{*}{ k } & tonê II & $\mathbf{L}$ & RV & $\begin{array}{l}\text { tree, large, } w \text {. reddish } \mathrm{fl} \text {, and } \\
\text { bitter small round fr. }\end{array}$ \\
\hline & toro' & $\mathbf{L}$ & RV & $=$ ketoro \\
\hline \multirow[t]{24}{*}{ k } & tou' & $\mathbf{L}$ & RV & Pterospermum diversifolium \\
\hline & tuak & Wh & $\mathrm{V}$ & $=$ koli \\
\hline & tuak pokang & Sl & $\mathrm{H}$ & $=$ koli \\
\hline & tuba (Ind.) & Lw & v & = pléa \\
\hline & tubé & Wh & V & Sonneratia alba \\
\hline & tuna ahane & $\mathrm{L}$ & RV & Hibiscus surattensis \\
\hline & tutung réng I & $\mathbf{L}$ & $\mathrm{RV}$ & Cassia tora \\
\hline & tutung réng II & $\mathrm{L}$ & RV & Crotalaria \\
\hline & ua & Wh & $\mathrm{V}$ & $=$ uar \\
\hline & ua/wua pajâ & $\mathrm{L}$ & RV & $=$ paja \\
\hline & uaj & Lw, Lr & $\mathrm{V}$ & $=$ uar \\
\hline & uar & $\mathrm{Bg}$ & RV & ?Calomus \\
\hline & uat bou' & $\mathbf{L}$ & RV & ?Calomus \\
\hline & uat manuk & $\mathrm{L}$ & RV & ?Daemonorops \\
\hline & ubal & Lw & $\mathrm{V}$ & $=$ magal \\
\hline & ué' I & $\mathbf{L}$ & RV & = uwé' \\
\hline & ué' II & $\mathbf{L}$ & RV & Ipomoea \\
\hline & ué' III & $\mathrm{L}$ & RV & = ué kajo \\
\hline & ué améng & $\mathrm{Bg}$ & RV & $=$ hura' $^{\prime}$ \\
\hline & ué' bél'ê & $\mathbf{L}$ & RV & = uwé' \\
\hline & ué' hura' & $\mathrm{L}$ & RV & $=$ hura' \\
\hline & ué jawâ & $\mathrm{Bg}$ & RV & = uwé' jawâ \\
\hline & ué kajo & $\mathrm{Bg}$ & RV & Manihot esculenta \\
\hline & ué' nou & $\mathrm{L}$ & RV & Dioscorea sarasinii \\
\hline \multirow[t]{12}{*}{$\mathrm{t}$} & ula' & $\mathrm{Lt}, \mathrm{Lr}$ & $\mathrm{V}$ & vine $w$. round yellow stem \\
\hline & ula talé & $\mathrm{Bg}$ & RV & $=$ ula $^{\prime}$ \\
\hline & ulu kuma & Sl & $\mathrm{dft}$ & Syzygium \\
\hline & unu & Wh & $\mathrm{V}$ & Wedelia urticaefolia \\
\hline & unu' & $\mathrm{Bg}$ & RV & = kakung baru \\
\hline & unu ale & $\mathrm{Bg}$ & RV & Wedelia biflora \\
\hline & unu wauk I & $\mathbf{L}$ & RV & Eupatorium \\
\hline & unu wauk II & $\mathbf{L}$ & RV & Lantana camara \\
\hline & unuj & Lw & & = kakung baru \\
\hline & ura & Wh & V & Cajanus cajan \\
\hline & ?urê & Wh & V & $=$ klewak \\
\hline & uror & Lw & V & $=\mathrm{au}^{\prime}$ \\
\hline
\end{tabular}




\begin{tabular}{|c|c|c|c|c|}
\hline & uru wadang & $\mathrm{Bg}$ & RV & Leea \\
\hline & utâ & Wh & V & = utâ niha'an \\
\hline & utâ bao & $\mathbf{W h}$ & $\mathbf{V}$ & = kipé \\
\hline & utâ blapit & $\mathrm{Lt}$ & RV & Canovalia \\
\hline & utâ blurat & $\mathrm{Lt}$ & RV & $=$ ura \\
\hline & utâ boto & $\mathrm{Bg}$ & RV & $\begin{array}{l}\text { pulse, cultivated, w. white } \\
\text { beans }\end{array}$ \\
\hline & utâ ipar & $\mathrm{Bg}$ & RV & $=\mathrm{ipa}$ \\
\hline & utâ kenolê & $\mathrm{Bg}$ & RV & = utâ niha'an \\
\hline & utâ niha'an & $\mathrm{Lt}$ & RV & Vigna unguiculata \\
\hline & utâ tana & $\mathrm{Lt}, \mathrm{Bg}$ & RV & Arachis hypogaea \\
\hline & uway & Sl & $\mathrm{H}$ & $=$ uar \\
\hline & uwé' & $\mathrm{Lt}$ & RV & Dioscorea alata \\
\hline & ?uwé holi & Wh & $\mathbf{V}$ & Dioscorea bulbifera \\
\hline & uwé' jawâ & $\mathrm{Lt}$ & RV & Ipomoea batatas \\
\hline & uwé karo & Wh & V & = ué kajo \\
\hline & uwé mure' & Wh & V & = uwé' \\
\hline & uwé wolanda & Sl & $\mathrm{H}$ & $=$ kentang \\
\hline & waha' & Lt, Wh & V & Oryza sativa \\
\hline & waha' ketâ & Wh & V & $=$ waha' naro \\
\hline & waha' naro & $\mathbf{L}$ & RV & Oryza sativa f. glutinosa \\
\hline k & wahér & $\mathrm{Lt}$ & RV & Morinda (tomentosa) \\
\hline $\mathbf{k}$ & wai & $\mathrm{Bg}$ & RV & Syzygium \\
\hline w & waing I & $\mathrm{Lt}$ & RV & ?Dryopteris \\
\hline w & waing II & $\mathrm{Lt}$ & RV & Nephrolepis radicans \\
\hline w & waing III & $\mathrm{Lt}$ & 5514 & Polypodiaceae \\
\hline ?k & wana & $\mathrm{Bg}$ & RV & $=$ koro boang \\
\hline $\mathrm{kl}$ & wana nuli'ing & $\mathrm{L}$ & 5523 & Hedyotis ?corymbosa \\
\hline & wa'o & Lt, Bg, Wh & V & Hibiscus tiliaceus \\
\hline & wao kesé'in & Wh & $\mathbf{V}$ & Ficus \\
\hline & warikit & $\mathrm{Sl}$ & $\mathrm{dft}$ & Mimusops elengi \\
\hline & waror & Lw & $\mathbf{V}$ & $=$ wa'o \\
\hline & wata & $\mathrm{Bg}, \mathrm{Sl}$ & H, RV & Zea mays \\
\hline & wata' & $\mathrm{Lt}, \mathrm{Wh}$ & RV & $=$ wata \\
\hline & wata belolo & $\mathrm{Bg}$ & RV & Sorghum saccharatum \\
\hline & wata' blolong & $\mathrm{Lt}$ & RV & $=$ wata belolo \\
\hline & $\begin{array}{l}\text { ?wata' blolong hokéng } \\
\text { wata' blolong kulung }\end{array}$ & $\mathrm{Lt}$ & RV & Sorghum propinquum \\
\hline & blo'ung & $\mathbf{L}$ & RV & = ipê gingé \\
\hline & wata solot & Wh & RV & $=$ wata belolo \\
\hline & wata wet̂̂ & $\mathrm{Lt}$ & RV & Fungi, Agaricales \\
\hline & watung & Sl & $\mathrm{dft}$ & Bischofia javanica \\
\hline $\mathrm{kl}$ & waû-ne & $\mathrm{Lt}$ & RV & Ageratum (conyzoides) \\
\hline
\end{tabular}




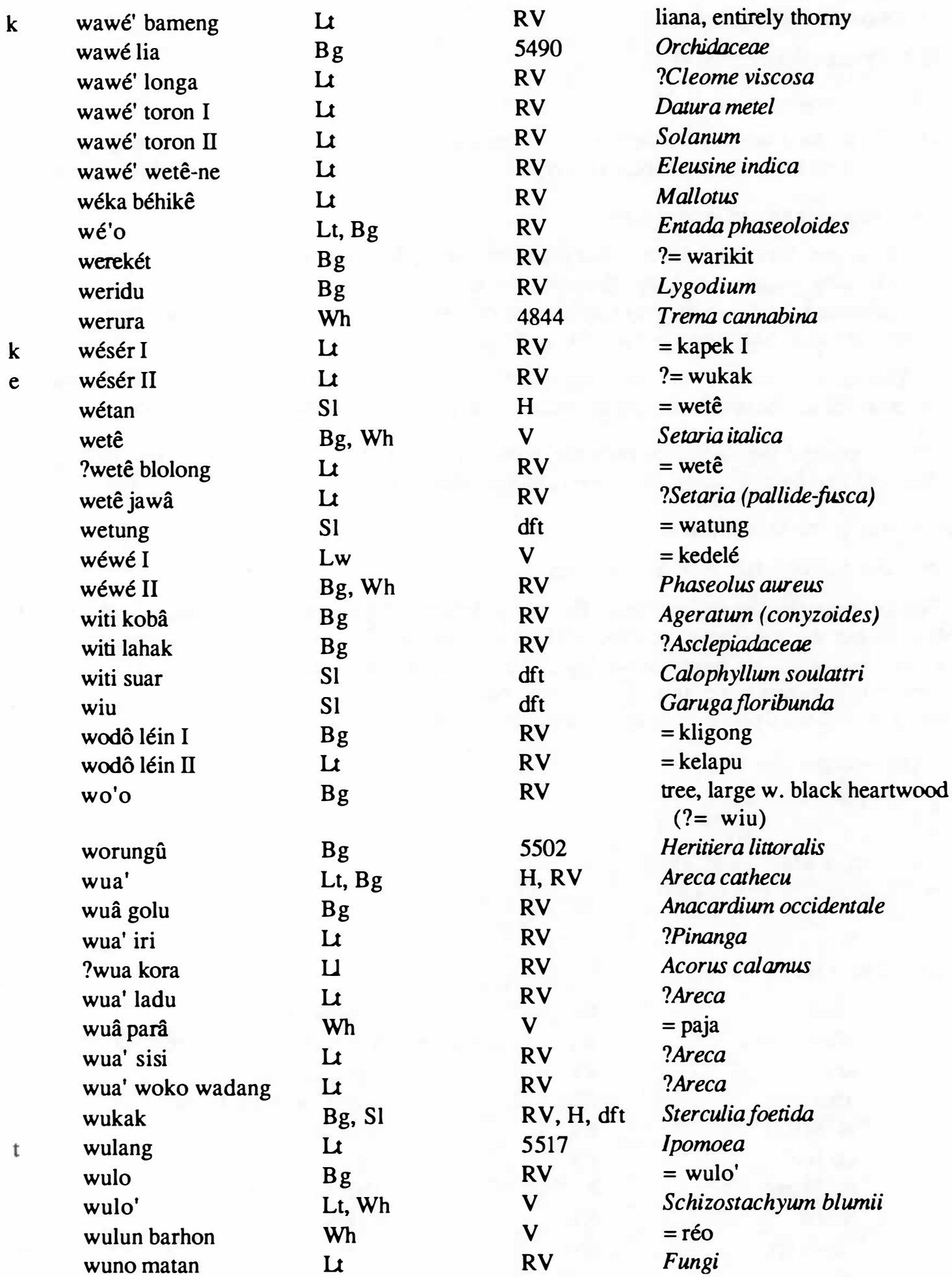




\subsection{KÉDANG - TAXONOMIC}

\subsubsection{PRELIMINARY REMARKS}

(a) The language

(i) The Kédang language differs greatly from the Solor and other neighbouring languages. No studies about it have as yet appeared except the lexicostatistical evaluation in Keraf $(1978,280 \mathrm{ff}$.).

(ii) Orthography and pronunciation

We found phonemic contrast between the unrounded higher-mid and the lower-mid front vowels: lébo 'waterpit' and lèbo 'a plant'. For the sake of conformity we spell the glottal stop as / /, although the (q), which was luckily introduced by the late Fr K. van Trier about 1950, is commonly used and known in the Kédang district.

The smooth onset is written - not quite consistently - with ( $\left.{ }^{\prime}\right)$ before the initial vowel, whereas the glottal stop is not spelled initially, but is spelled between vowels and word finally.

(iii) Almost all the names are from the dialect of Aliqur Obaq in Buyaq Suri; only two names, lèdu and lèu', come from Hoé Léaq (here spelled Hoéléa').

(b) Research and identification

No sources from elsewhere are known to me.

We lost a day by the difficult drive from Lewoléba to Aliqur Obaq (Aliuroba), though we were able to collect some plants on the way. Mr Ros and I worked feverishly with some six informants for one and a half days. He went into the field, whereas I checked my basic list to find out the scientific names of rather common plants. In the circumstances, our overall result was satisfactory. In the evening the collected plants were studied and (provisionally) prepared.

(c) Abbreviations for classifiers:

$\begin{array}{lll}\text { a } & \text { ayi } & \text { tree } \\ \text { r } & \text { ru' } & \text { grass, herb } \\ \text { r w } & \text { ru' wadé' } & \text { weed, shrub } \\ \text { w } & \text { wadé } & \text { vine, liana }\end{array}$

1.10.2 THE LIST

$\begin{array}{llll}\text { rw } & \text { abun } & \text { RV } & \text { Eleusine indica } \\ \text { a } & \text { ada } & \text { RV } & \text { Melanolepis multiglandulosa } \\ \text { a } & \text { aha } & \text { RV } & \text { Phaseolus pubescens } \\ & \text { ahung } & \text { RV } & \text { Schizostachyum blumii } \\ & \text { ala béhu } & \text { RV } & \text { Schleichera oleosa } \\ & \text { ala bélu } & \text { RV } & \text { = ala béhu } \\ \text { ala libang } & \text { RV } & \text { Averrhoa bilimbi } \\ \text { alèwu } & \text { RV } & \text { = alèwu' wua' } \\ & \text { alèwu apo } & \text { RV } & \text { apo' } \\ & \text { alèwu' 'au } & \text { RV } & \text { Dioscorea alata }\end{array}$


a

a

w

w

w

w

a

a

r w

a

a

a

a

a

a

r w
RV

RV

5545

RV

RV

RV

RV

RV

RV

RV

RV

RV

RV

RV

RV

RV

RV

RV

RV

RV

RV

RV

RV

RV

RV

RV

RV

RV

RV

RV

5534

RV

RV

RV

RV

RV

RV

RV

RV

RV

5560

RV

RV
Dioscorea esculenta

Ipomoea batatas

Maranta orundinacea

Manihot esculenta

Amaranthus

Trema orientalis

= gélang

Kleinhovia hospita

Passiflora foetida

Physalis

Polypodiaceae

Momordica charantia

Gossypium

Ipomoea

Dioscorea pentaphylla

Benincasa hispida

Luffa aegyptiaca

Luffa acutangula

?Tournefortia

Dioscorea

Poaceae

= alèwu' wua'

Gigantochloa ?apus

Bambusa ?blumeana

Macaranga ?tanarius

Ocimum basilicum

= awa ta'a riang

Ageratum conyzoides

Hyptis suaveolens

Nicotiana tabacum

Premna

Allium sativum

Allium cepa var. escalonicum

Barleria prionitis

Ficus balica

Gigantochloa?verticillata

Ficus benjamina

Ficus subcordata

Dioscorea sarasinii

Aegle marmelos

Aneilema

Solanum melongena (small fr.)

Solanum ?torvum 
a

a

a

\section{a}

\section{a}

a

r w

$$
\text { a }
$$

a

a

a

a

a

a

r w

a

w

$\mathbf{w}$

a

a

a

a bola rian

bola utun

bola wéa'

bo'ol

bo'ol botan

bono'

boro'

botan

botan loré

boyol

buata

bu'ér

bunga antonius

buru'

buya'

dalima

danung

ébol

èdu

'édu

éhu'

éré bitan maté'

éré matèn

éto

éto ayang

èyè' I

èyè' II

gélang

hapu

hé'ur

hulé

hupal

'i'a mato

iba'

inar I

inar II

ingo

ipil

ité

jati

johar

kabélung
RV

RV

RV

RV

5555

RV

RV

RV

RV

RV

RV

RV

RV

RV

RV

RV

RV

RV

RV

RV

RV

RV

RV

RV

5531

5556

RV

RV

RV

RV

RV

RV

5542

RV

RV

5538

RV

RV

RV

RV

RV

RV
Solanum melongena (large fr.)

$=$ bola

Solanum ?melongena

Morinda tomentosa

Morinda citrifolia

Melochia cf. umbellata

Artocarpus elasticus

$=$ bo'ol botan

$=$ bo'ol botan

Thespesia populnea

Sorghum propinquum

Ziziphus nwnmularia

Plumeria acuminata

tree, tall w. stinking fl.; fr. eaten by doves

Albizia procera

Punica granatum

Ficus

shrub

Lagenaria siceraria

Pennisetum purpureum

Entada phaseoloides

Leea ?indica

Solanum verbascifolium

Cassia fistula

Cassia (javanica)

Ficus

Ficus ?wassa var. obversifolia

Portulaca oleracea

Mallotus philippensis

Kaempferia galanga

?Sonneratia alba

?

Asteraceae

?Clerodendrum

?Mucuna pruriens f. pruriens

Mucuna

Sericocalyx asper

Intsia bijuga

Alstonia scholaris

Tectona grandis

Cassia siamea

Ricinus communis 


\begin{tabular}{|c|c|c|c|}
\hline $\mathbf{a}$ & kala 'olèn & RV & Pleomele \\
\hline \multirow[t]{2}{*}{ r w } & kanaru' & RV & Sida \\
\hline & kangkung & RV & Ipomoea aquatica \\
\hline \multirow[t]{2}{*}{$\mathbf{a}$} & kapo' & RV & Ceiba pentandra \\
\hline & kawaka' & RV & Eucalyptus alba \\
\hline \multirow[t]{3}{*}{ r w } & kéi rawa & RV & Urena \\
\hline & kiki-ring & RV & Crotalaria \\
\hline & kiri' ingo & RV & Wedelia \\
\hline \multirow[t]{2}{*}{ r w } & kiri' tahi' I & RV & Wedelia \\
\hline & kiri' tahi' II & 5546 & Tridax procumbens \\
\hline \multirow[t]{2}{*}{ r w } & ?kiringo & 5551 & $=$ kiri' ingo \\
\hline & kopi & RV & Coffea \\
\hline \multirow[t]{5}{*}{$\mathbf{a}$} & kulu bila & RV & Crescentia cujete \\
\hline & kulu jawa & RV & Psidium guajava \\
\hline & la' loran & 5557 & Vigna \\
\hline & la'a & RV & Bambuseae (small; for arrows) \\
\hline & labu jepang & RV & Sechium edule \\
\hline r w & ladi amé' & 5539 & Bidens \\
\hline a & lado(n) & RV & shrub, yellow fl., pinnate 1. \\
\hline \multirow[t]{2}{*}{$r w$} & ladu namé' & RV & Bidens \\
\hline & laku dara' & RV & Uvaria rıfa \\
\hline \multirow[t]{2}{*}{ a } & lamatoro & RV & Leucaena leucocephala \\
\hline & lamun & RV & Enhalus acoroides \\
\hline $\mathbf{a}$ & latèng & RV & Dendrocnide \\
\hline a & léa' & RV & Erythrina orientalis \\
\hline w & lèbo I & RV & Anamirta cocculus \\
\hline w & ?lèbo II & RV & Chonemorpha \\
\hline \multirow[t]{2}{*}{$\mathbf{a}$} & lèdu (Hoéléa') & RV & $=$ lèlu \\
\hline & léhéng & RV & Spondias malayana \\
\hline a & léka' sara & RV & ? Voacanga \\
\hline a & léla' & RV & Calotropis gigantea \\
\hline \multirow[t]{3}{*}{ a } & lèlu & RV & Homalium tomentosum \\
\hline & léra' & RV & Ipomoea pes-caprae \\
\hline & lèu' sawa (Hoéléa') & RV & = alèwu' sawa \\
\hline \multirow[t]{7}{*}{ a } & léur & RV & Casuarina equisetifolia \\
\hline & lèwu' & RV & $=$ alèwu' \\
\hline & ?lèwu' 'au & RV & $=$ alèwu' 'au \\
\hline & lèwu' 'otan & RV & Dioscorea aculeata \\
\hline & lèyè' & RV & Coix lacryma-jobi var. ma-yuen \\
\hline & lié & RV & Zingiber officinale \\
\hline & lié moli' & RV & Zingiberaceae \\
\hline a & liha & RV & Terminalia catappa \\
\hline r w & liliu & RV & Phyllanthus \\
\hline
\end{tabular}




\begin{tabular}{|c|c|c|c|}
\hline $\mathbf{a}$ & liti & 5550 & Wrightia \\
\hline $\mathbf{a}$ & lobo' & RV & Planchonella obovata \\
\hline \multirow[t]{2}{*}{$\mathbf{a}$} & lodo & RV & Cordia \\
\hline & loné & RV & $=$ bo'ol botan \\
\hline $\mathbf{a}$ & loté' & RV & Melia azedarach \\
\hline$r w$ & lotong & RV & Vernonia (cinerea) \\
\hline a & lu & RV & Eucalyptus urophylla \\
\hline w & luar & RV & Flagellaria indica \\
\hline $\mathbf{a}$ & luma sawa & RV & Anacardium occidentale \\
\hline \multirow[t]{2}{*}{$\mathbf{a}$} & lupang & 5537 & Helicteres (isora) \\
\hline & maha' 'ayang & RV & Toona ciliata \\
\hline $\mathbf{a}$ & mai I & RV & Desmodium \\
\hline \multirow{9}{*}{$\mathbf{a}$} & mai II & RV & Desmodium ?zonatum \\
\hline & ?mai III & RV & Triumfetta rhomboidea \\
\hline & $\mathrm{mal}$ & RV & Piper betle (fertile) \\
\hline & mal modung & RV & Piper ?retrofractum \\
\hline & mal nidun & RV & Piper ?retrofractum \\
\hline & malé & RV & Lichenes \\
\hline & manu' api' & RV & Polypodiaceae \\
\hline & manu' bu'an & RV & Cassia ?occidentalis \\
\hline & manu' la'a & RV & Phragmites karka \\
\hline \multirow[t]{2}{*}{$\mathbf{a}$} & mara & RV & Planchonia valida \\
\hline & mata mèr & RV & $?$ \\
\hline $\mathbf{a}$ & mato kolong & RV & Breynia \\
\hline r w & mawa rapu & RV & Euphorbia hirta \\
\hline $\mathbf{a}$ & mébung I & 5544 & Abutilon \\
\hline r w & mébung II & 5543 & Hibiscus \\
\hline $\mathbf{w}$ & mihéng maya' & 5553 & Centrosema \\
\hline \multirow[t]{5}{*}{$\mathbf{a}$} & mi'i tubar & RV & tree w. soft wood \\
\hline & mirè' & RV & Aleurites moluccana \\
\hline & miro' & RV & Colocasia \\
\hline & motong & RV & Moringa pterygosperma \\
\hline & mudé & RV & Citrus \\
\hline \multirow[t]{3}{*}{$\mathbf{a}$} & mular & RV & Ficus septica \\
\hline & mu'u & RV & Musa paradisiaca \\
\hline & mu'u 'ipu & RV & Musa \\
\hline a & mu'u lili' & RV & ?Hibiscus \\
\hline \multirow[t]{2}{*}{ a } & naha' & RV & Harrisonia brownii \\
\hline & nalo' & RV & Saccharum spontaneum \\
\hline a & naré I & RV & Pterocarpus indicus f. echinocarpus \\
\hline $\mathbf{a}$ & naré II & RV & Pterocarpus indicus f. echinatus \\
\hline w & nèli' I & $\mathbf{R V}$ & ?Chonemorpha \\
\hline $\mathbf{w}$ & nèli' II & RV & ?Ichnocarpus \\
\hline
\end{tabular}




\begin{tabular}{|c|c|c|c|}
\hline ?w & nèpi & $\mathrm{RV}$ & Cayratia \\
\hline a & nilé & RV & Grewia \\
\hline w & ning buru' & RV & Paederia scandens \\
\hline a & nita' & RV & Sterculia oblongata \\
\hline \multirow[t]{2}{*}{$\mathbf{a}$} & nona & RV & Annona muricata \\
\hline & nona timur & RV & Annona \\
\hline w & nua & RV & Derris ?elliptica \\
\hline \multirow[t]{7}{*}{ a } & nuling & RV & Alstonia spectabilis \\
\hline & obu' $^{\prime}$ & RV & Rhaphidophora \\
\hline & obu' lolon & RV & ?Scindapsis \\
\hline & odél & RV & Colocasia esculenta \\
\hline & oma' & RV & Mucuna pruriens f. utilis \\
\hline & oro & RV & Borassus sundaicus \\
\hline & padan & RV & Pandanus tectorius var. samak \\
\hline \multirow[t]{2}{*}{$\mathbf{a}$} & padu butu & RV & Jatropha curcas \\
\hline & pala paong & RV & Ananas comosus \\
\hline $\mathbf{a}$ & paloka & RV & Artocarpus altilis (søedless) \\
\hline \multirow[t]{3}{*}{$\mathbf{a}$} & para & RV & Voacanga \\
\hline & pata' manu' & RV & Pouzolzia zeylanica \\
\hline & pérung & RV & Dendrocalomus asper \\
\hline \multirow[t]{4}{*}{ r w } & péting 'élu & RV & Paspalum \\
\hline & pèu & RV & Mangifera indica \\
\hline & pèu oha' & RV & Mangifera \\
\hline & pilir hèi & RV & Datura metel \\
\hline a & polèn & RV & Arenga pinnata \\
\hline \multirow[t]{2}{*}{$\mathrm{rw}$} & popo' & 5552 & Cenchrus \\
\hline & $\mathrm{pu}$ & RV & Bombax ceiba \\
\hline $\mathbf{a}$ & pu'a & $\mathrm{RV}$ & Eucalyptus alba \\
\hline \multirow[t]{6}{*}{ w } & puhé tahan & RV & vine, small \\
\hline & puhun buya' & 5540 & Leucas \\
\hline & pulu' sapu & RV & Auricularia \\
\hline & putri malu & RV & Mimosa invisa \\
\hline & puyang & RV & Languas galanga \\
\hline & ranga & RV & Semecarpus forstenii \\
\hline \multirow[t]{3}{*}{ a } & rani & RV & Canarium vulgare \\
\hline & rémé & RV & Sesuvium portulacastrum \\
\hline & rèngal & RV & $=$ biar rèngal \\
\hline \multirow[t]{2}{*}{ a } & réo & RV & Lannea coromandelica \\
\hline & ring ring & RV & Crotalaria \\
\hline w & riti & RV & ?Tetracera \\
\hline \multirow[t]{3}{*}{ a } & ruha & 5536 & Solanum \\
\hline & ruha woi & RV & Platycerium \\
\hline & sakalai & RV & Lycopersicon lycopersicum \\
\hline
\end{tabular}




\begin{tabular}{|c|c|c|c|}
\hline & sili' butu sakalai & RV & = sakalai \\
\hline & sili' butu utun & RV & Lycopersicon lycopersicum (small) \\
\hline & sili' manu 'ai' & RV & Capsicum annuum var. \\
\hline & sili' mikel & RV & plant, medicinal \\
\hline & sili' rian & RV & Capsicum annuum var. \\
\hline & sili' sarani & RV & Capsicum frutescens \\
\hline & sukun & RV & Artocarpus altilis (sceded) \\
\hline & $\mathrm{ta}^{\prime}$ & RV & Cocos nucifera \\
\hline \multirow[t]{6}{*}{$r w$} & ta'a boléng & RV & $=$ awada \\
\hline & tali belanda & RV & Agave sisalana \\
\hline & taluma & RV & Sesbania grandiflora \\
\hline & tarnal & RV & Tamarindus indica \\
\hline & tara' méhil I & RV & Mussaenda frondosa \\
\hline & tara' méhil II & RV & ?Rauvolfia \\
\hline \multirow[t]{2}{*}{ a } & tatar & RV & tree, stinking fl. \\
\hline & taung & RV & Indigofera \\
\hline r w & taung otan & 5549 & Tephrosia \\
\hline $\mathbf{r}$ & tèbèng & RV & Cucurbitaceae \\
\hline a & tèbu' & RV & Corypha pinnata \\
\hline \multirow[t]{9}{*}{ a } & téhé' & 5558 & Pipturus \\
\hline & téhu & RV & Saccharum officinarum \\
\hline & télar & RV & Cucurbita moschata \\
\hline & timu rawang & RV & Citrullus lanatus \\
\hline & timu 'upén & RV & Cucumis sativus \\
\hline & tiri & RV & Amorphophallus campanulatus \\
\hline & tiri 'otan & RV & Amorphophallus variabilis \\
\hline & tiu' & RV & Pandanus \\
\hline & tuan ta' & RV & ?Amorphophallus \\
\hline \multirow[t]{2}{*}{ a } & tubar laén & RV & Clerodendrum buchanani \\
\hline & tubung & RV & Caesalpinia bonduc \\
\hline a & tulur & RV & tree, yellow fl. \\
\hline a & ua' (u'a) & RV & Sterculia foetida / oblongata \\
\hline $\mathbf{r} \mathbf{w}$ & uan tubi & 5541 & Corchorus trilocularis \\
\hline a & uar & RV & Ficus ?heteropleura \\
\hline a & ubang & RV & $\begin{array}{l}\text { tree, large, bark formerly for cloth, } \\
\text { twigs pithy }\end{array}$ \\
\hline a & ‘ubél & RV & Inocarpus edulis \\
\hline \multirow[t]{6}{*}{ a } & 'ué & RV & Areca cathecu \\
\hline & uhu' & RV & Canavalia \\
\hline & uma & RV & Curcuma viridiflora \\
\hline & upa' & RV & Lagenaria siceraria \\
\hline & ?'uré & RV & Peltophorum pterocarpum \\
\hline & ’uru & RV & Impatiens (platypetala) \\
\hline
\end{tabular}




\begin{tabular}{|c|c|c|}
\hline 'uru mèngi & RV & Cymbopogon ?citratus \\
\hline 'uru paé & 5533 & Heteropogon contortus \\
\hline 'uru tahi' & RV & Spinifex littoreus \\
\hline 'utan & RV & Vigna unguiculata \\
\hline 'utan édang & RV & = 'utan kédang \\
\hline 'utan kédang & RV & Phaseolus ?lunatus \\
\hline 'utan lalèng & RV & Arachis hypogaea \\
\hline 'utan rian & RV & Vigna unguiculata (pulse, very long) \\
\hline 'utan rowé & 5547 & Phaseolus lunatus \\
\hline 'utan téhé & RV & Phaseolus vulgaris \\
\hline u'un & RV & Schoutenia ovata \\
\hline 'uyé & RV & Cajanus cajan \\
\hline wahé & RV & Psophocarpus trigonolobus \\
\hline wahé 'étan I & RV & Coleus amboinicus (cj.) \\
\hline wahé 'étan II & 5548 & Canavalia \\
\hline wahé iwan & RV & Pachyrrhizus erosus \\
\hline wahé képa I & RV & = wahé 'étan I \\
\hline wahé képa II & RV & = wahé iwan \\
\hline wahén & 5532 & Sorghum nitidum \\
\hline walang I & RV & = walang 'ayang \\
\hline walang II & RV & Schizostachyum blumii \\
\hline walang 'ayang & RV & Schizostachyum brachycladum \\
\hline warun & RV & Hibiscus tiliaceus \\
\hline watar I & RV & Zea mays \\
\hline watar II & 5535 & Cassia \\
\hline watar holo' & RV & Sorghum saccharatum \\
\hline watar méngar & RV & Pennisetum purpureum \\
\hline waya & RV & Carica papaya \\
\hline wéi I & RV & ?Daemonorops \\
\hline wéi II & RV & Calamus \\
\hline wéla dapé' & RV & Fatoua pilosa / Phyllanthus \\
\hline wélé & 5559 & ?Dombeya \\
\hline wéra' & RV & Artocarpus integer \\
\hline wéré' & RV & Setaria italica \\
\hline wéré' la'u ébo & RV & Setaria \\
\hline wéwé & RV & Phaseolus aureus \\
\hline wiro' & RV & Colocasia \\
\hline witing umi & RV & Cyperus kyllingia \\
\hline wua' (?wu'a') & RV & Sterculia foetida \\
\hline wua nona & RV & Annona ?squamosa \\
\hline ?wué & RV & = 'ué \\
\hline wuhu' I & RV & ?Hibiscus \\
\hline wuhu' II & 5554 & Abutilon \\
\hline
\end{tabular}




\section{TAXONOMIC - VERNACULAR IN SOME 19 LANGUAGES}

\subsection{PRELIMINARY REMARKS}

(a) This list is a reversed simplified recapitulation of the separate lists in section 1. Just as it is usual with an index to a bilingual dictionary, so also here the chief volume (section 1) has to be consulted for details. In this case, that gives details of classifiers, the definitive orthographic form, the precise localities where dialectal names were found and the sources and quality of the identifications.

(b) In the first column of this list we find abbreviated names of families. (Their full names are given in section 3.) The higher taxa such as fungi and lichens are written in capitals. The knowledge of the families to which certain genera belong can be very useful for comparative etymology. Therefore I put the genera that figure in this study together, under the head of their respective families, in section 3. I use the nomenclature of families as practised in Flora of Java, e.g. Asteraceae instead of Compositae.

The second column gives the taxonomic names in alphabetical order, often followed by a species name, sometimes with an additional subspecies name. They are written in italics. Groups, like MUSCI, are also entered into this alphabetical order. Their genera and other taxa are put in brackets.

I tried to use the newest taxonomic names. In order to avoid doubts about the identity of plants a list of the most common taxonomic synonyms is given in Appendix 2.

Then, after a colon, we find the vernacular names preceded by abbreviations of the respective languages. They are separated by a semicolon. Semicolons are also used between vemacular names to separate different, though not scientifically identified, types of plants. The sequence of these languages is: Bima, Komodo, Far-east Manggarai, Kepo' (I and II), Waerana, Razong, Rembong, Rongga, Ngadha, Nagé, Kéo, Endé, Lio, Palu'é, West Sumba, East Sumba and Sawu, followed by Sika, the Solorese languages and Kédang from the Ambonese Group.

Where no vemacular-taxonomic list of a language exists, as is the case with Far-east Manggarai, Rembong and Nagé, the abbreviations of their dialects are put in parentheses.

(c) Abbreviations of names of languages and dialects (used in the list below).

$\begin{array}{ll}\text { Bm } & \text { Bima } \\ \text { Ed } & \text { Endé } \\ \text { ES } & \text { East Sumba group of dialects } \\ \text { FEM } & \text { Far-east Manggarai } \\ \quad(\text { Bé }) & \text { Békék } \\ (\mathrm{Ls}) & \text { Lengkosambi } \\ (\mathrm{Mb}) & \text { Mbai } \\ (\mathrm{Ml}) & \text { Mulu } \\ (\mathrm{Nn}) & \text { Nanganumba } \\ (\mathrm{Ri}) & \text { Riung } \\ (\mathrm{To}) & \text { Toring } \\ \mathrm{Kd} & \text { Kédang } \\ \mathrm{Ké} & \text { Kéo } \\ \mathrm{Km} & \text { Komodo }\end{array}$




\begin{tabular}{|c|c|}
\hline Kp & Керо' \\
\hline $\mathrm{Li}$ & Lio \\
\hline $\mathrm{Na}$ & Nagé \\
\hline$(\mathrm{Bw})$ & Bo'awaé \\
\hline$(\mathrm{Da})$ & Danga \\
\hline$(\mathrm{Ra})$ & Raja \\
\hline (Tm) & Tédamudé \\
\hline (Wd) & Wudu \\
\hline $\mathrm{Ng}$ & Ngadha \\
\hline $\mathrm{Pl}$ & Palu'é \\
\hline $\mathrm{Rg}$ & Rongga \\
\hline $\mathrm{Rm}$ & Rembong \\
\hline$(\mathrm{Ft})$ & Faté \\
\hline (Ri) & Ri'a \\
\hline$(\mathrm{Tr})$ & Térong \\
\hline$(\mathrm{Wk})$ & Wa(ng)ka \\
\hline (Wu) & Wué \\
\hline $\mathbf{R z}$ & Razong \\
\hline $\mathrm{Si}$ & Sika \\
\hline Sl & Solorese languages and dialects \\
\hline Sw & Sawu \\
\hline $\mathrm{Wr}$ & Waérana \\
\hline WS & West Sumba group of dialects \\
\hline
\end{tabular}

\subsection{THE LIST}

Malvac. $\quad$ Abelmoschus esculentus: Bm sokla; Ed kopi; Sl kopi soklat.

Malvac. $\quad$ Abelmoschus manihot ssp. tetraphyllus: WS zuru ndena II

Malvac. $\quad$ Abelmoschus moschatus: FEM nawung; $\mathrm{Kp}$ nawun; $\mathrm{Wr}$ nawu; $\mathrm{Rz}$ nawu; $\mathrm{Rm}$ nawun, (Wk) nawung; Rg ngawu; $\mathrm{Ng}$ ngawu, ngoa; $\mathrm{Na}(\mathrm{Tm})$ ngawu raka; Ed mbera nawu, nawu, rewo; Li nawu; Si nawu; Sl pepu.

Malvac. $\quad$ Abelmoschus moschatus ssp. tuberosus: Rg ngawu.

Stercul. $\quad$ Abroma: Ed mbera héko I, II.

Papil. $\quad$ Abrus precatorius: Bm sala n(g)ia; Km pipi'; Rg nggoré I; Ng gozé II, gojé I, kipi, li'é kozé; Ed mbutu mité; Li tali té'u II, wajo I; Pl nggoli; ES kalada nggangga; Sw poro loko; Si géra I; Sl be'oré, géi, segéi.

Malvac. $\quad$ Abutilon: $\mathrm{Ng}$ ?pedi; ES tai babu I, II; Sw walu goa; Kd mébung I, wuhu II.

Malvac. Abutilon auritum: ES tai babu I.

Malvac. Abutilon indicum ssp. albescens var. australiense: ES tai babu II; Sw walu goa.

Malvac. Abutilon javanense: $\mathrm{Km}$ bertai.

Mimos. Acacia farnesiana: Bm kara; Sw huki wou; ES rangga. 
Mimos. $\quad$ Acacia leucophloea: ES kawai I.

Mimos. Acacia oraria: Km nggersi' ntodoh; Sl akasia ta'é.

Mimos. $\quad$ Acacia cf. rugata: $\mathrm{Km}$ 'radah'.

Mimos. $\quad$ Acacia tomentosa: Bm ?kara; WS manggaléwa, manggalawa; ES kadara, kedara, kandara, manggalewa.

Mimos. $\quad$ Acacia verheijenii: $\mathrm{Kp}$ karot taor, $\mathrm{Rg}$ gera élo.

Mimos. $\quad$ Acacia villosa var. glabra: WS lamtoro jawa.

Euphorb. $\quad$ Acalypha: Rg kaso haki; $\mathrm{Ng}$ ?saka duli.

Euphorb. Acalypha caturus: ES kandinu kiku ahu.

Euphorb. $\quad$ Acalypha indica: $\mathrm{Km}$ (ngolo) sewuru; Ed wasa rima; ES ruta bai; Sw taba'i.

Euphorb. Acalypha rubra: Km sewuru kedi'.

Acanthaceae: Bm padu peo; arundae I; Wr lawi; Rg hinga mota, lawi; $\mathrm{Ng}$ kasi beku, hasi beku II, koi pada; Ed ora, ?tepu; Li koro II, kumu laka; WS bila wae jeje, miéjaka; ES kakaha.

Aster. $\quad$ Acanthospermum hispidum: Ngtadu banga; Ed banga.

Acer. $\quad$ Acer laurinum: Kp ratun pong; Rm ?ratun poso'; Ed kaju wawi, wela masa.

Amaran. $\quad$ Achyranthes aspera: Bm perdeke; Km ara ndaé degé'; Rg lando réndo II; $\mathrm{Ng}$ lasu mula, poso reké; Li suga sala; WS ka'ngurita, ka'ngorita; ES ha'lurutu I; Sw kaé huni; Si klorot apun, hugaléng.

Lamiac. $\quad$ Achyrospermum densiflorum: Kp kazu koja.

Arac. $\quad$ Acorus calamus: Bm ndango; FEM moro wio, komu; Wr mori wio; Rz mori wio; $\mathrm{Rm}$ muru wio, moro wio, (Wu) komu'; (Wk) kumu'; Rg moro wio; $\mathrm{Ng}$ danga, ?bhunga; $\mathrm{Na}(\mathrm{Tm})$ komu; Ed ako; Li kalé raga, kali raga; WS 'sekura, 'zekura, 'zekera, 'sikura, áku; ES 'sekuru, híkilu, 'hekulu, ?sedí; Sw na'i kébu; Si kali raga; Sl ?wua kora.

Rutac. $\quad$ Acronychia trifoliata (cp. Euodia): $\mathrm{Ng}$ aro; Ed pa.

Polyp. $\quad$ Acrostichum (aureum): ES ?pàpa; Sl ?boro.

Mimos. Adenanthera: Km pipi'.

Mimos. Adenanthera pavonina: Ed wajo; Li kalo wajo, wajo II; Pl mata méo; Si géra II.

Passifl. Adenia heterophylla: Km ngolo dangko, kedangko.

Polyp. $\quad$ Adiantum: Kp wo'or.

Polyp. $\quad$ Adiantum philippense: Rm niron.

Theac. $\quad$ Adinandra javanica: Kp rukus; Wr tia'; Rz tia'.

Myrsin. Aegiceras: Km peropa.

Myrsin. Aegiceras floridum: Km nggersi' tasi'. 
Rutac. $\quad$ Aegle marmelos: Bm bila; Km mamih; FEM mamis; Kp I mamis; Rmb mamis; $\mathrm{Rg}$ uté; $\mathrm{Ng}$ uté; $\mathrm{Na}(\mathrm{Tm})$ té'u, (Ra) uté; Li mboko para; WS bila, bilya; ES wabila, wua bila; Si ai dila; Sl kedila I, dila, kenare, karo nara; Kd bila.

Papil. cf. Aeschynomene: Km ngolo maja.

Agav. $\quad$ Agave sisalana: $\mathrm{Bm}$ aruna ai, aruna II; FEM pandang; $\mathrm{Kp}$ pandang; Wr pandan kazék; Rz pandang; Rm pandang; $\mathrm{Rg}$ penda jawa; $\mathrm{Ng}$ lema mori I; $\mathrm{Na}$ (Tm) pada, $(\mathrm{Ra})$ lema mori; Li lema mori; WS 'karota, manila II, pànda làma woya; ES rami I, manila II; Sw nena edu; Si kobu man(g); Sl ?wéwél, kobu wéwélê, sisal, suka'; Kd tali belanda.

Aster. $\quad$ Ageratum conyzoides: FEM (To) rewut woés (Ri) ghéla ghaé (Ls) rewut wau, $(\mathrm{Nn})$ rembé wau, (Ml) wéla wau, (Mb) tandus api; $\mathrm{Kp}$ wau; Wr lindang; $\mathrm{Rz}$ rukut wau; $\mathrm{Rm}$ reman wau, (Tr) kasi wang, (Wu) wéla wau, (Wk) rembung wau; $\mathrm{Rg}$ ?linda; $\mathrm{Ng}$ ora goa, wau I; $\mathrm{Na}(\mathrm{Tm})$ bené wau, $(\mathrm{Bw})$ bené wau; Ed ora ia, rubu ré'é; $\mathrm{Li}$ rumba ré'é II, wau ré'é; WS bou 'taiko, bou kàto, wu teyo; ES rumba wau; Sl witi kobâ, waû ne.

Meliac. Aglaia: WS ulu katáka; ES hulu katáka; nggoaka, nggoka, goka.

Meliac. Aglaia eusideroxylon: WS manera; ES ma'niera, manera.

Meliac. Aglaia ganggo: Ed ra'u mbopo; Sl hali I.

Meliac. Aglaia 'odorata': Bm kapanca cina.

Alang. Alangium chinense: ES kamba II, ?kambu II.

Alang.

Alang. Alangium villosum: Bm kamonca; FEM (To, Bé, Ls) wunis, (Ri) kunis pong, (Nn) kunis II, (Mb) ghaju kunis; Rm (kazu) kunis; Li kéu té'a; WS langìra, langirya; ES langira II.

Mimos.

Alangium villosum var. parviflorum: $\mathrm{Rg}$ kuné; Si lali mera.

Albizia chinensis: FEM waék, (Ls) wail, (Ml) fail, (Mb) fai; Kp kaé; Wr kaék; Rz kaé; Wr kaék; Rz kaé; Rm faé, (Wu) kaé, (Tr) waék, (Wk) faé; $\mathrm{Rg}$ fai; $\mathrm{Ng}$ fai; $\mathrm{Na}$ (Tm) boné; Ed fai; Li fai; WS marawìta; ES kawia II, kawiaka; Si ai béi; Sl baé.

Mimos. $\quad$ Albizia falcataria: $\mathrm{Ng}$ fai toro; Sl bajâ.

Mimos.

Albizia lebbeck: $\mathrm{Bm}$ ?temba; ES ?riut otan.

Mimos.

Albizia lebbekoides: $\mathrm{Bm}$ ?sareo; Km seréo; WS màreweta, ma'regheta II, ka'reweto; ES rútu I, rita II.

Mimos. $\quad$ Albizia procera: Bm ndaru I; Km lembuh; FEM dalo; Kp dalo', Wr dalo; Rm dalo'; Rg ndalu; Ng dalu, katu; $\mathrm{Na} \mathrm{(Tm)} \mathrm{hajo;} \mathrm{Ed} \mathrm{ranu,} \mathrm{lanu;} \mathrm{Li} \mathrm{lanu;} \mathrm{Si} \mathrm{ladur;} \mathrm{Sl} \mathrm{keladu,}$ kladu, kaladu; Kd buya'.

Mimos. $\quad$ Albizia saponaria: $\mathrm{Bm}$ ?isu; Si langir.

Euphorb. $\quad$ Aleurites moluccana: Bm Kaleli; Km kelili; FEM welu; Kp kaju kelu; Wr kelu; Rz kelu; Rm felu, welu, (Wk) felu, (Tr) welu; $\mathrm{Rg}$ felu; $\mathrm{Ng}$ felu, féo; $\mathrm{Na}$ (Tm, Bw, Ra) féo; Ed féo; Li féo; Pl welu; WS kanìi, kawàlu; ES kawàlu, kawìlu; $\mathrm{Sw}$ welu; $\mathrm{Si}$ gelo; Sl kemi'é, mi'é, mirék, kemi'é; Kd mirè'. 
ALGAE: $\quad$ FEM (To, Ls) lamung waé, (Bé, Nn) amuk waé, (Ri) lamun waé; Rm (Wu, Wk) lamun waé', (Tr) lamung waé; Ng lamu, lewi; Na (Tm) lamu waé; Li lamu, rogo; WS lu'muta; ES kangúrutu, 'lumutu, 'lumuto; Si lamung, tahi wakang, utang mérak, utang wetang, klogo; Sl lamu, lamû, kulat.

ALGAE Eucheuma: Km agah; ES kangoka.

ALGAE ?Padina: Km rambu'.

ALGAE Sargassurn: Km lato keriwa.

Amaryll. $\quad$ Allium cepa var. ascalonicum: Bm ḅawa; FEM somu; Kp sumu; Wr somu; $\mathrm{Rm}$ bawang sumu, (Wk) sumu; (Wu) somu, (Tr) sunga; $\mathrm{Rg}$ somu toro; $\mathrm{Ng}$ somu, suna toro; $\mathrm{Na}(\mathrm{Tm})$ somu, (Ra) sunga; Li somu; Pl somu; WS lona; ES lahuna, la'huana, lasona, lahóna; Sw lahuna, huna; Si somu; Sl bawâ mé'â, bawang mé'â, kebawang, bawâ mé'a; Kd bawang putu'.

Amaryll. $\quad$ Alliun cepa var. cepa: WS bawangu; ES bawa, kabawa.

Amaryll. $\quad$ Allium sativum: Bm ncuna; FEM sunga (puti); Rm bawang bakok; Rg somu, bara; $\mathrm{Ng}$ sunga, somu sunga; $\mathrm{Na}(\mathrm{Ra})$ somu; Li sunga; WS lona wasu, lona ghazu, lona ghayo, lona kaka; ES bawa kawau; Sw hunga; Si (hunga), sunga; Sl bawâ bura', kebawang bujak, bawân bura'; Kd bawang buya'.

Amaran. $\quad$ Allmania nodiflora: Sl aho wérẻ, aho wéwén, aho wéwér.

Sapind. $\quad$ ?Allophylus cobbe: $\mathrm{Rg}$ téwa ngamba.

Arac. $\quad$ ?Alocasia: $\mathrm{Ng}$ talé witu; ES suli wé, kadapu, tarúbu, tarebu.

Arac. $\quad$ Alocasia ?indica: WS mbula; ES mbula.

Arac. $\quad$ Alocasia macrorrhiza: Bm wira; FEM (Ri, Ls) kunggul, (Ml) koak, (To, Bé, Mb) wira, (Nn) tombang; Wr tobang; Rz tobang; Rm kobang, wira', (Tr) kunggul, (Ft, $\mathrm{Wu}, \mathrm{Wk}$ ) koak; Rg toba; $\mathrm{Ng}$ wira I; $\mathrm{Na}$ (Tm) toba; Ed wira toro; Li po lina; WS wi'a; ES wi, wia, uwi III; Si ?'lépo; Sl lete, ?kladar I, ?naluk I.

Liliac. $\quad$ Aloe: $\mathrm{Ng}$ lema mori.

Zingib. $\quad$ ?Alpinia: $\mathrm{Rg}$ sia; $\mathrm{Ng}$ séra bhara II.

Zingib. $\quad$ Alpinia (galanga): Kp kisok; Wr pu'u sia; Rm suduk, (Wk) fisok; ES winja, kalita búti.

Apocyn. $\quad$ Alstonia scholaris: Bm rida; Km rida; FEM sita; Kp rita'; Wr rita; Rm (Wk) rita', (Tr) sita; Rg rita; Ng rita, zita; Na (Tm, Ra) zita, (Bw) rita; Ed jita; Li jita; Pl rita; WS rita, ritya; ES rita I, marita, karita; Sw ged’i; Si rita; Sl rita, rétor, rita; Kd ité.

Apocyn. $\quad$ Alstonia spectabilis: Bm tula; Km tula; FEM loi, (Ls, Nn, Ml) koi, (Mb) kaloi; Kp lui; Wr koi'; Rz koi; Rm kui, (Wu, Tr) koi; Rg koi; Ng mari; Na (Tm) hoi; Li hapa loi, loi; WS èlo, halà(i)yo; ES halí, halai, salau, salai; Sw héliru; Si loi, téru; Sl lo'o I, ?kulitaba; Kd nuling.

Amaran. Alternanthera: Km sawé; Li lema nda'i; ES kàti tihu.

Amaran. Alternanthera pungens: Km degé'. 
Papil. $\quad$ Alysicarpus: WS kambé tana II.

Apocyn. ?Alyxia: ES polahari.

Amaranthaceae: $\mathrm{Rg}$ seko raké I.

Amaran. Amaranthus: Ed mboti ngadu rịiru; Si utan halé.

Amaran. $\quad$ Amaranthus (lividus): Bm nadu; Km sawé; FEM (To) sawé, ndawé, (Ml) mboti; Kp sawé; Wr sawé; $\mathrm{Rz}$ sawé; $\mathrm{Rm}$ (Wk) natén; ( $\mathrm{Tr}$ ) sawé; $\mathrm{Rg}$ mboti; $\mathrm{Ng}$ boti, boti modhé; $\mathrm{Na}(\mathrm{Tm})$ boti, (Ra) beci; Ed mboti, mboti pesa; Li boti, boti puti; Pl ud'a nio; WS paróna, pe'ruona, paróna; ES màja; Sw ru raé; Si arung (bura, méran); Sl a'u, wulû a'ung, buyâ; Kd 'alung.

Amaran. Amaranthus (spinosus): Bm nadu rui; FEM sawé, (Ls) sawé karét, (Ri) sawé wina, (Nn) sawé wawi; Kp karot natén; Wr nganda/o lizu; Rm (Wk) natén karot, (Tr) sawé; Rg nganda liru; $\mathrm{Ng}$ boti karo; $\mathrm{Na}(\mathrm{Tm})$ boti oka, (Bw) feci ga; Ed mobti karo, mboti ré'é; Li boti gulu; WS pa'ruona patara; $\mathrm{Si}$ arung atong; $\mathrm{Sl}$ wulung a'ung, klamâ, ?kuwék I.

Lythrac. Ammannia: Sw wodo, hodo.

Zingib. $\quad$ Amomum: Kp panas; Wr pané; Rz pané; Rm panas; léa pépan, (Wk, Wu) pikan; Rg pané; $\mathrm{Ng}$ ?léa rega II, séra, séra bhara I, séra ro; Li féndo, kepa; WS ka'paita; langgiri; ES kapulaga; Si 'luban; Sl lia' goa.

Zingib. Amomum maximum: Si popot.

Arac. $\quad$ Amorphophallus (species and races): Bm maja; FEM wanga, (Mb) kaka bharu; Kp lola; bokak; Wr lola'; Rz lola'; wanga; Rm wanga, (Wk) tiri; $\mathrm{Rg}$ lola, riti; $\mathrm{Ng}$ tobo tizo, lola, matu, tiri, tizo; $\mathrm{Na}(\mathrm{Tm})$ tiri, tiri ré'é, (Ra) ti; Ed adhé, tiri, tiri ré'é, robho; Li pudi I, kosi, tiri; gero; Pl d'ici; WS kawú, kawowo, kawowu, kabota koba, ndúma, kabota, ndore, nazo, kàba; ES ndúma kawini; nduma míni; nasu, nahu; $\mathrm{Sw}$ kebuta ped'i; kébuta nga'a; Si tiri; Sl muti', keti'é, obo, ti'é; menakâ tapo, tapo; Kd tiri, tiri 'otan.

Vitac. $\quad$ Ampelocissus: Rg sura II.

Vitac. $\quad$ Ampelocissus cf. arachnoides: Kp wanar wasé'; Wr ilur wawi; Rm ilur wawi; WS karupé II, marupé.

Anacardiaceae: ES lindu watu II.

Anac. Anacardium occidentale: $\mathrm{Ng}$ pau téo I; Sw péna'i holo; Si pau giréng, wuang golung; Sl jambu ménté, wuâ golu, pao rengi; Kd luma sawa.

Menisp. $\quad$ Anamirta cocculus: Bm wua podu; FEM lol (Mb) lor; Kp lol; Wr lol, lon; Rz lol; $\mathrm{Rm}$ (Tr) lol; (Wk, Wu) lon; Rg néta; $\mathrm{Ng}$ méta, néta, ngéta; $\mathrm{Na}$ (Tm) méta; Li néta; Pl méd'a mbola; WS pa'nétangu, panéta; ES pa'niatangu, pa'nétangu; Si 'lebo; S1 kelebo, klebo I; Kd lèbo

Bromel. $\quad$ Ananas comosus: Bm aru na, ?fanda I, fanda ngaha; $\mathrm{Km}$ aré na; FEM (To, Bé, Ls, $\mathrm{Ml})$ pala wandang, (Ri) béla banda, (Ls) pombong, (Nn) pala wanda; Kp ré'a jawa; Wr penda'; Rz penda; $\mathrm{Rm}$ tala wanda, $(\mathrm{Ft})$ zé'a wuni; ( $\mathrm{Tr}$ ) réa wuni; (Wu, Wk) pala wandan; $\mathrm{Rg}$ penda; $\mathrm{Ng}$ peda I, kéra zé'a; $\mathrm{Na}(\mathrm{Tm})$ pala wada (Ra) ana na; Ed ana na, 
Aster. $\quad$ Anaphalis longifolia FEM (To) lapa (Ri) mbako, muku té; Kp mbako roja; Wr mbako roja; $\mathrm{Rz}$ mbako roja; $\mathrm{Rm}$ mbako rozang, mbako razong, ( $\mathrm{Tr}$ ) mbako rango; $\mathrm{Rg}$ mbako roja; $\mathrm{Ng}$ wunu léwa; $\mathrm{Na}(\mathrm{Tm})$ pobo ré'é; Li ta'i nitu; ES bara pola.

Acanth. $\quad$ cf. Andrographis: WS (móro) ki'i; Sl peredu

Acanth. Andrographis paniculata: $\mathrm{Ng}$ sabi roto; Ed sambé ruto.

Commel. Aneilema: $\quad \mathrm{Rm}$ kepok; Ng mizu; WS kangúruku, ka'ngarika, ka'ngereko; ES ka'ngarika; Kd biru.

Polyp. $\quad$ Angiopteris evecta: Wr nakér; Rz nakér.

Lamiac. $\quad$ Anisomeles indica: WS kandángi pòdu; ES ?kaura, ?kunu.

Annon. Annona muricata: Bm garoso I, garoso rui; $\mathrm{Km}$ geroso belanda; FEM (To) kar kaza, (Bé) nanék, (Ls) kaju ata, (Nn) nonak, (Ml) kazu ata, (Mb) kanonak; Kp keroso; Wr keroso; Rz keroso; $\mathrm{Rm}$ keroso, wua nona, ( $\mathrm{Tr}, \mathrm{Wu}$ ) rosong, $(\mathrm{Ft})$ geroso, (Wk) kara kaza, kerosong; Na (Tm) kazu mi; Li naka belanda, naka kupa; WS naga 'lukuta, kalangga dawa, korondahi; ES harakayi, harakaya, sirikaya; Sw nona; Si nakat jawa; Sl ata belanda, ata, anona I, ata blada, lima dada; Kd nona timur.

Annon. $\quad$ Annona ?reticulata: Bm garoso ?mbolo; $\mathrm{Ng}$ ?mi goa; Li aré nona; $\mathrm{Sl}$ amona, anona II, ana noa; Kd nona.

Annon. $\quad$ Annona ?squamosa: Bm garoso II, garoso kaya; Km geroso; FEM (To) kanonak, (Ls) nonak, (Nn) kaju ata; Rm wua' nona; (Wk) kara kaza; Na (Tm) sara kai; Ed ata, (bua) nona; Li aé nona, aré nona, nona; WS kalangga ndima, kalángga II, kalangga lúlu; ES kalangga jawa, tariwangu, núnu; Sw aj’u jawa; Si antas, kaé nona, kapona(s), ka nona; Sl ata.

Annonaceae: $\quad$ Bm ?nonu II; Km lékéng mbéo'; Wr kazu lékéng; Ed gana wawi.

Rubiac. Anthocephalus cadamba: Bm konca II.

Rubiac.

Anthocephalus chinensis: Bm ?sancari; Kp kawak; Wr kawak; Rz kawak; Rm kawak; Rg kawa; Ng maja; ES mangawa.

Morac. $\quad$ Antiaris toxicaria: Sl newak.

Euphorb. $\quad$ Antidesma bunius: Bm ?sawone; Wr kazu wuné.

Euphorb. Antidesma ghaesembilla: Sl sili samo.

Apiac.

Apium graveolens (cp. Coriandrum sativum): Sw ?wila hégé.

Poac.

Apluda mutica: Ed isa I; WS kambila, ka'popoka; ES kapúmbu tai manu.

Icac. Apodytes: ES kakomba.

Papil.

Arachis hypogaea: Bm koja, rapa (mila); Km permila; FEM koja, (Nn) uté tana; Kp koja; Wr koza; Rz koza; Rm, (Wk, Tr) koza; Rg hobo tana; Ng hobho tana, sobho tana; $\mathrm{Na}(\mathrm{Tm})$ bué tana, ( $\mathrm{Ra}$ ) bué awa, bué tana; Ed bué tana; Pl redo, wéwé d'ama; 
Aral.

Myrsin.

Myrsin.

Arec.

Arec.

Arec.

Arecaceae:

Arec.

Poac.

Arist.

Morac.

Morac.

Morac.

Morac.

Morac.

Morac.

Morac.

Orchid.
WS kàbe tana, kàmbe tana, kembe tana; ES kabai tana, manila I, kambi tana; Sw ménila; Si tana unén, wéwé tana, wuék tana; Sl utâ tana, utâ naja onen, tana one; Kd 'utan lalèng.

Aralia montana: WS manda ngiyo; ES manda nu'u, mada nu'u I.

Ardisia: WS ndelo káka, ha'milungo.

Ardisia javanica: Rg ké'o.

Areca: Kp ?kabé' ratun; $\mathrm{Ng}$ héu laka, héu diwa; $\mathrm{Na}(\mathrm{Bw})$ 'éu; Li ladu, laka jawa; WS kabuaka; Sl ku'u I, wua' ladu, wua' sisi.

Areca cathecu: $\mathrm{Bm}$ u'a; Km rongkah; FEM wené; Kp ?kabé', rasi; Wr basi; Rm, ( $\mathrm{Wu}, \mathrm{Wk}$ ) wené', (Tr) wené; $\mathrm{Rg}$ héu; $\mathrm{Ng}$ héu, kéu I, séu; $\mathrm{Na}(\mathrm{Tm})$ héu, (Bw) 'éu, héu, oa zé'é; Ed 'éu; Li kéu; Pl wua; WS wino, mama, làba; ES winu; Sw kela, wenyi; Si wua; Sl wua', kléruk; Kd 'ué.

Areca cathecu var.: WS wino malandi, làbu malandi; ES winu malanja.

Si ('ai) biri.

Arenga pinnata: $\mathrm{Bm}$ nao; $\mathrm{Km}$ nao; FEM tuak; $\mathrm{Kp}$ tuak; Wr tuak; $\mathrm{Rz}$ tuak; $\mathrm{Rm}(\mathrm{Tr}$, Wk, Wu) tuak; Rg moké; $\mathrm{Ng}$ moké, tua; $\mathrm{Na}(\mathrm{Tm}, \mathrm{Bw})$ tua; Ed moké; Li moké; PI tua; WS karùdi; ES kanoru, kalotu; Sw moka, moké; Si tua utan; Sl kébo, kébol, pola'; Kd polè(n).

Aristida polyclados: ES wulu kalaü.

Aristolochia tagala: WS luwa rato.

?Artocarpus: nakat wéréng II.

Artocarpus altilis (seedless): Bm karara; $\mathrm{Km}$ kerara; FEM bakar, (Nn) sukun, (Mb) bhé; Kp bakar; Wr kerara; Rm (Wk) bakar, (Tr) sukun, (Wk) kerara; Rg kerara; Ng ka rara, kerara, mo, naka II; Ed karara; Li ?kara I, kerara; WS karara; ES karara; Sw kelara; Si baka, kala ara; Sl sukun; Kd paloka.

Artocarpus altilis (seeded): Bm kolo; Kp bakar; Ed ?kulu; ES kulu, nderi; S1 ?kwérak, ?kewérak I; Kd sukun.

Artocarpus elasticus: FEM (Bé, Ri) kelo, (Nn) kaok, (Mb) keraok, (Ml) lalé; Kp raé'; Wr raé'; Rz raé; $\mathrm{Rm}$ (Wu) lalé, (Tr) kaok, (Wk) kelo'; Rg teré; Ng teré; Ed teré; Li teré; WS terepa, terepo; ES tera, tíra; Si muné; Sl téét, borot, ?kumur; Kd boro'.

Artocarpus glaucus: Li ?ro kéi; WS mayela II; ES mayéla II.

Artocarpus heterophyllus: $\mathrm{Bm}$ nangga karao; $\mathrm{Na}(\mathrm{Bw})$ naka wara; Ed naka wara; $\mathrm{Li}$ koro ndé; Si nakat wéréng; Sl kwérak wato', ?naka ba'it, kaférak, kawéra.

Artocarpus integer: $\mathrm{Bm}$ nangga, nenggi, nanga; $\mathrm{Km}$ nangga; FEM mok; Kp mok; Wr pu'un mok; Rz mok; Rm (Wu, Tr, Wk) mok; Rg mo; $\mathrm{Ng}$ mo, naka I; $\mathrm{Na}$ (Tm) mo, (Ra) ?naka wa; Ed naka; Li naka I; Pl naka; WS nangga, naga; ES nangga; $\mathrm{Sw}$ (wo) naga, hoka; Si nakat; Sl kewérak lala', naka', kewérak II; Kd wéra'.

Arundina graminifolia: $\mathrm{Ng}$ lelu II; Sw wo libi mélai. 
Sapind. $\quad$ Arytera (litoralis): FEM ?dhakas; $\mathrm{Rm}$ (Tr) kazu sawar; Rg té'é; Ng doza III; Li bama; WS kalú ata.

Asclepiadaceae: FEM (Ri) sau, (Mb) sa'u; Kp lebo' sa'u; Rm lebo' sa'u, (Tr) sau; Ng kolé II, ?nidu; ES kawaka; Sw ?biko II, wué tédengi; Sl witi lahak.

Asclep. $\quad$ Asclepias curassavica: $\mathrm{Ng}$ ponga.

Liliac.

Asparagus: Ed tuku tobé.

Polyp. $\quad$ Aspleniun: $\mathrm{Ng}$ kaka kaju.

Polyp. $\quad$ ?Asplenium (nidus): Kp tikol; Wr kipé'; Rz tikol; Rm wangkéng; Rg kipé; Ng huku II, koré II, lebé raé II; Li koré I.

Asteraceae: $\quad \mathrm{Ng}$ guru hégé, ibu, mé'a ha'é, mé'a waé; Ed kinggo wawi; Li kigo, mbaka; Pl mbangga rai, ES pau maràda; Si gonang; Sl kebû II, puhû kerama; Kd 'i'a mato.

Acanth. Asystasia: $\mathrm{Ng}$ wonga ruru jawa.

Oxal.

Averrhoa bilimbi: Bm limbi I; Km belimbi; Rm (Wk) mbimbi; $\mathrm{Ng}$ bidi; $\mathrm{Na}(\mathrm{Ra})$ bidi; Ed mbindi I; Li mbindi (wala); Sw libi; Si blidi, bridi; Sl belimbí, klibang, belibê; Kd ala libang, 'i'a mato.

Oxal. $\quad$ Averrhoa carambola: Bm sarume II; $\mathrm{Km}$ serumé; Kp bibi; Wr bibi, bidi; $\mathrm{Ng}$ bidi; $\mathrm{Li}$ mbindi, sermélé; Sw melai, libi haramélé; Si balimbéng, sermélé, cerméle; Sl belibê.

Verben. $\quad$ Avicennia spp.: Km sia; Sw mengahi; $\mathrm{Sl}$ aur.

Verben. Avicennia marina var. resinifera: ES lindu watu.

Salvad. Azima sarmentosa: Km wéto'.

Poac.

Bambusa blumeana: Bm o'o rui; $\mathrm{Km}$ hau deki'; FEM (Ri) toé, to'é; $\mathrm{Kp}$ to'é; Wr to'é; Rz to'é; $\mathrm{Rm}$ (Wk) to'é, (Tr) toé; $\mathrm{Rg}$ to'é; $\mathrm{Ng}$ guru II, guru to'é, to'é; $\mathrm{Na}(\mathrm{Tm})$ to'é; Li gera, kengga I; Pl kengga; Sl ega', keka', kekaj; aru kramat; Kd 'aur woi.

Poac. $\quad$ ?Bambusa glaucescens: Kp kesa; $\mathrm{Rm}$ kiput; $\mathrm{Ng}$ ?kéa; Si nitu petun.

Bambuseae: $\quad$ Bm keu, o'o; Kp aur nua; Wr gurung kalok; Rz gurung kalok; Ng guru butu, guru kéro; Li beto laka; Pl diu wulu; WS palo; ES palu, ?au búku, oru, polu; Sw wo, kélaiwu jawa; Sl lekê.

Acanth. $\quad$ Barleria prionitis: Bm ?gandarisa II; Km deki' lili, lopoh; Ng sala luli; Ed koro rañu; WS tara wé'é; ES tara wé'é, kakaha míni; Si bahir; Sl rokak I, kehawu; Kd béhil.

Lecyth. $\quad$ Barringtonia racemosa: Rg loko waé.

Caesalp. Bauhinia: FEM ?matu; Li nggaké I.

Caesalp. $\quad$ Bauhinia (hirsuta): Kp pétak; Wr kazék kedang; Rm wazé' pétak; Li nggaké II; Si tu niluk; Sl keluk.

Caesalp. Bauhinia lingua: $\mathrm{Ng}$ waé eda.

Caesalp. $\quad$ Bauhinia scandens: $\mathrm{Ng}$ eda; Li ?weda lako.

Begon. Begonia (isoptera): Kp kembi watu; Wr lebo' mbilo; Rm wirok; Li onga; WS kapeka. 
Polyp. Belvisia mucronata: Rm wangkéng loé'.

Cucurb. Benincasa hispida: Bm hala; Km hala'; FEM (To) pelas, (Ls) ghelas, (Nn) kebok, (Mb) kelas; Kp kelas; Wr kelas; Rm zawong, kelé'; Rg kelu; $\mathrm{Ng}$ éjé, héa, izé, kelu, kéu V, séa I; $\mathrm{Na}(\mathrm{Tm})$ héa, (Ra) 'éa; Li héa, kéa; ES karàbu tunu, karobu tunu; Sw (wo) kérebo hawu; Si kléra, leja; Sı kela'a, kla'a, kerang; Kd ara.

Aster. $\quad$ Bidens: FEM (Bé, Ri) sawat, (Ls, Nn) cawat, (Mb) sawak; Kp sawat; Wr sawat; Rz sawat; Rm sawat; Rg sawa I; Ng lasé hui I, sawa; Na (Tm) sawa; Li mersawa, moro sawa, mura sawa, rolé fosé; WS kapáta I; ES karùngu, kiku ahu; Si 'rata 'oték; Sl kebû, kuda tain; Kd ladu namé', ladi amé.

Euphorb. $\quad$ Bischofia javanica: Kp rewu; Rm rewu; Ng na II; Ed kewu, na; Li na; WS memala; ES kóndu; Sl watung/?wetung.

Aster. $\quad$ Blumea: Ng bhaka bara, ruto I.

Aster. $\quad$ Blumea (balsamifera): FEM paja; Kp woang; Wr woang; Rz woang; Rm foang; Rg ?muku té'a; Ng baka bési, muku té'a I; Na (Bw) muku té'a; Ed pombo; Li muku té'a II; WS kapìra 'taiko, kapéri 'taiko mane, kapìri 'taiko, kapùru 'taiko, kapári 'teiyo; Sw ?na'i goi; Si ?'léro; Sl léi sédê, néhé wauk I.

Aster. $\quad$ Blumea lacera: WS kaliso, kalighu.

Aster. Blumea riparia: $\mathrm{Ng}$ baka.

Aster. Blumea riparia f. angustifolia: WS kanggaruka.

Urtic. $\quad$ Boehmeria: Kp silon tawu; Wr sulan tawu; Rz sulan tawu; Rm sulon tawu; lensing.

Urtic. Boehmeria (cp. Elatostema): Rg ponggo kura; Ng lesi II; zama nitu, ?masi, nigé III; Li mota ndoro I.

Nyctag. Boerhavia: Ed kangga au; WS katànda tana; ES 'turuku tana; Sw waka moné.

Zingib. Boesenbergia pandurata: Bm dumu kunci.

Bombac. $\quad$ Bombax ceiba: Bm ringi wuba; Km kawu puah; FEM (Bé, Ls, Mb) ?ka kawu, (Nn) lada jawa, (Mb, To, Bé) lada, (Ls) kewo; Kp lada; Wr lada; Rz lada; Rm ladar; Rg mboa lada; Ng boa jawa; $\mathrm{Na}$ (Tm) boa; Ed boa, mboa II, mboko ronggo I; Li boa ré'é; $\mathrm{Pl}$ lengé; WS ròngo matòmba, ròngo pi'a, rongo I; ES rànga, ràngu, kopa, ròngu; Sw kéweru liha; Si bola 'uta; Sl kajo kapek III, k puho, k wésér I, ebong puho', puho mé'an béle; Kd pu.

Boraginaceae: ES kamàla kóndu.

Arec.

Borassus sundaicus: Bm ta'a, soko; Km tah; FEM ta'al; Kp maghit; Wr maghit; Rz maghit; Rm ta'al; Rg maghi; Ng koi, koli, maghi, tua maghi; Na (Ra) koli; Ed kori; Li koli; Pl koli ('oli); WS 'mangita, mángico; ES manggita, manggitu, 'menggitu; Sw dué; Si koli; Sl koli, tuak pokang/kolér, tuak; Kd oro.

Rubiac. $\quad$ Borreria: $\mathrm{Ng}$ léda waé I, meré; WS rúta wùni II, ruta mète, 'taiko zapi; ES 'taiko sapi II.

Rubiac. $\quad$ Borreria levis: Ed remi maru.

Poac. Bothriochloa pertusa: ES mapu ka'hiriku. 
Poac. Brachiaria ramosa: ES kawongu au.

Poac. Brachiaria reptans: ES kandaïngu lúlu jungga II.

Poac. Brachiaria subquadripara: Ed kuru oro; Li bera bela.

Euphorb. $\quad$ Breynia: Rg neti; Sl ikâ matâ, marak; Ed mata rera I.

Euphorb. Breynia cernua: FEM (Ls) pau watu; Wr neti' kazék; Rm tilan wazé'; Ng gozé II.

Euphorb. $\quad$ Breynia racemosa: Kp tila; Wr neti'; Rz tila; $\mathrm{Rm}$ tilan; $\mathrm{Ng}$ toko weté I; ES paha wura II.

Euphorb. Breynia vitis-idaea: $\mathrm{Ng}$ toko weté II; Ed mata rera I; Si mata méra; Sl nileng.

Euphorb. Bridelia: Kp piras; piras pong; Wr piras; Rz pizas; Rm piras; Ed pira kéli, wunu meré.

Euphorb. Bridelia monoica: WS robasa kabála.

Euphorb. Bridelia ovata: $\mathrm{Km}$ rau; WS kapia I.

Euphorb. $\quad$ ?Bridelia tomentosa: $\mathrm{Rg}$ piras; $\mathrm{Ng}$ toko mité I; Ed mata rera II; Li mata leja.

Urtic.

Simar.

Rhizoph. Bruguiera gymnorrhiza: Km bangko muntu; Si krakat, klakat.

Rhizoph. Bruguiera parviflora: Sl boro.

Anac.

Buchanania arborescens: Bm ?pato I, ?mpelo, ?katawi; Km (mere) mpao; FEM pau watu; Wr pau manuk; Rg pau kéli, pau manu; Ng pau séko; Ed pau kéli; WS ?pou ùta; ES kamàla pau; Sw pau rutu; Sl pao moko, bera papaung.

Logan. Buddleja asiatica: Li ina koba.

Orchid. Bulbophyllum: $\mathrm{Ng}$ kadu, sunga nitu.

Stercul. Byttneria flaccida: Wr kazék dangé'; Ed kedho.

Caesalp. $\quad$ Caesalpinia: Ng ?titu; Pl karo; Sw hepé.

Caesalp. Caesalpinia (bonduc): Bm kadara; Km deki' nggoréng; Kp mata mbé; Rm konggang bakok; Rg dara; Ng wua dhara; Li ndaru; ES dàru, ndàru, hàru; Sl olok; Kd tubung.

Caesalp. Caesalpinia (crista): Bm rui peo; Kp kuku laku; Wr kuku laku; Rm kuku laku; $\mathrm{Rg}$ waké laku; Si ?tuwu(ng).

Caesalp. Caesalpinia (major): Bm kaḍara; FEM (Ls) létar; Rg dara; Ng dara; Li mboko néka I, mboko ona; SI keturu/olok, ke'olok.

Caesalp. Caesalpinia sappan: Bm supa; $\mathrm{Km}$ sepang; Wr molas po'as; $\mathrm{Rz}$ sepang; $\mathrm{Rm}$ sepang; Rg sepa; Ng koba karo, sepa; ES hàpangu; Sw hapé; Si hepang; Sl hepâ.

Papil. $\quad$ Cajanus cajan: FEM lusa; Kp lusa; Wr solang; Rz lusa; Rm uza; Rg uré; $\mathrm{Ng}$ hobho kaju, ujé, uré, uzé I; Na (Bw) 'uzé; Ed ura; Li ura; WS lawui, lawuji, laghui, wàla 
wiyo; ES wàlawina, wàlawinu, lawina, walawi, wi, katungga; Sw wo tori; Sl 'utâ blurat, ura; $\mathrm{Kd}$ 'uyé.

Arec.

?Calamus: $\mathrm{Bm}$ ?miro jopu; $\mathrm{Km}$ ué; FEM nanga; $\mathrm{Kp}$ nanga; $\mathrm{Wr}$ nanga; $\mathrm{Rz}$ nanga; $\mathrm{Rm}$ nanga; $\mathrm{Rg}$ nanga; $\mathrm{Ng}$ roda, ua maghi, ua wawi; $\mathrm{Na}(\mathrm{Ra})$ 'ua; Ed taga, ua taga; $\mathrm{Li}$ ?ngga ju soko, taga; Pl ua; WS uwe/?uwi, ughe; ES iwi manu, ui, iwi I, uwi II; Sw gui; Si gai; Sl uar, uaj, uat bou', ?uway, ua; Kd wasé' wéi.

Arec.

Calamus (cp. Daemonorops): Bm kaua I, miro; FEM wua; Kp wuas; Wr wuar; Rz wuas; $\mathrm{Rm}$ wuas; $\mathrm{Rg}$ ua; $\mathrm{Ng}$ ua: $\mathrm{Ed}$ ua; $\mathrm{Li}$ ua; $\mathrm{Pl}$ 'ua; ES iwi wé, uwi wé; mbúlu; si gai manuk; Sl uat manuk.

Orchid. $\quad$ Calanthe: Rm wua' la'a; WS wìno 'bioli.

Verben. $\quad$ Callicarpa: $\mathrm{Km}$ ?mbeloté; WS kùta ghazu.

Clusiac. Calophyllum: $\mathrm{Ng}$ muku té'a II.

Clusiac. Calophyllum inophyllum: Bm mantau, ?bintango na'é; $\mathrm{Ng}$ talo; WS kandora; ES kandora, mantau, mandora; Sl tawau.

Clusiac. $\quad$ Calophyllum soulattri: Bm bintango; Li kéu; ES widi ka'lauki; Si bintangur, taor; S1 witi suar.

Papil. Calopogonium: Kp séa' wura; wasé' wutun; Wr kowé' darat; $\mathrm{Rm}$ séa' wura I; $\mathrm{Ng}$ fili II; Li endu; Sl talé hépé.

Asclep. $\quad$ Calotropis gigantea: $\mathrm{Bm}$ kore; $\mathrm{Km}$ koré; FEM dagu dak; $\mathrm{Kp}$ ta'i ruék; Wr kolé; $\mathrm{Rz}$ lalok ruék; Rm wuntun; $\mathrm{Rg}$ kolé; $\mathrm{Ng}$ kolé I; Ed koré; Li kolé; Pl d'ina bolé; WS kambuta; ES wangukeli, wanggakelu, wnaggakúli; Sw kolé; Si kloko, kroko, kroko', koko; Sl kroko', klokô, krokor, keroko; Kd léla'.

Annon. $\quad$ ?Cananga odorata: Bm kananga; Rm kenanga; Sw ténaga.

Burser. ?Canarium: $\mathrm{Rg}$ koja nitu.

Burser. $\quad$ Canarium asperum: Bm ?heci; FEM (To) nana kojang, nggarit koza, (Bé) rondo wolo, $(\mathrm{Ri}, \mathrm{Ls})$ réndé wolo, $(\mathrm{Nn})$ pondo wolo, ponto wolo; $\mathrm{Kp}$ nduréng, kesi; $\mathrm{Wr}$ kesi, nduéng; Rz kesi, nduéng; Rm nana' koza, (Wk) réndo wolo; $\mathrm{Rg}$ nengi; $\mathrm{Ng}$ kesi I, nengi II; Ed koja wanda, nengi; Li koja nengi, nengi; WS kàhi I; ES kahi I, kàzi, kei; Si neni; Sl keretuk, ketuk.

Burser. $\quad$ Canarium vulgare: Bm kanari; FEM (To) kenari, (Bé, Ls, Nn, Mb) koja, (Ri) koza; Rm nana' koza; Rg koja; Ng koja; Ed koja; Li ?kitu koka, koja; Pl koca; WS karitaku kàhi; ES kariìtaku kàsi; Si koja, koja pepin; Sl koja', kenari; Kd rani.

Papil. $\quad$ Canavalia: $\mathrm{Bm}$ ḅoa, ?kope; $\mathrm{Km}$ lambé nanga napu; $\mathrm{Kp}$ wua kopé; lalong todo; $\mathrm{Wr}$ wua' baro'; wua' todo; Rm wua' su'a; $\mathrm{Rg}$ sara walu; $\mathrm{Ng}$ méké hé'é; Li bué duké; WS lùpe I; ES ka'papangu I; Sw képapa edu; Si lima kiat; Sl utâ blapit; Kd wahé 'étan II.

Papil. $\quad$ Canavalia maritima: $\mathrm{Km}$ lambé nanga.

Cannac. ?Canna: Ed koko woso ré'é. 
Cannac. $\quad$ Canna coccinea: FEM (To) kotak, (Ls) gozong, kota katak; (Nn) kota kata; Kp sangga ria; Wr pénu'; Rz nggozong; Rm (Ft, Wk) gozong, ( $\mathrm{Tr}$ ) gezong, ghezong, (Wu) kota kata; Rg kota; Ng kota; Na (Tm) kota kata; Ed koko, wonga kota; Li koko woso, woso; WS nggano, ganyo, kapàdi; ES gajung, ngganja, ngganju; Sw ganyo; Si kotak; Sl kotak, kotat.

Gentian. $\quad$ Canscora: $\mathrm{Ng}$ rora kuza; uta kuja.

Opil.

Cansjera leptostachya: ES kamàra wezi.

Poac. $\quad$ Capillipedium assimile: Wr rukut betong, ?mukon; Si 'urun aur.

Poac. $\quad$ Capillipedium parviflorum: $\mathrm{Kp}$ isa; $\mathrm{Rm}$ batis kombéng I; Li ?kuru sawu.

Cappar. $\quad$ ?Capparis: Bm cacingi; Si klorot regét.

Cappar. $\quad$ Capparis lucida: Km kenitu.

Cappar. $\quad$ Capparis micrantha: $\mathrm{Km}$ kerkoa' winé; $\mathrm{Ng}$ mudé nitu; Sl kewanga I.

Cappar. $\quad$ Capparis pubiflora: $\mathrm{Ng}$ mudé nitu kedhi; ES tara manu.

Cappar. $\quad$ Capparis cf. pyrifolia: Km kerkoak moné.

Cappar. $\quad$ Capparis quinifolia: Sl rokak II.

Cappar. Capparis sepiaria: Km kenitu tasi'.

Cappar. $\quad$ Capparis spinosa var. marina: Sw téi bahi.

Solan. $\quad$ Capsicum annuum: Bm saha na'e, sabia II; FEM (Ls) koro jawa, (Nn) koro tuang, (Mb) koro jawa tuang; Kp nggurus tuang; Wr nggurus méré', nggurus tuang; Rm nggurus toro; Rg koro méré; $\mathrm{Ng}$ koro mézé; $\mathrm{Na}(\mathrm{Tm})$ koro bhubhu; Ed koro méré; $\mathrm{Li}$ horo ria, koro ria; Pl 'oro cawa; ES mbaku ha'u bòkulu; Sw hili; Si koro, koro geté; SI sili bala, ku'u léléng, sili béle; Kd sili' manu 'ai, sili' rian.

Solan. $\quad$ Capsicum frutescens: Bm saha II; Km baruné; FEM (Ls) koro jawa loé, (Nn, Mb) koro jawa; Kp nggurus; Wr mburus; Rz nggurus; Rm nggurus; Rg koro ito; Ng koro; Na (Ra, Wd) ko; Ké kolo; Ed koro dhiki; Li horo, koro I; Pl koro 'uré; WS nggángga, nggánda, mbaku zaii; ES mbaku haii, mbaku haii (kudu), baku sawu; Sw hili j’ami; Si koro anak, koro blubuk; Sl sili, (ku'u) blurê, silér, sili busê; Kd sili' sarani.

Sapid. $\quad$ Cardiospermum halicacabum: Ed sendo I; WS póka.

Cyper. $\quad$ Carex: Li éra laé, éré laé.

Caric. $\quad$ Carica papaya: Bm panja, kampaja, mpaja; Km panja; FEM padut, padhut; Kp padut; Wr jawa; Rz zawa; Rm padut; Rg muku jawa I, ha'é; $\mathrm{Ng}$ ha'é, li'é ha'é, muku ngi'i ha'é, padu; $\mathrm{Na}(\mathrm{Tm})$ padhu, (Ra) 'a'é, (Wd) muku jawa; Ké g'é; Ed uta, uta ba'i; Pl kua; Li kajawa, ka'é jawa, kejawa, muku jawa, teka jawa, uta ba'i; WS kalowo dawa, kalogho dawa; ES kali jawa, kalelu, kalailu II, kajawa, kalowu jawa, kalú jawa; Sw hángo, mu'u jawa; Si jawan, mu'u jawa, padu, utang; Sl paja, wua pajâ, pajam, kasi, wua parâ; Kd waya.

Borag. Carmona retusa: Kp kazu witi. 
Arec.

Arec.

Caesalp.

Caesalp.

Caesalp.

Caesalp. Cassia floribunda: Wr mambang koé; Rm mambang; Ng bungé III.

Caesalp.

Caesalp.

Caesalp.

Caesalp.

Caesalp.

Caesalp.

Caesalp.

Laurac.

Laurac.

Casuar.

Casuar.

Apocyn.

Vitac.

Vitac.

Bombac.

Caryota: Mb moké nitu. kedudu; Sl bora. Kd watar II. I, ka'ndulangu, ?moro wuni; Sw helag'i beni. keluâ; $\mathrm{Kd}$ éto.

Cassia (javanica): Kd éto ayang.

Cassia occidentalis: Rg jata; Li mbéwo.

Cassia pluricapitata: $\mathrm{Kp}$ taor.

Cassia sophera: $\mathrm{Ng}$ béwo II, béwu. 'malangu; Sl tutung rê I.

Cassytha: Ed namo.

Cassytha filiformis: Bm njala-njala. wu; Si ru, semara; Kd léur. mberu; Ng beru, bu; Ed mbu; Li bu II; Si semara, $\mathrm{Sl}$ angin, semara.

Catharanthus roseus: Ed bunga tonjo. muku té'a II.

?Cayratia trifolia: $\mathrm{Ng}$ katé bara, nilu I; Sl oté kolang.
Caryota mitis: Wr tuak kodé', tuak wura; WS 'wuola, nába; ES 'wuala, wola; Sw

ro'a- WS karáwa; ES ai bàhi tánda 'malangu I; Sw behi; Si kata lari I; bunga méang:

Cassia alata: Rm landor; (Wk) koza kaba; $\mathrm{Ng}$ wonga sésé; WS langira I; ES langira

Cassia fistula: Bm kanahi; FEM (Bé, Nn, Mb) lembur, (Ri, Ls) kembur; Kp kembur; Wr kembur; Rz kembur; Rm kembur; Rg hembu; Ng hebu I, ngadu I; Na (Tm, Bw) hebu, (Ra) dalu; Ed dhombo; Li ndopo; Pl ndobo; WS 'ketoka, 'monjura, mojura; ES kúnjuru, mojoru, 'monjura; Si o'a; Sl teluâ, kluâ, kanda pistu, klowang,

Cassia mimosoides: $\mathrm{Km}$ ngolo maja; $\mathrm{Kp}$ (lebo) kodong toko'; Wr rukut kimot.

Cassia siamea: Rg jaga polo, jangga polo; Si johar, weru-wégé; Sl johar; Kd johar.

Cassia tora: $\mathrm{Kp}$ landor; Wr mambang koé'; Rm landor; $\mathrm{Ng}$ béwu ré'é I; ES tánda

Casuarina equisetifolia: Li bu I; Pl kacu angi; WS bùngga; ES kajú, kajiu; Sw kai

Casuarina (junghuhniana): $\mathrm{Kp}$ mao; $\mathrm{Wr}$ mao; $\mathrm{Rz}$ mao; $\mathrm{Rm}$ lélé aong, mao; $\mathrm{Rg}$

Cayratia: Bm karaka; Km mépéh; Kp mépés; Wr mépés; Rz mépés; Rm mépés; Rg

Ceiba pentandra: Bm ringi; Km kawu; FEM kawu; Kp kawu; Wr mboa; $\mathrm{Rz}$ mboa; Rm kawu, (Wk) kawu-kawu; $\mathrm{Rg}$ mboa; $\mathrm{Ng}$ boa; $\mathrm{Na}$ (Tm, $\mathrm{Ra}$ ) boa, (Bw) wonga boa; Ké mboa; Ed mboa I; Li boa, boaja jawa; Pl mbola; WS ?kába I, ròngo II, kamba watu; ES kamba hika, ?kaba lua, kopa lua; Sw kéweru jami; Si bola; S1 kapo', kape bola, kapok, kepok, puho kapo; Kd kapo'. 
Bombac. TCeiba pentandra: Sw kéweru liha.

Amaran. $\quad$ Celosia argentea: Rg lando kura; WS ghuni rara; ES wulu manu II.

Ulmac. $\quad$ Celtis tetrandra: Kp namut; Wr namut; Rz namut; Rm namut; $\mathrm{Rg}$ namu; $\mathrm{Ng}$ nigé I, nomu; Ed sati; Li seti I.

Ulmac. $\quad$ Celtis wightii: Bm pingga.

Poac. $\quad$ Cenchrus: Sl puhû terekat, klorot; Kd popo'.

Poac. $\quad$ Cenchrus (brownii): Ng ta'i kogha; Li repa; ES ka'pohaku II.

Apiac. Centella asiatica: FEM lakan sewé, (To) wuku zarang, (Ri) tilu sewé, (Ml) kinggo nipon, (Mb) mumus kusé, (Ls) sawéng; $\mathrm{Kp}$ tokak; Wr tokak; Rz tokak; Rm tongkak, (Wu) lakan sewé'; Rg toka; Ng hinga dhéké I; Ed mbisi kojo; Li tété kadho, tété nggebhé; WS koba wé'é; ES koba wai; Sw kaba loro I, kélai léré; Si puhé beta; SI maling I.

Poac. Centotheca latifolia: WS wu'u katòpa.

Papil. $\quad$ Centrosema: Si sépé sénak; Sl séntér séma, manu géwa'; Kd mihéng maya'.

Apocyn. Cerbera: Rm wuta'.

Apocyn. Cerbera manghas: Rg longko kodé; $\mathrm{Ng}$ futa; Ed huta; Si 'wuta; Sl futa', futa.

Rhizoph. Ceriops decandra: Sw bago.

Rhizoph. Ceriops (tagal): Km bangko nipih; ES ndongu.

Asclep. $\quad$ Ceropegia: Bm ?kaki'i; Sw rui; taba rui I.

Opil.

Champereia manillana: Bm silo; FEM (Ls, $\mathrm{Nn}$ ) cui, (Mb) wunu sui; $\mathrm{Kp}$ lebo' sui; Wr lebo' sui; Rm sui; Ng su'i; Ed ?sui; Li sui; WS kamàra wèzi I; Sl kué'.

Meliac. Chisocheton: ES lapali; ngoka.

Meliac. $\quad$ Chisocheton (cp. Elattostachys): Ng doza I.

Poac. Chloris barbata: WS wulu uru II; ES rumba penji, ka'ndaiingu, kawulu uru; Sw kedub'u.

Poac. $\quad$ Chloris virgata: ES wulu kamàmbi.

Apocyn. Chonemorpha (cp. Ichnocarpus): Bm kala moné I; Sl talé' maka ripi, talé' olak.

Polyp. $\quad$ Christella: Kp kedér; $\mathrm{Rm}$ kedér, (?Ft) pidang, kedér; $(\mathrm{Tr})$ totor, $(\mathrm{Wk})$ ?rundung ulu.

Polyp. Christella dentata: $\mathrm{Ng}$ fo'i féé.

Papil. Christia: ES muru wé.

Poac. Chrysopogon aciculatus: FEM (Ls) lonto garong, $(\mathrm{Nn})$ jepét, (Ml) wélang rano, $(\mathrm{Mb})$ garéng; $\mathrm{Kp}$ mberong; $\mathrm{Wr}$ rukut mberong; $\mathrm{Rz}$ mberong; $\mathrm{Rm}$ mberong, $(\mathrm{Tr})$ zepét; Rg lasi nggedo; $\mathrm{Ng}$ tezé; $\mathrm{Na}(\mathrm{Tm})$ rano; Li meké, paré I; WS wùlu ndaü II, wulu jawu; ES rumba mapu; Sl klélang.

Poac. Chrysopogon gryllus: ES mapu bara II.

Poac. Chrysopogon subtilis: ES mapu wulu ihi kamambi. 
Laurac.

Laurac.

Vitac.

Vitac.

Vitac.

Cucurb.

Rutac.

Rutac.

Rutac.

Rutac.

Rutac.

Rutac.

Rutac.

Rutac.

Rutac.

Rutac.

Euphorb.
Cinnamomum: Rm gambang.

Cinnamomum bumanni: Bm kaju mani(sa); FEM (To, Bé) ghaju manuk, (Ri) kazu manuk, (Ml, Bé) ghazu manuk, (Nn) kaju manuk; Kp ndingar; Wr ndingar; Rz ndingar; Rm dingar, kazu manuk, ( $\mathrm{Tr}, \mathrm{Wu}, \mathrm{Wn}$ ) kazu manuk; $\mathrm{Rg}$ ndinga; $\mathrm{Ng}$ manu I; Na (Tm, Bw) manu; Li mani; WS kanìnggo, kaninggyo; ES kaninggu,kanigu; Si ?manu/mano; Sl kleming.

Cissus: Km kusi; Wr tembong; Ng kabé azé, rura wawi; Ed merpara, sawa ra; WS kàmbe rara, kambe ra'a; ES pelu mánu; SI kepipi, nipi.

Cissus adnata: WS tambèdala II.

Cissus discolor: $\mathrm{Ng}$ tuki toko II; Sl ra'a.

Citrullus lanatus: Bm kalende; Km kaléndé; FEM (To, Bé) nggéjé, (Ls) ghéjé, (Ml) nggézé, (Mb) lasé rusa; Kp jawong; Rm (Wu) timba, $(\mathrm{Tr})$ zawong; Rg éjé; Li jawa ka; Na (Wd) nggéjé, wéjé; Pl nggécé; ES kadembi, kadimbu, kadébu, kadembu; Sw dimu dana; Si ?dimun(g), jawa; Sl sasi, dimû, kuka; Kd timu rawang.

Citrus: Bm ?dungga buti; FEM (To, Bé) lémo, (Ri) ndéru, (Ls) las rusa, (Nn) lacu rusa, (Ml) dhéru; Kp diru'; Wr déru; $\mathrm{Rz}$ déru; $\mathrm{Rm}$ ndiru, (Tr) diru, (Wu) dhéru.

Citrus spp.: Bm dungga, katungga; Km gero'; FEM raka'; Kp wua' muta; Wr mundé mutar; Rz munta; Rm raka', munté; Rg geru, lémo, mundé; WS mudi, munde; maroto; maróto kawìki, maroto kaghìki; ES mudi, jàriku lángga, jàriku wahìku, mundi, jarìku, nundi, maratu, rútu II; jariku wihiku; Sw jeru; jeru lému, jeru neta.

Citrus: Rz sambu raka; $\mathrm{Rm}$ raka'.

Citrus: Wr déru tungé, wua' témé; $\mathrm{Rz}$ mundé témé; $\mathrm{Rg}$ mundé jeru, mundé témé, muta; $\mathrm{Ng}$ déru, dhéru, jeru, mudé, rango, ringo, uté II; $\mathrm{Na}(\mathrm{Tm})$ tunga, $(\mathrm{Ra})$ mundé; Ké mundé; Ed tungo; Li mudé, muda geru, (mudé) mbangga, mudé meso, mudé mi, mudé soki, mudé telo, tuga, tuga lo'o, tuga soki; Pl mut'é; Si mudé min, mudé huré; mudé niluk, mudé telong; Sl mudat telung; mudat kniluk, muda manuk telun.

Citrus aurantifolia: $\mathrm{Bm}$ dungga ncia; Wr jeru; $\mathrm{Rg}$ jeru I; WS maroto kawiki, maroto kaghiki; ES jàriku, jàriku mayilu, jiriku, maroto kawiki.

Citrus aurantium: $\mathrm{Bm}$ dungga jurutuli.

Citrus grandis: Bm dungga ni'u; FEM mundé, lémo; Kp lémo; Wr lémo; Rz lémo'; Rm lémo;; $\mathrm{Ng}$ lémo, mudé ka II, mudé mézé; $\mathrm{Na}(\mathrm{Tm})$ mudé tua; $\mathrm{Ed}$ mundé; Li mudé ria, tuga ria, tuga sowa; WS maroto kaláda, mundé bokólo; ES jàriku 'tobungu, jàriku 'bokulu; Sw jeru kébau, jeru wo réna; Si mudé geté, mudé paré; SI muda wawé, mudat jemboa, muda sranin, muda karaban.

Citrus hystrix: FEM munta; Ng mudé koro I, mudé roka; Si mudé; Sl muda, mudat; $\mathrm{Kd}$ mudé.

Citrus nobilis: Sw jeru ki'i; Sl mudat klemi, muda sneruk, muda ké'in.

Citrus sinensis: Si mudé huré; Sl muda sina.

Cladogynos orientalis: WS móro kódi I. 
Euphorb. Claoxylon abbreviatum: Rg tepu; WS katunggu lengi.

Euphorb. Cleidion javanicum: ES tambùra II.

Euphorb. ?Cleistanthus: WS anggo kùra, ago kùra.

Ranunc. $\quad$ Clematis: Rm méa, mas; WS longge kabàketa; (lolo) kabàketa; ES wàla óna I.

Ranunc. Clematis (pickeringii): Ng gozé IV, moro etu; ED uwi ré'é I.

Ranunc. $\quad$ ?Clematis cf. smilacifolia: $\mathrm{Rm}$, (Wk) lebo' rentan; Kp raun repo'.

Cappar. Cleome gynandra: Ng lenga nitu; ES pa'yejangu.

Cappar. Cleome viscosa: $\mathrm{Km}$ kecah; wawé' longa.

Verben. $\quad$ Clerodendrum: Rm kazu ta'i; WS katùra lòdo, tangára lòdo; ES mangàndingu.

Verben. $\quad$ Clerodendrum buchanani: FEM (Ri) wéla méntar, (Ml) wéla' méntar; Kp wéla' métar; Wr wéla' métan; $\mathrm{Rz}$ wéla' métar; $\mathrm{Rm}(\mathrm{Wu})$ wéla' méntar, $(\mathrm{Tr})$ wéla méntar; $\mathrm{Rg}$ wéla méta; $\mathrm{Ng}$ ka beka, kala beka, ra'a beka, wowa II; $\mathrm{Na}(\mathrm{Tm})$ boro ra; Ed soki, soro mbika, suru mika; Li wonga bingu, wonga londa II; Si kajo wair; Sl nakâ bi'a ubeng.

Verben. $\quad$ Clerodendrum (inerme): $\mathrm{Km}$ romo dato.

Papil. ?Clitorea ternatea: Sl puhû ajû.

Cucurb. Coccinia grandis: Ng papo; Ed nindi; Li kala.

Cucurb. $\quad$ Coccinia grandis (cp. Gymnopetalum): Kp saot; Wr bumbu.

Arec. $\quad$ Cocos nucifera: Bm ni'u, ?tupa; Km niu; FEM mo', nio; Kp nio'; Wr nio'; Rz nio'; Rm (Wk) nio', (Tr) nio; Ng nio I; Na (Tm, Ra) nio; Ed nio; Li nio I; Pl nio; WS nu'u, ?nyou, ngiu; ES 'kokuru, langai, ní; Sw nyiu; Si kabor, niur; Sl tapo, tapo', tapor; Kd ta'.

Arec. $\quad$ Cocos nucifera var.: Rg nio; ES 'kokuru (ka'wulanga); Sw nyiu hango.

Poac. $\quad$ Coelorachis rottboellioides: Ed gai ara.

Rubiac. $\quad$ Coffea (arabica): Bm kahawa; FEM kopi, kupi; Kp ghawa, kopi; Wr kopi manggarai; Rz kopi manggarai; $\mathrm{Rm}$ hawa, kupi; Rg kopi; $\mathrm{Ng}$ kopi; $\mathrm{Na}$ (Tm, Ra) kopi; Ed kopi; Li kopi; Pl kopi; WS kopi; ES kopi; Si kopi; Sl kopi; Kd kopi.

Rubiac. $\quad$ Coffea robusta: FEM (Ri) kupi rebusta, (Mb) kupi rebustan; Kp ghawa, kopi; Wr kopi tuang; Rz kopi robustan, kopi kawak; Rm hawa rebustan, (Wk) kupi rebustan; WS kopi; ES kopi; Sw kowi.

Poac. Coix lacryma-jobi var. agrestis: Wr elas, wura; $\mathrm{Rz}$ elas, wura; Rm sela géléng, ké'o kongkak, (Wk) ké'o kokak; Ng ?képa; Li ké'o mui, ké'o ota; WS kamàngge rewa I, kamànggé rewa I, matu nuhi II; ES wua júli, jolu; si mo'i; Sl lekâ tenga, delat.

Poac. $\quad$ Coix lacryma-jobi var. ma-yuen: Bm lere; Km gandong; FEM (To, Bé, Ri, Mb) sela pisi, (Ls, Nn) sela pici, ké'o kokak, (Ml) sela loké, ké'o peké'; Kp sela; WR elar; Rz elas; $\mathrm{Rm}$ ké'o, (Tr) kéo, (Wu) ké'o kongkak; Rg ela, ké'o I; $\mathrm{Ng}$ ké'o; $\mathrm{Na}$ (Tm, $\mathrm{Bw}, \mathrm{Ra})$ ké'o; Li ké'o I, mbapé; Pl ké'o mboka; WS kamanggisa, kamàge, 
ka'menggeho; ES kama, kamagisu, kamànggihu, kamàngge; SW ké'o; Si lelé, lelé goan, lelé o'on; Sl dela, delat goa; Kd lèyè'.

Lamiac. $\quad$ Coleus amboinicus: Li ro tela; Sl képa I; Kd wahé étan, wahé képa.

Lamiac. Coleus tuberosus (cp. Dioscores esculenta):: WS ?tandé'i, laghuta; ES ?litangu, ?lutangu.

Arac. $\quad$ Colocasia: Kp teko' menado, teko' tuang; Bm majakale II.

Arac. $\quad$ Colocasia: FEM ?teko wira waé; Kp mboa; Wr mboa, tédér, tagil, nggéron; Rz nggéron; Rg nggéro; $\mathrm{Ng}$ bo ba, boé baé, ridhu, wira waé; Ed jara ndora, wira; Li duli II, rosé aé, uta go; WS uli máne, huli kaka, huli dawa; ES mbukutu; piuru II, ka'wadaru, hili làtangu; ka'maimbungu, ka'mimbingu; Si wutik; Sl kladar I, kladar mé'â; Kd wiro'.

Arac. Colocasia esculenta: Bm uwi eja; FEM (Ls, Mb) teko ka, (Nn) koto kata, teko; Kp teko'; Wr teko'; Rz teko'; Rm teko' ka', (Wu, Tr) teko; Rg teko; Ng ho'i, so'i II, talé I, talé jawa; $\mathrm{Na}(\mathrm{Tm})$ ho'i, (Ta) 'osé; Ed rosé, uju bima; Li rosé, wira II, wira rua; WS uli, ùli, huli; ES hili, sili, hili jawa, selu, hili bara; Sw ané; Si rosét; S1 keladi, kladar II, naluk II; Kd odél.

Combr. Combretum latifolium: WS tali rara, tei rara.

Commel. Commelina: Kp monggot; Rg monggo; $\mathrm{Ng}$ léda, léda mézé, ?mizu, uta guru II; Ed monggo; Li lénda au, mogo, monggo; Si mogot.

Commel. Commelina (diffusa): $\mathrm{FEM}(\mathrm{Ri})$ ranga waé; $\mathrm{Kp}$ reték; Wr reték; $\mathrm{Rz}$ reték; $\mathrm{Rm}$ lampa' waé', (Tr) landor alo', (Wk) landor alo'; ES kalíku.

Convolvulaceae: Ed mbonggi I; ES katàbi lúku.

Tiliac. $\quad$ Corchorus: Rg ?mbeté jara; Ng rama, zepé; Ed meké jara; Li mboko meké; Sl kapek II.

Tiliac. $\quad$ Corchorus aestuans: S1 rora.

Tiliac. $\quad$ Corchorus (capsularis): $\mathrm{Bm}$ guni; $\mathrm{Rm}$ longa bapu'; $\mathrm{ES}$ kamàla lànga, kamàla lànga maràda.

Tiliac. $\quad$ Corchorus trilocularis: Kd uan tubi.

Borag. $\quad$ Cordia: Rg bolo; Ng na I; Li kepo kéli; Si ?papang.

Borag. $\quad$ Cordia dichotoma: Bm nuna; $\mathrm{Km}$ nunang; FEM nunang; $\mathrm{Kp}$ nunang; Wr nunang; $\mathrm{Rz}$ nunang; $\mathrm{Rm}$ nunang; $\mathrm{Rg}$ nuna; $\mathrm{Ng}$ kedha I, nuda; $\mathrm{Na}(\mathrm{Tm}) \mathrm{keda}$; $\mathrm{Ed}$ epo; $\mathrm{Li}$ hepo, kepo; WS lùnda, lundángo; ES tawai; Si nunang; Sl kenuna, nunâ, kewanga.

Borag. Cordia monoica: $\mathrm{Km}$ ?dangé; Rg mesé; $\mathrm{Ng}$ kedha II.

Borag. $\quad$ Cordia subcordata: Bm binawa; Km genawa; Si nunang; Sl kanona, kena, kewanga II.

Liliac. $\quad$ Cordyline fruticosa: FEM (To, Ri) nao, (Ml) nao'; Kp nao'; Wr nao'; Rz nao'; Rm nao', (Tr) nao; Ng lanu II, ngusu; Ed gau; Li bengu, gau; WS karenggu ala; Si kébar; Sl kèdibu. 
Apiac. $\quad$ ?Coriandrum sativum: Bm katumba; WS bengge; ES benggi, wàla benggi; Sw ?wila hégé.

Apiac. $\quad$ Coriandrum sativum (cp. Apium graveolens): WS zànggi, wàla hànggi; ES sàgi, henggi, sànggi, wàla hànggi; $\mathrm{Sw}$ hégé.

Arec. $\quad$ Corypha utan: Bm laju; Km sowang; FEM cowang, sowang; Kp sowang; $\mathrm{Wr}$ borong; Rm sowang, borong; Rg mboro; $\mathrm{Ng}$ bhoro; $\mathrm{Na}(\mathrm{Tm})$ boro, ( $\mathrm{Ra})$ bo; Ké mbolo; Li boro; Pl poro; WS mboro, mboru; ES mburungu, ?iwés, 'borungu, mbúrungu, mbórungu, mboro; Sw boro; Si tebuk; Sl ketebuk, tebuk, ktebuk, tawu pokang; Kd tèbu'.

Aster. Cosmos caudatus: WS kapáta II.

Zingib. $\quad$ Costus speciosus: Kp lebo' tepok; Wr tepok; $\mathrm{Rz}$ tepok; $\mathrm{Rm}$ sempok; $\mathrm{Rg}$ tepo; $\mathrm{Ng}$ pudi, wiku waé III; Ed ?pudi, puri; Li laja II, pana; WS mòlu mane; Sl manuk méî.

Bignon. $\quad$ Crescentia cujete: $\mathrm{Bm}$ ponda mbolo; $\mathrm{Km}$ wila; $\mathrm{Kp}$ bilas; $\mathrm{Wr}$ bila; $\mathrm{Rz}$ bilas; $\mathrm{Rg}$ bila; Li bila; WS mangatu; ES tapusa, tapuha; Si bilan(g), ?gila, dila I; Sl kedila II, dila' bélé I, keludu; Kd kulu bila.

Liliac. $\quad$ Crinum asiaticum: FEM (Ml) wingku wangkung; Kp wakung; Wr wakung; $\mathrm{Rz}$ wakung; $\mathrm{Rm}$ wangkung, molor, wingku wangkor, wiko wako, (Wk) wakung; $\mathrm{Rg}$ wa(ng)ku; Ng molo; $\mathrm{Na}(\mathrm{Tm})$ molo; Ed sebé wéa; WS kaghailo tana; ES mangata, mangàta; $\mathrm{Sw}$ kelia beri; $\mathrm{Si}$ ?bunga baku.

Papil. $\quad$ Crotalaria sp.: Bm kakapi; $\mathrm{Kp}$ mambang; $\mathrm{Wr}$ ?mambang; $\mathrm{Rz}$ ?mambang; $\mathrm{Rg}$ wonda méo; $\mathrm{Ng}$ woda dheké, regha regha; Ed andé ponda, andé wonda, wonda ro'a; WS langgóro meràda; ES karàngu ahu; langgiri meu, langgoro maràda, ?wala nggiri miau, wàla nggìri meu; Si saru 'wowong, kata lari II, kota lari II; Sl éga; keteli, ruha ta'û, tutung réng; Kd ring ring, kika ring.

Euphorb. $\quad$ Croton tiglium: Bm duwa wua; Km duwa; FEM tuwa; Kp tuwa; Wr tuwa; Rz tuwa; Rm tuwa; Rg tuwa I; Ng duwa I, tuwa I; Na (Tm) tuwa jawa; (Bw) tuwa; Sl ruho'.

Crypter. ?Crypteronia paniculata: $\mathrm{Na}(\mathrm{Bw})$ maja I.

Laurac. Cryptocarya: Li wéa nana.

Laurac. $\quad$ Cryptocarya (triplinervis): $\mathrm{Kp}$ wughar; $\mathrm{Wr}$ wughar; $\mathrm{Rz}$ wughar; $\mathrm{Rm}$ wughar; ES kadúru landu kaka.

Cucurb. $\quad$ Cucumis sativus: Bm dimu, timu; $\mathrm{Km}$ timung; FEM timung, timus; $\mathrm{Kp}$ timun; $\mathrm{Wr}$ timun; Rz timun; Rm tinum, (Tr) timung; Rg ndaka, timu; $\mathrm{Ng}$ timu; $\mathrm{Na}$ (Tm, Ra) katé; Ed daka, ?ndaka, ndimu; Li daka, koto II, timu; Pl t'aka; WS kareri, karere; ES kariri II, ka'dinggiru, ?kareru; Sw dimu poro; Si ?dimun, 'oto, timun; Sl dimu, keri, dimû wata'; Kd timu upén.

Cucurb. Cucurbita moschata: Bm ponda; Km kondang; FEM (To) dési, (Bé, Ri, Mb) togo, (Ls, Ml) tonggo; Kp robo; $\mathrm{Wr}$ ndési; $\mathrm{Rz}$ robo; $\mathrm{Rm}$ timba, (Tr) togo, (Wu) zawong, (Wk) tonggo; $\mathrm{Rg}$ mbési; $\mathrm{Ng}$ bési II, bési jala; $\mathrm{Na}(\mathrm{Ra})$ 'uta; Ed bési; $\mathrm{Li}$ bési; $\mathrm{Pl}$ 'opo cawa; WS karòbo, karòbo jawa; ES karàbu, karobu hàmu, karobu rara, kàlahu; Sw kérebo jawa; Si bési; SI bési; Kd télar. 
Cucurbitaceae: Bm hala henggo; $\mathrm{Km}$ éjé; $\mathrm{Rg}$ timu ka; $\mathrm{Ng}$ fo'a, kopa dhola, kuwu, lu lima I, sama waru, sao I, timu nitu, timu polo, waro dua; $\mathrm{Na}$ (Tm) héa ba'i; Ed ?nagha; Li bela ba'i, kuwu, ndimu dolo II, ?puli, waro; ES kariri kalau, kadinggiru kalaü, tai 'muanga, tai monga; Sw doro, doro wa; Si soro kowa'.

Lamiac. Cuminum cyminum: Bm jinta.

Lythr. Cuphea hookeriana: Li déré mera.

Zingib. Curcuma: Bm duma; $\mathrm{Km}$ tewoa talu; FEM léa rengga, lia rengga, (Ml) lia renggang; $\mathrm{Kp}$ mapos; $\mathrm{Wr}$ léa naron; $\mathrm{Rz}$ naron; $\mathrm{Rm}$ léa naron, (Wk) léa renggang; $\mathrm{Ng}$ léa rega I; $\mathrm{Na}(\mathrm{Tm})$ léa rega.

Zingib. $\quad$ Curcuma viridiflora: $\mathrm{Bm}$ huni; $\mathrm{Km}$ gunis; FEM kunis I; $\mathrm{Kp}$ kunis; Wr lemok; Rz kunés; $\mathrm{Rm}$ kunis I; Rg kuné; $\mathrm{Ng}$ kuné; $\mathrm{Na}$ (Tm, Ra) kuné; Ed kuné; Li kuné; PI (k)uni; WS kunita, kúni, 'dingira, 'hingiro, kabùni; ES 'wìnguru, 'kaungi, 'wingira, 'kunyi; Sw kewunyi; Si guni; Sl kuma, kumas, uma.

Zingib. $\quad$ Curcuma zedoaria: Kp sumang; ES lamboya, laboya.

Commel. Cyanotis axillaris: Wr lema manuk.

Cyath. $\quad$ Cyathea: FEM (Ri) wuni, (Ml) kemut, (Ls) wené nitu; Kp puni, kemut; Wr puni; Rz puni; $\mathrm{Rm}$ puni, (Wu) kemut; $\mathrm{Rg}$ puni; $\mathrm{Ng}$ poni; $\mathrm{Na}(\mathrm{Ra})$ poni; Li poni, poni lo; $\mathrm{Kd}$ ?apan.

Cyath. $\quad$ Cyathea ?angiopteris (?glabra): Rm puni pong; WS paü karambo.

Amaran. Cyathula prostrata: Rg lando réndo; Ed meké reké II; Si baing tuhung, béing tuhung.

Cycad. $\quad$ Cycas (rumphii): Km angkor; FEM akor, (Ri) akur; Wr wéit; Rz ?wéit; Rm akur, ( $\mathrm{Tr}, \mathrm{Wu}$ ) akor; $\mathrm{Rg}$ (wunu) wéi; $\mathrm{Ng}$ laé III; $\mathrm{Na}(\mathrm{Tm})$ lai; Li léi, lo lai; WS kère; ES kàri, karu; Si blé'it, ramu, bré'it; Sl blé'it, kewaki.

Poac. Cymbopogon ?citratus: Bm pataha mpori I; FEM (Ri) kasi téu; Kp kasi' tiu, lasi' tiu; Wr kasi téu; Rz kasi téu; Rm kasi tiu, (Wu) kasi téu, nawéng; Rg kasi téu; $\mathrm{Ng}$ wako mengé, wako nawé; $\mathrm{Na}(\mathrm{Tm}, \mathrm{Ra})$ nawé; Ed nawé; Li nawé; WS mandawa bùka, mandagha bùka; ES kandangu buku, kandángu I; Sw ?heré; Si klahi rotan(g); Sl seré, seré wangi, notâ; $\mathrm{Kd}$ 'uru mèngi.

Poac. Cymbopogon nardus: ES kandali, kandaïngu wítu.

Asclep. Cynanchum: $\mathrm{Ng}$ mumu mapa.

Poac.

Cynodon dactylon: ES kandaüngu lúlu jungga I.

Caesalp. Cynometra cauliflora: $\mathrm{Bm}$ puci anggi; $\mathrm{Km}$ puci anggi.

Caesalp. Cynometra ramiflora: Si kéam.

Cyperaceae: $\quad$ Bm (mpori) kaḅisa na'e, kaḅisa to'i, kalalu, ngame; Kp iko ngga'éng; pézos; $\mathrm{Wr}$ rukut lawi; $\mathrm{Rz}$ rukut lawi; $\mathrm{Rm}$ mungkis; $\mathrm{Rg}$ résa, résa haki; $\mathrm{Ng}$ lemu, manu lalu II, saka béka, tuka lako; ES kahomba; Sw ?heré/héré; Sl sina séping; kedubu, lalu meran, ?méran. 
Cyper. $\quad$ Cyperus: $\mathrm{Ng}$ pu, pulu III, rabhu II, rabhu wawi, résa; Ed mbu'a huja; Li kasa kamba.

Cyper. $\quad$ Cyperus (brevifolius): $\mathrm{Ng}$ rabhu III; Ed kuru mengé; Li ana baga; WS wulu wawi; ?ma'regheta; ES rútu I; Si wiré.

Cyper. $\quad$ Cyperus kyllingia: Kp mbili mbon II; FEM keté mélén I; Rm mélén, (Tr) penduruk (Wk) ?tebhuk; Rg kumba résa; Ng rabhu II, sela kedhi; Li modu; Sw ?j'u'u méngi, ?wou méngi; Si ndéru dédé, wujo berékét, déru dédé.

Cyper. $\quad$ Cyperus monocephalus: WS mareza, mareha; ES kareha, ?karera, karesa, ?reha, kahomba, 'bokulu.

Cyper. $\quad$ Cyperus pilosus: $\mathrm{Ng}$ lasu lako.

Cyper. $\quad$ Cyperus rotundus: Bm (mpori) sisi II; FEM (Ri) wuk wawi, (Ls, Nn) rembung; Kp rukut rabéng; Wr rukut satar; Rm wut wawi I, (Tr) wuk wawi, (Wk) rembung; $\mathrm{Ng}$ fu lemu II, weté nitu I; Li ?bara kowé, berkowé; ES kareha wai, kahomba kudu; Si wujo ?kumi; Sl kujo' kuni.

Cyper. $\quad$ Cyperus tenuiculmis: Kp mbili mbon I; Wr ngelas waé; Rm pusék; Ed reru ré'é.

Gesner. $\quad$ Cyrtandra: FEM (Mb) wintong?; Kp kaju repo'; Rm rempok; Ng beko; WS wàngga I.

Poac. $\quad$ Cyrtococcum accrescens: $\mathrm{Ng}$ kuru bheto IV.

Poac. Dactyloctenium aegyptium: Ng siku deké II, waba; Ed kabi; ES kandaiingu mbata kambàku; Sw gagé.

Arec. $\quad$ Daemonorops (cp. Calamus): $\mathrm{Rg}$ ua; $\mathrm{Ng}$ ha'o, ua manu; Sl uat manuk.

Arec. $\quad$ Daemonorops melanochaetes: Bm ?miro, ?kana.

Solan. $\quad$ Datura metel: Bm ḅembe; FEM mbungéng; Kp mbungér; Wr mbungér; Rz mbungér; $\mathrm{Rm}$ mbungér, ( $\mathrm{Tr}$ ) mbungéng; $\mathrm{Rg}$ mbungé; $\mathrm{Ng}$ bungé I; $\mathrm{Na}(\mathrm{Tm})$ mbungé, $(\mathrm{Bw})$ bungé; Li mbungé; WS 'puaku, nggule; ES 'mbunguru, 'mbungura, 'bungaru, la'mbunguru, wàla 'mbunguru, kalibu waï, la'bunguru; Sw kolé wawi; Si wawi ta'in; Sl wawé' toron III, kepunga'; Kd pilir hèi.

Urtic. Debregeasia: Ng nigé IV; WS ridi wówo, rindi ghogho I, rindi paré; ES kapàra luku.

Myrtac. Decaspermum: ES 'lobungu I, labu, ka'lobungu.

Myrtac. Decaspermum triflorum: $\mathrm{Rg}$ pui; Wr puis.

Amaran. Deeringia (amaranthoides): Km belentang; Ng kedha; ES kamíti I.

Orchid. $\quad$ Dendrobium sp.: Rm koza wo'or; WS katówa kedu.

Poac.

Dendrocalamus asper: ?Bm o'o todo; Km betong; FEM betong, (Mb) bhetong; $\mathrm{Kp}$ betong; Wr betong; Rz betong; Rm betong, (Wk) bhetong; $\mathrm{Rg}$ beto; $\mathrm{Ng}$ beto I, bheto; Na (Tm, Ra) bheto; Ed beto, ?bheto; Li bheto; Pl béd'u; WS pòto, wo'o pòto katilu; ES tàringu, tèringu, 'tiringu, pitungu, au 'bokulu, 'pitingu, pàtungu; Sw kelaiwu; Si petung; Sl petû; Kd pérung. 
Urtic. $\quad$ Dendrocnide (cp. Laportea (forstenii)): Bm maladi; maladi ara; $\mathrm{Km}$ lanténg; FEM lantong, ( $\mathrm{Nn}$ ) lanténg; $\mathrm{Kp}$ latong; Wr latong; $\mathrm{Rz}$ latong; $\mathrm{Rm}$ lantong, (Wk) latong; Rg laté I, laté II; $\mathrm{Ng}$ adé I, landé I, laté I; $\mathrm{Na}(\mathrm{Tm})$ ladé pu'u; Li landé I; Pl ladhé; WS langgate, lagate; ES ju'latingu; Si 'ladang; Sl klateng laking, latèng.

Loranth. Dendrophthoe: Ed ndombo ndo'o; Li moro ro'a; WS kahala managhe.

Papil. ?Derris: Bm duwa; Km duwa runca.

Papil. Derris ?elliptica: Bm duwa tutu; Km ndaréng; FEM (To, Mb) raréng, (Bé, Ri, Ls, $\mathrm{Nn}$ ) laréng; Kp laréng; Wr laréng; Rz laréng; Rm laréng; $\mathrm{Rg}$ ?mesa; $\mathrm{Ng}$ téngé, tuwa, tuwa ika; $\mathrm{Na}(\mathrm{Tm})$ laré; Ed ?tuwa I; Li moro; Pl d'uwa 'ud'a; WS tuwa, tuwa', tugha; ES túwa, cuwa; Sw ?keb'edu; Si pléa; Sl pléa, tuba, nuwa; Kd nua.

Papil. Derris polyphylla: Rg tuwa II.

Papil. Desmodium: FEM (Ri, Ls) kepé, (Ls, Nn) moro kélas; Kp boto' tuka; sepé lemét; $\mathrm{Rm}$ koza wura, kepé rapét; $\mathrm{Rg}$ lepi I; $\mathrm{Ng}$ pépi II, uné ika, weka I, weka (raké); $\mathrm{Na}$ (Tm) luba; Ed kubi dhéké hai, mata ika, una ika; Li ?lege fé'a, repé II, lege fésa, wuso weka; WS kandúruko; ?ka'pelata ndende; wème ndara I, wème ndara II; kapi'élata, kapelata; Sl kewéra.

Papil. Desmodium heterophyllum: Bm mpori sisi I.

Papil. Desmodium laxiflorum ssp. laxiflorum: Rg pele ki'a haki.

Papil.

Desmodium repandum: Kp kélas; Wr kélas; Rz kélas; Rm kepén, (Tr) kepé.

Papil.

Desmodium strigosum: Rm kepén kazu.

Papil.

Desmodium triflorum: Rg mata mela; WS wème ndara; Sl manakâ merâ.

Papil.

Desmodium velutinum ssp. velutinum: Ed kubi dhéké I, meké ré'é.

Papil.

Desmodium zonatum: $\mathrm{Ng}$ weka, weka raké.

Poac.

Dichanthium ?annulatum: WS kapúmbu I.

Poac.

Dichanthium caricosum: ES kahanga kiri.

Poac.

Dichanthium erectum: WS kapumbu II; ES mapu kangúrutu I, kapumbu.

Acanth. Dicliptera: ES kakaha II, kasa II; ka'pohaku míni I, kapohaku I.

Gleichen. Dicranopteris pubigera: Wr kera; Rz serat; $\mathrm{Rm}$ serat; $\mathrm{Ng}$ paja I, paza; Li kipa I.

Poac. Digitaria adscendens: ES kandaüngu bàkulu.

Poac. Digitaria setigera: $\mathrm{Ng}$ toré faga neka.

Dillen. Dillenia pentagyna: FEM (To, Bé) robok, (Ri) bilas, (Ls) koék, (Ml) koé; Kp bilas, kuis; Wr bilas; Rz bilas; Rm bilas, kuis; Rg mbila; $\mathrm{Na}$ (Tm) koé; Li wira I; WS zake, ?katanggara, áke; ES kananggaru, ka'nanggaru; Sl kadu ria.

Poac. Dimeria ornithopoda: Kp wuk ela; $\mathrm{Rm}$ wut wawi II.

Poac. Dinochloa scandens: $\mathrm{Bm}$ ?o'o ai; $\mathrm{Km}$ hau isi; $\mathrm{Ng}$ bheto nitu; Si nitu petun; $\mathrm{Sl}$ au ora, orâ. 
Diosc. $\quad$ Dioscorea sp.: Bm eja wilu; Ko nalun asu, koék; Wr koék; Rz koék; Rm koék, (Tr) koak; WS katilara, katilyóro; ES ?lawuhu; luwa tana.

Diosc. $\quad$ Dioscorea sp.: FEM (To) koton, (Ls, Nn) koto, (Ml) peca mela; weké; Kp bolo'; Wr bolo'; Rm pesa', (Tr) koto, (Wu) pesa mela.

Diosc. $\quad$ Dioscorea spp.: $\mathrm{Rg}$ séwu; $\mathrm{Ng}$ fiso, hiso; lowa II; tenu; séwu; Ed ndepu; Li asa uwi, kéwa, teni, tenu, (uwi-)monda; Pl wanga; Si buli bowa uta, buli bowa nata, pinda, wangar; Sl kolé'; Kd biar rèngal.

Diosc. Dioscorea ?aculeata: $\mathrm{Bm}$ (uwi) sura II; Km séwo; FEM séwu, (Ri) séwo; Kp ndaté séwo; Wr séwo; Rm séwo, (Tr) séwu; Rg sura kumba; $\mathrm{Ng}$ kebhu, kebu, séwo, sura II; Na (Bw) ?sura, (Ra) ?kéwa; Li mbanggo; Pl 'ura ud'a; WS kabusu, kabuzu, laghùta; ES lita rumba; Sw woké/huré edu; Si hura edu, hura nurong, hura 'uta; S1 ?hura utan, hura' lado, sura rotan, kemede'; Kd lèwu' 'otan.

Diosc. Dioscorea alata: $\mathrm{Bm}$ uwi sura I; FEM uwi, (Ml) uwi; Kp uwi, uwi répong, uwi jékong; Wr uwi; Rz uwi; Rm uwi; Rg uwi; Ng uwi; Na (Tm, Bw) uwi; Ed uwi; Li uwi; Pl 'uwi; WS lua (pi'a), luwa (pi'a), lugha piyo; ES luwa; Sw hiwu; Si ohu, ohu krowé; Sl hura I, ué belê, ué' I, ué' bél'ê, 'uwé', sura amen, ?surar, uwé mure'; Kd alèwu' au.

Diosc. Dioscorea ?bulbifera: FEM mola; Kp rokong; Wr rokong; Rz rokong; $\mathrm{Rm}$ rongkong, (Tr, Wk) mola; $\mathrm{Ng}$ gola; $\mathrm{Na}(\mathrm{Tm})$ mola; Li modi; WS kamenggo; $\mathrm{ES}$ ka'menggala, ka'menggalu, ka'megalu; Sl kolo énga I, kang tupateng, ?uwé holi.

Diosc. Dioscorea esculenta (cp. Coleus tuberosus): Bm uwi tandi'i; uwi sura I; FEM tesé, ( $\mathrm{Ri}, \mathrm{Ls})$ sezan, (Ml) tesé, kérang; $\mathrm{Kp}$ ndaté; $\mathrm{Wr}$ ndaté; $\mathrm{Rz}$ ndaté; $\mathrm{Rm}$ sezan, $(\mathrm{Tr})$ tesé; Rg sura I; Ng éjo, suja, supu, sura, suza I, 'uza; Na (Tm) suja, (?Ra, Tm) suza; Li suja, uwi suja; Pl 'ura; WS tande'i, laghuta; ES litangu, litangu, lùtangu; Sw kéhuré; Si hura epa'; SI hura II, ué améng, ué' hura', hura', sura mojek, hura'; Kd lewu'.

Diosc. Dioscorea hispida: Bm lede, ?gadu; Km rao'; FEM raut, (Nn) ondo; Kp raut; Wr kou'; Rz kou; Rm nangkon, (Tr, Wk) raut; $\mathrm{Rg}$ kou; $\mathrm{Ng}$ kou, odo; $\mathrm{Na}$ (Tm) odho, (Ra) odo; Ed ondo; Li ondo; Pl ondo; WS ùwi I, ughi; ES iwi II, auwi, àwi, iwi maïku; Sw guwi; Si magar, pida; Sl magal, ondo, ubal.

Diosc. Dioscorea pentaphylla: FEM cué, suan, sué; suan melir, cué bhuang; cué wulu, cué léwé, sué wulu, suan wulu; $\mathrm{Kp}$ siwu; $\mathrm{Wr}$ séwu; $\mathrm{Rz}$ séwu; $\mathrm{Rm}$ suan ghelis, suan telo' kaba, suan wulu, (Tr) suan laso; Rg kéwa; $\mathrm{Ng}$ kéwa; $\mathrm{Na}(\mathrm{Tm})$ kéwa; Ed ?nggéo; Li ?mboko uwi; Pl ?mola; WS langgódo II, lágódo; Si ?kéwa; Sl odo, kubak; Kd apo'.

Diosc. Dioscorea sarasinii: FEM (To, Ls, Nn) kéngal, (Bé, Ri) éngal, (Mb) kéngar, (Ml) 'ingal, 'uwi ndiris; Kp éngal; Wr kéngal; Rz kéngal; Rm ingal, (Tr) éngal, (Wk) kéngal; $\mathrm{Rg}$ kénga; $\mathrm{Ng}$ sama jawa; $\mathrm{Na}(\mathrm{Tm})$ koto; Ed ndepu; Li losa; $\mathrm{Sw}$ ?hiwu inga; $\mathrm{Si}$ 'ohu kolé; Sl ué' nou, nou; Kd rèngal, biar réngal; WS 'engala, 'engolo; ES 'engala/u, 'engalu.

Eben. Diospyros: ES ai míteng(u), ai mítingu.

Eben. Diospyros ebenum: Si 'ai mitang. 
Eben. $\quad$ Diospyros javanica: Bm tonggu ala; Km bosa'; Rm lentun.

Eben. $\quad$ Diospyros littorea: Bm haju me'e I; FEM (Ri) léléng, $(\mathrm{Mb})$ lélé; $\mathrm{Rm}$ léléng.

Eben. $\quad$ Diospyros malabarica: Bm ?kalaku, mpiu I; Km ?kalaku, mpiu I.

Eben. $\quad$ Diospyros maritima: Sw ai.

Eben. Diospyros 'toposia': Bm haju me'e II; Km (haju) mé'é II.

Polyp. $\quad$ ?Diplazium: FEM (Bé, Ri, Ls) maku, (Nn) paku; Kp paku; Wr paku; Rz paku; Rm maku; $\mathrm{Rg}$ paku; $\mathrm{Ng}$ paku, rori waé, uta paku; $\mathrm{Na}(\mathrm{Ra})$ paku; WS pawu, paghu; ES paii, paku; Sw paku; Si paku; Sl paku.

Cucurb. Diplocyclos palmatus: $\mathrm{Ng}$ baru.

Polyp. Dipteris conjugata: WS kùru kára Ii.

Asclep. $\quad$ Dischidia: Ed fi'i ji.

Hamam. Distylium stellare: Ed fo koki.

Bignon. $\quad$ Dolichandrone spathacea: Bm (n)tui.

Papil. Dolichos junghuhniana: Rm ku'u.

Papil. $\quad$ Dolichos lablab: Bm wure.

Stercul. Dombeya: Kd wélé.

Marant. Donax cannaeformis: Wr nini'; Rz nini.

Euphorb. Doryxylum spinosum: $\mathrm{Rg}$ mbeba; $\mathrm{Ng}$ lima léwa.

Anac. Dracontomelon edule: Bm ?rau; Wr ra'u; $\mathrm{Rz}$ ra'u; $\mathrm{Rg}$ ra'u; $\mathrm{Ng}$ za'u I; Ed ra'u I; Li ra'u; Si ra'u.

Asclep. $\quad$ Dregea volubilis: $\mathrm{Km}$ poa; $\mathrm{Rg}$ kupé II.

Caryoph. Drymaria cordata: Kp lebo' liti'; Wr kinga té'us; $\mathrm{Ng}$ mata mela; $\mathrm{Na}(\mathrm{Bw})$ mela mité; Ed mera gera; Li mela ré'é.

Polyp. $\quad$ Drynaria: FEM (To, Ls, Nn) lukuk, lukup, (Bé) tekap, (Ri) lukut; Kp rembé raé; Wr lebé raé; $\mathrm{Rz}$ lembé raé; $\mathrm{Rm}$ rombo raé, (Tr) lukut, (Wk) kukut; $\mathrm{Rg}$ lembé raé; $\mathrm{Ng}$ belé biza, koré IV, nara hara; $\mathrm{Na}(\mathrm{Tm})$ belé biza; WS lukuta; ES lukutu I; Si klukut; Sl ko'it I.

Polyp. $\quad$ ?Dryopteris: FEM limé lipang, lebé raé, raé; Kp lema kipan; Wr lema kipan, liméng kipan; Rz liméng kipan; Rm lema kipan; $\mathrm{Na}(\mathrm{Tm})$ lima hipa; Sl wulû waing I.

Polyp. $\quad$ Dryopteris microsorium: $\mathrm{Ng}$ paku wolo.

Polyp. $\quad$ Dryopteris purpurascens: Ng kungu sama roga; Ed soso; Li paku I.

Euphorb. Drypetes: Bm safare rangga; ES kanùnu.

Euphorb. Drypetes (longifolia): Rg téwa ngamba; Ed lalu; Li ana bara.

Papil. Dumasia villosa: Li tali té'u I.

Verben. Duranta erecta: Si bunga raja. 
Bombac. Durio zibethinus: Bm duria, aduria.

Meliac. $\quad$ Dysoxylum: FEM asong; Kp dora; Wr dora; $\mathrm{Rz}$ dora; $\mathrm{Rm}$ dora; $\mathrm{Rg}$ wua pu'u fai; Ed alu jawa, anudi, até roa, bonggi boja, gagé kéli, kaka rawa, rima réwa, ?oja, singgi bara; Li ?ma, moka, nila, nio II; ES lapále; kìru bára; lándu káka 'omangu; Si ?kurok; Sl amakéan; hali.

Meliac. $\quad$ Dysoxylum arborescens: Kp lokor kodé'; Wr lokor kodé'; Rz lokor kodé; Rm longkor lawo', mbaring; Rg wua pu'u haki; Sl telir II?, laku mukung, ?karo.

Meliac. Dysoxylum caulostachyum: Bm kapu'u; Kp wua' pu'un; Wr wua pu'u; Rz wua' pu'u; Rm wua' pu'u, (Tr) wua pung, (Wk) ?nua pu'un; Rg wua pu'u II.

Meliac. Dysoxylum gaudichaudianum: Rg hapé léwa; $\mathrm{Ng}$ hopa béwa I; Es lándu káka.

Meliac. $\quad$ Dysoxylum cf. nutans: Km besola; Wr worok; $\mathrm{Rz}$ worok.

Meliac. Dysoxylum parasiticum: Rg wu'a pu'u I; Ed ?kesa, ngaga ié; WS kìru, kiru; ES kàti katàru II; kìru.

Poac. $\quad$ Echinochloa colonum: Rm woza paké'; $\mathrm{Ng}$ weté nitu; ES randi, rendi, uhu rendi; Sw hérégo.

Poac. $\quad$ Echinochloa crus-galli: Rm batis kombéng; Li kuru paré II.

Aster. $\quad$ Eclipta alba: Sl bési kélén.

Aster. $\quad$ Eclipta prostrata: Ng léda waé II, moro paké; WS katara kàde.

Borag. $\quad$ Ehretia (acuminata): Bm ?mboro; Ng kéu II; Ed ana kéa, kobé.

Borag. $\quad$ Ehretia microphylla: Rg ta'i tibo I; Na (Bw) bongo, mbongo.

Elaeoc. $\quad$ Elaeocarpus floribundus: Bm sapompo, ?sapombo; FEM (Ls) lecong, (Nn) lecéng; Kp damu; Wr damu; Rz damu; Rm damu; Ed buju, kawu, mbuju; Si daé.

Elaeoc. $\quad$ Elaeocarpus (petiolatus): Rg nito; Sl munak paong.

Elaeoc. $\quad$ Elaeocarpus sphaericus: Bm ?majakale; FEM (To) ?nintok; ?wol; Kp nito; Wr nito'; Rz nito; Rm ninto, (Tr, Wk) ?wol; Ed maja II.

Urtic. $\quad$ Elatostema: Rm mumus kuzang.

Urtic. $\quad$ Elatostema (cp. Boehmeria): $\mathrm{Rg}$ ponggo kura; $\mathrm{Ng}$ lesi II, nigé II, wié waé II; Li mota ndoro I.

Urtic. $\quad$ Elatostema: Kp wiko'; Rm ?wikul, wiko'.

Sapind. $\quad$ Elattostachys: FEM (Bé, Ri) ?lowé api; Rm (Tr, Wk) ?lowé api.

Sapind. $\quad$ Elattostachys (cp. Chisocheton): $\mathrm{Ng}$ doza I.

Cyper. $\quad$ Eleocharis dulcis: Bm karebe: Sw waka.

Aster. $\quad$ Elephantopus scaber: Rg hinga mota II; Ng kabu sengasu; Ed kere kéba; WS wi'a mareda, wi'a 'langita, kuru wawi, ngura wawi; ES mbúlungu pingi.

Poac. $\quad$ Eleusine indica: FEM kurun; Kp reman kurun; Wr kurun kaba, nepé né'ét; Rz ?kurun; Rm kurun; Ng ngadé I; Na (Bw) ngada badha; Li kuṛu kamba; WS kabia 
karabo, ?kabiya karambo; ES mápu 'mbelaru pingi I; Si 'urun, népar, 'urun paré; S1 wawé wetê ne.

Lamiac. $\quad$ Elsholtzia pubescens: $\mathrm{Ng}$ wonga wau; $\mathrm{Si}$ ?lonok.

Myrsin. $\quad$ Embelia: FEM (Ls) moro méki, (Nn) moro méci, (Mb) nagé rana; Kp lebo' moro mik;; Wr lebo' liti, lémbar, sirok; Rz moro méki; Rm moro miki; Rg lando kata I; Na (Tm) nagé rana, (Bw) komba katé; Ed komba katé, ?mbéré wawi; WS ka'niluk(a) I.

Aster. Emilia sonchifolia: Bm padu peo; Kp rénggong; Wr soit; Rz rénggong; $\mathrm{Rm}$ rénggong; Rg kinggo; $\mathrm{Ng}$ kigo I, kigo wio I, koké; $\mathrm{Na}(\mathrm{Bw})$ kigo; Ed kinggo; Li higo, kigo, kigo paré; WS makíku/makiku, ka'buruko; Si kligong; Sl wodo léin, kligong.

Jugland. $\quad$ Engelhardia spicata: Ed neta batu, tawu I.

Hydroch. $\quad$ Enhalus acoroides: Km lamung, satah tasi'; Pl lamu; Kd lamun.

Gentian. $\quad$ Enicostemma axillaris: $\mathrm{Ng}$ manu lalu I; ES muru njara.

Musac. $\quad$ Ensete glaucum: Bm ?kalo linci; FEM (Bé) bujung, (Ri) buzun, (Ls, Nn, Mb) mbujung, (M) mbuzung; Kp bozon; Wr lozon; Rz bozon; Rm bozon I, buzun; Ng muku buju; Na (Tm) buzu, (Ra) buju; Ed rojo; Li lojo; Pl muku ?uru; WS kalowo kedu, kaloho koki; ES kalú yúmbulu, kalú húmbulu, húmbulu, kalowi kuki, yumbulu, kàlowu sobulu; Si baki, bakér; Sl baki, muko baki.

Mimos. $\quad$ Entada phaseoloides: Bm humpa leu; FEM ajo, (To, Bé) azo, (Ls) luit, (Ml) 'azo'; Kp azo;; Wr azo'; Rz kazék azo'; Rm azo', (Wu) loit, (Tr) azo, (Wk) luit; Rg léké; $\mathrm{Ng}$ léké; $\mathrm{Na}(\mathrm{Tm}, \mathrm{Ta})$ léké; $\mathrm{Li}$ léké; $\mathrm{Pl}$ kili; Si 'énak; Sl wé'o, kélféor, késo; $\mathrm{Kd}$ éhu'.

Equiset. $\quad$ Equisetum debile: $\mathrm{Kp}$ raun kedu; $\mathrm{Rm}$ sun; Li bu béwa, lesé.

Poac. $\quad$ Eragrostis: ES wàla penggi.

Poac. $\quad$ Eragrostis (amabilis): ES híbu manginu; Sw méngi/mengu.

Poac. $\quad$ Eragrostis elongata: $\mathrm{Ng}$ bupu moku.

Poac. $\quad$ Eragrostis japonica: $\mathrm{Ng}$ pubu neka kedhi.

Poac. $\quad$ Eragrostis pilosa: Ng fu lamu I.

Poac. $\quad$ Eragrostis tenella: $\mathrm{Ng}$ kuru peti.

Poac. $\quad$ Eragrostis (warburgii): Li kuru weté I; ES wulu ùru I, kawulu ùru II.

Acanth. $\quad$ Eranthemum: WS tòdola; Es kayàmbi 'omangu, ?balaria.

Aster. Erechtites valerianifolia: FEM (To, Ls) rewut kepal, (Ml) bozot; Kp bonggéng, nipon; Wr bozél; Rz bozé; Rm bozot, (Tr) kinggong, (Wk) tewon kepal; Rg bojé; $\mathrm{Ng}$ hingo, hingo nipo, ?ingo, kigo nipo, mogo, susu wawi I, ta'i wawi I, ta'i wawi II, tibo, uta bomo, wonga dhonu; $\mathrm{Na}(\mathrm{Tm})$ baké mézé, ( $\mathrm{Bw}$ ) kigo nipo; Ed kinggo, kinggo nipo, mbaka; Li mbaka jégha, mbaka (nipo); WS 'buoki, mbówila, 'kapala lera, 'kapalo; ES kóndu 'urangu II; Si lemur, pobo; Sl sola, ?kuwék II. 
Aster. $\quad$ Erigeron (sumatrensis): $\mathrm{Ng}$ baka éu, baka lélu, pobo, rama ruba; Ed mbaka kerị; Li bu mbéwa, toko sora; Sl ribu wulû.

Poac. $\quad$ Eriochloa ramosa: Sw nyeb’u délo.

Apocyn. $\quad$ Ervatamia: Ed jaga sa'i, sega sa'i, sege sa'i.

Apocyn. $\quad$ Ervatamia (see Pagiantha and Appendix 2).

Apocyn. $\quad$ Ervatamia (pubescens): Sl teburô I.

Papil. $\quad$ Erythrina: FEM jejak, (Ri) zezat, (Ml) zezak; Kp zezat; Wr zezat, kalo zezat; $\mathrm{Rm}$ kalo ghan, (Wu, Wk) zezat; Sw karé nga'a.

Papil. $\quad$ Erythrina: FEM (To, Ls) toal, (Ri) minsi, (Ml, Nn) méci, waké téré; $\mathrm{Kp}$ misi; $\mathrm{Wr}$ mési; Rz (kalo) mési; Rm minsi, (Wu) mési, ( $\mathrm{Tr}, \mathrm{Wk}$ ) misi; $\mathrm{Ng}$ bhu mési; $\mathrm{Na}(\mathrm{Ra})$ mési, (Bw) dhéo mési.

Papil. $\quad$ Erythrina (orientalis): Bm péto; FEM (To, Ls) mbérang; Wr kalo; Rz kalo; Rm kalo; $\mathrm{Rg}$ dero II; $\mathrm{Ng}$ dero II, mési II; $\mathrm{Na}(\mathrm{Tm})$ déro, (Bw) dhéo, (Ra) dho; Li dero, hero; WS rèdapa, wòla kari, ràdapa, wàla kare; ES wàla keri, wàla kari, wàla karu; Sw karé; Si blata, blawang, brata; SI blata', lejap, rerap; Kd léa'.

Papil. $\quad$ Erythrina subumbrans: Bm péto radarasa; FEM (Ls) menado, (Ml) dadap menado; Kp kalo (me)nado; Wr kalo menado; Rz menado; Rm (kalo) menado, (Wu) dadap menado, ( $\mathrm{Tr}$ ) dadat; $\mathrm{Rg}$ kalo menado; $\mathrm{Ng}$ dero I; $\mathrm{Na}(\mathrm{Tm})$ dhéro tua, $(\mathrm{Bw})$ mési menado, (Ra) dhéo belanda; Li dero belanda; Sw karé melanu; Si bla'at.

Myrtac. $\quad$ Eucalyptus alba: Si ?pelawan, popo bura; Sl keronga, pukat, pukaj, kapuka, kapape, pelawan, puka, popo I, puka'.

Myrtac. $\quad$ Eucalyptus urophylla: Rg ampupu; Si popo mérak; Sl lu; puka lelaki, gerung; Kd lu.

Myrtac. Eugenia (see Syzygium).

Poac.

Eulalia fulva: ES rara I, mapu rara.

Poac.

Eulalia leschenaultiana: $\mathrm{Ng}$ pubu neka kedhi; Sl kemu'un.

Rutac. $\quad$ Euodia: Bm mpa(ng); FEM (To, Bé, Nn, Ls) ?pak; Kp pak; Wr pak; Rz pak; Rm pak; $\mathrm{Rg}$ peko pa; $\mathrm{Ng}$ 'aro pa, bé'o, nipa nai; Ed pa wau.

Celastr. $\quad$ Euonymus (javanicus): Kp ngapur; Rm ngampur; WS wangkura.

Aster.

Eupatorium inulifolium: FEM ganéfo, (Ri, Ls) sénsus; Kp merdéka, sénsus; Wr oka dé; Rz sénsus; $\mathrm{Rm}$ merdéka', (Wk) ganéfo, $(\mathrm{Tr}, \mathrm{Wu})$ sénsus, $(\mathrm{Tr})$ merdéka; $\mathrm{Ng}$ séko dé'u I, séko si'é, siko si'é, sésu, wonga bhara III; Ed rinu; Li kerinu; Sl kada waning.

Aster. $\quad$ Eupatorium odoratum: Bm kemba(ng), oka dé; Km bara wau; FEM (Ls) sénsus, ganéfo; $\mathrm{Rm}$ sinsus, (Wu) sénsus; $\mathrm{Rg}$ sésu (sénsus); $\mathrm{Ng}$ sé(n)su(s), séko déu II; $\mathrm{Na}$ (Tm) ganéfo, (Bw) wonga ba, wonga boa; Ed mburi mbedo, mbutu mité, rinu, wonga bhara II; Li golkar; Sl puhû burâ, unu wauk I.

Euphorb. Euphorbia: $\mathrm{Rg}$ lando réndo I; $\mathrm{Ng}$ lado rédo I, taga kolo II, kaju ular; Ed kumi rongo II; Li ?sé'é II; Si ?kaha, ai padun; Sl sebo'ut. 
Euphorb. Euphorbia cf. antiquorum: ES kalàla jawa; SI ?dila bélê.

Euphorb. ?Euphorbia barnhartii: FEM (Ls, Nn) lelak; Kp tanggo éndé; Rm tanggo' éndé; $\mathrm{Ng}$ susu benu, susu penu I, susu témé; Sl pekok.

Euphorb. ?Euphorbia hirta: $\mathrm{Kp}$ susu mbenu; $\mathrm{Wr}$ susu mbenu; $\mathrm{Rm}$ nunu naéng; $\mathrm{Ng}$ lado rédo, rédo-rado; $\mathrm{Na}(\mathrm{Bw})$ ego ma'u, léwa rora; Li kumi rusa I; Si koja temuk; Sl lima bolak, ké'a méî.

Euphorb. Euphorbia hypericifolia: Rg lando réndo I; WS ruta wùni I; ES rùngu rutuhu; Sw hou mengi.

Euphorb. Euphorbia levis: Sw lilo géro moné.

Euphorb. Euphorbia prostrata: Ng dhari boka, li'é logo I; ES ?moro I, rumba rara II.

Euphorb. Euphorbia prunifolia: Pl koca nggelo; Sl kesâ.

Euphorb. Euphorbia schuhmannii: Ed taru ndu'a.

Euphorb. Euphorbia tirucalli: Bm kabatula; FEM (Ri, Bé) kaba tula, (Ls) lelak wasék; Rm ?kaba tula, (Wk) kamba tula; Sw taba rui II; Sl futa'.

Euphorbiaceae: Bm belere; $\mathrm{Km}$ belere; Ed kitu; Sw méa.

Theac. Eurya: Li éko ndé'u.

Euphorb. Excoecaria agallocha: ES tabùka; Sl kawuta'.

Santal. $\quad$ Exocarpus latifolius: Bm ncambu wera; WS moro lóngge I.

Logan. $\quad$ Fagraea: Ed boré gaga.

Logan. $\quad$ Fagraea ceilanica: Kp lema kaba; Wr lema kaba.

Morac. $\quad$ Fatoua pilosa: $\mathrm{Rg}$ seko raké II; Ng paka do I; Ed meké reké III; Li ?meka reké; WS kadito wòla, kadito wàla; Sl kelapu, wodô léin; Kd wéla dapé'.

Morac. $\quad$ Ficus: Bm wudiwonca; Km naa; naa mbisu; FEM kipo; mesé; nunuk (wulang); nunuk; Kp ara kipo; Wr kelo', purus boko'; ara kanun; Rm kipo', kaka; (Wk, Wu, Tr) nunuk; kelo; niron; Rg ara; Ng bhuru bhara, hebu II, héga, kaka kaju I, kéngo, nunu mézé, rabu, rhebu I, rura kogha I, ruto jawa, ruto nunu, séga, ta'i peti II, wua pu'u, reké; $\mathrm{Na}(\mathrm{Ra})$ nunu; Ed ara, ero, moru, nunu ra, réré, réré mberé, rẹéé méré, ra; Li baké, funga, hewo, hoki, kewo, koki, lélé dhéndé, lélé halo, lélé kaki, lélé kalo, lélé panda, seti II; WS kambálaka, ka'balaka, kadoki, ka'pulota, kapuloto; wazu loma karambo, wasu lòma karabo; pèdi mète; Es kabala; kaduki, kadauki, kaduku, kadóke, kapulutu, ka'pulitu, ka'pilítu, ka'pulutu, kapulita, kapilutu; wangga II; wangga III, handa/hànda; manjailu, ta'nganangu, ta'ngananga; maloki, maloku; karúku, pìdi kalauki, kàroku, wunuta; Sw keb’unu, meluki, médiri, menyaro, méhia, wega, hega, luki; Si tolén(g), liro; Sl wao kesé'in, ke'ulek, kewoà, klawê I.

Morac. $\quad$ Ficus sp.: Wr kelo'; Rm ?kelo'; Rg ara helo; $\mathrm{Ng}$ helo, kelo, kéu IV, selo; Li helo, kelo.

Morac. $\quad$ Ficus sp.: Rg baso; $\mathrm{Ng}$ bhaso; Ed baso; Li baso. 
Morac. $\quad$ Ficus sp.: Kp kabé' alas; Wr kabi alas; Rz kabé' alas; Rm kabé' alas; Rg kabé ala.

Morac. $\quad$ Ficus sp.: Km rah, ras; FEM ras; Kp ras; $\mathrm{Rm}$ ras.

Morac. $\quad$ Ficus ampelas (cp. F. wassa): FEM (To, Ls, $\mathrm{Nn}$ ) baka kolo, (Ri, Ml) pelas; $\mathrm{Ng}$ nara séga, pela, pidi; Li mboi; ES karaki, karaku; Kd ?para.

Morac. $\quad$ Ficus balica: FEM (To) kobo, (Bé) kobok, (Ls) kewo, kébhék, (Nn) kébhék, (Mb) khobok; Kp kobok; Wr kobok; Rz kobok; Rm, (Wk) kobok, (Tr) kebok; Rg kobé; $\mathrm{Ng}$ hewo, sewo; $\mathrm{Na}$ (Tm, Bw) hewo; Li kinga kewo; ES yumbul, jimbu; Si koang; Sl kwoâ, foâ.

Morac. $\quad$ Ficus benjamina: Bm due; $\mathrm{Km}$ weringing; FEM rutong, (Ls) ruton, (Mb) ruténg; $\mathrm{Kp}$ riton; Wr ruton; $\mathrm{Rz}$ ruton; $\mathrm{Rm}$ riton, ruton, $(\mathrm{Wu})$ ruton, $(\mathrm{Tr})$ rutong, (Wk) rutan, nélo; Rg nunu; $\mathrm{Ng}$ ngedhu, nunu, ruto III; $\mathrm{Na}(\mathrm{Tm})$ titu widu, (Bw) 'a, (Bw, Ra) nunu; Ed nunu ra; Li lélé, lélé fai; Pl nunu I; WS ?kabalaka, ?kambalaka I, wangga; ES wangga, waga; Si bao; Sl bao, baor; Kd bèu.

Morac. $\quad$ Ficus benjamina var. bracteata: FEM (To, Bé) ara tia, (Ri) tia, (Ls, Nn) ti'a; Kp ti'a; Wr ti'a; Rz ti'a; Rm (Wu) ti'a, (Tr) tia, (Wk) kekas.

Morac. $\quad$ Ficus depressa: Kp beka; Wr tawa'; Rm (Wu) bengka, (Wn, Tr) beka; Rg bega; WS malíti I; ES kalamba.

Morac. $\quad$ Ficus fistulosa: Kp kabé' I; Wr kabé; Rz kabé; Rm (Tr) kabé'; Rg kabé; $\mathrm{Ng}$ boko nawé, li'é nawé, nawé; Ed meru I.

Morac. $\quad$ Ficus ?fulva: Kp rakas; Wr rakas; Rz rakas; $\mathrm{Rm}(\mathrm{Wu}) \mathrm{kaka}$ ( $\mathrm{Tr}, \mathrm{Wk}) \mathrm{kelo}$; $\mathrm{Ng}$ gaka II; Li tanggo; WS 'wewera.

Morac. $\quad$ Ficus ?heteropleura: KP kapél; Wr koki; Rm (Wu) kampél, (Wk) kapél; Ng nélo, nunu ta'i peti, ta'i peti I; Ed koki; Li ?lélé II; WS pèdi káka; Sw keb’o I; Sl perék; Kd uar.

Morac. $\quad$ Ficus 'myriocarpa': Sl tata.

Morac. $\quad$ Ficus ?pilosa: WS maliti II; ES kalihi, kalìti, kalisi.

Morac. $\quad$ Ficus polyantha: $\mathrm{Rg}$ ?data; $\mathrm{Ng}$ data.

Morac. $\quad$ Ficus pubinervis: Ed kaju ai; ES harama njara; Sl numo I.

Morac. $\quad$ Ficus ?punctata: Rm lulu; Ed seri; Li tangi mura ro'a.

Morac. $\quad$ Ficus religiosa: WS wudu, ghudi, ?karimbyoyo; ES wudi, wudu.

Morac. Ficus rumphii: Bm wodi.

Morac. $\quad$ Ficus septica: FEM (To, Bé, Nn, Mb) kilit, (Ri) ghilor, (Ls, Ml) kilor; Kp sulit; Wr silor; Rz silor; Rm sulit, (Tr) sulin, (Wu, Wk) kilor; $\mathrm{Rg}$ sulé; $\mathrm{Ng}$ léo, sulé; $\mathrm{Na}$ ( $\mathrm{Tm}$, $\mathrm{Bw}$ ) sulé; Ed suré; Li sulé; WS kambùkela mene, kabùkela, kabùkelo, kambùkelo; ES kalambàki; Si ai hulér; Sl kehulo', hulé', kehulé.

Morac. $\quad$ Ficus subcordata: FEM nélo; Kp nélo; Wr purus; Rz burus; Rm nélo; Sl kenapa; Kd bèu.

Morac. $\quad$ Ficus subulata: Kp lékas; Rz lékas. 
Morac. $\quad$ Ficus (variegata): $\mathrm{Ng}$ ara, helo, reké; $\mathrm{Na}(\mathrm{Tm})$ ara; $\mathrm{Ed}$ ara; $\mathrm{Li}$ ara; $\mathrm{Si}$ 'ara; $\mathrm{S} 1$ kû, klawê II; Kd danung.

Morac. $\quad$ Ficus variegata var.: $\mathrm{Km}$ ara; FEM ara, (Ml, Ls) 'ara; Wr ara; $\mathrm{Rz}$ ara; $\mathrm{Rm}$ ara II, (Wu, Wk) 'ara; WS kandélu; ES kanjilu, kencalu, ka'anjailu, ka'jailu.

Morac. Ficus virens var. glabella: WS 'ghunuta, wunuto; ES wunutu, wundutu, 'wunuta.

Morac. $\quad$ Ficus ?wassa (cp. F. ampelas): Rg peri; $\mathrm{Ng}$ héga réa, nara héga, nara séga, séga, zi'o; Ed meru II; Li meru; Si peri; Kd èyè' I.

Morac. $\quad$ Ficus ?wassa var. obversifolia: FEM (To, Nn) boka kolo, (Ri, Ml, Ls) pelas; Kp pelan; Wr kazu pelan; Rz pelan; Rm pelan, ( $\mathrm{Tr}$ ) baka kolong, (Wk, Wu) pelas; WS pèdi; ES pèdi, pìdi.

Cyper. $\quad$ Fimbristylis sp.: Rm kembang kata.

Cyper. $\quad$ Fimbristylis miliacea: Sw hiha manu.

Cyper. $\quad$ Fimbristylis ovata: $\mathrm{Ng}$ fu lemu.

Asclep. $\quad$ Finlaysonia obovata: Sw jeru menanga.

Flacourt. Flacourtia (indica): Bm ntimu; $\mathrm{Km}$ timur, timus; FEM wuék; Wr mata wara; $\mathrm{Rm}$ wuék.

Flagel. $\quad$ Flagellaria indica: Bm kaua II; FEM kuar; Kp kuar; Wr kuar; Rz kuar; Rm (Tr, Wk, Wu) kuar; Rg kua; Ng kua I; Na (Tm) kua; Ed kua I; Li kua; Pl rab'i; WS wàwo, ghàgho, wùgha; ES nggai; Si kuar; Sl lua, lua'; Kd wadé' luar.

Lamiac. Foeniculum vulgare: ES wàla wunga.

Oleac.

Fraxinus griffithii: FEM (To, Nn, Ls) rerit, (Bé) wul, (Ls) lerit, mulu; Kp lui; Wr lui; $\mathrm{Rz}$ lui; $\mathrm{Rm}$ (Wk) mulu, (Tr) rerit, (Wu) lulu; $\mathrm{Ng}$ lui I; Ed ladu.

Pandan. $\quad$ Freycinetia: $\mathrm{Ng}$ hiké, hipa, midu; Li seli I.

Pandan. $\quad$ Freycinetia cf. insignis: FEM (Ri, To, Bé) réa, (Nn) ré'a; Kp réa kodé; Wr zé'a darat, zé'a kodé'; Rm zé'a lawo', zé'a pong, (Wk) zé'a, (Tr) réa pong, Rg zé'a nitu; $\mathrm{Ng}$ zé'a nitu; WS lùri I, ranu I; ES kìku mánu I; Sw lùri I, rànu I.

Pandan. $\quad$ Freycinetia scandens: FEM (To) lulu, (Bé) lulu waé; Kp mulu waé; Wr mulu waé; $\mathrm{Rz}$ mulu waé; Rm lulu waé, (Wu) lulu, (Wk) lulu'.

FUNGI Kp wua but; Wr wua' but; Rm but.

FUNGI Bm cacoro; Km cacoro; FEM wung kokak, (Bé) nung ta'i zarang, (Ls) ndéghing, nung mborong, numé, numé tala, numé tangis, (Bé, $\mathrm{Ri}$ ) wung, ( $\mathrm{Nn}, \mathrm{Mb}$ ) nung, nung ghang; wung mésé, $(\mathrm{Nn})$ nung mésé; $\mathrm{Kp}$ wua' lulang, wung tangis, wung kokak, wua tuak, wua' uran; Wr wua wung tangis; wua wung kokak; wua' uzan; Rz wung; Rm waé' nuzan, wua' nuzan; wung kokak; wung tangis; (Wk) dondor, wung dondor, rorat; $\mathrm{Rg}$ déngé ta'i jara, lando réndo II; $\mathrm{Ng}$ bhe'é, bu tana, deki, deki tua, duri kuru, gulé, gulé pau, haha hoé, hinga dhéké, hinga hongo, kato jara, kato kadhé, kato kodhé, kupé II, lado kobo, lebé denu, liko du'é, logo ngélu, logo ngéu, lua méo, mazo pubu, pura bheto, rano tua, rapu bheto, réri A, ruru, susu kaba, susu wawi II, ta'i ha II, ta'i jara, ta'i kaba, ta'i laja, ta'i su'a, tiro taro, toko kosu, turu 
FUNGI

FUNGI

FUNGI

FUNGI

FUNGI

FUNGI

FUNGI

FUNGI

FUNGI

FUNGI

FUNGI

FUNGI

FUNGI

FUNGI

FUNGI

FUNGI

FUNGI

FUNGI

FUNGI lulu, wajo péli, wié waé I, witu I; Na (Tm) bu tana, deghi, déghi ta'i jara, (Bw) ga suda, (Ra) ki'i; Ed kura; Li eri, fako, hongo, jala pébé II, jata, ?(uta) kengé, ?kenggé, ki'i, (uta) kongo, (uta) kula, mboko féo, ngeku ro'a II, nduru II, ndutu, tembu, tembu moké, tepé tana, wora bata, wora moké; WS kalia; kalia taiko karàmbo, ka'ripito tai ndara; minu we'e; karipita, karipico, karipico ma'rapu; ES kapuda; kakembalu, kariki; kúlu tana, karipitu I, karipatu; ka'hauki, kahaiku; jaü, ndaü, wulu ndaü I, manjailu; maloki, maloku; ta'nganangu; Sw hég'udu jara; Si bokak 'lon(g), bokak 'lihuk, kbokat, kbokat bura, kbokat méran, kbokat mitak, dudu, 'ré(h)i bokat, 'réi wawi, 'réi wi'ing, 'ré'i jaran, jarang 'réi, 'lon(g), koting, kowang, sabu rongo, jarang kowang; Sl kélu, kélur dang, klomé tilun I, klonuk, lengê, wuno matâ.

Kp lara'.

Kp wua' betong; Wr wua' betong.

Kp wua' tuak; Rz wua' tuak; Rm nggoro' kazu.

Kp wung; Wr wua' wung; Rz wung; Rm wung.

Wr lalir kata'.

Kp wua' mbora; Wr wua' mbora; $R m$ kora.

Kp wua' nggaré; Wr wua' nggaré; Rz lélé nggaré; Rm nggaré'.

Kp tawa ghé; FEM (To) nung, (Bé) nung tai zarang, (Ls, Nn) ndéghing; Wr tawa ghé; Rz tawa ghé; Rm kaka tawa; (Wk) kora', (Tr) nggaré.

Kp wua' ri'i; Wr wua' ri'i; $\mathrm{Rm}$ isi' ri'i.

Kp laso ular; Wr wiwir jaran.

(ground mushrooms): $\mathrm{Ng}$ bhu tana, fako + attributes; $\mathrm{Na}$ (Tm) bu tana, fako; Ed fako, hako, hako jara, hako tana, fako tana; Sl wuno mataâ.

(mould): $\mathrm{Ng}$ apu.

(Agaricales): Kp wung meros; Wr wua' meros; Rz meros; Rm wung mezos, ( $\mathrm{Tr}$ ) meros; $\mathrm{Ng}$ duri, duri kéri; Sl wata wetî.

(Auriculariaceae): Rg déngé ta'i jara; $\mathrm{Na}(\mathrm{Ra})$ ki'i; Si té'u tilu(ng); Sl dokal, ?kpukut/melak kingan, keromé tilun; Kd ?ulu' sapu.

(Daedalea palisoti): FEM (To) ndodor, (Bé, Ri) rorat, (Ls) tongol; $\mathrm{Rm}$ tongol.

(Gasteiomycetes): FEM ?monggot I; Kp I butul; Wr wua' mus; Rm busu jangang; $\mathrm{Na}(\mathrm{Tm})$ mogo.

(Hirneola): FEM tilu kendong, (Ri) tilu ngiung; $\mathrm{Kp}$ tilu ngiung; Wr tilu ngiung; Rz tilu ngiung; Rm kinga rok, (Tr) tilu ngiung, (Wk) mboko po; WS karipita; ES karipitu, ka'ripitu II.

(Lenzites): FEM (to) dondor ghang, (Ls) konggul, (Ml) gilok; Kp gilok; Wr gilok; $\mathrm{Rz}$ (wua') gilok; Rm gilok I, (Tr) dulok, (Wk) golok, $(\mathrm{Ft})$ girok.

(Microporus): Kp gilok tana; Rm gilok II, gilok tana. 
FUNGI ('Polyporus'): FEM (Bé, Ls) korak, (To) nung ghang, (Ls) numé, (Nn) nung; Rm (Wu) kora, (Wk) ndighing, (Tr) nung.

FUNGI (Pycnoporus): Km dangé haju; FEM jit, (Ml) zéngér, (Nn) ki'ik, (Ls, Mb) jik, zit; $\mathrm{Kp}$ déngér; Wr déngér; $\mathrm{Rz}$ déngér; $\mathrm{Rm}$ déngér, $(\mathrm{Wu})$ zengér, $(\mathrm{Tr}, \mathrm{Wk}) \mathrm{zit} ; \mathrm{Rg}$ déngé; $\mathrm{Ng}$ fitu + attributes; $\mathrm{Na}(\mathrm{Tm})$ ki'i, ki'i tua.

FUNGI (Pycnoporus sanguineus): FEM (Ri) jit wara, $(\mathrm{Mb})$ jik wara; Kp déngér nggaras; Wr déngér torong; $\mathrm{Rz}$ déngér torong; $\mathrm{Rm}$ déngér, déngér wara, $(\mathrm{Tr}, \mathrm{Wk})$ zit wara; $\mathrm{Rg}$ déngé toro; WS kalia rara, karipico maràpu.

FUNGI (Schizophyllum commune): Kp piri pipi; Wr pépi répi; $\mathrm{Rz}$ piri pipi; $\mathrm{Rm}$ piri pipi.

FUNGI (Xylaria): Kp wa'i ka; Rm wa'i ka', wa'ing ka'.

Aster.

Sapind. Ganophyllum falcatum: Bm sangi, ?karana isu.

Clusiac. $\quad$ Garcinia: Bm ?lembiara; katipu doro; FEM (Ml) wirok; Kp sirok; Wr sirok; Rz sirok; $\mathrm{Rm}(\mathrm{Tr}, \mathrm{Wu})$ wirok; Si buri wawi.

Clusiac. $\quad$ Garcinia (dulcis): Bm lalende; WS ka'niluka II; ES ?laru II.

Clusiac. Garcinia mangostana: Bm mangguta.

Rubiac. Gardenia: Rg mondo.

Burser. Garuga floribunda: Bm wiu; Km kedondo watu; FEM mondo; Kp mondo; Wr mondo; Rz mondo; Rm mondo; Li ?weru; Si ?weru (weru); Sl wiu, wo'o.

Aral. Gastonia papuana: Ng hepa éwa, hepa léwa II, lobo toro I, toro I.

Acanth. Gendarussa vulgaris: Bm gandarisa I; FEM (Ls) lanur; Kp lanur; Rm lanur waé, (Wk) nggilo waé'; Rg ?kaju laka; $\mathrm{Ng}$ lawi; Ed toko tenggo.

Logan. Geniostoma rupestre: Kp tepong ta'i; Rm kazu ta'i; WS 'taiko malawo.

cf. Gesneriaceae WS kapu ndala I.

Poac. $\quad$ ?Gigantochloa apus: Bm o'o; Km hau(s); FEM (To) aur, (Bé, Ri) gurung, (Ls, Nn, Ml) nggurung; Kp gurung; Wr gurung; Rz gurung; Rm aur, (Wk, Wu) gurung, (Tr) nggurung; Rg ngguru; $\mathrm{Ng}$ guru I; $\mathrm{Na}(\mathrm{Ra})$ gu; $\mathrm{Ed} \mathrm{au} ; \mathrm{Li}$ au; $\mathrm{Pl}$ 'ao; WS wo'o (pi'a), gho'o, onggalu; ES au, ou, ú; Sw kalaiwu wa; Si aur; sl au, au', uror; Ed 'aur.

Poac. $\quad$ Gigantochloa ?verticillata: Bm o'o ?kunci, o'o pa'i; Wr pering; Rm pering; Rg peri; $\mathrm{Ng}$ peri; $\mathrm{Na}(\mathrm{Ra})$ ?pezi; Ed peri; Li peri I; WS wo'o jawa; ES au jawa; Si peli II; Sl pen̂, peri; Kd béring.

Ulmac. Gironniera cuspidata: Li wua wulu.

Zingib. ?Globba marantina: FEM (Ml) lia acu, (To, Bé, Ri, Mb) lia asu, (Ls, Nn) lia wakar; $\mathrm{Kp}$ léa asu; Wr léa ka'o; $\mathrm{Rm}$ léa ka'o, (Wu) lia wakar, $(\mathrm{Ft}, \mathrm{Wk})$ léa wakar; $\mathrm{Rg}$ ?kiso; $\mathrm{Ng}$ léa lako; $\mathrm{Na}(\mathrm{Tm})$ léa lako; $\mathrm{Sl}$ kuma burâ.

Euphorb. Glochidion: FEM (To, Bé, Ls, Nn) rengit; Kp ngatol; Wr rewu; Rm paka dara', peké dara', (Tr) dara beka; WS kapia II; ES tambúlu. 
Euphorb. Glochidion angulaturn: WS kalàki kambe.

Euphorb. Glochidion glabrum: WS kambùkela, kandaü luku.

Euphorb. Glochidion (philippicum): Ng hobho II, lobo toro II, ngadu II; Na (Tm) keké; Ed ?lombo toro, ?rombo toro; Li longgo baja.

Papil. $\quad$ Glycine soja: Bm kadele, ?lawui; Wr koza kesang; Ng rori II; Li (bué-) nggodho; $\mathrm{Sl}$ kedelé, ?wéwé I.

Rutac. Glycosmis: WS ?lino, linu I, linyo; ES linu I.

Rutac. Glycosmis zippelii: Rg ngelo maju.

Verben. Gmelina asiatica: Bm kananga wuḅa.

Verben. $\quad G$ melina elliptica: FEM (To, Mb) rerit, (Ri, Bé) wul, (Ls, Nn) lerit; Rm (karot) wul, (Tr) lerit; $\mathrm{Rg}$ dingga; $\mathrm{Ng}$ dingga; $\mathrm{Na}(\mathrm{Tm})$ garo hanga.

Gnetac. Gnetum (gnemon): Rg suka; Ng suka; Ed ?suka; WS ùka, hugha; ES huwa, suwa; payeti.

Icac. Gomphandra mappioides: FEM (To) ghazu téu; Kp kaju tiu'; Wr kazu téu; Rm (Wk, Tr) (kazu) tiu, (Wu) kazu téu; Rg muku jawa; Ng tewu I.

Urtic. Gonostegia hirta: $\mathrm{Ng}$ ranga lala I.

Malvac. Gossypium: Bm wolo, wunta; FEM kampas; Kp kapas; Wr lélu; Rz lélu; Rm kampas, (Tr, Wu) lélu, (Wk) kapas; $\mathrm{Ng}$ bhena, lélu, lélu (késo); $\mathrm{Na}(\mathrm{Tm})$ rawi, (Ra) bhena; Li lélu; Pl kab'a; WS kamba I; ES kaba II, kamba I; Sw wengu I; Si kapa; SI kapek, kape lolon, kapes, kapek I; Kd apé.

Malvac. Gossypium: Wr lélu nipon; $\mathrm{Rg}$ lélu jawa, lélu nipo; $\mathrm{Ng}$ bhena nipo, lélu nipo.

Malvac. Gossypium hirsutum var. hirsutum: $\mathrm{Rg}$ lélu késo.

Acanth. Graptophyllum pictum (hortense): ES kakaha II.

Tiliac. Grewia: Bm rino wadu; FEM nila; Kp sono; Wr nilar, somo; Rz lumut; Rm (Wu, Tr, Wk) kazu nila; Rg soro; Ed junu; Li gunu, ?junu; WS langgétu II, langgítu, langgùtu; ES hamaili, langgoti; Sl kanila.

Tiliac. Grewia ?acuminata: Bm rinoai.

Tiliac. $\quad$ Grewia (eriocarpa): Bm ?rino; $\mathrm{Km}$ nila; $\mathrm{Rg}$ nila I; $\mathrm{Ng}$ nila; $\mathrm{Na}$ (Tm) nila; Ed nira; ES linu II; Si ?nila, ?nilo; Sl nilat; Kd nili.

Tiliac. Grewia 'salutaris': SI nila, kanila.

Rubiac. Guettarda speciosa: Ed béo ré'é.

Asclep. Gymnanthera: Km tali kemoné.

Cucurb. cf. Gymnopetalum: see ?Coccinia grandis.

Cucurb. Gymnopetalum cochinchinensis: $\mathrm{Rg}$ ghumbu; $\mathrm{Ng}$ papo I.

Aster. Gynura (grandifolia): Ed pombo; Li kigo ria.

Thymel. Gyrinops (cumingiana): Ed soké; ES homa, 'huama; wila I. 
Hernand. Gyrocarpus (americanus): Bm ?bara I; Km ?bara I; ES wila II; kalitu ahu; Sl buit.

Poac. Hackelochloa granularis: ES kóndu 'kurangu I.

Hydroch. Halophila ovalis: Km sawé tasi'.

Aral. $\quad$ Harmsiopanax aculeatus: Kp keré; Wr ngeré; Rz keré'; Rm padut pong; Ed kaju nio; Li nio.

Sapind. Harpullia: FEM (Bé, Ri) néngké, ( $\mathrm{Nn}, \mathrm{Mb}$ ) lelak; Rm néngké, (Wk) néké'.

Simar. $\quad$ Harrisonia brownii: Km mbela'; $\mathrm{Rg}$ mbarị; $\mathrm{Ng}$ basi; Sl pahadé.

Zingib. Hedychium coronarium: Bm arundae I.

Rubiac. Hedyotis: Sl bési kélét.

Rubiac. Hedyotis (corymbosa): $\mathrm{Ng}$ woja paké; Li lema manu; Sl wana muli'ing.

Proteac. Helicia: Rm lemos; Ed uwi réé II; ES tebu.

Stercul. Helicteres (isora): Km mboté', tangé; WS kapolého, kapoleho; ES kapúli, kapúlihu, ?púlihu; Sl lupâ; Kd lupang.

Borag. Heliotropium: Sl keruku I.

Borag. Heliotropium indicum: WS rábuka; ES mbúlungu kawuku.

Acanth. Hemigraphis: Rg hinga mota I.

Hepaticae: $\quad$ Rm lamun kazu, lamun watu.

Stercul.

Heritiera littoralis: ES ?'rurungu; Sl worungû, baner, regu.

Poac.

Heteropogon contortus: Rg nderé; Ed susu meti; WS buka bilara; ES wulu nggàduku I, wulu kalala; Sw kepoké; Si urun tebé; Sl ladoméra, ko; Kd 'uru paé.

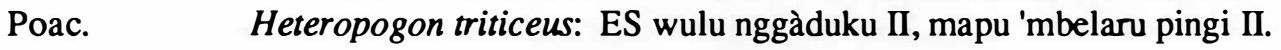

Asclep. Heterostemma acuminatum: Rg bé, li'é léwa.

Convolv. Hewittia sublobata: $\mathrm{Rg} \mathrm{ma}$; $\mathrm{Ng}$ gangé bhara; Li sé'é I.

Malvac. Hibiscus: Bm tampoli; ES waru; waru waka; Sl apu, kenoé; Kd ?wuhu I, ?mu'u lili', mébung II.

Malvac. Hibiscus rosae-sinensis: S1 kembang sepatu.

Malvac. Hibiscus sabdariffa: Rm rosila.

Malvac. Hibiscus surattensis: Km ngolo jong; Kp lebo keraéng; $\mathrm{Ng}$ kungu méo; Ed gosa gora, gora gosa; Es lúlu karara; Si gosa gora; Sl tuna ahane.

Malvac. Hibiscus tiliaceus: $\mathrm{Bm}$ wau; $\mathrm{Km}$ waru nanga; FEM (To, Ml) waru, (Bé, Ri, Ls, $\mathrm{Nn}, \mathrm{Mb}$ ) waso; $\mathrm{Kp}$ waru; Wr waru; $\mathrm{Rz}$ waru; $\mathrm{Rm}$ ( $\mathrm{Tr}, \mathrm{Wu}, \mathrm{Wk}$ ) waru; $\mathrm{Rg}$ waru; $\mathrm{Ng}$ azé watu I, waru I; Na (Tm) waru, (Ra) wau; Ed waru; Li waru; Pl wacu; WS kawàngo/u; ES kabaru, kawua, kawaü, kawawu; Sw hau, waru, wau; Si 'lago, waru; Sl wa'o, waror; Kd warun.

Malvac. Hibiscus vitifolius: Ed mbararoto, ?mbera roto, Sw wengu kod'é 
Euphorb. Homalanthus: ES ka'bébaku.

Euphorb. Homalanthus (peltatus): FEM (Ri) lenté; Kp leté; Wr leté'; Rz leté'; Rm lenté', (Tr) lenté, (Wk) leté'; Ed ngendi mité, nggendi; Li hebu, ?kabo, kebu I.

Euphorb. Homalanthus populneus: WS 'wolora.

Flacourt. Homalium tomentosum: Bm ndangu/a mada; FEM (Bé, Ri) ledu, (Ls, Nn, Ml, Mb) lendu; Kp ledu; Wr ledu; Rz ledu; Rm ledu, (Tr) lendu; Ng ledu; PI let'u; Si ledu; SI keledo, kledo', kalado; Kd lèlu, lèdu.

Arac. $\quad$ ?Homalomena: Km keladi napu; FEM (To, Bé, Mb) wira, (Nn) tombang, (Ri) wira waé; Kp wira' waé'; $\mathrm{Rm}$ (Wk, Wu) wira' waé, (Tr) wira; $\mathrm{Ng}$ ?toba.

Arac. Homalomena latifrons: ES hili manggaü, manggaï I.

Asclep. Hoya: Kp kupul; Ng rara; WS makèbela.

Violac. Hybanthus enneaspermus: Rg lando kata II.

Rubiac. $\quad H y d n o p h y t u m$ formicarum (cp. Myrmecodia): Si nitu ohun I?; SI ko'it III.

Hydrocharitaceae: Km satah tasi'.

Rubiac. Hymenodictyon: Wr kazu mbako; Rz kazu mbako; Rg mbako II.

Acanth. Hypoestes: Ed ingga II; WS ki'i II.

Acanth. Hypoestes malaccensis: Km baéh, baés.

Polyp. Hypolepis / Microlepis: Kp sewo kawar.

Cyper. Hypolytrum: WS ndei.

Lamiac. Hyptis: FEM (To) mbujung, (Bé) bozong, (Ri) tai zarang; Kp ta'i ela, ta'i jaran, merut manuk; Wr longa' bapu', longa' wau, ta'i wawi; Rz ta'i zaran; Rm bangka bu, malén manuk, ta'ing kobor, (Wk) ta'i zaran, ta'i zarang, (Tr) tai kobor, (Wu) ta'i wawi; WS taiko ndara, katúa duoda (ruoda), 'bubulo; ES taiko ndara, kapuwa hambaku.

Lamiac. Hyptis (brevipes): Kp ta'i zaran; $\mathrm{Rm}$ ta'i kobor; $\mathrm{Ng}$ isi toko bhara, rura kogha II, ta'i ha'é, ta'i wawi I.

Lamiac. Hyptis longipes: Ed toko mbonga I.

Lamiac. $\quad H y p t i s$ rhomboidea: Kp ta'i ela; Wr longa' bapu'; Rz ta'i zaran; Rg lenga wau II; $\mathrm{Ng}$ isi toko laka; Ed toko mbenga I; Li toko mbenga; Si mbako ma'an.

Lamiac. Hyptis suaveolens: Bm ?pata-ha mpori II; Km teringao; FEM (Ri, Bé) lindang poti, (Ls, Nn) kaput, (Mb) monggot II; Kp lasi' nggorang; lindang reghén; Wr kasi kaba; Rz kasi kaba; Rm lindang pezuk, (Tr) rewut wau, (Wk) tewon wau; Rg ta'i wawi, kasi kamba; $\mathrm{Ng}$ sewa rongo, wau II; $\mathrm{Na}(\mathrm{Bw})$ oa goa; Ed bobo ré'é, ruba ré'é; $\mathrm{Li}$ bu boré; WS kandángi, ?kadángi, ndàngi; ES kunu búti, kalawau, kamàru làngga; Sw kunu roté; Si klaté; Sl unu', unuj, kukung baru, segelai.

Apocyn. Ichnocarpus: $\mathrm{Ng}$ sui, sui koba II; $\mathrm{Na}$ (Tm) wewu; Ed komba nana; Sl klebo II. 
Apocyn. Ichnocarpus (cp. Chonemorpha): Bm kala mone; Km tai kelemoné; FEM (Tr, Bé, Ri) sewu, (Ls, Nn, Mb) wewu, (Ml) cewu; Kp sewu'; Wr sewu'; Rm (Wk, Wu) sewu', (Tr) sewu; Sw roro aj'u.

Apocyn. Ichnocarpus moluccanus: Ng gogo; Ed (komba-)nana.

Aquif.

Ilex: ES tebu bara.

Balsam. Impatiens (platypetala): Kd 'uru.

Poac. Imperata cylindrica var. major: $\mathrm{Bm}$ (mpori) ati; $\mathrm{Km}$ ki; FEM ri'i; Kp rii; Wr rii; $\mathrm{Rz}$ ri'i; Rm ri'i, (Tr) rii; Rg keri; Ng kéri, keri, ki; Na (Tm, Ra) ki; Ed ki; Li hi, ki; Pl ci'i; WS 'ngaingo, nginyo; ES witu, wicu, wucu, wusu; Sw rou widu; Si ri'i; SI lu'o, réar.

Papil. Indigofera: $\mathrm{Bm}$ dau; FEM tajung, (Ri) tao, (Ml) tarung, (Tr, Bé) tazung; Kp tao; Wr tao'; Rz tao'; Rm (Wu, Wk) tao'; Rg taru; Ng tao, taru; Na (Tm) taru, (Wu, Ra) tau; (Ké) talu; Ed taru; Li taro, taru; Pl d'acu; WS kanùbu, kanàbu; ES kamàla wóra, wuara, kámbu lawuara, wóra; Sw dao; Si tarun(g); Sl ta'û, ta'o, taong, taum, gemalo; Kd taung.

Papil. Indigofera linifolia: Km safi; Ed rema mori lo'o.

Papil. Inocarpus fagiferus: $\mathrm{Ng}$ ghaja, ka'é; Li ?nggaja; Sl gaé, gému.

Caesalp. Intsia bijuga: Bm fimpi; Si 'ipir; Sl ipis, ipij, ipi; Kd ipil.

Convolv. Ipomoea: Bm humpa langguru, kamboja II; Ed ho'a; Li loti, mbonggi; Sw kaba loro II.

Convolv. Ipomoea alba: FEM (Bé) ramut tesé; Kp lombong laé; Wr lamba laé, lombo laé; Rm lombong laé', (Tr) lombong, (Wk) kotok; Ng mako; Li ndora ndu'a.

Convolv. Ipomoea aquatica: $\mathrm{Bm}$ lara; FEM (To, Bé) kangkong, (Ri, $\mathrm{Ls}, \mathrm{Nn}, \mathrm{Ml}, \mathrm{Mb})$ kangkung; Kp kakung; Wr kangkung; Rz kakong; Rm kangkung, (Wk) kakung; $\mathrm{Ng}$ kako; Na (Tm) kako; Li nggako, séba nggako; ES tambúki, taboku, tamboku, tamboka, kapongge; Sw hajo kako; Si kangkong; SI kangkung; Kd kangkung.

Convolv. Ipomoea batatas: Bm uwi (gunu), uwi rama; Km tété; FEM (To) gulé romba, (Bé) ubi zawa, (Ls, Nn, Mb) ubi jawa, (Ri) gulé, (Ml) lué komba; Kp gulé; Wr dao kaké; Rm gulé', (Wk) gulé' komba; Rg dao (aré); $\mathrm{Ng}$ dhao, ranga I, tété; $\mathrm{Na}$ (Tm, Ra) uwi jawa; Li kisa I, ndora; Pl ndora; WS ròpu, katété, ràpu; ES katàbu, katàbi, katéti; Sw hiwu jawa; Si ohu jawar, tuka; Sl ué jawâ = ué', uwé jawâ, sawan, kewoit; Kd léu' sawa.

Convolv. Ipomoea (nil): $\mathrm{Rg}$ boro I; $\mathrm{Ng}$ foza; Ed mako; $\mathrm{Li}$ iju ra; $\mathrm{Si}$ tali bogin; $\mathrm{Sl}$ teburô II, kolo énga (I, II, III), ué' II, kojâ, wulang, romé tilu.

Convolv. Ipomoea obscura: Km tali landa; $\mathrm{Rg}$ tuma; Li komba monda; ES katàbi 'omangu; $\mathrm{SI}$ krome tilun, klomé tilû II.

Convolv. Ipomoea pes-caprae: Bm lamba nanga; $\mathrm{Ng}$ dhao zé'é, tété zé'é; Pl tala; ES (wua) ndia; Si holo loti, kolo loti; Sl keréra; Kd léra'.

Convolv. Ipomoea pes-tigridis: Ed uwi jawa ré'é; WS lólo kabóna I; ES ruwa kapu, (lúlu) karua kapu. 
Convolv. Ipomoea quamoclit: Sl keboja II.

Convolv. Ipomoea sepiaria: WS kali kúwa.

Convolv. Ipomoea triloba: $\mathrm{Ng}$ faré mengé; $\mathrm{ES}$ wàla katàbi.

Poac.

Isachne beneckii: FEM (Nn) cecér; Rm (Tr) sesor I.

Poac. Ischaemum muticum: Ng kuru bheto III; Ed ua ré'é.

Poac. Ischaemum timorense: ES apet.

Saxifr. Itea macrophylla: Kp pandang; Rm padang.

Rubiac. Ixora: Rg loko; WS malóko; ES kapalia.

Oleac. Jasminum sambac: Bm mundu; Sw bunga loro.

Euphorb. Jatropha curcas (cp. Ricinus communis): Bm katanga, tatanga II; Km kadung; FEM pandu pung, pandu tompong, (Ls) pandu mésé, ( $\mathrm{Nn}$ ) pandu, (Ml) pandur; Kp pandu topong; Wr kazu padut; Rz pandu waé'; Rm pandu tompong, (Tr) pandu kazu, (Wu) pandu wara, (Wk) pandu pa: Rg mbaru jawa; $\mathrm{Ng}$ goa, mbaru jawa, waru jawa I, waru wégé I; Na (Bw, Ra) padu goa; Ed ira; Li dama I, melu; WS kadàmo (pi'a), ?madòmo; ES padàmu humba; Sw lolo raé dou; Si damar jawa I, damar nilon; Sl padu jawâ, padu dima, padu wuré, kblung, kebelû; Kd padu butu.

Euphorb. Jatropha gossypifolia: FEM (To, Bé) pandu puung, (Ls) pandu mésé, (Ri) pandu tompong; Rm pandu tompong; Rg goa toro, waru wégé toro; Ed ira meru II; Li ila melu II; Pl b'at'u ra'a; Sw biko II, lolo wa; Si damar laka; Sl padu jawâ me'â.

Pedal. Josephinia imperatricis: Km lenga watu; Rg lenga wau I.

Zingib. $\quad K a e m p f e r i a:$ Kp sekur bakok; sekur wara; Wr sekur bakok; sekur torong; Rz sekur bakok; sekur torong; Rm sekur zat; sekur ka'o.

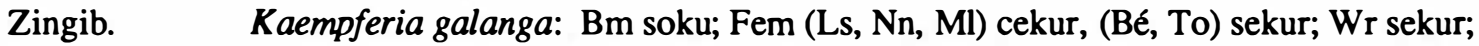
Rz sekur; Rm (Wu, Wk, Tr) sekur; Rg seku; Ng dodho bhara, dodho toro, seku; Na (Tm, Ra) seku; Li seku; Pl seku;WS òkura, hakuro; ES hahuru, cekir, sakura, sikuru; Si hekur; Sl seku, hekur, seku; Kd hé'ur.

Crassul. Kalanchoe integra: Rg kumba lebo, wunu kupu; $\mathrm{Ng}$ tewa I; Ed ta'i ra.

Crassul. ?Kalanchoe pinnata: ES maneni; Sl kerbau tilû.

Stercul. Kleinhovia hospita: Bm ntanga; $\mathrm{Km}$ ?ndangé; FEM dangé, (Ml) dhangé; Kp dangé; Wr kazu dangé'; Rz dangé; Rm (Tr) dangar, (Wu, Wk) dangé; Rg ndanga; $\mathrm{Ng}$ danga II, keda I; Na (Tm) danga; Li pu'u janga; Pl t'anga; WS ka'ndángara, ka(n)dángara, ka'dangar; ES anjángi, (n)jangi, nundangu, parau ramingu, wojangi, kanjangi; Si dagar; Sl kedanga, danga', kadanga; Kd angar.

Myrist. $\quad K n e m a$ (cinerea var. sumatrana): Kp ?laru; Wr ?laru; Rz laru; Rm laru, laru ko€; WS laru I; ES lari.

Cucurb. Lagenaria siceraria (var.): Bm ponda nono, ?ponda naru; $\mathrm{Km}$ ponda ndala; FEM (To) bonggo buang, (Bé, Ls) tawu, (Ri) tawu bonggo, tawu sewak, $(\mathrm{Nn})$ tawu léwé, (Bé, Ls, Nn) tawu bhuang, (Ls) bhuang; Kp ?tawu zawa, sewak; Wr tawu I, sewak; Rz sewak; Rm tawu sao, tawu bonggo, (Tr) tawu sewak, (Wk) sewak, 
bonggo; $\mathrm{Rg}$ tora, tawu; $\mathrm{Ng}$ boka, bhoka, kua II, kula, ngemé; kéna, kubu, tawu, toja; $\mathrm{Na}(\mathrm{Tm})$ có, toja, (Ra) kula; Li hénga, kénga; ?go salu; Pl 'obo cawa moku, d'awu; WS karòbo, karàbo; ES ?tambàla, tabàla, katíti, tambàla, tapusa, tapuha, karobu, karàbu; Sw deb 'a; d'eba ped'u; Si poté, tawu; Sl kela, kela', klewak, ?uré; belekâ, doto', ékot; Kd èdu, upa'.

Lythrac. $\quad$ Lagerstroemia flos-reginae: Bm rondu; FEM munting, (Ls) muntin; Kp muting; $\mathrm{Wr}$ muting; Rz munting; Rm (Tr) munting, (Wu) muntin, (Wk) mutin; Rg muti; $\mathrm{Ng}$ mudhi; Ed mundi; Li mundi; Si budi; Sl kenutî, nuting.

Lythrac. $\quad$ Lagerstroemia ovalifolia: Bm ndaru II; Km ndaru II.

Lamiaceae: $\quad \mathrm{Ng}$ foro II, hasi ka, hasi kaba, kasi beku, taka ora; Sw kunu wa; Si tana ra'ing I; SI kenoé.

Zingib. $\quad$ Languas galanga: Bm lao; FEM (Ls, Nn) laja, (Ri) laza, (Ml) laza'; Kp laja'; Wr léa laza; Rm (Wu) laza', léa laza', (Tr) laza; Rg léa laja; Ng laja, léa laja; $\mathrm{Na}$ (Tm, Bw, $\mathrm{Ra}$ ) laja; Li laja I, nato; WS lisa ruoda, liza rónda, lisa ráta, ligha rónda; ES languasu; Sw lakua; Si léa laja, laja; Sl laot, tomu!; Kd puyang.

Anac. Lannea coromandelica: Km kedondo; FEM kedondo; Kp kedondo; Wr kesi'; Rz kedondo; Rm (Wu, Wk, Tr) kedondo; Rg indo waé; $\mathrm{Ng}$ kesi II; Na (Tm, Ra) kesi; Ed kesi; Li kesi; Pl kacu sina; WS kàsi, kèzi, kazi; ES kahi II, kàhi jawa; Si réo, kepi; Sl réo, wulun barhon; Kd réo.

Meliac. Lansium: Bm garu.

Meliac. $\quad$ Lansium domesticum: Bm lasa; $\mathrm{Km}$ lasa; Sw lasa.

Verben. $\quad$ Lantana camara: Bm taride; kamboi; Kp kambui; Wr kawéng; Rz kawéng; Rm kawéng, (Tr) merdéka; Si kara munti, kurmunting, ?kota lari; Sl wana, koro boâ, unu wauk II.

Theac. $\quad$ Laplacea: Ed nggura.

Urtic.

Laportea: $\mathrm{Ng}$ adé II, ladé II, laté(-meré), paka do I; Li landé, landé wuja, laté.

Urtic.

Laportea ?aestuans: Si 'lada; Sl klatê waî.

Urtic. $\quad$ Laportea (forstenii) (cp. Dendrocnide): Bm héi; $\mathrm{Km}$ lanténg; FEM lantong, (Nn) laténg; Wr latong rébak; Rz latong kébak; $\mathrm{Rm}(\mathrm{Wu}, \mathrm{Tr})$ lantong, (Wk) latang.

Urtic. Laportea intermupta: Sl klatê.

Lauraceae: $\quad$ Li ?usu.

Lamiac.

Lavandula officinalis: ES lawanda.

Lythrac.

Lawsonia inemis: ES laka.

Polyp.

Lecanopteris carnosa: Rg lembé raé II; Ed sara.

Vitac.

Leea: Ed nipa nai.

Vitac.

Leea (angulata): $\mathrm{Km}$ tangga waja; $\mathrm{Kp}$ diru pong; $\mathrm{Rm}$ rabas; $\mathrm{Rg}$ karo mori, saru walu; Ed sara waru; Li ?gulu gua, ngguru waé, ngguru wa'i; WS timu tèke I, timbu tèke; ES ha'njokaru tàki; Sl kwiki, uru wadang, kewikit, kewik. 
Vitac. Leea (indica): Wr lutu'; Rz malir.

Vitac. $\quad$ Leearubra: Kp malir, Wr mali; Rz ?malir; Rm lalis manuk.

Leguminosae: $\quad \mathrm{Rg}$ séko kala; $\mathrm{Ng}$ sama séko II, sama wago; Ed hinga té'u, mata mité, ségo régo, wa'i ré'é; Li ndémbi, nggéndi, nggolu.

Acanth. Lepidagathis: Rg lawi; $\mathrm{Ng}$ bolé kogo I, bungé api III, figo boté, waru waé; Ed tepu I, wonga mata; WS langgapa lolo; kambúta tagho.

Acanth. $\quad$ cf. Lepidagathis: WS langgapa lolo; kambúta tagho.

Myrtac. Leptospermum flavescens: FEM liti; Kp kaju liti; Rm (Wk, Tr, Wu) liti.

Mimos. Leucaena leucocephala: Bm lamantoro, ?lémbantaro, kopi cina; FEM (To) tér, (Bé, $\mathrm{Ls)}$ kopi rewut, ( $\mathrm{Ri}, \mathrm{Nn}$ ) lamin toro; $\mathrm{Kp}$ lain toro; Wr gegé, lamén toro; $\mathrm{Rz}$ limén toro; $\mathrm{Rm}$ lamin toro, ( $\mathrm{Tr}, \mathrm{Wu}, \mathrm{Wk}, \mathrm{Ft}$ ) tér; $\mathrm{Rg}$ gegé; $\mathrm{Ng}$ gegé, lamu toro; $\mathrm{Na}(\mathrm{Tm})$ lamu toro, (Ra) lama toro; Ed bibi, gegé; Li gegé II; WS lantoro; ES kopi 'riutu, kopi nggangga, kopi rútu, lantoro, lamtoro; Sw hélag'i ara; Si lamêntoro; Sl lama toro, lamtoro, peté sina; Kd lama toro.

Lamiac. $\quad$ Leucas (flaccida): ES tandúla pua I; Kd puhun buya'.

Lamiac. Leucas javanica: Rm maza ngizung.

Urtic. $\quad$ Leucosyke ?capitellata: Bm ḅiru; FEM (Ls, Nn) reca, (To, Bé) resa, (Ri) rasi; Kp rasi kaju, kabé; Wr basi; $\mathrm{Rz}$ basi; $\mathrm{Rm}$ kazu rasi, (Wk) resa; $\mathrm{Rg}$ wio; $\mathrm{Ng}$ woi, zama witu; Li mbawu.

LICHENES: Kp lamu; Rz ramun; Rm lamun.

LICHENES: $\quad$ FEM (To, Bé, Nn) amuk watu, (Ri, Ls, Ml) lamung watu; Kp ramun watu; Wr ramun watu; $\mathrm{Rz}$ ramun watu; $\mathrm{Rm}$ (Wu, Wk) lamun watu, ( $\mathrm{Tr}$ ) lamung watu; $\mathrm{Ng}$ foa; $\mathrm{Na}(\mathrm{Tm})$ lamu watu; $\mathrm{Kd}$ malé.

LICHENES (Usnea): Km tai anging; FEM mumus poso, lambé ka, témbé ulas, témbé kodé, randak ghaju; Kp sago kodé'; Wr sago kodé'; Rz sago kaju; Rm sago nitu, (Tr, Wu) zanggok nitu, (Wk) mumus nitu; $\mathrm{Rg}$ sanggu sa; $\mathrm{Ng}$ tebé sa; $\mathrm{Na}(\mathrm{Tm})$ tébé ha; $\mathrm{Li}$ kumi lamu I, kumi lélé, kumi nitu; Sl angin taing.

Liliaceae: $\quad \mathrm{Ng}$ koto I, nio nitu.

Butom. Limnocharis flava: $\mathrm{Ng}$ talé wolo II.

Scroph. $\quad$ ?Lindernia: Li bewu, lepé kata.

Scroph. $\quad$ Lindernia (crustacea): Sw kaka lobo; Sl tana rak̂.

Oleac. $\quad$ Linociera: Li singgi mité; WS kaci katáro; ES kawìta kaba.

Rubiac. $\quad$ Litosanthes biflora: WS minu we'e.

Laurac. $\quad$ Litsea: $\mathrm{Bm}$ ntonu bura; $\mathrm{Kp}$ lowang; $\mathrm{Rm}$ kazu toko ikang, lowang; $\mathrm{Ng}$ haro beté, telo; Ed aré nana, kéu té'a, talo, unu aja, uru talo, waru jawa; Li ?aré nana.

Laurac. $\quad$ Litsea accedentoides: Bm konca doro, ?mpiu II; FEM (To, Bé, Ls) wunis, (Nn) kunis; Rm (Tr) kunis pong, kazu kunis, (Wu, Wk) kunis. 
Laurac. Litsea tomentosa: Li mara pau.

Laurac. Litsea velutina: $\mathrm{Ng}$ ?kuju, ?kuza; Ed uru waté; Li uru, uru waté; ES bakara wato, ?bakara wai, tambùra I.

Arec.

?Livistona rotundifolia: Bm sandonawa I.

Loranthaceae: FEM (To, Bé) tai tala, (Ri) taing tala, (Ls, Nn) ta'i tala, (Ml) ta'ing tala'; Kp ta'ing tala'; Wr ta'ing tala, ta'i tala; Rz ta'i tala'; Rm (Wu) ta'ing tala', (Wk) ta'i tala', (Tr) tai tala; $\mathrm{Rg}$ ta'i ndala; $\mathrm{Ng}$ moro dala; $\mathrm{Na}(\mathrm{Tm})$ ta'i dala; Ed ndombo ndo'o, WS wu kahìi; madùbu, mandùbu; Sw d’adi lai; Si wero pléan.

Onagr. $\quad$ cf. Ludwigia: Rg kumba sawa I; $\mathrm{Ng}$ kuza II.

Onagr. $\quad$ Ludwigia octovalvis: $\mathrm{Km}$ anting-anting; $\mathrm{Kp}$ ratun waé.

Cucurb. $\quad$ Luffa acutangula: $\mathrm{Bm}$ ?patola, koropa'i; Kp réwak; Wr tawu réwa; $\mathrm{Rz}$ tawu réwa; $\mathrm{Ng}$ sao II; Li go salu, pepua; WS karere kóro, koru pòdu, kóru; Sw patola; Si kamodo, tawu 'lewén; Sl kedola I, wulû tolâ, modok; Kd ara bia 'otan.

Cucurb. Luffa aegyptiaca: Bm ponda ndali; Kp ruwuk; Rg héa; $\mathrm{Ng}$ boda, lu lima II, lulu lima; Ed boda; Li peri ula, perula, peta pepua, tola; WS karòbo pùti, kalaho ?uti; ES ?patola; Sw wo pitola, tola; Si patola, pantola, sola; SI kedola II, modok II; Kd arbia, ara bia.

Combr. Lumnitzera (littorea): Sw biko II.

Combr. Lumnitzera racemosa: Km rumput atu.

Rutac. Lunasia ?amara: Rg néta mbatu; WS lagápa.

Solan. $\quad$ ?Lycianthes banahaensis: Ng mbenga, pesu benga; Sl néhé wauk II.

Solan. $\quad$ Lycianthes leve: Ed reri.

Solan. Lycopersicon lycopersicum (small fruit): $\mathrm{Bm}$ (kadui) paranggi; $\mathrm{Km}$ perenggi, peringgi; FEM (To, Nn) tagha lai, (Bé, Ri, Mb) taga lai, (Ls) taga lais, (Ml) tagha laé; Kp mbarang ko€́; Wr mbarang kó́; Rm mbarang loé', mbarang tuang, tomat, ( $\mathrm{Tr}, \mathrm{Wu}, \mathrm{Wk}$ ) tangga lai; $\mathrm{Rg}$ mbara woni; $\mathrm{Ng}$ taga lai I, taga lai kedhi; $\mathrm{Na}(\mathrm{Tm})$ tagha lai dhiki, (Bw) taga lai; Ed daga lai dhiki; Li dege lai; Pl daga lai; WS toro dánggalasa, danggalaza, toro manggalawa, toro lae, manggalawa I; ES anggalaï; Sw wo maka balé; Si daga lai; Sl kematir, dagalaing, sagalai; Kd sili' butu.

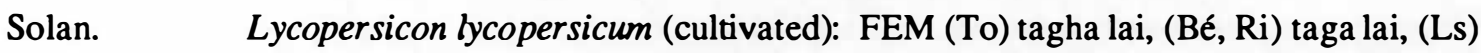
taga lais; Kp mbarang mézé; Wr mbarang mézé; Rz mbarang; Rm mbarang mézé'; Rg mbara woni; Ng bara II, bara lai, taga laé, taga lai goa, taga lai mézé'; Ed daga lai méré; Li dege lai; Pl daga lai; Si daga lai(s); Sl kemati, daga laing bélê; Kd sakalai.

Lycop. Lycopodium: Sl keléla.

Lycop. $\quad$ Lycopodium cernuum: $\mathrm{Rm}$ pora; $\mathrm{Ng}$ kanga paké I, kungu paké II, kungu ro'a; Li kuku mbeku II, kumi rusa II; WS kata I; ES kata I, káta I.

Lycop. $\quad$ Lycopodium phlegmaria: Rm luzu lénggong.

Schizae. $\quad$ cf. Lygodium: Kp rekot; Wr lerot; $\mathrm{Rm}$ rekot. 
Schizae. $\quad$ Lygodium ?circinnatum: FEM (To) cuba, (Bé) midu, (Nn) nido, (Ml) midhu', (Mb) nedho; Kp mindu'; Wr mindu'; Rz mindu'; Rm mintu', (Tr) nintu, werok, (Wu) midu, (Wk) selir; $\mathrm{Rg}$ mindu; $\mathrm{Ng}$ midho, ngido, nidho, nidu; $\mathrm{Na}$ (Tm) nidho; $\mathrm{Li}$ ngidho; WS káta II, kata; ES káta II; Sl nidu'.

Schizae. $\quad$ Lygodium (japonicum): Ed ngidho ré'é; Sl weridu.

Schizae. Lygodium microphyllum: WS kata lolo, lataho.

Caesalp. Lysiphyllum binatum: Km daléng tasi'.

Lythraceae: $\quad$ Bm kapanca; Ed laka toba; ES laka; Sw laka.

Euphorb. $\quad$ cf. Macaranga: WS mandonu II; ES tàda ka'pihaku; kandanu II, madinu, kandinu II.

Euphorb. $\quad$ Macaranga tanarius: FEM noa, (Ri) kébak; Wr rébak; Rz kébak; Rm kébak; Rg remba; $\mathrm{Ng}$ ?éko lako I, rebha; Ed wura; Li teru, wuja; WS kubùkela manu; ES kandinu bara; Si wuré; Sl kerewak, ferak.

Morac. Maclura cochinchinensis: FEM (Bé, Ls, Nn, Mb) nggulung, (Ri) nggulun, (Ml) gulung; Kp gulun; Wr gulun; Rz gulun; Rm gulun; Rg nggulu; $\mathrm{Ng}$ diga, gulu I; Li gulu béwa; WS idu; ES iju; Sl dulo kumang.

Sapot. $\quad$ Madhuca (malaccensis): Si tutur, waroting; Sl kuma léu.

Myrsin. Maesa: WS kabáli biedu.

Magnol. $\quad$ Magnolia glauca: Kp lumu; Wr lumu; Rz lumu; Rm lumu; Ng lumu I.

Euphorb. ?Mallotus: Ng po II; Sl wéka béhikê.

Euphorb. Mallotus moritzianus: Sw pesesa.

Euphorb. Mallotus ?philippensis: FEM pusor, (Nn) pusér; Kp pijor; Wr pizor; Rz pizor; Rm pizor, ( $\mathrm{Tr}$ ) pusor, (Wu) puzor, (Wk) puzar; $\mathrm{Rg}$ puré; $\mathrm{Ng}$ manu, polé, puré; Li la; WS wulu manu I, ghulu manu; ES kamàla kamba; SI kedo'ok, maku'.

Euphorb. Mallotus ricinoides: Kp kaso; Wr kaso; Rz kaso; Rm kaso'; Rg ?kaso fai; $\mathrm{Ng}$ éko lako II, po I, reba; Ed remé, tutu po; Li wonga londa I; WS kiku bòngga marapu, kiku bòngga; Sl lité.

Malvaceae: $\quad$ Ng saka kélé; Ed kamba mbonggi II; Li lélu ré'é; WS kadóke tana.

Clusiac. Mammea timorensis: WS kadoké tana; Si tour II.

Anac. Mangifera: $\mathrm{Rg}$ pau nengi; $\mathrm{Ng}$ pau ro'a; Ed pau katé; Li koto koto, pau koto, pau ndu'a; Si pau ?ta'a.

Anac. $\quad$ Mangifera indica: Bm fo'o; Km pau; FEM pau; Kp pau'; Wr pau; Rz pau'; Rm pau'; $\mathrm{Rg}$ pau; $\mathrm{Ng}$ pau; $\mathrm{Na}(\mathrm{Tm})$ pau; Ed pau; Li pau; Pl b'au; WS ùpo, pou, poyo; ES pau, po; $\mathrm{Sw}$ wo pau; Si pau; Sl pao, paor; Kd pèu.

Anac. $\quad M a n g i f e r a$ (longipes): $\mathrm{Bm}$ fo'o ?doro; Kp pau' watu; Wr pau' pong; Rm pau' pong; WS upo rúta; ES pau 'omangu; Si pao; Kd pèu oha'.

Anac. Mangifera timorensis: Bm fo'o ?wuḅa; Ed pau rẹu. 
Euphorb. Manihot esculenta: Bm uwi haju, daem se; Km bojo; FEM (Bé, Nn, Mb) uwi ghaju, (Ri) uwi ghazu, (Ls) 'uwi kazu, (Ml) lué ghasu; Kp uwi kazu; Wr dao kazu; Rz uwi kazu; Rm uwi kazu, (Tr, Wu, Wk) gulé(') kazu; Rg dao mboa, uwi mboa; Ng uwi jawa; Na (Tm) uwi kaju, (Ra) uwi ai, uwi lo; Ed uwi kaju; Li uwi boa, uwi kaju; Pl 'uwi kacu; WS luwa wasu, luwa ghazu, lugha dawa; ES luwa ai, lùwa ia, lua ai; Sw wéi képaka, éi képaka; Si 'ai 'ohu, ohu ai; Sl ué' kajo, ué' III, uwé kajo, sura kajor, uwé karo; Kd au wua', alèwu'.

Euphorb. Manihot glazovii: Sl geta.

Sapot. Manilkara achras: Bm sawo.

Sapot. $\quad$ Manilkara kauki: Bm sawo kala; Km natu.

Urtic. $\quad$ Maoutia: Kp walék; $\mathrm{Rz}$ walék; $\mathrm{Rm}$ walék; $\mathrm{Ng}$ lélé I; $\mathrm{Na}(\mathrm{Tm})$ feba; Ed mbawu; Li mbawu noko.

Marant. Maranta arundinacea: Kd aléwu' tuang.

Asclep. Marsdenia tinctoria: $\mathrm{Rg}$ wéra; $\mathrm{Ng}$ nodé.

Asclep. $\quad$ cf. Marsdenia villosa: ES kamba kadu.

Pedal. ?Martynia annua: Rg ?mbungé; WS ku'u wódo, ku'u ghódo; ES kàriku (hu), ngàndu kàriku; Sw kaé ku'u.

Cornac. Mastixia rostrata: Bw tapa seké; Ed ra'u II, sié, tapa seké.

Melast. ?Medinilla: WS meli káka.

Myrtac. Melaleuca leucadendra: WS àlango; ES kayu puti, yàlangu, àlongu.

Euphorb. Melanolepis multiglandulosa: $\mathrm{Km}$ wajuh; $\mathrm{Kp}$ léré; $\mathrm{Wr}$ rewa; $\mathrm{Rz}$ rewa; $\mathrm{Rm}$ rewa; $\mathrm{Rg}$ rewa; $\mathrm{Ng}$ rewa; Ed ewa; Li lemé; WS rowa I, karara, marapu; ES 'uowa, owa; Si lewa; Sl lewa'.

Melast. Melastoma: Kp dusuk; Wr pu'u dusuk; Rz rangat; Rm rangat II, rangat pada', (Wk) runu rangat; $\mathrm{Rg}$ ndusu; $\mathrm{Ng}$ jéko, moro bétu I, saka, saka kaju, butu; WS benda kada yìbi, katilu yibi, rindi ghogho II; ES ghasu ghula.

Meliac. $\quad$ Melia azedarach: Bm menga; Km mera; FEM mbera; Kp mera; Wr mera; Rz mera; Rm mera'; Rg mbera; $\mathrm{Ng}$ lowa I; $\mathrm{Na}(\mathrm{Tm})$ ba; Ed mbera; Li bera; WS làme, lomo, ?lème; ES lamua; Si berang; $\mathrm{Sl}$ manuk ra'an; Kd klaét I, belilé.

Meliaceae: $\quad$ FEM (Bé, Ri, Nn) raok (cp. Artocarpus elasticus): Kp raok; Rm raok.

Sabiac. Meliosma (ferruginea): Ed mboja, tanggo, tawu; Li kapok kéli, nuja tewu.

Stercul. Melochia corchorifolia: ES kawindu.

Stercul. $\quad$ Melochia cf. umbellata: FEM teno; Kp teno; Wr teno; Rz teno; Rm teno; Rg ndenu; $\mathrm{Ng}$ dhenu, denu, ko'u; Na (Tm) denu; Ed dhenu, denu; Li denu; Pl 'enu; WS mandònu I, manduyo; ES kandinu mítingu, kandànu I, kandínu I; Si denu; Sl feno.

Cucurb. Melothria: FEM (Nn, Bé) timus, (Ml) timung ka; Kp karong ka'; Wr mbarang ka; Rm karong ka', timun karong ka', (Tr) timung ka, (Wk) boang ka'. 
Cucurb. Melothria (perpusilla): Ng duré éjo, kala boba, kobho, pera; $\mathrm{Na}(\mathrm{Bw})$ papo; Ed rẹi I; Li ndimu ndolo I.

Melast. Memecylon: Bm samela.

Menispermaceae: Li moro nipa; WS kùru kára I.

Convolv. Merremia emarginata: Sw kelawa rai.

Convolv. Merremia tridentata: Sw kajepé.

Convolv. Merremia umbellata: Bm uwi jara; Ed sepi muku.

Convolv. Merremia (vitifolia): FEM (To) wasé' moko', (Bé) wejang, (Ri) ozong mat, (Nn) komba, (Mb) wanéng; $\mathrm{Kp}$ wazé mat II; Wr kazék bozot; Rm wazé' mat, (Tr) mat wozang; $\mathrm{Rg}$ boṛo II; $\mathrm{Ng}$ faré mengé; $\mathrm{Na}$ (Tm) pojo nga'o; ES karakápu.

Caesalp. Mezoneuron cucullatum: FEM (Bé) nampi, (Ri, Mb) ghili ghi, (Ls) taor, (Nn) saru; $\mathrm{Kp}$ ndatar; Wr ndatar; $\mathrm{Rz}$ ndatar; $\mathrm{Rm}$ ghili ghi.

Magnol. Michelia champaca: Sw hépaka.

Tiliac. Microcos: Rm nila.

Tiliac. Microcos paniculata: $\mathrm{Km}$ soro; Rg nila II.

Rutac. Micromelum hirsutum: Ed ndawa II.

Rutac. Micromelum minutum: Rg neti lélu.

Aster. $\quad$ Mikania: $\mathrm{Kp}$ mat; $\mathrm{Wr}$ ?kazék wulan; Rm wazé' wulan.

Aster. Mikania (cordata): $\mathrm{Ng}$ foja.

Bignon. Millingtonia hortensis: Bm tangga; ES ka'tanggaru; Si 'ai bunga.

Mimos. Mimosa invisa: Bm putri malu; FEM (Bé, Ri, Ls, Nn) bibit, (Ml) bhibhit, (Mb) putri malu; Kp ndango rang; Wr ringot; Rz ringot; $\mathrm{Rm}$ (karot) kia'n, (Tr) kia kodong, (Wk) bibit; Rg ringo; $\mathrm{Ng}$ gulu II, kaba, méa, mé'a I; $\mathrm{Na}(\mathrm{Tm})$ lema tuna, (Ra) séma; Ed bunga malu, mimosa; Li degu lengu, mimosa; Pl putri malu; WS tara maròmba, tara maràmba, tara kàha; Sw kobo deré II; Si bunga méang, oho ai, wa'an bain; Sl bemia, ai baî, bunga kerama'; Kd putri malu.

Mimos. Mimosa pigra: ES rí kowa.

Sapot. $\quad$ Mimusops elengi: Bm arunana, tanju; Ed wunu butu; Sl warikit, ?werekèt.

Nyctag. Mirabilis jalapa: Bm bila wau; Si bila 'wau.

Sapind. $\quad$ Mischocarpus: Kp té'ér; Wr té'ér; Rz té'ér; Rm (kazu) sawar II.

Sapind. $\quad$ Mischocarpus sundaicus: FEM (Ls) cimpar, (Ml) cipar; Kp sipar; Rm sipar; $\mathrm{Ng}$ ghé'é.

Logan. Mitrasacme pygmaea var. malaccensis: ES kareha lúku.

Papil. Moghania: Ed kéra, kubi dhéké II, loko féa; WS ka'pelato.

Papil. Moghania (lineata): Rg lepi I.

Mollug. Mollugo pentaphylla: WS ma'ranaka. 
Cucurb. Momordica charantia: Bm paria; Km pai'; FEM (Bé) ampa réa, (Ri) réa, (Ls) panggat, $(\mathrm{Nn})$ nambat, $(\mathrm{Ml})$ péa, $(\mathrm{Mb})$ peréa; $\mathrm{Kp}$ réa; Wr péa; $\mathrm{Rz}$ réa; $\mathrm{Rm}$ pia, $(\mathrm{Tr}$, Wu) péa, (Wk) sapé réa; $\mathrm{Rg}$ péa; $\mathrm{Ng}$ paga, paga péa, péa, peria, timu ba'i; $\mathrm{Na}(\mathrm{Tm})$ paga, (Ra) 'iga; Ed ?réba; Li léba; WS kadíra, pandéra, kapàdu; ES ri 'paita, ?kapu ndala, pandéra; Sw péringi éi; Si léba, ?blé'on, kela mé'on; Sl peria, léba', teria; Kd aparia.

Ponted. Monochoria vaginalis: Bm ?buka(ng); Li séba aé; ES hahilu wúya.

Aster. ?Moonia moluccana: Ed ingga I; Li bora.

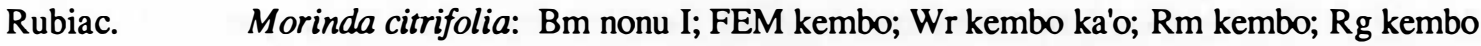
lako; Pl kepo; ES ?nggoko; Si kebo; Sl keroré, keroré' kloré, klorér; Kd loré, botan loré, bo'ol botan.

Rubiac. $\quad M o r i n d a$ tomentosa: Km nggudu; Kp kembo; Wr kembo; Rz kembo; Rg kembo; $\mathrm{Ng}$ kebo; Na (Tm, Ra) kebo; Ed kembo; Li kembo; WS kómbo; ES kombu, kàmbu I, kobu; Sw keb'o II; Si buké; Sl kebuké, kebuko, kebuke', wahér.

Moring. Moringa pterygosperma: Bm paronggé; Km peronggé, kélor; FEM (Bé, Ri) roé, (Ri) kélor, (Ls, Nn, Ml, Mb) ro'é; Kp kélor; Wr kazu wonga; Rm kélor, (Tr) kilor, (Wu) ro'é; $\mathrm{Rg}$ wona, wonga; $\mathrm{Ng}$ wona, wonga; $\mathrm{Na}(\mathrm{Tm}, \mathrm{Ra})$ wona; Ed wona; $\mathrm{Li}$ wona; Pl kacu wau; WS kawóna, kaghóna, marunggu; ES ka'woana, ka'wuana, ?marunggi, mawona, kélor, (ka)wóna; Sw wona, maronggé; Si motong; Sl motô, motong I, moltong; Kd motong.

Morac. $\quad$ Morus australis: Wr teno tuang; $\mathrm{Rm}$ pesar; $\mathrm{Ng}$ hapa (jawa), jawa III, waru jawa; $\mathrm{Li}$ mora-a, mura-a; WS molas; ES moras; Sw serbéi; Sl moras.

Papil. $\quad$ Mucuna a): FEM (Bé) leba kowé, (Ls) leba wura, (Nn) leba bhoru, (Mb) segé; $\mathrm{Rm}$ leba' wura.

Papil. $\quad$ Mucuna b): Km ?ajo; FEM (Bé) kékos, (Ml) telo rangga, (Mb) lenggang; Kp konggang; Wr konggang kazék; Rz konggang; Rm konggang, konggang bakok, konggang mitong, (Tr) kékos, (Wk) kogang; Ng léké nitu, léké salo; Sl kakô bolo'; Kd inar II.

Papil. Mucuna acuminata: Ed mosa.

Papil. $\quad$ Mucuna pruriens f. pruriens: Bm ?handa; FEM ndétok; Kp této'; Wr této'; Rz této'; $\mathrm{Rm}$ této; $\mathrm{Rg}$ ndéto; $\mathrm{Ng}$ déto, wago I; $\mathrm{Na}(\mathrm{Tm})$ déto; $\mathrm{Ed}$ ndéto; $\mathrm{Li}$ ndéto, wonga ndéto; Pl tau; WS ode, onde; ES lìpi pana, úndi, ?undi pana, onde, odi, ondi, úndu; Sw winga lé edu, (wo) lé edu; Si 'lawé; Sl keda', keda, kedar; Kd inar I.

Papil. Mucuna pruriens f. utilis: Wr kundung; Rm kuas; $\mathrm{Ng}$ lipé; Li bué ria, bué rumbi, lipé; Pl 'ib'é; Ws lìpé, lupyo, lùpe II; ES lipu, lupi; Sw lé; Si ?péku; Sl uta ipar, bengo', ipaj, ipa; Kd oma'.

Cucurb. $\quad$ Mukia maderaspatana: Sw timu taka.

Rutac. $\quad$ Murraya paniculata: Bm kamuni; Km kemuning; FEM (Bé, Ri, Ls Nn) kemuning; Kp kemuning; Wr kemuning; Rz kemuning; Rm kemuning; Rg kemuni; $\mathrm{Na} \mathrm{(Tm)}$ muni; WS karìri, karìzi, kariho; ES kahàbaru, karisi, kahabaru; Sl telir I. 
Musac. $\quad$ ?Musa: $\mathrm{Ng}$ ladu; Li éwu; $\mathrm{Kd} \mathrm{mu'u} \mathrm{'ipu;} \mathrm{Bm} \mathrm{kalo} \mathrm{goa.}$

Musac. $\quad$ Musa cf. acuminata: Kp réndok; Wr réndok; Rz réndok; Rm laran; Li muku penggi, penggi; Pl muku b'egi; Si popot, popo II; Sl popo II.

Musac. $\quad$ Musa paradisiaca: Bm kalo; Km kalo; FEM (Bé, Ri, Nn, Mb) muku; Kp muku; Wr muku; Rz muku; Rm muku; Rg muku; Ng muku; Na (Tm, Ra) muku, (Wd) ka ngeta; Ed muku; Li muku; Pl muku; WS kalowo, kalógho; ES kalú, kalailu I, muku, kàli, kalowu; Sw mu'u; Si muku; Sl muko, mukor; Kd mu'u.

MUSCI: $\quad$ Kp ramun (watu); Wr ramun; $\mathrm{Rz}$ ramun; $\mathrm{Rm}$ lamun (watu); $\mathrm{Ng}$ lumu II, lumu méo; $\mathrm{Na}(\mathrm{Ra})$ lamu; Ed ramu; Li kumi lamu I, lamu ka'u; ES ?kangurutu II; Si tana ra'in(g) II; Sl kulat II (Ind.).

Rubiac. $\quad M$ Mussaenda frondosa: Kp suwul; Wr suwul; Rz suwul; Rm suwul; Rg wonga bara; Ng suwo, ?suwu, wonga bhara I; Ed wonga bhara I; ES mata manu; Si puhu bura; Kd tara' méhil.

Myrist. Myristica fatua: Si solo.

Myrist. $\quad$ Myristica fragrans: Bm kapala; ES magalaga, manggalawa III; Sw manggalawa III; Si pala; Sl kéam batu.

Myrist. $\quad$ Myristica (teysmanni): Wr laru; Rm laru mézé'; Rg laru; Ng gajo kéli; Ed kabo.

Rubiac. $\quad$ Myrmecodia: WS katowa kedu II.

Rubiac.

Myrmecodia armata (cp. Hydnophytum formicarum): Si ?nitu 'ohung.

Myrsinaceae: Li taga kata, taga manu.

Brass. Nasturtium: WS rágata.

Brass. $\quad$ Nasturtium officinale: Li lada.

Rubiac. $\quad$ Nauclea: Bm langge maju; Km konca maju; Si 'ai telo; SI lo'o.

Rubiac. $\quad$ Nauclea coadunata: Bm konca I; Km ?konca; FEM (Bé) wunis, (Ri) kunis, (Ls) wunis, (Nn, Mb) ghaju kunis (cp. Alangium villosum); Kp kazu wunis; Wr kazu wunis; Rz kazu wunis; Rm (kazu) kunis II; Na (Tm) bheka.

Rubiac. $\quad$ Nauclea orientalis: Sl keloré.

Nymph. Nelumbo nucifera: Bm tonjo.

Laurac. $\quad$ Neolitsea: $\mathrm{Km}$ mengih; $\mathrm{Rg}$ ndinga lako; $\mathrm{Ng}$ manu II; WS 'yawila, 'yághila; ES hamui, samot, yawilu, (kalìta wásu), (kaìta ghazu), 'yawila, kandoro.

Rubiac. $\quad$ Neonauclea calycina: $\mathrm{Bm}$ pato II; Kp paté; Wr paté(k); Rz paté; $\mathrm{Rm}$ panté; $\mathrm{Rg}$ paté; Ed bapa; Si balu, wekat nalu; Sl ?keloré.

Rubiac. $\quad$ Neonauclea excelsa: Bm (n)timu II; Km (n)timu II; ES tùmbu dàba.

Rubiac. Neonauclea lanceolata: ES wìt 'wundungu.

Sapind. $\quad$ Nephelium lappaceum: Bm rambuta.

Polyp. $\quad$ Nephrolepis biserrata: Ed paku téki I.

Polyp. $\quad$ Nephrolepis (hirsutula): Ng hedé, kabi jata, lebé biza I, lebé raé I; Ed paku IV. 
Polyp. $\quad$ Nephrolepis radicans: Sl wulung waing II.

Poac.

Neyraudia arundinacea: Ed éko méo I.

Solan.

Nicotiana tabacum: Bm tambaku; Km mbako; FEM mbako; Kp mbako; Wr mbako; Rm mbako; Rg mbako; Ng bako; Na (Tm, Ra) bako; Li bako, mbako; Pl mbako; WS baku, mbaku; ES hambaku, hambeu, tambaku; Sw na'i; Si bako; Sl kebako, bako, kabako; Kd bako.

Arec. $\quad$ Nypa fruticans: Bm nipa; Km nipa; ES nipa, ?libra.

Lamiac. $\quad$ Ocimum: Bm pataha; Km pataha; FEM (Mb) lasi mama; $\mathrm{Ng}$ hasi I, hasi beku I, kasi; Li faté; Sw meda'a bela, meda'a kemu; Sl keruku II.

Lamiac. Ocimum ?basilicum: Bm pataha mengi; pataha londe; Km kelasi, lasi; FEM (To) lasi bengé', (Bé) lasi orang, (Ri) lasi wuwur, (Ls) laci, (Nn) laci tolok, (Ml) kaci ting, (Mb) lasi; Kp lasi' bengé; Wr kasi belang; Rz kasi belang; Rm kasi bengé', (Wk) ?kasi wiang, (Tr) kasi ghang; Rg kasi waé; $\mathrm{Ng}$ foro I, hasi, kasi; $\mathrm{Na} \mathrm{(Tm)} \mathrm{hasi,} \mathrm{hasi}$ jara, (Bw) hasi mesi, (Ra) 'asi; Li faté mela; Pl la(hi); WS mandawa, mandagha, ndagha; ES kandangu hàmu, kandnagu II; Sw meda'a duku; Si klahé, klahi; Sl ketaka I, taka', ketaka be'ê; Kd awa ta'a.

Lamiac. Ocimum gratissimum: $\mathrm{Ng}$ hasi jara.

Lamiac. Ocimum ?sanctum: Sl ketaka II.

Lamiac. Ocimum cf. tenuiflorum: $\mathrm{Km}$ kelasi mama.

Menisp. $\quad$ cf. Omphalea: ES móro II.

Convolv. Operculina turpethum: ES katàbi mondu; SI keboja I.

Ophiogl. Ophioglossum pendulum: FEM lawé lénggong; $\mathrm{Kp}$ luju lénggong; $\mathrm{Wr}$ siar; $\mathrm{Rm}$ lawé lénggong; $\mathrm{Ng}$ lai léwa.

Opil. Opilia: WS kamàra wèzi II.

Poac. Oplismenus compositus (cp. Isachne beneckii): Bm penggampa; FEM (Bé) sosor, (Ls) cecor, (Nn) cecér, (Ml) mungkis; Kp rembun totok; Rm sosor II, (Wu) ?mungkis, (Wk) ?kuri'; Ng beza; Li sawu I; ES karìtaku I, ùhu mangìnu II.

Cactac. Opuntia elatior: Bm rui ntangu: FEM (To) karot éndé, (Bé, Ri) tanggo éndé, (Ls, $\mathrm{Nn}, \mathrm{Mb}$ ) tonggo éndé; $\mathrm{Kp}$ tanggo éndé; Rm tanggo' éndé, karot éndé, ( $\mathrm{Tr}$ ) tanggu éndé; $\mathrm{Rg}$ (karo) dapé; $\mathrm{Ng}$ sela, togo édé; $\mathrm{Na}$ ( $\mathrm{Tm})$ hinga dhéké, togo édé, togo édhé; Ed pera; Li karo pela; WS ka'lelapa, ka'lalapa, kalalapo; ES kalàla; Sw dudu káha, kala káha, aj’u kéwawo; Si pedan(g) bain(g), pedan(g) wawa, pedan(g) goan; Sl kaha'.

Orchidaceae: $\quad \mathrm{Rg}$ lanu manu; $\mathrm{Ng}$ bhogi bheka, bowo; Li nio nitu; ES kata III; Sl wawé lia.

Annon.

Orophea polycarpa: WS maghela, mazela; ES mayela I.

Bignon. Oroxylum indicum: Rm émpas lélos, ?kinga lélos; Rg kowa.

Lamiac. Orthosiphon aristatus: Ng kumis kucing; Li ora; ES madáwa. 
Poac. Oryza sativa: $\mathrm{Bm}$ fare; $\mathrm{Km}$ paré; $\mathrm{FEM}$ (To, Ri) woza, (Bé, Ls, $\mathrm{Nn}, \mathrm{Mb}$ ) woja; $\mathrm{Rm}$ woza, (Wk) kusu, (Wu) kosu; $\mathrm{Rg}$ paré; $\mathrm{Ng}$ aré, kosu, mama ngagha, paré; $\mathrm{Na}(\mathrm{Tm})$ paré, (Ra) paé; Ké palé; Ed aré, paré ui; Li paré I; Pl lama; WS pare, pari; ES uhu, pari, wiaha, auhu, ausu, 'wiaza; Sw aré; Si nalu, paré; Sl tahâ, waha.

Poac. Oryza sativa f. glutinosa: Bm fare keta; $\mathrm{Rg}$ paré raké; $\mathrm{Ng}$ mama raké; $\mathrm{Na}(\mathrm{Tm})$ mama raké; Li paré dhaki; WS ?uhu kadito, pare kadito; ES usu kadita, (uhu) kadita; Sw aré laka; Si nalu lépu, paré 'regét, nalu épu; Sl tahâ tenaro, waha' naro, waha' ketâ.

Melast. Osbeckia ?dolichophylla: Kp dusuk sélék; Rm ?rangat pada'; WS kamia marèda.

Myrtac. Osbornia octodonta: Km bergelang.

Oxal. Oxalis corniculata: FEM (Bé, $\mathrm{Nn}$ ) mela, (Ls, Mb) wunu mela, (Ml) peca mela; $\mathrm{Kp}$ pesa mela; $\mathrm{Wr}$ poso mela, pesu mela; $\mathrm{Rz}$ pesu mela; $\mathrm{Rm}$ pesang mela, $(\mathrm{Wu})$ pesa mela, (Wk) mela; $\mathrm{Rg}$ susu waé; $\mathrm{Ng}$ mata mela I, rompa, rora; $\mathrm{Na}(\mathrm{Tm})$ wunu mela, (Bw) bené mela; Ed niru mera, wunu mera; Li mela, mela ji'é, nilu mela; Si mela I; Sl aho melâ.

Rubiac. $\quad$ Oxyceros miquelii: Rg nggélu.

Papil. Pachyrrhizus erosus: Bm buri; Ng sama, sama séko, sama tora; Ed ndora bima; $\mathrm{Li}$ bima, ndora bima; ES wóhu, kawuasa, 'uwahu, uwasa; Sw wué kota; Si tuka timu; Sl képa II, kepa'; Kd wahé képa, wahé iwan.

Rubiac. $\quad$ Paederia scandens: Km ngolo pesu; FEM wangér, (Ls) wanggér, (Ml) fangér; Kp wangér; Wr kazék kangor; $\mathrm{Rz}$ kangor; $\mathrm{Rm}$ fangor, wangér, (Tr) wangér, (Wk) fangar; $\mathrm{Rg}$ fangé; $\mathrm{Ng}$ fangé I; $\mathrm{Na}(\mathrm{Tm})$ fangé; Ed hangé, fangé; Li fangé I; Pl b'ecu wenga; Sw pena'i ki'i; Si tali poho; Sl kolô pohô, talé' paho; Kd ning buru'.

Apocyn. Pagiantha (Ervatamia) sphaerocarpa (cp. Voacanga grandifolia): Km sepada ntodoh; Kp gaka'; Wr gaka' ?ranar, gaka'; Rg nggaka ?haki; Ng gaka I, ?ghaka, lésé II, paja II, paza; Ed basé (bhasé), segé sa'i; Li basé; ES wota kamàmbi I; Si para; Sl kepara, para, keleka' para.

Sapind. Palaquium: Bm sada kala; FEM (Ri) suru nana; Wr natu; Rz natu; Rm suru nana', ( $\mathrm{Tr}, \mathrm{Wk}$ ) ?kazu nana('); Ed waen na; WS naho; ES nahu II.

Sapind. $\quad$ Palaquium (amboinense): Bm lahodo.

Sapind. Palaquium obovatum: Bm ?kantusu; Kp képo'; Wr képo'; Rz képo'; Rm kémpo'; WS ndélo; ES ka'ndoruku, kadúru.

Pand. $\quad$ Pandanus sp. (broad-leafed): FEM wako; Kp wako'; Wr wako'; Rz wako'; Rm wako'; Ng dika, waku; $\mathrm{Na}(\mathrm{Tm})$ waku; Li ndoko, waku; WS renu, rienu, ranu II; ES 'wakihu; Si toké; Sl doko; Kd tiu'.

Pand. Pandanus (wild): Rg pedé; Ng peda II, pégo; Li peda I; Si éman; Sl reta II.

Pand. Pandanus (thornless): ES iri.

Pand. Pandanus amaryllifolius: Bm fanda mengi; Sw lata méngi; Si pedan(g) uta.

Pand. $\quad$ Pandanus (tectorius var.): WS luri II; ES uri, saluri. 
Pand. Pandanus tectorius var. laevis: WS landa.

Pand. $\quad$ Pandanus tectorius var. littoralis: Km landis; Wr landis; Rz landis; Sw lata (banga).

Pand. $\quad$ Pandanus tectorius var. samak: Bm fanda dipi, fanda II; Km ré'a; FEM ré'a, (Bé, Ri) réa; Kp ré'a; Wr zé'a; Rz zé'a; Rm zé'a, (Tr) réa; Rg zé'a; Ng jé'a, ré'a, zé'a; Na zé'a, (Da) pada; Ké ré'a; Li ré'a; Pl ré'a; WS pònda, pòda, pànda, ES pàndangu, pàda; $\mathrm{Sw}$ lata; $\mathrm{Si}$ reta; $\mathrm{Sl}(\mathrm{Bg}, \mathrm{Wh})$ pedâ, reta I; Kd padan.

Poac. Panicum maximum: ES rumba jawa.

Poac. Panicum miliaceum: FEM (Bé, Ri, Ls) watar; Rm watar; Rg ghédo; Ng ghédo, ?wata; Pl ?wad'a.

Poac. Panicum notatum: Ng kuru bela; Ed au ré'é.

Poac. Panicum repens: WS ?'deisa, ndeiza, ndaica, ndeha; ES daisa, ndeha, ndesa; Sw ju'u kai.

Poac. Panicum sarmentosum: Rm laka waé'.

Papilionaceae: Bm ro'o koja; Rg nggeli polo; $\mathrm{Ng}$ hidhé, hi'é, hobho nitu, howé, ibu ngiu, koba teli, koro kowé nitu, kowé I, lebha nitu I, nidu imu, sidhi, zéko; Ed hésa kungu jata, mata mité; Li éndu I, kowé, ndelo; ES kambí maràda; Sw képapa edu; Si kédang, komak, guwang, ruha buén(g), tilu gelir, wuék wué.

Morac. $\quad$ Paratrophis philippinensis: Ed lélé wula; ES mayéla kalú.

Rosac. Parinari corymbosum: Bm ?lere II; ES huluku, ai rára.

Mimos. Parkia roxburghii: Bm kopa.

Mimos. $\quad$ Parkia speciosa: Bm pode; ES ?puti; Sw peté.

Poac. Paspalidium flavidum: Km satah arandaé; ES rumba míni.

Poac. $\quad$ ?Paspalum (conjugatum): FEM (Ri) kurun tuang; Kp reman ruténg; Wr rukut legi; $\mathrm{Rz}$ legi; Rm (reman) ruténg, (Wk) kurun tuang; Rg pasa; $\mathrm{Ng}$ bheto II, ngadé II, siku deké I; Na (Bw) ego beto; Ed kuru ba'i, mbindi II; Li kuru ba'i; Si 'urun lanang.

Poac. Paspalum scrobiculatum: ES kamàla litapu.

Passifl. Passiflora edulis: Ng sengsara.

Passifl. Passiflora ?foetida: Bm anggu; $\mathrm{Km}$ anggur; $\mathrm{Rg}$ aré anggu(r), nawu; $\mathrm{Ng}$ tébé ha; Ed nggara ngganda, rewo; Li anggo I; WS wíra, zuru ndéna I; ES rambútan; Si tali anggur, tali 'lawat, anggor; Sl nipô, anggur, bibis; Kd anggur 'otan.

Passifl. $\quad$ Passiflora quadrangularis: Sl bua sengsara.

Rubiac. $\quad$ Pavetta: FEM (Ri) toko ikang, (Nn) toko ikak; Rm toko ikang.

Rubiac. Pavetta montana: $\mathrm{Na}(\mathrm{Tm})$ bamé.

Euphorb. $\quad$ Pedilanthus (cp. Pereskia): ES kalala humba; Sw puri raho.

Caesalp. $\quad$ Peltophorum pterocarpum: Bm mbora; FEM (Ri) enot, (Ml, Ls) nok, (Nn) enék; Wr épa; Rm enot, (Wu) not; Rg ko'u; Ng ené; $\mathrm{Na}(\mathrm{Ra})$ ko'u I; Li waé II; ES loba; Si ?bur; Kd 'uré. 
Poac. Pennisetum macrostachyum: Ed éko méo I.

Poac. Pennisetum purpureum: $\mathrm{FEM}(\mathrm{Nn}, \mathrm{Ml})$ rumbét; $\mathrm{Kp}$ wéténg; $\mathrm{Wr}$ wéténg; $\mathrm{Rm}$ wéténg; $\mathrm{Ng}$ helé II; Li kipa II, soko belanda, jara; Si 'lité, bawa latang I; Sl kalikèt, klikét.

Poac. Pennisetum spicatum: FEM (Bé) latun jepang, (To) gandrung nipong; Wr lépang nipon; $\mathrm{Rz}$ watar; $\mathrm{Ng}$ haé léwa nipon; Li ?lolo kowé, lolo ngité, lolo poé; Si 'watar girén; Sl wata belolo, ipê gingé, wata' blolong kulung blo'ung; Kd watar holo' (méngar).

Cactac. $\quad$ Pereskia (cp. Pedilanthus): ES ?kalala humba.

Menisp. $\quad$ Pericampylus glaucus: Kp wazé' wulan; Wr kazék wulan; Rz kazék wulan; Rm wazé' wulan, (Tr) sobok, (Wk) konggé kaba.

Acanth. Peristrophe: ES kakaha III.

Orchid. ?Peristylis: WS pùtika.

Laurac. Persea americana: $\mathrm{Ng}$ ata foka; $\mathrm{Na}(\mathrm{Ra})$ ata foka; $\mathrm{Si}$ atapokat; $\mathrm{Sl}$ atpokat, atfokat.

Annon. Phaeanthus: Rg nona II.

Thymel. Phaleria octandra: Rg pura I; Ed suka ria; Li sui suka.

Papil.

cf. Phaseolus: Km gelguda'; FEM (Ls) leba giri, (Nn, Mb) leba mbiring; nggoli rewut, wuék wura; Rm papan; $\mathrm{Rg}$ ?kowé nitu; $\mathrm{Ng}$ fili, gozé III, hobho mami, kowé, lebha kedi II, sawa lebha; $\mathrm{Na}(\mathrm{Tm})$ boci; Li bué fésa, bué kaju roja, bué sé'a, nggoli; Si ?tali finé; Sl magé jawâ, maling II.

Papil. $\quad$ Phaseolus aureus: Bm kaḅoó jao; Kp ?kowé'; Rm keboé, (Wk) rumbét; Rg rumbé; $\mathrm{Ng}$ hobho kaju, rubhé; $\mathrm{Na}(\mathrm{Ra})$ bué; $\mathrm{Li}$ bué kaju; Pl pué; WS kàmbe móro wiasa, kàmbe móro wìaza, kèmbe móro wiha; ES kambi múru, kambí haii, kambí hànu; $\mathrm{Sw}$ kébui iki; Si bué, bué lengi; Sl uta wéwé II; wéwé, wéwé II; Kd wéwé.

Papil. Phaseolus lathyroides: Rg ma.

Papil. $\quad$ Phaseolus ?lunatus: FEM leba; Kp lomba; Wr leba'; Rm (Tr) lembot, (Wk) ?kékos; $\mathrm{Rg}$ leba; $\mathrm{Ng}$ fésa, lebha, lebha fésa; $\mathrm{Na}(\mathrm{Tm})$ fésa, kowé mézé; Li domba, bué fésa, hésa; WS ka'papa, ka'papa rí I+II; ES ka'papangu II, ka'papangu ùhu; Sw kepapa nga'a; Si biha, biha natar; Sl kipé, képa' glého' utâ bao; Kd utan édang, utan ?kédang, utan rowé.

Papil. $\quad$ Phaseolus pubescens: FEM (To) nggewo, (Bé) nggoli, (Mb) wuék lớ; Kp séa'; Wr kowé'; Rz gowé; Rm séa', (Wk) ngguli; Rg nggoré II, kowé; Ng ?gozé III, koro kowé, kowé, séa II; $\mathrm{Na}(\mathrm{Tm})$ céa; Li nggoli bali; Kd aha.

Papil. Phaseolus radiatus: Bm kaboe.

Papil. $\quad$ Phaseolus vulgaris: FEM (Bé, Ri, Ml, Mb) boncis; Kp bunsis; Wr bonsis; $\mathrm{Rm}$ bunsis; $\mathrm{Rg}$ hobo; $\mathrm{Ng}$ hobho II; $\mathrm{Na}(\mathrm{Ra})$ boci; Li beréné, beréné bon, bué bosi; ES kapapa rí II; Si boncis, wéwé kléréng; Sl boncis.

Arec. Phoenix dactylifera: Bm koroma.

Orchid. Pholidota: $\mathrm{Ng}$ léké solé. 
Rosac. Photinia: Kp leri'; Rm leri.

Poac. $\quad$ Phragmites karka: Bm kenu; FEM (Ls, Nn, Mb) lelu; Kp lelu'; Wr kiput; Rz lelu'; Rm lelu'; Rg timbu; Ng lelu I; Na (Tm) ghai; Li kela; WS ka'bounyu, kabúnu, kabóna II; ES katambuni, katambini, kabaunu; Sw téwo; Si dalo, ?dama; Sl tebé, tebo.

Papil. $\quad$ ?Phylacium bracteosum: Rg lepi II; $\mathrm{Ng}$ séko si'é IV, weka koba; ES tugha pare.

Euphorb. $\quad$ Phyllanthus: Kp ndoréng ndorik; Rm ?kia'n II; Ng li'é logo II, ?sunga wolo, taga kolo I; Ed mboko ronggo, muku ronggo; Li mboko longgo I; ES tánda malangu III, tánda malangu II; Sl lolô tobi, tobi lolô, tobi ronê?, tobi makî; Kd ?wéla dapé'.

Euphorb. Phyllanthus acidus: Bm sarume I; Sw haramélé.

Euphorb. Phyllanthus amarus: WS móro rewa; ES màrahu rau, màrahu rú.

Euphorb. Phyllanthus hypericifolius: WS mama lakáwa; ES pahawura I.

Euphorb. Phyllanthus pulcher: ES wura wolu.

Euphorb. Phyllanthus reticulatus: WS mbora; ES pahaura.

Euphorb. $\quad$ Phyllanthus virgatus: Rg kambi ndari; Ed mboko ronggo II; Sl karcinta.

Polyp. $\quad$ Phymatodes scolopendria: Ed kuku manu; WS ma'wailo, ma'ghailo, ma'yailo.

Solan. $\quad$ Physalis: Bm tobe langgiri; FEM (Ls) wua bo, (Nn) bo sa'i; Kp bo sa'i; Wr wua' bo' sa'i; Rz wua' bo' sa'i; Rm bo' ulu, kepok; Rg pura II, ?nggoré II; $\mathrm{Ng}$ bolé kogo, boné, butu hiku, wua soi; Ed ana wonda, wonga wonda; Sl ketelak.

Solan. $\quad$ Physalis micrantha: Ed wendi wonda; WS kapóka, poka-poka; ES kapoku, hapoku.

Urtic. $\quad$ Pilea microphylla: $\mathrm{Ng}$ lewi watu.

Caesalp. $\quad$ Piliostigma malabaricum var. acidum: Bm rufé; $\mathrm{Km}$ daléng; Wr kupé; $\mathrm{Rm}$ kupé; $\mathrm{Rg}$ kupé; Ng kupé; Li kupé; WS wasu kamba, ghazu kamba, karùpe, mùne; ES karìpi, karupi, karipu; Sw dupé; Si tu niluk; Sl ?kalukê, kepi', nyanyilu.

Arec. $\quad$ Pinanga (coronata): FEM (Nn) mbonga nai; Kp pinggong; Wr pinggong; Rz pinggong; Rm pinggong; $\mathrm{Ng}$ héu nitu, héu piki, séu nitu; $\mathrm{Na}(\mathrm{Tm})$ héu nitu; Li biji; WS dàwara; ES dàwaru; Sw wenyi edu; Si wua biri; Sl wua' iri.

Piper. $\quad$ Piper: FEM wunu mbeké, kala tanggo, (Bé, Nn, Mb) nggurus, wunu saung, wunu, (To) rebo wé'an, (Ls) wunu wura; Kp rebo remang; rebo kodé, rebo mbéké; rebo tanggo; Wr kélan rukut; $\mathrm{Rz}$ rebo rukut; kélan tanggo; $\mathrm{Rm}$ rebo mbéké, rebo reman, rebo tanggo', (Wk) ndana; $\mathrm{Rg}$ kuru kodé; $\mathrm{Ng}$ koro wolo, méngi, mota, nitu I; Na (Tm) horo; Ed koro wuro, ndoro; Li koro wolo, mota ndoro II, ndoro; Pl kucu; WS uta we'e, uta 'waiyo; uta kalara, ro (u)ta kalara, ES kuta lara, kuta búti, ndau luku; kalara; Sw kélara jami; Si koro 'iling; Sl lolô buri.

Piper. $\quad$ Piper aduncum: Si bana, ta'a koro; Sl ku'u krowéng, ku'u taling.

Piper. $\quad$ Piper betle (races): Bm nahi, bulu; Km sepa; FEM wunu, wunu saung; Kp rebo; Wr kélan, kélan wua'; Rz rebo; rebo wu'a; Rm rebo; $\mathrm{Rg}$ nata, nata tanggo; $\mathrm{Ng}$ nata, nata béké; Na (Tm) wunu zi'a; Ed ?égo légo, nata; Li mota, nata(-moka); Pl mutu; WS 
ùta; rútu III; ES kuta I; kuta haü, kuta kawaü; Sw kénana, hiri; Si ta'a uta; Sı lolô, malu; Kd mal.

FEM wunu méngi, (Ri) kala mengi, $(\mathrm{Mb})$ wunu mengi; Kp rebo mingi; Wr kélan méngi; $\mathrm{Rm}$ rebo mingi; $\mathrm{Rg}$ nata méngi; $\mathrm{Ng}$ méngé, nata méngi; $\mathrm{Na}(\mathrm{Tm})$ wunu mengi; (Bw) méngi; Ed méngi; Li méngi.

Piper. $\quad$ Piper nigrum: Bm saha I, marica; Ed mboko sa; Li mboko sa; Sw kélai linga laka, kélai linga jawa; Si sang; Sl sa'ang.

Piper. $\quad$ Piper retrofractumn: Bm sabia I; Km sabia; Rg kuru kodé; Ng nata butu I, nitu I; Ed wuro; Si ta'a koro; Sl ?ku'u III; Kd mal modung, malo nidun, téhé'.

Urtic. $\quad$ Pipturus: Kp sama ular; Rm laméng ular, (Tr, Wk) lamé ular; $\mathrm{Ng}$ lesi I, zama witu; WS rame; ES remi, rami II; Kd téhé'.

Urtic. $\quad$ Pipturus argenteus: FEM sama, (To) wasé' sama; Kp sama isi'; Wr rébak; Rm kébak/sama, (Wk) lamé; $\mathrm{Rg}$ lesi; $\mathrm{Ng}$ zama; $\mathrm{Na} \mathrm{(Tm)} \mathrm{zama;} \mathrm{Ed} \mathrm{rama;} \mathrm{Li} \mathrm{rama.}$

Nyctag. $\quad$ Pisonia (aculeata): Km nggalong; Rg tindu; WS ghòlo.

Nyctag. $\quad$ Pisonia grandis: Si halé; Sl ?motong II.

Nyctag. Pisonia sylvestris: ES ai hei.

Nyctag. $\quad$ Pisonia umbelliflora: Rg nuli; Sl duling.

Mimos. $\quad$ Pithecellobium angulatum: Kp balas; Wr lalar; Rz balas; Rm balak, (Wk) ?wulu'; Rg lala.

Mimos. Pithecellobium tomentellum: Ed segé; Sl mata ba'a.

Mimos. Pithecellobium umbellaturn: $\mathrm{Km}$ warang; Ed pira; Sl tenunu.

Pittosp. Pittosporum: Sl okak.

Pittosp. Pittosporum moluccanum: Bm luha; FEM (To) longkor wé'an; Kp légar; Rm longkor lawo', (Wu) kongkor lawo'; Rg ndaka kodé, ndeka kodé; Ng lasé kodhé; $\mathrm{Na}(\mathrm{Tm})$ moro méké; Ed angi, mara méké; Li muku té'a I; ES mènde, ngùdu wawi.

Sapot. Planchonella: Ed naka kéli.

Sapot. $\quad$ Planchonella duclitan: Bm ?nggala; Ng bhaka III; Ed keta, wolo angi; WS katango; ES kàtangu.

Sapot. Planchonella firma: Si amana kak.

Sapot. $\quad$ Planchonella linggensis: Ed ndopo.

Sapot. $\quad$ Planchonella obovata: Bm nangga doro; Kp ketang; Wr ketang; Rz ketang; Rm ketang; Rg keta; Ng bhaka II, keta; Ed keta, naka ré'é; Li naka ré'é; Si amana.

Lecyth. $\quad$ Planchonia valida: Bm katipu; Km kendau; FEM ngancar, (Bé, Ri) ngansar; Kp ngasar; Wr ngasar; Rz ngasar; Rm ngansar; Rg wala; $\mathrm{Ng}$ bhaka I, wala; $\mathrm{Na}$ (Tm) repu; Ed wara I; Li wala; WS langae, ngae, mbáha; ES langáha, wàla ngaha, langasa; Si tour I; Sl manaha, naha'.

Plantag. $\quad$ Plantago major: ES kahilu wéi. 
Icac. $\quad$ Platea excelsa: Kp kelu pong; Wr kelu pong; Rm welu pong; Ed taro kéli.

Polyp. $\quad$ Platycerium bifurcatum: Kp wawé; Wr wawé; Ng huku I; Si 'ohu do'én; Sl manu kepi, ?kakung baru.

Liliac. $\quad$ ?Pleomele: Li ?waki; WS karénggu; ES kìku mánu II.

Aster. $\quad$ ?Pluchea indica: $\mathrm{Kp}$ rowé'; Wr rowé; $\mathrm{Rm}$ rowé'; $\mathrm{Ng}$ wonga bhara II; $\mathrm{Sl}$ bluntas.

Apocyn. Plumeria acuminata: Bm kamboja; Wr kemboja; ES bunga, ?mbunga; Si ?kemuni, sikun jaga, bunga tou; $\mathrm{Sl}$ kamboja; Kd bunga antonius.

Polyp. $\quad$ ?Pneumatopteris sp.: FEM $(\mathrm{Nn})$ limé lipang, $(\mathrm{Mb})$ tédhér; Rm kedér; Rg kumba sawa II.

Poaceae: $\quad$ Bm ?kéu; Km ?kéu; FEM (Ls) kelé, (Nn, Mb) kela; ghas, ngéok, ngé'ok; litot; Rm ferat, kuri'; litot, pézos, kuru tuang; Rg mé'a; Ng ?kuju I, pubu, toko ika; Ed kuru kamba, pumbu; Li éndu II, gai géla, kumi rongo, lolo péga, paké siku, peke siku, rega; WS déisa; naripa, naripyo; ES kuku, nderi; ka'pumbungu, kamàla uhu; màritu; 'naripu, 'nyariku, 'neripu, 'narupu; mapu múru kapuka; Sw bela kepué, kaé, wila; Si té'u helon, ba basar; keremét; kemu'u au, mu'ung 'au, rata burâ.

Podoc. Podocarpus amarus: FEM (Ri) long, (Nn) ?mbera; Kp ngelong; Wr ngelong; Rz ngelong; Rm ngelong; Ng pini, ?pinis; ES bok.

Podoc. $\quad$ Podocarpus (imbricatus): Ng danga III; Ed peto; WS dòyo; ES kajú 'omangu, kajú II, mbaku haü II, kadiu; Si ?(e)mbu.

Podoc. $\quad$ Podocarpus neriifolius: $\mathrm{Km}$ jati talu; Li aé.

Poac. Pogonatherum crinitum: $\mathrm{Ng}$ pubu waé.

Lamiac. cf. Pogostemon: WS nggolé; ES remi kawini.

Commel. Pollia: Si kéru, kléru; Sl kéru.

Caryoph. Polycarpaea sumbana / zollingeri: Ng momi, uwi momi, Ed rẹi aré.

Polygal. Polygala malesiana: ES wura kapáki.

Polyg. Polygonum: $\mathrm{Rg}$ mundung kura, wunu kura; $\mathrm{Ng}$ lodé kedhi; Li uta sala.

Polyg. $\quad$ Polygonum chinense: $\mathrm{Ng}$ kuza I, lodé; Li sala; WS kandàlu, tìbu dalu.

Saxifr. $\quad$ Polyosma: Ed baru aé, jawé II; Li balu aé; ES malai rau.

Polypodiaceae a): FEM (To, Nn) limé lipang, (Bé) raé, (Ri) lebé raé; kénda; Kp raun kénda; $\mathrm{Wr}$ lebo' rimang; lalir kata', kipé; Rm kidang, wangkéng, (Wk) rundung ulu, maku wéwo, (Tr) maku mampang; Ng kipé; fo'i fé'é, kanga paké, logo mité, meso beki, ogo mité, patola, roté, sama roga, sigu migu, tigo, toko mité II, mu, wunu rori; Ed reru, sara koré; Li dala, lelu, lelu kela II, ?paku, soso; WS káta; ES káta IV; Si hera, roni, teké liman; Sl wulung waing III.

Polypodiaceae b): FEM (Ml) céngka lélang; Kp zaka lélang; lara; Wr zaka lélang; Rm zangka lélang.

Aral. Polyscias scutellaria: $\mathrm{Ng}$ ?wiku. 
Sapind. $\quad$ Pometia pinnata f. tomentosa: Kp maras; Wr maras; Rz maras; Rm maras; Rg kipi; $\mathrm{Ng}$ hopa béwa I, mara, maza; Li ?keba, ?péto; ES ta'méangu; Sl ra'ong; ma'a.

Papil. Pongamia pinnata: Bm ?bawe; Si bla'at.

Portul. $\quad$ Portulaca oleracea: Bm soka; Km ngolo saka; FEM bélang; Rm bélang; Ng moro bowo; Ed rẹi; Li leri, wunu kapa; Pl wawi 'élo; WS ?pádu bola, katìri dála; ES lamata, katàlari; Sw láma; Si arung wélang; Sl gélâ burâ, gélâ; Kd sang gélang, 'amang.

Piper. Pothomorphe: Li éru.

Piper. Pothomorphe subpeltata: WS ndàbo.

Arac. Pothos: Rm mando lebé.

Urtic. Pouzolzia: Ed goru, kumi rongo I, nggoru; Li ranga II.

Urtic. Pouzolzia hirta: Si ina réo.

Urtic. $\quad$ Pouzolzia zeylanica: Kp rangat; Wr rangat; $\mathrm{Rm}$ rangat kazu; $\mathrm{Ng}$ kabu ranga, ranga III, ranga lédha; $\mathrm{Na}(\mathrm{Tm})$ koba waba; Li ranga I; WS wòla wawi, wàla wawi.

Verben. $\quad$ ?Premna sp.: Bm rondu keha; Km (haju) mbéla'; Ed mbapa; Kd ba'ung.

Verben. Premna tomentosa: Bm mpafa.

Burser. Protium javanicum: Bm loa.

Rosac. $\quad$ Prunus (cp. Pygeum): ES tàda katàbi.

Rosac. $\quad$ Prunus wallaceana: Kp kénda; Wr kénda; Rz kénda; Rm kénda; Rg lepi IV; $\mathrm{Ng}$ hedhé.

Acanth. $\quad$ ?Pseuderanthemum: Sl tepo.

Myrtac. $\quad$ Psidium guajava: Bm jambu; FEM (Bé, Ri) jembu, (Ls, Mb) goawa, (Nn) jebu, (Ml) nggoé 'awa; Kp zembu; Wr zembu; Rz zembu; Rm zembu; Rg jambu; $\mathrm{Ng}$ boé awa, bu awa, bué awa, goé awa, guawa, zabu, zebu; Na ( Tm) goawa, goé awa, (Ra) goa 'awa; Ed nggoé nggawa, guawa; Li ga awa, nggo awa, nggoa awa, nggoé awa, nggoé nggawa; Sw woko jawa, lub'a; Si goi awas, konjawa, kebojan, regon; Sl gewawa, koa jawa, mogo; Kd kulu jawa.

Psilot. Psilotum complanatum: WS moro parìnggi.

Papil. $\quad$ Psophocarpus tetragonolobus: FEM (Bé) lojok, (Ri) bengur, (Ls, Nn, Mb) segé, (Ls, Nn, Ml) céko, (Mb) séko; Kp ku'u; Wr ku'u; Rz ku'u; Rg ku'u; Ng séko; Na (Tm, Ra) séko; Li dowé, séko; Bm salira; Si hé'o.

Papil. $\quad$ Psoralea tamborensis: Km gambong; ES 'muara.

Rubiac. Psychotria: Ng tepu.

Polyp. $\quad$ Pteris biaurita: Ed memu.

Polyp. $\quad$ Pteris (ensiformis): $\mathrm{Rg}$ kumba sewo; $\mathrm{Ng}$ hedé toko laka, ?midhu.

Polyp. $\quad$ Pteris venulosa: WS wulu manu mane.

Polyp. Pteris vittata: Ed paku II. 
Papil. $\quad$ Pterocarpus indicus: Bm nara; Km nara; FEM nara; Kp nara; Wr déndé; Rz nara; $\mathrm{Rm}$ nara; $\mathrm{Rg}$ d'éndé; $\mathrm{Ng}$ dhédé; $\mathrm{Na}(\mathrm{Tm})$ nara; Li nara; ES kenáwa; Sw aré II; $\mathrm{Si}$ campaga, sendana laki, sampaga; Sl kena'a, kenaha, ansana (Ind.); Kd naré.

Papil. Pterocarpus indicus f. echinocarpus: Bm nara wadu; Kp nara sepang; Wr déndé watu.

Aster. Pterocaulon sphacelatum: ES ?kahilu kamemi, kahilu kamambi; Sl pelé'.

Euphorb. ?Pterococcus corniculatus: Bm maladi ai; FEM (To) latong wasé, (Bé, Ri) lantong wasék (cp. Dendrocnide); Wr latong kazék; Rz latong kazék; Sl munak klatê.

Stercul. Pterospermum diversifolium: Bm sala; $\mathrm{Km}$ wajuh lamé; FEM (Bé, Ri) damér, (Ls, $\mathrm{Nn}, \mathrm{Mb}$ ) wajur; Kp damér; Wr damér, $\mathrm{Rz}$ damér; $\mathrm{Rm}$ damér; $\mathrm{Rg}$ waro; $\mathrm{Ng}$ waé, waro I; Na (Tm) ko'u II (cp. Peltophorum); Ed waé; Li waé I; WS wero; ES yú karambua, tada wero; Sl tou'.

Stercul. Pterospermum javanicum: Ng luba; Sl dami.

Stercul. $\quad$ Pterygota horsfieldii: Rg kuwu; Li ?wuwu; ES ?malubu, malumbi.

Punic.

Punica granatum: Bm talima, ?dalima; Sw jelima; Si dalima; Sl dalima; Kd dalima.

Amaran.

Pupalia lappacea: Rg ta'i tibo II.

Menisp.

cf. Pycnarrhena: ES lólo móro II.

Menisp.

Pycnarrhena (longifolia): Ng su'i koba I; Li lolo moro.

Rosac.

Pygeum (cp. Prunus): Ed kuro, mbaja, mbéwa; Li mboja II, uru bara.

Polyp.

?Pyrrosia: Kp repo'; Wr wua' kimit; Ed fi iji, fi'i ji; Li mboko kusi; WS us réwa; ES pula kalémbi.

Polyp. $\quad$ Pyrrosia longifolia: Ng lai II.

Bignon. Radermachera: Kp lombo; $\mathrm{Ng}$ tui, tuli; ES tui, toyu, towi.

Rubiac. Randia: Rg ?nganga; Ed ango, lodo.

Rubiac. Randia patula: Km dikik ngalong.

Myrsin. Rapanea: Ed ana bua.

Brass. Raphanus sativus: Li loba I.

Apocyn. Rauvolfia: Rg keropo; Ng zita ré'é; Li ?kewi; Sl klekar.

Arac.

Remusatia vivipara: $\mathrm{Rm}$ mangkang; $\mathrm{Ng}$ tiri kazu; Li sara mbira.

Arac.

Rhaphidophora (cp. Scindapsis): FEM (Bé) melung, (Nn) belur; Kp ndulu'; Wr ndolu'; Rz ndolu'; Rb melung, (Tr) melun, (Wk) belung; Rg melu; $\mathrm{Ng}$ melu I; Ed ira meru II; Li ila melu I; WS mòlu; ES katúku malungo; Si melu moét; Sl belû.

Rhizoph. Rhizophora apiculata: Bm wako; Km bango; Si bangkok.

Rhizoph. Rhizophora stylosa: Sl ladi.

Anac.

Rhus taitensis: $\mathrm{Bm}$ ?enggo/?nggo; Kp garét; Wr garét; Rz garét; Rm garét, (Wk) panda lari; Rg nggari; Ng gari; Ed gari, nggari; Li gari; ES wùru wàla; Sl gari'. 
Papil. Rhynchosia minima: Rg nggeli.

Cyper. Rhynchospora: $\mathrm{Ng}$ résa waé.

Cyper. Rhynchospora corymbosa: WS 'tiemu.

Meliac. Ricinocarpodendron polystachyum: Si tulén.

Euphorb. Ricinus sp.: Sl padu aho tain.

Euphorb. $\quad$ Ricinus communis (cp. Jatropha): Bm tatanga, katanga jawa; FEM (Bé) pandu nggadung, (Ri, Nn, Mb) pandu kadung, (Ls) padu, (Ml) pandu; Kp pandu kadul, pandu kadung; Wr pandu; (Rz) pandu waé; Rm pandu kadung; (Wk) pandu wuni, (Wu) pandu rangga; $\mathrm{Rg}$ pandu; $\mathrm{Ng}$ dhao goa I, padu goa, ta'i bara; $\mathrm{Na}(\mathrm{Tm})$ padu kadhu; Li dama II, pandu; Pl b'at'u; WS kadàmo ndima, madòmo dawa; ES madamu, padàmu; Sw lolo (bangi); Si damar jawa II, damar nilon, padu II; Sl padu klou, padu', pador; Kd kabélung.

Brass. $\quad$ Rorippa: $\mathrm{Ng}$ faka tana.

Rosac. Rosa: Bm jene mawa (ua), bunga mawa; Si bunga mawa.

?Rosaceae: $\quad$ Ed noki.

Poac. Rottboellia exaltata: WS 'litopa, 'litopo, licopo; ES lìtapu, 'litapu, litapa.

Connar. ?Rourea minor: $\mathrm{Ng}$ katé toro, koba.

Arec. Roystonea regia: Si wua ladu.

Rubiaceae: Sw tédengi.

Rosac. $\quad$ Rubus spp.: Kp borong; Rm diong pong, (Wn) ?riting.

Rosac. $\quad$ Rubus alceaefolius: Li mburi mbombo.

Rosac. $\quad$ Rubus (moluccanus): Kp wazé' borong; Wr wua' karot; Rz wua' karot; Rm diong, (Tr) saru, (Wu) saru la, (Wn) karat saru; $\mathrm{Ng}$ baba, bési I, kébé; Ed karo bési; Li mberi mbombo,.mbira mbombo, mbiri mbombo.

Rosac. Rubus moluccanus var. discolor: WS tara wìri lolo.

Rosac. Rubus rosaefolius: WS tara wèri.

Flacourt. cf. Ryparosa: WS wasu 'reketa.

Malpigh. Ryssopterys: WS 'kotera, 'kótera.

Malpigh. Ryssopterys timoriensis: Ed tuwa II.

Poac. Saccharum arundinaceum: WS katina; ES tàbu hala, tíbu hala, ka'tinahu; Sw debu loko, ?deb'u wéro; Sl bawa batang.

Poac. Saccharum officinarum: Bm dobu; FEM téu, (Ri) tiu; Kp tiu'; Wr téu; Rz téu; Rm tiu, (Wu) téu; Ng tewu II; Na (Tm, Ra) tewu; Ed tewu; Li tewu; Pl d'ewu; ES tìbu, tàbu; Sw debu; Si tewu; Sl tewo, tefo; Kd téhu.

Poac. Saccharum spontaneum: Bm ati (ke)ndolo, ndolo; FEM (Bé, Ri) tebor, (Nn) tebér, (Ml) kelé', (Mb) tebhér; Kp tebor; Wr tebor; Rz tebor; Rm tebor, faluk, (Wk) faluk; 
Rg tewu; Ng sulu I, witu II; Na (Tm) kela, wiza; Ed witu; Li éko lako, gai, nggaju; Pl 'oko; WS ílyaho; ES 'ilahu; Sw la, ila; Si rotan; Sl tebe', tewa'u.

Arec.

Salacca edulis: Bm sala.

Salic.

Salix tetrasperma: FEM (Bé, Ls, Nn, Ml) ratung, (Ri) ratu; Kp ratun; Wr ratun; Rz ratun; $\mathrm{Rm}$ ratun, (Wk) ratung; Ed ?rana, rena.

Chenop. Salsola kali: Sw karo'.

Lamiac.

Salvia: ES karìtaku II; Sw ru kunu wango; Sl permé.

Lamiac.

Salvia misella: $\mathrm{Rg}$ ru.

Lamiac.

Salvia (riparia): $\mathrm{Ng}$ meré wau; Ed fangé, hangé; Li ?lako raki; fangé II.

Mimos.

Samanea saman: Sl karo gula.

Caprif.

Sambucus canadensis: Li bunga.

Meliac.

Sandoricum koetjape: Bm ?suntu.

Santal. Santalum album: Rg mbawa; WS ndana, dána, handána; ES sandana, rita II, nítu, saḍana; Sl cendana.

Sapindaceae: ES kàti katàru I.

Sapind.

Sapindus saponaria: WS ?mboro II; ES kàhi kataru.

Sarcosp.

Sarcosperma paniculatum: Ed kua II, lama kamba, wowo; Li ngala kutu.

Saurau.

Saurauia verheijenii: FEM ilur wawi, (To) ilur ela, (Ls) 'ilur wawi; Kp giro'; Wr giro'; Rz giro'; Rm ilur wawi, (Wk) ilur kui; Ng lema kaba; Li lema kamba.

Aral.

?Schefflera: Kp repa' paké; $\mathrm{Ng}$ lima ro'a.

Aral.

Schefflera elliptica: WS 'ndatara, ndátara.

Aral.

Schefflera cf. rigida: FEM (Ml) lombo nio; Kp lombo nio; Wr lombong nio'; ?lombong wené; Rm lombong nio', (Tr) kazu nio, (Wk) nio rékang.

Poac. $\quad$ Schizachyrium brevifolium: ES mapu kangúrutu II.

Poac. $\quad$ Schizostachyum blumii: Bm mila, o'o hamia; Kp sawar; Wr sawar; Rz sawar; Rm sawar; Rg ila; Ng ila, wulu II; Na (Ra) wulu; Ed wuru; Li wulu; WS tàme, tamia; ES tamiangu; Sw témié; Si 'wiro, wulu; Sl wulo, wulo', fulor; Kd walang.

Poac. $\quad$ Schizostachyum brachycladum: Bm o'o nteri; Km belang; Kp belang; Wr belang; $\mathrm{Rz}$ belang; Rm belang; Rg mbela; $\mathrm{Ng}$ bela I; $\mathrm{Na} \mathrm{(Tm)} \mathrm{bela;} \mathrm{Li} \mathrm{bela,} \mathrm{?bela} \mathrm{wula;} \mathrm{Pl}$ ?’oka; Si belan(g); Sl belâ; Kd walang 'ayang.

Sapind. $\quad$ Schleichera oleosa: Bm sambi; Km sambi; FEM sambi, (Ri) kodol; Kp sambi; Wr sambi; Rz sambi; Rm sambi; Rg sambi; Ng sabi; Na (Tm, Ra) sabi; Ed sambi; Li sambi; Pl hapi; WS 'kuomi, komi; ES kahámbi, kahembi, kasambi, kahambu, komisa; Sw kéhabé, kohabé; Sl kebahi, bahi, kabahi, kasambi, kesambi, bahi; Kd ala béhu, ala bélu.

Tiliac. $\quad$ Schoutenia ovata: Bm luhu; Km mbuhung; FEM kukung, (Ri) kukun; Kp kukun; Wr kukun; Rz kukung; Rm kukun; Rg kuku; Ng kuku; Na (Tm, Ra) kuku; Ed 
kuku;Li kuku; WS ghungo, laghùngo, laghughu; ES ?laungu, wunga I, lawungu; Si gu'un(g); Sl kukû, kukung; Kd u'un.

Arac. $\quad$ Scindapsis (cp. Rhaphidophora): WS nggaü, manggaü.

Cyper. $\quad$ Scirpus: FEM (To) ngelas, (Ri, Ls) la; WS ka'suoba; wulu wae mète.

Cyper. $\quad$ Scleria (terrestris): $\mathrm{Rm} \mathrm{la;} \mathrm{Rg}$ ghela; $\mathrm{Ng}$ wako laé; Ed éra, mbu'a huja; WS malingata; ES maninggatu.

Loranth. $\quad$ Scurrula: $\mathrm{Rg}$ ta'i ndala.

Lamiac. Scutellaria discolor: WS ketunggu lawa ndende.

Cucurb. $\quad$ Sechium edule: FEM (Bé) togo jepang, (Nn, Mb) labu jepang, (Ri, Ml) labu zepang; Kp labu zepang; Wr labu zepang; Rz labu zepang; Rm labu, (Tr) zepang, (Wu) labu zepang; $\mathrm{Rg}$ labu; $\mathrm{Ng}$ labu jepa; $\mathrm{Na}(\mathrm{Tm})$ toza, (Ra) labu jepa; Ed labu jepa; Li uta jepa; WS karobo jawa, karòbo dawa; Si labu jepan(g); Sl labu jepâ; Kd labu jepang.

Poac. $\quad$ Sehima nervosum: ES mapu bara I.

Selag. $\quad$ Selaginella: Ng hetu I; Si wau mu'an, tana ra'i.

Selag. Selaginella plana: Ed répa reko.

Selag. $\quad$ Selaginella willdenowii: Ed repo; Es kara'wùrangu.

Anac. $\quad$ Semecarpus (forstenii): FEM (To) pilus, (Bé, Ri, Ls, Nn) bélél, (Mb) bélék; Kp pilus; Wr pélus; Rz pélus; Rm bélél, (Wk) bélén; $\mathrm{Ng}$ moja, ngara, ngara ré'é; Li méra pélé, mer(e) pélé; WS 'ruota; ES 'ruata, rota; Si 'wéru; Sl bérut, kelomo I; Kd ranga.

Acanth. Sericocalyx: FEM tepu.

Acanth. Sericocalyx asper: $\mathrm{Rg}$ runu waé; $\mathrm{Ng}$ figo, meru; Li ingga; Sl terata wulun loko, klapu tébang.

Pedal. $\quad$ Sesamum orientale: $\mathrm{Bm}$ ringa; FEM longa; $\mathrm{Kp}$ longa kazu; Wr longa kazu; $\mathrm{Rz}$ longa kazu; Rm longa kazu, (Wu) longa, (Wk) sunding; Rg lenga; $\mathrm{Ng}$ lenga; $\mathrm{Na}(\mathrm{Ra})$ lenga; Li doba, doba lenga, lenga; PI longa; WS lànga; ES lànga; Si koja lona(ng); SI longa.

Papil. Sesbania grandiflora: Bm palawu; FEM (Ls) huma; Ng fai zé'é I, huma, rewu, suma; Ed huma; Li kuma; Pl luma; WS wunga III, ?taminu, ghùnga; ES wunga III, wàla wunga I; Sw wengu II; Si 'luma; Sl teluma, luma'; Kd taluma.

Ficoid. $\quad$ Sesuvium portulacastrum: Bm ?kumbe; Sw d'ad'alu; Sl gélâ mé'â, gélâ jawa; Kd rémé.

Poac. Setaria: Si wetan ahu; Kd wéré' la'u ébo.

Poac.

Setaria adhaerens: $\mathrm{Ng}$ ?nalu, weté; Ed kuru weté.

Poac. $\quad$ Setaria italica: Bm witi; FEM (To) jewawut, (Nn) sané koé; Kp ghusu; Wr ghosu; $\mathrm{Rz}$ weton; $\mathrm{Rm}$ weton; $\mathrm{Rg}$ hosu, weté; $\mathrm{Na}(\mathrm{Ra})$ weté, $\mathrm{Li}$ jo wawo; Pl ?lolo, wed'é; WS 'watara kajíji, bòla; kameli, bàla; ES uhu kani, usu kani, uhu kanu, kanu; 
ka'maili; Sw uhu; Si i'ur wetan, wetang wéting, wetan sigar; Sl wetê, ?wetê blolo'ng, wétan; Kd wéré'.

Poac. Setaria laxa: ES uhu manginu I.

Poac. $\quad$ ?Setaria (pallide-fusca): Sl wetê jawâ.

Poac. $\quad$ Setaria palmifolia: FEM (Ri) mezang, (Ls, Nn) mejang; Kp mejang; Wr mezang; Rz mezang; Rm mezang; Ng beza, meja, meza; Ed meja; Li ?lité I, meja; Si 'léna.

Poac. Setaria verticillata: ES kamàla uhu kanu.

Malvac. $\quad$ ?Sida: FEM (Ml) maki mincé; Kp mata lero' II; Wr mata lejo'; Rm nalun mbé', (Wu) turak mbé, (Wk) turak wura; Ed mbaté kamba, mbeté jara, Li beté jara, ngeti kamba, paju kutu; WS (ro'o) susu nana, mada nu'u II; Si hada buri; Sl herâ lolô, tahi lolo.

Malvac. $\quad$ Sida acuta: Wr mbeté jaran; $\mathrm{Rg}$ mbeté jara; $\mathrm{Ng}$ beté kaba, ura kogha I; $\mathrm{Na}(\mathrm{Bw})$ ?potu polo meka; Li beté kamba; Si paré wenit; Sl namo' I.

Malvac. Sida elongata var. balica: Ed meké reké I.

Malvac. Sida javensis ssp. expilosa: Km raké'.

Malvac. $\quad$ Sida javensis ssp. javensis: Tr susu nana

Malvac. Sida parvifolia: ES pahìndu ka'rambua.

Malvac. $\quad$ Sida rhombifolia: Bm paramau, permau; FEM mata leso; Kp mata lero' I; Rz mata lezo; Rm nalun mbé'; Ng ura kogha II; $\mathrm{Na} \mathrm{(Bw)} \mathrm{?potu} \mathrm{polo} \mathrm{haki;} \mathrm{Ed} \mathrm{mata} \mathrm{rera} \mathrm{II;} \mathrm{Li}$ ngadu liru, ngara liru; WS dìkira, dagha lára; ES hayìndu, hunani, kahindu; Si 'ai aning; Sl nadé; Kd namo II.

Malvac. $\quad$ Sida subcordata: $\mathrm{Km}$ ara ndaé.

Celastr. Siphonodon celastrineus: Ed oja kapa.

Smilac. Smilax zeylanica: $\mathrm{Kp}$ wanar; $\mathrm{Wr}$ wanar; $\mathrm{Rz}$ wanar; $\mathrm{Rm}$ karot dongkong; $\mathrm{Rg}$ wana, kénga; $\mathrm{Ng}$ nata wolo, nilu II; Ed ?wana.

Papil. Smithia: WS kàti káka.

Solan. $\quad$ Solanum: Bm kadui rui, kanturu; FEM (Bé) toro mboré, (Ri, Mb) toro wura, (Ri) toro tous, (To) karot dongkong; Kp toro tok, toro wura; $\mathrm{Wr}$ toro bapu'; $\mathrm{Rz}$ toro wura; $\mathrm{Rm}$ toro tok, toro wura; $\mathrm{Rg}$ mbara polo; $\mathrm{Ng}$ bara ba'i, bara karo, benga, li'é bara, toro karo; $\mathrm{Na}(\mathrm{Bw})$ doso ga; Ed mberi ré'é II; Li toro pisi; Pl d'ou; WS 'kapala kura; toro bongga, toro ka'mauta, toro pòdu; ES turu 'paita, kanduru 'paita, ndùla; kandúru ahu; Si wawi toron; Sl wawé toron I; kelupu, lebok, lolô kebakô; Kd lola 'otan; ruha.

Solan. Solanum erianthum: Rg denga; Ed mberi ré'é III.

Solan. Solanum indicum: Ed mberi ré'é I.

Solan. $\quad$ Solanum melongena: Bm kadui (na'é), kadui dolu janga; FEM toro; Kp toro; $\mathrm{Wr}$ toro'; Rz toro; Rm toro; $\mathrm{Ng}$ bara I, bara(-kedhi), toro II, woni; Na dhoso; Ké dhoso; 
Ed mberi; Li mberi, mberi wula, toro; Pl mberi; WS toro; ES kandúru, toru, túru; Sw têré; Si toro I; Sl ketoro, toro'; Kd bola.

Solan. $\quad$ ?Solanum nigrum: Kp raun kenti; $\mathrm{Rm}$ kenti; $\mathrm{Ng}$ bolé kogo III, soi I; hoi.

Solan. $\quad$ Solanum torvum: Bm kadui ?lako; Km turu deki'; Kp toro wura; Wr toro' bapu'; Rz toro bapu'; Rm toro bapu'; Rg mbara; WS toro padu; ES ?toro wùli; Sw teré edu.

Solan. Solanum tuberosum: ES katàbi jawa; Si tuka wolanda/wawa; Sl kentang, uwé wolanda.

Solan. Solanum verbascifolium: Bm kadui ?tarende; Rm teras.

Aster.

Sonchus: Rm rénggong mézé'; Ng kigo wio II, ?susu penu II.

Sonner. $\quad$ Sonneratia alba: Bm paropa, ropa; $\mathrm{Km}$ ?paropa; Sw boa; Sl tubé.

Poac.

Sorghum (nitidum): Si 'watar gahar; Kd wahén.

Poac.

Sorghum plumosum: ES mapu múru kapuka.

Poac.

Sorghum ?propinquum: Kp kolo; $\mathrm{Rm}$ ferat; $\mathrm{Ng}$ helé I, ngili, pepa, selé; Ed éko rako; Li lité II; WS ròbo; ES kamàla 'wataru, 'maripu; Si 'lité, ?rité; Sl ?wata' blolong hokéng.

Poac. $\quad$ Sorghum saccharatum: Bm latu, latu séra; Km boka; FEM (To) pezak, (Bé) latun rakot, $(\mathrm{Ri}, \mathrm{Ml})$ sela rakot, (Ls, $\mathrm{Mb}$ ) latung, $(\mathrm{Nn})$ sela pici; $\mathrm{Kp}$ peja; Wr lépang; $\mathrm{Rz}$ lépang; Rm pangin rakot, (Mbawar) metos, (Wu) ké'o rakot, (Wk) ké'o rakat; $\mathrm{Rg}$ lépa; $\mathrm{Ng}$ haé léwa, jawa I, pangi, saé léwa, jawa; $\mathrm{Na}(\mathrm{Ra})$ 'olo wolo; Ké yolo léwa; Ed oro; Li lolo; PI lolo, wad'a; WS 'watara pi'a, 'wataro pìyo; ES 'wataru hàmu ('unjuku), 'wataru wuli; Sw téraé hawu; Si dela, watar gahar, watar II; Sl wata belolo, wata' blolo'ng, kwaru olot, wata solot; Kd watar holo'.

Bignon. $\quad$ Spathodea companulata: Ed ?kesi éndé; Sl réo belanda.

Orchid. Spathoglottis cf. plicata: Ng bhogi bhéka; Li nio ata mata; Sl bahik.

Aster. Spilanthes grandiflora: WS ka'madala ndara.

Aster. $\quad$ Spilanthes iabadicensis: FEM (To, Bé, Ri) méa, (Ls, Nn, Mb) mé'a; Kp mé'as; Wr rukut mé'ar; $\mathrm{Rz}$ mé'as; $\mathrm{Rm}$ mé'a; $\mathrm{Rg}$ mé'a; $\mathrm{Ng}$ mé'a II; $\mathrm{Na}$ (Tm, Bw) mé'a; Li mé'a II; WS ka'madala; Si soro wowo; Sl 'alér I, ke'alé.

Poac. Spinifex littoreus: Bm garanji; Km nggalnggoang, nggele-nggoang; ES landusa, ka'pidihu; Sl segé laâ; Kd 'uru tahi'.

Anac. Spondias dulcis: Bm kedondo; Km kedondong; Ng kedondo; WS ende dáwa, ede dawa, dinjo dawa; ES inju jawa, ede I, injungu; Sw 'ehi; Sl kedondô I.

Anac. $\quad$ Spondias malayana: Bm inci; Km leséng; FEM (Ls) lecong, (Nn) lecéng, (Mb) leséng; Rm (Mbajang) ?leson; Rg indo; Li lesa; Pl lé; WS ende, ede II, dinjo; ES iji, ?inju, ?injungu; Si lahan(g); Sl éhẻ, leséng, ?kedondô II; Kd léhéng.

Verben. $\quad$ Stachytarpheta indica: Kp lasu ular; Wr lasu' ular; Rg mépo I; ES kawúru wàla, halurutu II; Sw kunu wa; Si lain tuhun; Sl ?boru.

Stemon. ?Stemona: Ng tuki toko I; Li monda réé; Sl kolê. 
Icac.

Menisp.

Menisp.

Stercul.

Stercul.

Stercul.

Sterculiaceae:

Morac.

Morac.

Acanth.

Logan.

Logan.

Simar.

Sympl.

Sympl.

Aster.

Myrtac.

Myrtac.

Myrtac.

Myrtac.

Myrtac.

Myrtac.

Myrtac.

Myrtac.
Stemonurus: Ed saka.

Stephania: $\mathrm{Ng}$ suza ré'é; Ed toko monda.

Stephania japonica var. (timorensis): Ng telo; WS pupu; ES ?kambu lawora II.

Sterculia: Ed sawo kaka II.

Sterculia foetida: Bm ?léu, wuwu; Km woh, wos; FEM (Bé, Ri, Ls, Nn) wol, (Mb) wor; Kp wuwur, ?paka; Wr paka; Rz paka; Rm wuwur, (Tr, Wk) wol; Rg paka; Ng paka, wuwu; Ed wuwu; Li wuka; Pl wuwu; WS kapaka, mata api; ES ka'lumbangu, ka'yumbangy, kapaka; Sw képaka; Si wukak; Sl wésér II, wukak, kepo, képoh, owa; Kd wua' (wu'a).

Sterculia oblongata: Bm feli; Km nita'; FEM (To) kontos, (Ls) ?matu; Kp litat; Wr kagok; Rz litat; Rm konto', kontos, (Wk) koto'; Rg hanggo; Ng hago; Ed 'ago; Li hago, kago; Si nita; Sl nitat; Kd nita'.

\section{WS ?langgétu I.}

Streblus: Ng wulu I; tara 'kaito I.

Streblus asper: WS pèdi nggángga.

Strobilanthes (cp. Sericocalyx): Kp tepu; Rm tepu; WS kamara II, meraka.

Strychnos: FEM saré maté; Kp soro maté; Rm saré maté.

Strychnos lucida: Bm songga.

Suriana maritima: Sw beku.

Symplocos: $\mathrm{Ng}$ lobha.

Symplocos cochinchinensis (ssp. philippensis): $\mathrm{Kp}$ lait; $\mathrm{Wr}$ lait; $\mathrm{Rz}$ lait; $\mathrm{Rm}$ lait; $\mathrm{Ng}$ lai.

Synedrella nodiflora: Li mé'a I; WS taiko sapi I.

Syzygium a): Bm ?bohobangi, ?langgua, libi bura, libi itam, monggo; FEM (Bé) baka kolong, (Ri) bakar kolong; lokon, lokong; lokom pong; lokon ghan; més; Kp lokon; Wr lokon; Rz lokon; Rm lokon, kolong, baku kolong; més; Rg loko; $\mathrm{Ng}$ loko; Ed baru, lalu mité, lawi éré, luku, ruku singgi mité, taro; Li singgi; WS wasu we'e, ghazu we'e; ES poda I, púnda; tàda muru, kamíti II; Si lalu, berungun, rao; Sl wai, beluwak, ulu kuma, katué.

Syzygium b): Rg loko waé; $\mathrm{Ng}$ rada, rengo, rengo waé; Li balu, luku réé; $\mathrm{Si}$ bilong; Sl bilong, jambulan, balu, naka uta, tima, ?belo, numo II.

Syzygium aqueum: Ng luku, pau téo II; Li luku; Si 'lubér.

Syzygium aromaticum: Bm cangké; Si singké; Sl sengké.

Syzygium cumini: Bm duwé; Kp ?lokon ghan; $\mathrm{Wr}$ ?lokan ghan; $\mathrm{Rm}$ ?lokan ghan. Syzygium jambos: Sw lub'a raé dou.

Syzygium (lineatum): WS lobungu II, labungo; ES lobungu, labungu, 'lobo.

Syzygium zeylanicum: $\mathrm{Km}$ ?nggulung. 
Apoc.

Taccac.

Taccac.

Taccac.

Portul.

Caesalp.

Rubiac.

Rubiac.

Polyp.

Polyp.

Verben.

Papil.

Combr.

Combr.

Combr.

Combr.

Dillen.

Datisc.

Vitac.

Vitac.

Poac.

Poac.

Poac.

Poac.

Poac.

Stercul.
Tabernaemontana orientalis: WS ríti kàngga, kangga ríti; Sw péna'i ki'i.

Tacca: $\mathbf{N g}$ ?tiré.

Tacca leontopetaloides: $\mathrm{Bm}$ ?kacunda; $\mathrm{Rm}$ ?lola.

Tacca palmata: ES langgúdu, ?langgódo I.

Talinum triangulare: Ng uta kupa; Ed nasi, sasi; Si sayur kupang.

Tamarindus indica: $\mathrm{Bm}$ manggé; $\mathrm{Km}$ doli, duli; FEM maki; Kp maki; Wr nanggé; $\mathrm{Rz}$ nanggé; $\mathrm{Rm}$ maki', (Tr) maki; $\mathrm{Rg}$ nanggé; $\mathrm{Ng}$ nagé I; $\mathrm{Na}(\mathrm{Ra})$ nagé; Ed magi, nanggé; Li magé; Pl malé; WS kása I, káza; ES kamàru, àmba, kamàru, kameru, kaha; Sw hélag'i; Si magé; Sl tobi; Kd tamal.

Tarenna: Bm sala/i moto.

Tarenna incerta: Ed kata roko, toko mbako, wunu gaga.

Tectaria crenata: Ed paku manu II.

Tectaria siifolia: $\mathrm{Ng}$ logo teké; Ed paku manu I.

Tectona grandis: Bm jati; FEM jati, (Ri) zati; Wr zati; $\mathrm{Rz}$ zati; $\mathrm{Rm}$ zati; $\mathrm{Na}(\mathrm{Ra})$ jati; WS njati; Sl jati; Kd jati.

Tephrosia noctiflora: Rg taru kala; ?Kd taung otan.

Terminalia: Ed wara II; Sl ipek.

Terminalia catappa: $\mathrm{Bm}$ sarisé; $\mathrm{Km}$ ?ketapang; $\mathrm{Rm}$ menggé; $\mathrm{Ng}$ ketapa, koja; Ed isa II; ES talíhu; Sw katapa nga'i; Si lihé, llihé; Sl keliha, ketapâ; Kd liha.

Terminalia 'mollis': Ed sawokaka I.

Terminalia zollingeri: Bm sarisé doro; Km menggé; Si ipak.

Tetracera scandens: Kp ?legi rusa; Wr kazék pelan; Ed pendi, ?penggi; Li pedi; S1 pi'a.

Tetrameles nudiflora: Bm bara II.

cf. Tetrastigma: Km mépéh; Wr kazék lesa'; Ng bié, koba katé; Li katé; WS kenda kabela; dungga, kapopóko; ES kámbu lawora I; 'mulungu.

Tetrastigma papillosum: FEM (Ml) ndawang; Kp dawan; Wr kazék dawan; Rz ndawan; Rm dawan; WS tambèdala I.

Themeda: WS kamàngge rewa II, matu nuhi I; ES mapu.

Themeda arguens: Km satah.

Themeda imberbis: ES mapu penji.

Themeda intermedia: $\mathrm{Km}$ satah wakah.

Themeda villosa: FEM waka, (To) wakas, (Bé) wakos, (Ri) wakot; $\mathrm{Kp}$ wakas; $\mathrm{Wr}$ wakar; Rz wakas; Rm fakat, wakat, (Wu, Wk) waka; Rg wako; Ng fao wako, wako I; Ed gai; Li sawu II; WS nalo; ES nara; Sw nara; Si bawa latang II; Sl lalat.

Theobroma cacao: FEM (Bé) cokelat; Rm sokelat; Sl coklat. 
Malvac. $\quad$ Thespesia populnea: Bm wau moti; $\mathrm{Km}$ mbeloti('); Rm waru tacik; Li waru jawa I; Sw mangi (?mengi).

Poac. $\quad$ Thysanolaena maxima: FEM (To) sengkar, (To, Ml) ké'o, (Bé, Ri, Nn) ketus; Kp riung; Wr mbajik; Rm sengkar, (Wu) ké(')o-ketus, ( $\mathrm{Tr}, \mathrm{Wk}$ ) ketus; $\mathrm{Ng}$ wizu; Li bheto nitu, gai kiu, nggaju kiu; Sl tengal.

Rubiac. Timonius timon: Wr kuér; Rm kuér; $\mathrm{Rg}$ kué; $\mathrm{Ng}$ tasi, upé; $\mathrm{Na}(\mathrm{Ra})$ ?tasi; Ed upé; WS timo, timu, ?timbu, ndimu; ES nggaï, timu; Sl timu.

Menisp. Tinospora: $\mathrm{Ng}$ ?tuka kutu.

Aster.

Tithonia diversifolia: FEM (Bé, $\mathrm{Ri}, \mathrm{Mb}$ ) bunga mata hari, (Ls) mata leso mésé, (Ml) bunga; Kp bunga matahari; Rm bunga matahari, (Wk) bunga mata lezo; $\mathrm{Ng}$ bunga ba'i I, wonga ba'i II, wonga bunga; Li bunga ba'i.

Rutac. $\quad$ Toddalia asiatica: Kp karot kalo; Rm ra'an teké'; Ng gi, teké; Ed nigi sogi; WS tara kuku mète.

Meliac. $\quad$ Toona sureni (ciliata): Bm mengi; FEM (Bé, Ls, Mb) ojang, (Ri, Nn) ozang, (Ml) 'ozang; Kp ajang; Wr azang; Rz azang; Rm azang, (Tr, Wu) ozang; Rg oja; Ng oja, oza; Na (Ra) oja; Ed jawé I; Li oja; Pl 'aca; WS kasósa, kazóza, ?linu III, ?karánu, huranu; ES huréni, hurani, surani, huranu; Si ara nana, 'én(g); Sl nahi ajâ, jajang, suréng, aurarâ; Kd maha' 'ayang.

Borag. Tournefortia: Ng wonga wau; Ed wora mbata.

Borag. Tournefortia argentea: Bm ladu ?boling.

Asclep. Toxocarpus: Ed dhoi kamba.

Ulmac.

Trema (cannabina): Sl blurat, werura.

Ulmac.

Ulmac.

Trema orientalis: FEM (Ri) derong, (Ls, Nn) redong, (Mb) redhong; Kp derong; Wr derong; Rz derong; Rm derong, ( $\mathrm{Tr}$ ) dhéo/?ndéo; $\mathrm{Rg}$ dero, minggé; $\mathrm{Ng}$ déo, loto toro III; Ed dhéo; Li déo; WS ka'poraka, kapàraka; ES kapàra, kapàraku.

Hymen. $\quad$ Trichomanes: Kp kedér; $\mathrm{Rm}$ kedér; $\mathrm{Ng}$ wua nua; Li néro.

Cucurb. Trichosanthes: Sl lima pait.

Cucurb. Trichosanthes anguina: Si salur.

Cucurb. $\quad$ ?Trichosanthes villosa: Km kedangka; FEM (To) pelas, (Ls) ghelas kamba, (Nn) kebok, (Mb) kebhok; Kp suda; Wr suda; Rm suda, (Wk) pelas, (Tr) tawu réwa; Rg timu ka; Ng bési solo, ghubu I, kuwu; Ed kuwu; WS taghu réda, kalaho; ES kapu ndala II; Sl kela' utâ.

Aster. $\quad$ Tridax procumbens: Ed toko retu; Sw pudi wila; Sl kuda ta'in; Kd kiri' tahi'.

Sapind. $\quad$ Tristiropsis canarioides: Sl tenai, mara.

Tiliac. $\quad$ Triumfetta (cp. Urena lobata): Kp maso; Wr nilur; Rm kutan; Rg pele ki'a, pulu II; $\mathrm{Ng}$ pulu II, pulu haki; Ed puru I, puru II; Li hai latu, pulu II; Sw ?pulu téi. 
Tiliac.

Staphyl.

Asclep.

Typhac.

Ulmac.

Rubiac.

Papil.

Malvac.

Urticaceae:

Annon.

Ericac.

Orchid.

Aster.

Aster.

Aster.

Aster.

Aster.

Aster.

Caprif.

Papil.

Papil.

Triumfetta ?rhomboidea (cp. Urena lobata): FEM (Bé, Ri, Ls, Mb) kempo rona; Kp sigir; Wr ?sepot; Rz sigir; Rm kempo', (Wn) kepo'; Rg pulu II, pulu haki; ES ka'poroto II; WS kapohaku mini II, ?ka'pirihu; Si klorot; Sl karorot (klorot), kloro; Kd ?mai.

Turpinia (sphaerocarpa): Ed aé, kua III; Li tepe déké.

cf. Tylophora sp.: Rm bansul; Sl kenobak.

Typha angustifolia: Bm kabisa na'e; FEM (Bé) woja onang; Ng ngagha ai, paré nitu, wako waé.

Ulmus lanceaefolia: Ed namu; Li namu.

Uncaria gambir: WS nggambiryo; ES 'nggamuru, 'nggambira, 'gabiru; Si gambér; Sl gabe.

Uraria lagopodioides: Rg éko lako; $\mathrm{Ng}$ ?weka II.

Urena lobata: $\mathrm{Km}$ puduk; FEM kempo, (Ls, Ml) pulut, (Nn) kempo wina; $\mathrm{Kp} \mathrm{litot;}$ Wr ta'ing koa'; Rm kempo', kepo'; Rg pulu I, pulu fai; $\mathrm{Ng}$ pulu I, pulu fai; $\mathrm{Na}$ $(\mathrm{Bw})$ pulu moka, $(\mathrm{Ra})$ pulu; Ed puru fai; Li pulu I; WS ka'poroto I; ES ka'pohaku I, kaporatu, ka'pohaku bai, kaporeta; Si ?pulu watu; Sl kuda klorot, kepulut.

Rm rama, lantong wazé', (Tr) lantong wazék; $\mathrm{Ng}$ zama dhéké, wiku waé I, kazu zama; $\mathrm{Na}(\mathrm{Tm})$ koba ladé; Li mata ndoro.

Uvaria (rufa): $\mathrm{Km}$ lékéng; Wr lékéng kazék; Rz kazék lékéng; $\mathrm{Rg}$ lésé; $\mathrm{Ng}$ ?lésé; $\mathrm{Ed}$ ?ragé; Li kara II; Pl léké; WS leke, wu maleke; ES alaku, jàla 'alaku, alaka; Si manu; Sl la'ak, léké; Kd laku dara'.

Vaccinium timorense: Li aré ngoni, kaba anak ko'o.

Vanda: Ng koré IV.

?Vernonia: $\mathrm{Ng}$ baka koké, baka mité, bhara; Ed bu boré; Li ta'i wawi; $\mathrm{Sw}$ té.

Vernonia arborea: Kp dewo; $\mathrm{Rm}$ dewo; $\mathrm{Ng}$ kéu III.

Vernonia cinerea: $\mathrm{Rg}$ sapu; $\mathrm{Ng}$ bungé api, taga ko, uza zé'é; Ed ta'i manu; $\mathrm{Si}$ klotong; Sl klotô, bési kélé.

Vernonia cinerea var. parviflora: FEM (To) sapu, (Bé, Ri, Mb) rawang tana, (Ls, $\mathrm{Ml)}$ rawang tana'; Kp reman sapu; Wr sapu; Rm rawan tana', (Tr, Wu, Wk) rawang tana'; Sw wila ona.

Vernonia moluccensis: ES wàla óna II.

Vernonia wetarensis: WS tùdu lawa, ka'tunggu lawa; ES tandala pua.

Viburnum sambucinum: FEM (Bé) timung, (Ri) timun, (Ml) ghazu timung, (Mb) ghaju timung; Rm (kazu) timun; $\mathrm{Ng}$ ghésa, késa; Li muku té'a III; WS katunggu láwa, tùdu lawa; ES tandala pua.

Vigna: Kd la' loran.

Vigna unguiculata: Bm ḅué; FEM wuék, (Ml) kembar; Kp tago'; Wr ?lolé; $\mathrm{Rz}$ kembar; Rm tago', (Ri) wué, (Tr) sampo, (Wu) mbué, (Wk) wuék; Rg hobo naké, 
tanggo; Ng heba, hobho I, hobho azé, hobo, obho, sobho; $\mathrm{Na}(\mathrm{Tm})$ bué, (Bw) sobho, $(\mathrm{Ra})$ bué; Li bué rako, bué uta; $\mathrm{Pl}$ wéwé; WS kàmbé lólo; $\mathrm{Sw}$ kebui aé; $\mathrm{Si}$ wéwé; wéwé glonak; Sl utâ kenolê, utâ niha'an, utâ; Kd 'utan rian.

Urtic. Villebrunea: $\mathrm{Ng}$ paka do III.

Vitaceae:

$\mathrm{Ng}$ (koba) bhara; saru walu; Li kuku mbeku I; WS timu tèke; ES pelu; Sl klaru méra.

Verben.

Vitex paniculata: Sl keménî.

Verben.

Vitex parviflora: ES kulá.

Verben.

Vitex pubescens: $\mathrm{Bm}$ pampa; Km pampa; Wr mbapa'; Rg mbapa; WS katilu kawìmbi; ES ?kiketaru.

Verben. Vitex trifolia: Bm sangari; FEM (To) ghui, (Bé) karkaja, (Ri) ata, (Ls, Ml) repot, (Nn, Ri) kaju ata; Kp ghui; Wr woi; Rz kaju ata; Rm kazu ata, (Tr) wora; Ng ngela, pépi I, séko si'é II; Na (Tm) ghela; Ed éko ndé'u; Li langga; ES kalali ngangu; SI tonê I.

Verben. $\quad$ Vitex trifolia var. littoralis: Sw kalai ngela bangi.

Vitac.

Vitis vinifera: $\mathrm{Li}$ anggo II; $\mathrm{B}$, anggo; $\mathrm{Si}$ ?atar; $\mathrm{Sl}$ anggor.

Apocyn. Voacanga grandifolia (cp. Pagiantha): Bm wudu jara; Km sepada; FEM (To, Bé, Ls, $\mathrm{Nn}$ ) boto, (Ls) paja, (Mb) bhoto; Kp gaka' loé', ?lui lembak; Wr gaka' ?winar; Rz gaka'; Rm gaka', (Tr) boto; WS karimboyo; ES wota kamàmbi II, wota kamemi, wota kamembi.

Aster. Wedelia (biflora): Ed runu jawa; Sl unu ale, kikê; Kd kiri' ingi, kiringo.

Aster. Wedelia ?urticaefolia: FEM runu, (To) runus; $\mathrm{Kp}$ runus; $\mathrm{Wr}$ runus; $\mathrm{Rm}$ runu; $\mathrm{Rg}$ runu; $\mathrm{Ng}$ runu; $\mathrm{Na}(\mathrm{Tm})$ runu; Ed runu; Li runu, wonga runu; WS malere; $\mathrm{ES}$ tandùla pua II, ?malere; Si runu; Sl 'aler II, unu.

Cunon. Weinmannia: Li ana wéa.

Cunon. Weinmannia blumei: WS móro longge II.

Rubiac. Wendlandia: FEM (Ls, Nn, Mb) mondo; Kp mondo(ng), wodong; Wr wodo'; Rm mondong; $\mathrm{Na}(\mathrm{Tm})$ kesi; Ed kangga, kesi tana; WS katilu simbi; Sl klikar.

Scroph. Wightia borneensis ssp. ottolanderi: Rm naru.

Thymel. Wikstroemia androsaemifolia: S1 klikar.

Lythrac. Woodfordia fruticosa: WS wagha.

Apocyn. Wrightia: Kd liti.

Apocyn. Wrightia (pubescens): Km niti; ES 'hubuku; Sw alo.

Arac. Xanthosoma nigrurn: $\mathrm{Ng}$ talé dungu, talé wolo; $\mathrm{Na}(\mathrm{Tm})$ pangi.

Olac. Ximenia americana: Km ntimi.

Meliac. Xylocarpus granatum: $\mathrm{Km}$ haju sia.

Meliac. Xylocarpus moluccensis: Km butung. 
Aster. Youngia: Ed kere kéba haki.

Poac. Zea mays: Bm jago; FEM sela, (To) latun; Kp latun; Wr kadéa'; Rz kedéa'; Rm pangin, (Tr) kéo, (Ri, Wu, Wk) ké'o; Rg zawa; Ng haé, ho, jawa II, pangi jawa, saé; Na jawa, holo, ’olo, ké'o, ké'o jawa; Ké yolo; Ed jawa, oro jawa; WS 'watara; ES 'wataru, ka'mborungu, ka'mburungu; Sw téraé (j'awa); Si lelé, watar II, aja, ajawa; Sl wata, wata', kwaror, fata; Kd watar.

Zingib. $\quad$ Zingiber: FEM (Bé) mas, (Ls) lia rengga; $\mathrm{Rm}$ suduk; $\mathrm{Rg}$ léa naro; $\mathrm{Ng}$ léa kaba, léa kogha, léa manu, léa naro, léa rega III.

Zingib. Zingiber aromaticum: Bm kampuja.

Zingib. Zingiber officinale: Bm réa; Km lia; FEM lia; Kp léa; Wr léa; Rz léa; Rm léa, (Tr) lia; Rg léa; $\mathrm{Ng}$ éa, léa; $\mathrm{Na}(\mathrm{Tm})$ léa, $(\mathrm{Ra})$ léa; Ké déa; Li léa; Pl léa; WS lisa, liza; ES ?ladi, wàla lia, layia; Sw kelia; Si léa; Sl lia'; Kd lié.

Zingib. Zingiber purpureum: Bm ḅanggulae.

Zingiberaceae a): FEM (Bé, Ri) suduk, (Ls) dagu dak; Rm suduk; géang, mbuza, (Wk) dagu dak; $\mathrm{Ng}$ séra toro; $\mathrm{Na}(\mathrm{Tm})$ wélo; $\mathrm{Si}$ wai talo, 'ai ta'i; Sl kéwuk.

Zingiberaceae b): FEM (To, Ri, Bé) lia asu, (Nn, Ls) lia wakar, (Ml) lia acu; Kp léa kodé'; Rm léa ka'o, léa naron, (Wu) léa ka'o, (Wk) léa naron; WS tòmu, tàmu.

Rhamn. $\quad$ Ziziphus: Km lengkang moné; Rg lui, ?ngnaga; ES wunga II; Sl babesi.

Rhamn. Ziziphus celtidifolia: Bm sara'a.

Rhamn. Ziziphus horsfieldii: Km kalangka.

Rhamn. Ziziphus nummularia: Bm rangga; Km lengkang; WS tára kaito II; ES kalangga I; Sw bidara, ko; Si bidara, pindara; Sl darat, bidara; Kd bu'ér.

Rhamn. Ziziphus 'talanai': Sl babesi putih.

Rhamn. Ziziphus timoriensis: Sl kelomo II. 


\section{GROUPS AND FAMILIES WITH GENERA}

\section{ACANTHACEAE}

Andrographis

Asystasia

Barleria

Dicliptera

Eranthemum

Gendarussa

Hedychium

Hemigraphis

Hypoestes

Lepidagathis

Peristrophe

Pseuderanthemum

Sericocalyx

Strobilanthes

ACERACEAE

Acer

AGAVACEAE

Agave

AIZOACEAE v.

Molluginaceae

Ficoidaceae

ALANGIACEAE

Alangium

ALGAE

Eucheuma

Padina

Sargassum

AMARANTHACEAE

Achyranthes

Allmannia

Altemanthera

Amaranthus

Celosia

Cyathula

Deeringia

Pupalia
AMARYLLIDACEAE

Allium

ANACARDIACEAE

Anacardium

Buchanania

Dracontomelon

Lannea

Mangifera

Rhus

Semecarpus

Spondias

ANNONACEAE

Annona

Cananga

Orophea

Phaeanthus

Uvaria

APIACEAE

(UMBELLIFERAE)

Apium

Centella

Coriandrum

APOCYNACEAE

Alstonia

Alyxia

Catharanthus

Cerbera

Chonemorpha

Ervatamia

Ichnocarpus

Pagiantha

Plumeria

Rauvolfia

Tabernaemontana

Voacanga

Wrightia

AQUIFOLIACEAE

nex
ARACEAE

Acorus

Alocasia

Amorphophallus

Colocasia

Homalomena

Pothos

Remusatia

Raphidophora

Scindapsis

Xanthosoma

ARALIACEAE

Aralia

Gastonia

Harmsiopanax

Polyscias

Schefflera

ARECACEAE (PALMAE)

Areca

Arenga

Borassus

Calamus

Caryota

Cocos

Corypha

Daemonorops

Livistona

Nypa

Phoenix

Pinanga

Roystonea

Salacca

ARISTOLOCHIACEAE

Aristolochia

ASCLEPIADACEAE

Asclepias

Calotropis

Ceropegia 
Cynanchum

Dischidia

Dregea

Finlaysonia

Gymnanthera

Heterostemma

Hoya

Marsdenia

Toxocarpus

Tylophora

ASTERACEAE

(COMPOSITAE)

Acanthospermum

Ageratum

Anaphalis

Bidens

Blumea

Cosmos

Eclipta

Elephantopus

Emilia

Erechtites

Erigeron

Eupatorium

Galinsoga

Gynura

Mikania

Moonia

Pluchea

Pterocaulon

Sonchus

Spilanthes

Synedrella

Tithonia

Tridax

Vernonia

Wedelia

Youngia

BALSAMINACEAE

Impatiens
BEGONIACEAE

Begonia

BIGNONIACEAE

Crescentia

Dolichandrone

Millingtonia

Oroxylum

Radennachera

Spathodea

BOMBACACEAE

Bombax

Ceiba

Durio

BORAGINACEAE

Carmona

Cordia

Ehretia

Heliotropium

Tournefortia

BRASSICACEAE

(CRUCIFERAE)

Nasturtium

Raphanus

Rorippa

BROMELIACEAE

Ananas

BUDDLEJACEAE v.

Loganiaceae

BURSERACEAE

Canarium

Garuga

Protium

BUTOMACEAE

Limnocharis

CACTACEAE

Opuntia

Pereskia
CAESALPINIACEAE (LEGUMINOSAE)

Bauhinia

Caesalpinia

Cassia

Cynometra

Intsia

Lysiphyllum

Mezoneuron

Peltophorum

Piliostigma

Tamarindus

CANNACEAE

Canna

CAPPARACEAE

Capparis

Cleome

CAPRIFOLIACEAE

Sambucus

Viburnum

CARICACEAE

Carica

CARYOPHYLIACEAE

DTymaria

Polycarpaea

CASSYTHACEAE v.

Lauraceae

CASUARINACEAE

Casuarina

CELASTRACEAE

Euonymus

Siphonodon

CHENOPODIACEAE

Salsola

CLUSIACEAE

(GUTTIFERAE)

Calophyllum 


$\begin{array}{lll}\text { Garcinia } & \text { Gymnopetalum } & \text { ERICACEAE } \\ \text { Mammea } & \text { Lagenaria } & \text { (VACCINIACEAE) } \\ \text { COMBRETACEAE } & \text { Luffa } & \text { Vaccinium } \\ \text { Combretum } & \text { Melothria } & \text { EUPHORBIACEAE } \\ \text { Lumnitzera } & \text { Momordica } & \text { Acalypha } \\ \text { Terminalia } & \text { Mukia } & \text { Aleurites } \\ \text { COMMELINACEAE } & \text { Sechium } & \text { Antidesma } \\ \text { Aneilema } & \text { Trichosanthes } & \text { Bischofia } \\ \text { Commelina } & \text { CUNONIACEAE } & \text { Breynia } \\ \text { Cyanotis } & \text { Weinmannia } & \text { Bridelia } \\ \text { Pollia } & \text { CYATHEACEAE } & \text { Cladogynos } \\ \text { COMPOSITAE v. } & \text { Cyathea } & \text { Claoxylon } \\ \text { Asteraceae } & \text { CYCADACEAE } & \text { Cleidion } \\ \text { CONIFERAE v. } & \text { Cycas } & \text { Cleistanthus } \\ \text { Podocarpaceae } & \text { CYPERACEAE } & \text { Croton } \\ \text { CONNARACEAE } & \text { Carex } & \text { Doryxylum } \\ \text { Rourea } & \text { Cyperus } & \text { Drypetes } \\ \text { CONVOLVULACEAE } & \text { Eleocharis } & \text { Euphorbia } \\ \text { Hewittia } & \text { Fimbristylis } & \text { Excoecaria } \\ \text { Ipomoea } & \text { Hypolytrum } & \text { Glochidion } \\ \text { Merremia } & \text { Rhynchospora } & \text { Homalanthus } \\ \text { Operculina } & \text { Scirpus } & \text { Jatropha } \\ \text { CORNACEAE } & \text { Scleria } & \text { Macaranga } \\ \text { Mastixia } & \text { DATISCACEAE } & \text { Mallotus } \\ \text { CRASSULACEAE } & \text { Tetrameles } & \text { Manihot } \\ \text { Kalanchoe } & \text { DILLENIACEAE } & \text { Melanolepis } \\ \text { CRUCIFERAE v. } & \text { Dillenia } & \text { Pedilanthus } \\ \text { Brassicaceae } & \text { Tetracera } & \text { Phyllanthus } \\ \text { CRYPTERONIACEAE } & \text { DIOSCOREACEAE } & \text { Pterococcus } \\ \text { Crypteronia } & \text { Dioscorea } & \text { Ricinus } \\ \text { CUCURBITACEAE } & \text { EBENACEAE } & \text { FICOIDACEAE } \\ \text { Benincasa } & \text { Diospyros } & \text { Sesuvium } \\ \text { Citrullus } & \text { ELAEOCARPACEAE } & \text { FLACOURTIACEAE } \\ \text { Coccinia } & \text { Elaeocarpus } & \text { Flacourtia } \\ \text { Cucumis } & \text { EQUISETACEAE } & \text { Homalium } \\ \text { Cucurbita } & \text { Equisetum } & \text { Flarosa } \\ \text { Diplocyclos } & & \text { Flagellaria } \\ & & \\ & & \end{array}$


FUNGI

Agaricales

Auriculariaceae

Daedalea

Gasteiomycetes

Himeola

Lenzites

Microporus

'Polyporus'

Pycnoporus

Schizophyllum

Xylaria

GENTIANACEAE

Canscora

Enicosternma

GESNERIACEAE

Cyrtandra

GLEICHENIACEAE

Dicranopteris

GNETACEAE

Gnetum

GRAMINEAE v.

Poaceae

GUTTIFERAE v.

Clusiaceae

HAMAMELIDACEAE

Distylium

HEPATICAE

HERNANDIACEAE

Gyrocarpus

HYDROCHARTTACEAE

Enhalus

Halophila

HYMENOPHYLLACEAE

Trichomanes
ICACINACEAE

Apodytes

Gomphandra

Platea

Stemonurus

JUGLANDACEAE

Engelhardia

LABIATAE v.

Lamiaceae

LAMIACEAE (LABIATAE)

Achyrospermum

Anisomeles

Coleus

Cuminum

Elsholtzia

Foeniculum

Hyptis

Lavandula

Leucas

Ocimum

Orthosiphon

Pogostemon

Salvia

Scutellaria

LAURACEAE

Cassytha

Cinnamomum

Cryptocarya

Litsea

Neolitsea

Persea

LECYTHIDACEAE

Barringtonia

Planchonia

LEGUMINOSAE v.

Caesalpiniaceae

Mimosaceae

Papilionaceae
LICHENES

Usnea

LILIACEAE

Aloe

Asparagus

Cordyline

Crinum

Pleomele

LOGANIACEAE

Buddleja

Fagraea

Geniostoma

Mitrasacme

Strychnos

LORANIHACEAE

Dendrophthoe

Scurrula

LYCOPODIACEAE

Lycopodium

LYTHRACEAE

Ammannia

Cuphea

Lagerstroemia

Lawsonia

Woodfordia

MAGNOLIACEAE

Magnolia

Michelia

MALPIGHIACEAE

Ryssopterys

MALVACEAE

Abelmoschus

Abutilon

Gossypium

Hibiscus

Sida

Thespesia

Urena 


\begin{tabular}{|c|c|c|}
\hline MARANTACEAE & MORACEAE & Pisonia \\
\hline Donax & Antiaris & NYMPHAEACEAE \\
\hline Maranta & Artocarpus & Nelumbo \\
\hline MELASTOMATACEAE & $\begin{array}{l}\text { Fatoua } \\
\text { Ficus }\end{array}$ & OLACACEAE \\
\hline Medinilla & $\begin{array}{l}\text { Ficus } \\
\text { Maclura }\end{array}$ & Ximenia \\
\hline $\begin{array}{l}\text { Melastoma } \\
\text { Memecvlon }\end{array}$ & Morus & OLEACEAE \\
\hline Memecylon & Paratrophis & Fraxinus \\
\hline Osbeckia & Streblus & Jasminum \\
\hline MELIACEAE & MORINGACEAE & Linociera \\
\hline $\begin{array}{l}\text { Algaia } \\
\text { Chisocheton }\end{array}$ & Moringa & ONAGRACEAE \\
\hline Dysoxylum & MOTACEAE & Ludwigia \\
\hline Lansium & Streblus & OPHIOGLOSSACEAE \\
\hline Melia & MUSACEAE & Ophioglossum \\
\hline Ricinocarpodendron & Ensete & OPILIACEAE \\
\hline Sandoricum & Musa & Cansjera \\
\hline $\begin{array}{l}\text { Toona } \\
\text { Xylocarpus }\end{array}$ & MUSCI & Champereia \\
\hline MFNISPFRMACFAF & MYRISTICACEAE & Opilia \\
\hline $\begin{array}{l}\text { MENISPERMACEAE } \\
\text { Anamirta }\end{array}$ & Knema & ORCHIDACEAE \\
\hline $\begin{array}{l}\text { Anamirta } \\
\text { Omphalea }\end{array}$ & Myristica & Arundina \\
\hline $\begin{array}{l}\text { Omphalea } \\
\text { Pericampylus }\end{array}$ & & Bulbophyllum \\
\hline Pericampylus & MYRSINACEAE & Calanthe \\
\hline Pycnarrhena & Aegiceras & Dendrobium \\
\hline Stephania & Ardisia & Peristylis \\
\hline Tinospora & Embelia & Pholidota \\
\hline MIMOSACEAE & Maesa & Spathoglottis \\
\hline (LEGUMINOSAE) & Rapanea & Vanda \\
\hline Acacia & MYRTACEAE & OXALIDACEAE \\
\hline Adenanthera & Decaspermum & Averrhoa \\
\hline Albizia & Eucalyptus & Oxalis \\
\hline Entada & (Eugenia) & PALMAE v \\
\hline Leucaena & Leptospermum & Arecaceae \\
\hline Mimosa & Melaleuca & Avecaleate \\
\hline Parkia & Osbomia & PANDANACEAE \\
\hline Pithecellobium & Psidium & Freycinetia \\
\hline Samanea & Syzygium & Pandanus \\
\hline MOLLUGINACEAE & NYCTAGINACEAE & PAPILIONACEAE \\
\hline (AZOACEAE) & Boerhavia & (LEGUMINOSAE) \\
\hline Mollugo & Mirabilis & Abrus \\
\hline
\end{tabular}


Aeschynomene

Alysicarpus

Arachis

Cajanus

Calopogonium

Canavalia

Centrosema

Christia

Clitorea

Crotalaria

Derris

Desmodium

Dolichos

Dumasia

Erythrina

Glycine

Indigofera

Inocarpus

Moghania

Mucuna

Pachyrrhizus

Phaseolus

Phylacium

Pongamia

Psophocarpus

Psoralea

Pterocarpus

Rhynchosia

Sesbania

Smithia

Tephrosia

Uraria

Vigna

PASSIFLORACEAE

Adenia

Passiflora

PEDALIACEAE

Josephinia

Martynia

Sesamum
PIPERACEAE

Piper

Pothomorphe

PITTOSPORACEAE

Pittosporum

PLANTAGINACEAE

Plantago

POACEAE (GRAMINEAE)

Apluda

Aristida

Bambusa

'Bambuseae'

Bothriochloa

Brachiaria

Capillipedium

Cenchrus

Centotheca

Chloris

Chrysopogon

Coelorachis

Coix

Cymbopogon

Cynodon

Cyrtococcum

Dactyloctenium

Dendrocalamus

Dichanthium

Digitaria

Dimeria

Dinochloa

Echinochloa

Eleusine

Eragrostis

Eriochloa

Eulalia

Gigantochloa

Hackelochloa

Heteropogon

Imperata

Isachne
Ischaemum

Neyraudia

Oplismenus

Oryza

Panicum

Paspalidium

Paspalum

Pennisetum

Phragmites

Pogonatherum

Rottboellia

Saccharum

Schizachyrium

Schizostachyum

Sehima

Setaria

Sorghum

Spinifex

Themeda

Thysanolaena

Zea

PODOCARPACEAE

(CONIFERAE)

Podocarpus

POLYGALACEAE

Polygala

POLYGONACEAE

Polygonum

POLYPODIACEAE

(sensu lato)

Acrostichum

Adiantum

Angiopteris

Asplenium

Belvisia

Christella

Diplazium

Dipteris

Drynaria

Dryopteris 


\begin{tabular}{|c|c|c|}
\hline Hypolepis & Borreria & SANTALACEAE \\
\hline Lecanopteris & Coffea & Exocarpus \\
\hline Nephrolepis & Gardenia & Santalum \\
\hline Phymatodes & Guettarda & SAPINDACEAE \\
\hline Platycerium & Hedyotis & Allophylus \\
\hline Pneumatopteris & Hydnophytum & Arytera \\
\hline Pteris & Hymenodictyon & Cardiospermum \\
\hline Pyrrosia & Ixora & Elattostachys \\
\hline Tectaria & Litosanthes & Ganophyllum \\
\hline PONTEDERIACEAE & Morinda & Harpullia \\
\hline Monochoria & Mussaenda & Mischocarpus \\
\hline & Myrmecodia & Nephelium \\
\hline $\begin{array}{l}\text { PUR TULACACEAE } \\
\text { Portulaca }\end{array}$ & Nauclea & Palaquium \\
\hline Portulaca & Neonauclea & Pometia \\
\hline Talinum & Oxyceros & Sapindus \\
\hline PROTEACEAE & Paederia & Schleichera \\
\hline Helicia & Pavetta & Tristiropsis \\
\hline PSILOTACEAE & Psychotria & SAPOTACEAE \\
\hline Psilotum & $\begin{array}{l}\text { Randia } \\
\text { Tarenna }\end{array}$ & Madhuca \\
\hline PUNICACEAE & Timonius & Manilkara \\
\hline Punica & Uncaria & Mimusops \\
\hline RANUNCULACEAE & Wendlandia & Planchonella \\
\hline Clematis & RUTACEAE & SARCOSPERMACEAE \\
\hline RHAMNACEAE & Acronychia & Sarcosperma \\
\hline Ziziphus & Aegle & SAURAUIACEAE \\
\hline RHIZOPHORACEAE & Citrus & Saurauia \\
\hline Bruguiera & Euodia & SAXIFRAGACEAE \\
\hline Ceriops & Glycosmis & Itea \\
\hline Rhizophora & Lunasia & Polyosma \\
\hline ROSACEAE & $\begin{array}{l}\text { Micromelum } \\
\text { Murraya }\end{array}$ & SCHIZAEACEAE \\
\hline Parinari & Toddalia & Lygodium \\
\hline Photinia & SABIACEAE & SCROPHULARIACEAE \\
\hline Prunus & $\begin{array}{l}\text { SABIACEAE } \\
\text { Meliosma }\end{array}$ & Lindernia \\
\hline Pygeum & & Wightia \\
\hline Rosa & SALICACEAE & SELAGINELLACEAE \\
\hline Rubus & Salix & Selaginella \\
\hline $\begin{array}{l}\text { RUBIACEAE } \\
\text { Anthocephalus }\end{array}$ & $\begin{array}{l}\text { SALVADORACEAE } \\
\text { Azima }\end{array}$ & $\begin{array}{l}\text { SIMARUBACEAE } \\
\text { Brucea }\end{array}$ \\
\hline
\end{tabular}


Harrisonia

Suriana

SMILACACEAE

Smilax

SOLANACEAE

Capsicum

Datura

Lycianthes

Lycopersicon

Nicotiana

Physalis

Solanum

SONNERATIACEAE

Sonneratia

STAPHYLEACEAE

Turpinia

STEMONACEAE

Stemona

STERCULIACEAE

Abroma

Byttneria

Dombeya

Helicteres

Heritiera

Kleinhovia

Melochia

Pterospermum

Pterygota

Sterculia

Theobroma

SYMPLOCACEAE

Symplocos

TACCACEAE

Tacca
THEACEAE

Adinandra

Eurya

Laplacea

THYMELAEACEAE

Gyrinops

Phaleria

Wikstroemia

TILIACEAE

Corchorus

Grewia

Microcos

Schoutenia

Triumfetta

TYPHACEAE

Typha

ULMACEAE

Celtis

Gironniera

Trema

Ulmus

UMBELLIFERAE v.

Apiaceae

URTICACEAE

Boehmeria

Broussonetia

Debregeasia

Dendrocnide

Elatostema

Gonostegia

Laportea

Leucosyke

Maoutia

Pilea

Pipturus
Pouzolzia

Villebrunea

VACCINIACEAE v.

Ericaceae

VERBENACEAE

Avicennia

Callicarpa

Clerodendrum

Duranta

Gmelina

Lantana

Premna

Stachytarpheta

Tectona

Vitex

VIOLACEAE

Hybanthus

VITACEAE

Ampelocissus

Cayratia

Cissus

Leea

Tetrastigma

Vitis

ZINGIBERACEAE

Alpinia

Amomum

Boesenbergia

Costus

Curcuma

Globba

Hedychium

Kaempferia

Languas

Zingiber 


\section{APPENDIX I: INFORMANTS}

I am pleased to give below the names of the men and women who kindly provided me with data about plants and their names in certain cultures and languages. Unfortunately, it is impossible to mention the hundreds who rather incidently gave information; alas, even the names of some important informants are lost. To all of them I tender my heart-felt thanks. Some of their names are:

in the Bima language: Daéng Melai, Dg. Mastua, Mr B. Nembo (Réo); Dg. Balanda, Dg. Pesita, Mr H. Héko (Pota);

in Ende:

Messrs Y. Ngado, W. Wérang;

in Kédang:

Messrs M. Maka, L. Lèu, A. Lalang, A. Pulang, Domi Ai, J. Kalaké, L. Poya, G. Béni Labi, K. Eto Ehaq.

in Kepo':

Messrs H. Tala, P. Bolo, T. Saro, F. Ronggong;

in Komodo:

Messrs Abdulrajab, Mahu and Alo Sahu;

in Lio:

Mrs Veronika Aés, Ms W. Nggana, Messrs A.W. Soré, M. Ulé;

in Ngadha:

Messrs I. Dahus, Kr., Is. and Ruf. Balo, F. Raï Balo, Kl. Kapi; the ladies M. Uwa, M. Sawo, L. Bupu, M. Maza; Mr Y. Taru Waé (So'a);

in Raja:

Mr John Egho Wolo;

in Rembong:

Messrs E. Dadu, A. Nanggé, P. Mangga, Dam. Nda'u, M. Mbaring, A. Nggawang, A. Sipi;

in Rongga:

Messrs Lamb Gasa, Joh Jani, Mark Ndoa, L. Lalu, T. Ndu;

in the Sawu language:

in Sika:

Messrs Lukas Rihi (Melolo), Lorés Wadu;

Messrs Juling Lewuk, F. Juwa, M. Sino, Etm. Mundus, J. Wua, T. Tara, Fr A. Mitan;

in Solor:

Messrs A. Sabon, M. Juang Sogé, A. Wagét, R.R. Tukan, A. Jogo Balo, A. Tolok Kotén, S. Ratu Héwèn, B. Boli Ujan;

in Sumba: West -

Messrs Kanis Jowa Kanisa (Wewewa), Y. Dowa (Totok), T. Bili Rambi, (We'e Pangali); Fr Boro Wolo (Kodi); 'Matias' (Loura);

East - $\quad$ Messrs K. Windi (Kambera), John Rara Mata (Karera); Ama Rawa (Rindi) Oe. H. Kapita (Kambera). 


\section{APPENDIX II: SYNONYMOUS TAXA}

Immediately below, in alphabetical order are the relevant outdated or facultative synonyms of genera and species, followed by their modern forms. It is hoped in this way to prevent possible misunderstandings and doubts. Also rather common names that are of ten missppelled, such as Areca cathecu or Albizia, are included.

We should be aware that mostly only part of the species out of a genus is renamed, for example from the genus Eugenia one species is left (in Java); all the others, such as the clove-tree (E. aromatica) and rose-apple (E. aquea), have been classed in the genus Syzygium.

\begin{tabular}{ll} 
Abarema & Pithecellobium \\
Achasma & Amomum \\
Achras zapota & Manilkara achras \\
Actinophorafragrans & Schoutenia ovata \\
Afzelia bijuga & Intsia bijuga \\
Albizzia & Albizia \\
Andropogon & Cymbopogon, Sorghum, Heteropogon, Chrysopogon \\
Aneilema & Murdannia \\
Aphanamixis & Ricinocarpodendron \\
Areca catechu & A. cathecu \\
Arenga saccharifera & A.pinnata \\
Artocarpus communis & A. altilis \\
Artocarpus champeden & A. heterophyllus \\
Arundina bambusifolia & A. graminifolia \\
Bambusa spinosa & B. blumeana \\
Bauhinia & Piliostigma, Lysiphyllum \\
Bischoffia & Bischofia \\
Borassus flabbellifer & B. sundaicus \\
Brucea amarissima & Br.javanica \\
Buddleia & Buddleja \\
Canarium commune & C. vulgare \\
Canna indica & C. coccinea \\
Cathormium umbellatum & Pithecellobium umbellatum \\
Cedrela & Toona \\
Clerodendron & Clerodendrum \\
Cordyline terminalis & C. fruticosa \\
Corypha gebanga & C. utan \\
Cudrania javanensis & Maclura cochinchinensis \\
Datura fastuosa & D. metel \\
Dioscorea esculenta (partly) & D. aculeata (partly) \\
Dracontomelum & Dracontomelon \\
\hline
\end{tabular}


Dracontomelum-lon mangiferum

Dracontomelum-lon puberulum

Erianthus arundinaceus

Ervatonia

Eugenia

Flemingia

Flewrya

Glycine max

Gossampinus heptaphylla

Gyrinopsis

Heleocharis

Hydrocotyle

Homalanthus

Inocarpus edulis

Jussiaea

Justicia

Kyllinga monocephala

Lagenaria leucantha

Laportea

Leucaena glauca

Litsea

Lochnera

Ludwigia pubescens

Luffa cylindrica

Mezoneurum

Moringa oleifera

Musa glauca

Nauclea

Nipa

Nothopanax

Oldenlandia

Paederia foetida

Pagiantha sphaerocarpa

Panicum

Pennisetum glaucum

Pisonia excelsa

Pithecellobium saman

Pollinia

Pygeum

Randia
Dracontomelon edule

Dracontomelon edule

Saccharum anundinaceum

Pagiantha; Tabernaemontana

Syzygium

Moghania

Laportea

Gl. soja

Bombax ceiba

Gyrinops

Eleocharis

Centella

Omalanthus (disputed)

I. fagiferus

Ludwigia

Gendarussa, Graptophyllum, Peristrophe

Cyperus kyllingia

Lagenaria siceraria

Dendrocnide

L. leucocephala

Neolitsea

Catharanthus

L. octovalvis

L. aegyptiaca

Mezoneuron

M. pterygosperma

Ensete glaucum

Neonauclea

Nypa

Polyscias

Hedyotis

$P$. scandens

Ervatamia sphaerocarpa

Brachiaria, Pennisetum, Setaria

$P$. spicatum

$P$. umbelliflora

Somanea saman

Eulalia

Prunus

Oxyceros 


$\begin{array}{ll}\text { Rhizophora conjugata } & \text { R. apiculata } \\ \text { Rhyssopteris } & \text { Ryssopterys } \\ \text { Salmalia } & \text { Bombax } \\ \text { Spilanthes acmella } & \text { Sp. iabadicensis } \\ \text { Sterculia horsfieldii } & \text { Pterygota horsfieldii } \\ \text { Stramonium } & \text { Datura } \\ \text { Strobilanthes } & \text { ?Sericocalyx } \\ \text { Tabenaemontana } & \text { Ervatamia, Pagiantha } \\ \text { Vitis } & \text { Cayratia, Cissus, Tetrastigma, Ampelocissus } \\ \text { Zizyphus } & \text { Ziziphus }\end{array}$




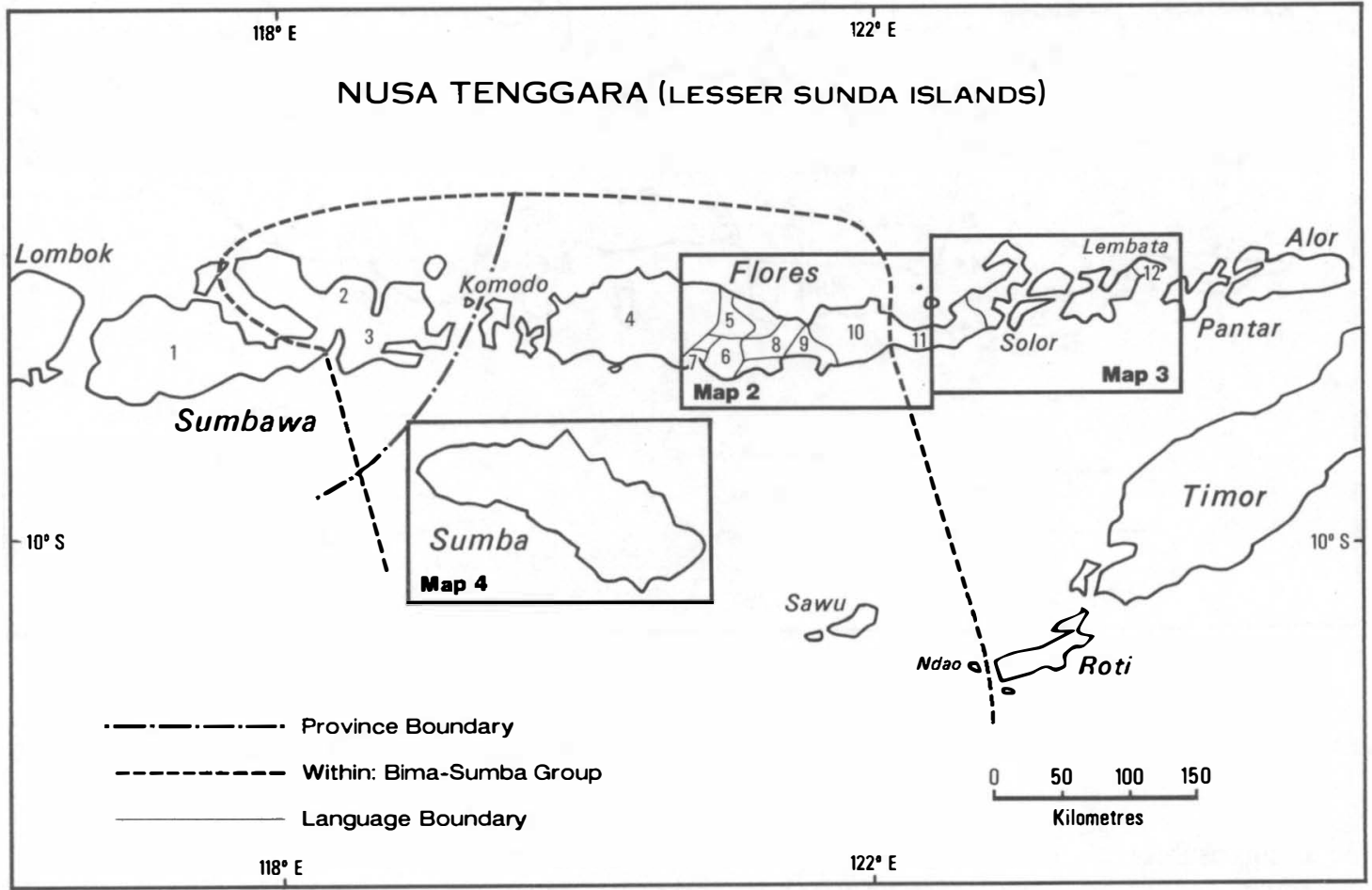

1. Sumbawa Besar

2. Sanggar

3. Bima

4. Manggarai
5. Rembong

6. Ngadha

7. Rongga

8. Nagé
9. Endé

10. Lio

11. Sika

12. Kédang

\section{MAP 1}




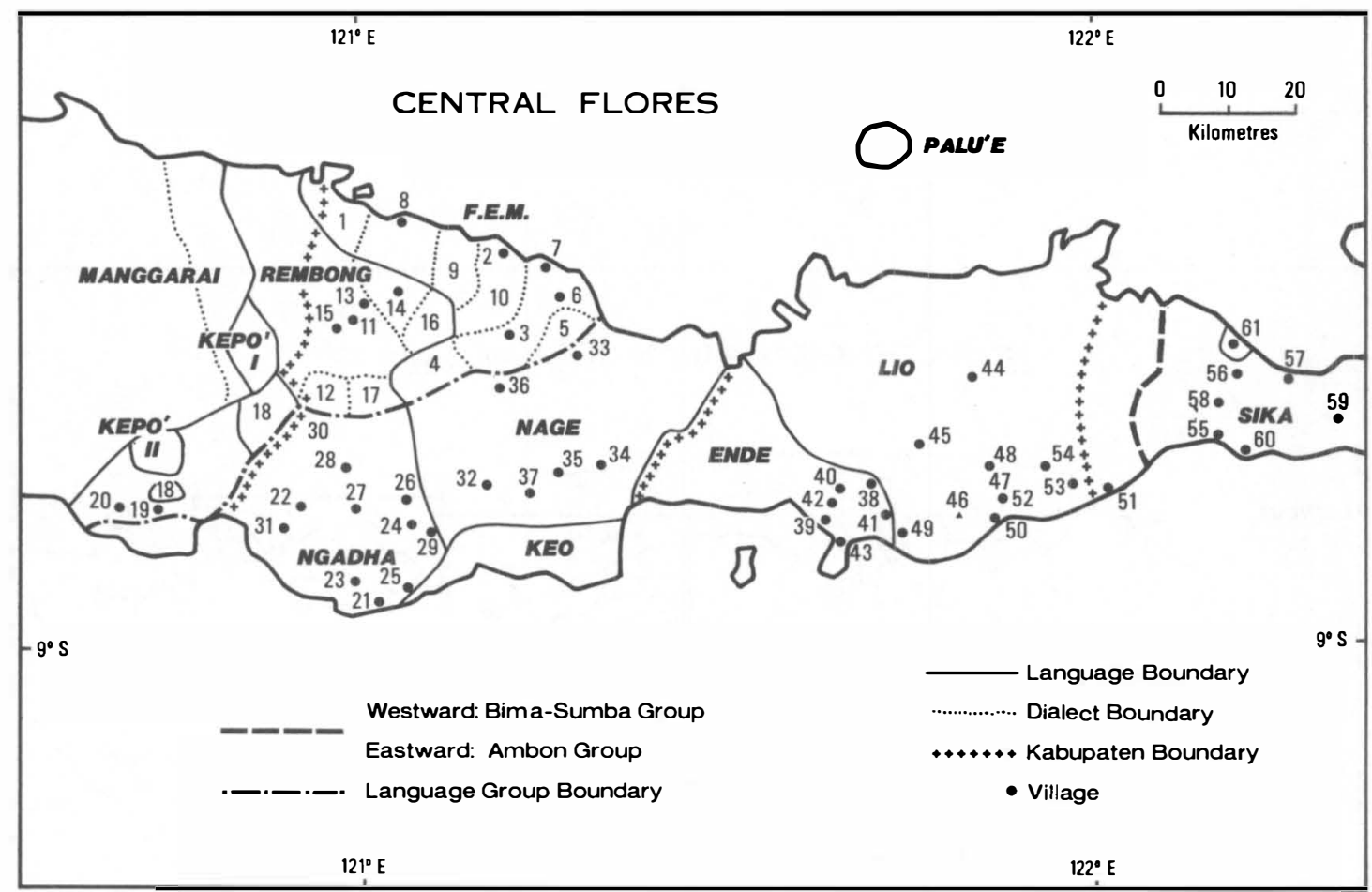

FEM Far-east Manggarai

1. Bar

2. Békék

3. Lengkosambi

4. Mulu

5. Mbai

6. Nggolonio

7. Nanganumba

8. Riung

9. Tado

10. Toring

(Manggarai Group)

KEPO'

REMBONG

11. Faté

12. Namu

13. Ri'a
14. Térong

15. Warukia

16. Wa(ng)ka

17. Wué

18. RAZONG

19. WAÉRANA

20. Kisol

NGADHA

21. Boba

22. Bajawa

23. Jérébu'u

24. Laja

25. Ma'ubawa

26. Mataloko

27. Manguléwa

28. So'a

29. Takatunga

30. Tanawolo
31. Watujaji

NAGE

32. Bo'awaé

33. Danga

34. Ndora

35. Raja

36. Tédamudé

37. Wudu

ENDÉ

38. Lianggéré

39. Mbomba

40. Nuabosi

41. Roworéké

42. Watusipi

43. Endé

LO

44. Detukéli

45. Detusoko
46. Kéli Mutu (volcano)

47. Lisé

48. Moni

49. Ndona

50. Nggéla

51. Paga

52. Wolojita

53. Watunéso

54. Wolowaru

SIKA

55. Léla

56. Lédalero

57. Ma'uméré

58. Nita

59. Watublapi

60. Sika

PALU'É

61. Nangahuré 


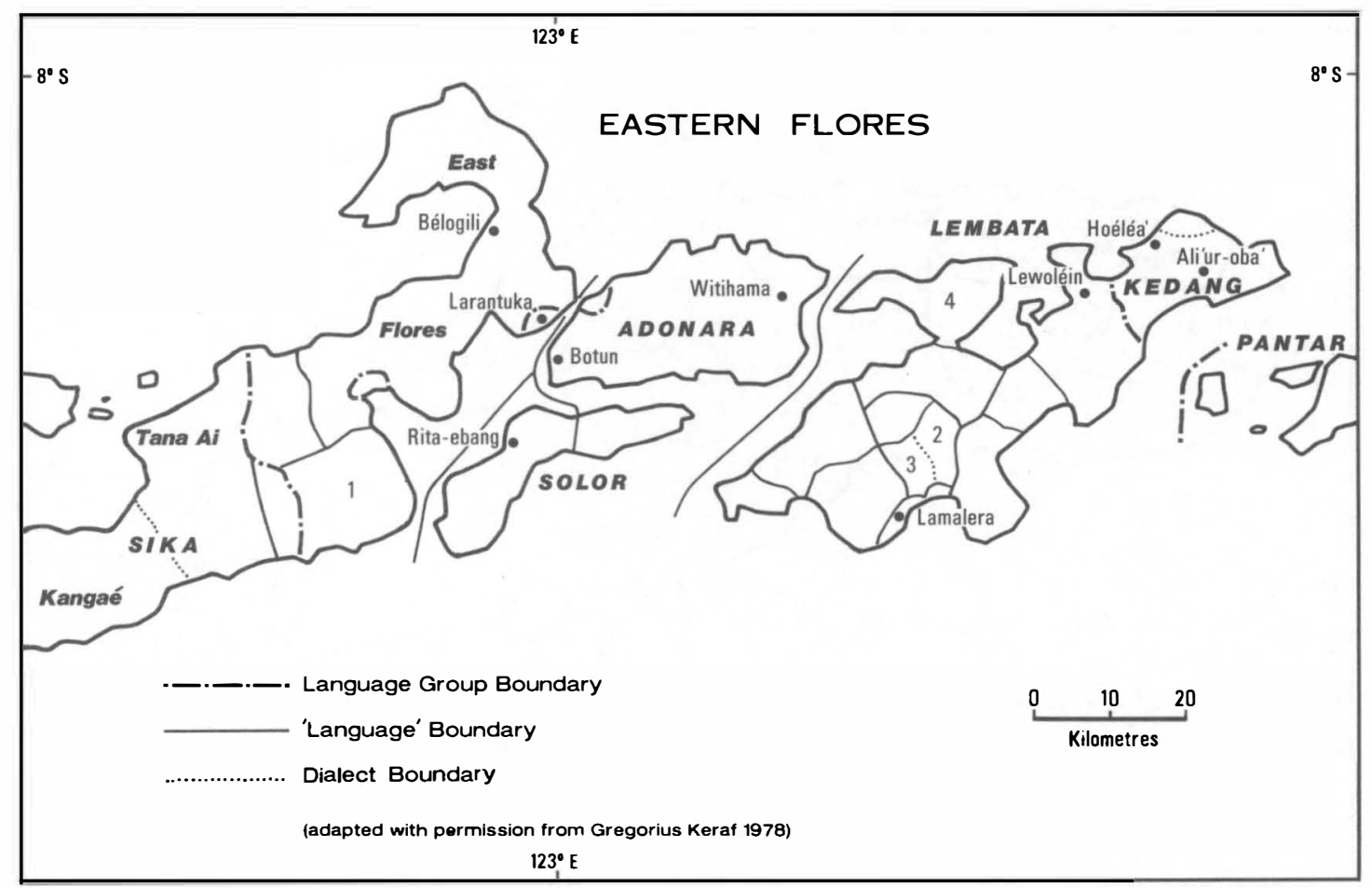
1. Lewotobi
2. Kalikasa
3. Lewuka
4. Ilé Mandiri

MAP 3 


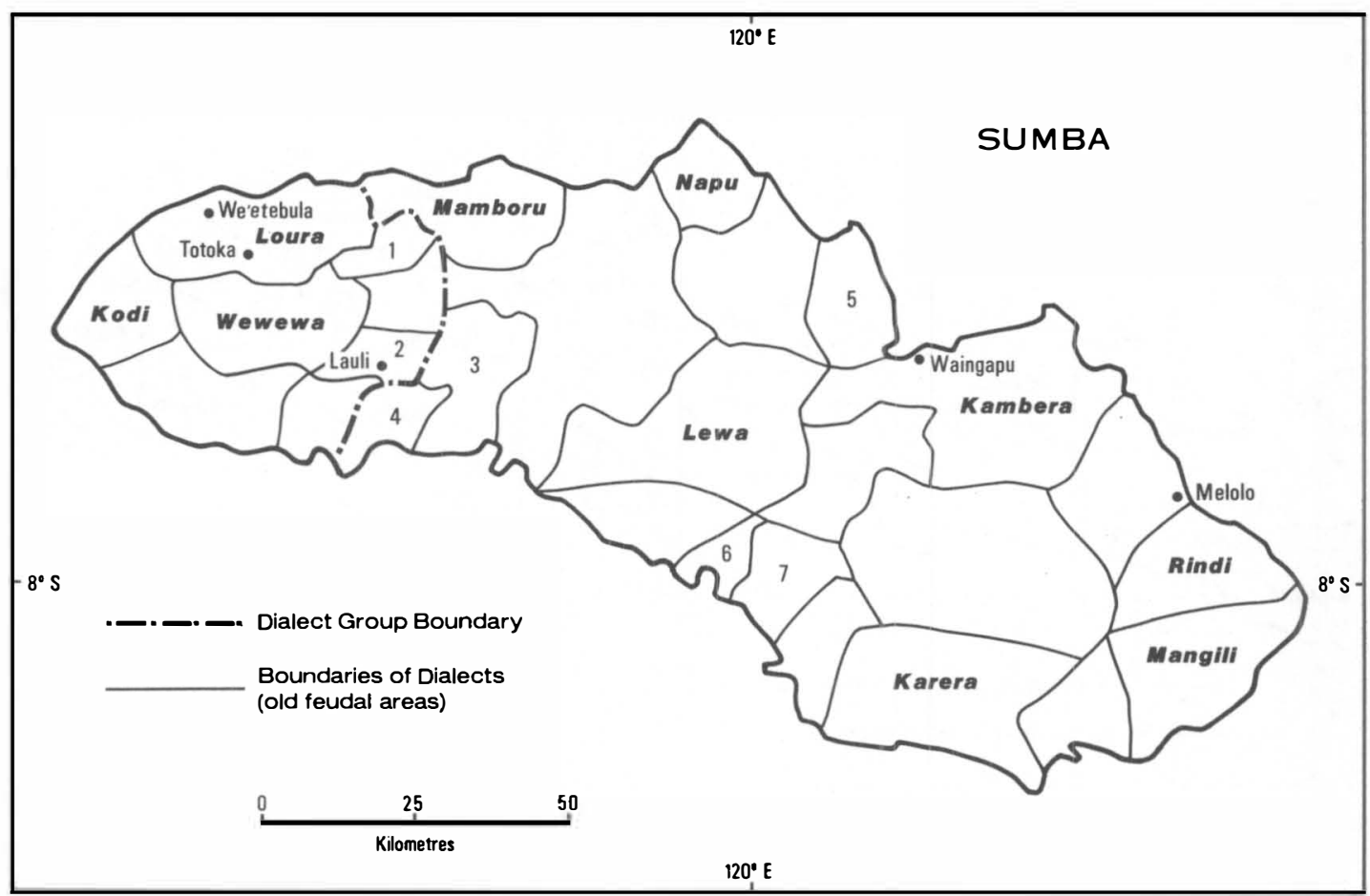

1. Tanarighu

5. Kanatangu

2. Waikabubak

6. Tarimbangu

3. Anakalangu

7. Tabundungu

4. Wanukaka

MAP 4 


\section{BIBLIOGRAPHY}

ARNDT, Paul, 1934,Li'onesisch-Deutsches Wörterbuch. Ende, Flores: Amoldus.

1961, Wörterbuch der Ngadha-Sprache. Fribourg.

BACKER, C.A. and R.C. BAKHUIZEN van den BRINK Jr, 1963-68, Flora of Java, vols I-III. Groningen.

BLUST, Robert, 1980, Austronesian etymologies. Oceanic Linguistics 19:1-181.

BOLSCHER, H., 1985, Tati lalang sara Sika. (A privately stencilled Indonesian-Sika dictionary with a thematic tendency, especially for religious usage, 475pp. + additions.)

CENSE, A.A. with ABDOERRAHIM, 1979, Makassaars-Nederlands woordenboek, met Nederlands-Makassaars register en voorwoord door J. Noorduyn. The Hague.

DAMMERMAN, W., 1926, Soembaneesche planten- end dierennamen. Tijdschrift voor Indische Taal-, Land-en Volkenkunde 66:105-239.

DIRKZWAGER, Margaretha, 1978, Esser lists with vocabularies from Ngadha dialects. MS.

DJAWANAI, Stephanus, 1977, A description of the basic phonology of Nga'da and the treatment of borrowings. In Ignatius Suhamo, ed. NUSA 5:10-18. Jakarta: NUSA.

HEYNE, K., 1950, Nuttige planten van Indonesië (3rd edn.). The Hague/Bandoeng: Van Hoeve.

HILDEBRAND, F.H., 1940,1952, Daftar nama-nama pohon-pohonan 'Timor'. Stencilled. Bogor.

HOEKSTRA, Pieter, 1948, Paardenteelt op het eiland Sumba. PhD Thesis, Batavia: Kappee.

JONKER, J.C.G., 1893, Bimaneesch-Hollandsch woordenboek. VBG 48/1. Batavia/The Hague.

KAPITA, Oemboe H., 1976, Sumba di dalam jangkauan jaman. Waingapu (Jakarta).

1982, Kamus Sumba/Kambera-Indonesia. Waingapu (Ende-Flores).

KERAF, Gregorius, 1978, Morfologi dialek Lamalera. Ende-Flores: Amoldus.

MEYER, Frans, 1937,1946, Sikkaneesch-Nederlandsch woordenboek. (Stencilled from a typewritten copy.)

ONVLEE, L., 1973, Cultuur als antwoord. VKI 66. The Hague: M. Nijhoff.

ONVLEE, L. with Oe.H. KAPITA and P.J. LUIJENDIJK, 1984, Kamberaas(Oost-Soembaas)-Nederlands woordenboek met Nederlands-Kamberaas register. Dordrecht.

SAHIDU, Abdul Karim, 1987, Kamus Bima-Indonesia. Mataram. (mimeograph.)

SCHMUTZ, Erwin, 1977-80, Die Flora der Manggarai, 5 vols. Ruteng (mimeographed private edition).

SUCHTELEN, B.C.C.M.M., 1921, Endeh (Flores). Weltevreden: Papyrus.

VERHEIJEN, J.A.J., 1974-77, Field-notes and vernacular - taxonomic lists of plant names in the languages of

Rongga, Ngadha, Ende, Lio, Sawu, Sumba, and Bima. MS.

1976a, Logat nama-nama tumbuhan. Ilmiah-Indonesia dan Indonesia-llmiah. Sebuah naskah. Ruteng.

1976b, Some data on the avifauna of the island of Roti, Lessser Sunda Islands, Indonesia. Zoölogische Mededelingen

50:1-21.

1977, Bahasa Rembong I: Kamus Rembong-Indonesia. Ruteng. (mimeograph.)

1978, Bahasa Rembong III: Kamus Indonesia-Rembong. Ruteng, Flores. (mimeograph.)

1982a, Dictionary of Manggarai plant names. PL, D-43.

1982b, Komodo: het eiland, het volk en de taal. VKI 96. The Hague.

1984, Plant names in Austronesian linguistics. NUSA 20.

1986-87, Field notes and lists of plant-names in Ngadha, Sika and the Solor achipelago. MS.

1987, Pulau Komodo. Tanah, rakyat, dan bahasanya. Jakarta. (Enlarged Indonesian translation.)

WALKER, Alan T., 1982, A grammar of Sawu. NUSA 13.

WIELENGA, D.K., 1909, Soembaneesche woordenlijst (dialect van Kambera). In: Schets van een Soembaneesche spraakeunst (naar het dialect van Kambera), 145-336. Batavia.

WIJNGAARDEN, J.K., [1896], Sawuneesche woordenlijst. [The Hague.] 
